\title{
DOCUMENTATION OF THE STATUS OF INTERNATIONAL GEOTHERMAL POWER PLANTS AND A LIST, BY COUNTRY, OF SELECTED GEOTHERMALLY ACTIVE GOVERNMENTAL AND PRIVATE SECTOR ENTITIES
}

\section{Topical Report}

October 1992

\section{DISCLAIMER}

This report was prepared as an account of work sponsored by an agency of the United States Government. Neither the United States Government nor any agency thereof, nor any of their employees, makes any warranty, express or implied, or assumes any legal liability or responsibility for the accuracy, completeness, or usefuiness of any information, apparatus, product, or process disclosed, or represents that its use would not infringe privately owned rights. Reference herein to any specific commercial product, process, or service by trade name, trademark, manufacturer, or otherwise does not necessarily constitute or imply its endorsement, recommendation, or favoring by the United States Goverament or any agency thereof. The views and opinions of authors expressed herein do not necessarily state or reflect those of the United States Government or any agency thereof.

Work Performed Under Contract No. DE-FG0T-89ID12850

For

U. S. Department of Energy Office of Industriar Technologies Washington, D.C.

By

National Geothermal Association Davis, CA 95617-1350
,

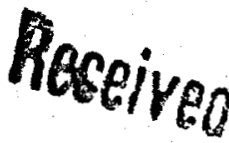

Nov

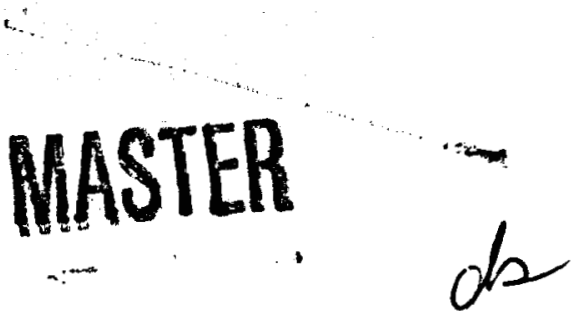




\section{DISCLAIMER}

This report was prepared as an account of work sponsored by an agency of the United States Government. Neither the United States Government nor any agency Thereof, nor any of their employees, makes any warranty, express or implied, or assumes any legal liability or responsibility for the accuracy, completeness, or usefulness of any information, apparatus, product, or process disclosed, or represents that its use would not infringe privately owned rights. Reference herein to any specific commercial product, process, or service by trade name, trademark, manufacturer, or otherwise does not necessarily constitute or imply its endorsement, recommendation, or favoring by the United States Government or any agency thereof. The views and opinions of authors expressed herein do not necessarily state or reflect those of the United States Government or any agency thereof. 


\section{DISCLAIMER}

Portions of this document may be illegible in electronic image products. Images are produced from the best available original document. 
DOE/ID/12850-6

\title{
DOCUMENTATION OF THE STATES OF INTERNATIONAL GEOTHERMAL POWER PLANTS AND A LIST, BY COUNTRY, OF SELECTED GEOTHERMALLY ACTIVE GOVERNMENTAL AND PRIVATE SECTOR ENTITIES
}

Topical Report

October 1992

Work Performed Under Contract No. DE-FG07-89ID12850

\author{
Prepared for the \\ U.S. Department of Energy \\ Under DOE Idaho Field Office \\ Sponsored by the Office of the Assistant Secretary \\ for Conservation and Renewable Energy \\ Office of Industrial Technologies \\ Hashington, D.C. \\ Prepared by \\ National Geothermal Association \\ P. 0. BoX 1350 \\ Davis, CA $95617-1350$
}




\section{CONTENTS}

\section{section}

Tab Number

Introduction $\ldots \ldots \ldots \ldots \ldots \ldots \ldots \ldots \ldots \ldots \ldots \ldots \ldots \ldots$

1985 Geothermal status Reports .............. 2

1990 Geothermal status Reports .............. 3

Explanation of the Abbreviations used in the International Geothermal Power Plant Data Base .. 4

International Geothermal Power Plant Data Base (including USA installations) $\ldots \ldots \ldots \ldots \ldots \ldots \ldots \ldots$

Geothermally Active Entity Data Base .......... 6

Maps of Geothermal Installations, by country .... 7

Data Base Questionaires and Mailing Lists ....... 8

ngadb.int 


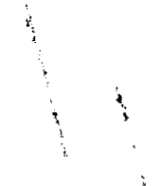

\section{INTRODUCTION}




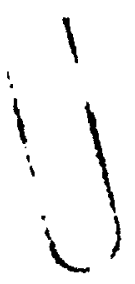

DOCUMENTATION OF THE STATUS OF INTERNATIONAL GEOTHERMAL POWER PLANTS AND A LISTING, BY COUNTRY, OF SELECTED GEOTHERMALLY ACTIVE GOVERIMENTAL AND PRIVATE SECTOR ENTITIES

\section{Introduction}

Steam; produced as a result of the exploitation of geothermal energy resources, has been used to fuel electric power generation facilities since 1904 when dry steam from the fields at Lardarello, Italy was first harnessed. Since then, and especially since the decade of the 1950s, energy from a growing number of geothermal fields has been put to commercial use internationally. of significant importance has been the growth in exploitation of geothermal resources that are liquid dominated, of moderate temperature and whose energy can be tapped using Binary power cycles.

since 1973, when the first Arab oil embargo shocked the world with its dramatic effect on petroleum prices, there has been increasing, if somewhat episodic, international awareness of the need to decrease dependance on petroleum as a fuel source for power generation for economic and environmental reasons. Accordingly, exploration and development of geothermal resources has increased significantly.

Traditionally, power generation facilities have been built by national governments pursuant to their mandates to provide infrastructure for the needs of their citizens. Recently, many developing countries have fallen upon difficult economic times, with very large debt burdens and little cash with which to build, enlarge and/or improve their much needed power systems. This situation has opened windows of opportunity for infrastructure investment by private entities previously precluded from ownership of such facilities.

In order for private investors to consider participation in geothermal projects vis-a-vis other investment opportunities, reliable, up-to-date information must be readily available regarding technical aspects of the geothermal facilities in place internationally. There should 
also be one source of information regarding the identities of the agencies responsible for geothermal development in each country and the names and telephone/FAX numbers of the people currently in charge.

In late 1989, the National Geothermal Association recognized these needs, submitted a proposal to the U. S. Department of Energy, and received funding under Grant No. DE-FG0789ID12850 to develop a database containing the information described above. The document that follows is the first edition of what is planned to be a biannually updated series of publications available to interested parties through the National Geothermal Association.

Included, as a prologue to this document, are reproductions of two articles, written in 1985 and in 1990, to document the status of international geothermal exploration and development. These papers, published in the Transactions and Bulletins of the Geothermal Resources Council, are intended to give readers significant background information relevant to the data presented in the main body of the database.

The data bases presented below have been compiled using "PC-File", Version 5.0, (Copyright 1982-1990 by Jim Button and Copyright 1984-1990 by ButtonWare, Inc. All Rights Reserved). The software is designed for use on IBMcompatible computers and the format allows import and export of data to most other data base programs. PC-File is especially compatible with the several versions of "dBASE".

The PC-File subdirectory contains about 1.22 MB of information as of June 1991, therefore a user requiring the raw data should have available at least this much memory to begin with. The current formats are designed for use with a Hewlett Packard LaserJet III printer, however any reasonably modern printer can be substituted with minor code modifications.

GWH

June, 1991

ngadb.int 
1990 GEOTHERMAL STATUS REPORTS

reprints ensued. 
EXPLANATION OF ABBREV. IN INTERN'L

GEOTHERMAL POWER PLANT DATABASE 
EXPLANATION OF THE ABBREVIATIONS USED IN THE DATA BASE FOR INTERNATIONAL POWER PLANTS

PP Name

PP site

status

operDate

Nation

subDivision

Res Owner

Res Devel

$A$ and $\mathbf{E}$

Pp Owner

Turb source

PwrPurchaser

Ppcontractor

PPownrcntact
The name of the Power Plant.

The name of the city nearest the power plant. $\mathrm{P}=\mathrm{Planned}, \mathrm{O}=$ operating, $\mathrm{U}=$ Under construction, $\mathrm{N}=$ Non-operating, $\mathrm{D}=$ Decommissioned.

Date that the power purchaser accepted power on a firm basis. (mm/dd/yy)

The name of the country in which the power plant is located.

The political subdivision of the nation in which the power plant is located.

The name of the geothermal resource owner.

The name of the entity that has leased, or has a concession or that is developing the geothermal resource.

The name of the firm responsible for design of the power plant.

The name of the owner of the power plant.

The name of the manufacturer or supplier of the turbine.

The name of the entity purchasing the produced power, steam or hot water.

The name of the entity responsible for construction of the power plant.

The name of the person to contact for information related to this power plant. 
His Employer The name of the entity employing the power plant contact person.

His Phone The telephone number of the power plant contact person.

His Fax The Telefacsimile (FAX) number of the power plant contact person.

His TELEX

The TELEX alphanumeric code for the power plant contact person.

Gross Mw

The Gross power that the power plant was designed to produce on an average.

Net Mw

Totwellprod

TDS

The Net power that the power plant was designed to deliver on an average.

The total production of steam and/or hot water from all wells used to generate power in thousands of pounds per hour.

The Total Dissolved Solids content of the fluids produced as of the operation date in PPM (Parts per Miliion).

PP Iifetime The economic life span over which the power plant was designed to function.

Inlet pres The initial design inlet pressure as measured at the outlet of the gathering system in psig (Pounds per square inch gauge)

Initrestemp The initial temperature of the geothermal reservoir as measured at depth in degrees $F$.

Totexplcost An approximation of the total exploration costs associated with the identification and characterization of the geothermal resource used to generate power in millions of \$us.

Av WeIl cost The average cost of completed production, injection and standby wells, not including pumps, in millions of \$US. 
subsurf Area The approximate subsurface area, in square kilometers, if directional wells extensions are considered.

Tot PP cost Total cost of the power plant, including the "switch yard", but not including the transmission line, in millions of \$US.

TotProjcost Total cost (equity and debt) of the project, without including the transmission line, in millions of \$US.

Availability The number of hours that the power plant was available to produce power during the most recent year for which data is recorded as divided by 8760 and multiplied by 100 .

Yr. of Avail The year for which the availability percent was calculated.

PPCycletype Dry Steam, Single Flash, Double Flash or Binary cycles.

Inlet Temp. The temperature of the resource at the point where it enters the turbine in degrees $F$.

Av well Depth The average depth of all used production, injection and stand-by wells in feet.

No. Prodwells The number of production wells needed to fully supply resource to the power plant without considering stand-by wells.

No. Injwells The number of injection wells needed to dispose of fluids at full power plant operation, without considering stand-by wells.

Fall_Abdwells The number of non-producable exploration wells plus production, injection and standby wells unsuccessfully drilled or abandoned for the power plant in question.

Total Wells The total number of wells able to function as per design for the beneficial use of the power plant in question. 
R_C sludge The number of Tons produced per month by a Reactor-clarifier, if one is in use.

Financorganiz The name of the organization that took the lead in providing funds for the power plant.

Pwrsaleprice The average price, in sus per month, received for electricity generated by the power plant in 1989.

Ppcmpltnyear The year in which construction of the power plant was completed.

PPshutDownyr The year in which the power plant stopped all operations and was closed.

Genrtrsource The name of the manufacturer or supplier of the generator.

Resownrcntact The name of the person to contact for information regarding the geothermal resource.

His Employer The name of the entity employing the resource contact person.

His Phone The telephone number of the resource contact person.

His FAX The telefacsimile (FAX) number of the resource contact person.

His TELEX The TELEX alphanumeric code for the resource contact person.

PP Operator The name of the power plant operator, in case that it is not the same as that of the owner.

Gathsystcost The cost of the fluid gathering and disposal systems for both production and injection, in millions of \$US.

Av Well Life The average number of years that the wells function properly with normal maintenance. 
H2s sludge The tons per month of sludge produced if an $\mathrm{H}_{2} \mathrm{~S}$ scrubber is used.

Power sales The number of Kilowatt hours of power sold in 1989 (or another designated year).

PowersalesYr The year for which the number of Kilowatts sold was recorded (see Power Sales).

InitialTotNCG The weight percent of the total amount of NonCondensible Gasses extracted from the produced resources when the power plant first went into operation.

Initial $\cos$ The total weight percent of carbon dioxide produced initially.

Initial H2s The total weight percent of hydrogen sulfide produced initially.

CurrentrotNCG The weight percent of the total amount of NonCondensible Gasses extracted from the produced resources in 1989 (or other designated year).

Currentco2 The total weight percent of carbon dioxide produced in 1989 (or other designated year).

currenti2s The total weight percent of hydrogen sulfide produced in 1989 (or other designated year).

NcGTrtmtsyst The name or type of system used to treat NonCondensible Gasses entrained in the resource

NOTES:

1. The unavailability of information is shown by either $A$ BLANR SPACE or a "o".

2. N/A means "Not Applicable"

ngaabrv.exp 


\section{CHINA}




\begin{tabular}{|c|c|c|c|}
\hline $\begin{array}{l}\text { PP Name } \\
\text { PP site } \\
\text { Status } \\
\text { OperDate } \\
\text { Nation } \\
\text { SubDivision } \\
\text { Res Owner } \\
\text { Res Devel } \\
\text { A and E } \\
\text { PP Owner } \\
\text { Turb Source } \\
\text { PwrPurchaser } \\
\text { PPContractor } \\
\text { PPOwnrCntact } \\
\text { His Employer } \\
\text { His Phone } \\
\text { His Fax } \\
\text { His TELEX } \\
\text { oss Mw } \\
\text { t Mw } \\
\text { IotWellProd } \\
\text { TDS } \\
\text { PP Lifetime } \\
\text { Inlet Pres } \\
\text { InitResTemp } \\
\text { TotExplCost } \\
\text { Av Well Cost } \\
\text { Subsurf Area } \\
\text { Tot PP Cost } \\
\text { TotProjCost } \\
\text { Availability } \\
\text { Yr. of Avail }\end{array}$ & $\begin{array}{l}\text { DENGWU, UNIT } 1 \\
\text { FENGSHUN } \\
0 \text {, /70 } \\
\text { CHIN } \\
\text { GUANGDONG }\end{array}$ & 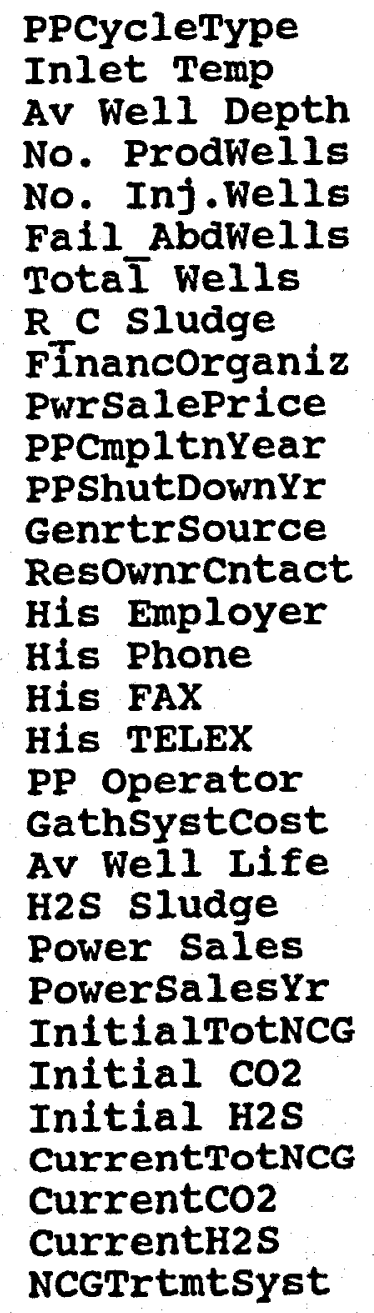 & $\begin{array}{l}\text { o \$US Millions } \\
\text { Years } \\
0 \text { Tons per Month } \\
0 \text { KWH per Year } \\
\text { Weight } \% \\
\text { Weight o } \\
\text { Weight } \% \\
\text { Weight } \% \\
\text { Weight } \% \\
\text { Weight } \%\end{array}$ \\
\hline
\end{tabular}




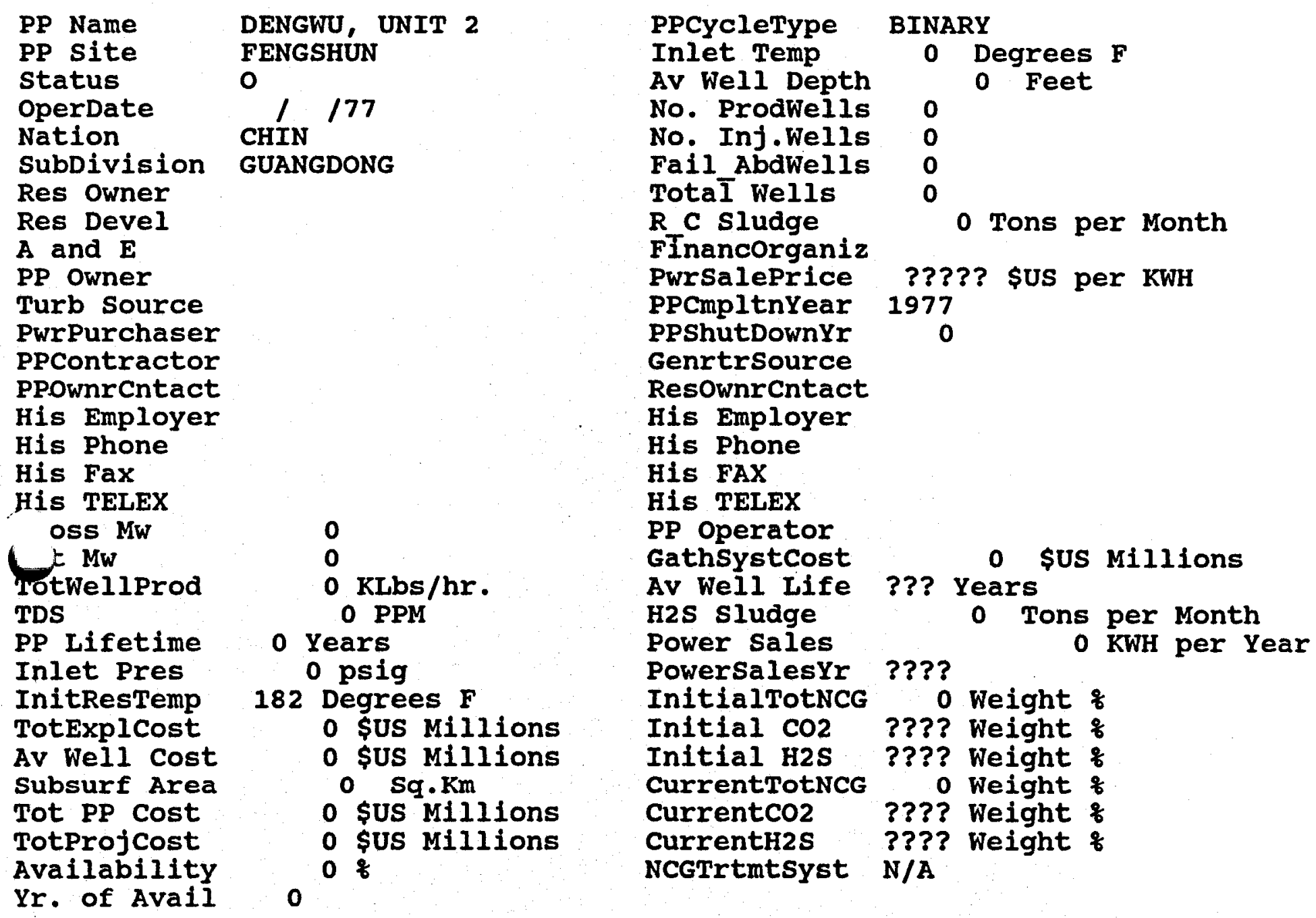




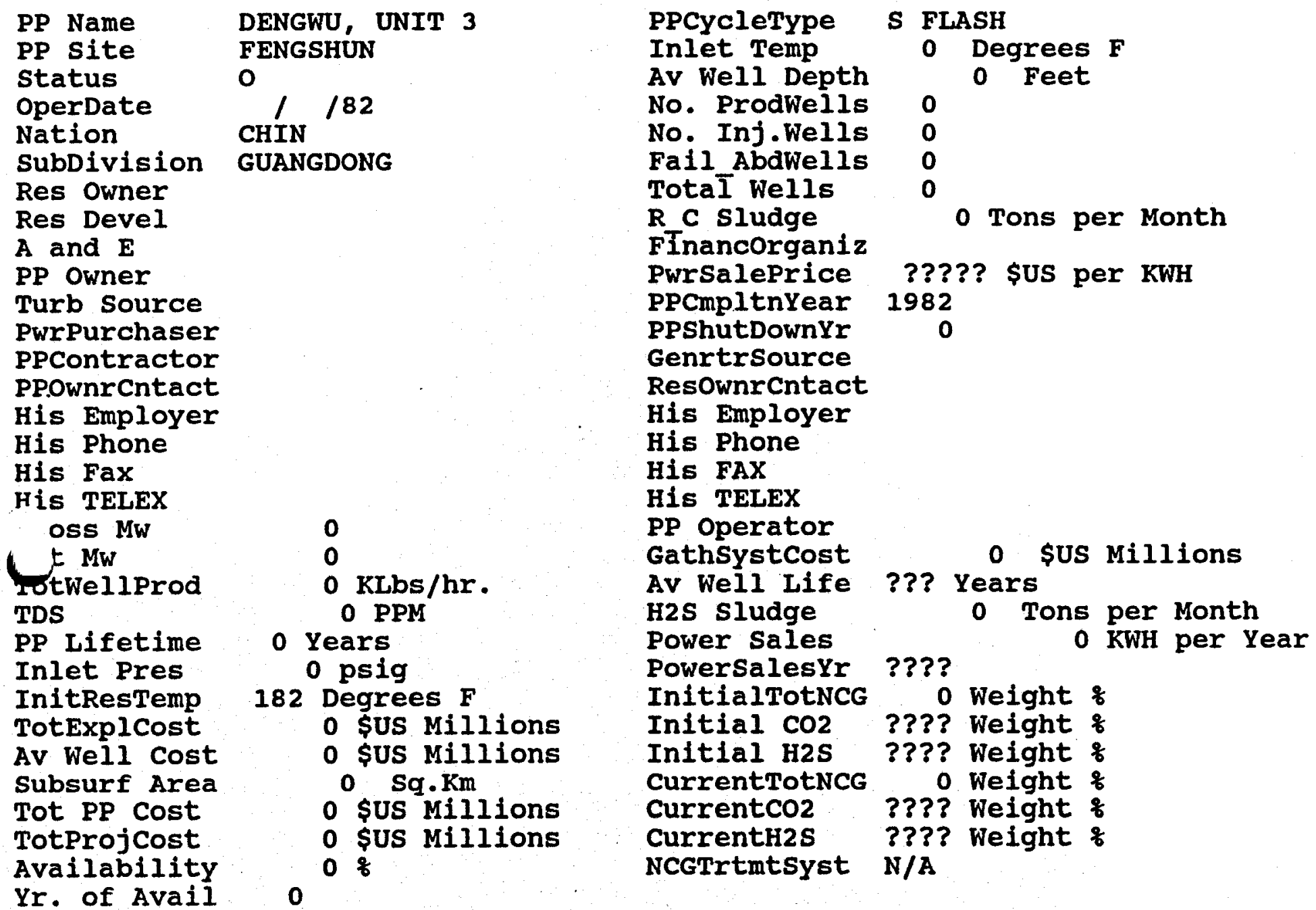




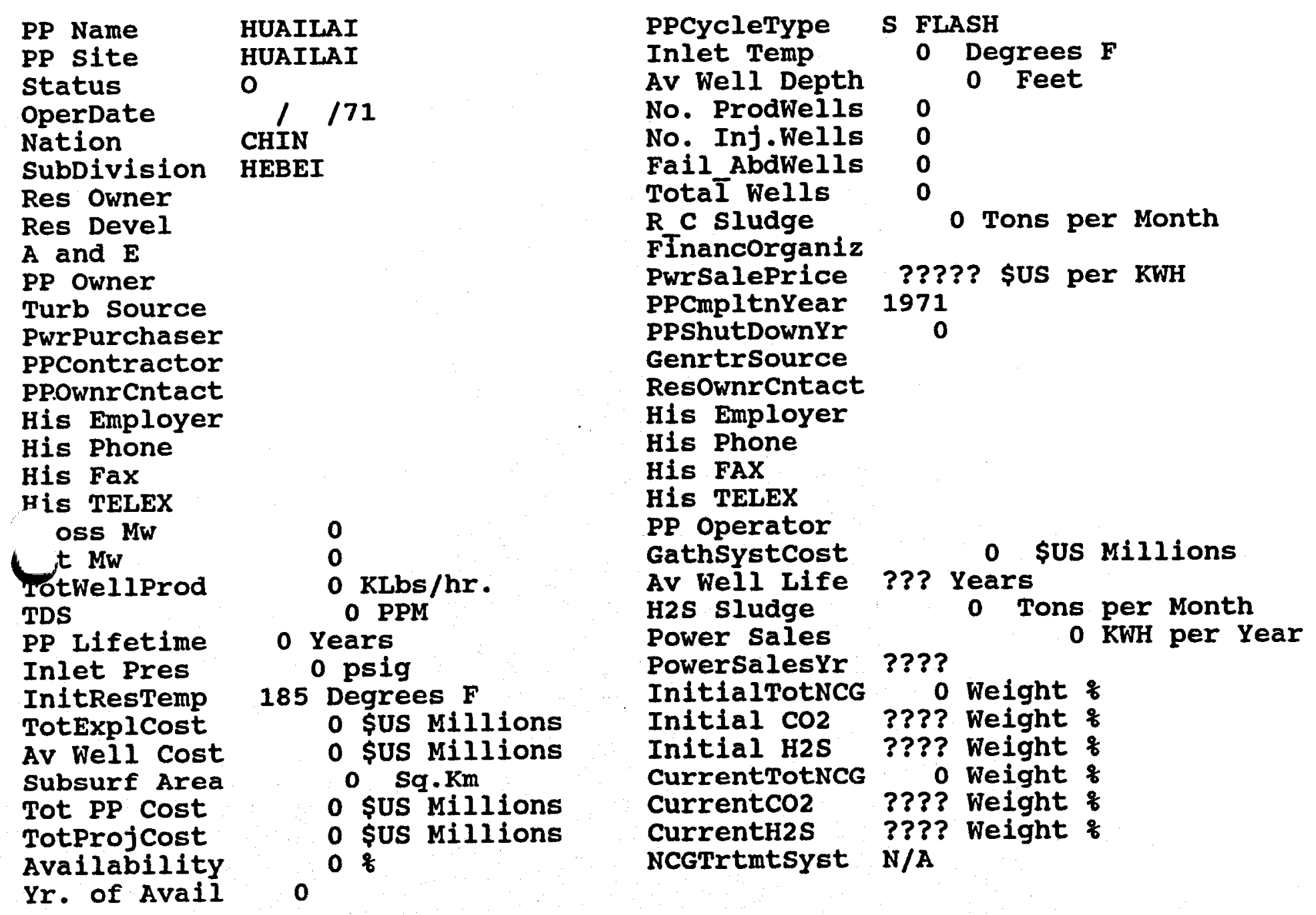




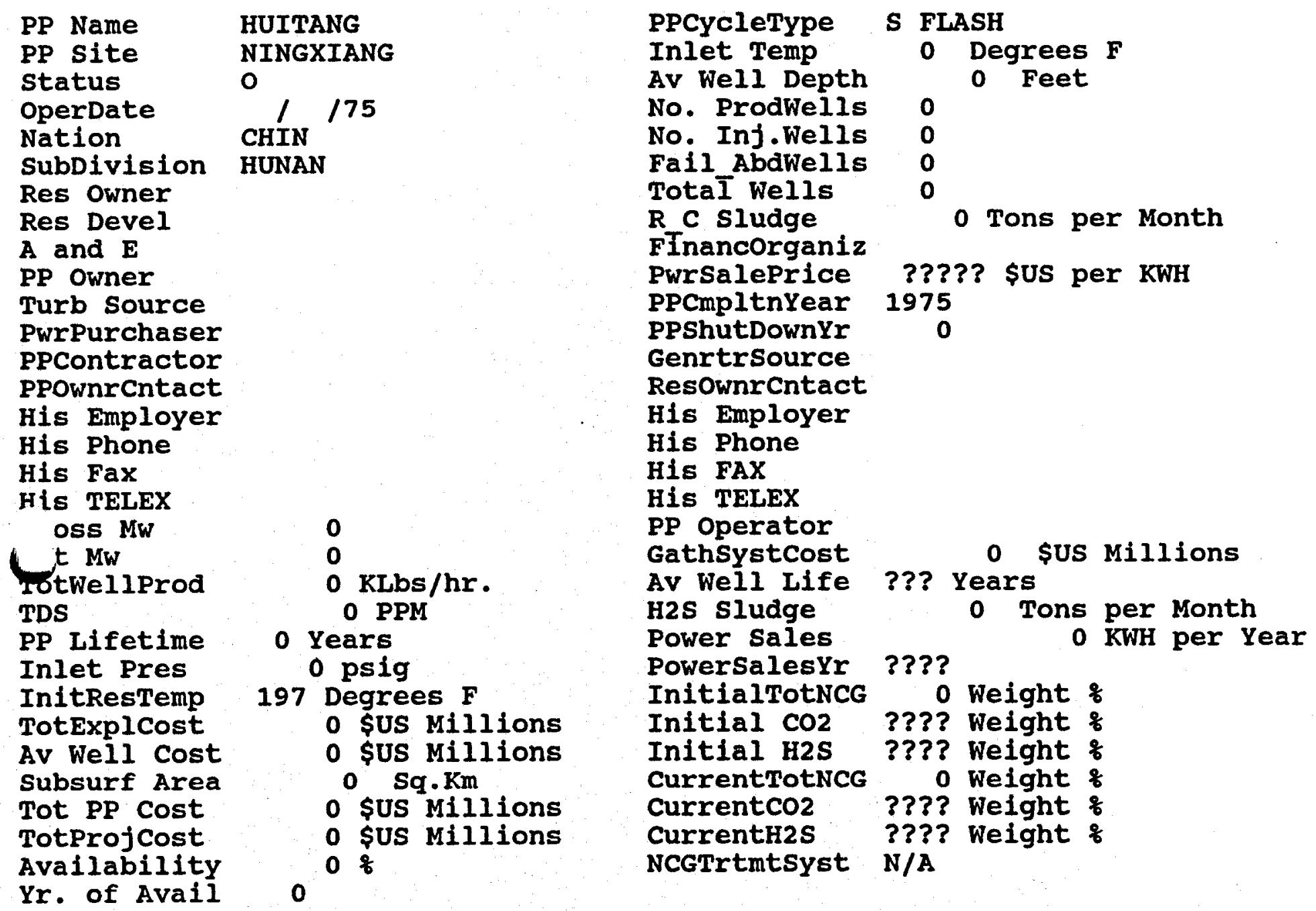




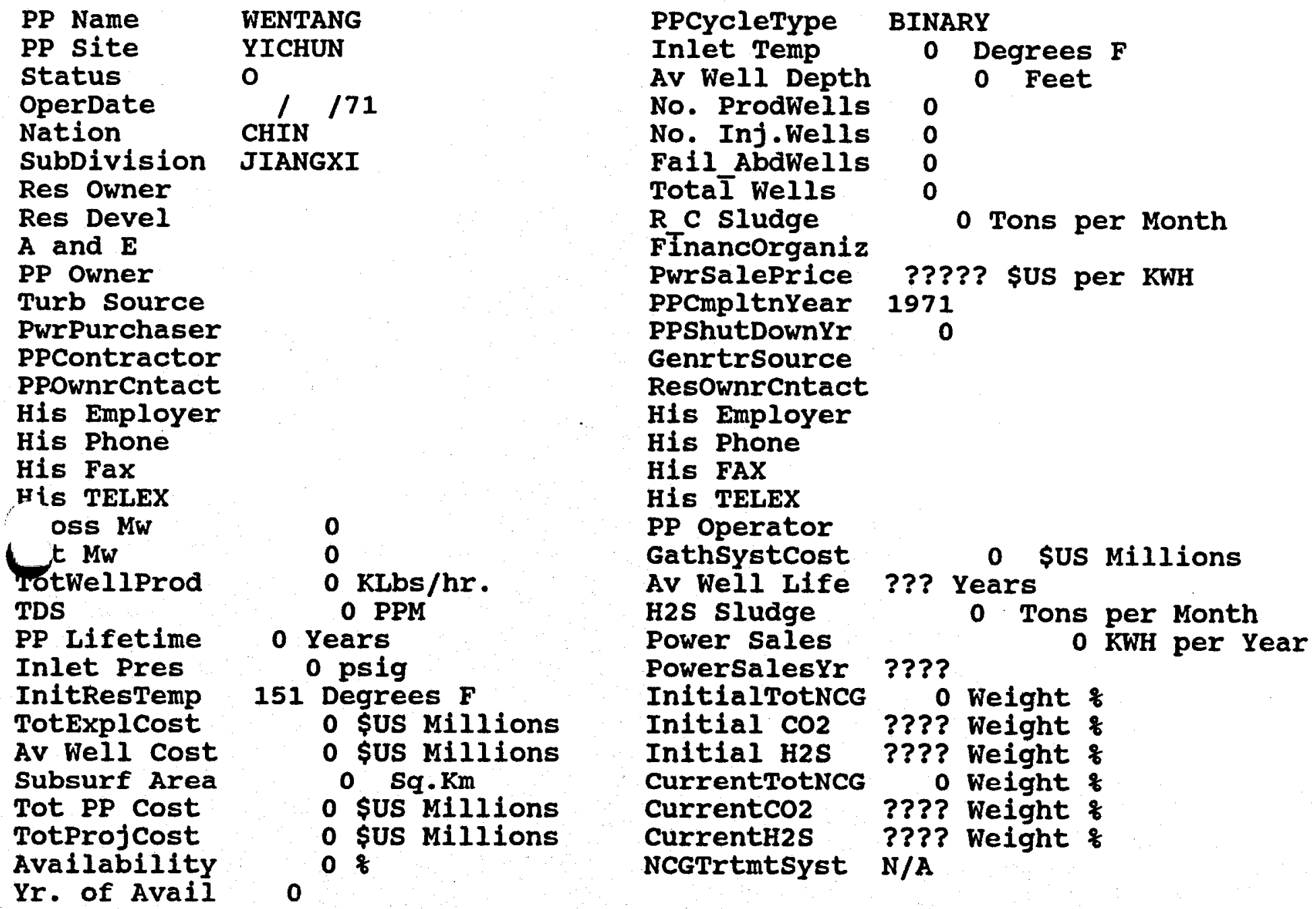




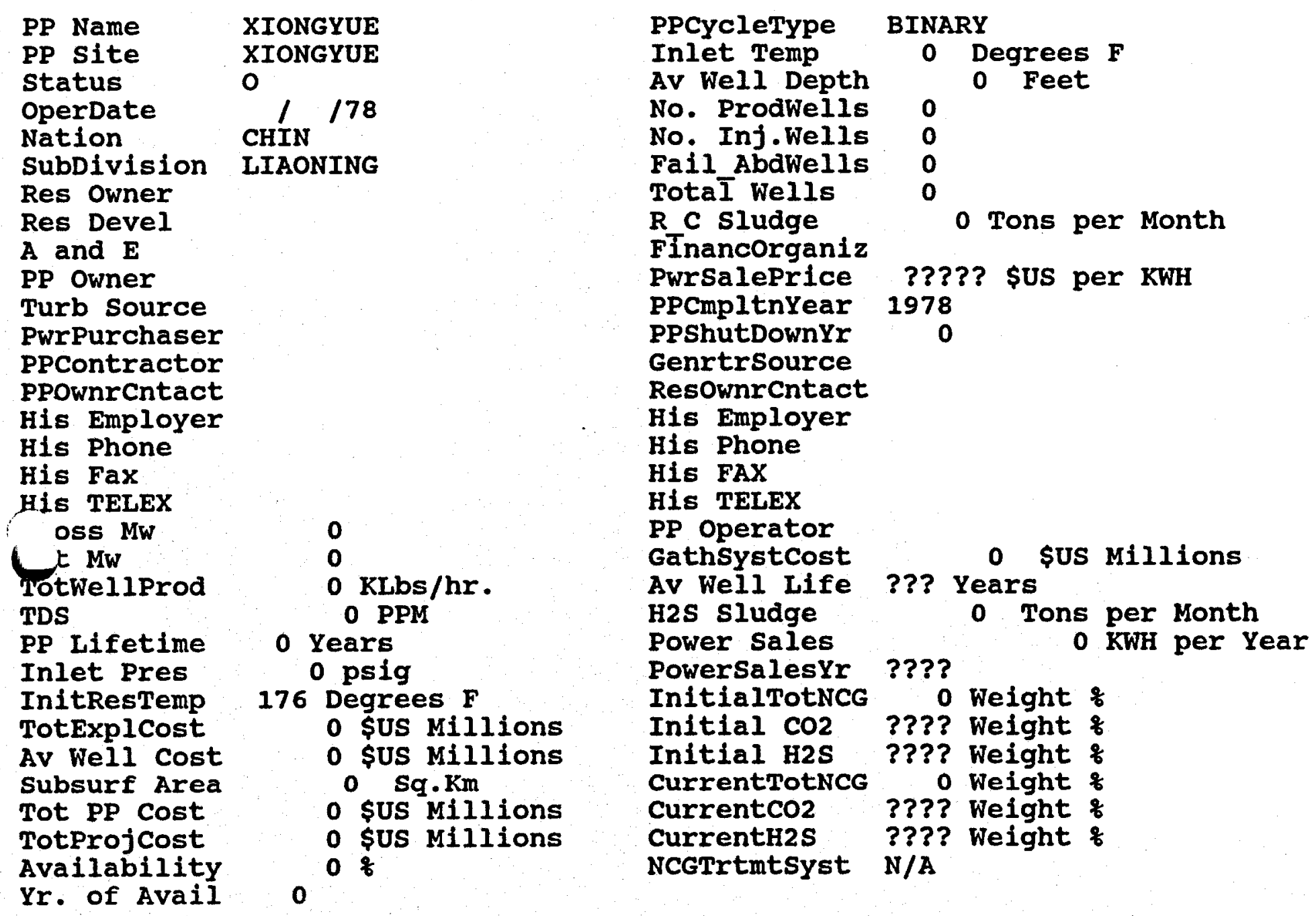




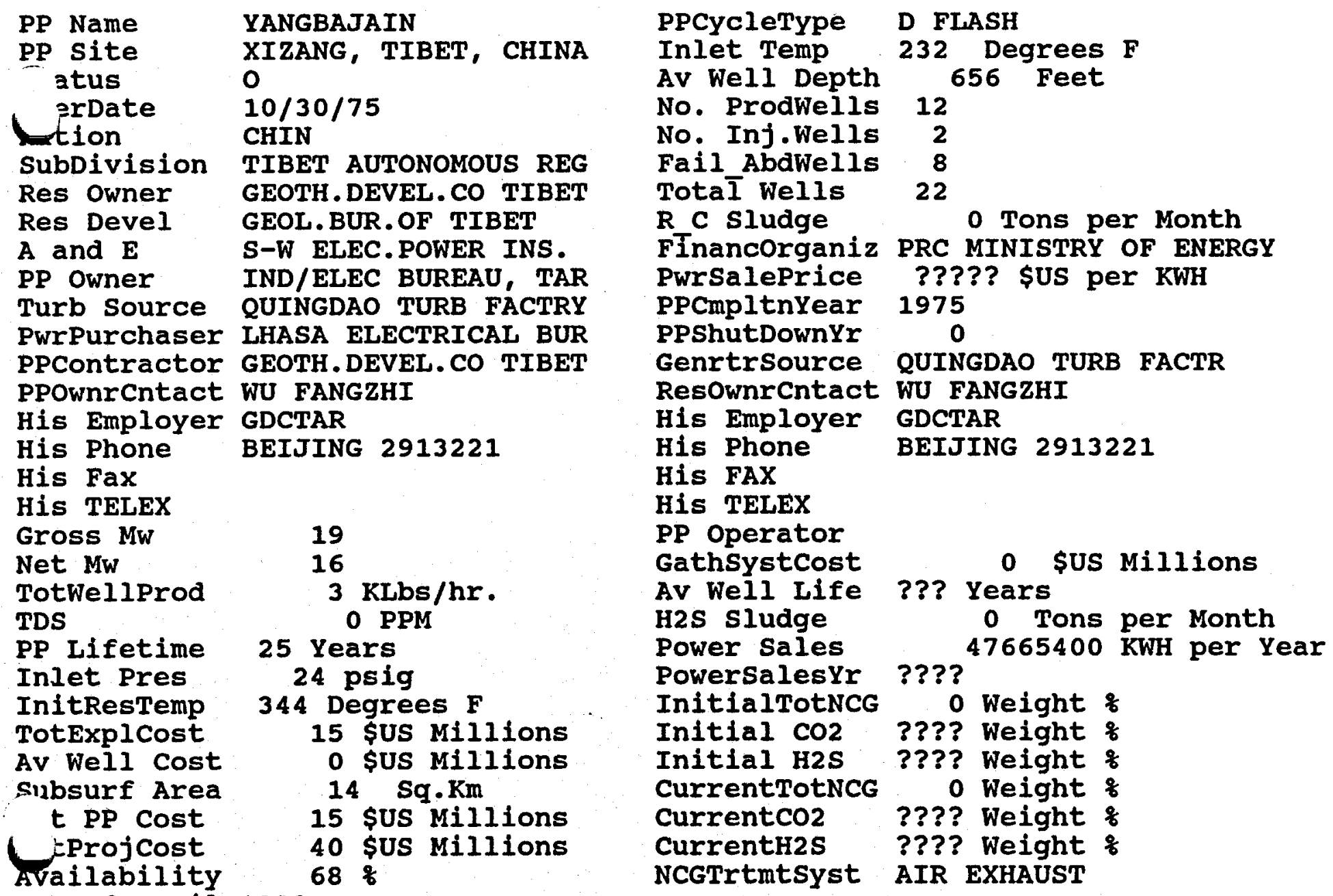




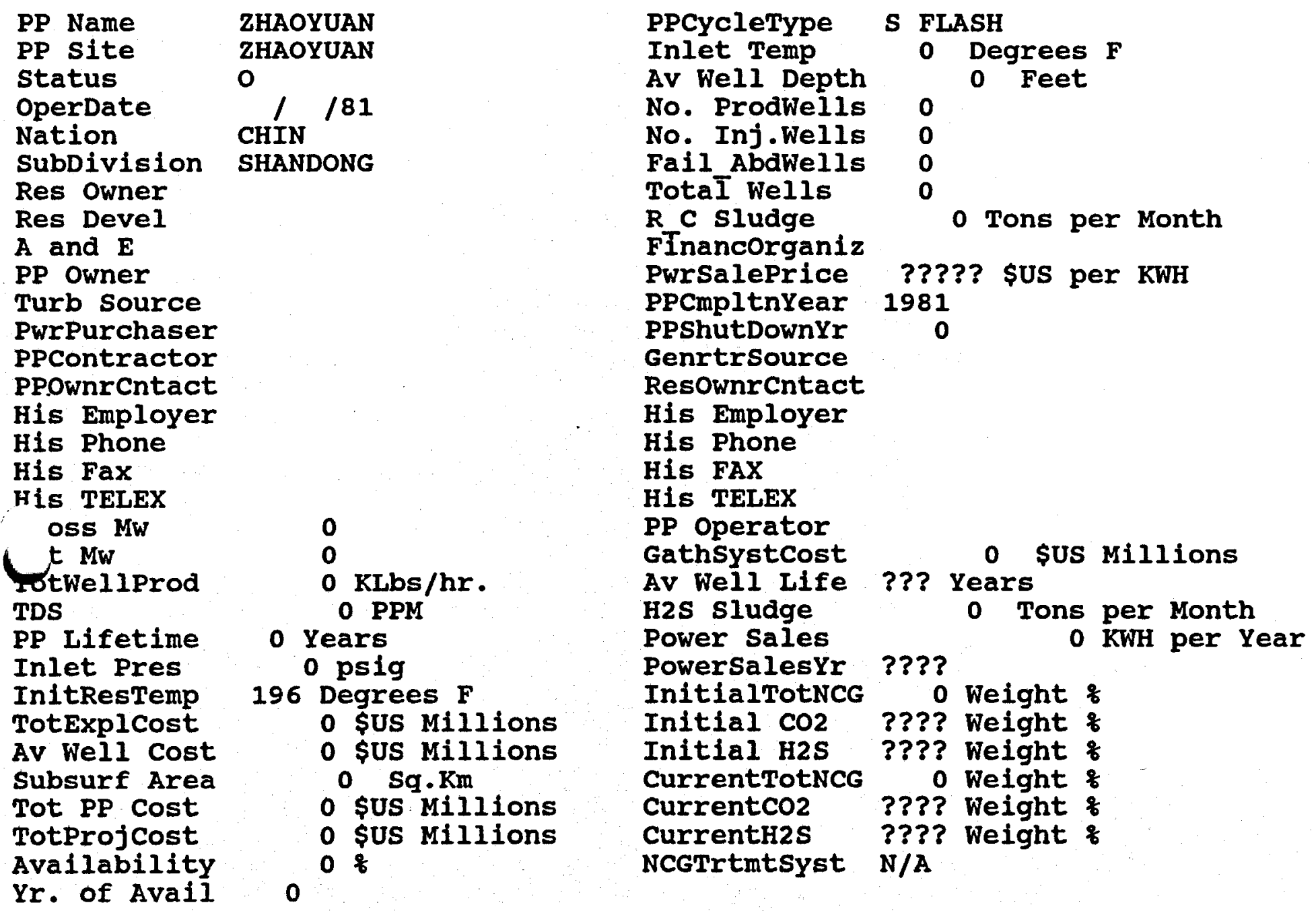


COSTA RICA 


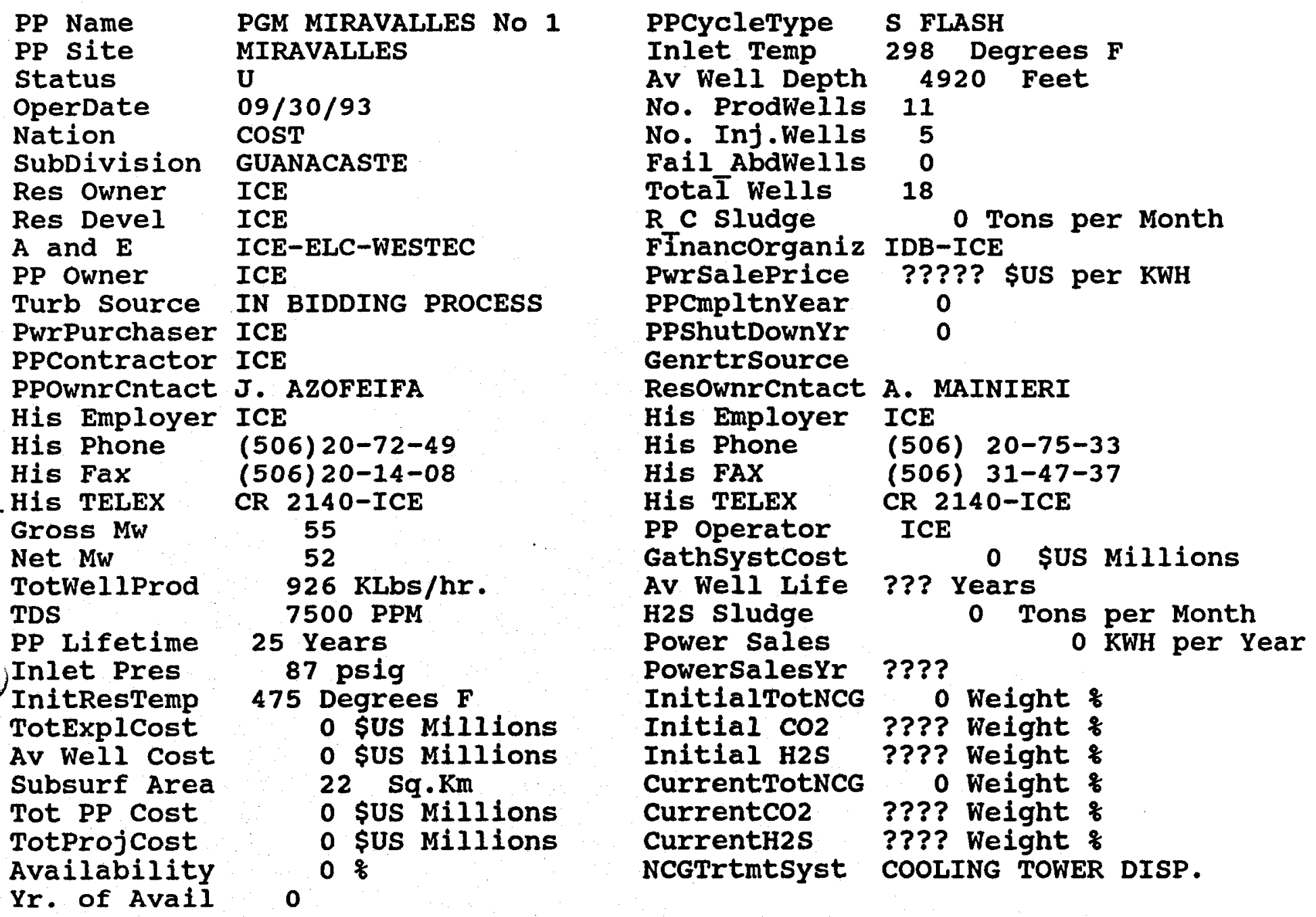




\begin{tabular}{|c|c|c|c|}
\hline 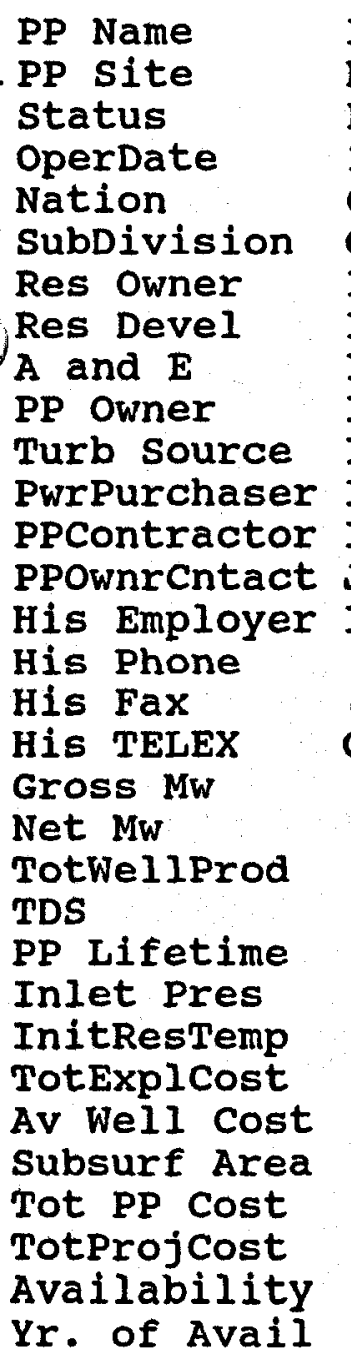 & $\begin{array}{l}\text { PGM MIRAVALLES NO } 2 \\
\text { MIRAVALLES } \\
\text { P } \\
\text { 10/01/95 } \\
\text { COST } \\
\text { GUANACASTE } \\
\text { ICE } \\
\text { ICE } \\
\text { ICE-ELC_WESTEC } \\
\text { ICE } \\
\text { IN BIDDING PROCESS } \\
\text { ICE } \\
\text { ICE } \\
\text { J AZOFEIFA } \\
\text { ICE } \\
(506) 20-72-49 \\
(506) 20-14-08 \\
\text { CR } 2140-I C E \\
55 \\
52 \\
926 \text { KLbs/hr. } \\
7500 \text { PPM } \\
25 \text { Years } \\
87 \text { psig } \\
475 \text { Degrees F } \\
0 \text { \$US Millions } \\
0 \text { \$US Millions } \\
22 \text { Sq.Km } \\
0 \text { \$US Millions } \\
0 \text { \$US Millions } \\
0 \text { \% } \\
\text { a }\end{array}$ & $\begin{array}{l}\text { PPCYcleType } \\
\text { Inlet Temp } \\
\text { Av Well Depth } \\
\text { No. ProdWells } \\
\text { No. Inj. Wells } \\
\text { Fail AbdWells } \\
\text { TotaI Wells } \\
\text { R C sludge } \\
\text { Financorganiz } \\
\text { PWrSalePrice } \\
\text { PPCmpltnYear } \\
\text { PPShutDownYr } \\
\text { GenrtrSource } \\
\text { ResOwnrCntact } \\
\text { His Employer } \\
\text { His Phone } \\
\text { His FAX } \\
\text { His TELEX } \\
\text { PP operator } \\
\text { GathSystCost } \\
\text { Av Well Life } \\
\text { H2S Sludge } \\
\text { Power Sales } \\
\text { PowerSalesYr } \\
\text { InitialTotNCG } \\
\text { Initial CO2 } \\
\text { Initial H2S } \\
\text { CurrentTotNCG } \\
\text { CurrentCO2 } \\
\text { CurrentH2S } \\
\text { NCGTrtmtSyst }\end{array}$ & 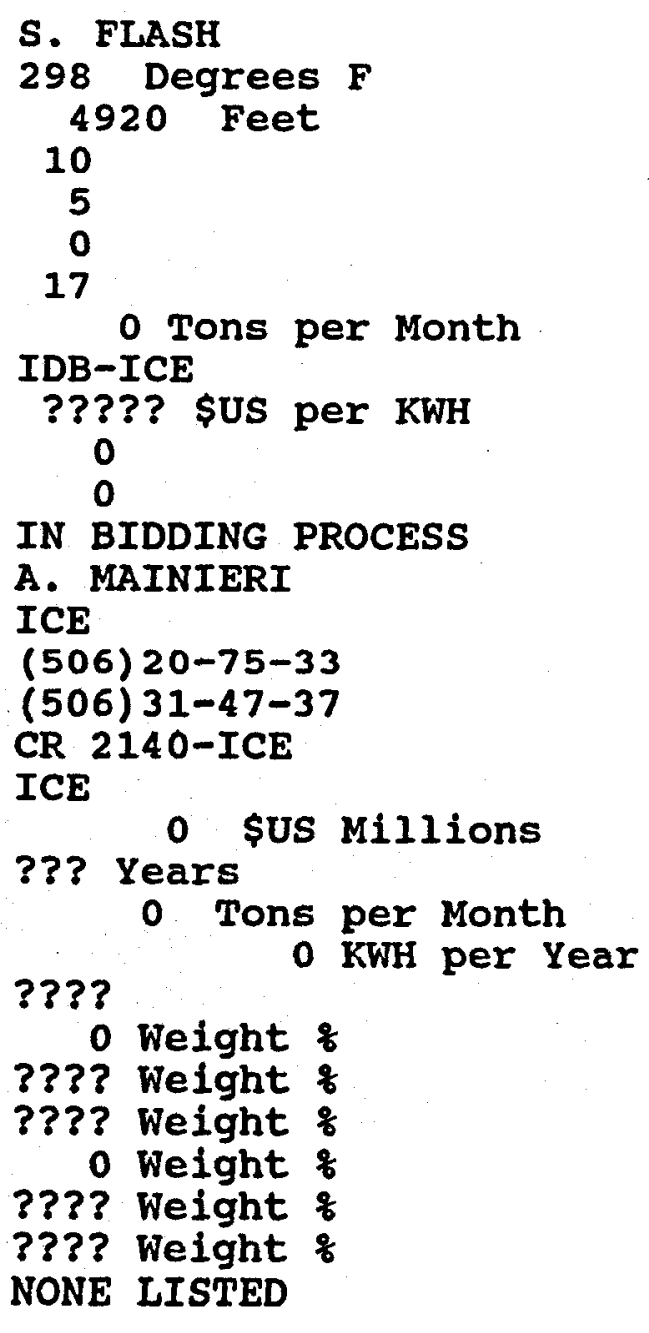 \\
\hline
\end{tabular}


EL SALVADOR 


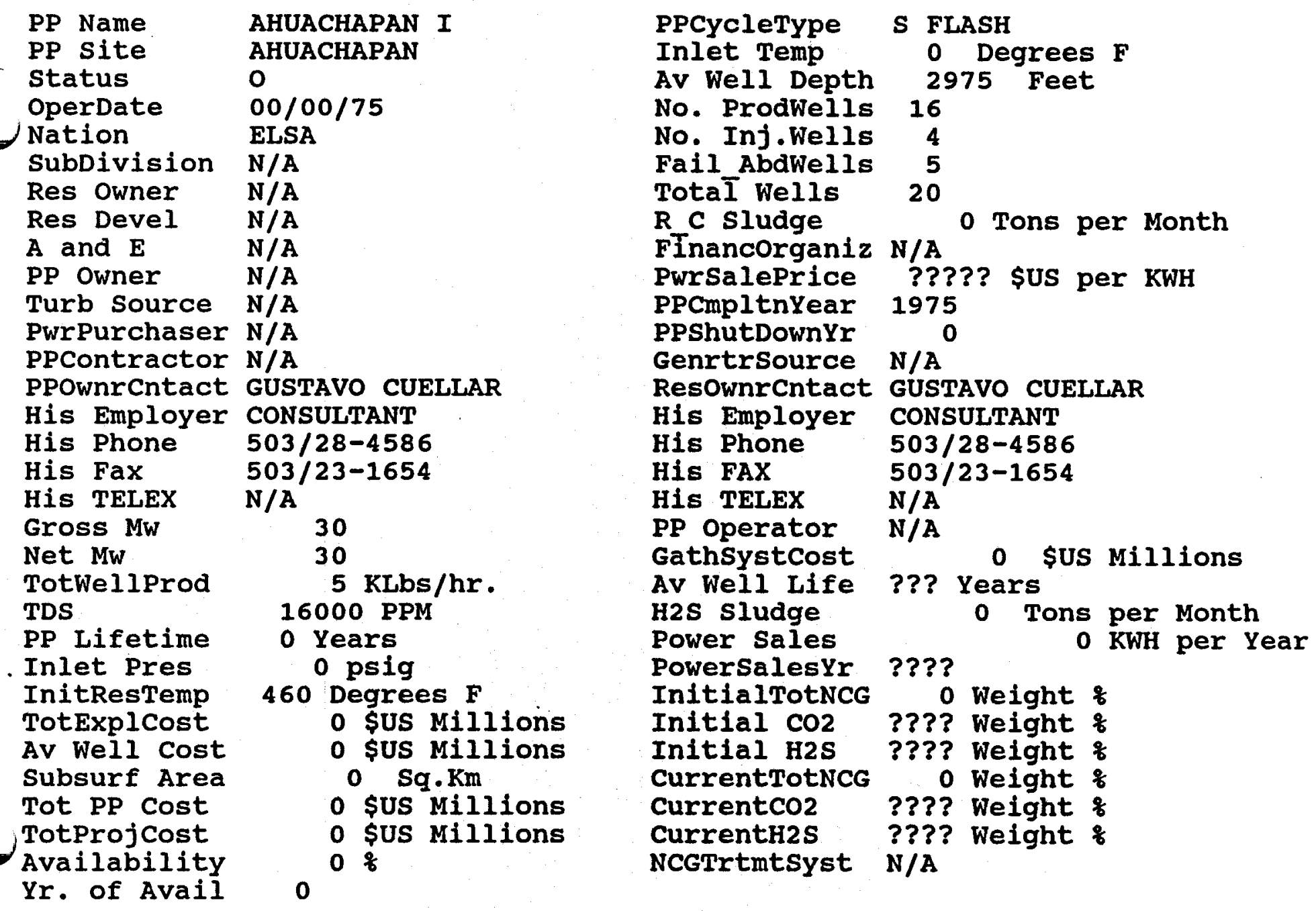




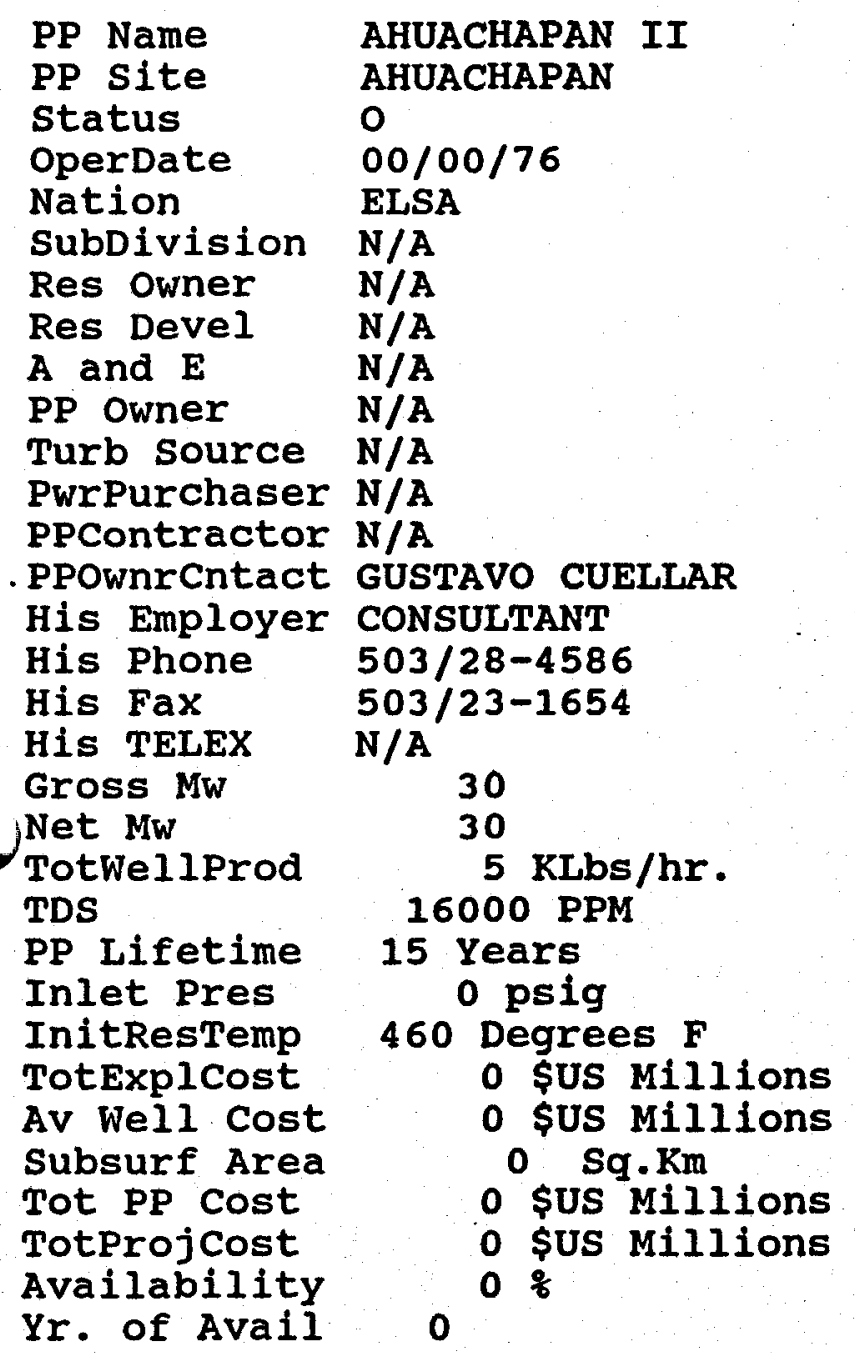

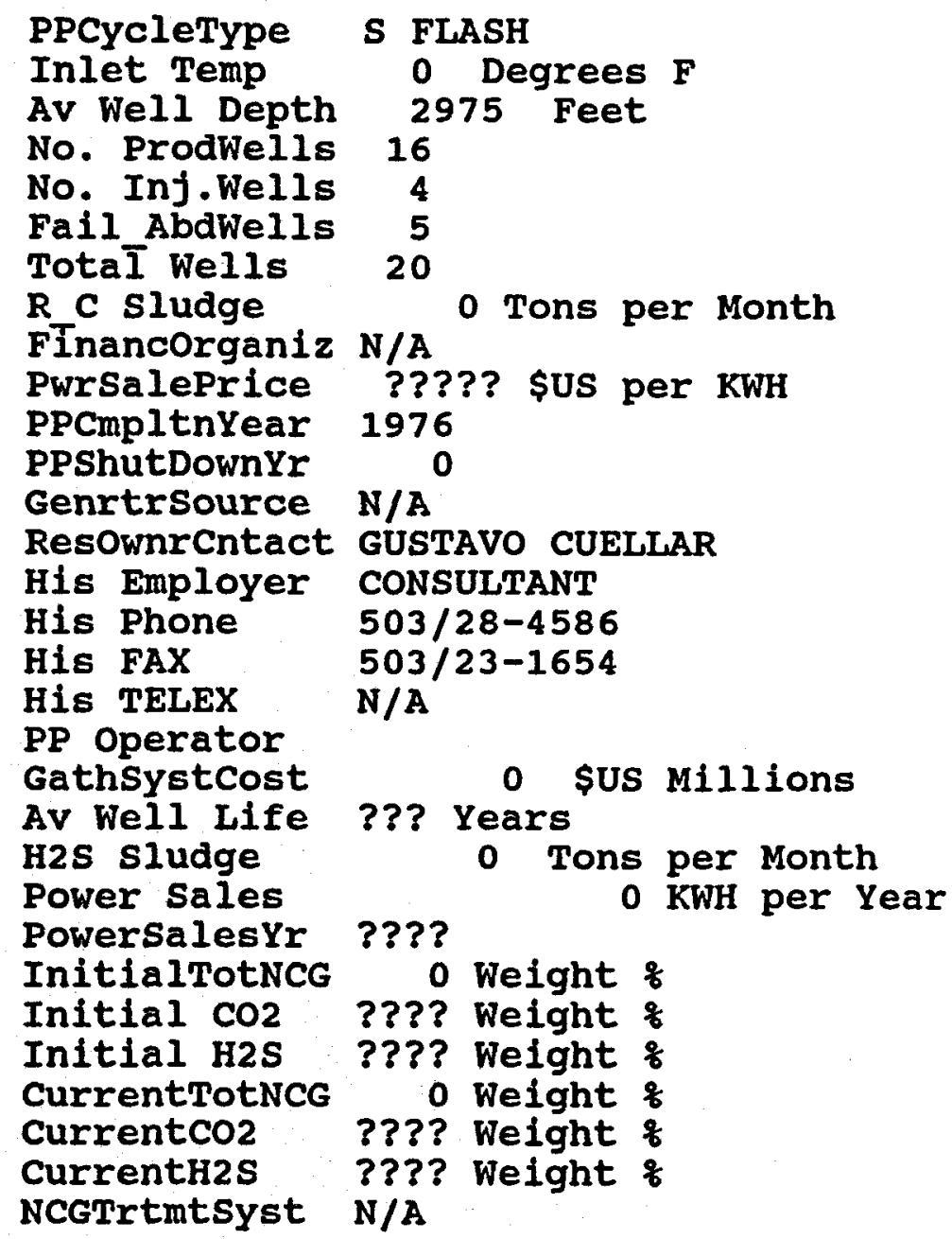




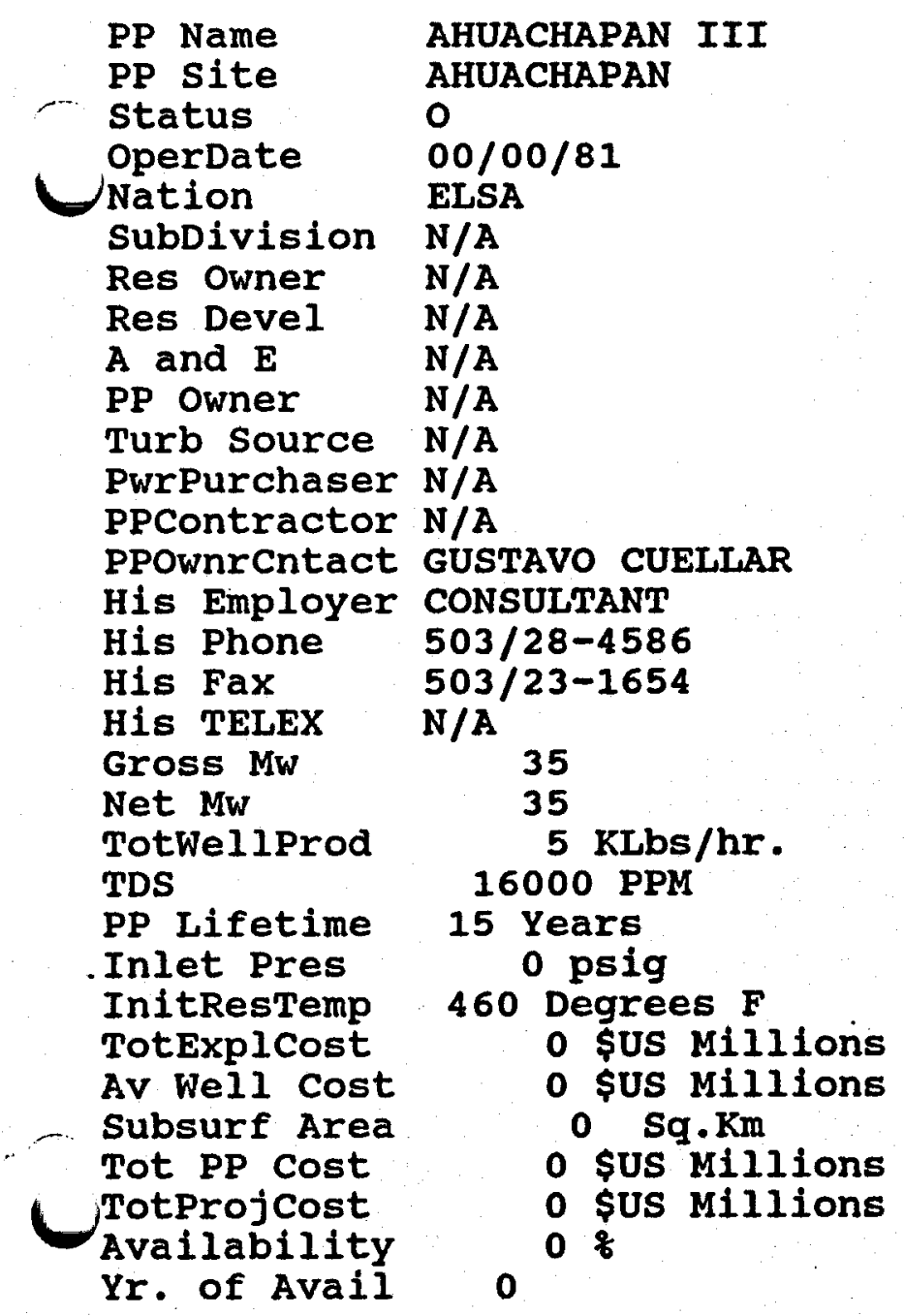

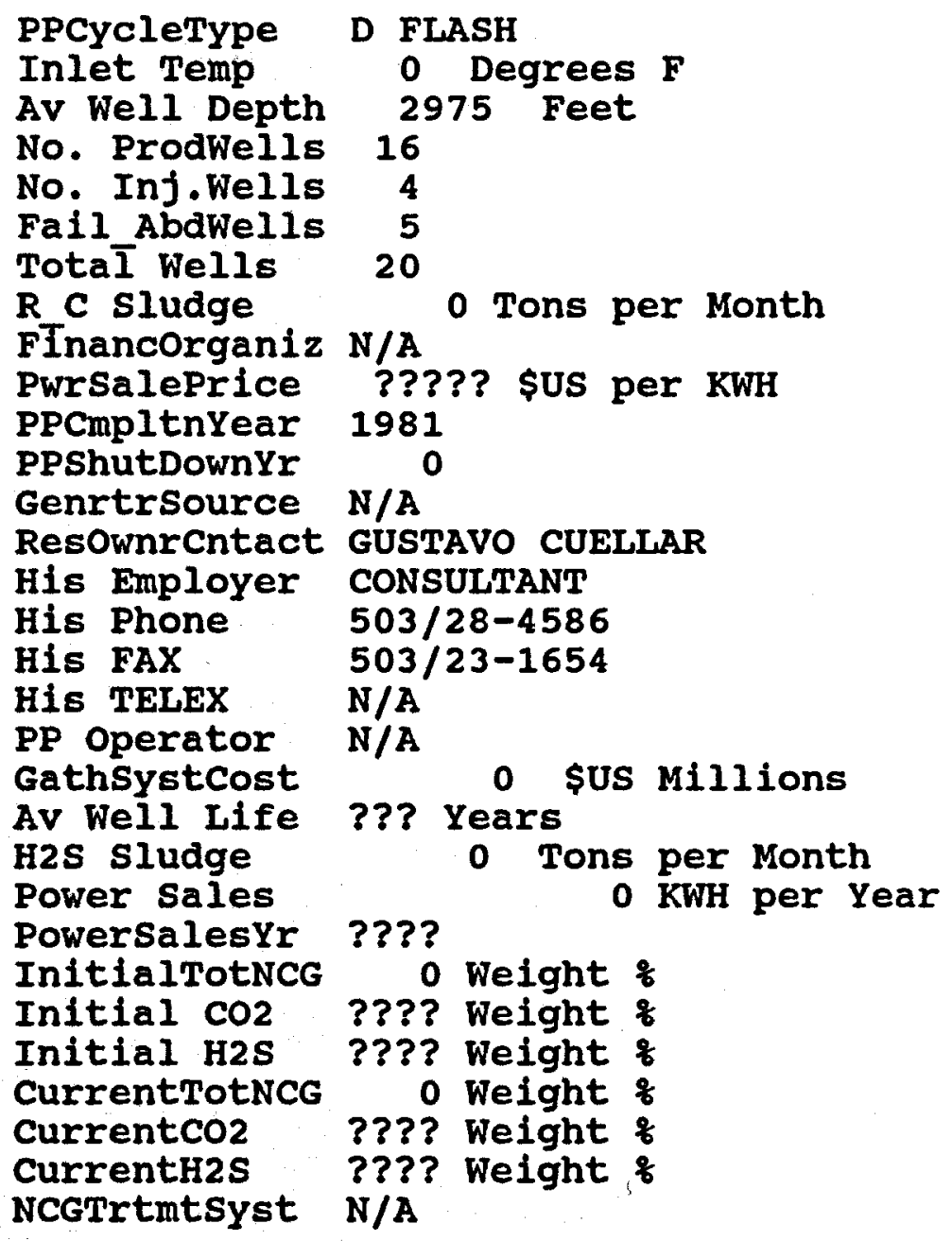


FRANCE 


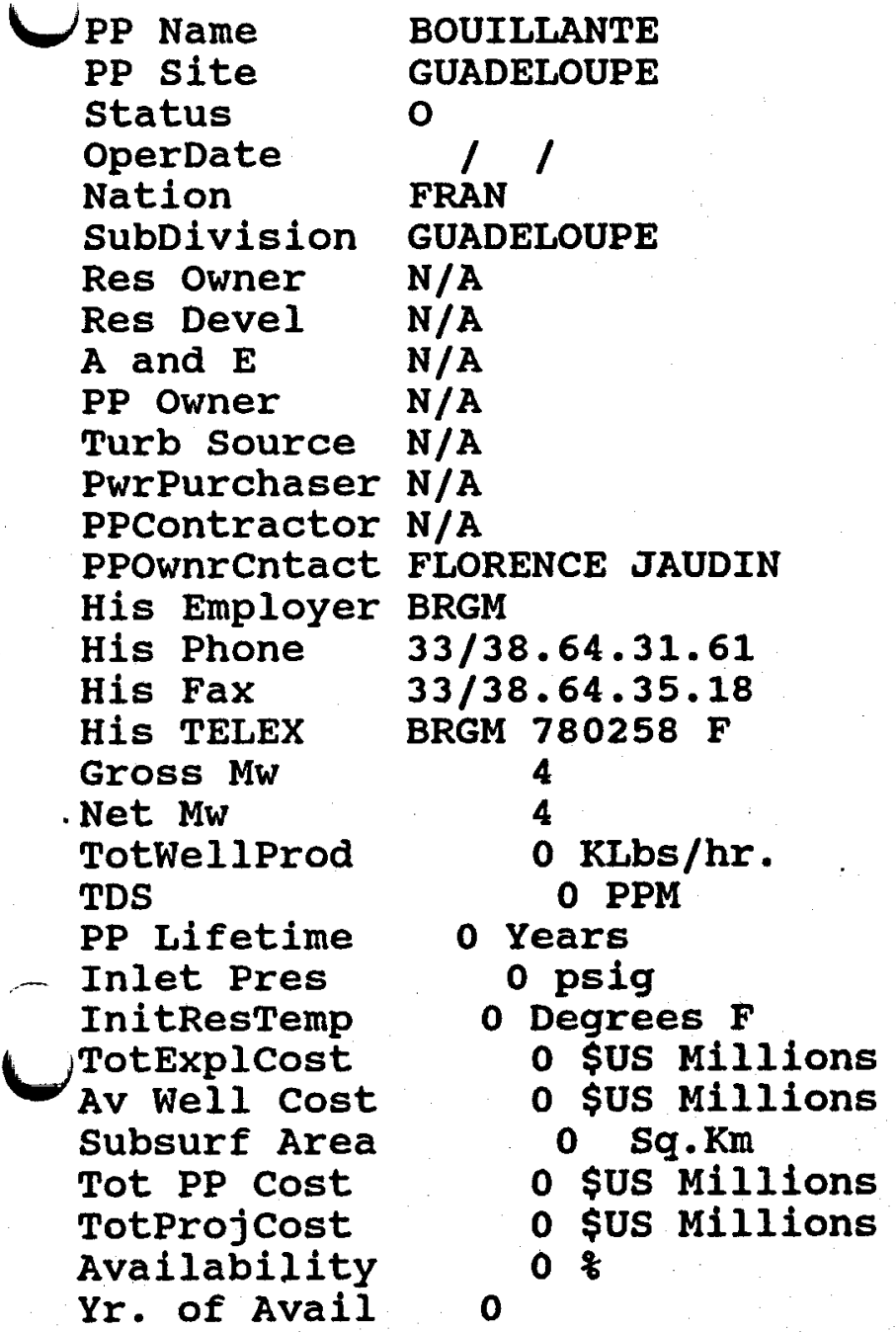

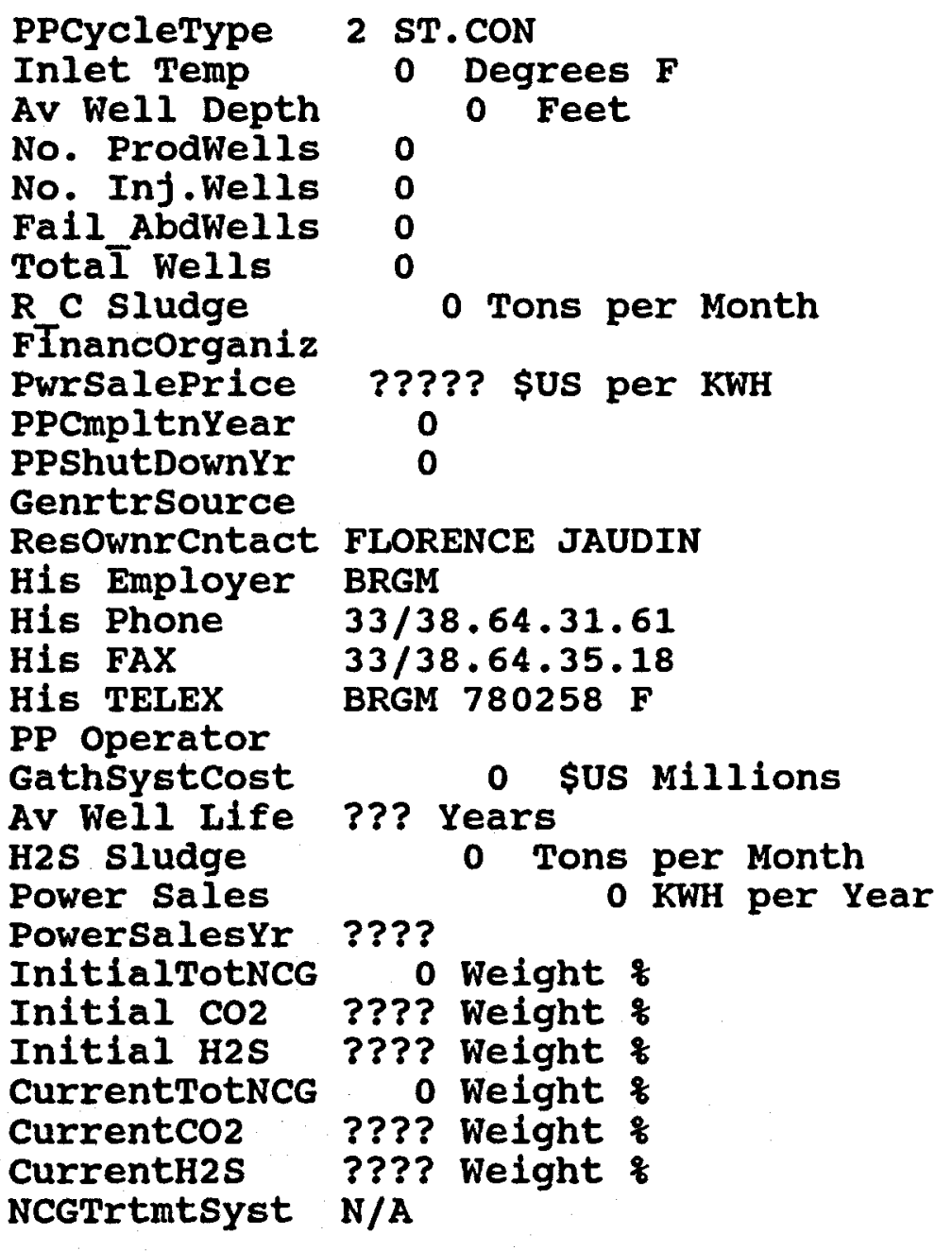


GREECE 


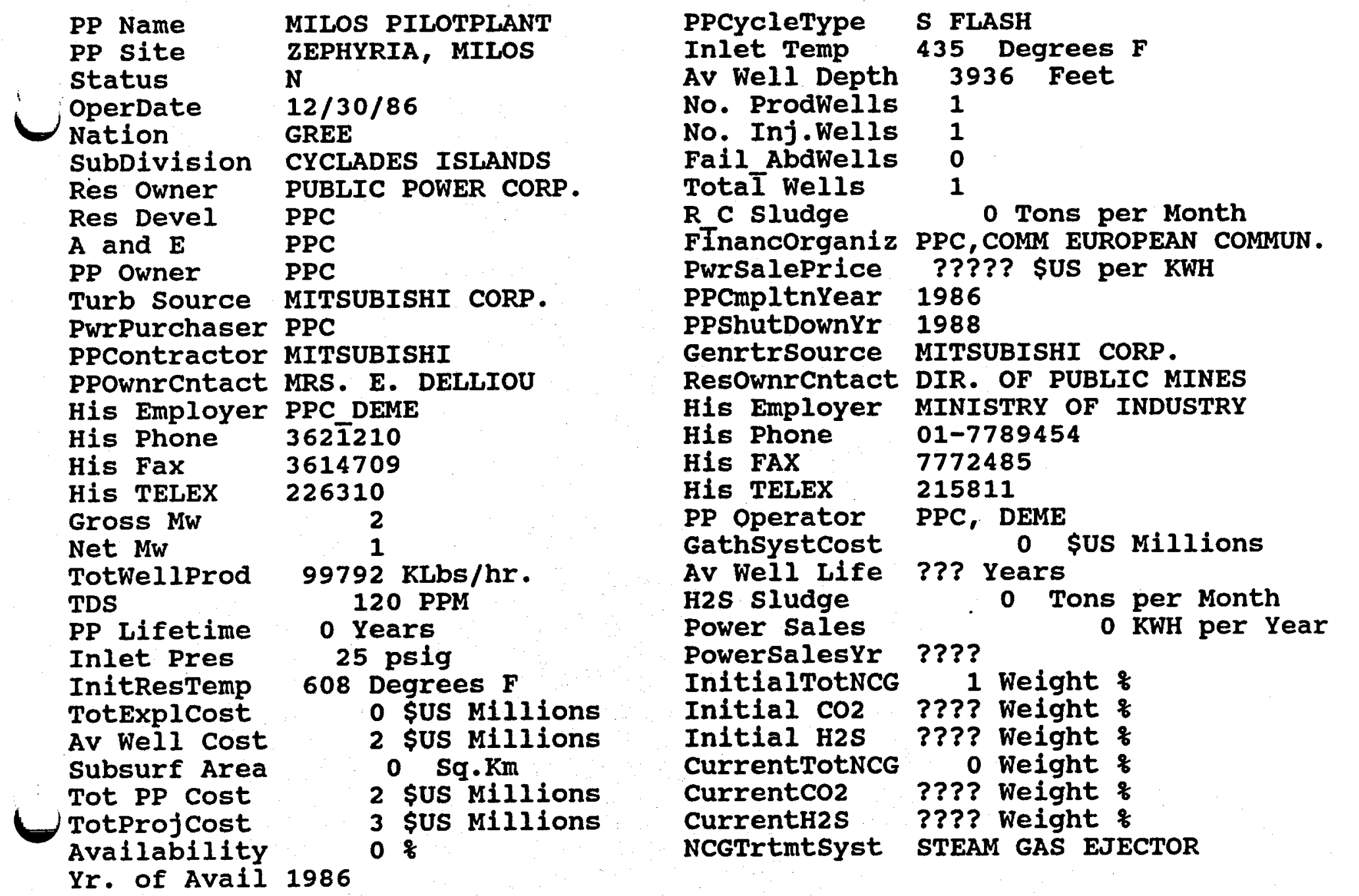

$\begin{array}{lll}\text { PP Name } & \text { ZUNIL } 1 & \text { PPCYcleType }\end{array}$ 
GUATAMELA 


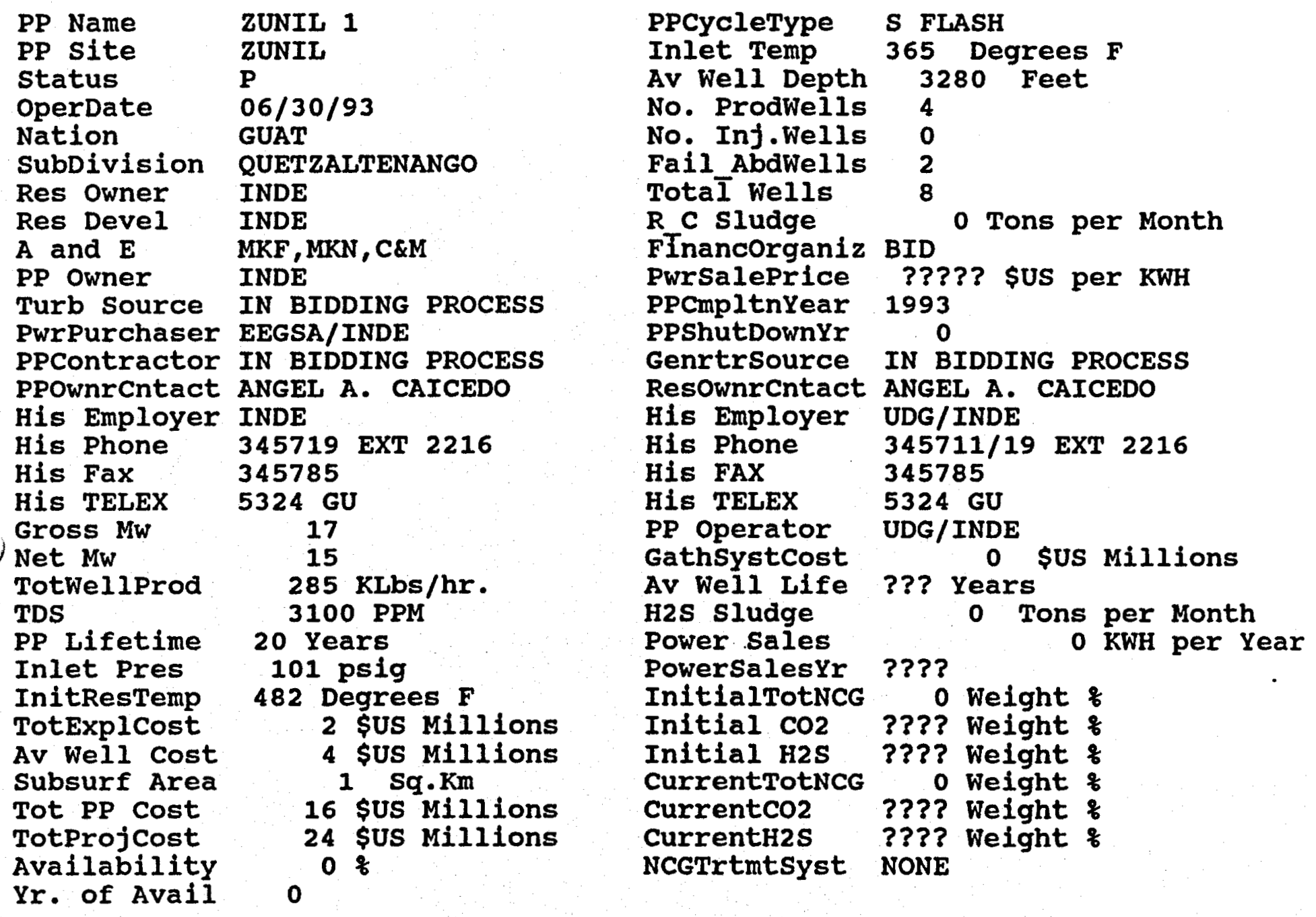




\section{ICELAND}




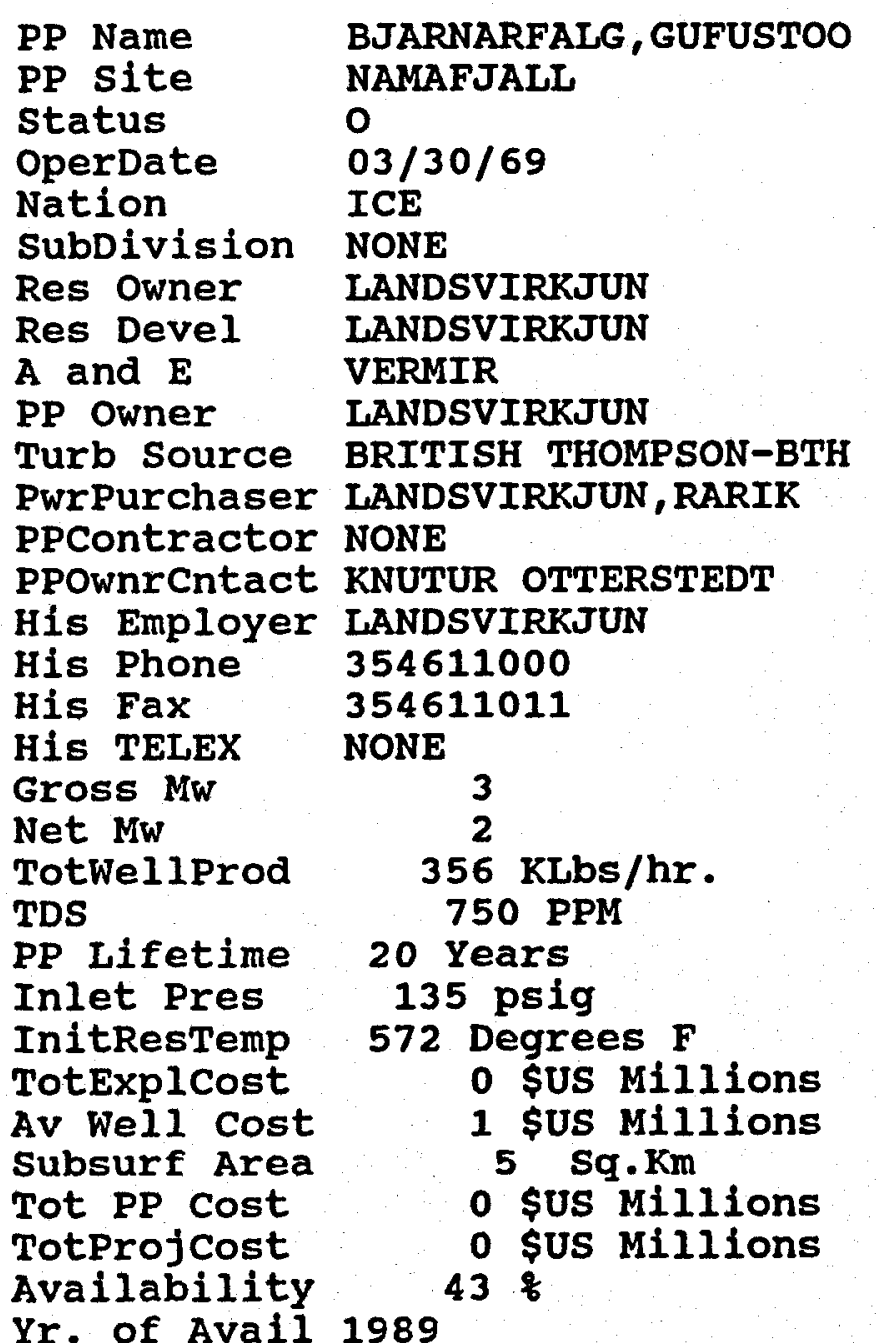

Yr. of Avail 1989

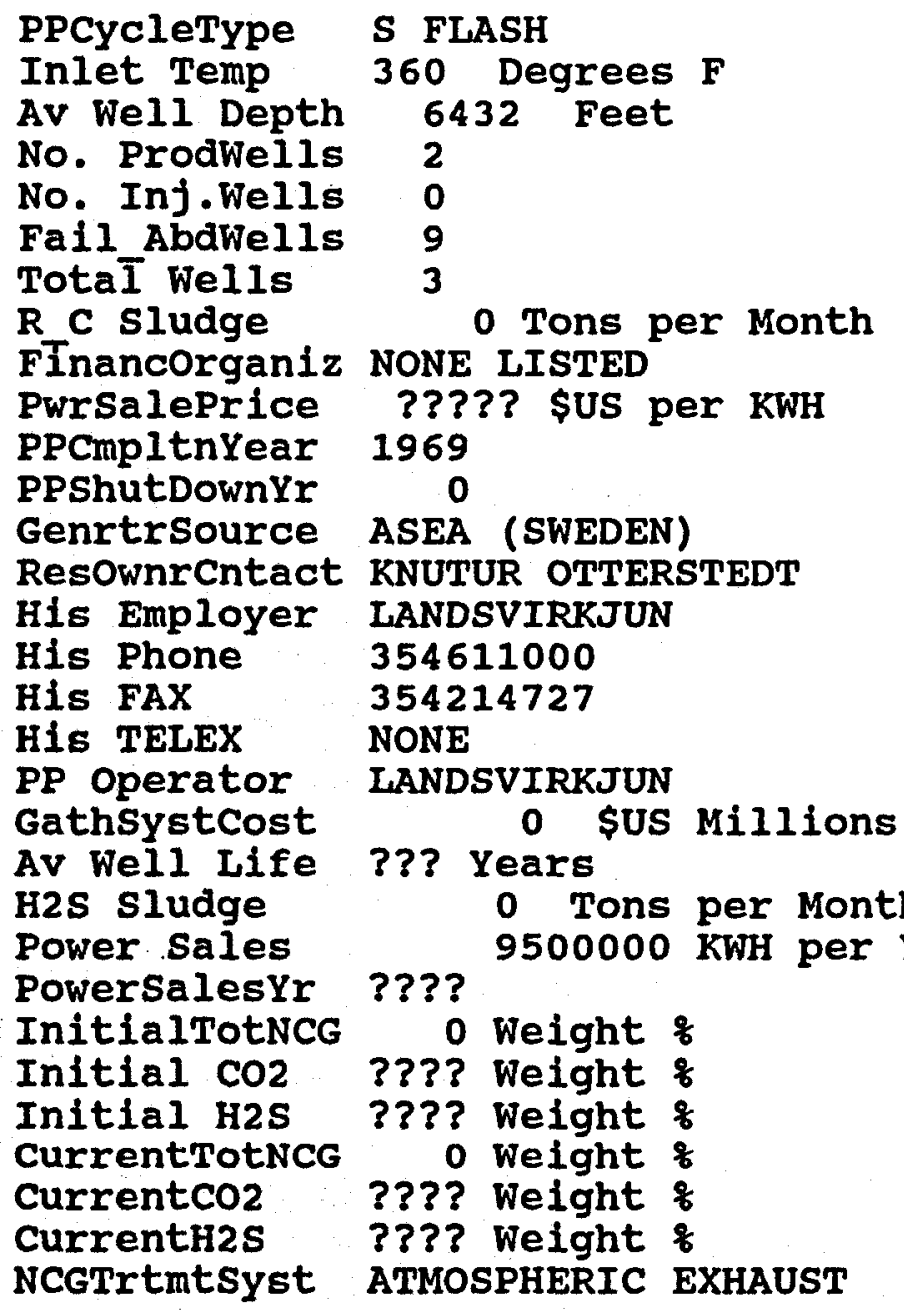

PPCycleType Inlet Temp Av Well Depth No. ProdWells No. Inj.Wells Fail AbaWells TotaI Wells R C sludge Financorganiz NONE LISTED PwrSalePrice ????? \$US per KWH PPCmpltnYear 1969 


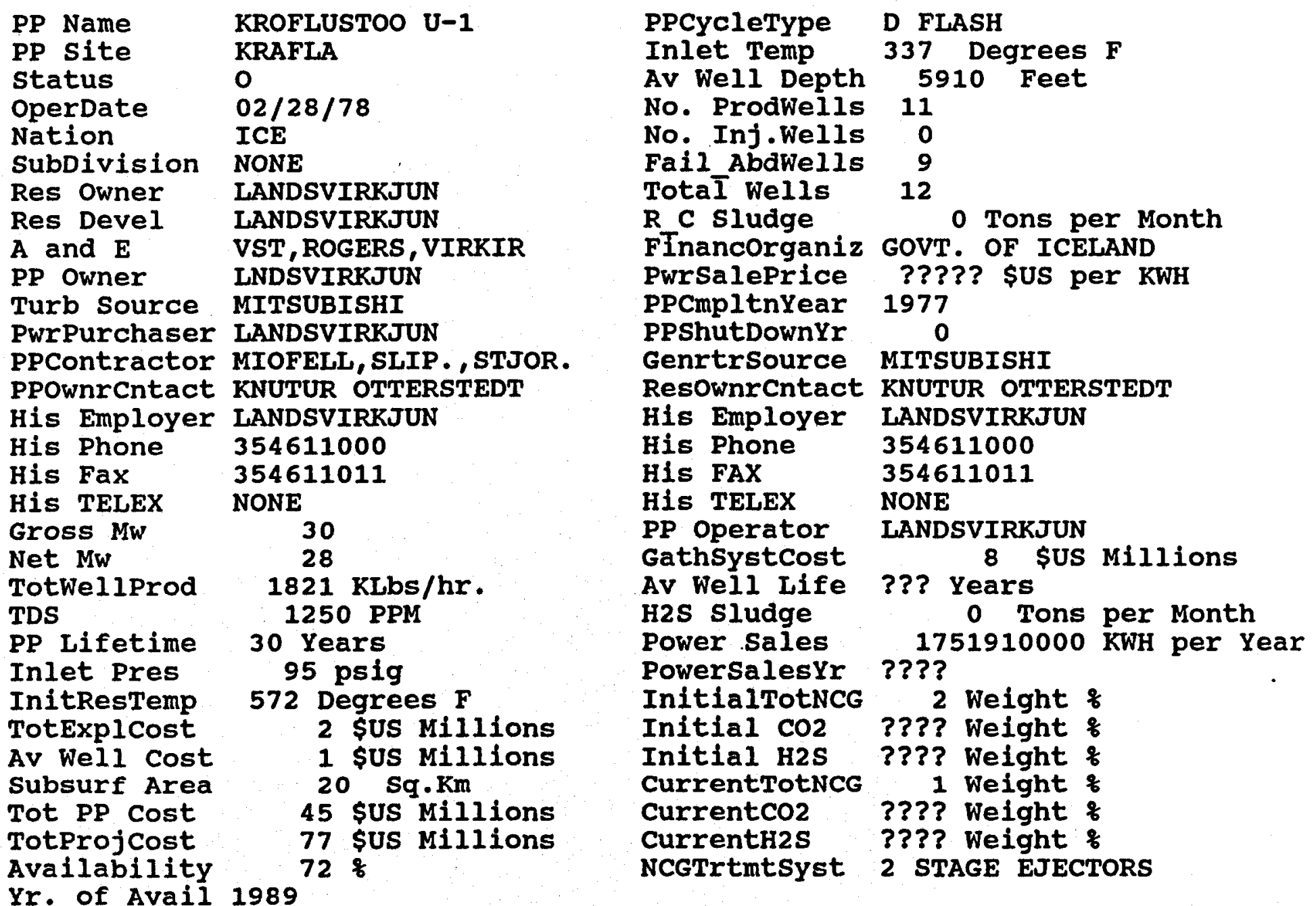




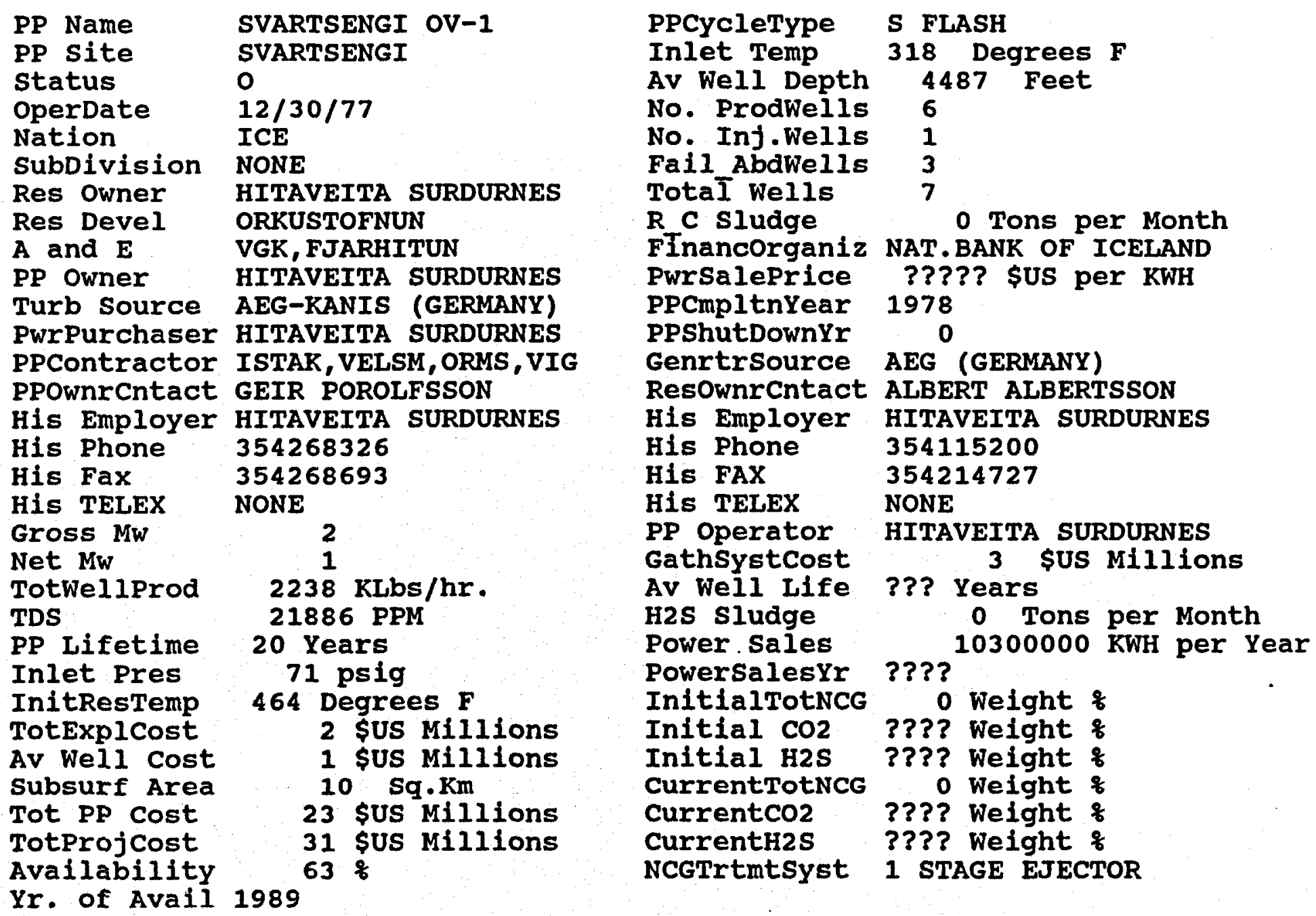




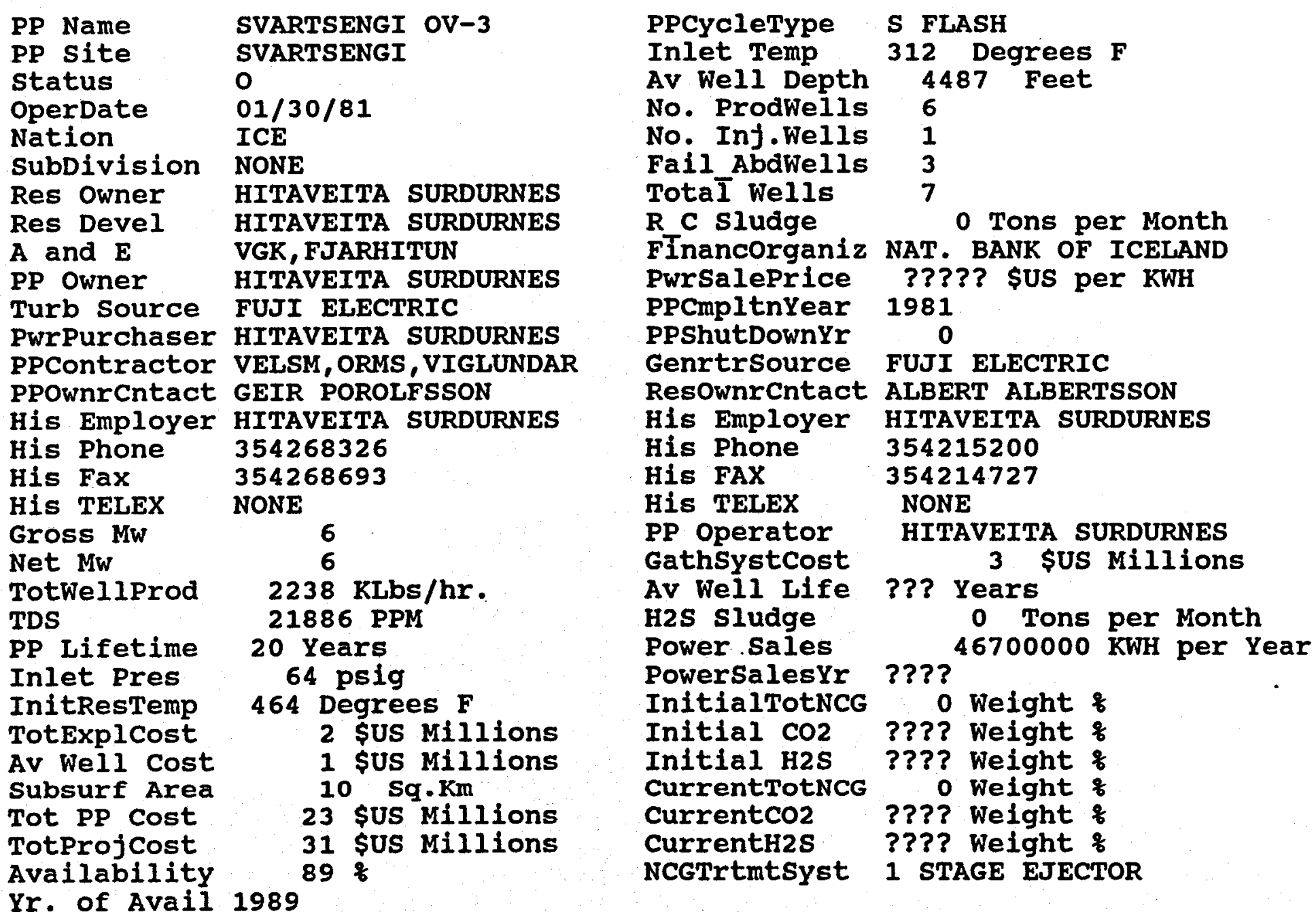




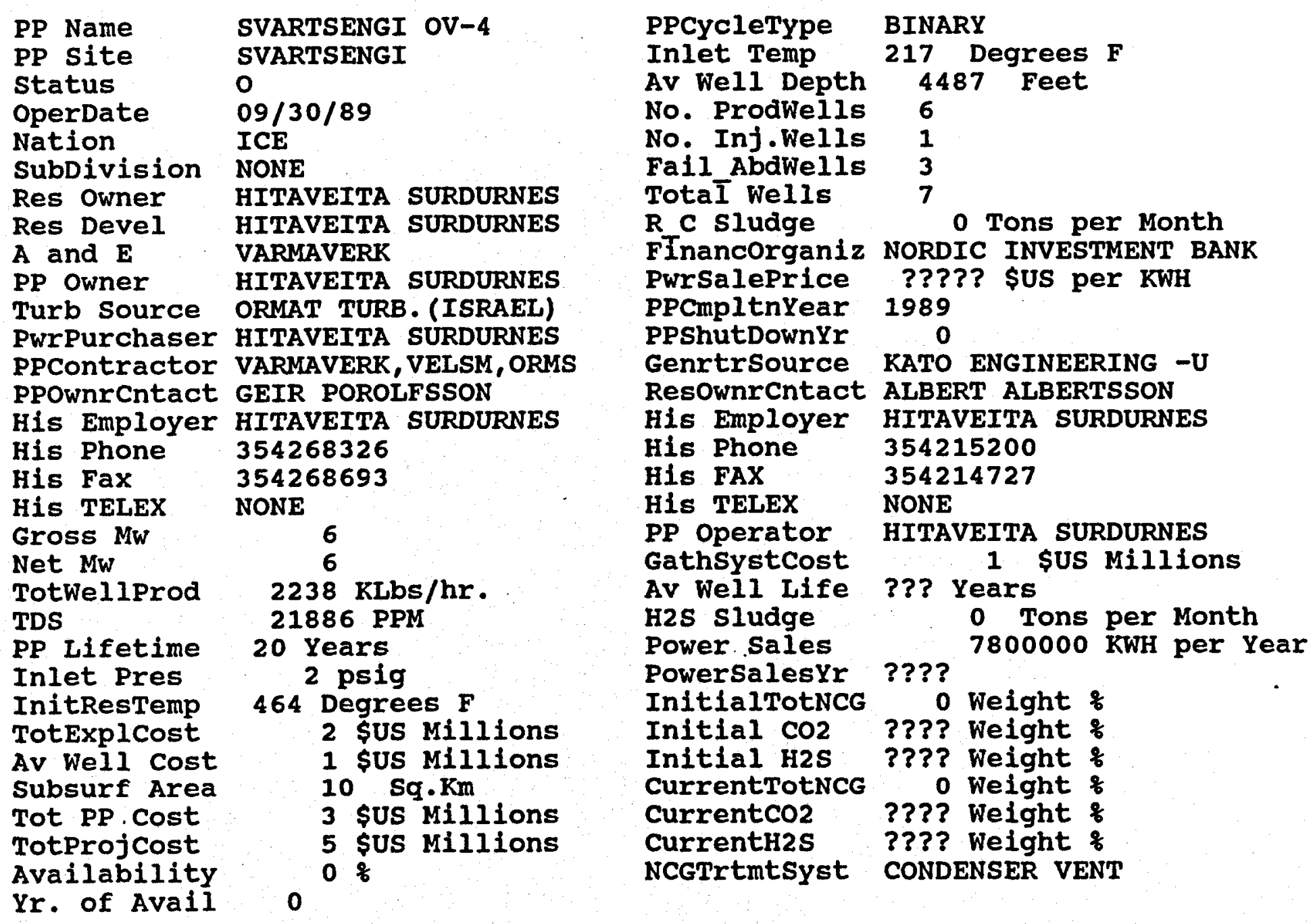


INDONESIA 


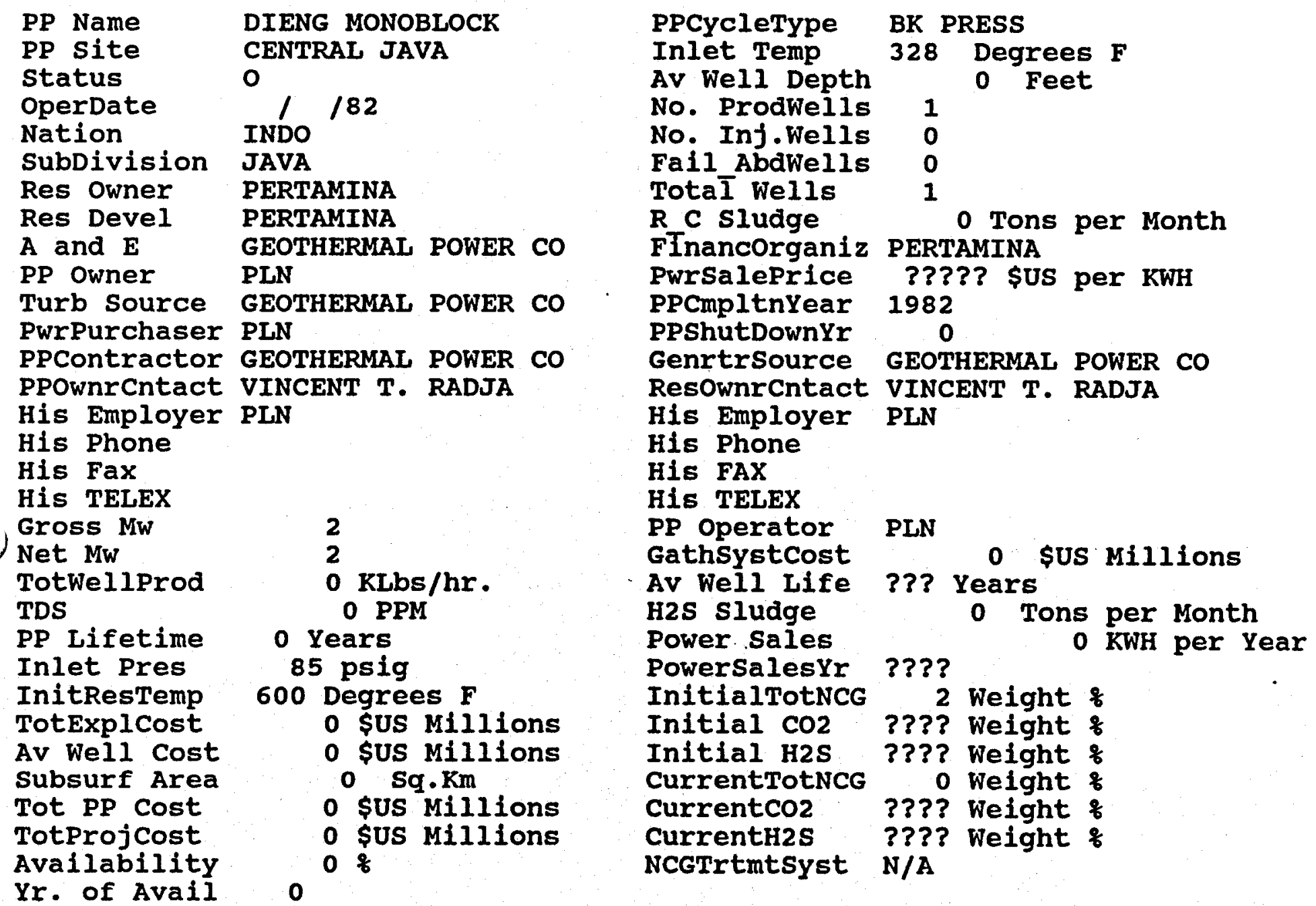




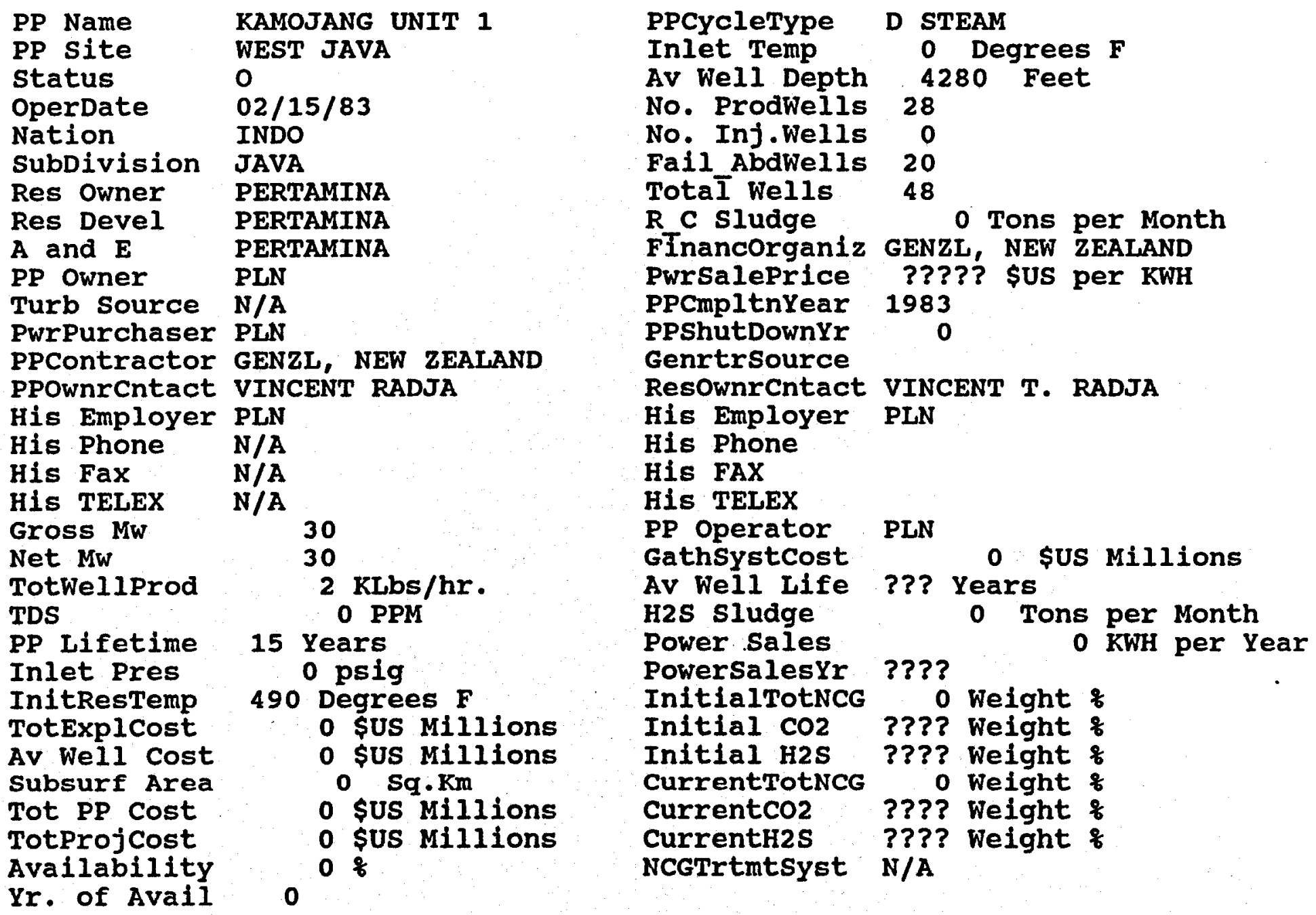




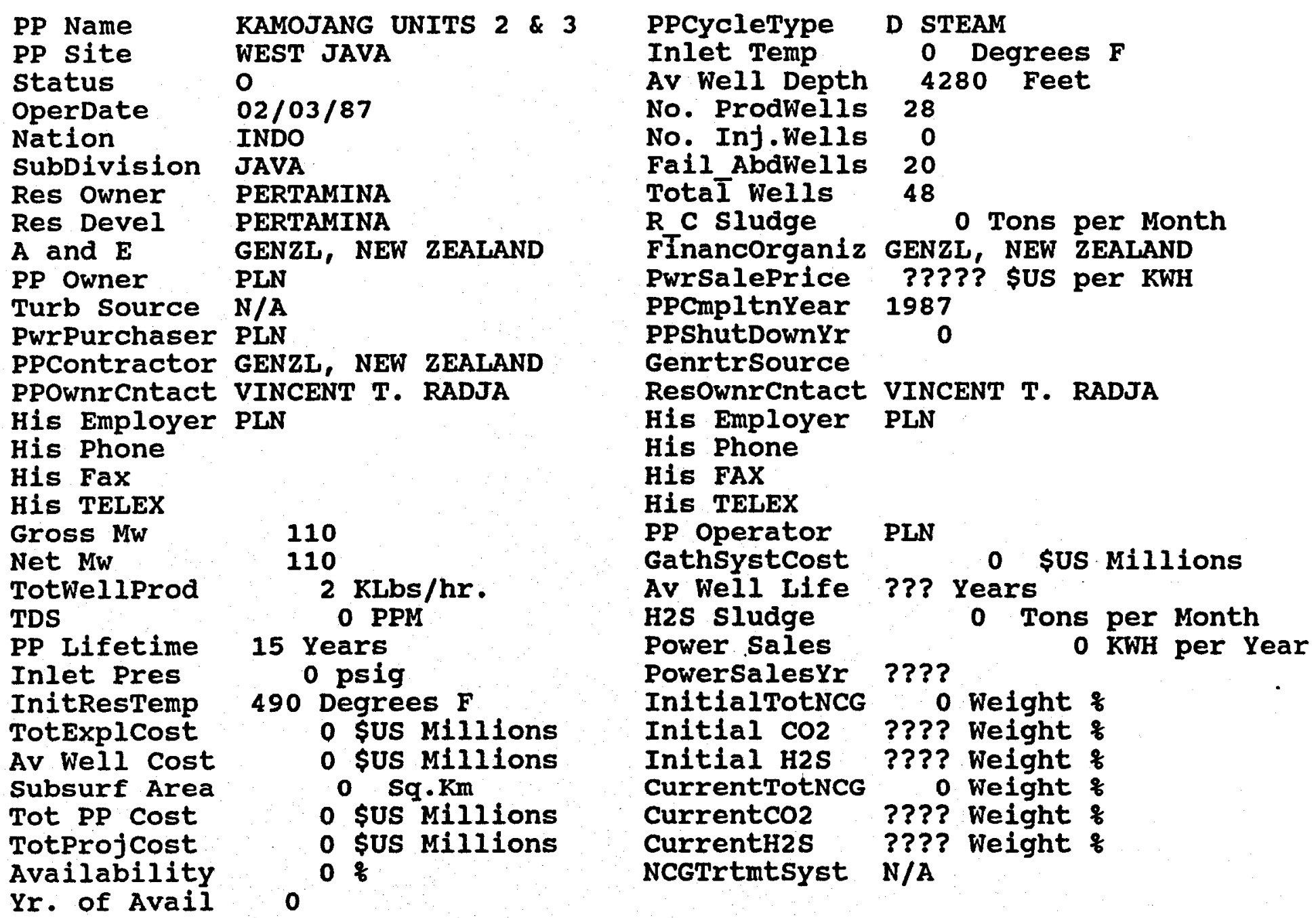




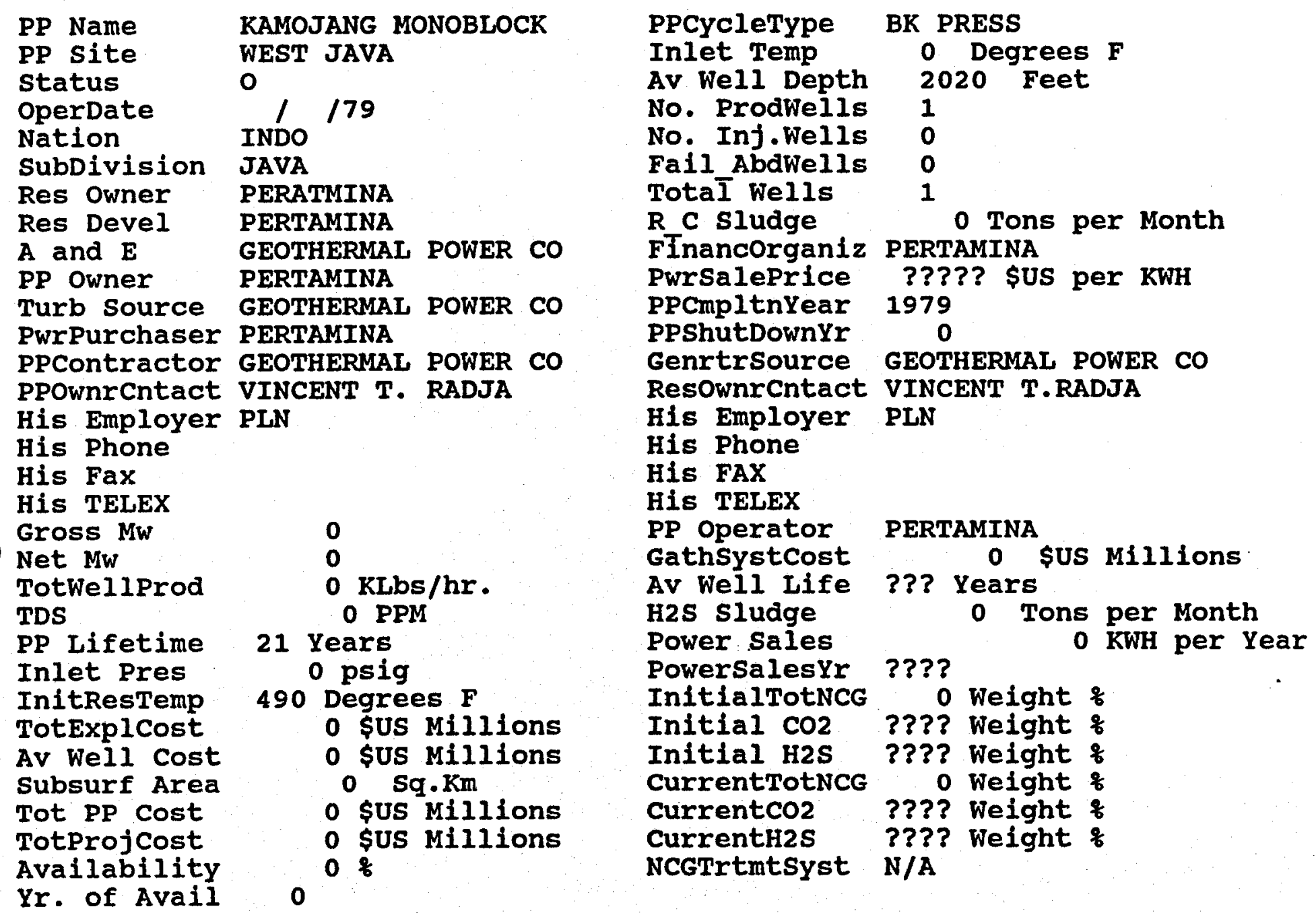


ITALY 


\begin{tabular}{|c|c|c|c|}
\hline & $\begin{array}{c}40 \\
40 \\
0 \text { KLbs/hr. } \\
0 \text { PPM } \\
0 \text { Years } \\
0 \text { psig } \\
650 \text { Degrees F } \\
0 \text { \$US Millions } \\
0 \text { \$US Millions } \\
\text { o Sq.Km } \\
0 \text { \$US Millions } \\
0 \text { \$US Millions } \\
0 \%\end{array}$ & $\begin{array}{l}\text { PPCycleType } \\
\text { Inlet Temp } \\
\text { Av Well Depth } \\
\text { No. ProdWells } \\
\text { No. Inj. Wells } \\
\text { Fail AbdWells } \\
\text { TotaI Wells } \\
\text { RC Sludge } \\
\text { FInancorganiz } \\
\text { PwrSalePrice } \\
\text { PPCmpltnYear } \\
\text { PPShutDownYr } \\
\text { GenrtrSource } \\
\text { ResOwnrCntact } \\
\text { His Employer } \\
\text { His Phone } \\
\text { His FAX } \\
\text { His TELEX } \\
\text { PP Operator } \\
\text { GathSystCost } \\
\text { Av Well Life } \\
\text { H2S sludge } \\
\text { Power Sales } \\
\text { PowerSalesYr } \\
\text { InitialTotNCG } \\
\text { Initial CO2 } \\
\text { Initial H2S } \\
\text { CurrentTotNCG } \\
\text { CurrentCO2 } \\
\text { CurrentH2S } \\
\text { NCGTrtmtSyst }\end{array}$ & 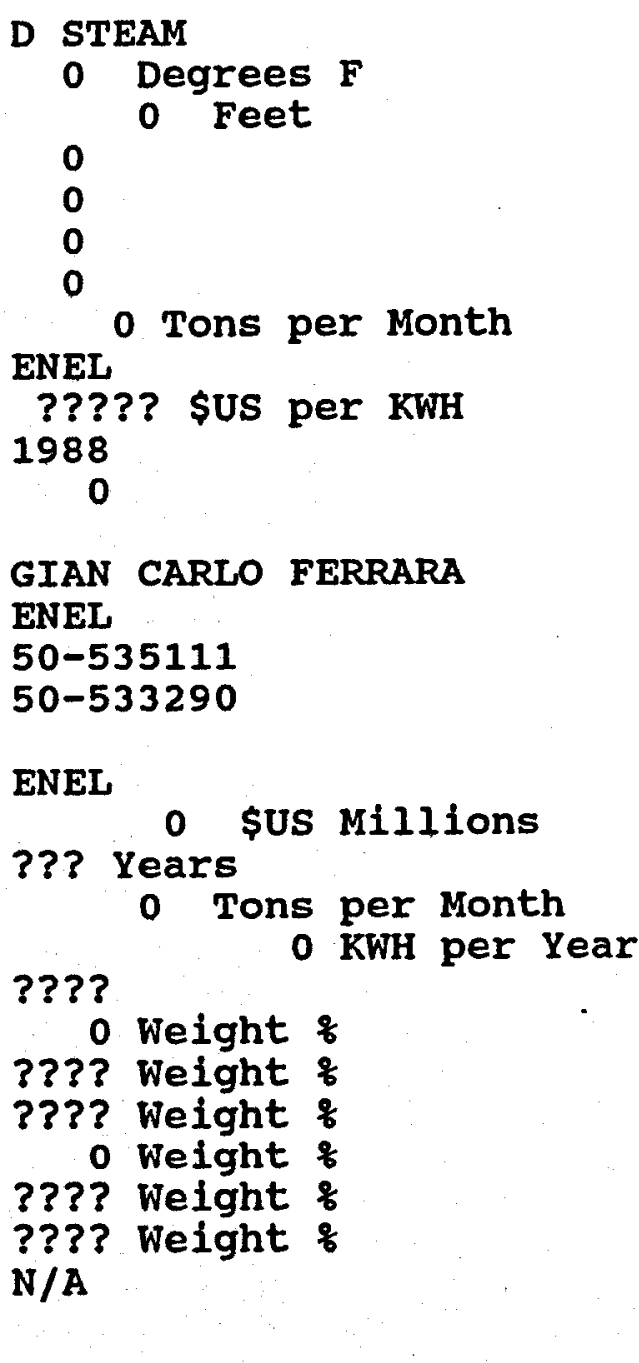 \\
\hline
\end{tabular}




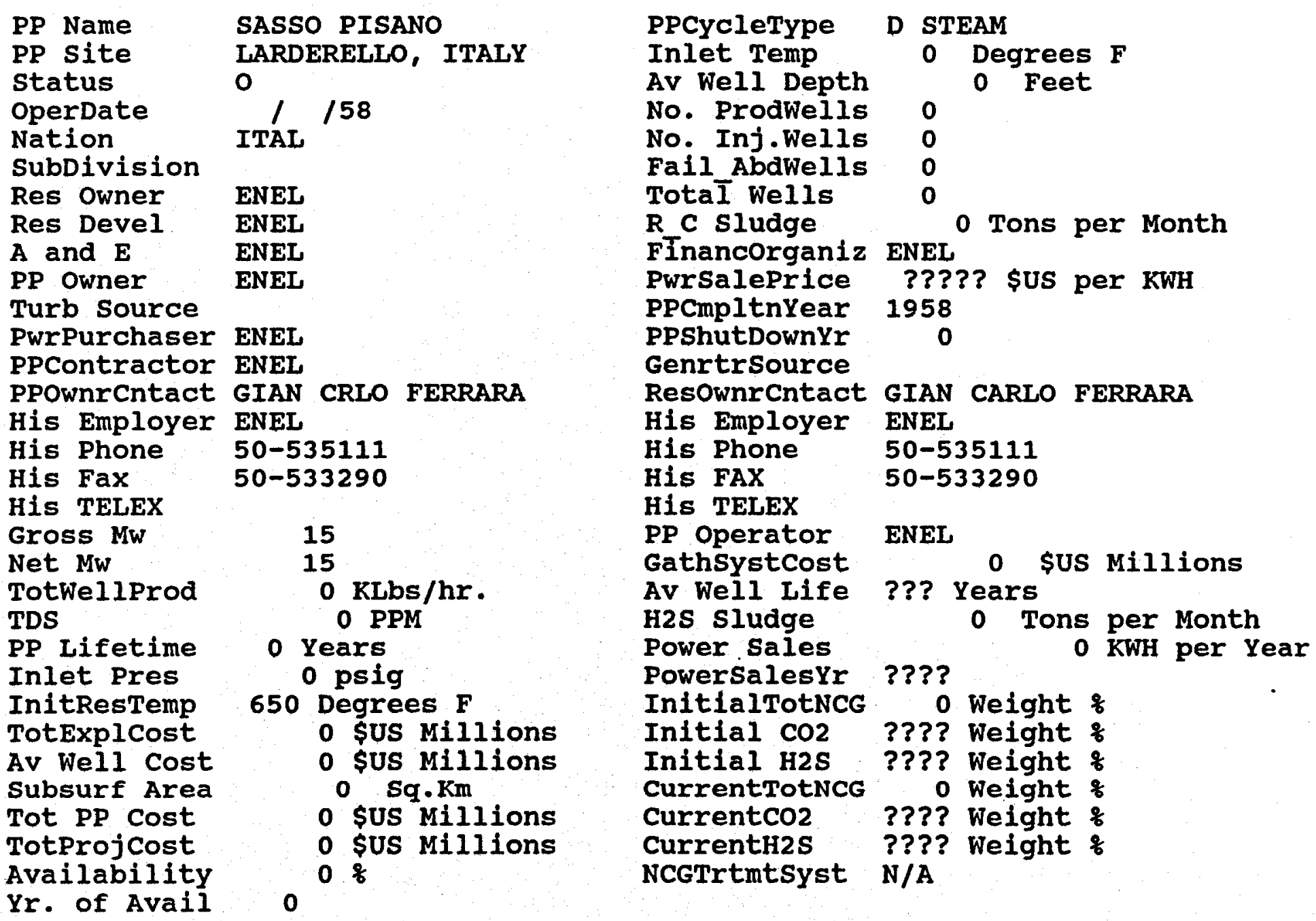




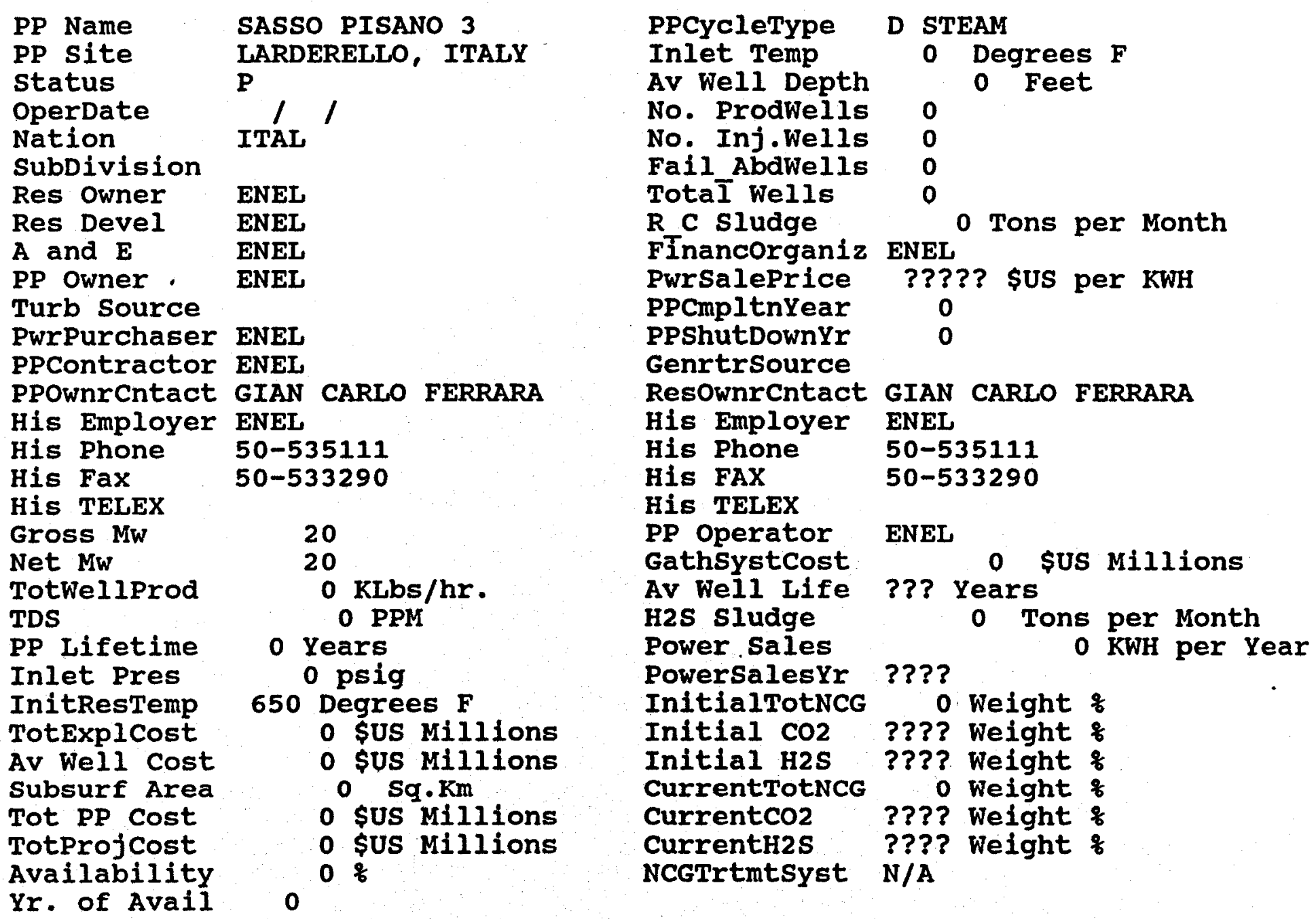




\begin{tabular}{|c|c|c|c|}
\hline $\begin{array}{l}\text { bDivision } \\
\text { s Owner } \\
\text { s Devel } \\
\text { and E } \\
\text { Owner }\end{array}$ & $\begin{array}{l}\text { SELVA } 1 \\
\text { LARDERELLO, ITALY } \\
\mathrm{P} \\
\text { ITAL } \\
\text { ENEL } \\
\text { ENEL } \\
\text { ENEL } \\
\text { ENEL } \\
\text { ENEL } \\
\text { ENEL } \\
\text { GIAN CARLO FERRARA } \\
\text { ENEL } \\
50-535111 \\
50-533290 \\
\quad 20 \\
20 \\
0 \text { KLbs/hr. } \\
0 \text { PPM } \\
0 \text { Years } \\
0 \text { psig } \\
650 \text { Degrees F } \\
0 \text { \$US Millions } \\
0 \text { \$US Millions } \\
\text { o Sq.Km } \\
0 \text { \$US Millions } \\
0 \text { \$US Millions } \\
0 \text { o } \\
0\end{array}$ & $\begin{array}{l}\text { PPCycleType } \\
\text { Inlet Temp } \\
\text { Av Well Depth } \\
\text { No. ProdWells } \\
\text { No. Inj.Wells } \\
\text { Fail AbdWells } \\
\text { Total Wells } \\
\text { R C sludge } \\
\text { FinancOrganiz } \\
\text { PwrSalePrice } \\
\text { PPCmpltnYear } \\
\text { PPShutDownYr } \\
\text { GenrtrSource } \\
\text { ResownrCntact } \\
\text { His Employer } \\
\text { His Phone } \\
\text { His FAX } \\
\text { His TELEX } \\
\text { PP Operator } \\
\text { GathSystCost } \\
\text { Av Well Life } \\
\text { H2S sludge } \\
\text { Power. Sales } \\
\text { PowerSalesYr } \\
\text { InitialTotNCG } \\
\text { Initial CO2 } \\
\text { Initial H2S } \\
\text { CurrentTotNCG } \\
\text { CurrentCo2 } \\
\text { CurrentH2S } \\
\text { NCGTrtmtSyst } \\
\end{array}$ & $\begin{array}{l}\text { GIAN CARLO FERRARA } \\
\text { ENEL } \\
50-535111 \\
50-533290\end{array}$ \\
\hline
\end{tabular}




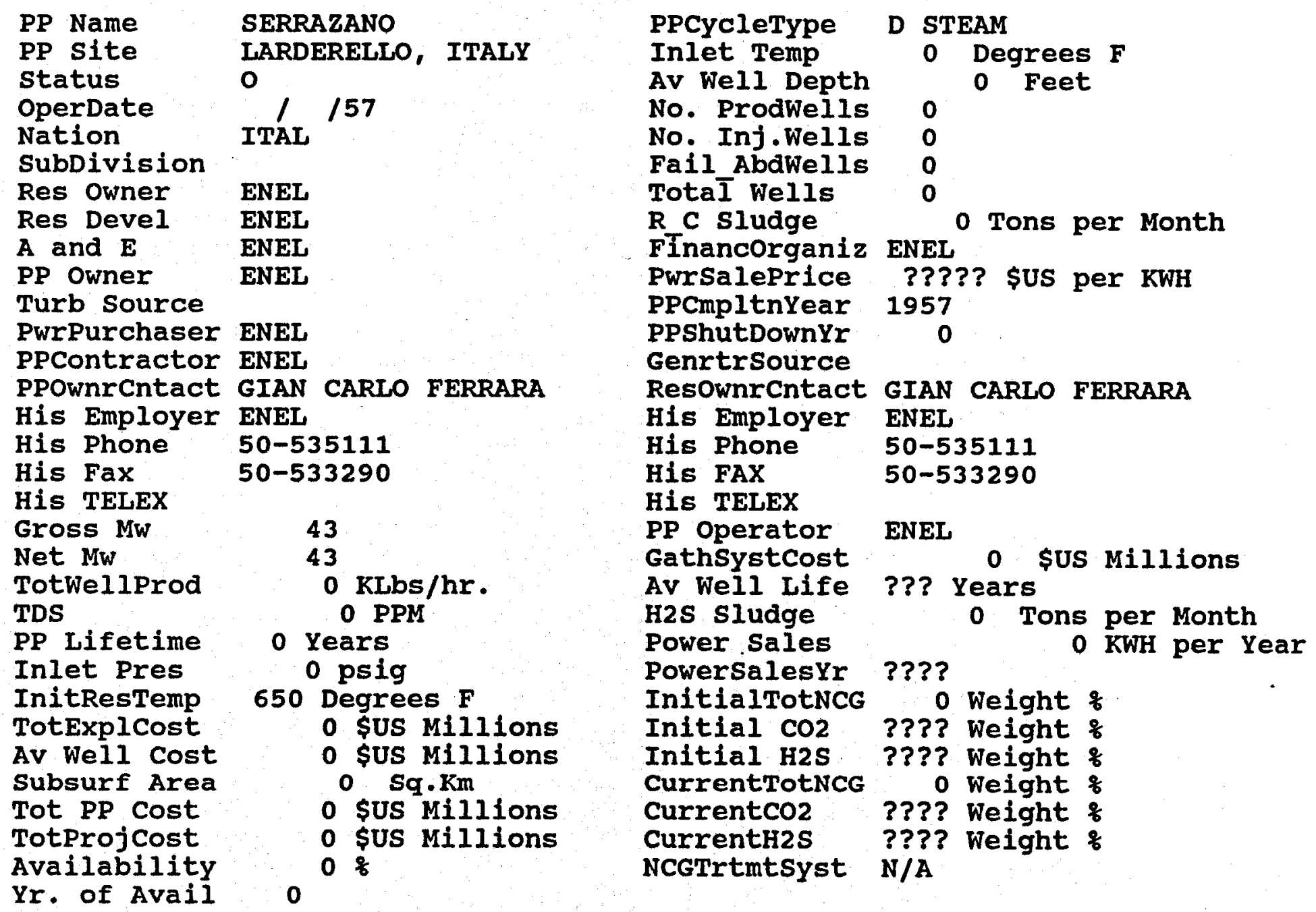




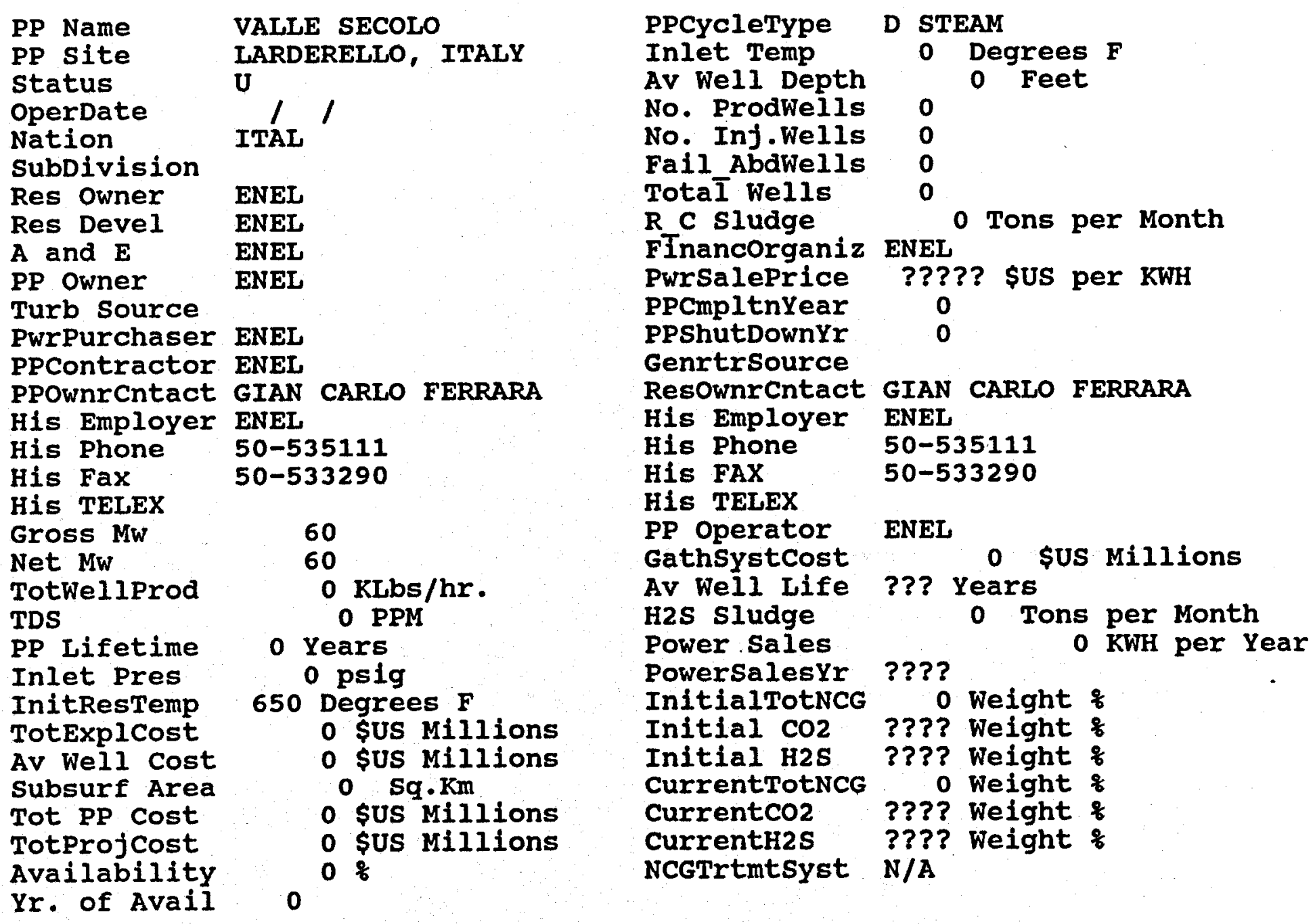




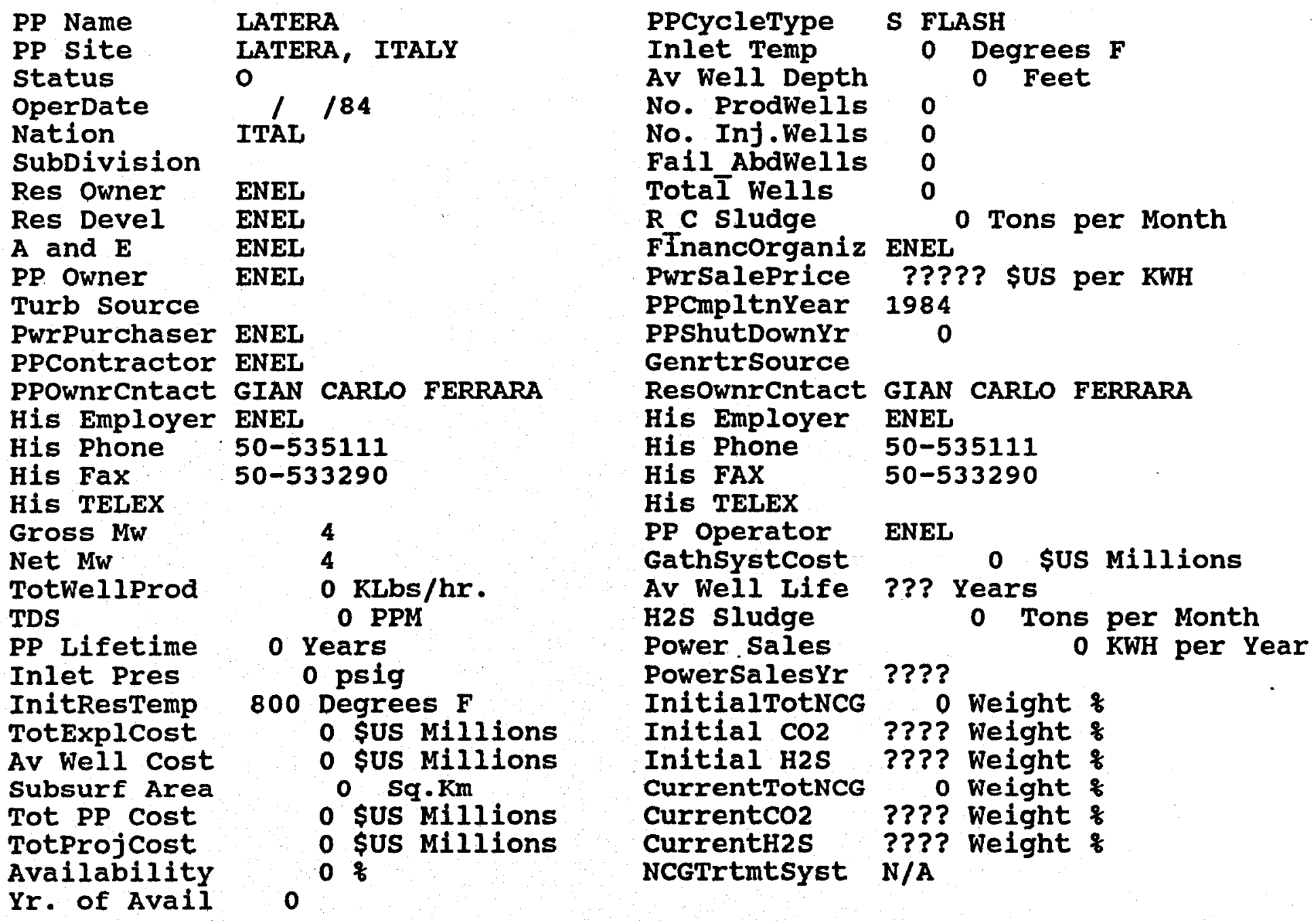




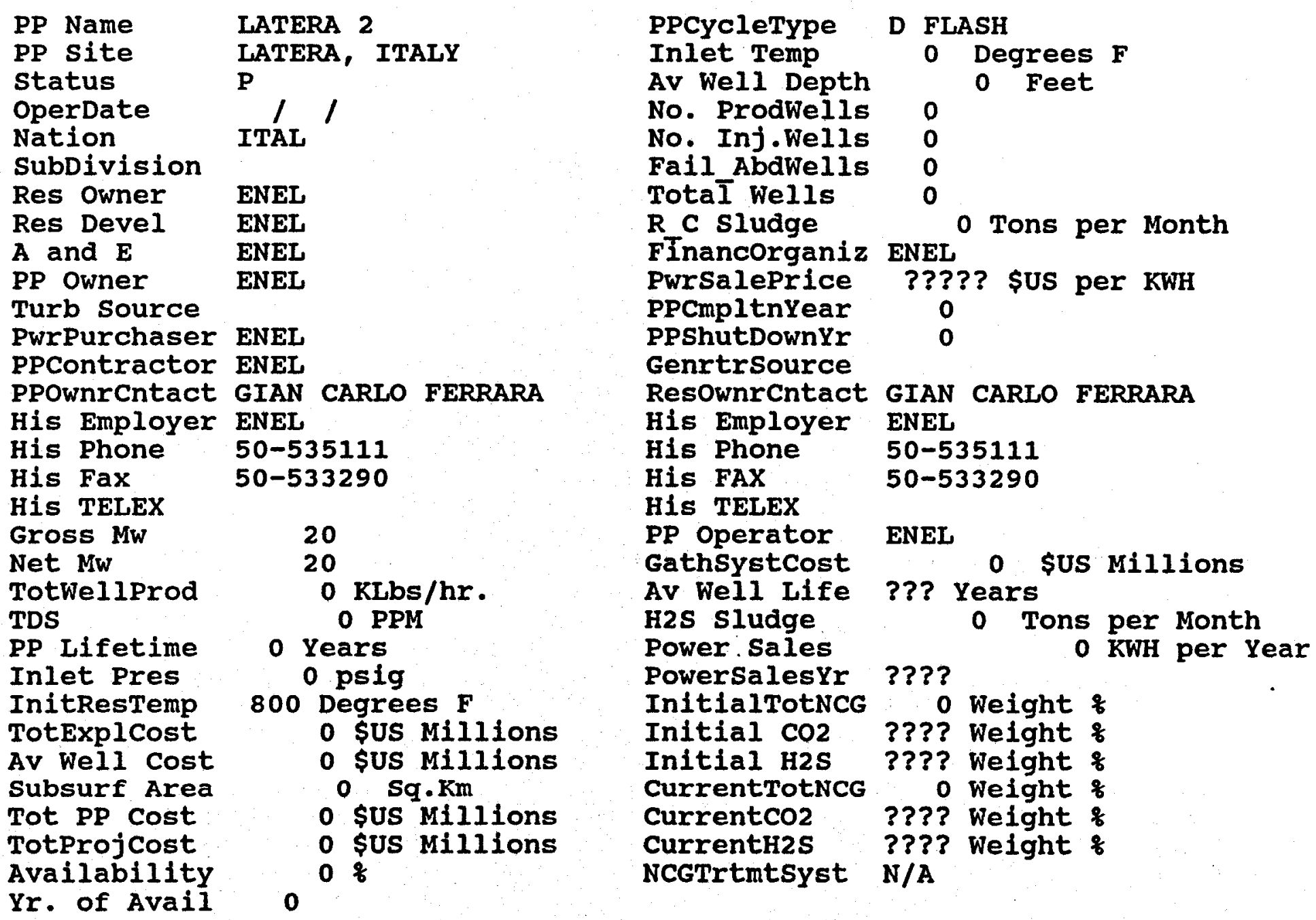




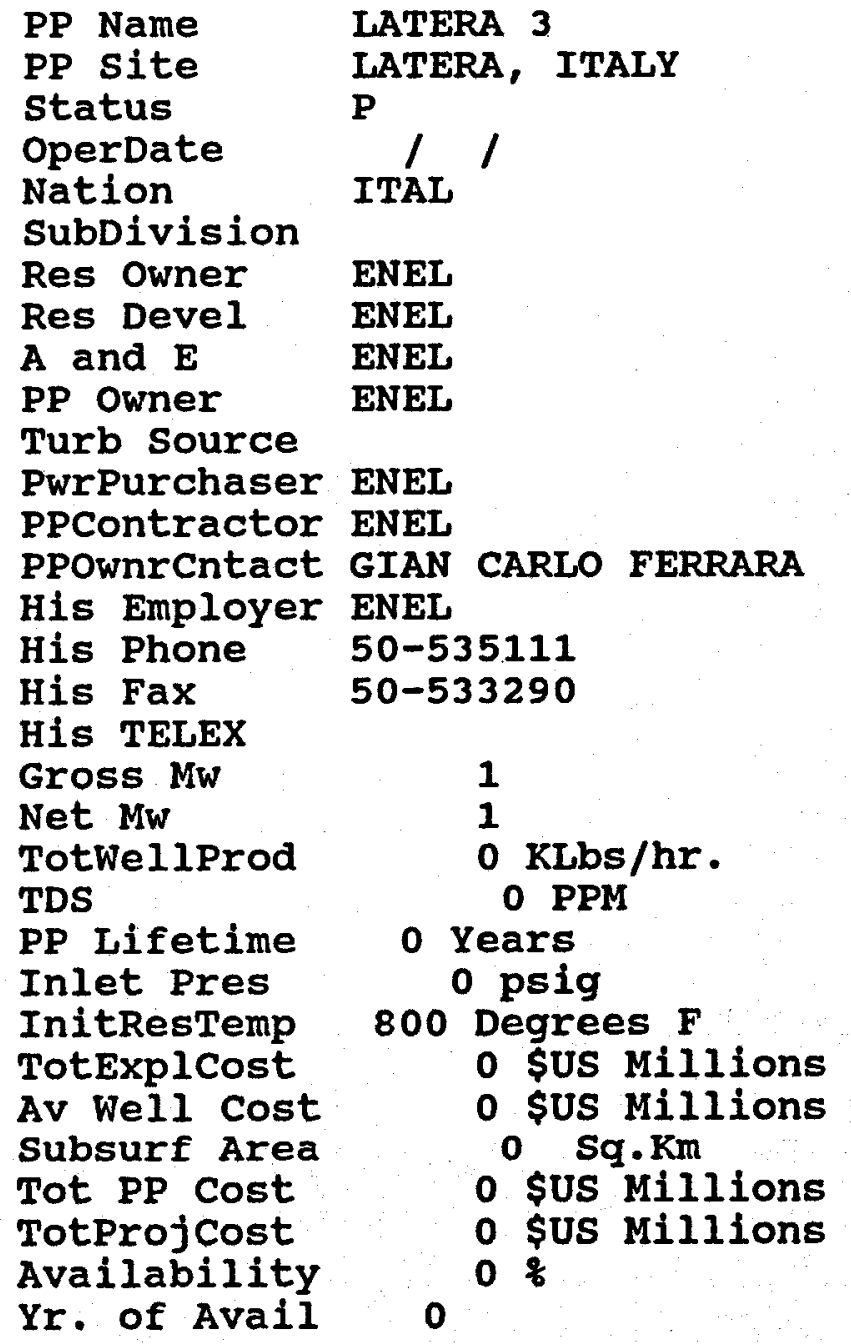

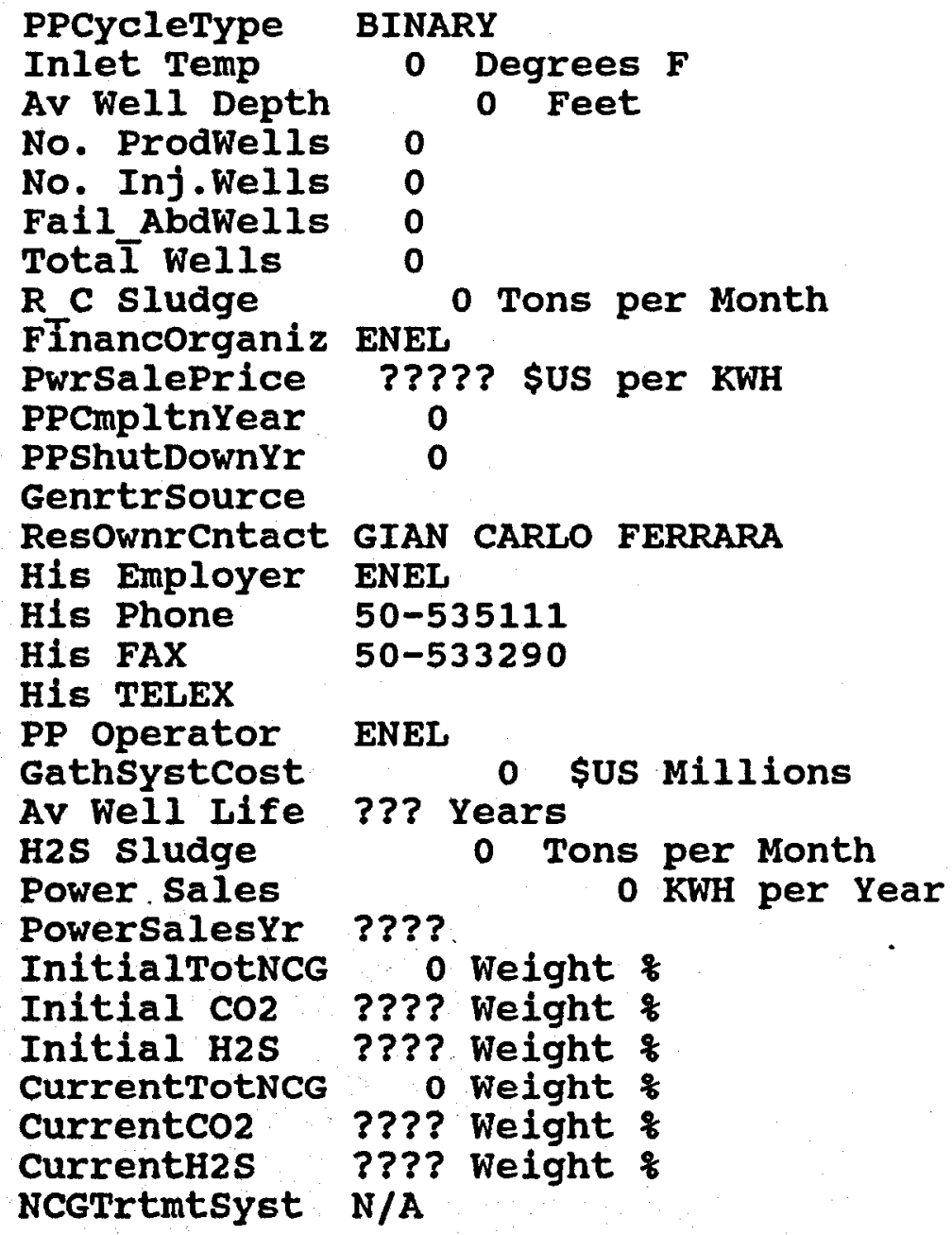




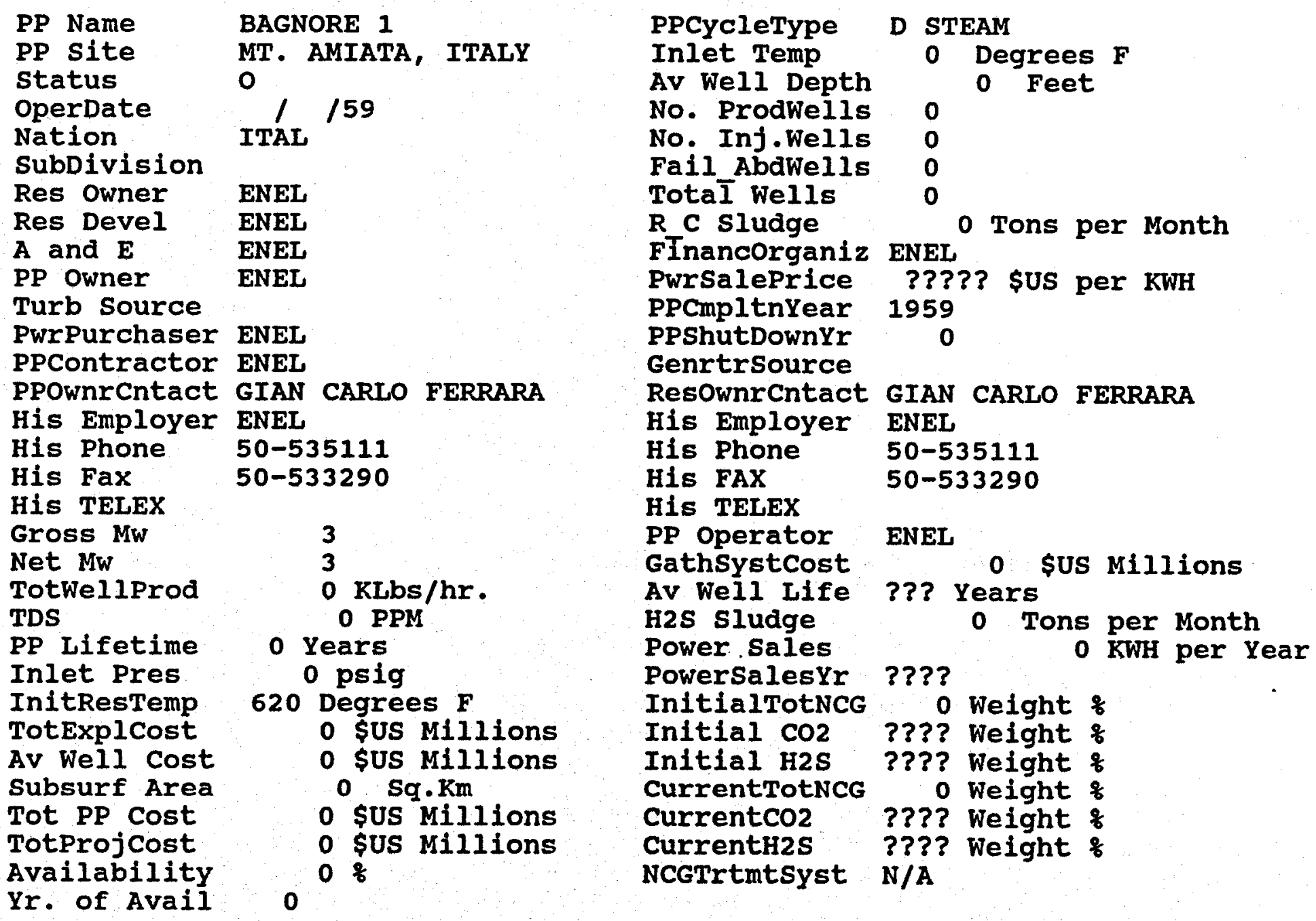




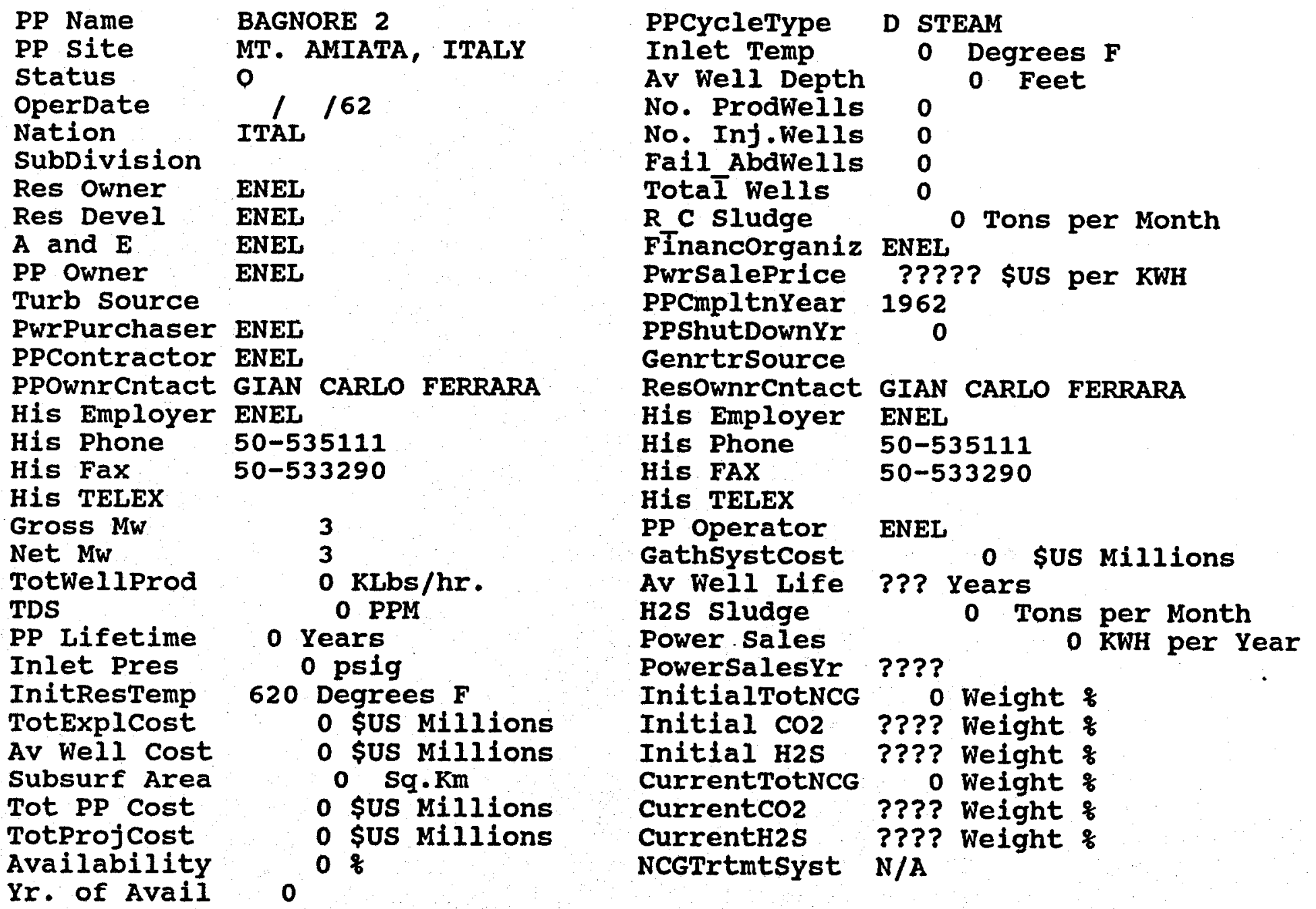




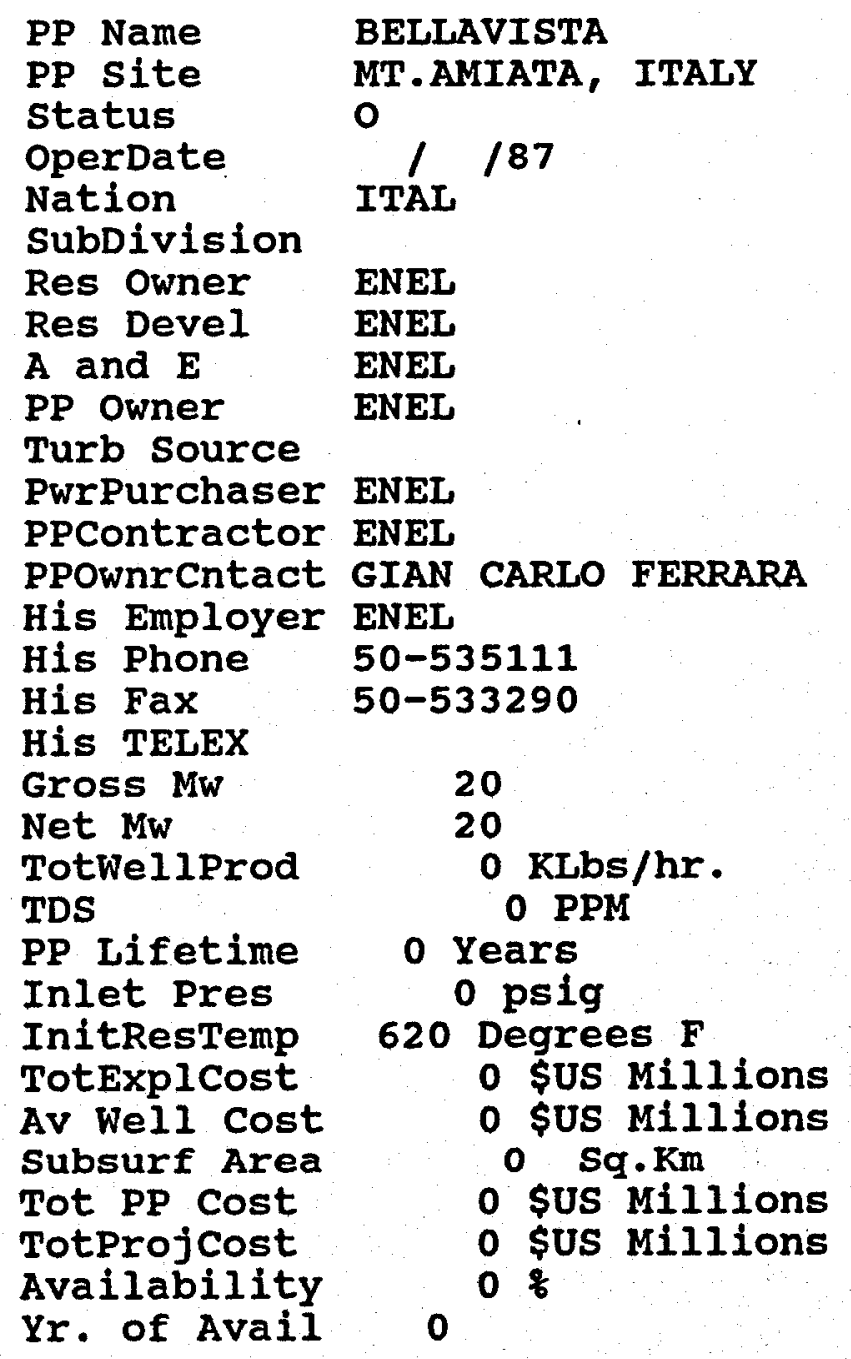

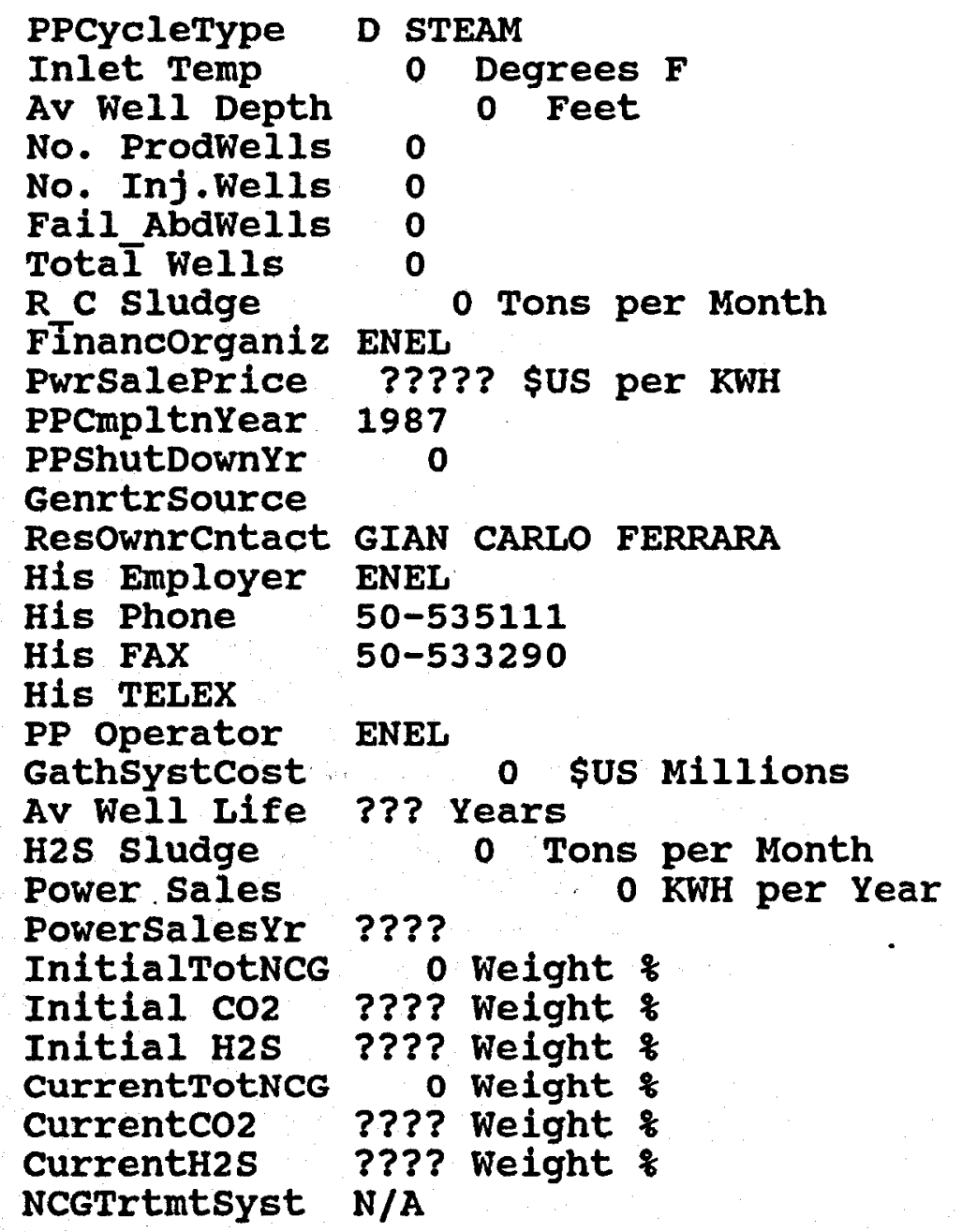




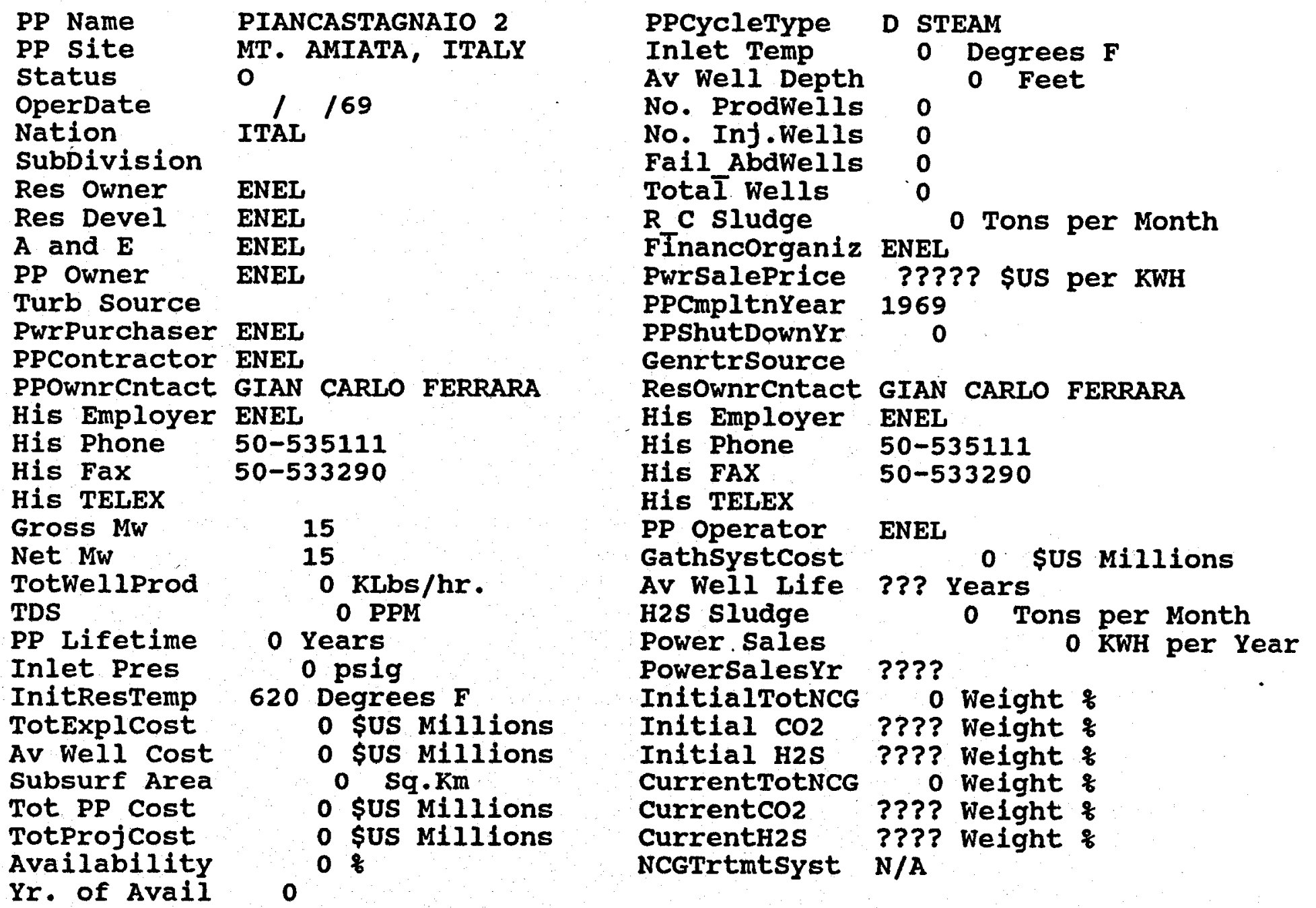




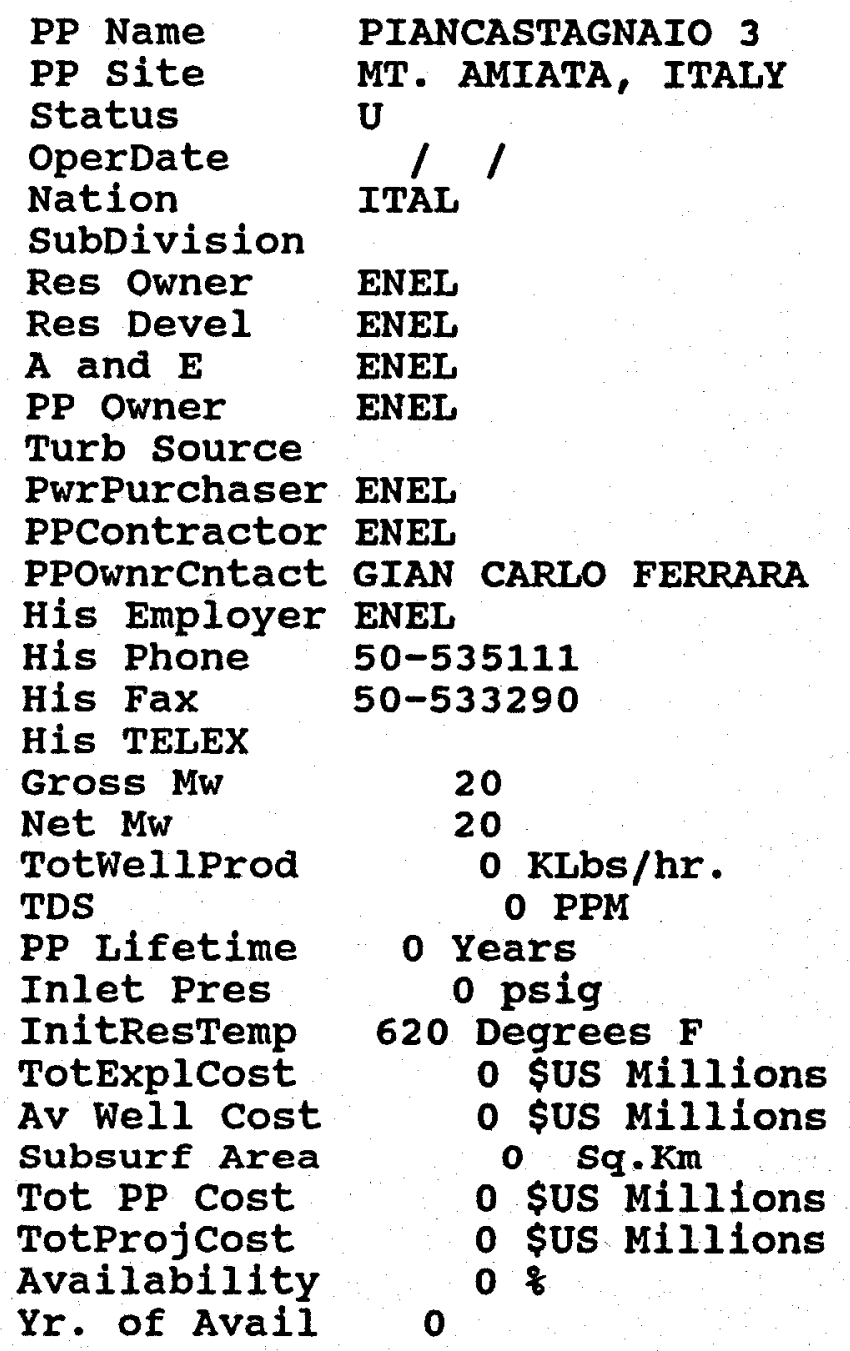




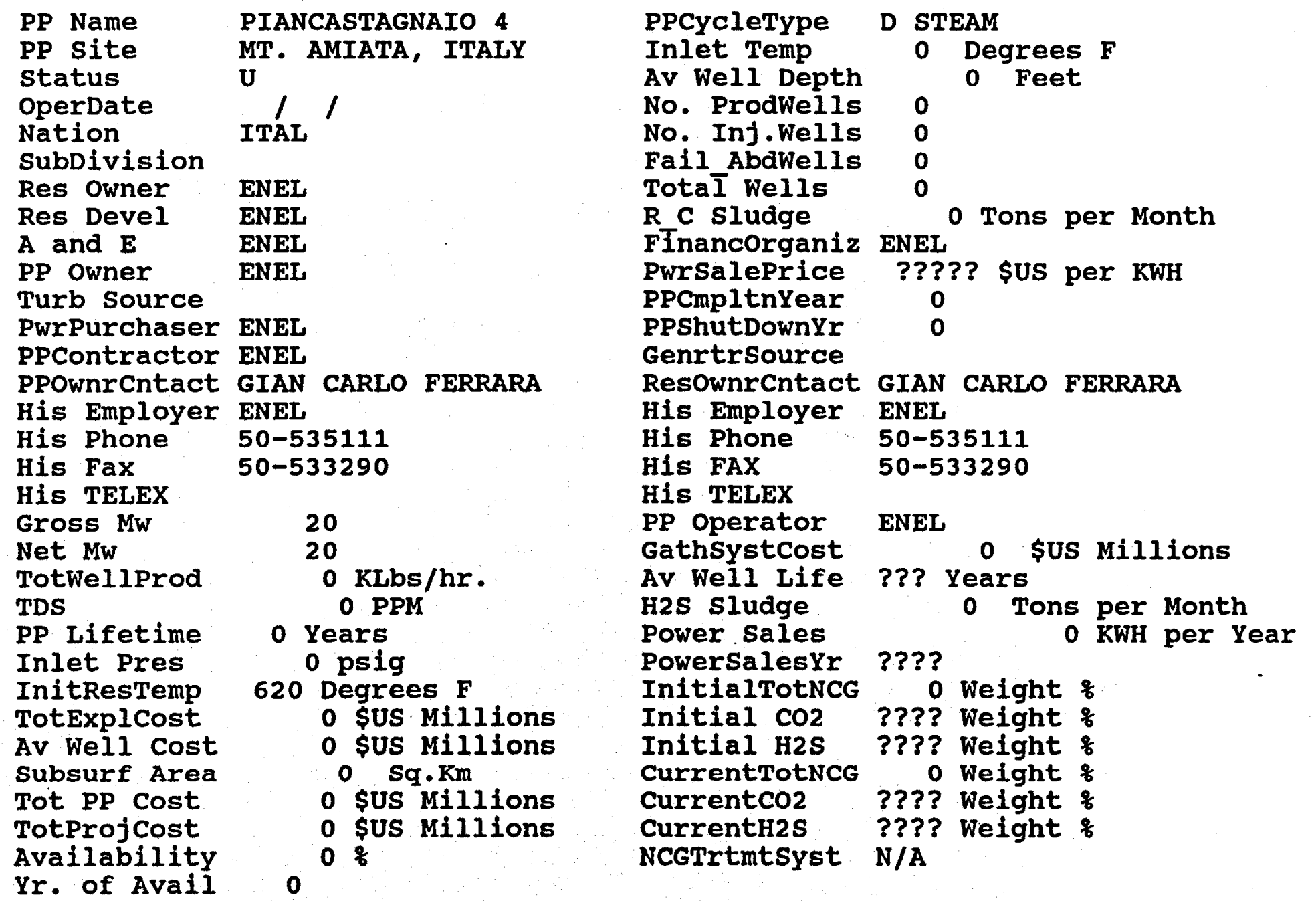




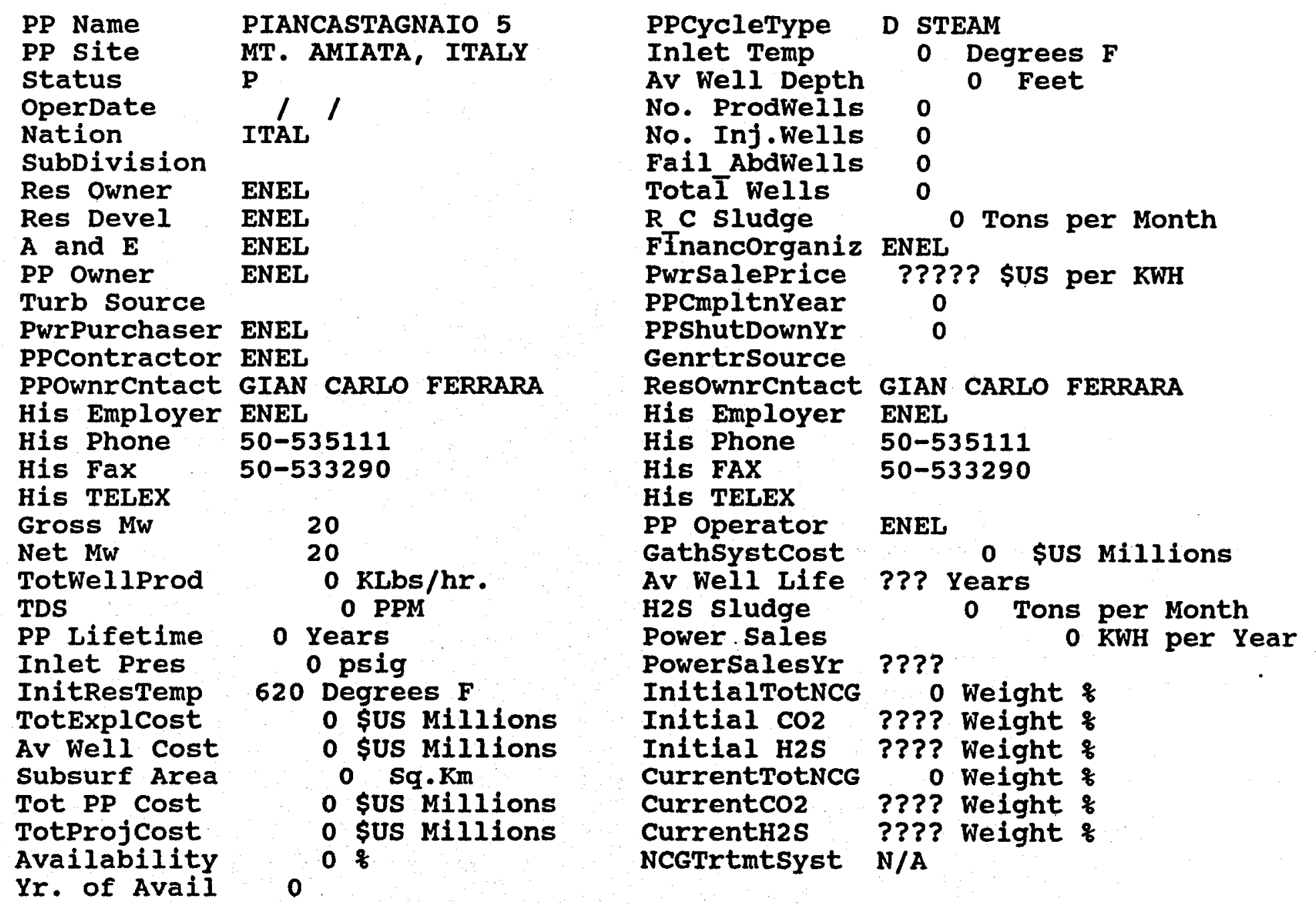




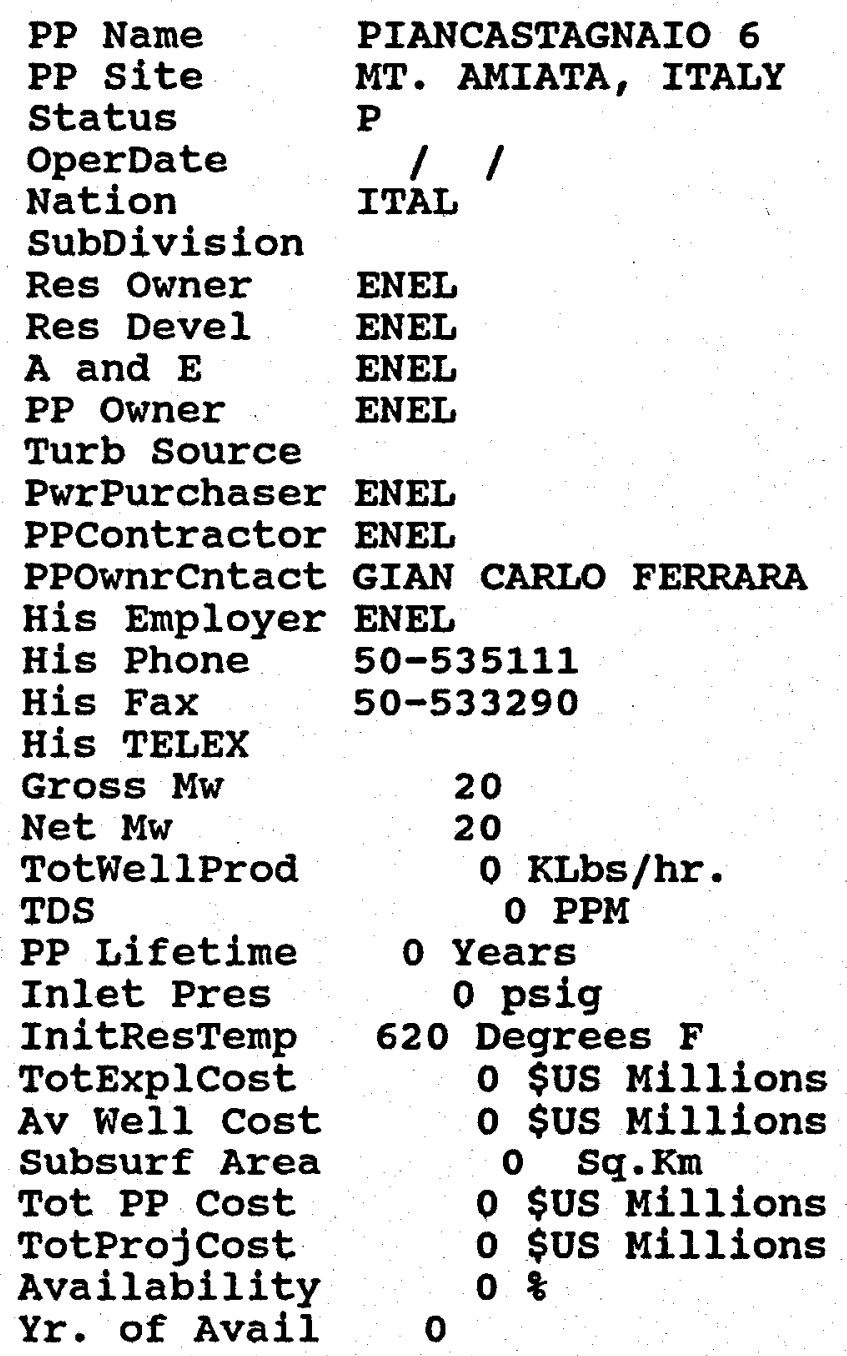




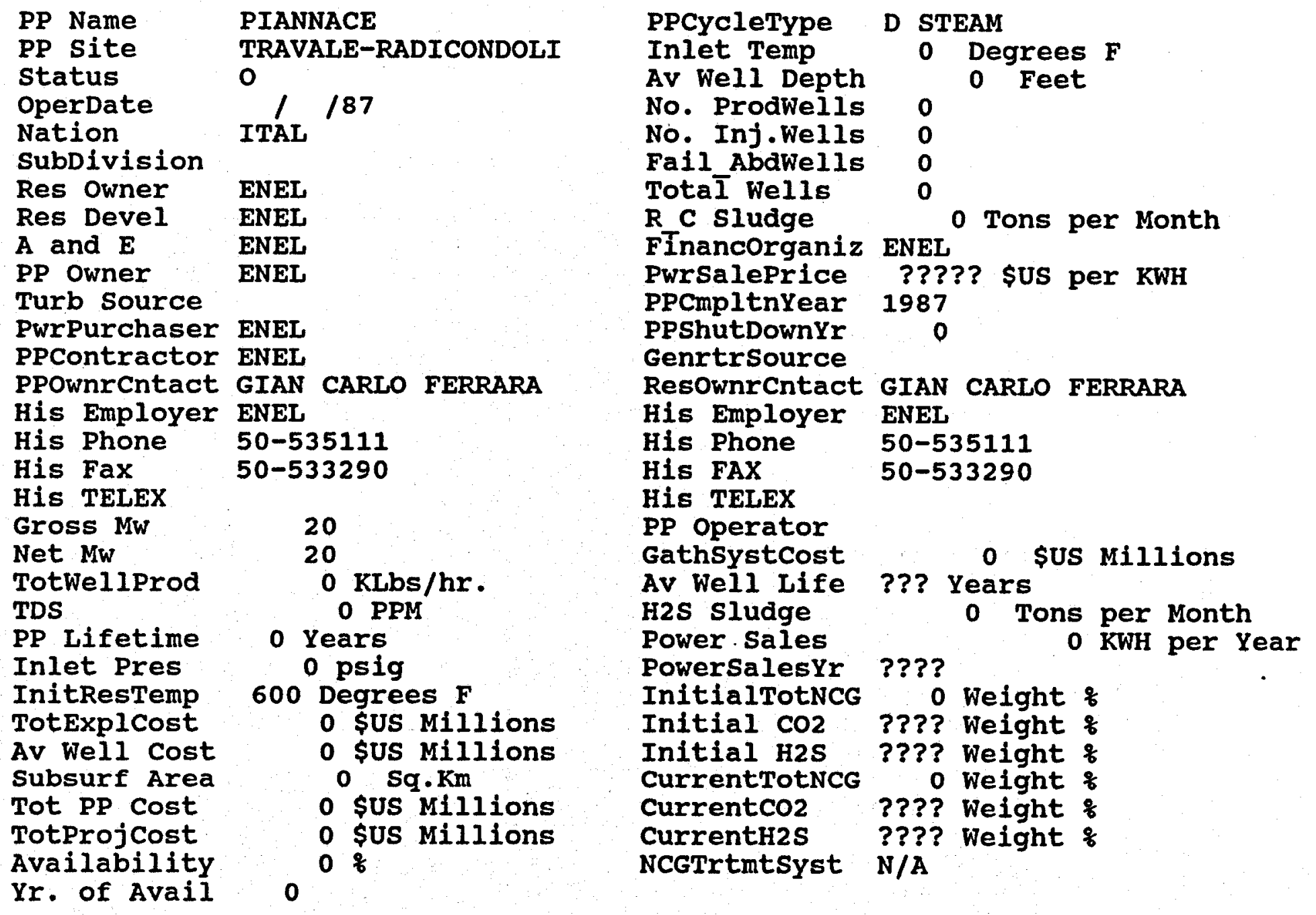




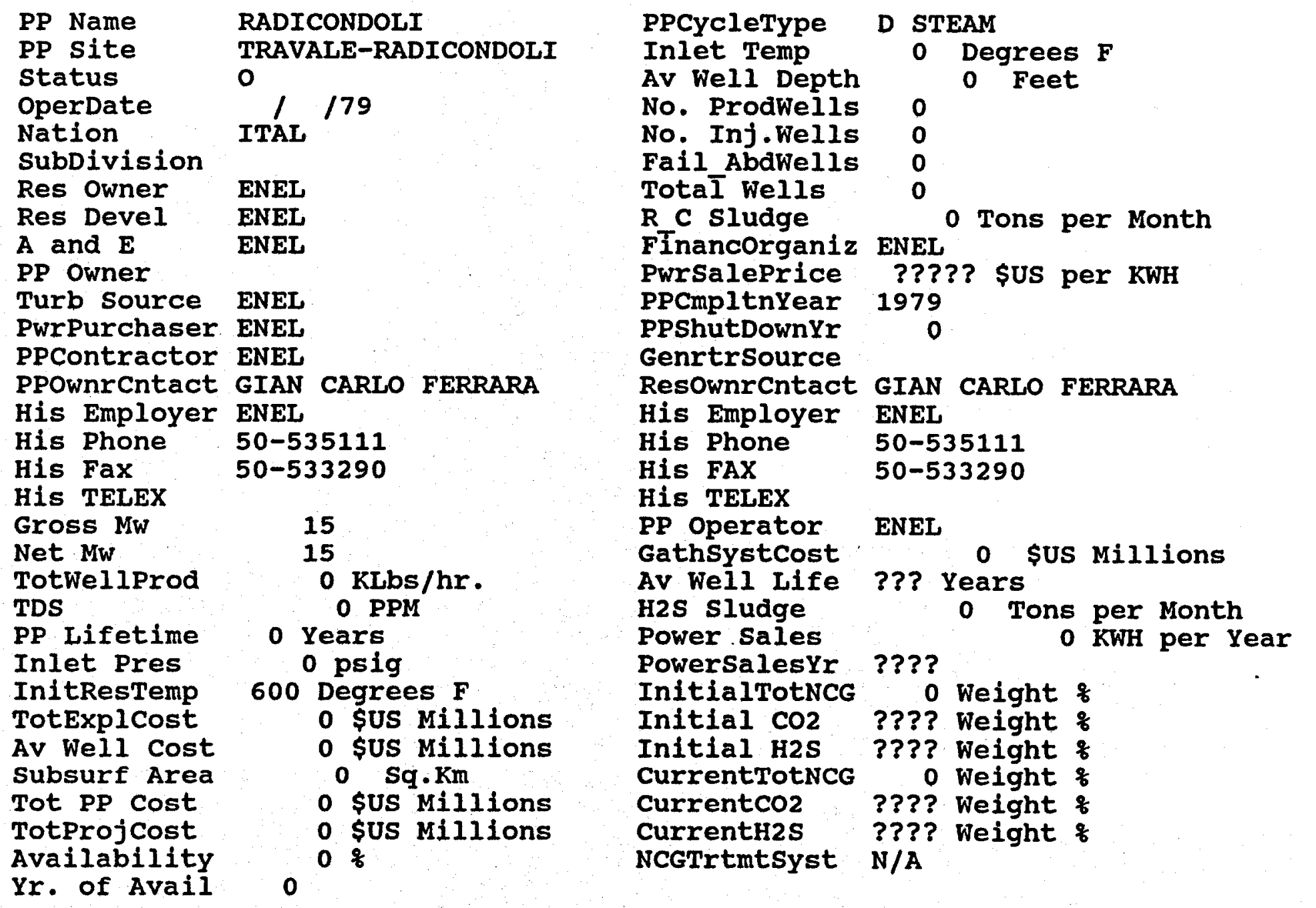




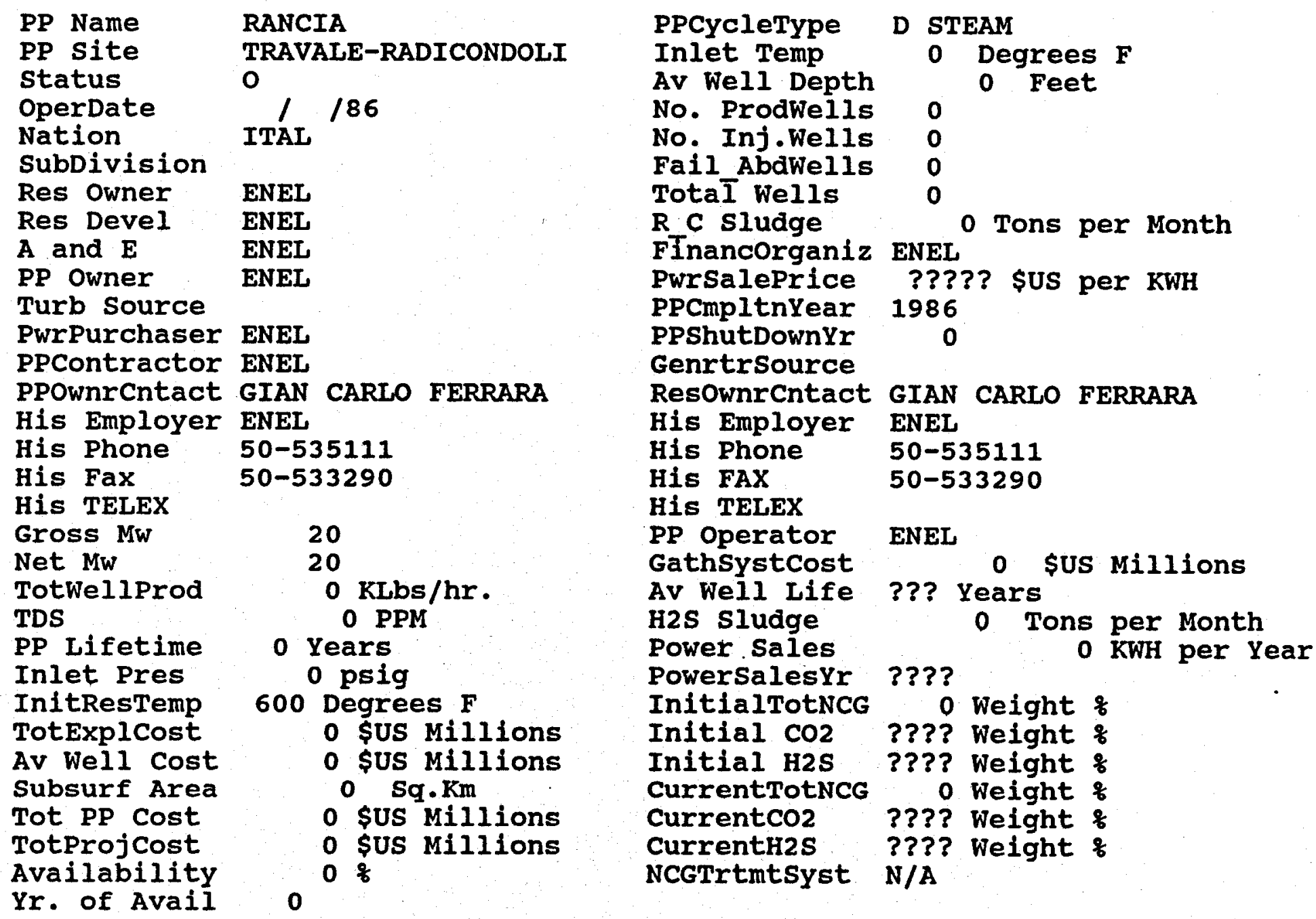




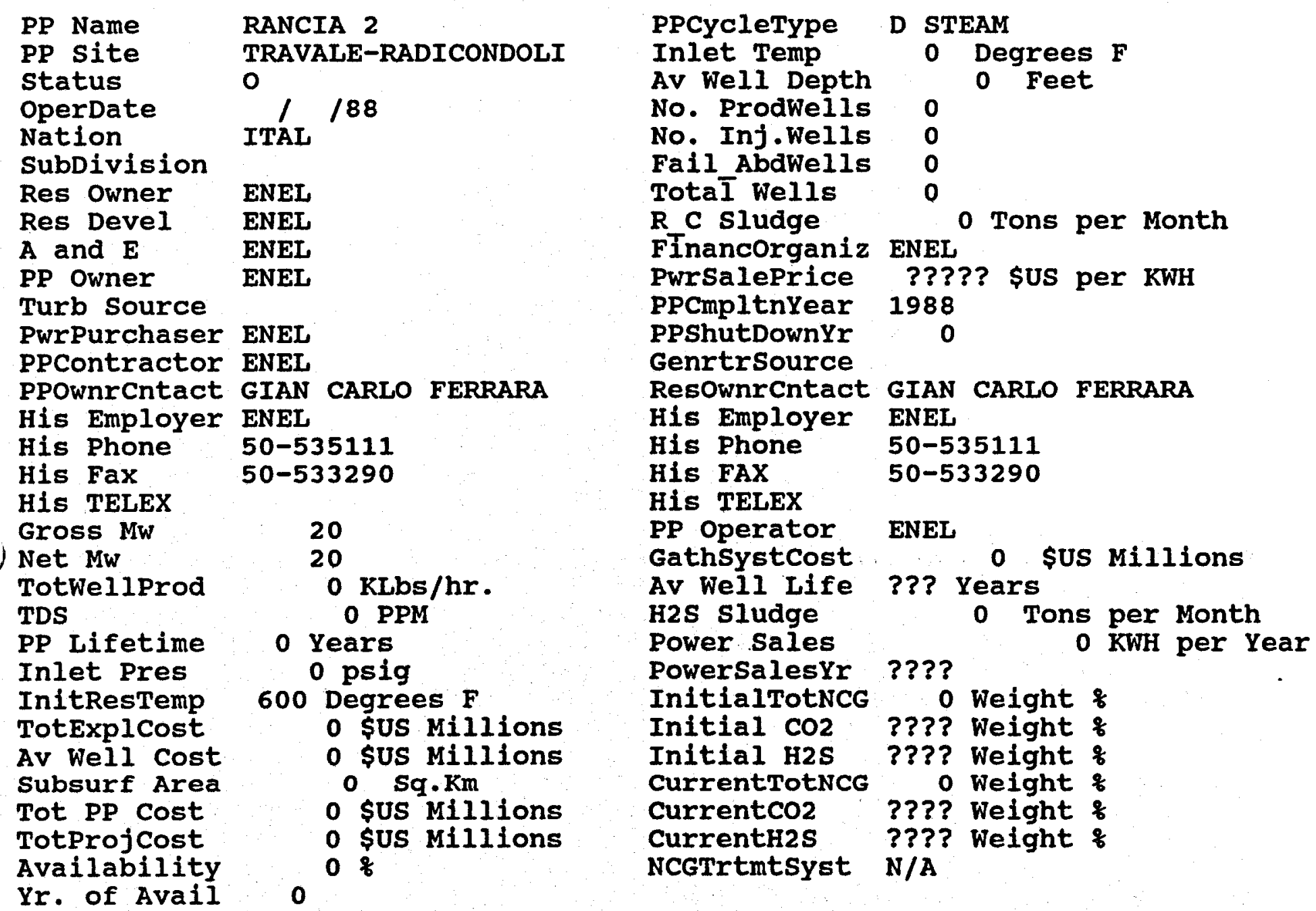




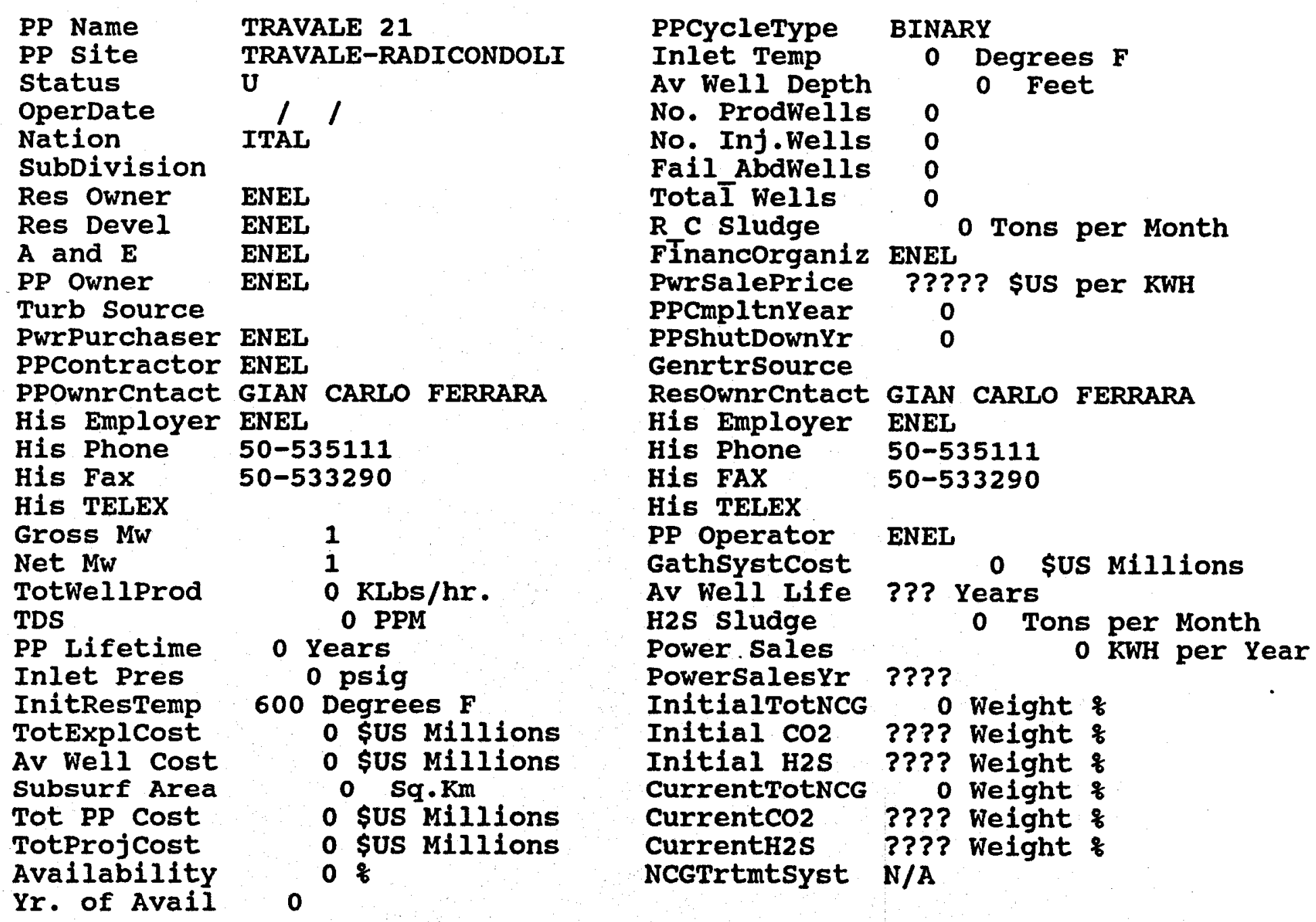




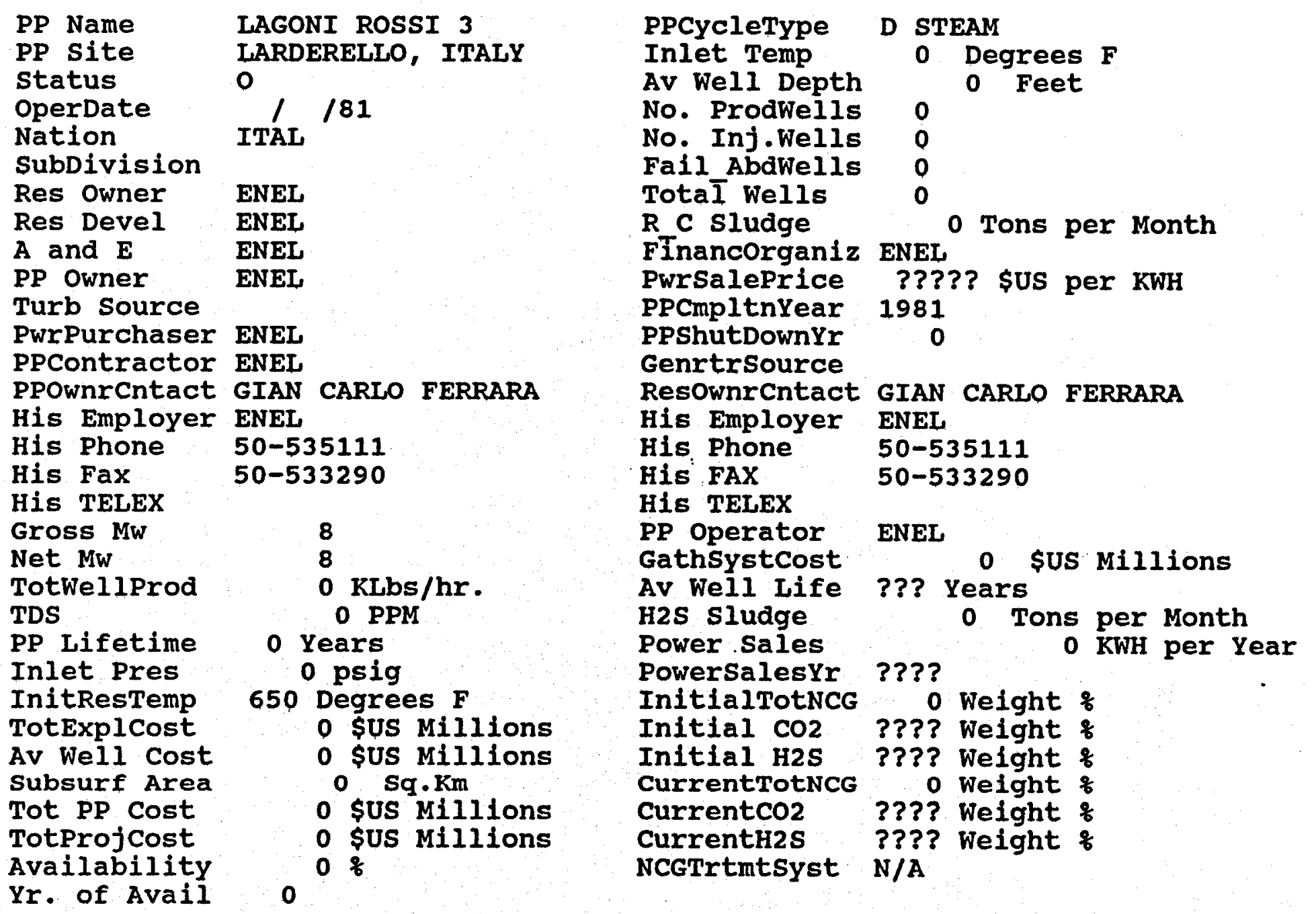




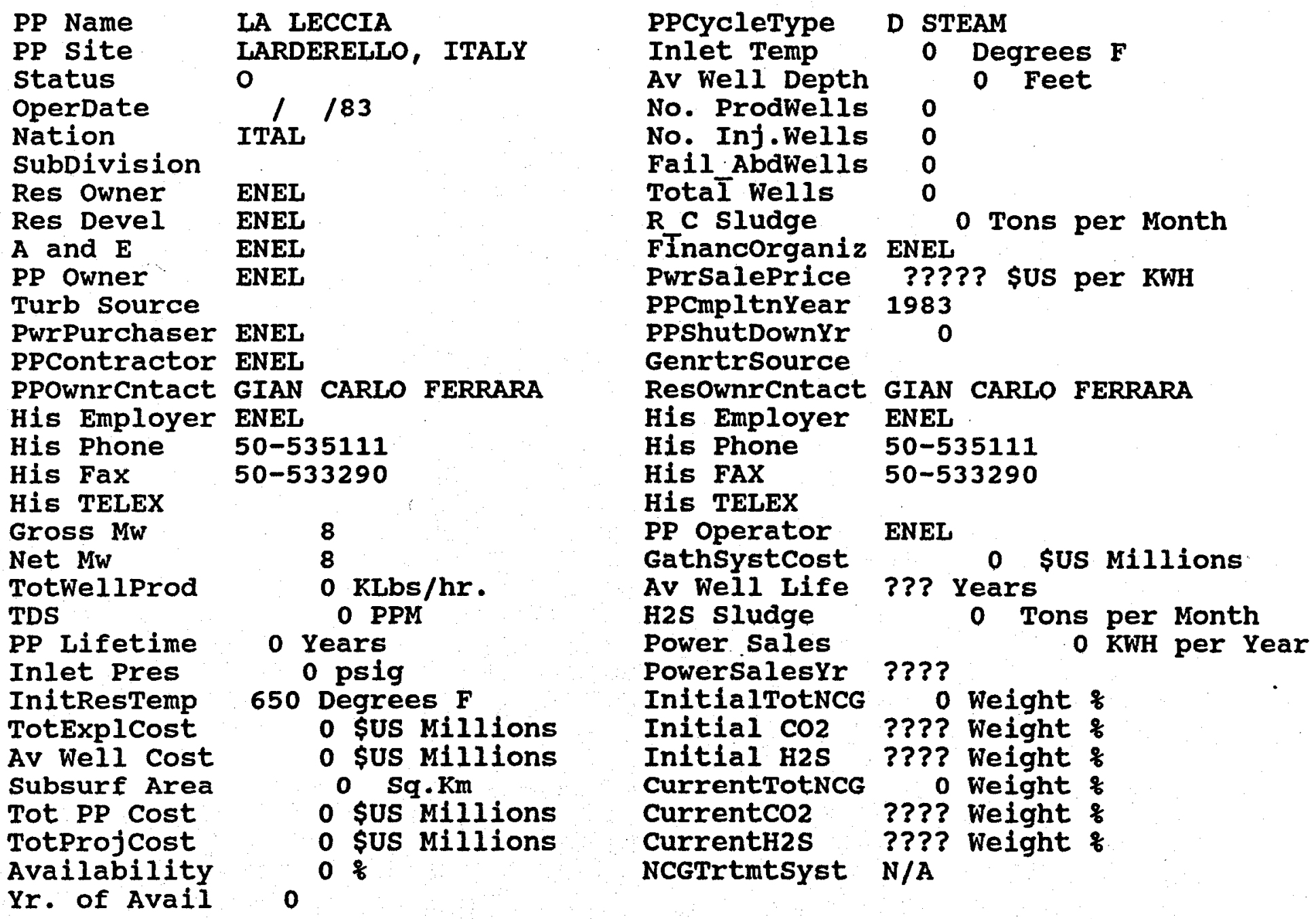




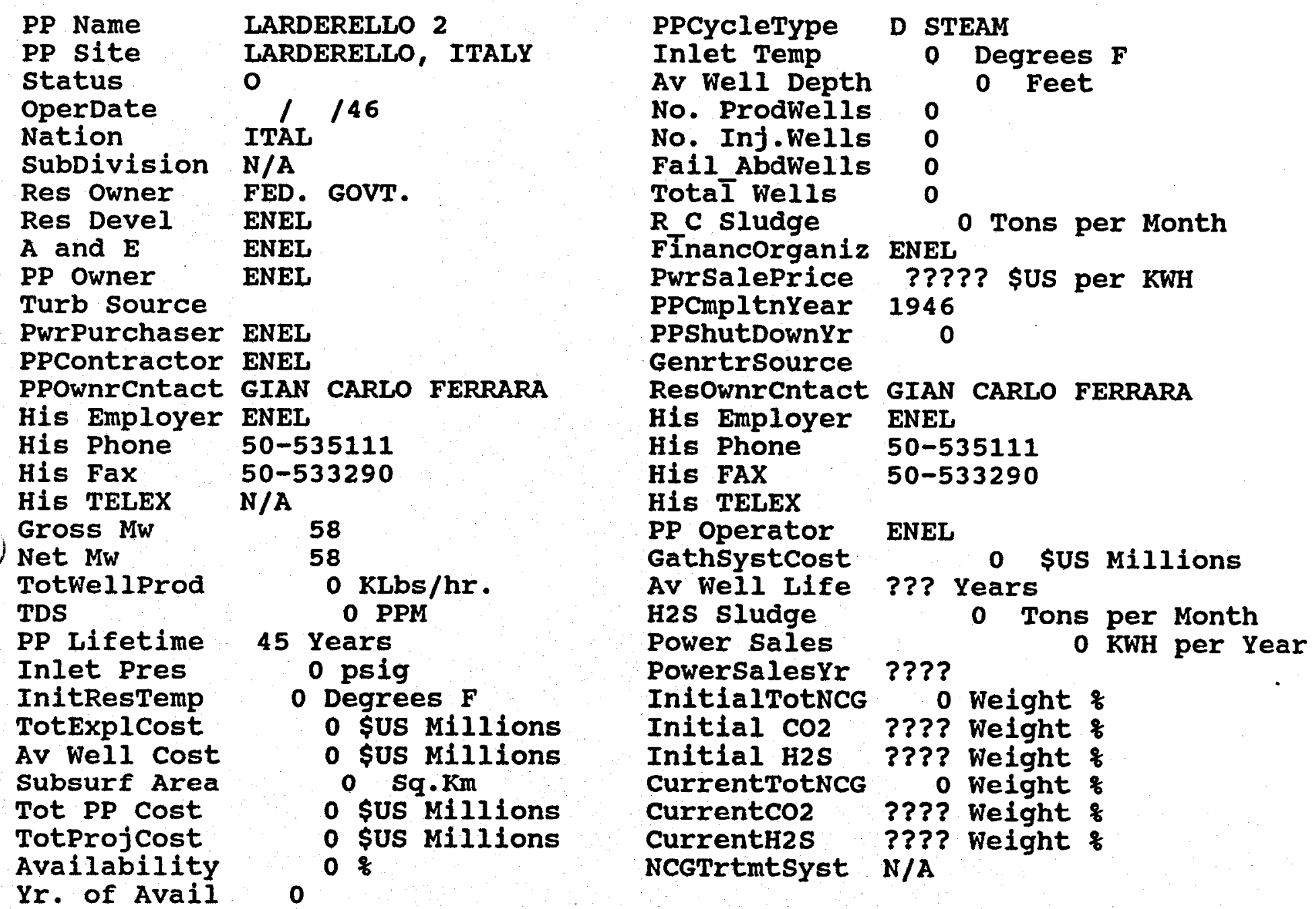




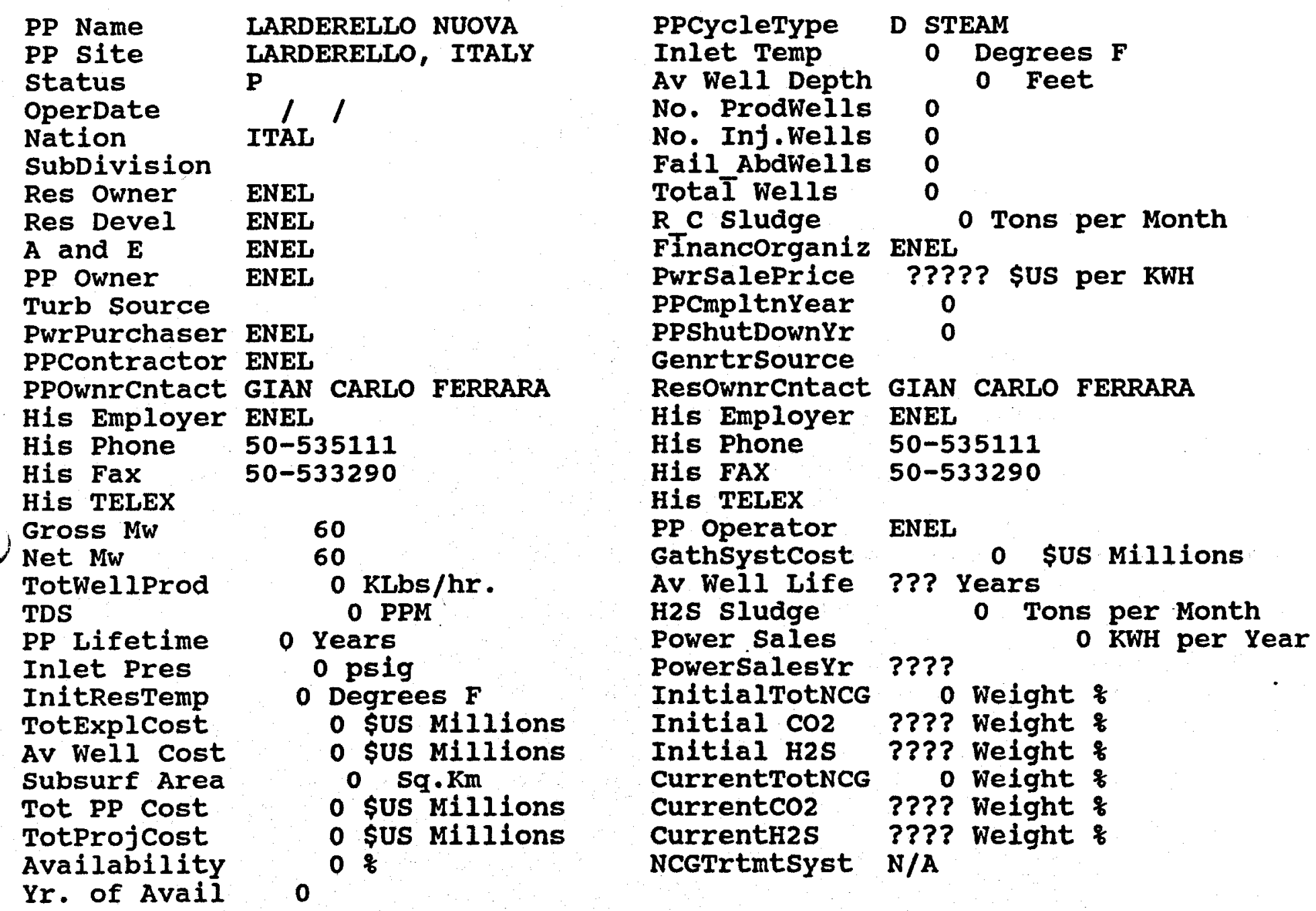




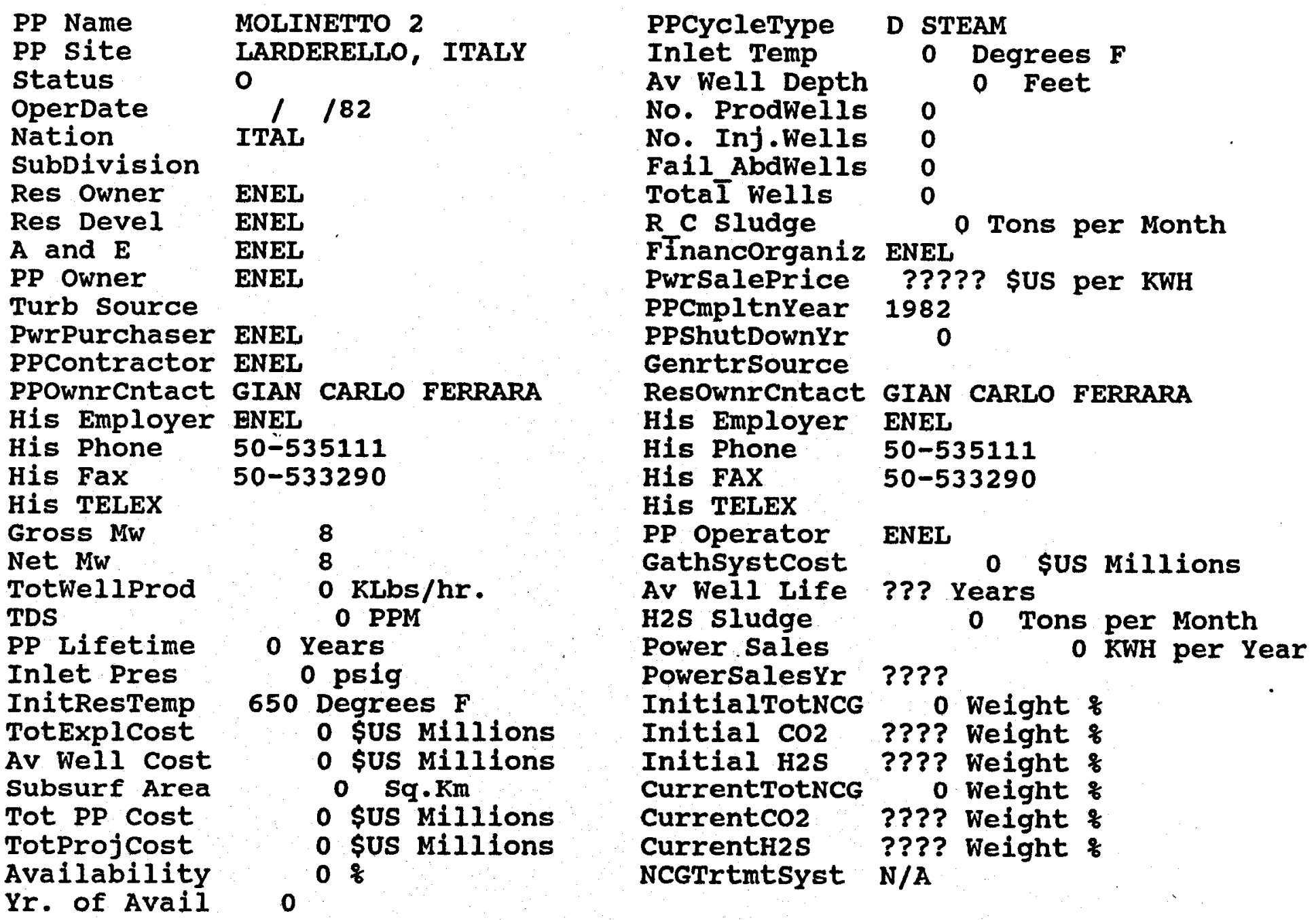




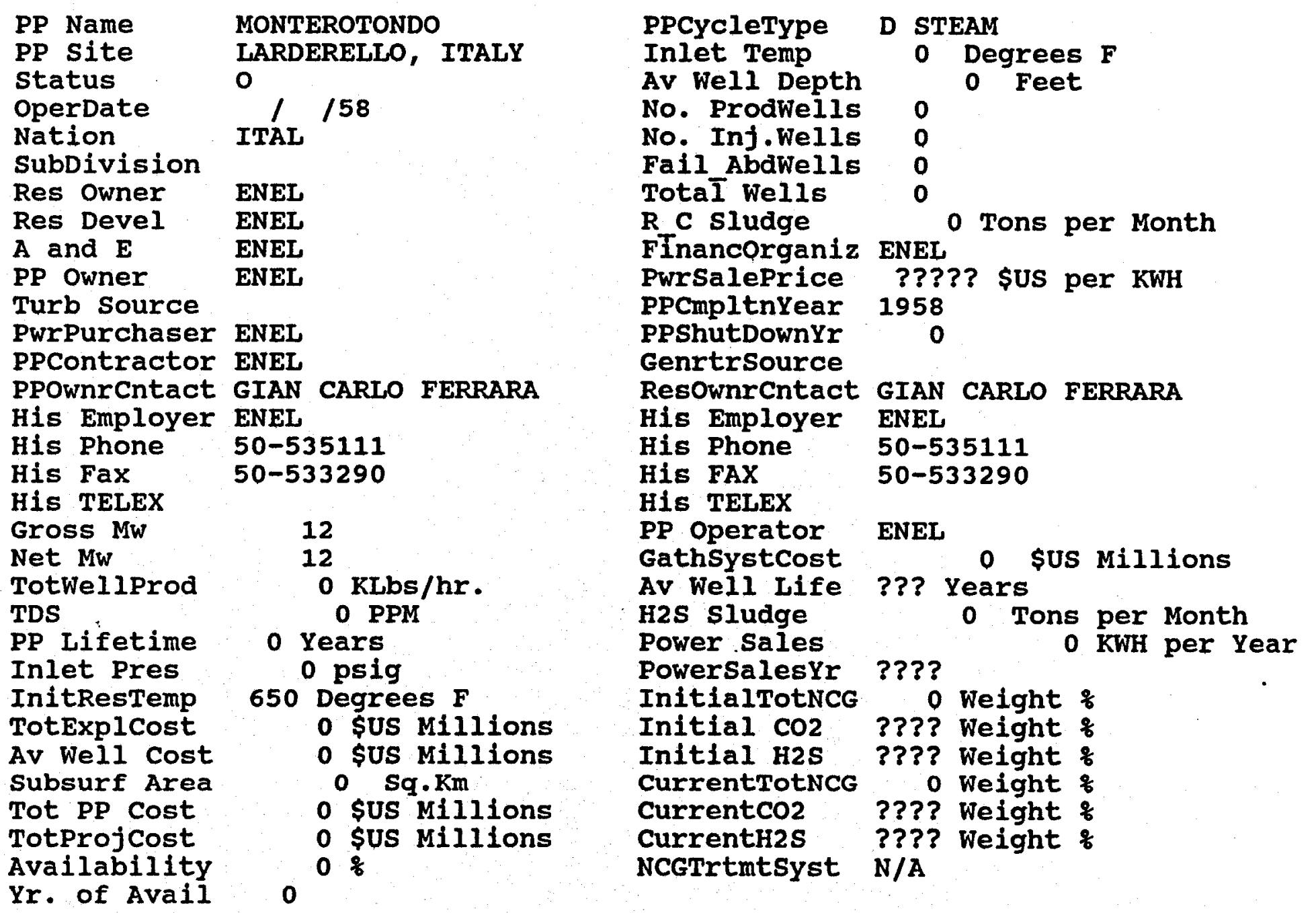




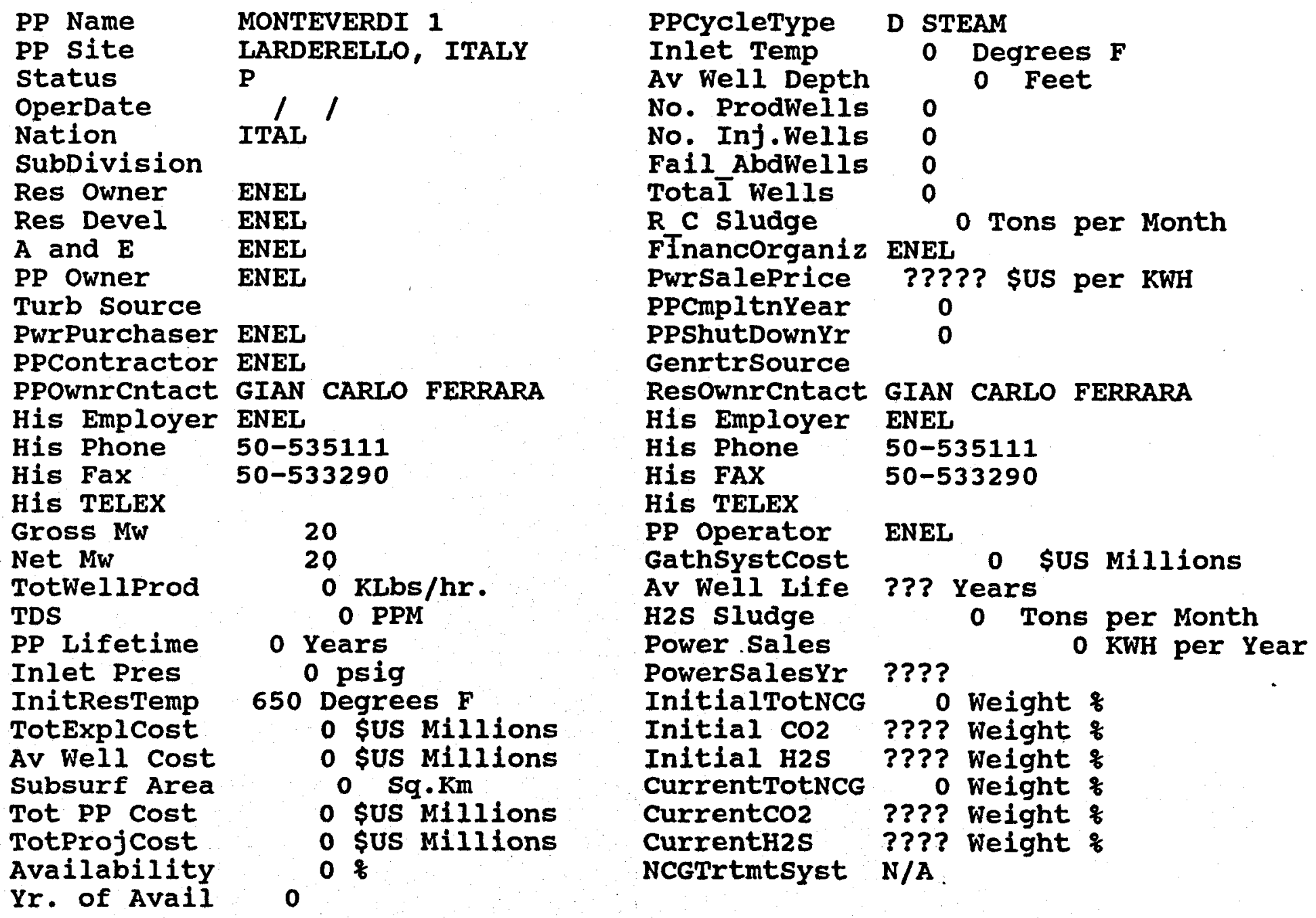




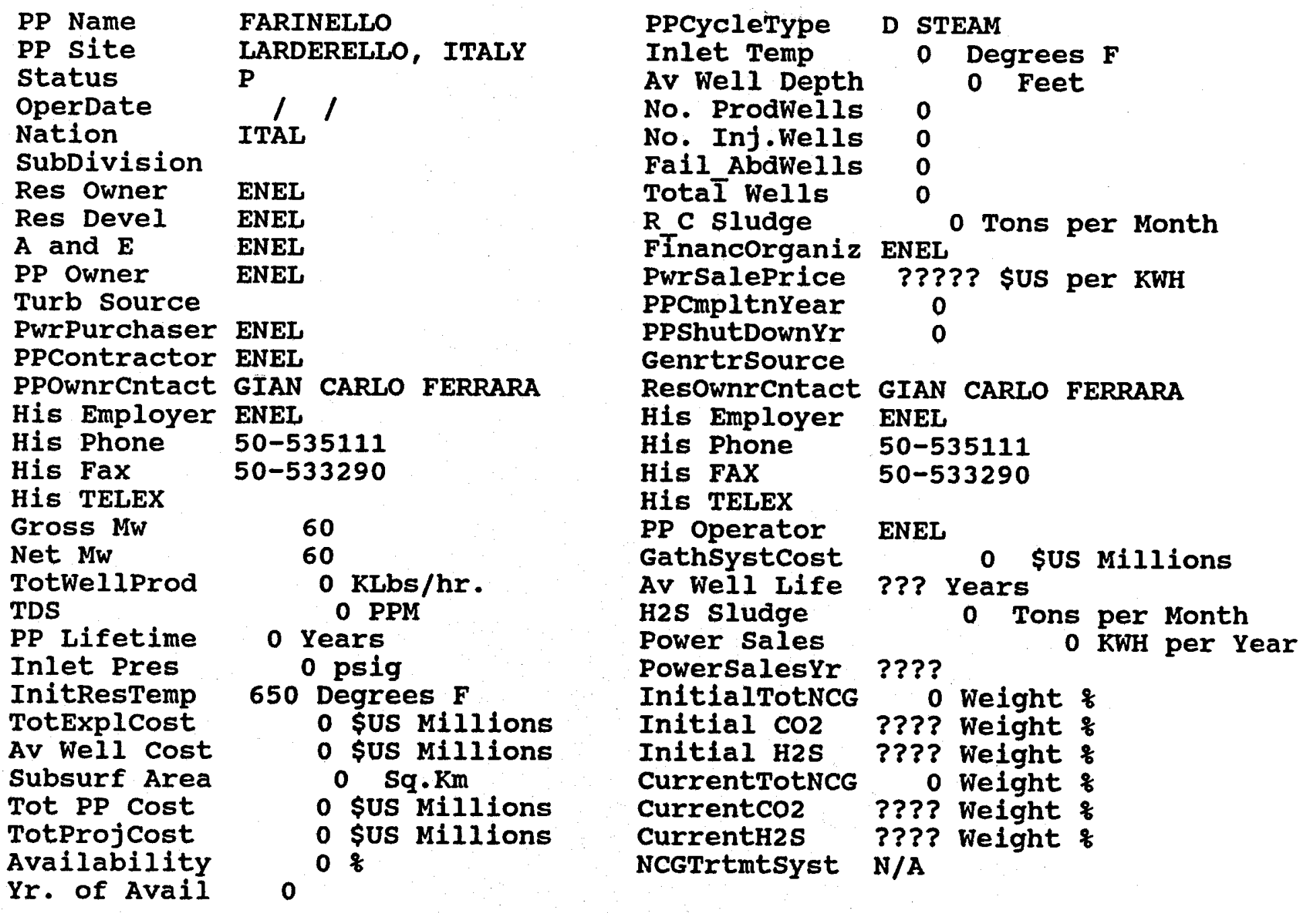




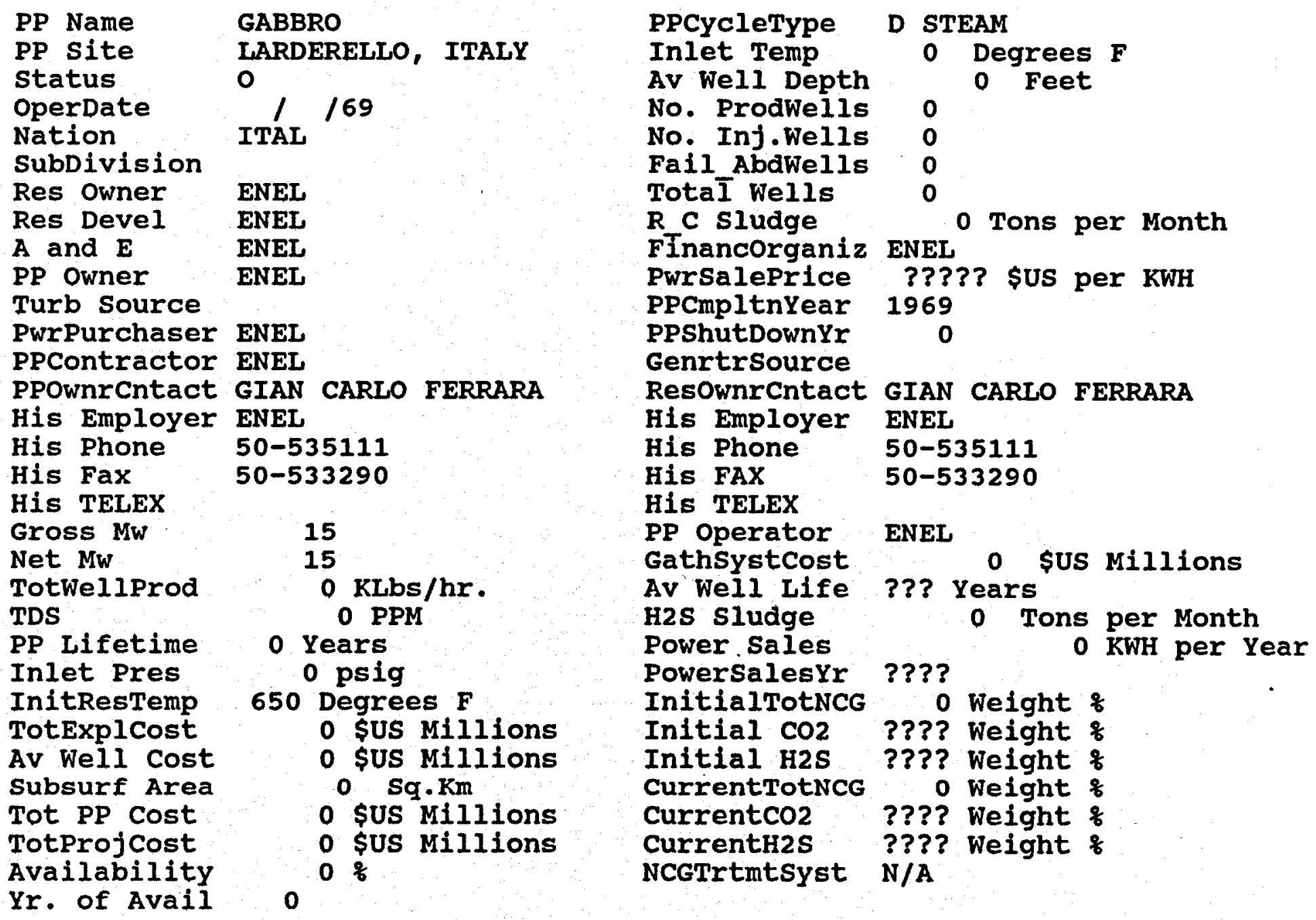




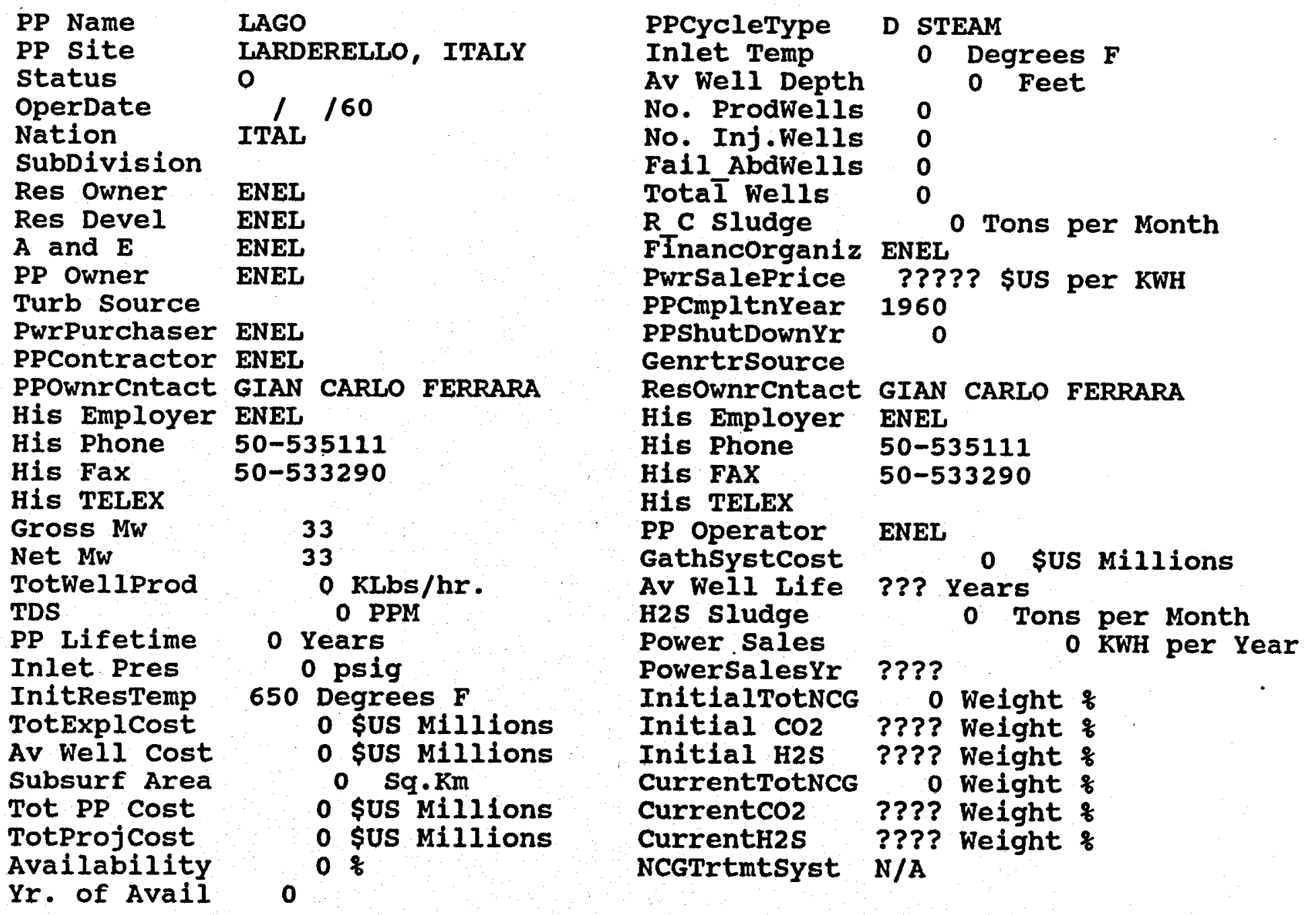




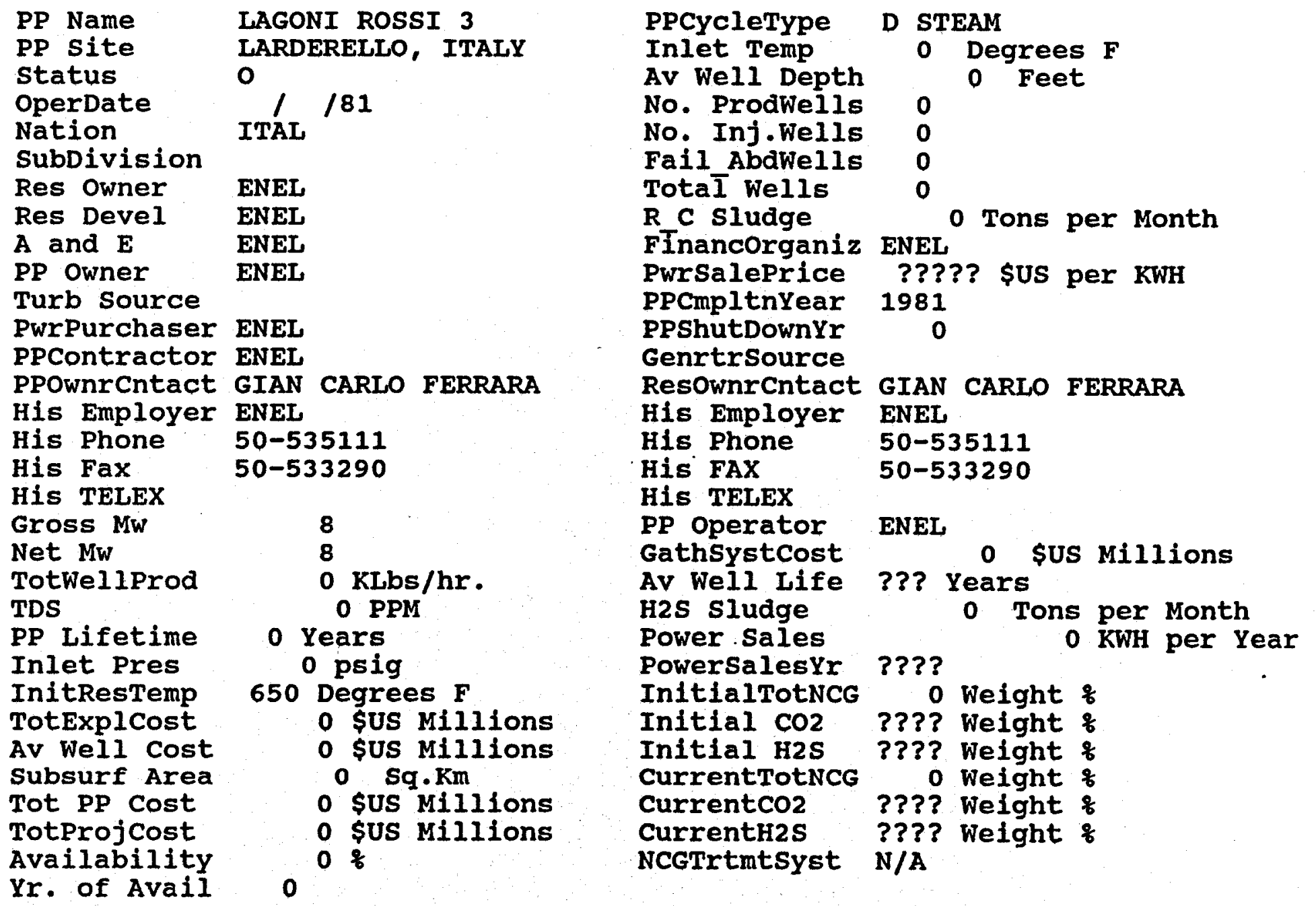




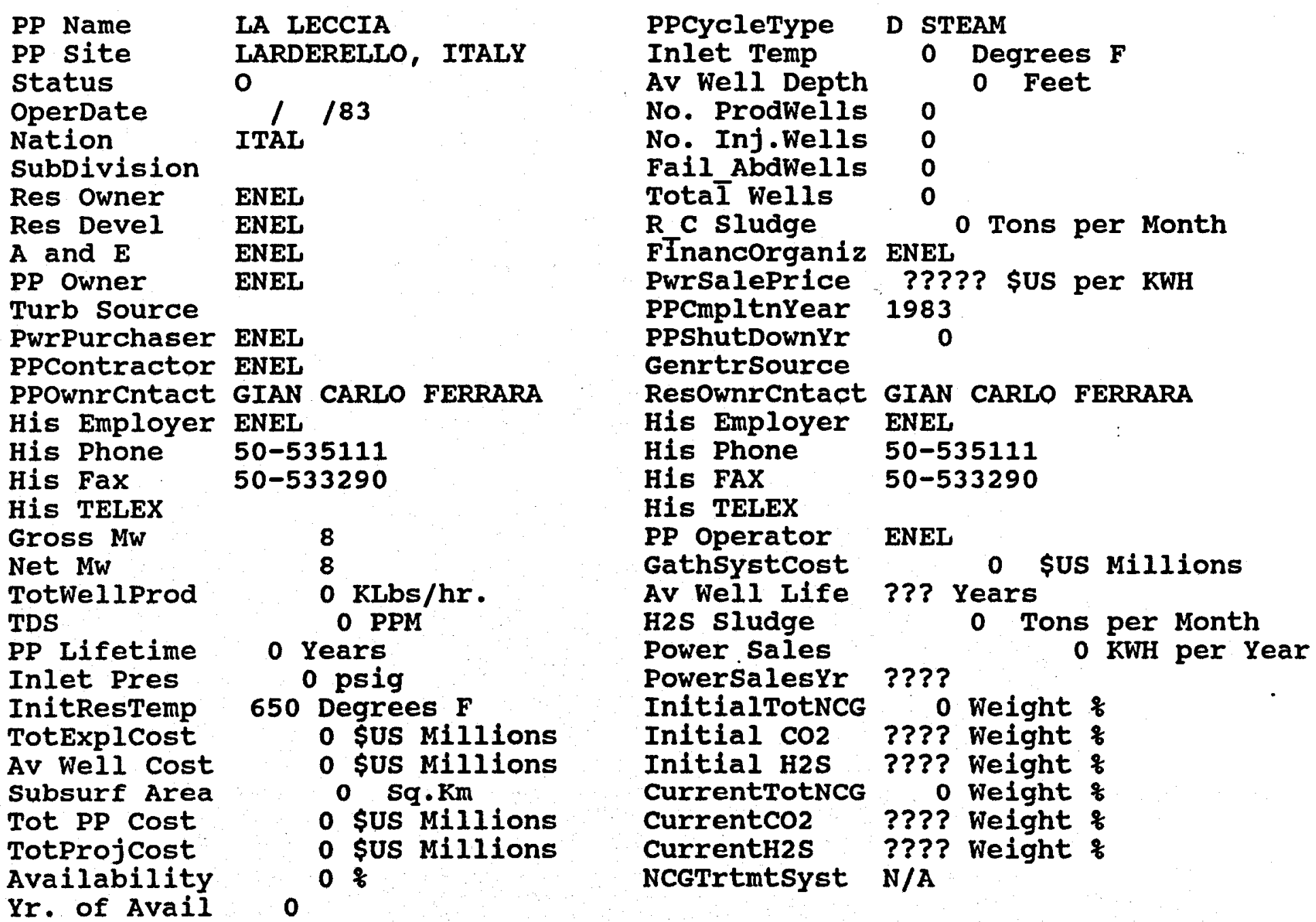




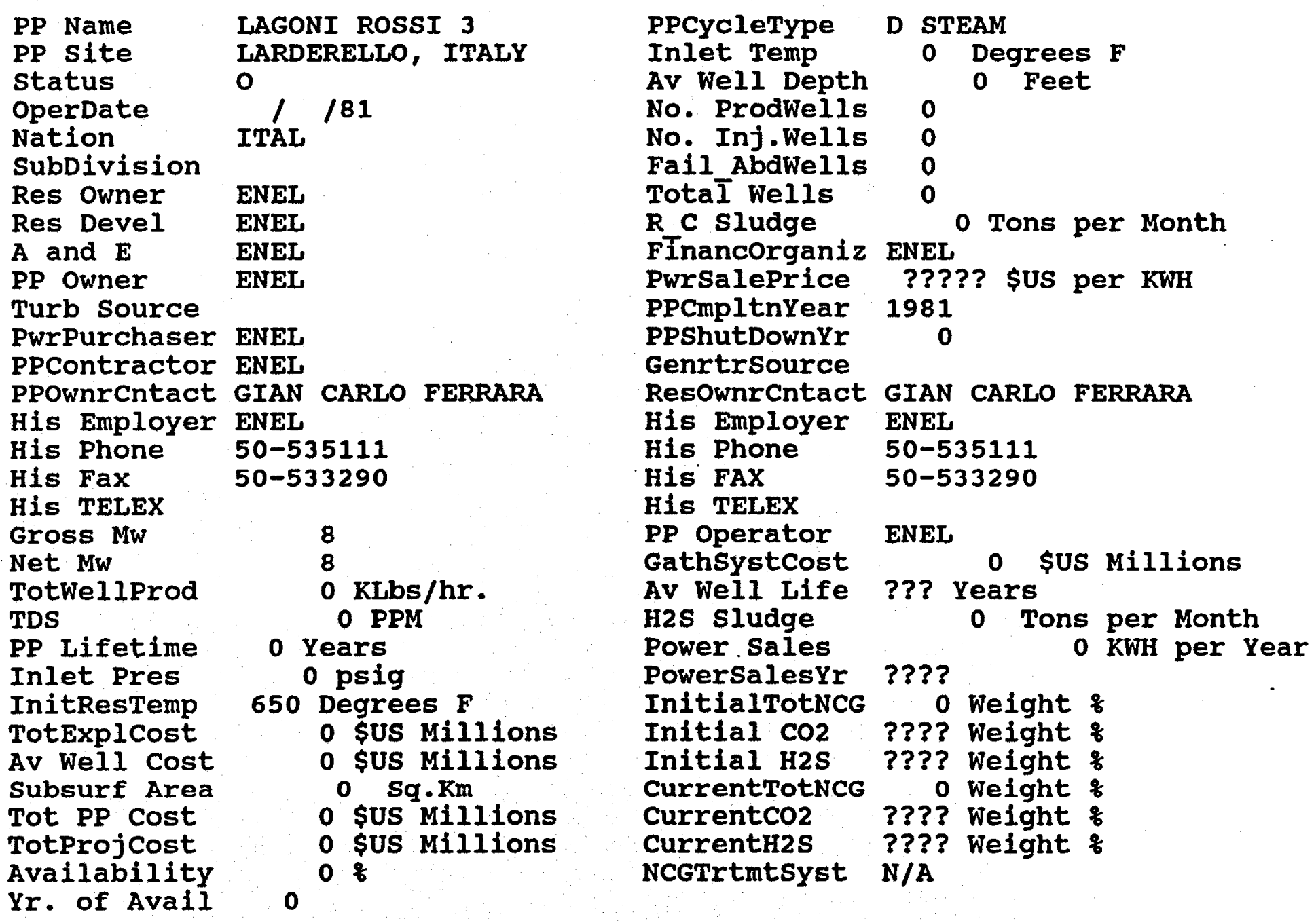




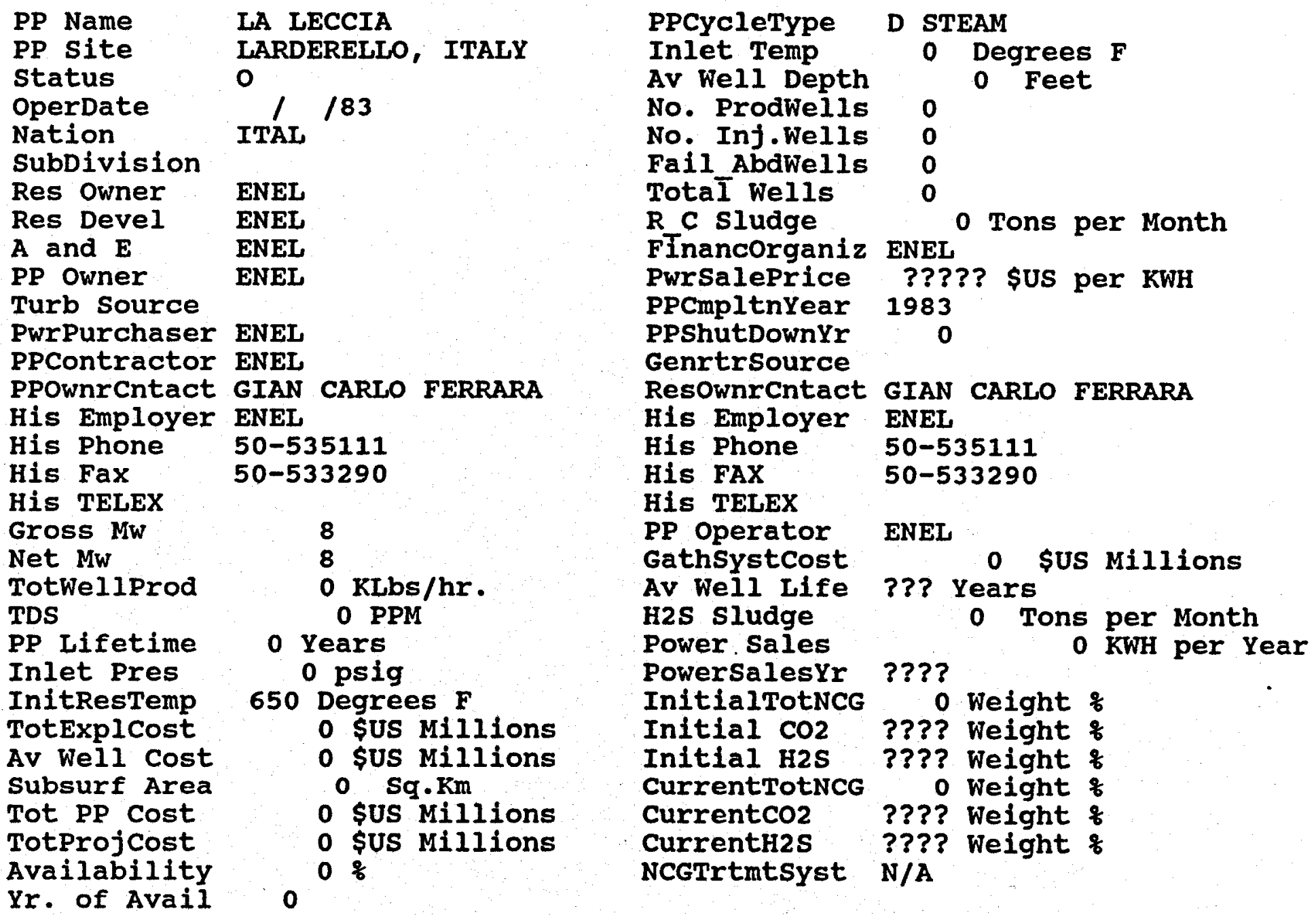




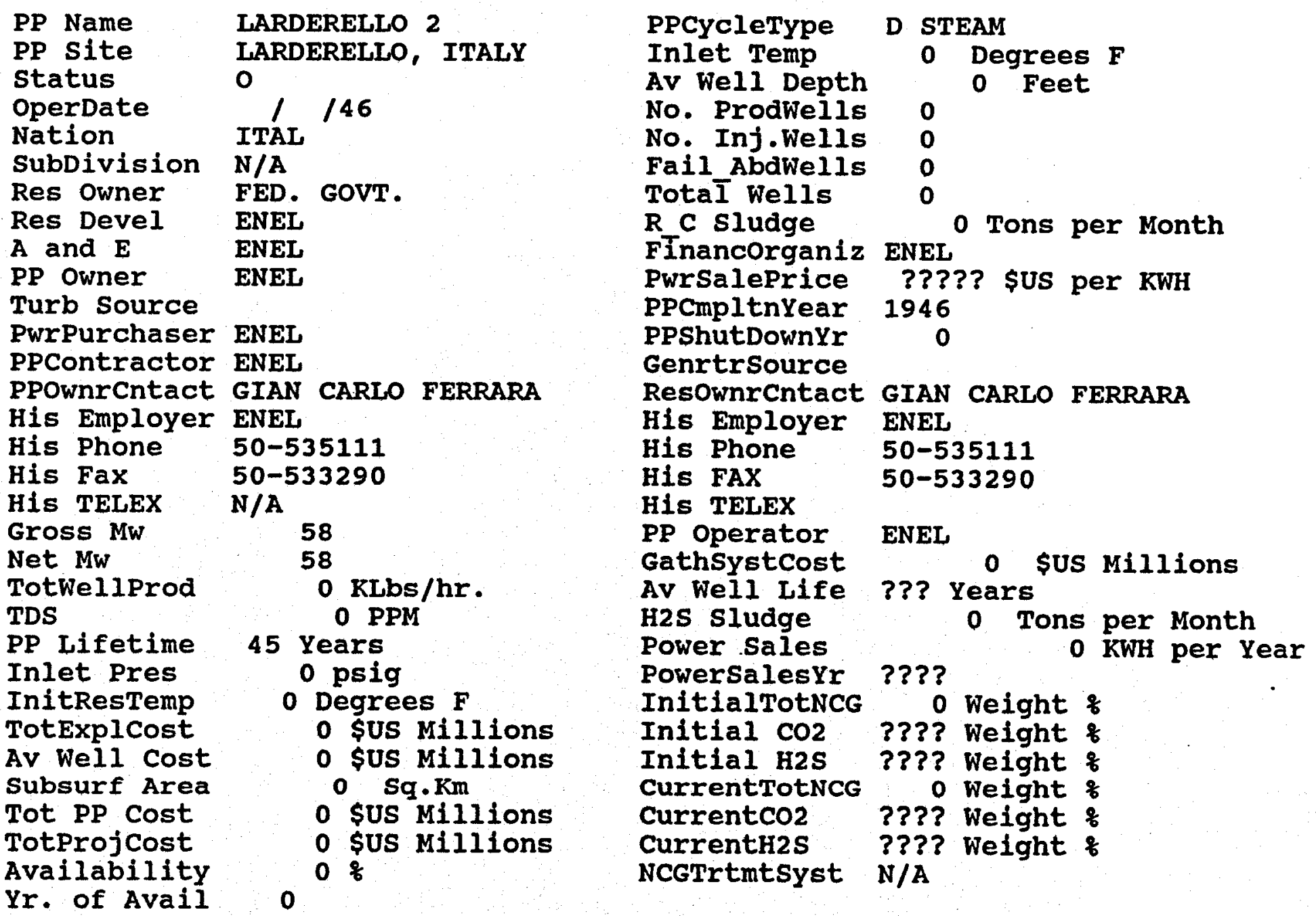




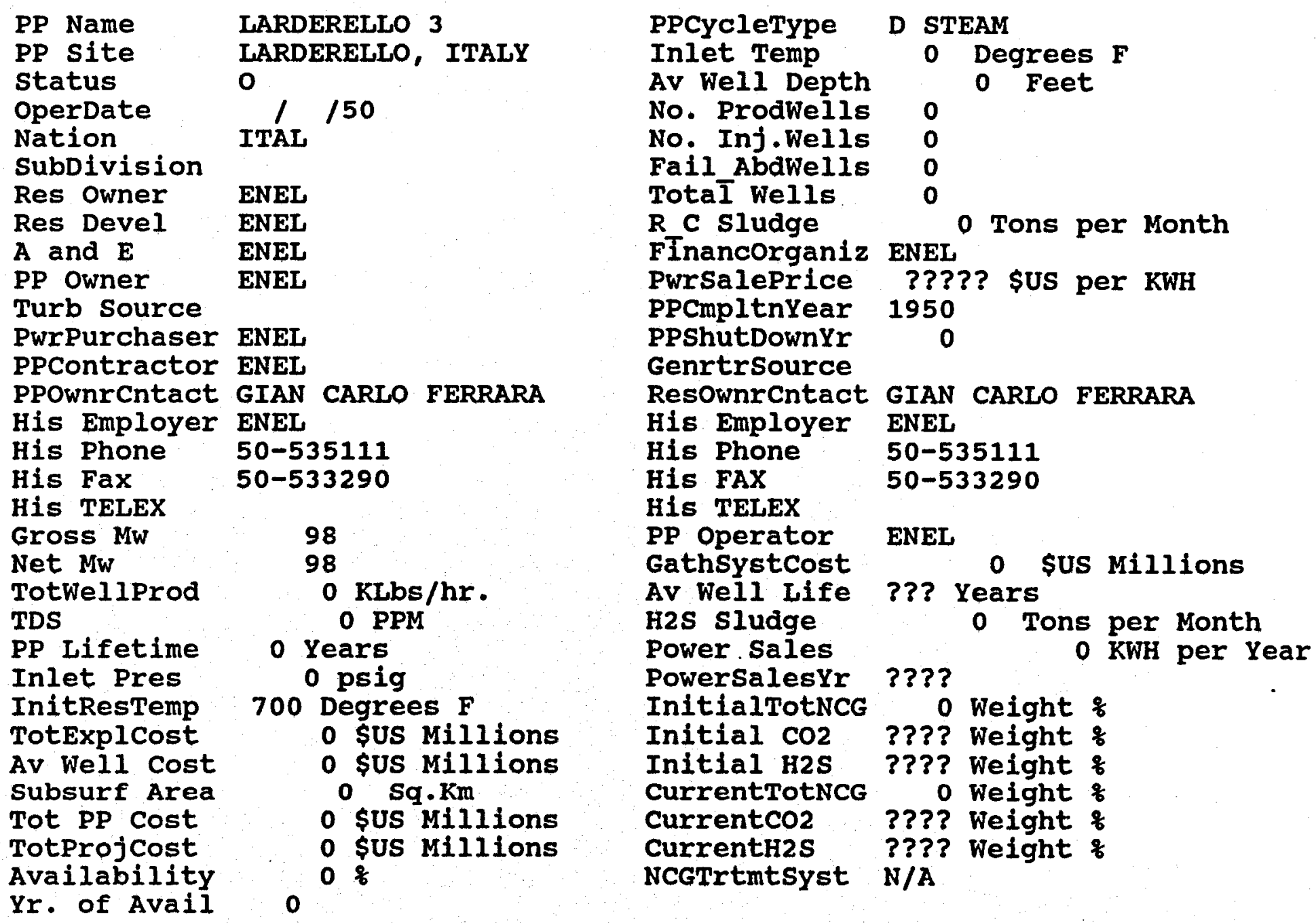


JAPAN 


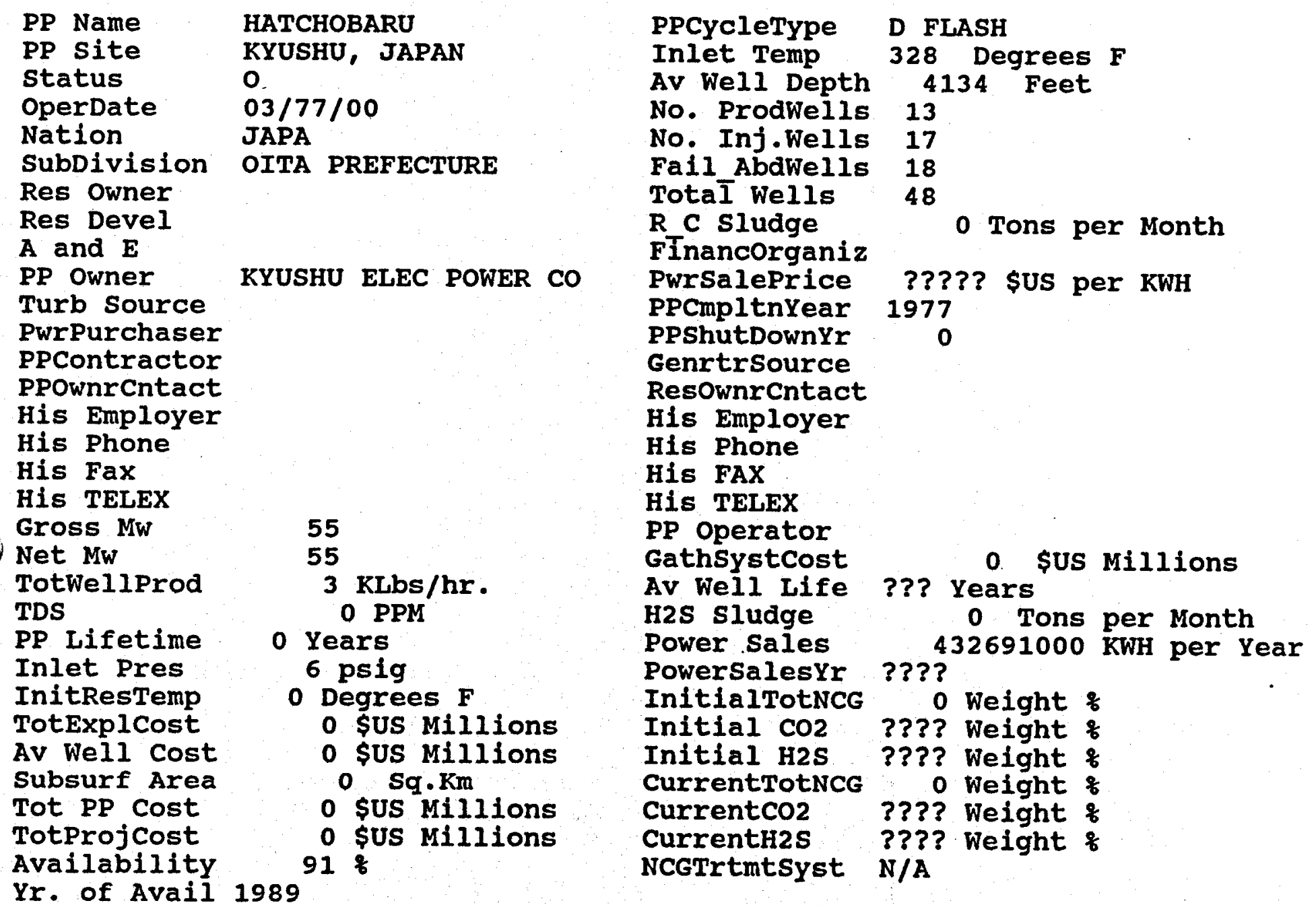




\author{
PP Name \\ PP site \\ status \\ OperDate \\ Nation \\ SubDivision \\ Res Owner \\ Res Devel \\ $A$ and $E$ \\ PP Owner \\ Turb Source \\ PwrPurchaser \\ PPContractor \\ PPOwnrCntact \\ His Employer \\ His Phone \\ His Fax \\ His TELEX \\ Gross MW \\ Net $M W$ \\ TotWel1Prod \\ TDS \\ PP Lifetime \\ Inlet Pres \\ InitResTemp \\ TotExplCost \\ Av Well Cost \\ Subsurf Area \\ Tot PP cost \\ TotProjcost \\ Availability \\ Yr. of Avail 1989 \\ KAKKONDA \\ N . HONSHU, JAPAN \\ 0 \\ 05/78/00 \\ JAPA \\ IWATE PREFECTURE \\ JAPAN METALS \& CHEM \\ TOHOKU ELECT. POWER
0 Years 3 psig
0 Degrees $F$
o \$US Millions
0 \$US Millions o $\mathrm{Sq} \cdot \mathrm{Km}$
o \$US Millions
0 \$US Militons $95 \%$

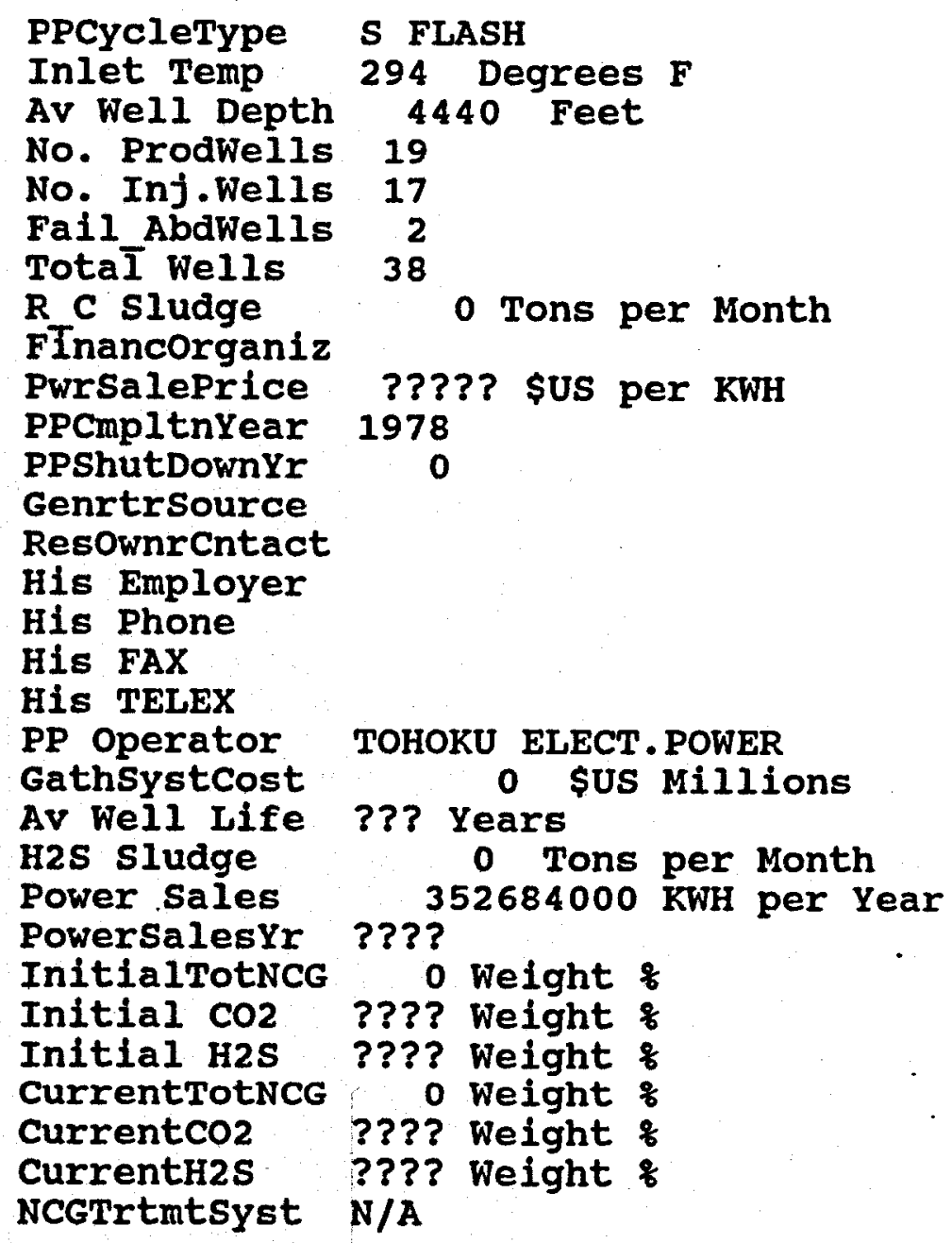




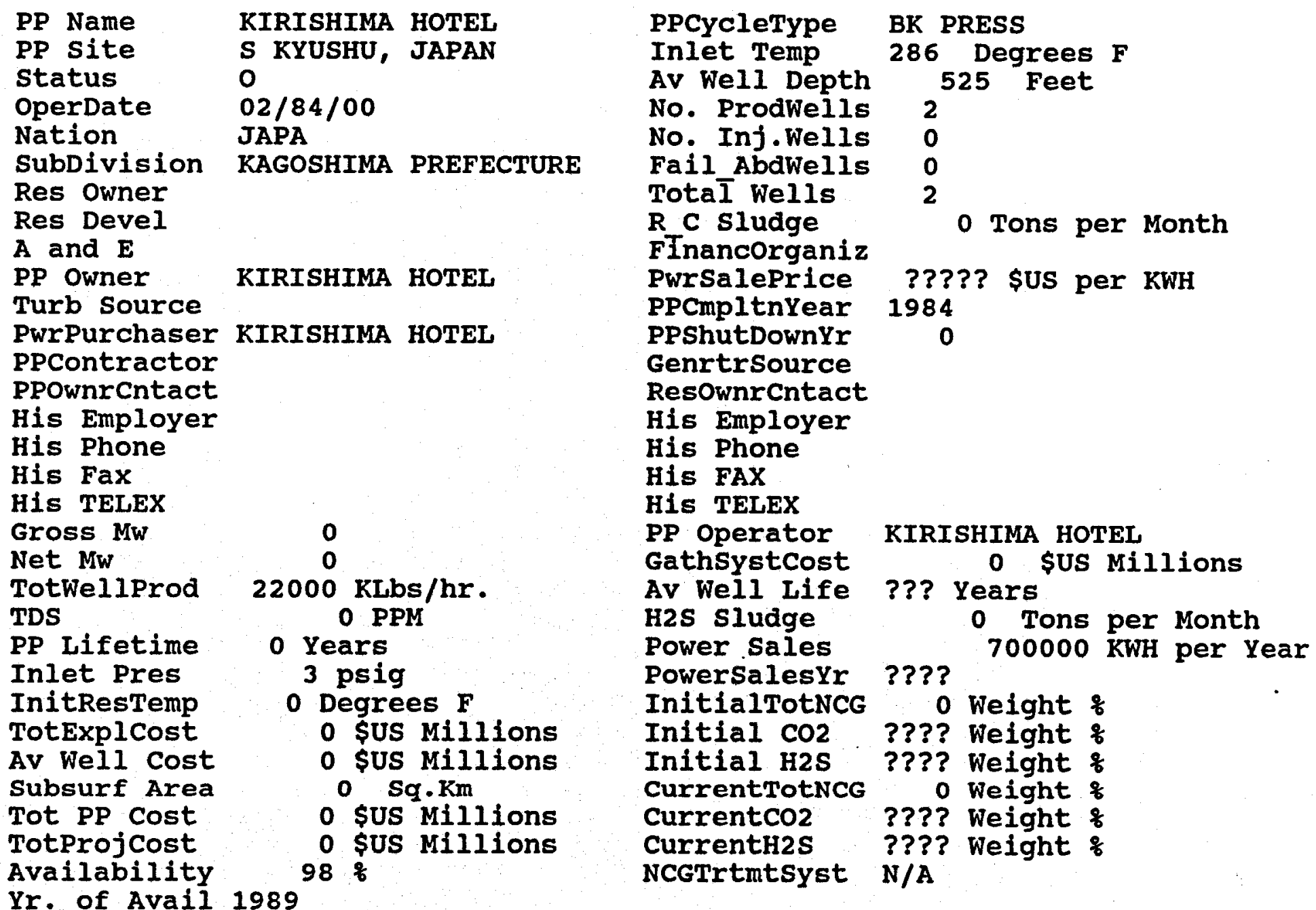




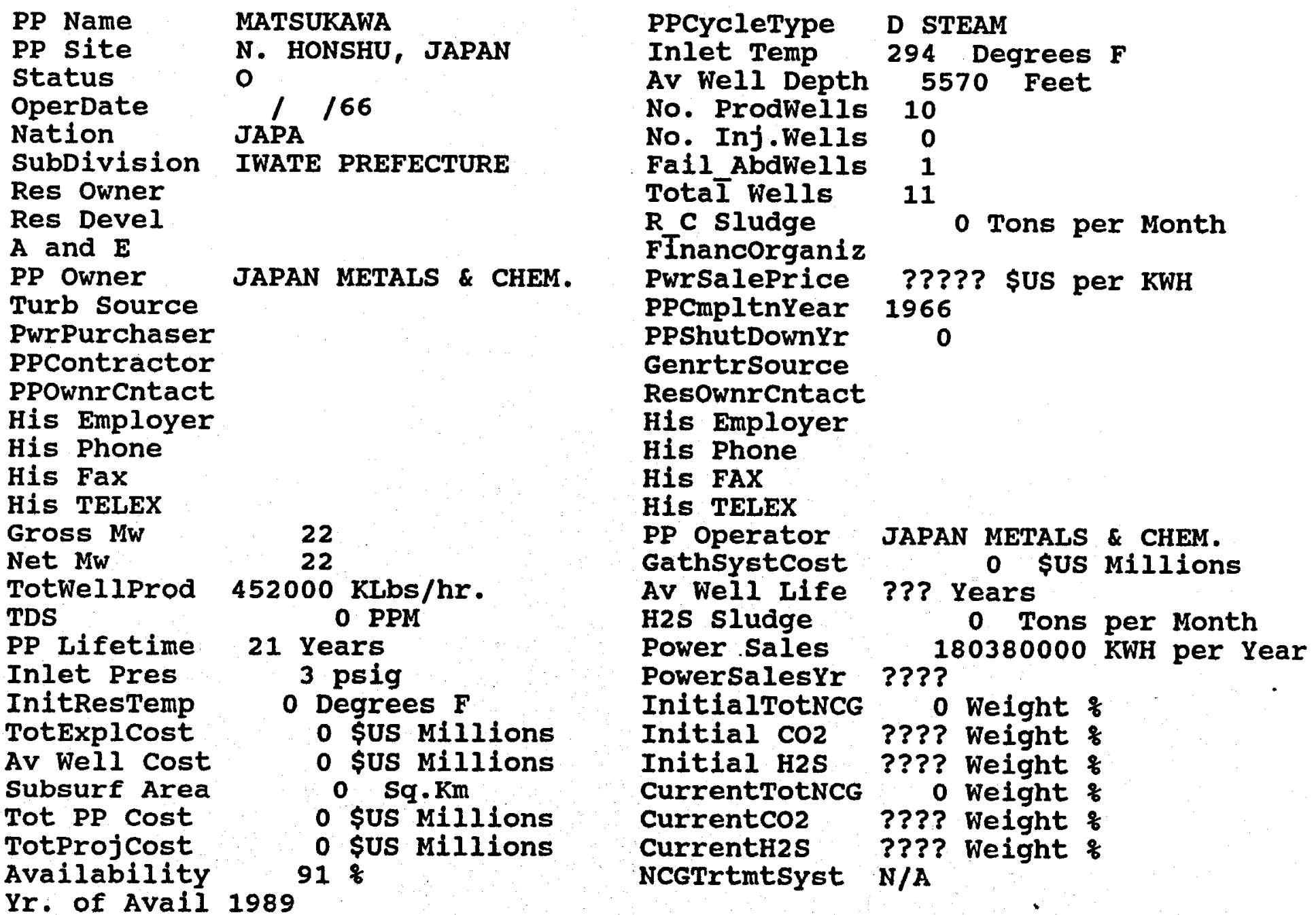




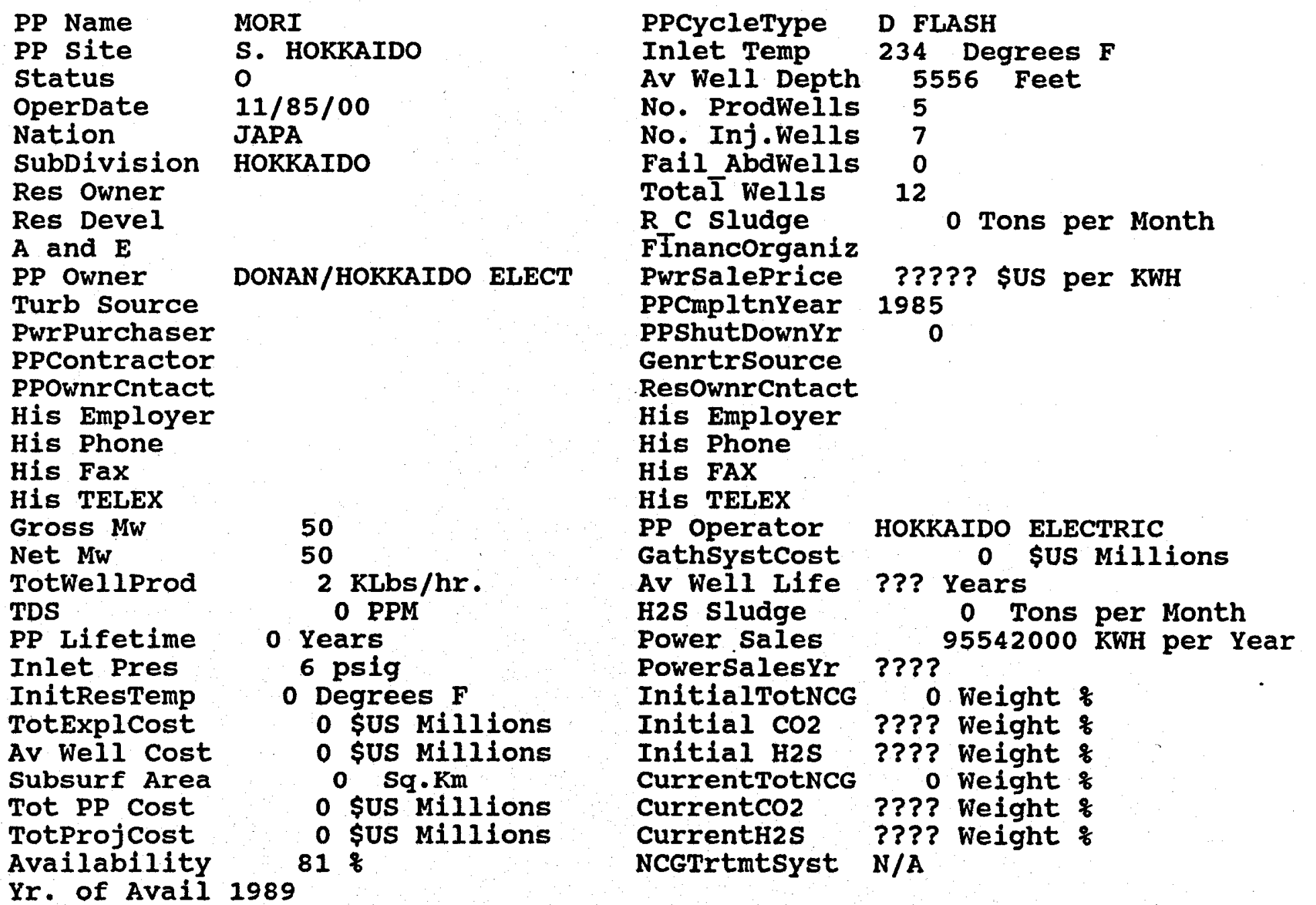




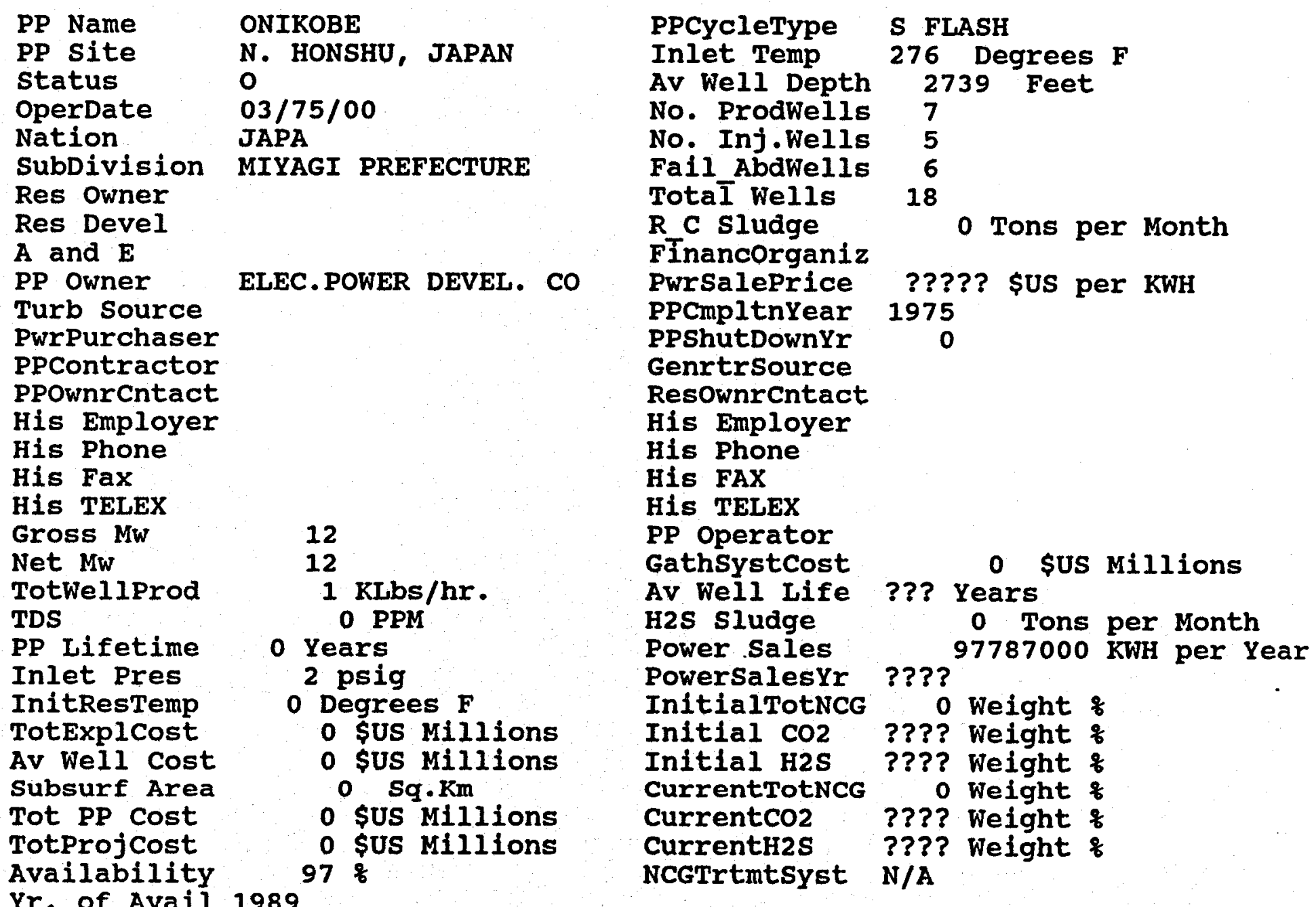




\author{
PP Name \\ PP Site \\ status \\ OperDate \\ Nation \\ SubDivision \\ Res Owner \\ Res Devel \\ $A$ and $E$ \\ PP Owner \\ Turb Source \\ PwrPurchaser \\ PPContractor \\ pPownrcntact \\ His Employer \\ His Phone \\ His Fax \\ His TELEX \\ Gross MW \\ Net MW \\ TotWellProd \\ TDS \\ PP Lifetime \\ Inlet Pres \\ InitResTemp \\ TotExplcost \\ Av Well cost \\ Subsurf Area \\ Tot PP cost \\ TotProjcost \\ Availability \\ Yr. of Avail 1989 \\ ONUMA \\ N. HONSHU, JAPAN \\ 0 \\ 06/74/00 \\ JAPA \\ AKITA PREFECTURE \\ MITSUBISHI METALS CO

9
9
980000 KLbs/hr
0 PPM
0 Years
1 psig
0 Degrees F
0 \$US Millions
0 \$US Millions
o Sq.Km
0 \$US Militons
0 \$US Millions
95 q

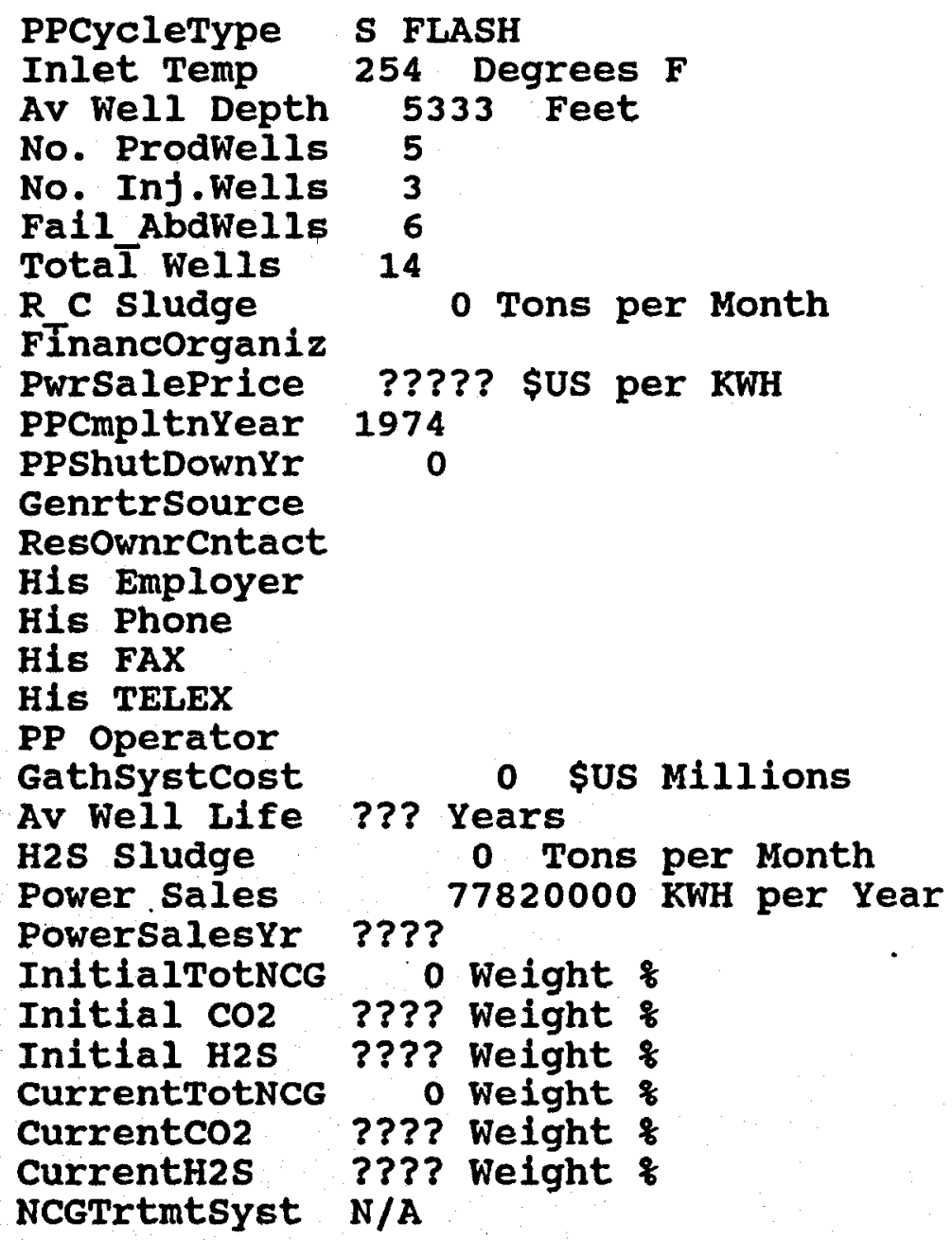




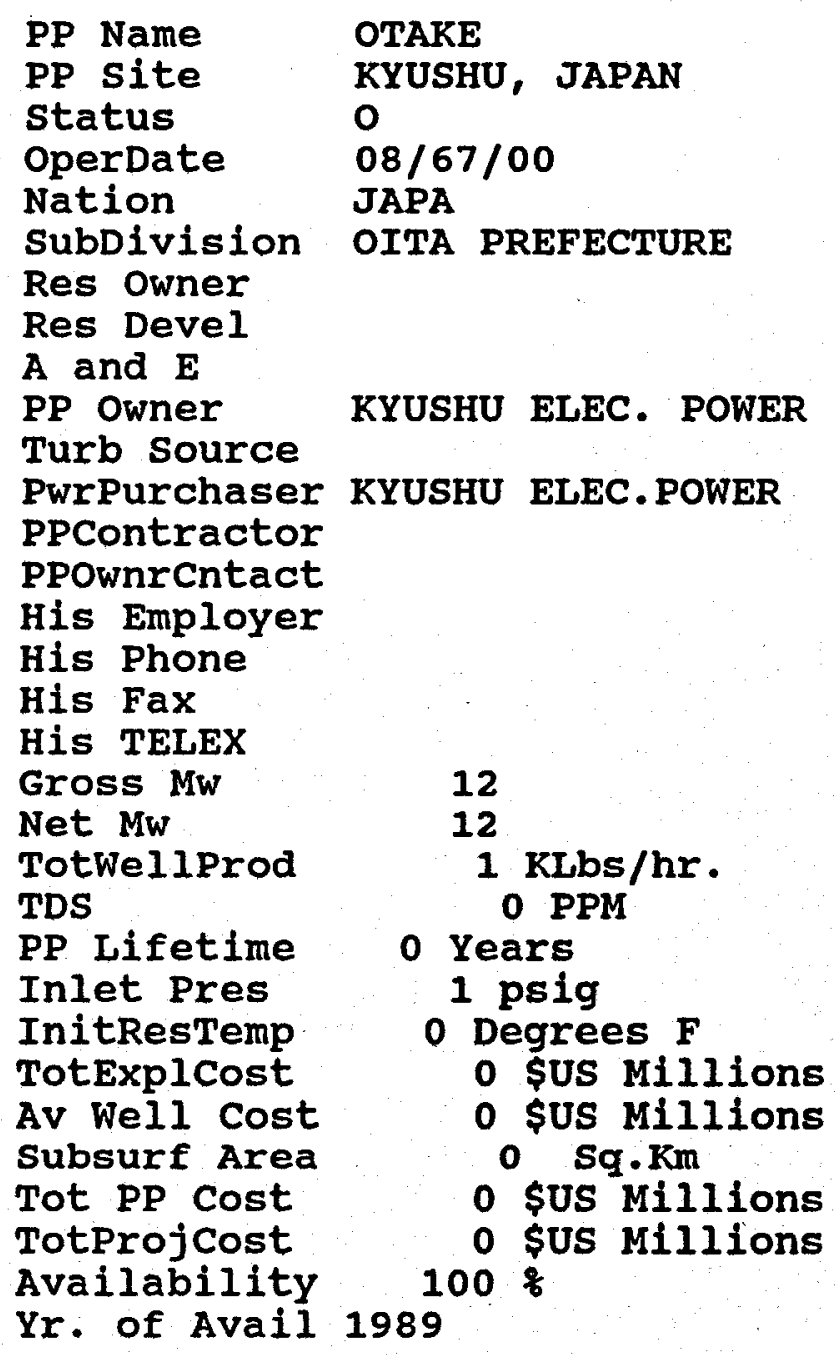

PwrPurchaser KYUSHU ELEC.POWER

OTAKE

KYUSHU, JAPAN

0

$08 / 67 / 00$

JAPA

OITA PREFECTURE

KYUSHU ELEC. POWER

0 Years

1 psig

0 Degrees $F$

o SUS Millions

0 \$US Millions

$0 \mathrm{Sq} \cdot \mathrm{Km}$

o \$US Millions

0 \$US Millions $100 \%$

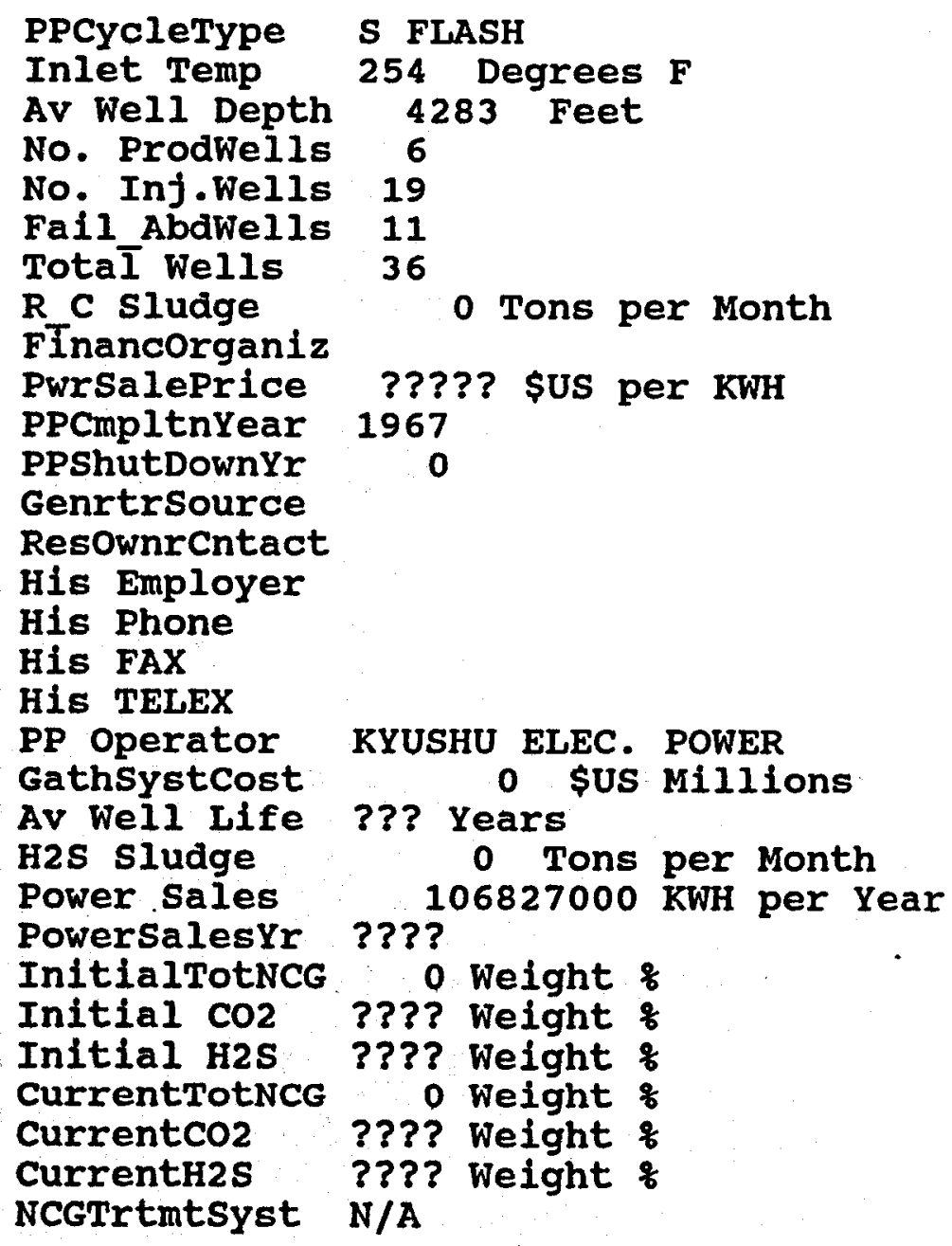




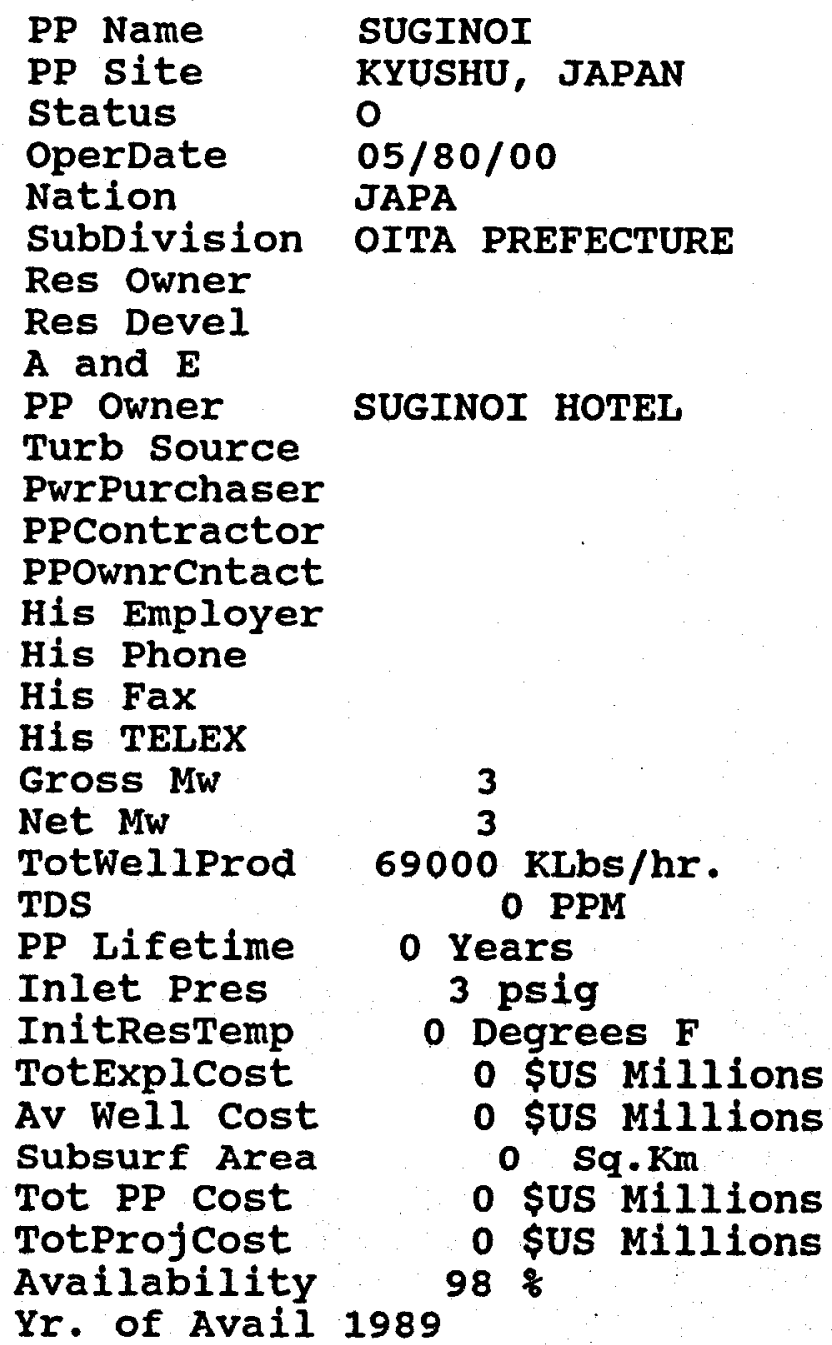

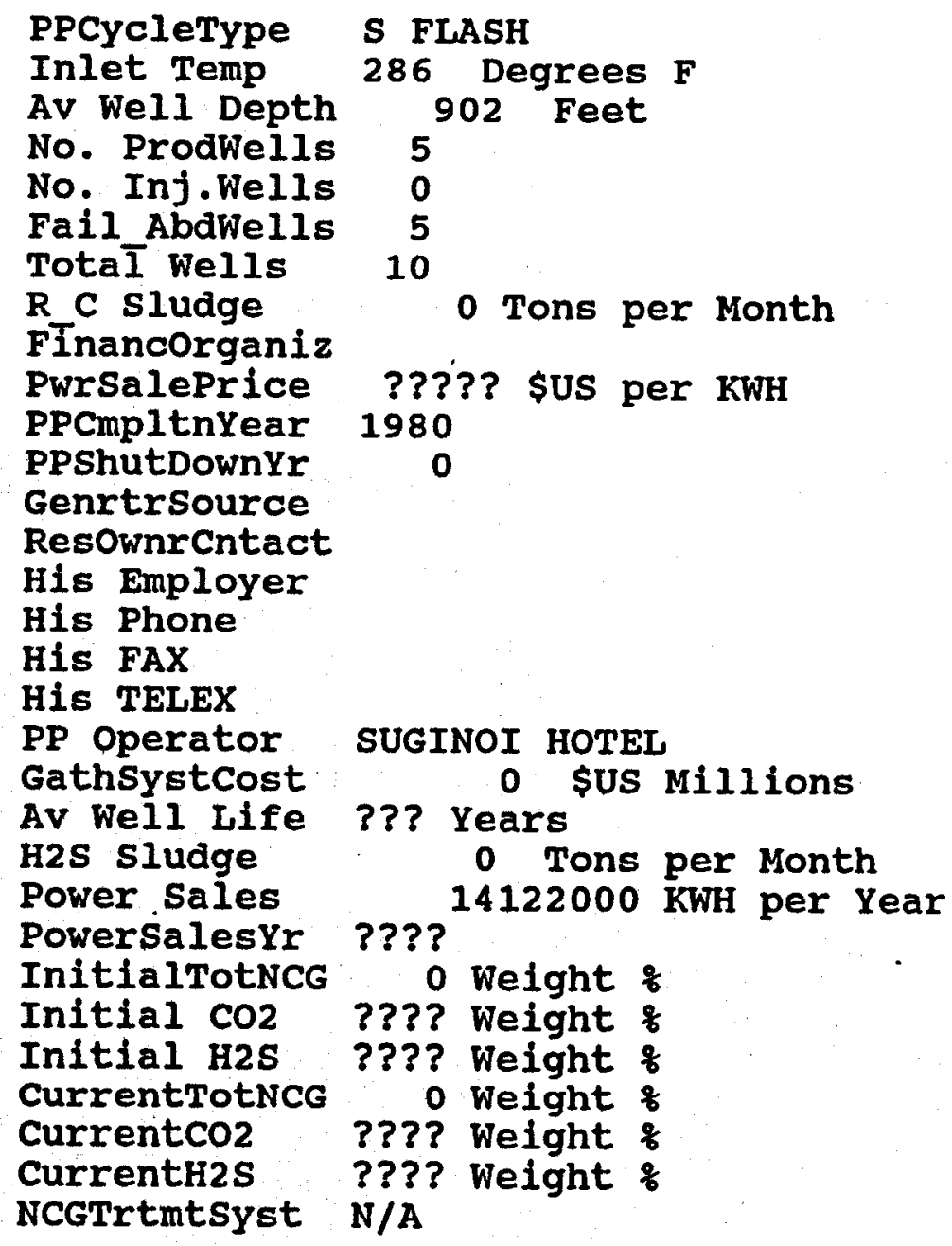


KENYA 


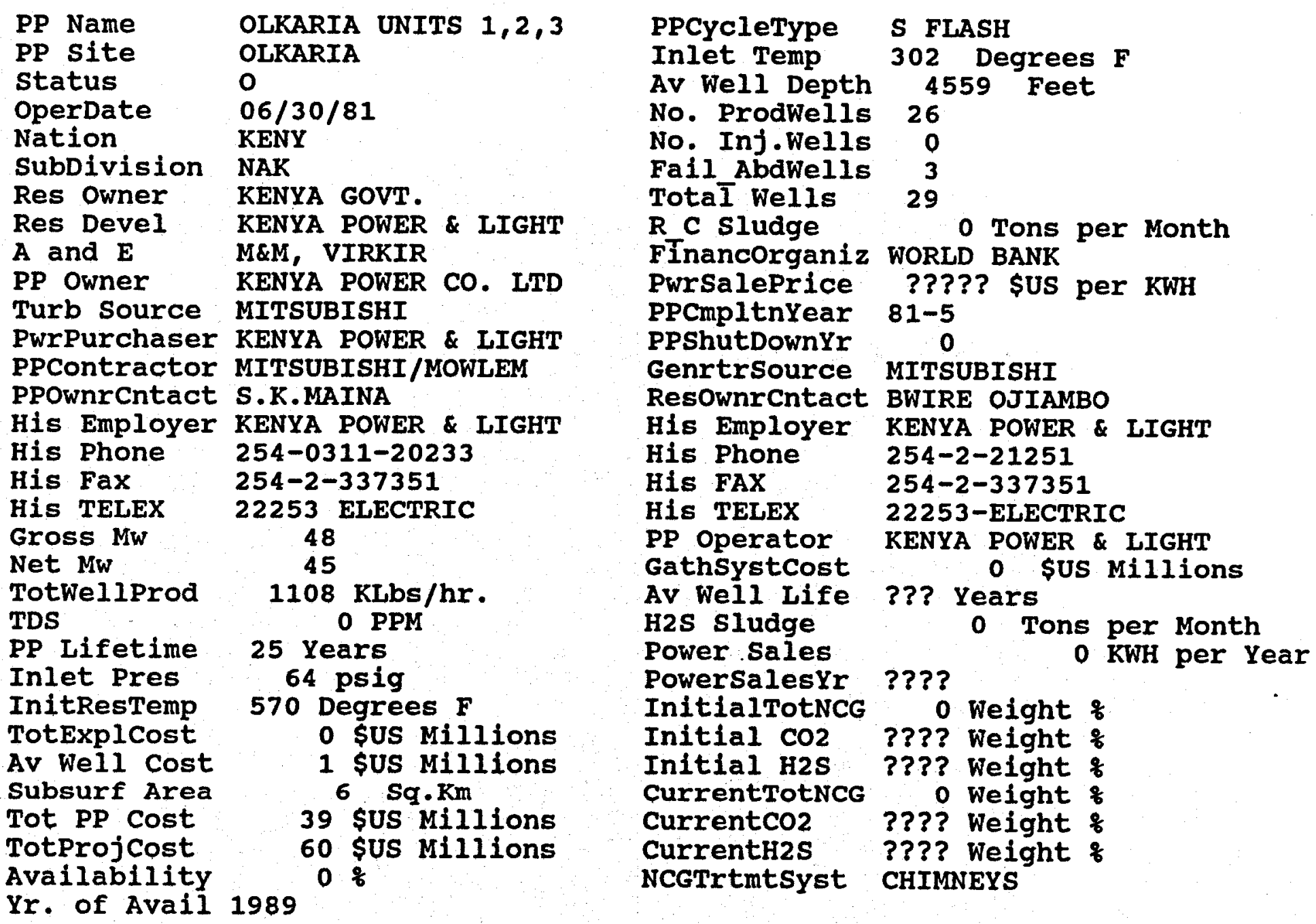


MEXICO 


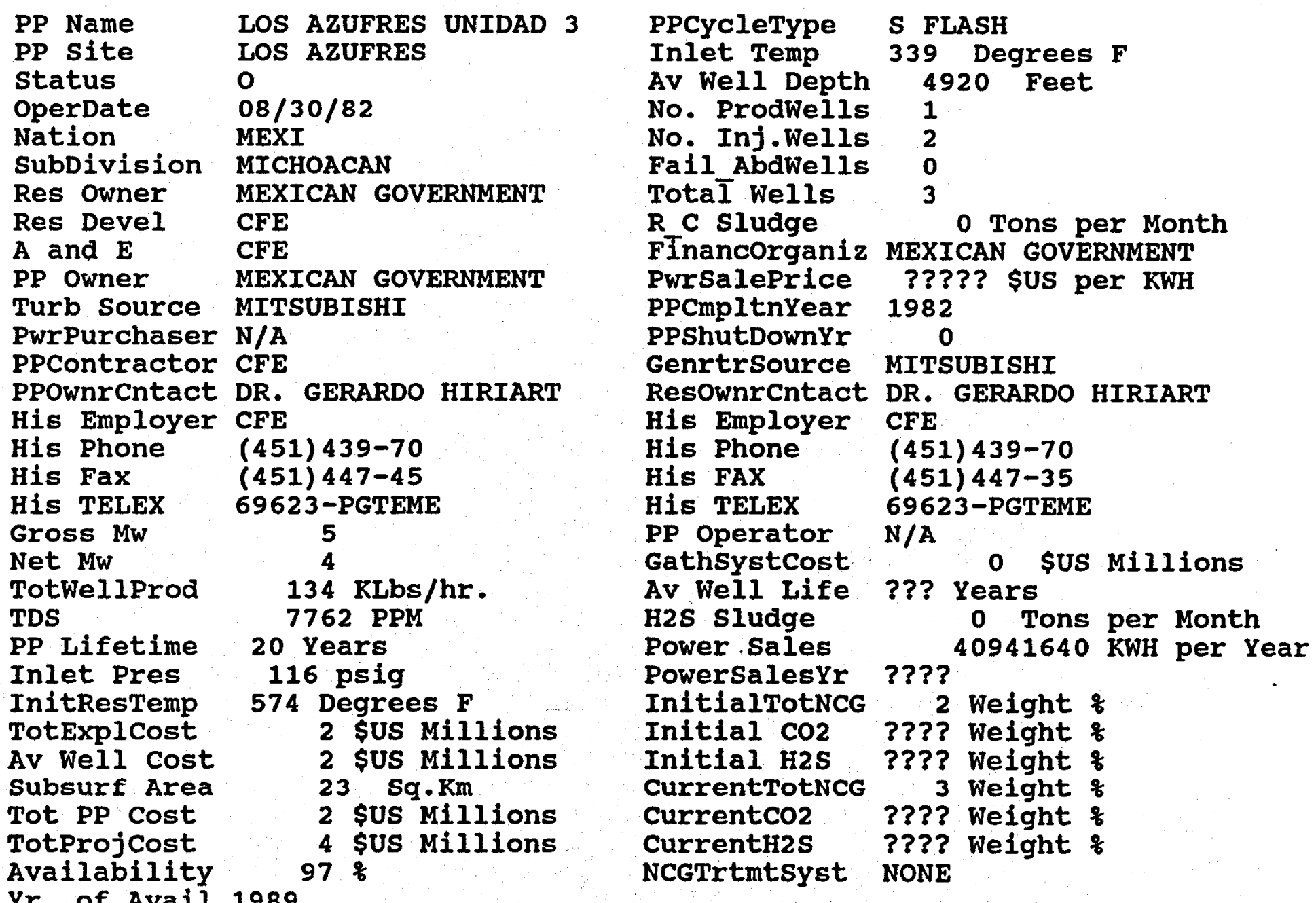




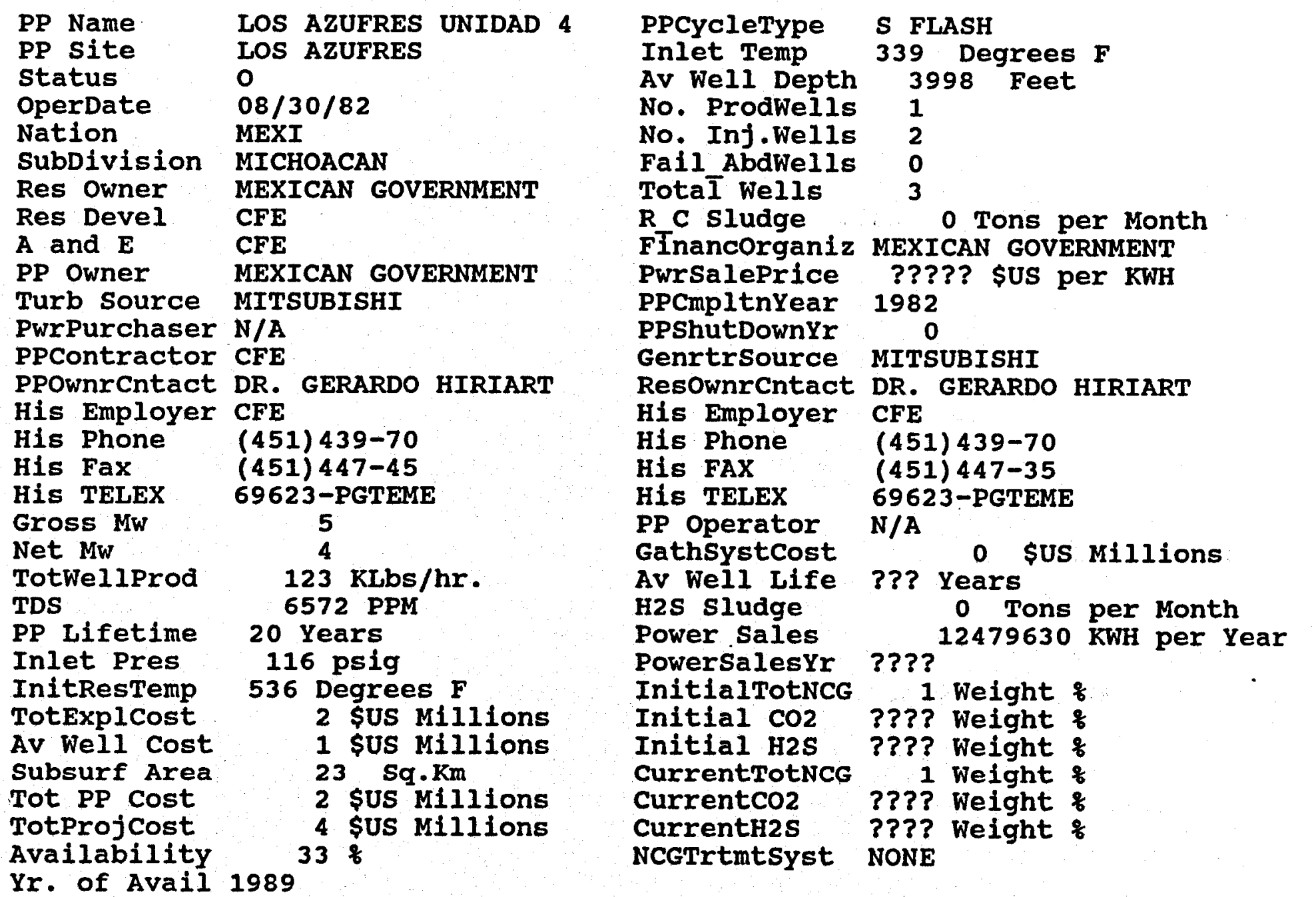




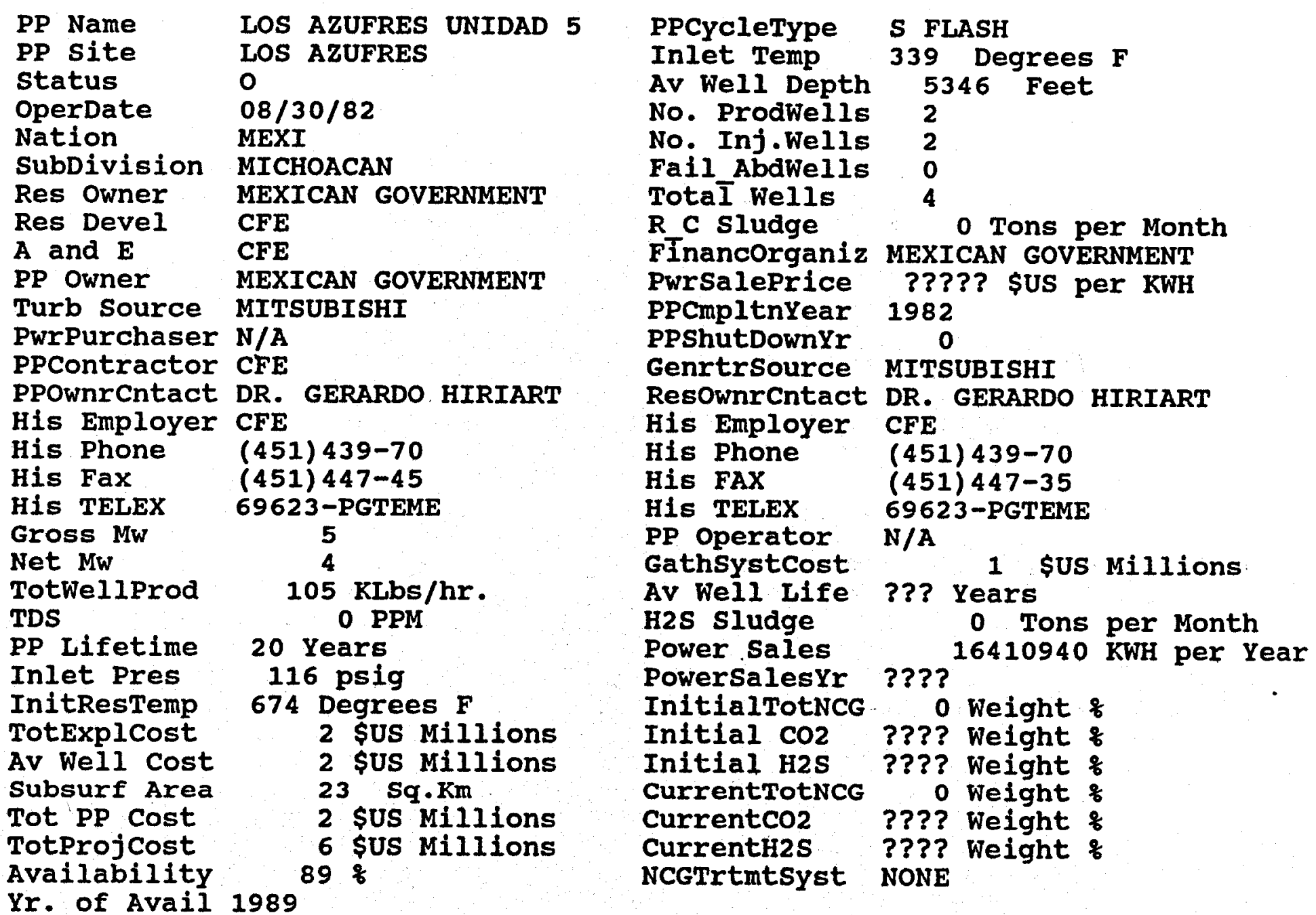




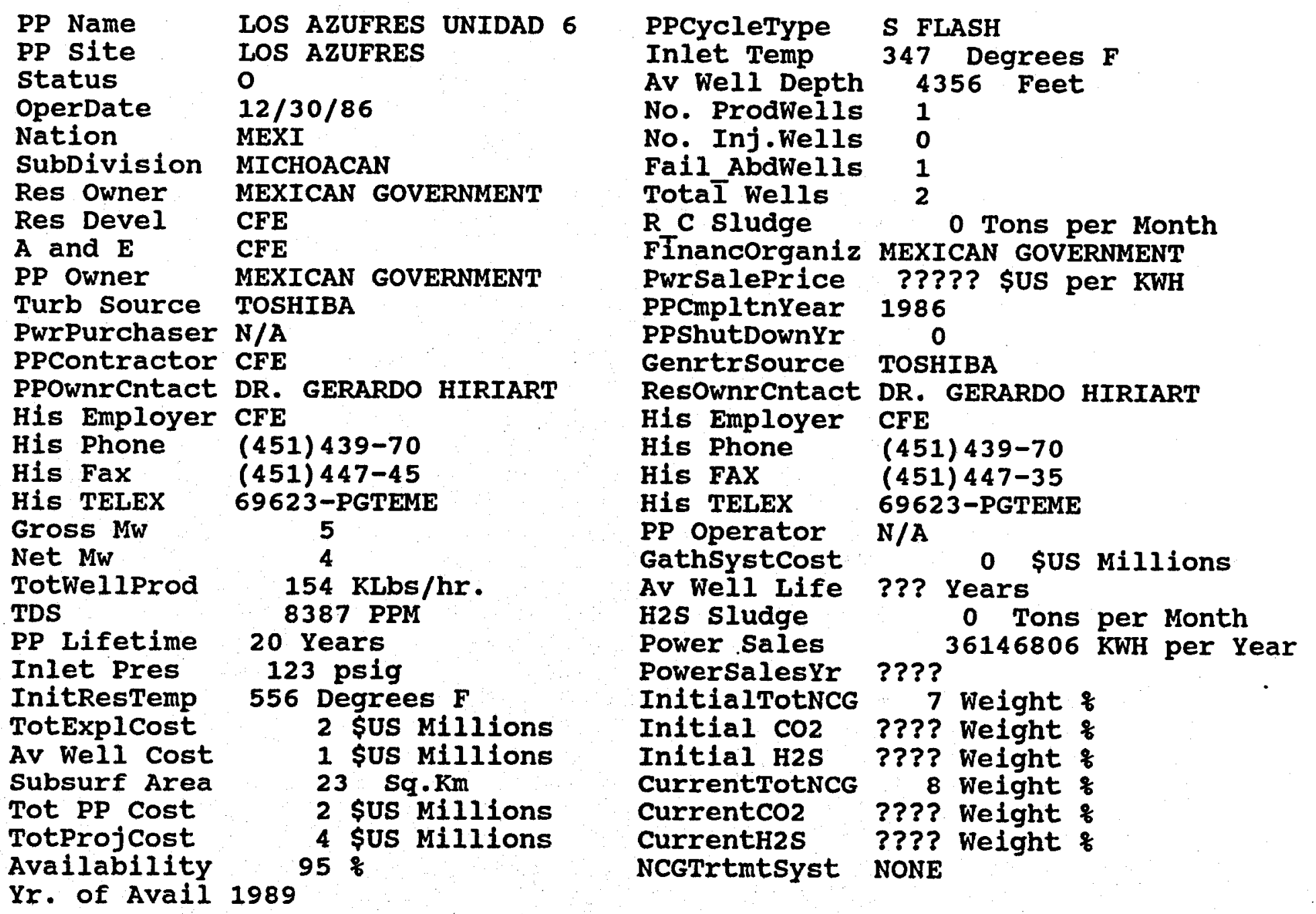




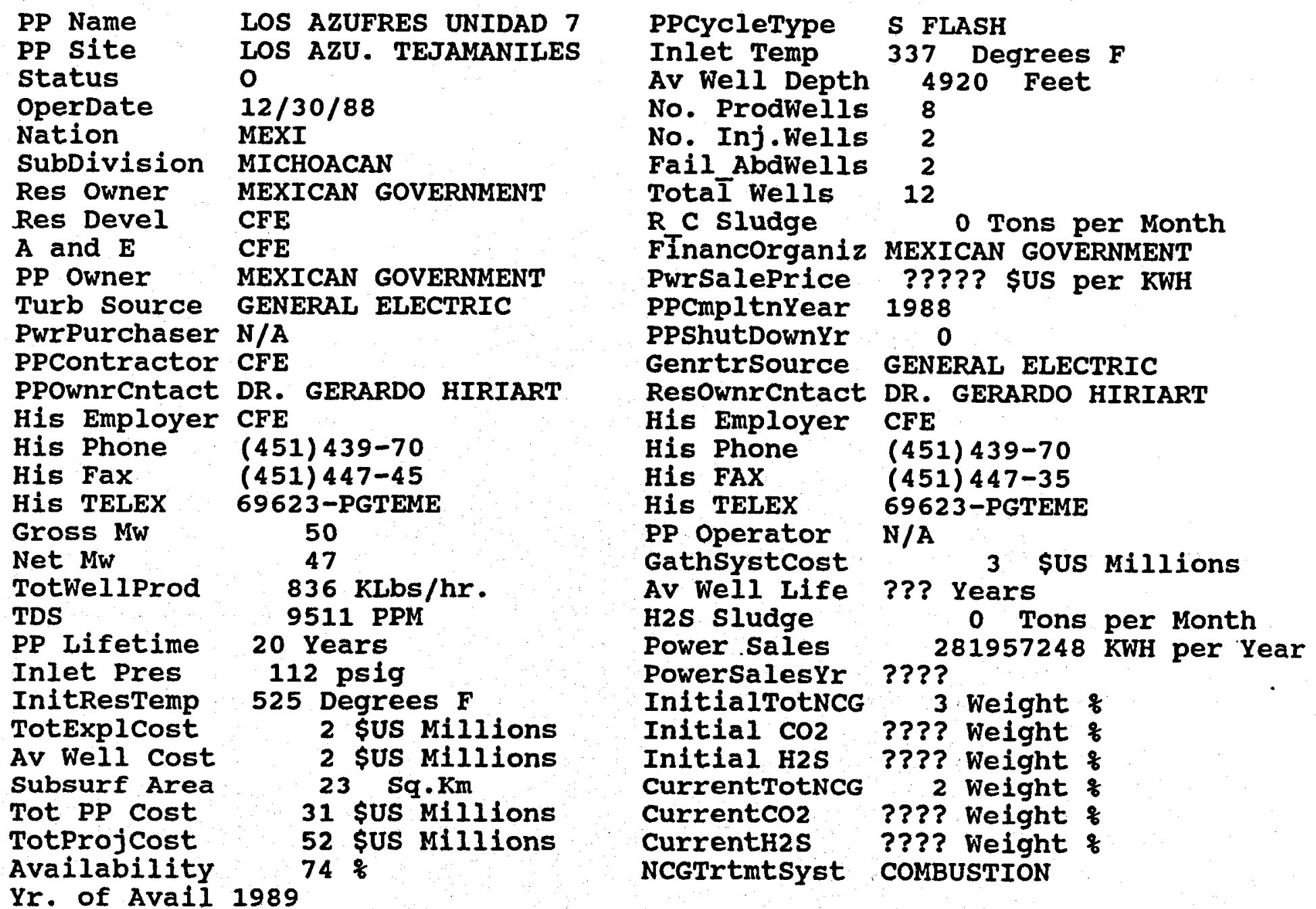




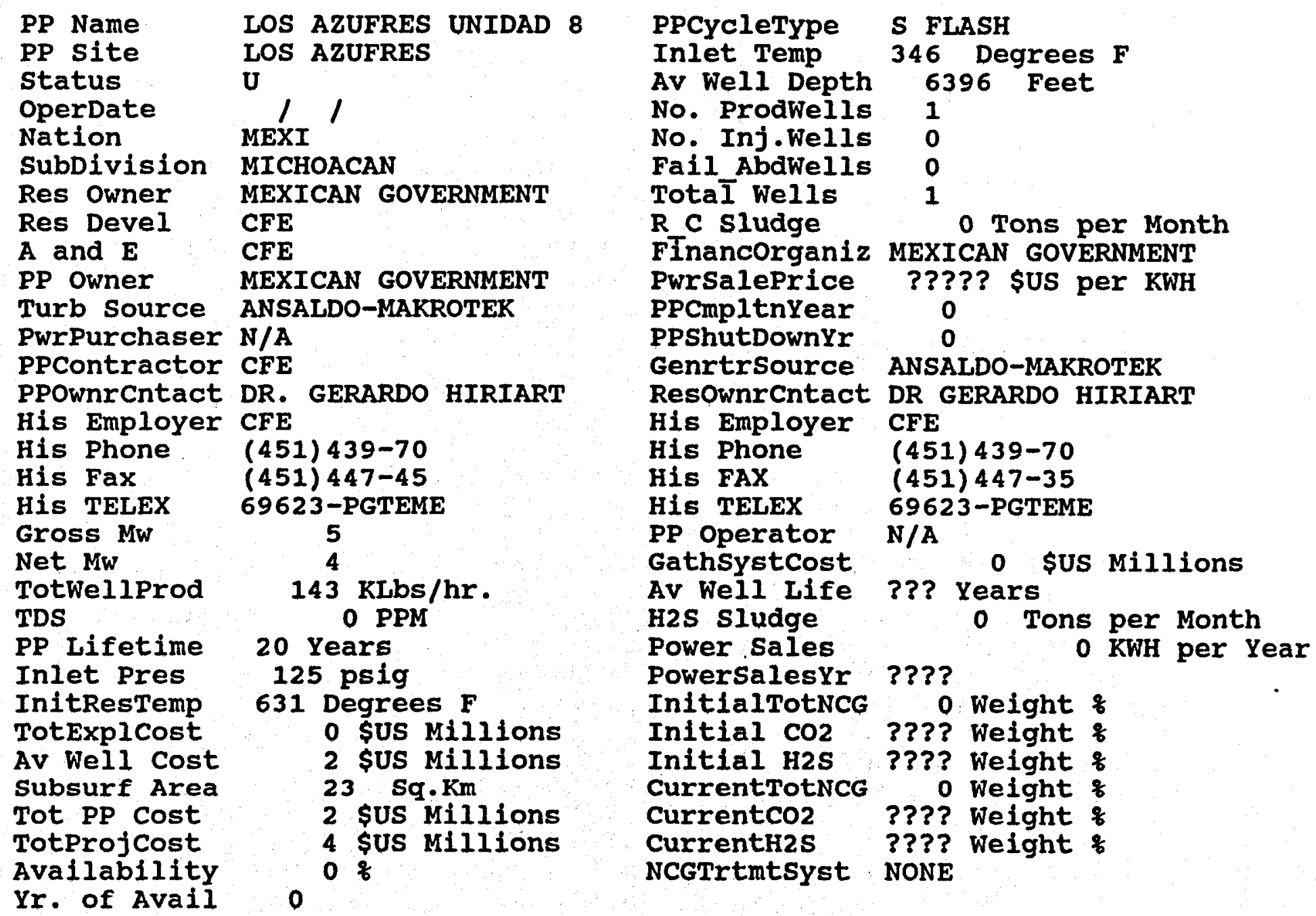




\begin{tabular}{|c|c|c|c|}
\hline $\begin{array}{l}\text { P Name } \\
\text { P site }\end{array}$ & 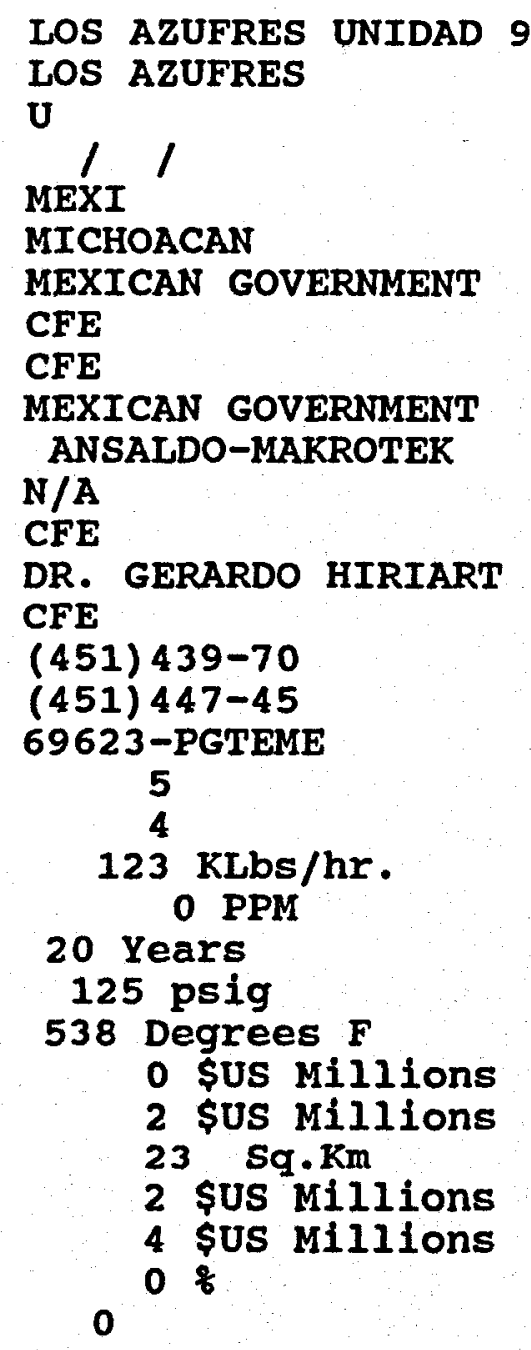 & $\begin{array}{l}\text { PPCycleType } \\
\text { Inlet Temp } \\
\text { Av Well Depth } \\
\text { No. ProdWells } \\
\text { No. Inj. Wells } \\
\text { Fail AbdWells } \\
\text { Total Wells } \\
\text { RC sludge } \\
\text { Financorganiz } \\
\text { PwrSalePrice } \\
\text { PPCmpltnYear } \\
\text { PPShutDownYr } \\
\text { GenrtrSource } \\
\text { ResOwnrCntact } \\
\text { His Employer } \\
\text { His Phone } \\
\text { His FAX } \\
\text { His TELEX } \\
\text { PP Operator } \\
\text { GathSystCost } \\
\text { Av Weli Life } \\
\text { H2S Sludge } \\
\text { Power Sales } \\
\text { PowerSalesYr } \\
\text { InitialTotNCG } \\
\text { Initial CO2 } \\
\text { Initial H2S } \\
\text { CurrentTotNCG } \\
\text { CurrentCO2 } \\
\text { CurrentH2S } \\
\text { NCGTrtmtSyst }\end{array}$ & 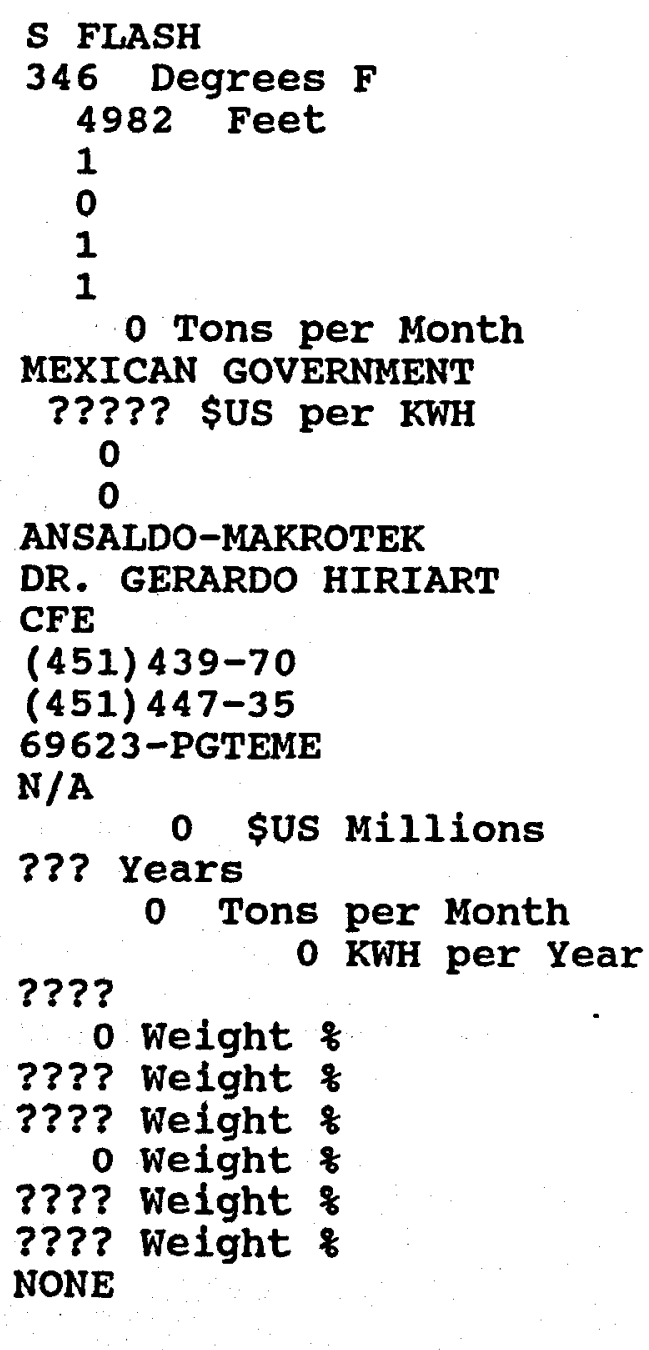 \\
\hline
\end{tabular}




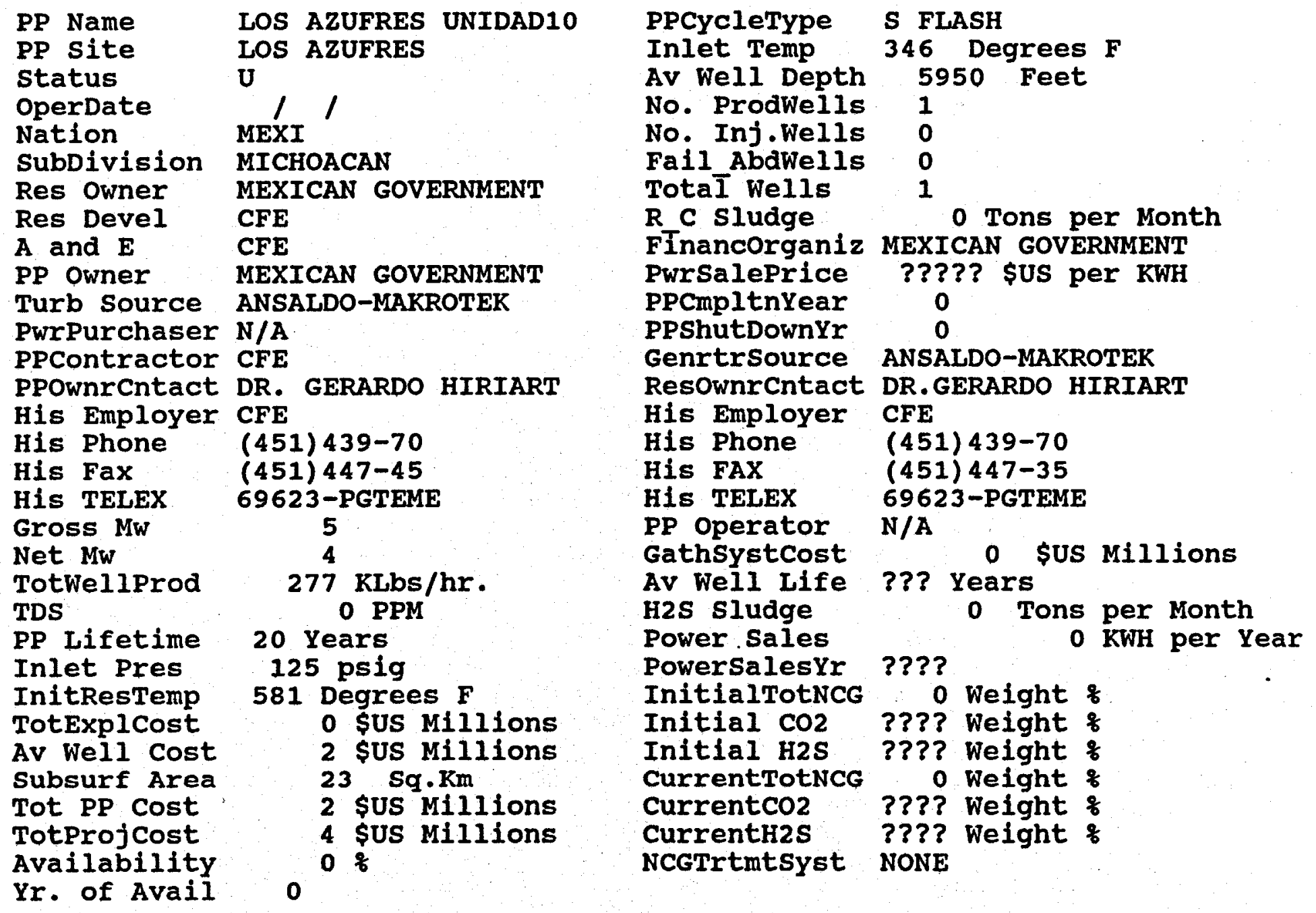




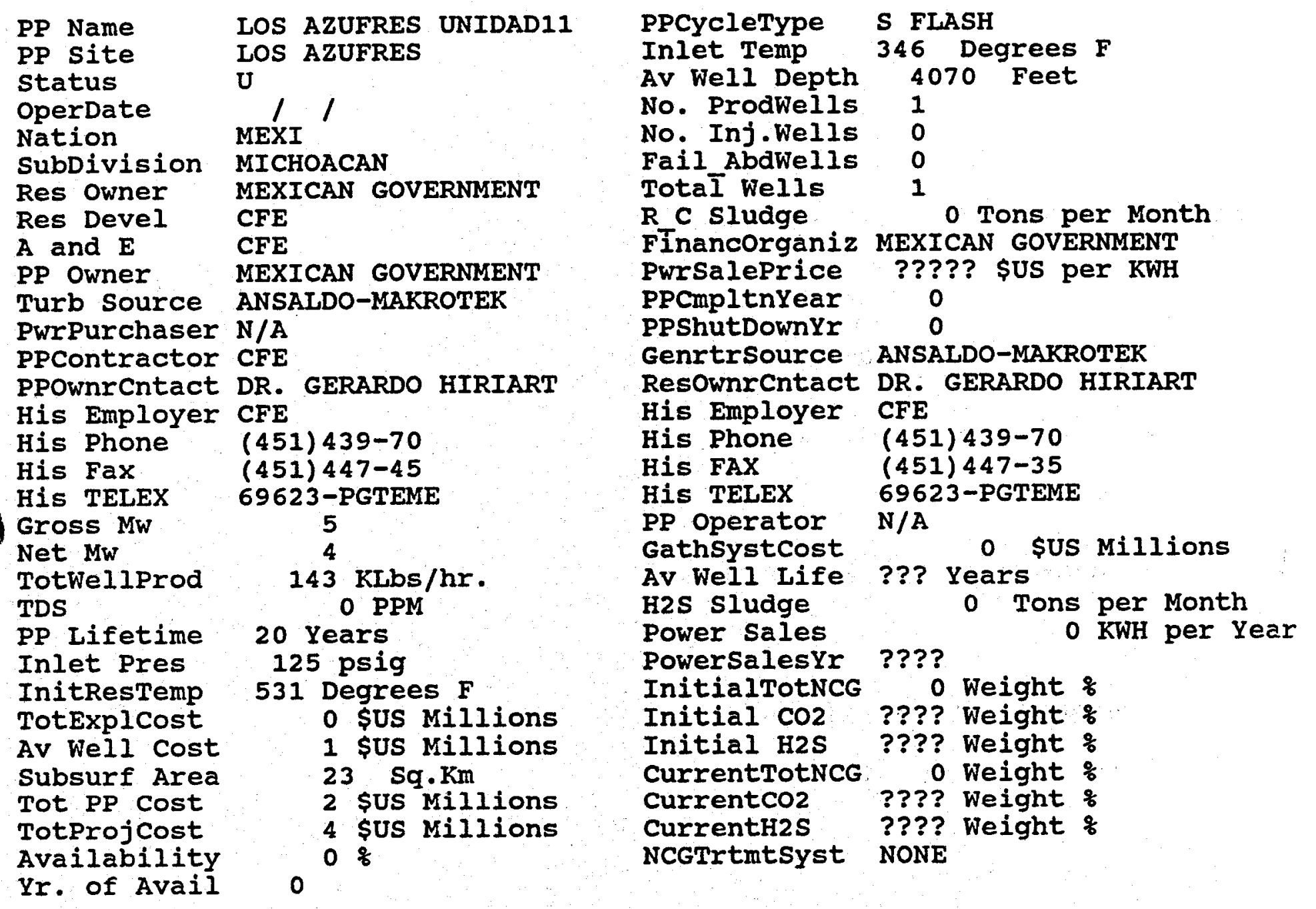




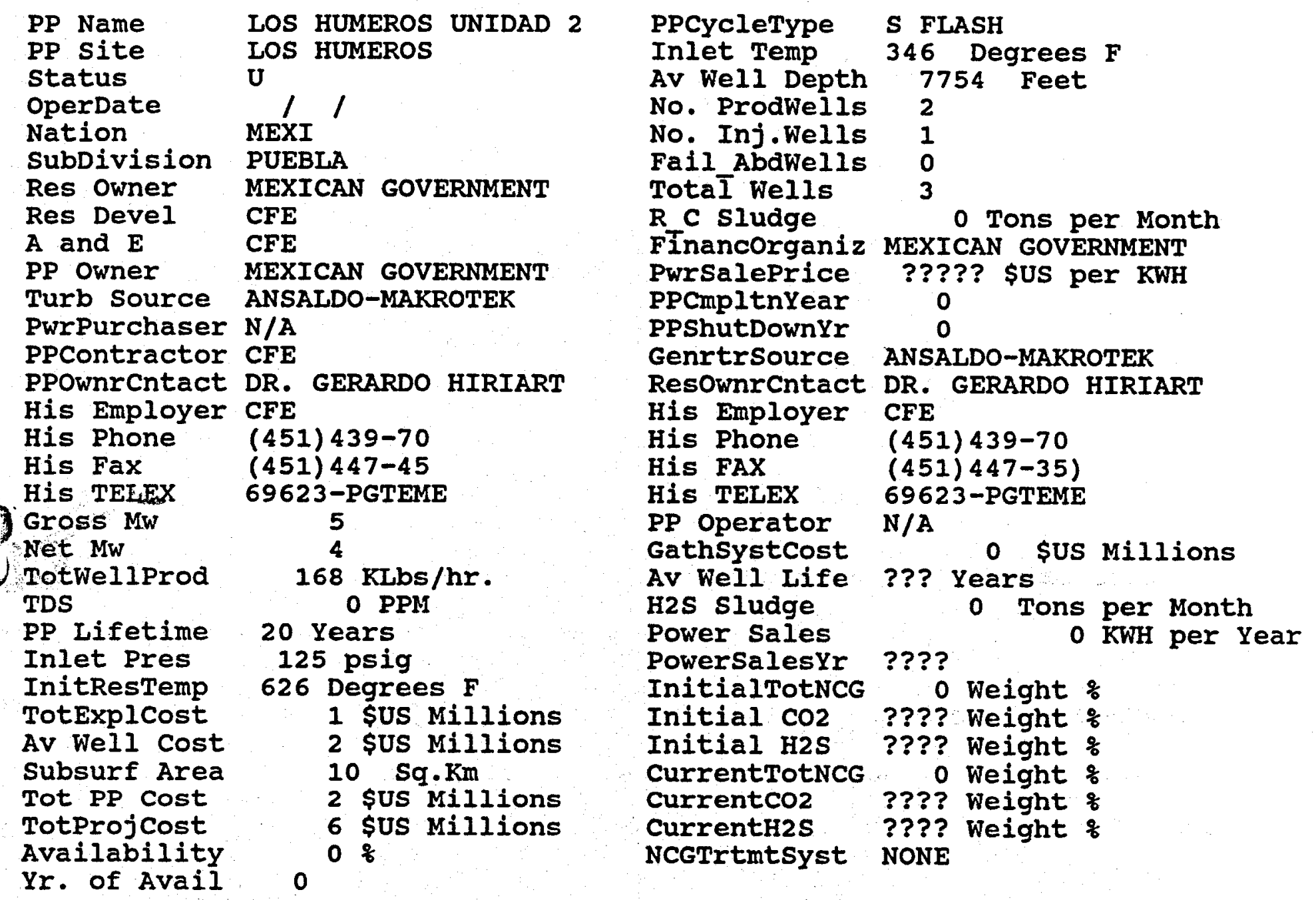




\begin{tabular}{|c|c|c|c|}
\hline P Name & $\begin{array}{l}\text { LOS HUMEROS UNIDAD } 1 \\
\text { LOS HUMEROS } \\
\text { U } \\
\text { MEXI } \\
\text { PUEBLA } \\
\text { MEXICAN GOVERNMENT } \\
\text { CFE } \\
\text { CFE } \\
\text { MEXICAN GOVERNMENT } \\
\text { ANSALDO-MAKROTEK } \\
\text { N/A } \\
\text { CFE } \\
\text { DR. GERARDO HIRIART } \\
\text { CFE } \\
(451) 439-70 \\
(451) 447-45 \\
69623-\text { PGTEME } \\
5 \\
4 \\
194 \text { KLbs/hr. } \\
0 \text { PPM } \\
20 \text { Years } \\
125 \text { psig } \\
597 \text { Degrees F } \\
1 \text { \$US Millions } \\
2 \text { \$US Millions } \\
10 \text { Sq.Km } \\
2 \text { \$US Millions } \\
6 \text { \$US Millions } \\
0 \text { \% } \\
0 \text {. }\end{array}$ & $\begin{array}{l}\text { PPCycleType } \\
\text { Inlet Temp } \\
\text { Av Well Depth } \\
\text { No. ProdWells } \\
\text { No. Inj. Wells } \\
\text { Fail AbdWells } \\
\text { TotaI Wells } \\
\text { RC Sludge } \\
\text { Financorganiz } \\
\text { PwrSalePrice } \\
\text { PPCmpltnYear } \\
\text { PPShutDownYr } \\
\text { GenrtrSource } \\
\text { ResOwnrCntact } \\
\text { His Employer } \\
\text { His Phone } \\
\text { His FAX } \\
\text { His TELEX } \\
\text { PP Operator } \\
\text { GathSystCost } \\
\text { Av WeIl Life } \\
\text { H2S Sludge } \\
\text { Power Sales } \\
\text { PowerSalesYr } \\
\text { InitialTotNCG } \\
\text { Initial CO2 } \\
\text { Initial H2S } \\
\text { CurrentTotNCG } \\
\text { CurrentCO2 } \\
\text { CurrentH2S } \\
\text { NCGTrtmtSyst }\end{array}$ & 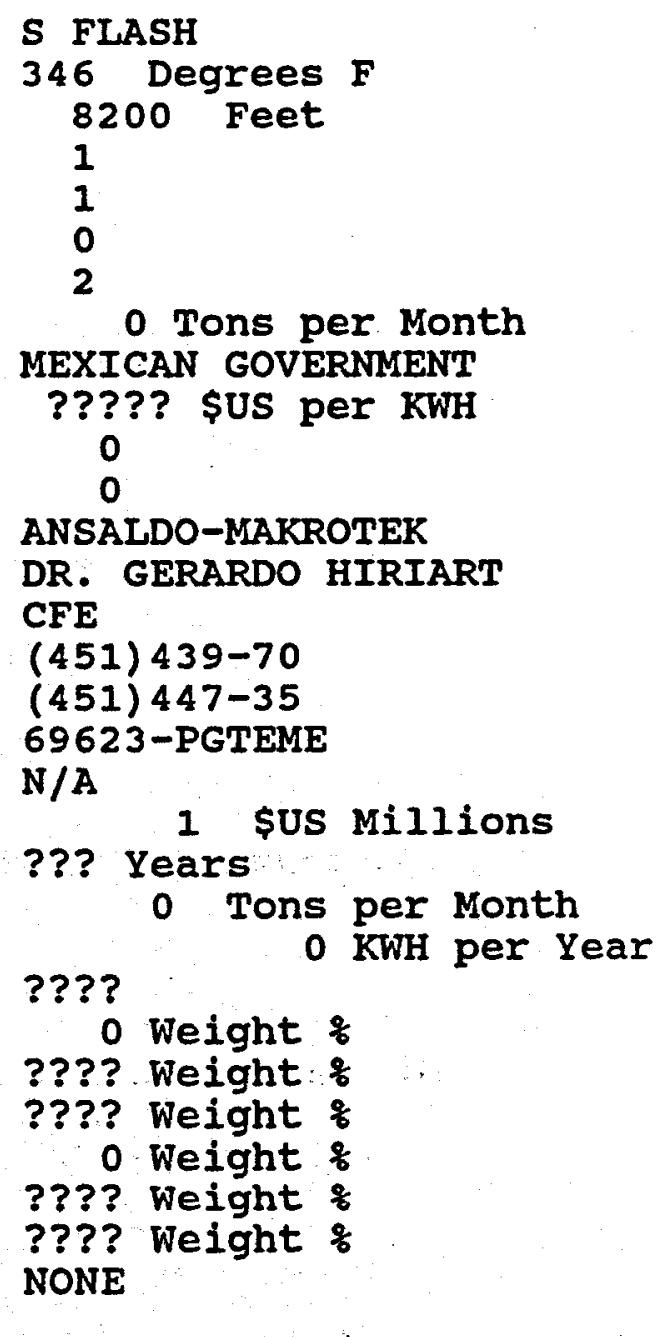 \\
\hline
\end{tabular}




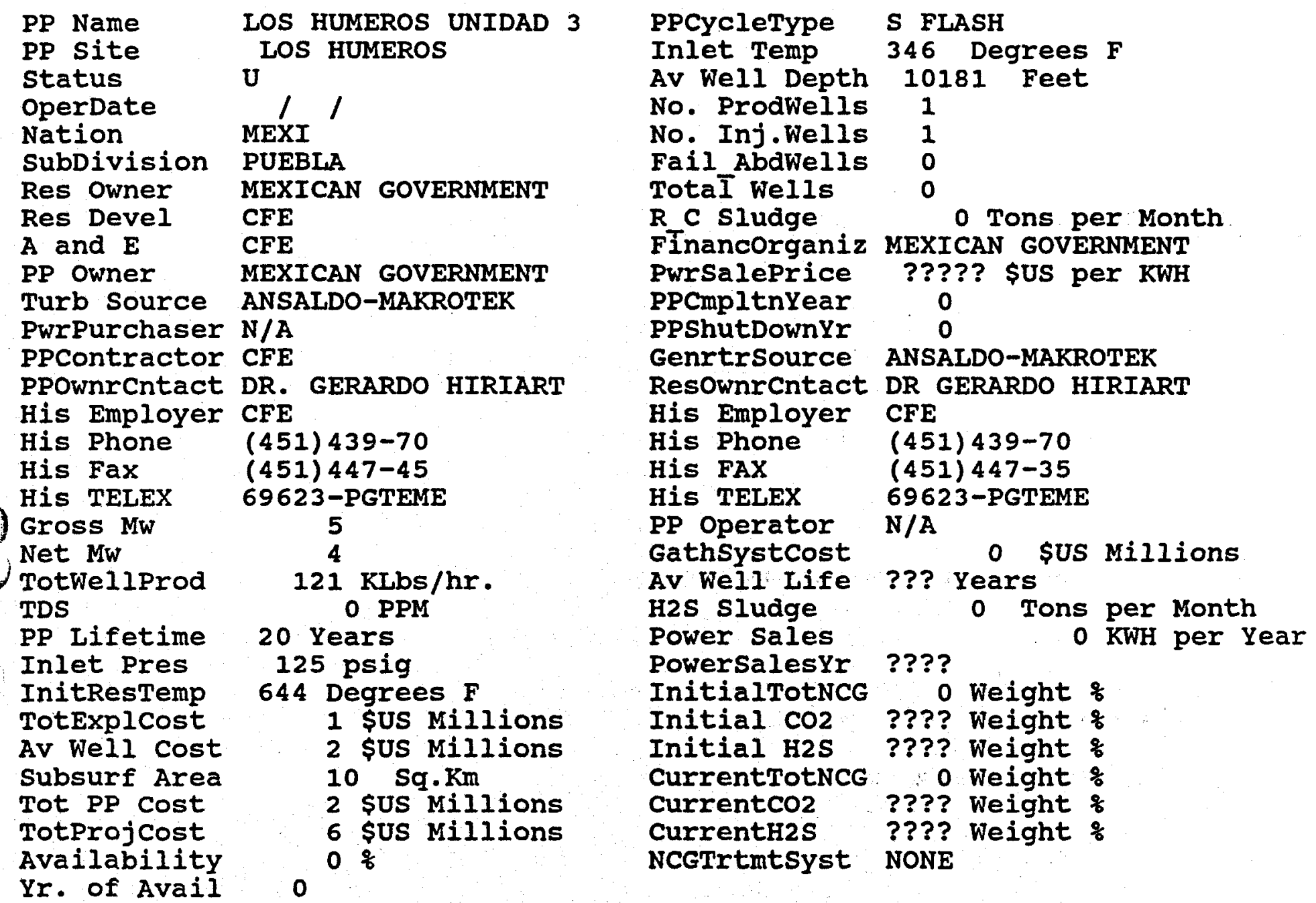




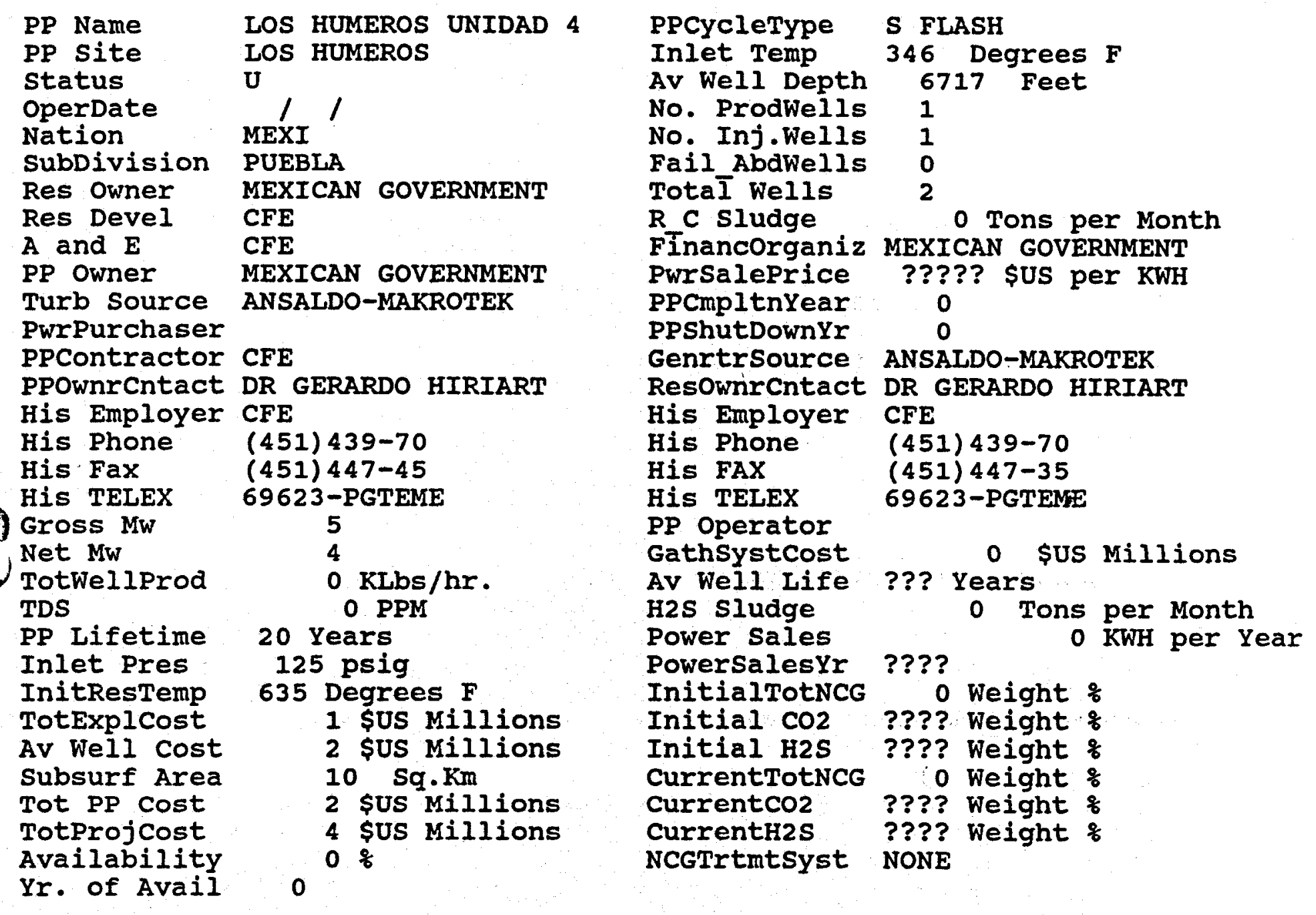




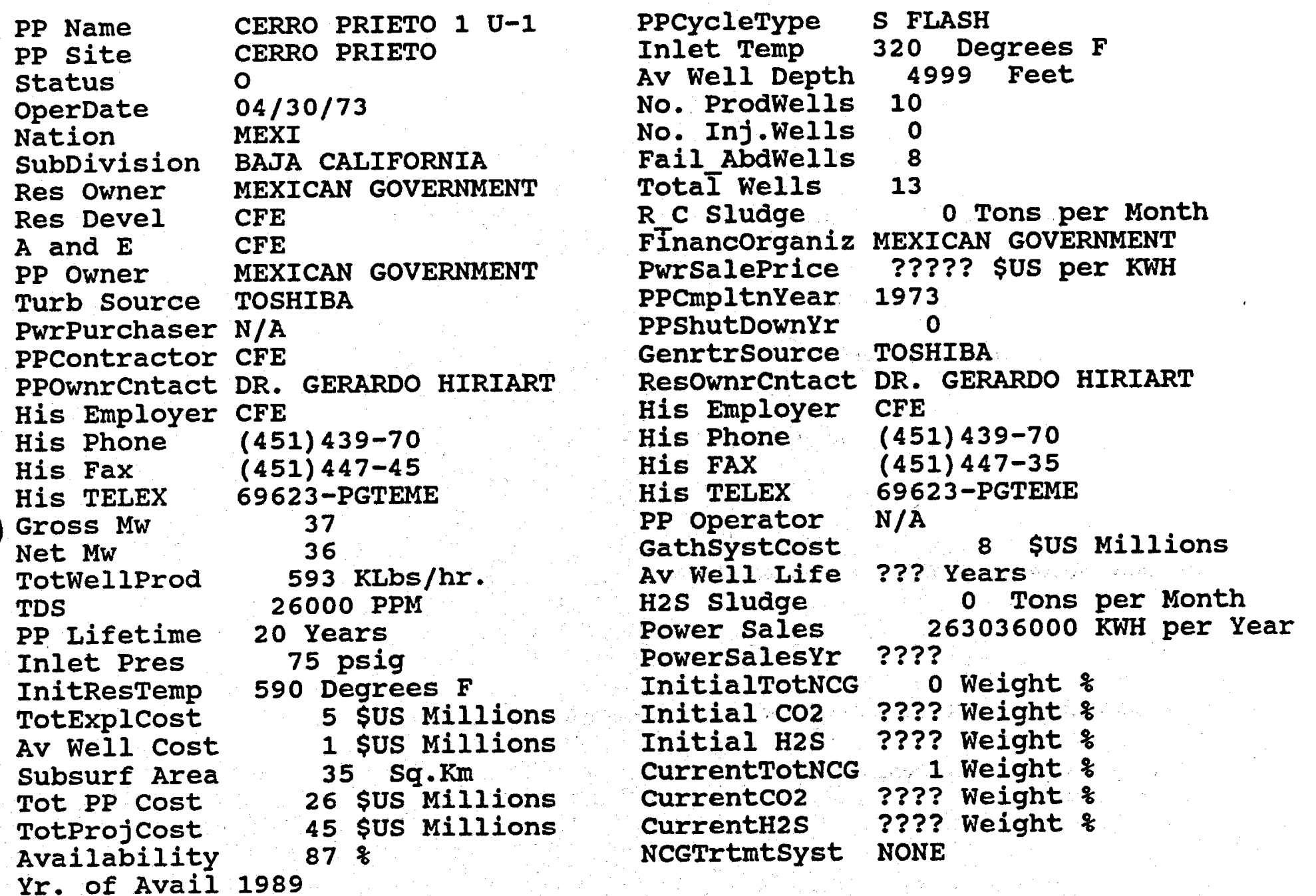




\begin{tabular}{|c|c|c|c|}
\hline $\begin{array}{l}\text { PP Name } \\
\text { PP Site }\end{array}$ & $\begin{array}{ll}\text { CERRO PRIETO } 1 \mathrm{U}-2 \\
\text { CERRO PRIETO }\end{array}$ & $\begin{array}{l}\text { PPCycleType } \\
\text { Inlet Temp }\end{array}$ & $\begin{array}{l}\text { S FLASH } \\
320 \text { Degrees F }\end{array}$ \\
\hline status & & Av Well Depth & 4953 Feet \\
\hline OperDate & $09 / 30 / 73$ & No. ProdWeils & 6 \\
\hline ation & MEXI & No. Inj.Wells & 0 \\
\hline SubDivision & BAJA CALIFORNIA & Fail_AbdWells & 9 \\
\hline $\begin{array}{l}\text { Res Owner } \\
\text { Res Devel } \\
A \text { and } E\end{array}$ & $\begin{array}{l}\text { MEXICAN GOVERNMENT } \\
\text { CFE } \\
\text { CFE }\end{array}$ & $\begin{array}{l}\text { TotaI Wells } \\
\text { RC sludge } \\
\text { Financorganiz }\end{array}$ & $\begin{array}{l}11 \text { o Tons per Month } \\
\text { MEXICAN GOVERNMENT }\end{array}$ \\
\hline PP Owner & MEXICAN GOVERNMENT & Pwrsaleprice & ????? \$US per KWH \\
\hline $\begin{array}{l}\text { Turb source } \\
\text { PwrPurchaser }\end{array}$ & $\begin{array}{l}\text { TOSHIBA } \\
\text { N/A }\end{array}$ & $\begin{array}{l}\text { PPCmpltnYear } \\
\text { PPShutDownYr }\end{array}$ & $\begin{array}{r}1973 \\
0\end{array}$ \\
\hline PPContractor & CFE & Genrtrsource & TOSHIBA \\
\hline $\begin{array}{l}\text { His Employer } \\
\text { His Phone } \\
\text { His FaX } \\
\text { His TELEX }\end{array}$ & $\begin{array}{l}\text { CFE } \\
(451) 439-70 \\
(451) 447-45 \\
69623-P G T E M E\end{array}$ & $\begin{array}{l}\text { His Employer } \\
\text { His Phone } \\
\text { His FAX } \\
\text { His TELEX }\end{array}$ & $\begin{array}{l}\text { DRE } \\
\text { (451) } 439-70 \\
(451) 447-35 \\
69623-\text { PGTEME }\end{array}$ \\
\hline $\begin{array}{l}\text { Gross Mw } \\
\text { Net Mw }\end{array}$ & $\begin{array}{r}37 \\
36 \\
538\end{array}$ & $\begin{array}{l}\text { PP Operator } \\
\text { Gathsystcost } \\
\text { Av Well Iife }\end{array}$ & $\begin{array}{l}\text { N/A } 5 \text { \$US Millions } \\
\text { ??? Years }\end{array}$ \\
\hline $\begin{array}{l}\text { TDS } \\
\text { PP Lifetime } \\
\text { Inlet Pres }\end{array}$ & $\begin{array}{l}26000 \text { PPM } \\
20 \text { Years } \\
75 \text { psig }\end{array}$ & $\begin{array}{l}\text { H2S Sludge } \\
\text { Power Sales } \\
\text { PowersalesYr }\end{array}$ & $\begin{array}{c}0 \text { Tons per Month } \\
236055000 \text { KWH per Year } \\
\text { ???? }\end{array}$ \\
\hline $\begin{array}{l}\text { InitResTemp } \\
\text { TotExplCost } \\
\text { Av Well Cost }\end{array}$ & $\begin{array}{l}590 \text { Degrees } F \\
5 \text { SUS Millions } \\
1 \text { SUS Millions }\end{array}$ & $\begin{array}{l}\text { InitialTotNCG } \\
\text { Initial CO2 } \\
\text { Initial H2S }\end{array}$ & $\begin{array}{l}\text { o Weight } \% \\
\text { ???? Weight } \% \\
\text { ???? Weight \% }\end{array}$ \\
\hline $\begin{array}{l}\text { Subsurf Area } \\
\text { Tot PP cost } \\
\text { TotProjcost } \\
\text { Availability }\end{array}$ & $\begin{array}{l}35 \text { Sq. Km } \\
26 \text { \$US Militions } \\
44 \text { \$US Millions } \\
84 \text { \% }\end{array}$ & $\begin{array}{l}\text { CurrentTotNCG } \\
\text { Currentco2 } \\
\text { CurrentH2S } \\
\text { NCGTrtmtSyst }\end{array}$ & $\begin{array}{l}1 \text { Weight : } \\
\text { ???? Weight \% } \\
\text { ???? Weight \% } \\
\text { NONE }\end{array}$ \\
\hline & & & \\
\hline
\end{tabular}




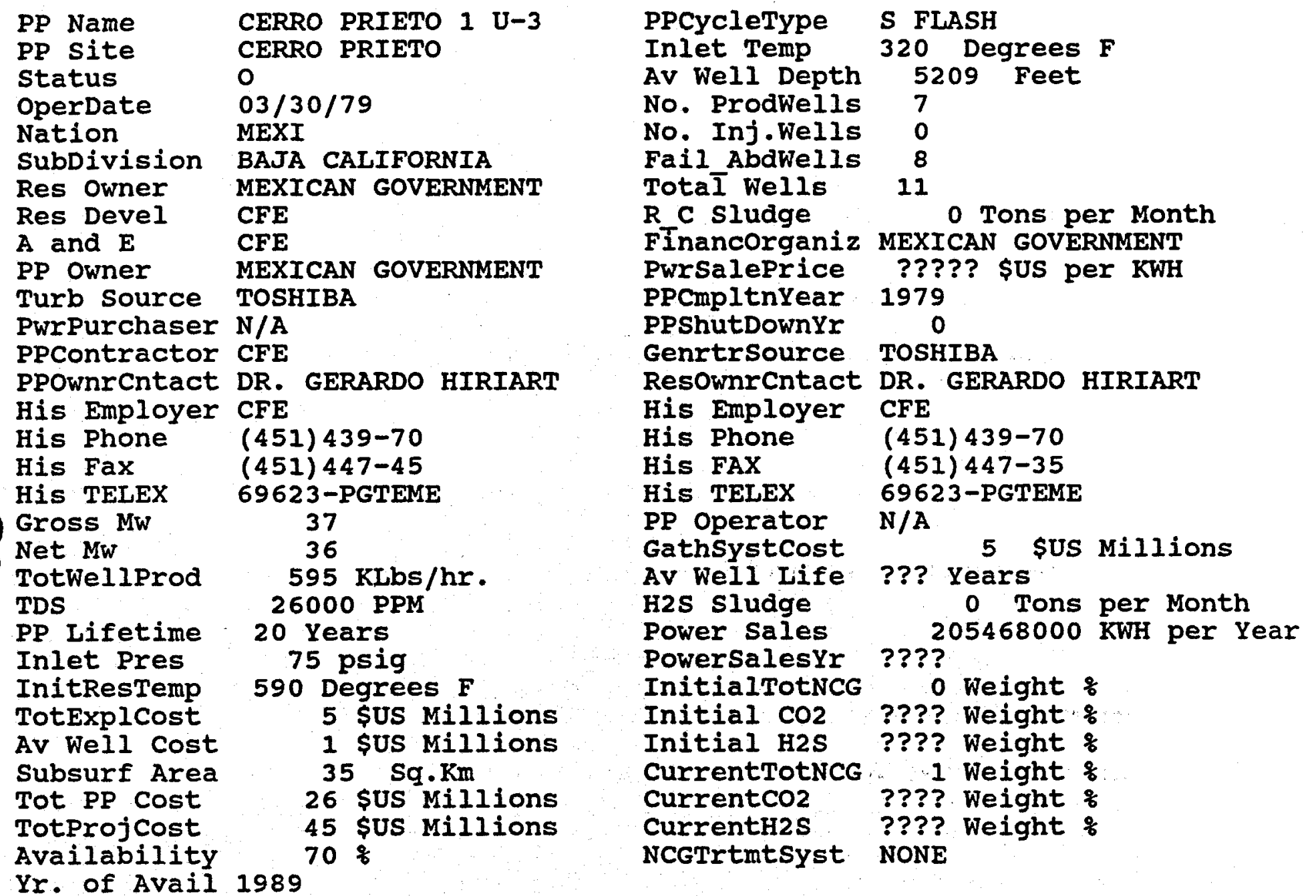




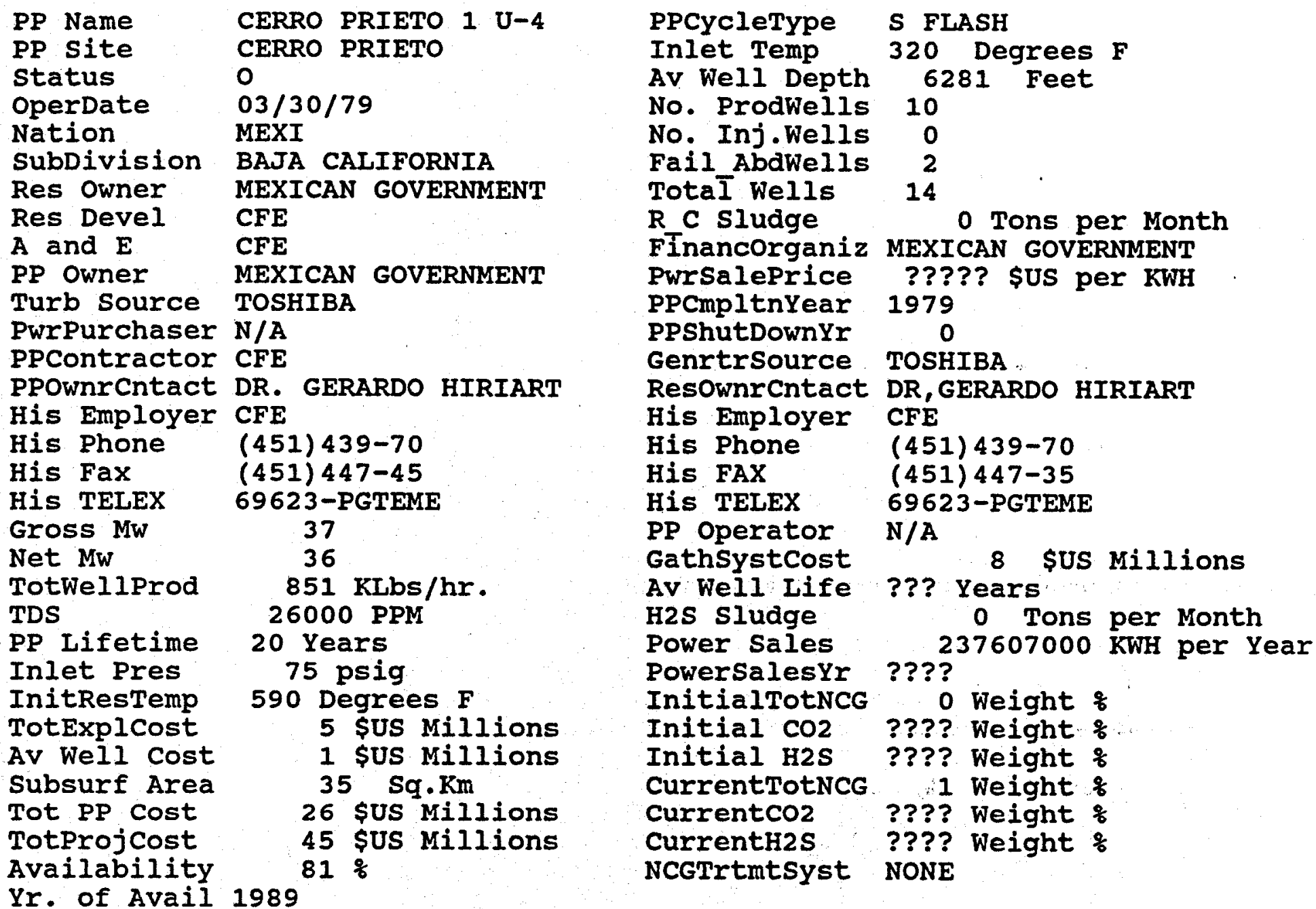




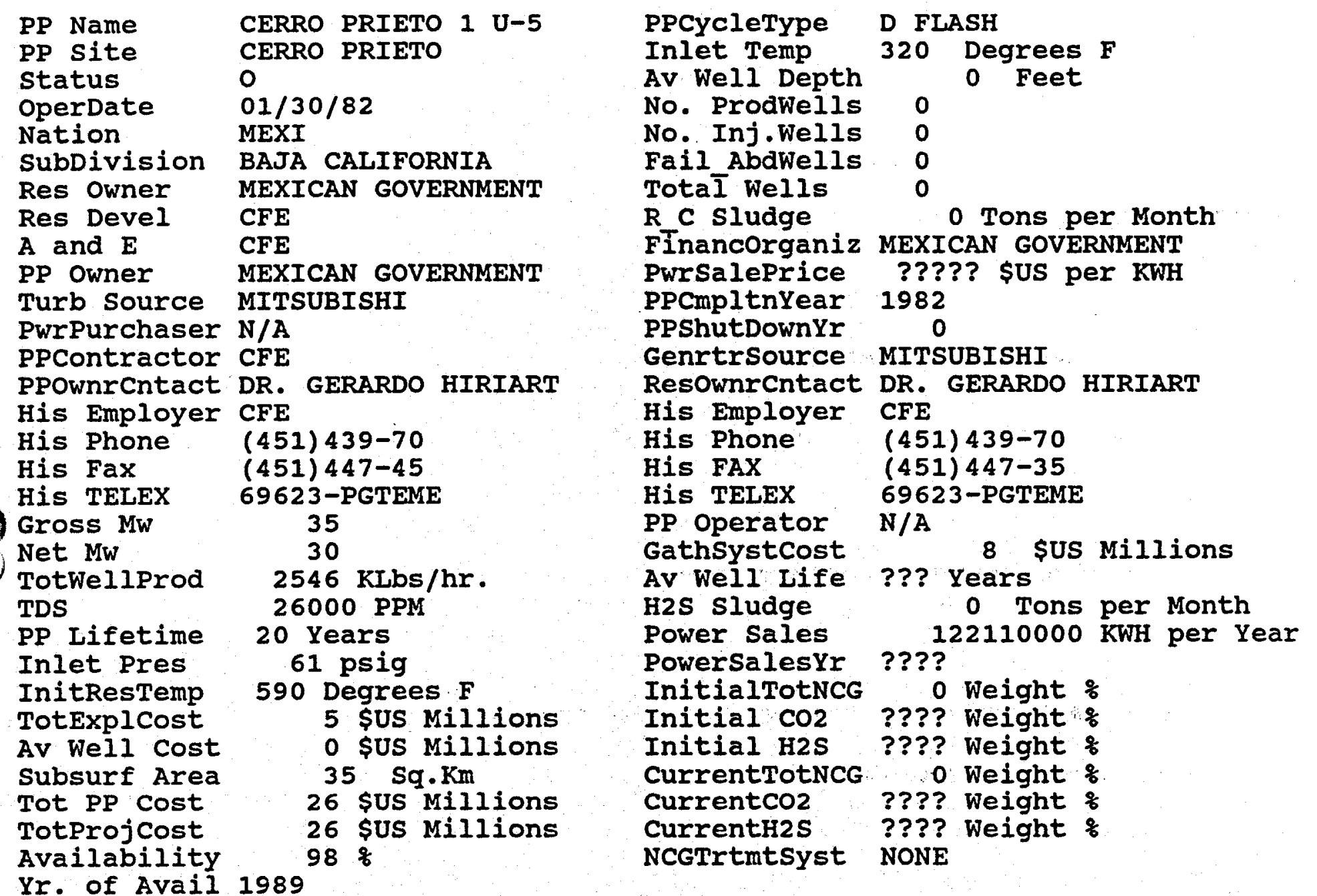




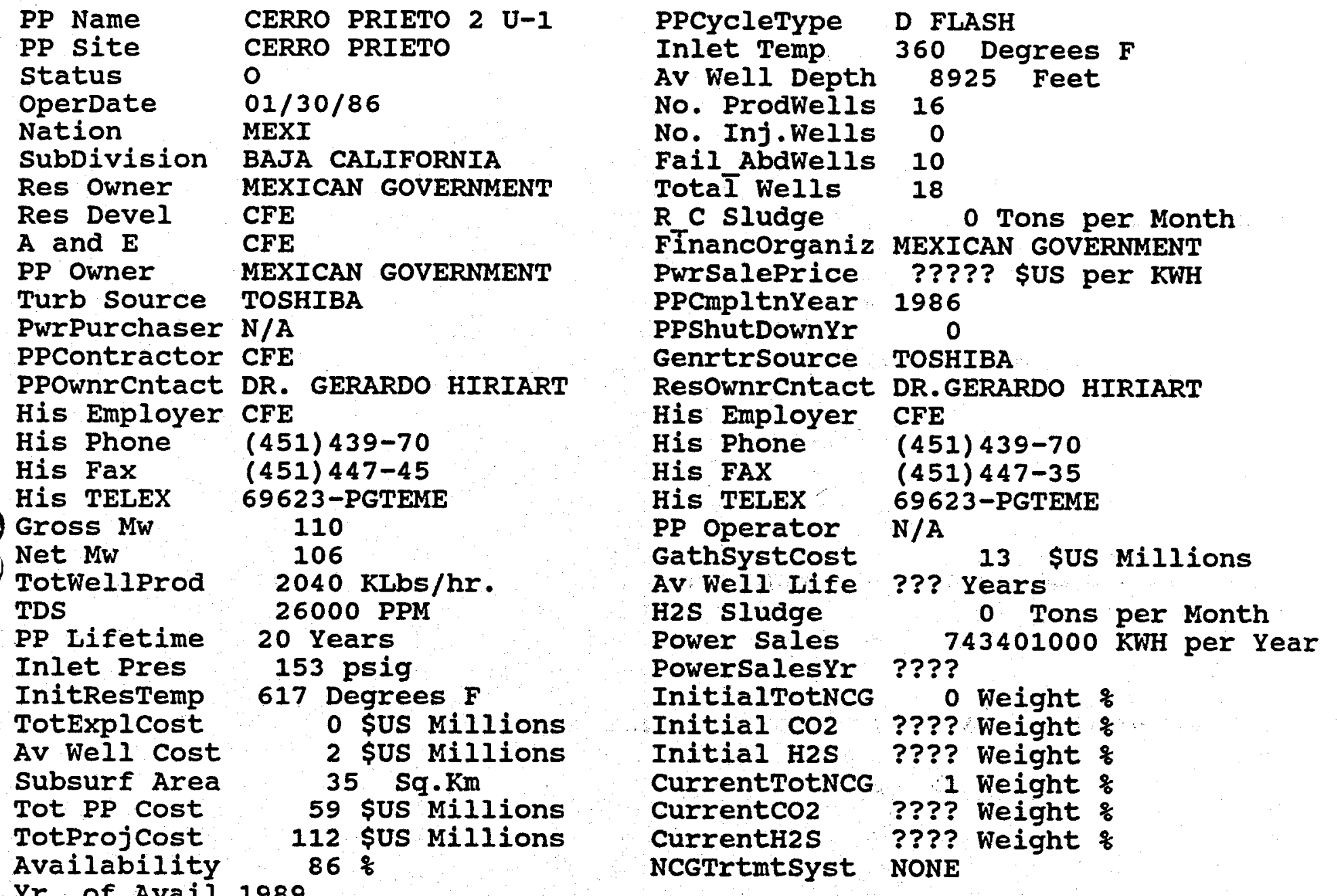




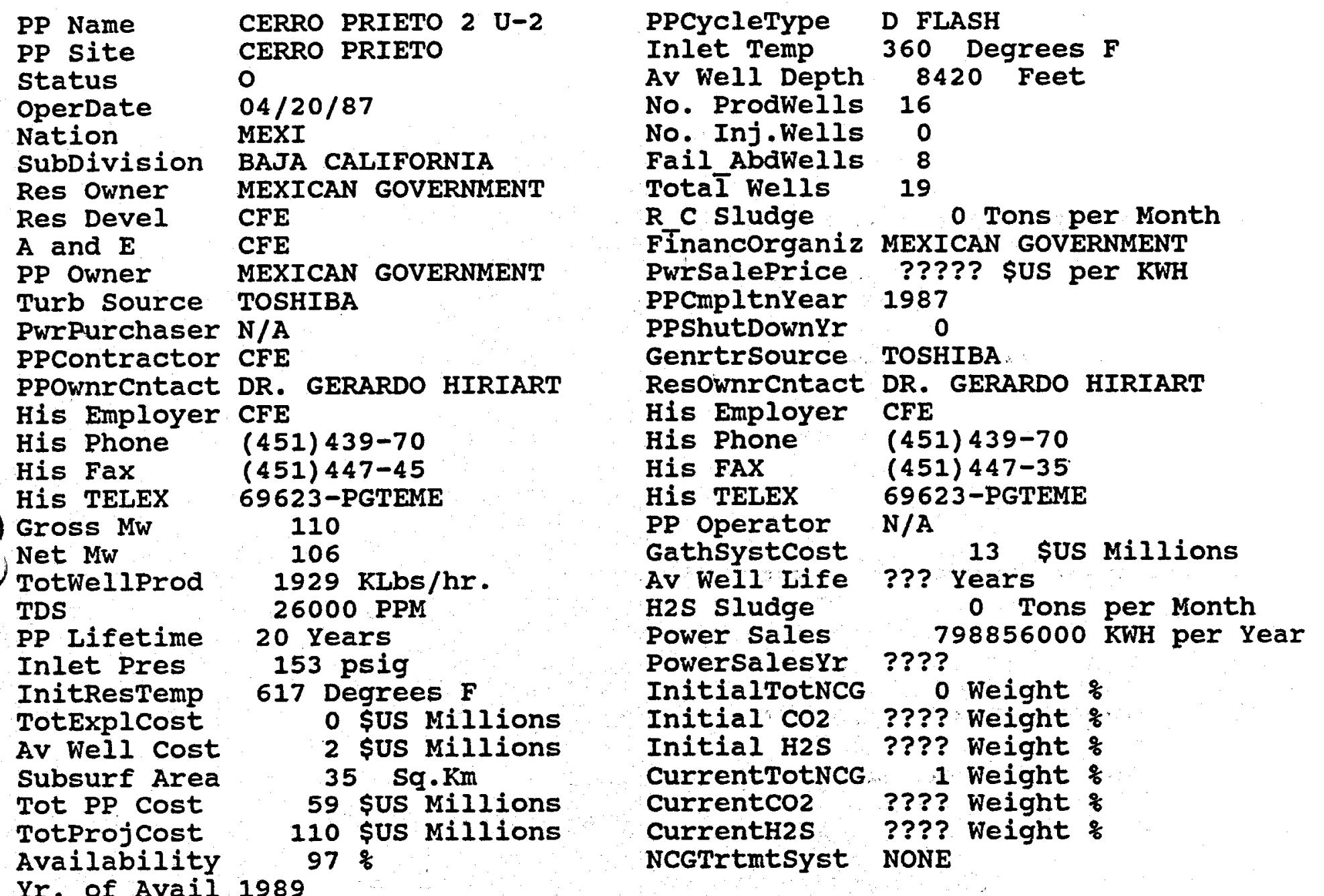




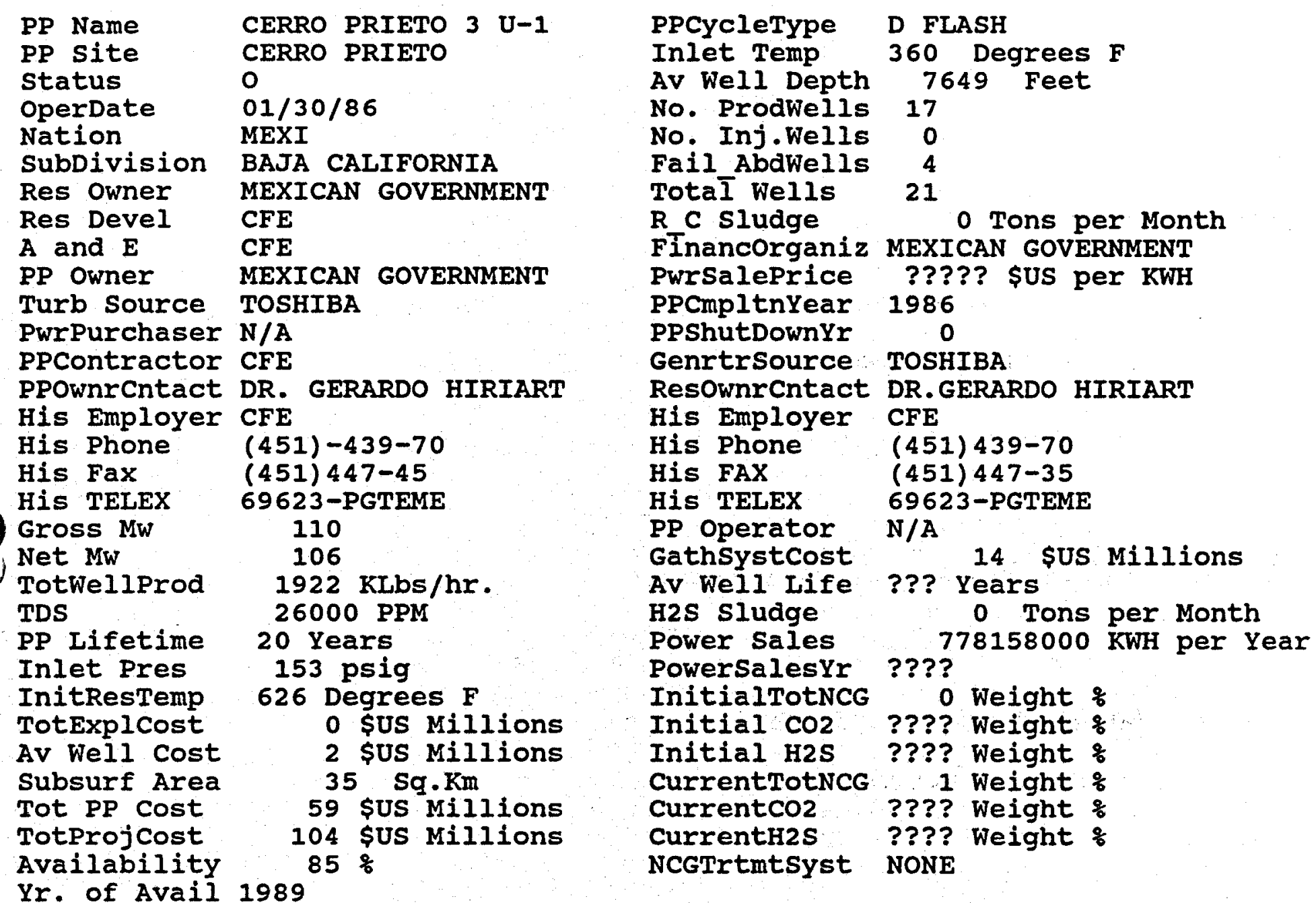




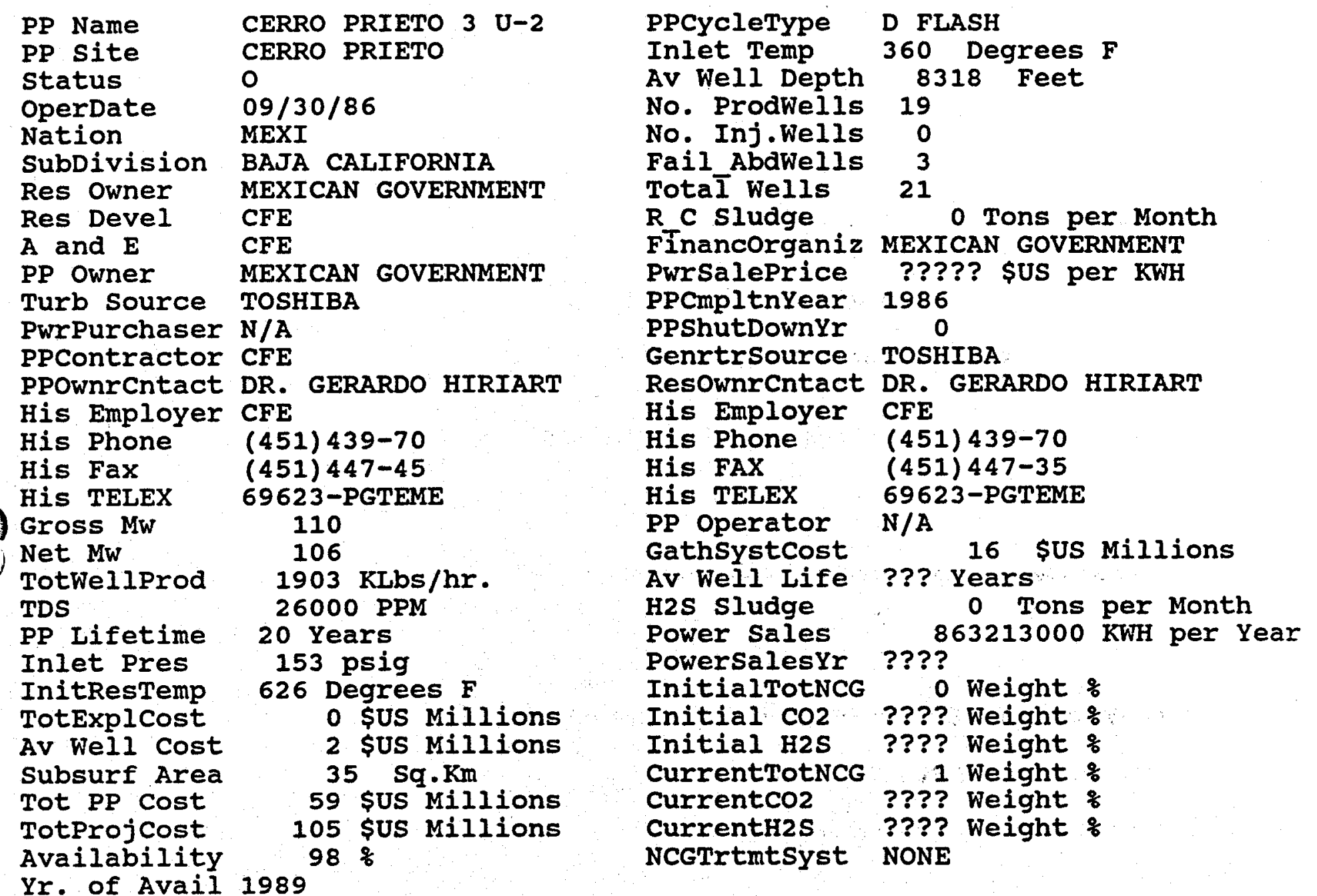




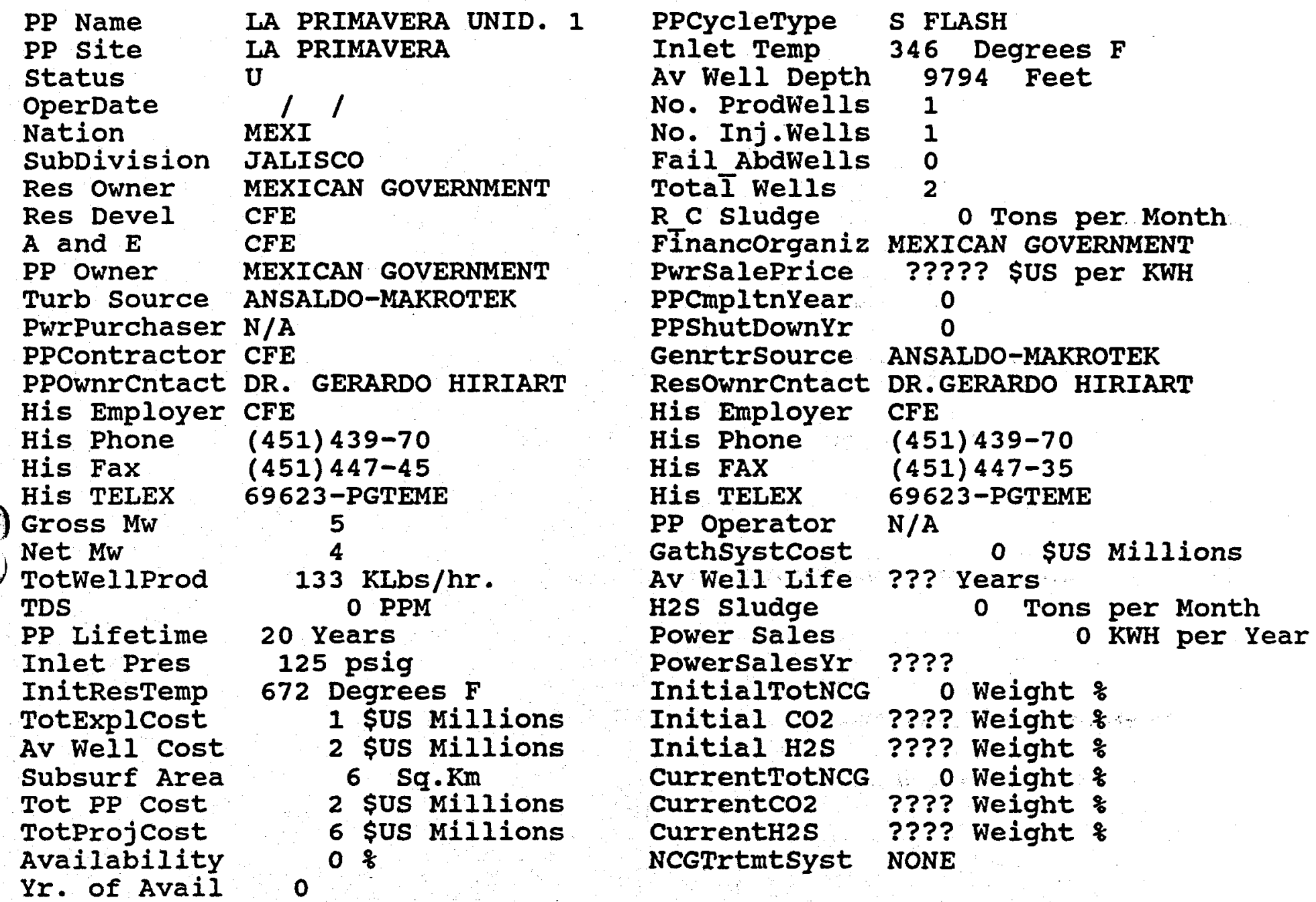




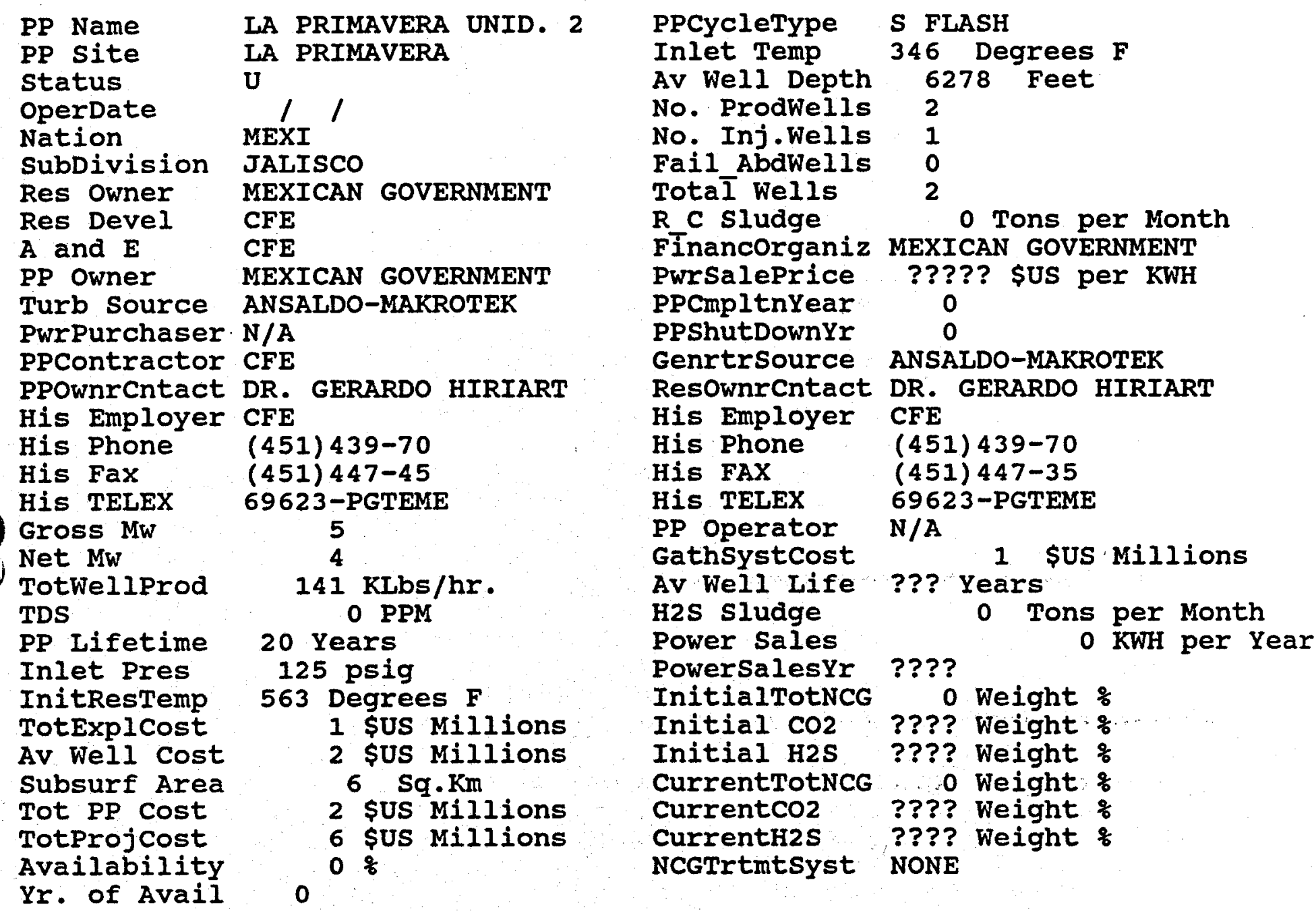




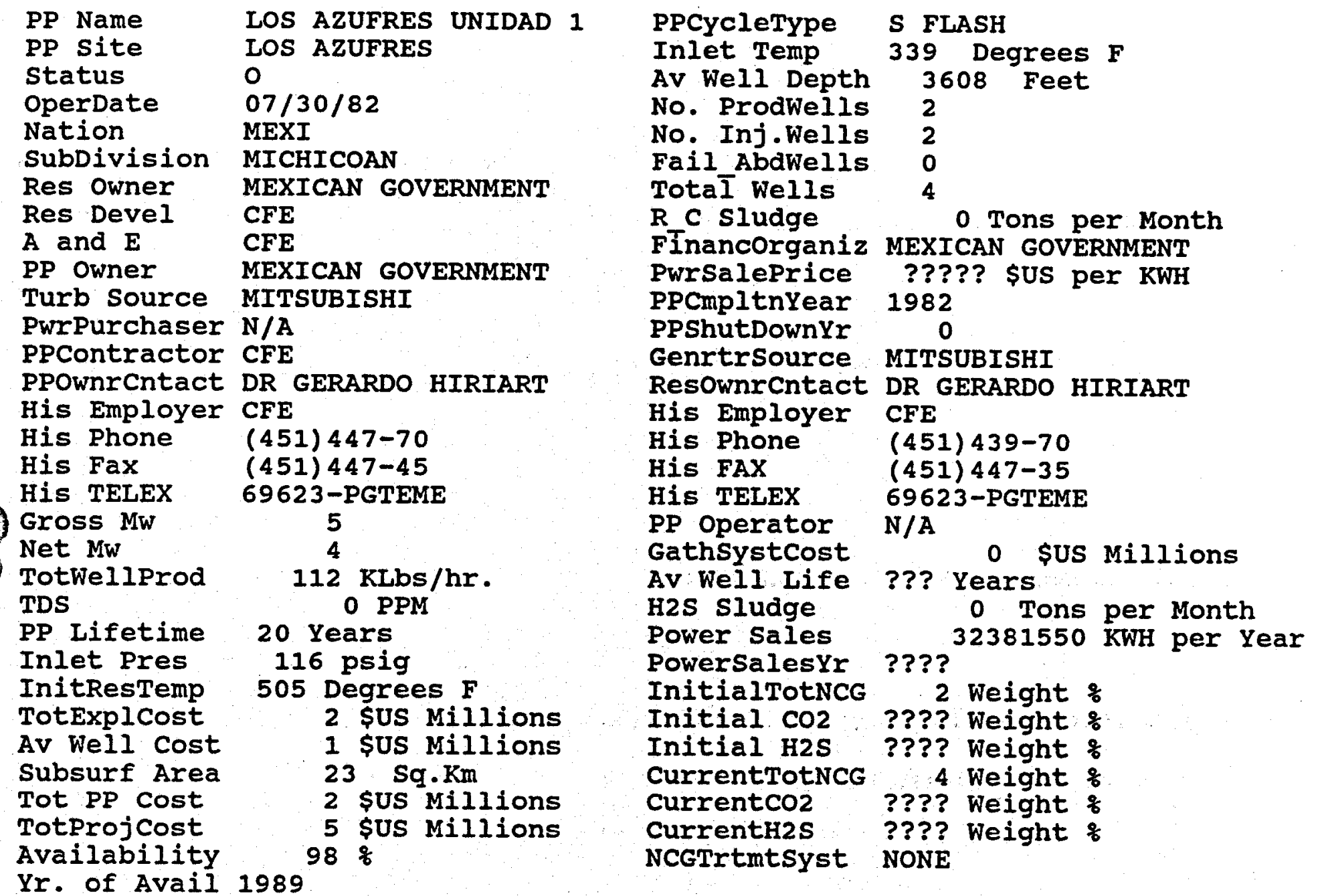


NEW ZEALAND 


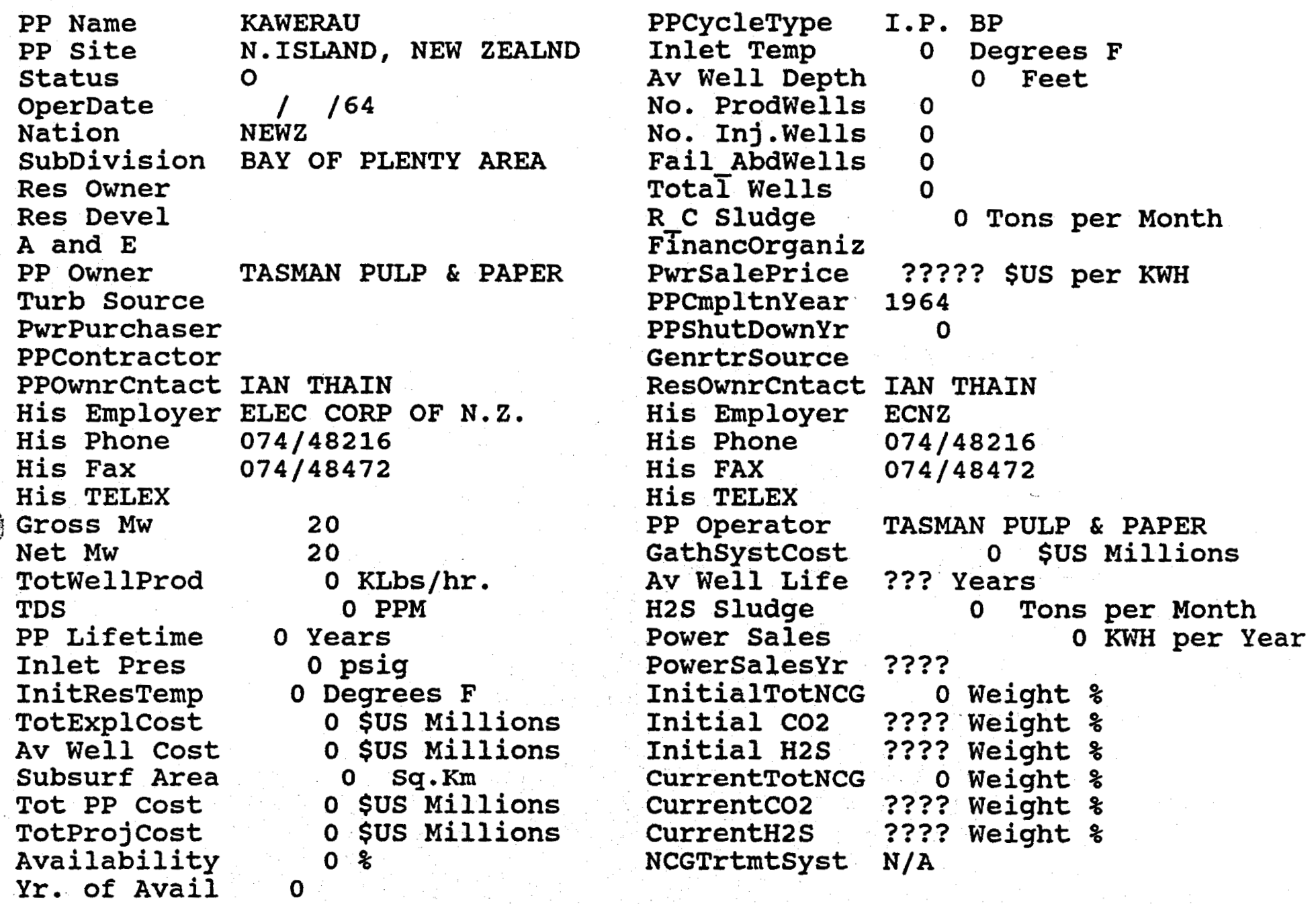




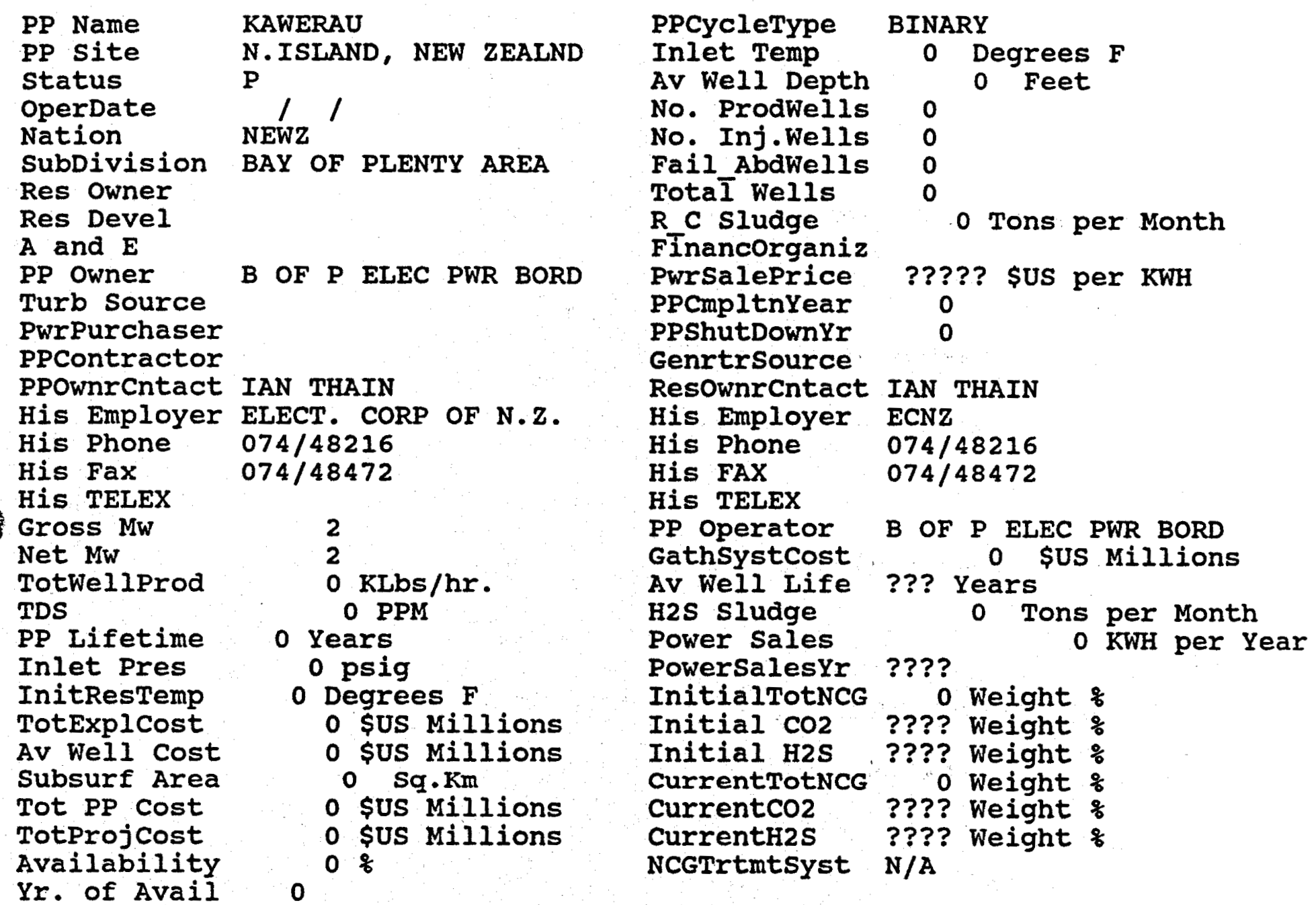




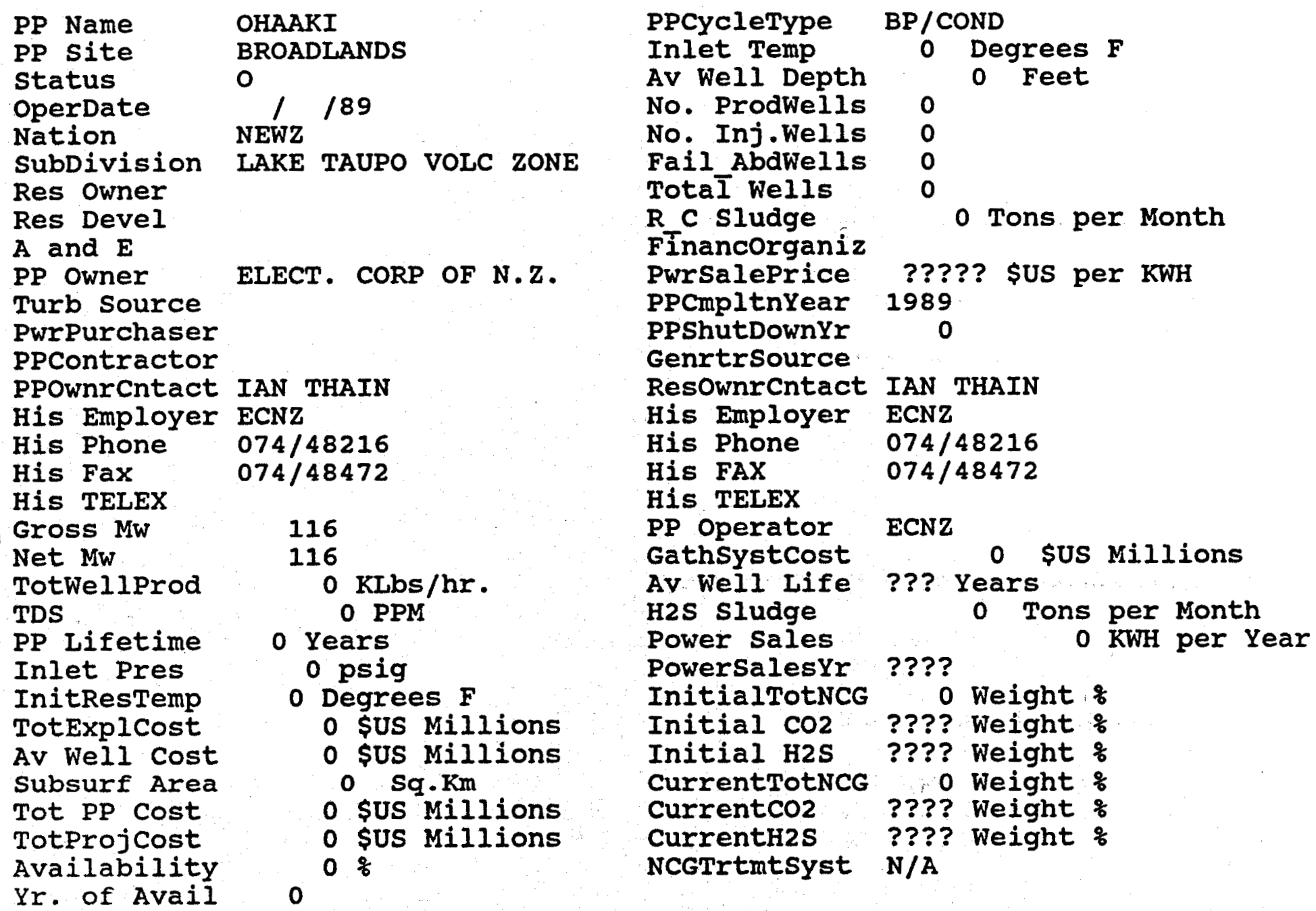




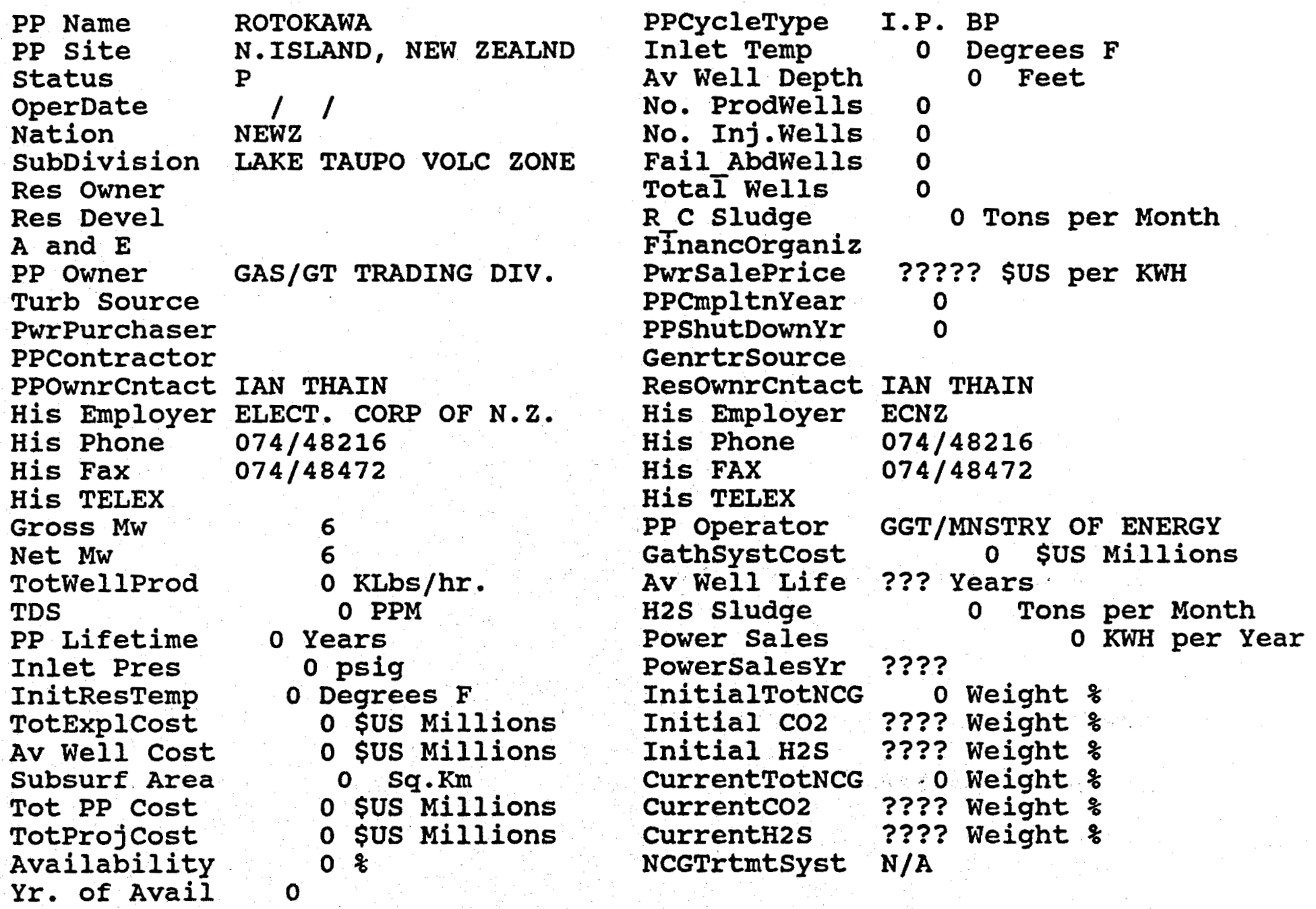




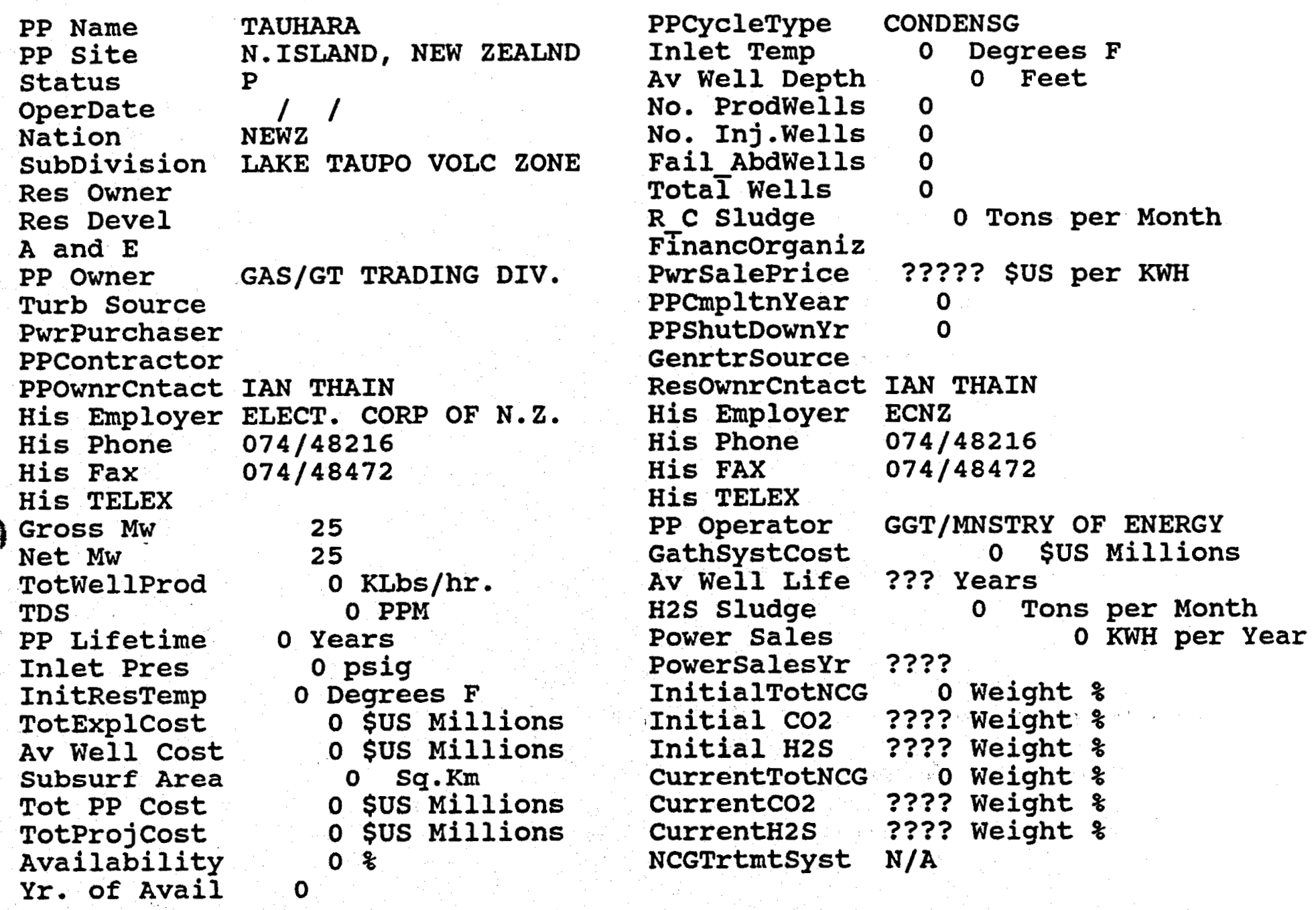




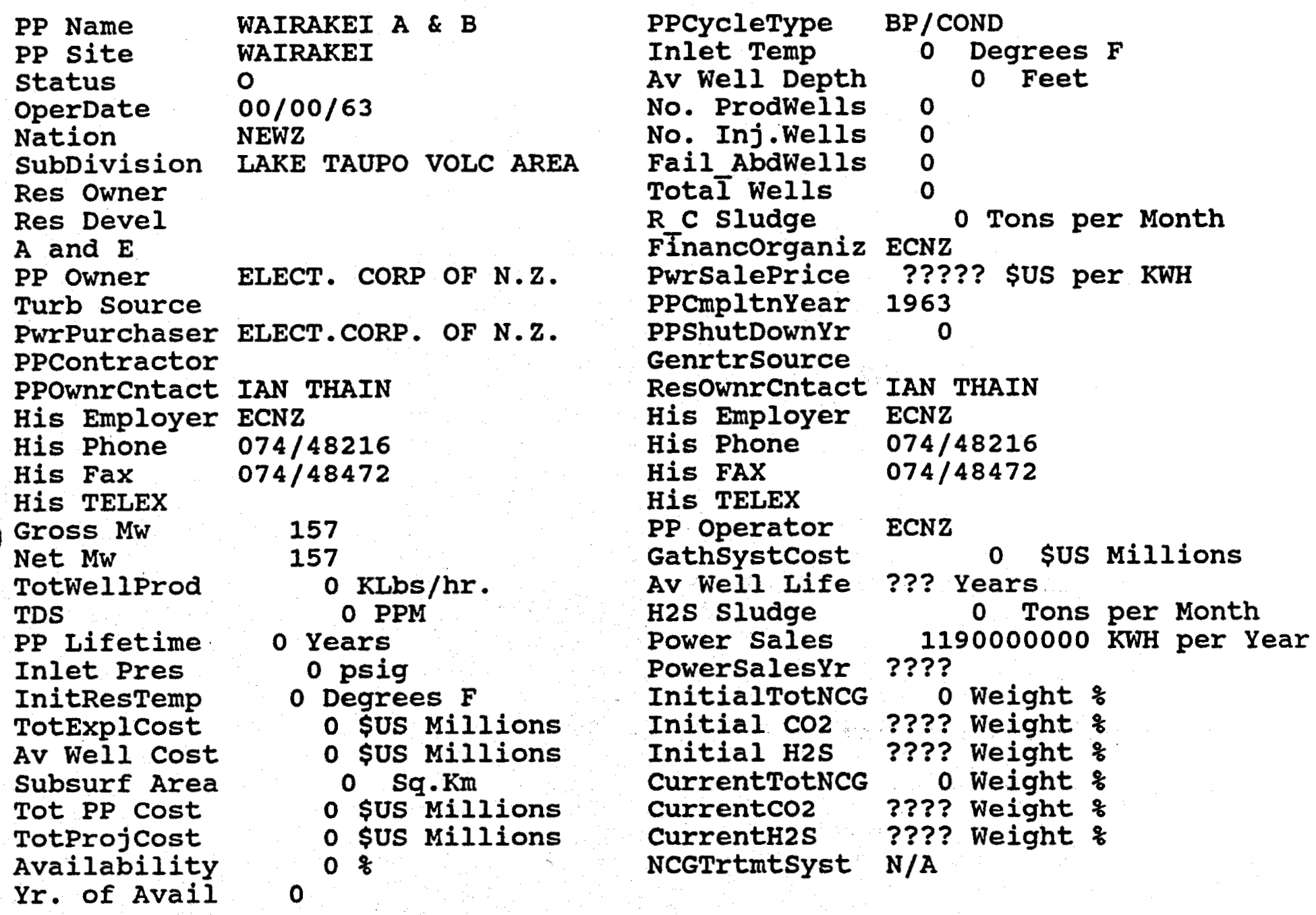




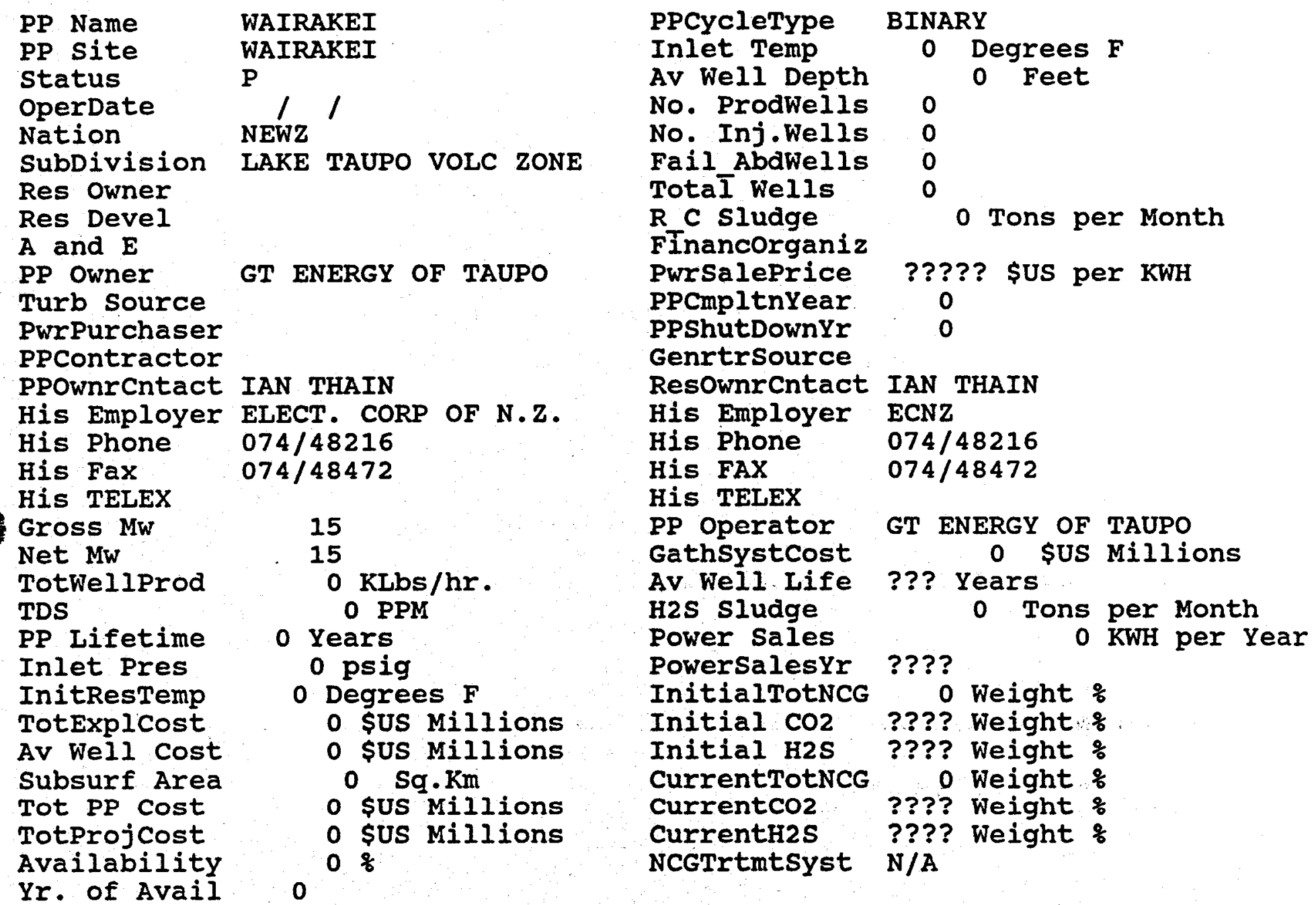


NICAGRAGUA 


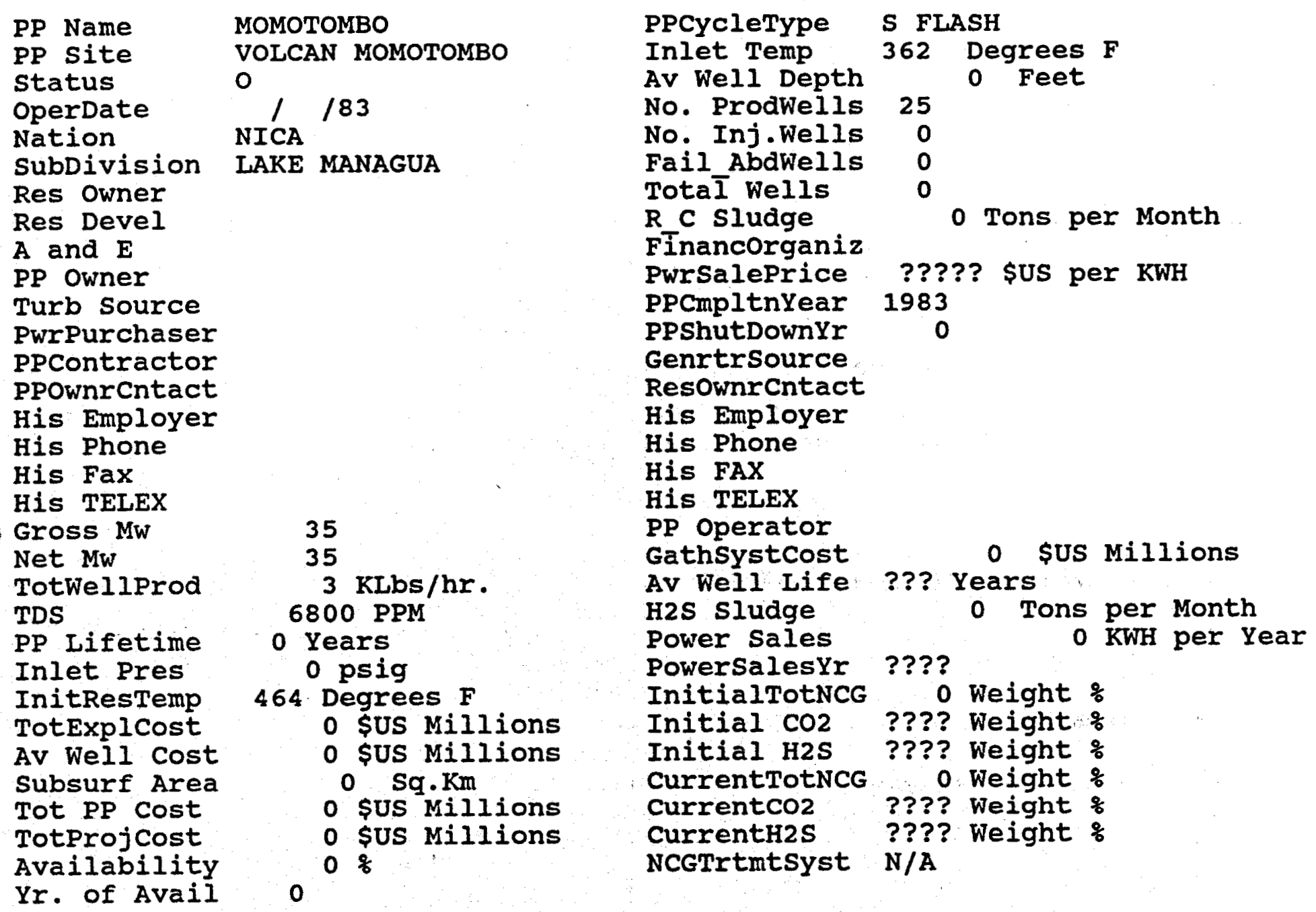


PHILIPPINES 


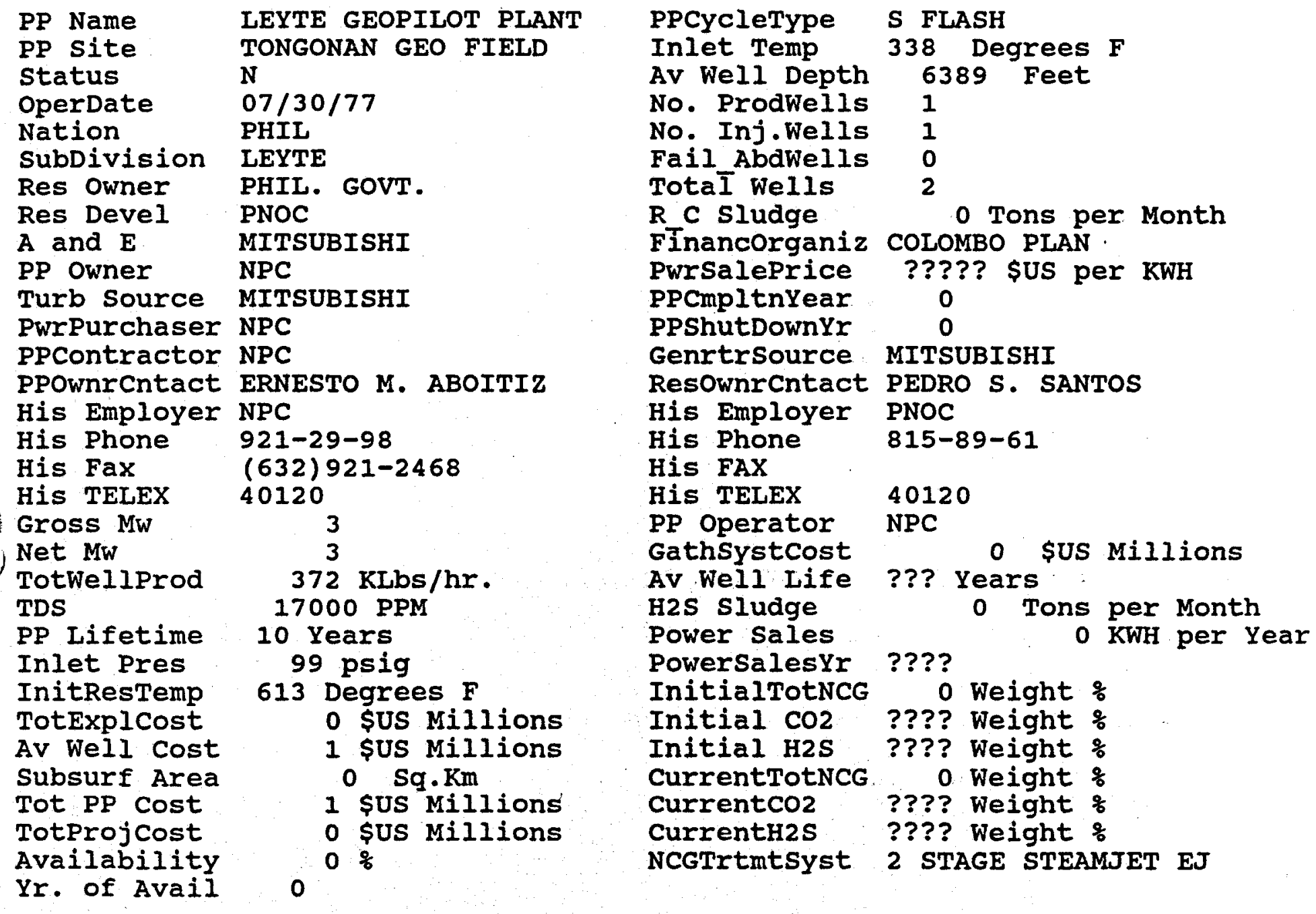




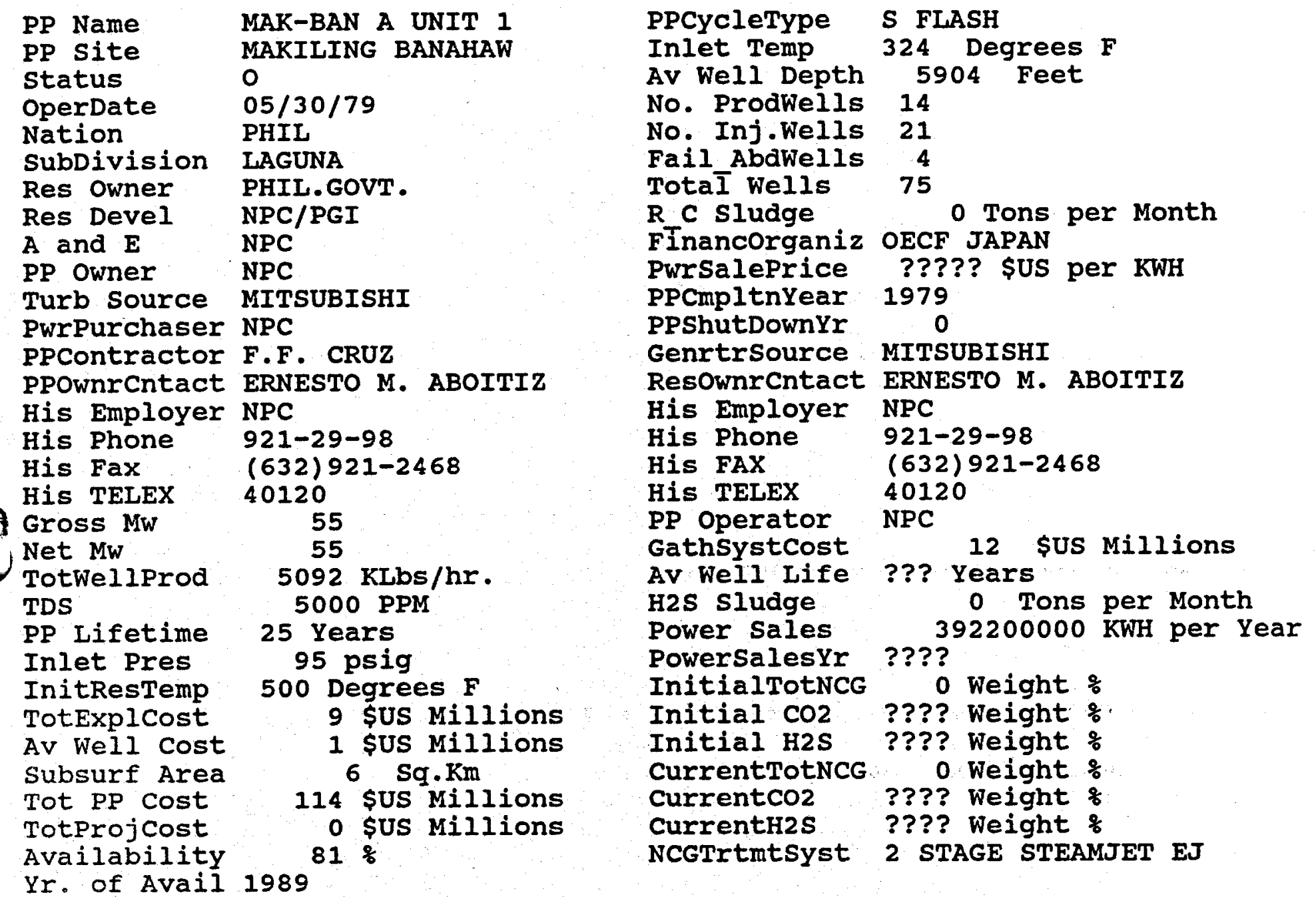




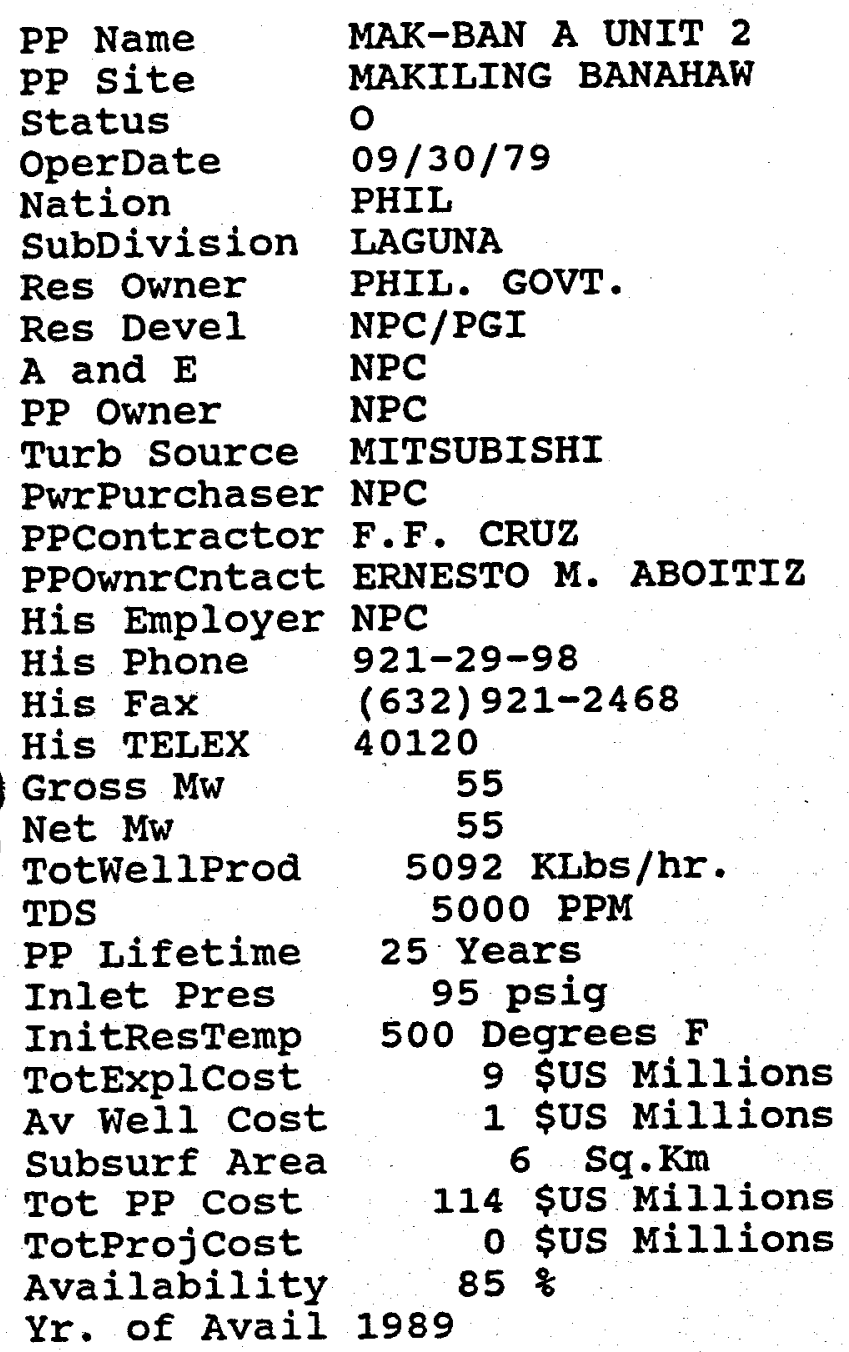

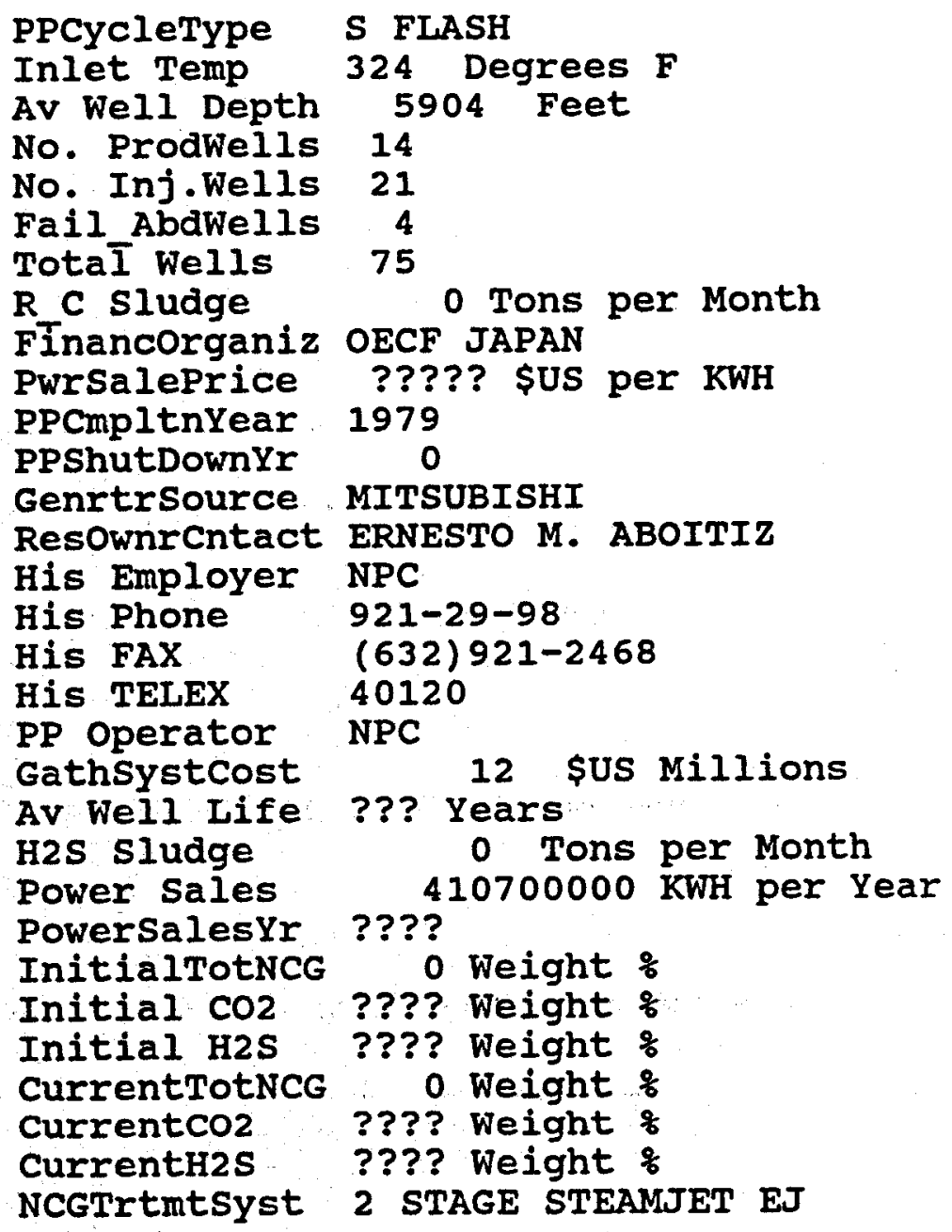




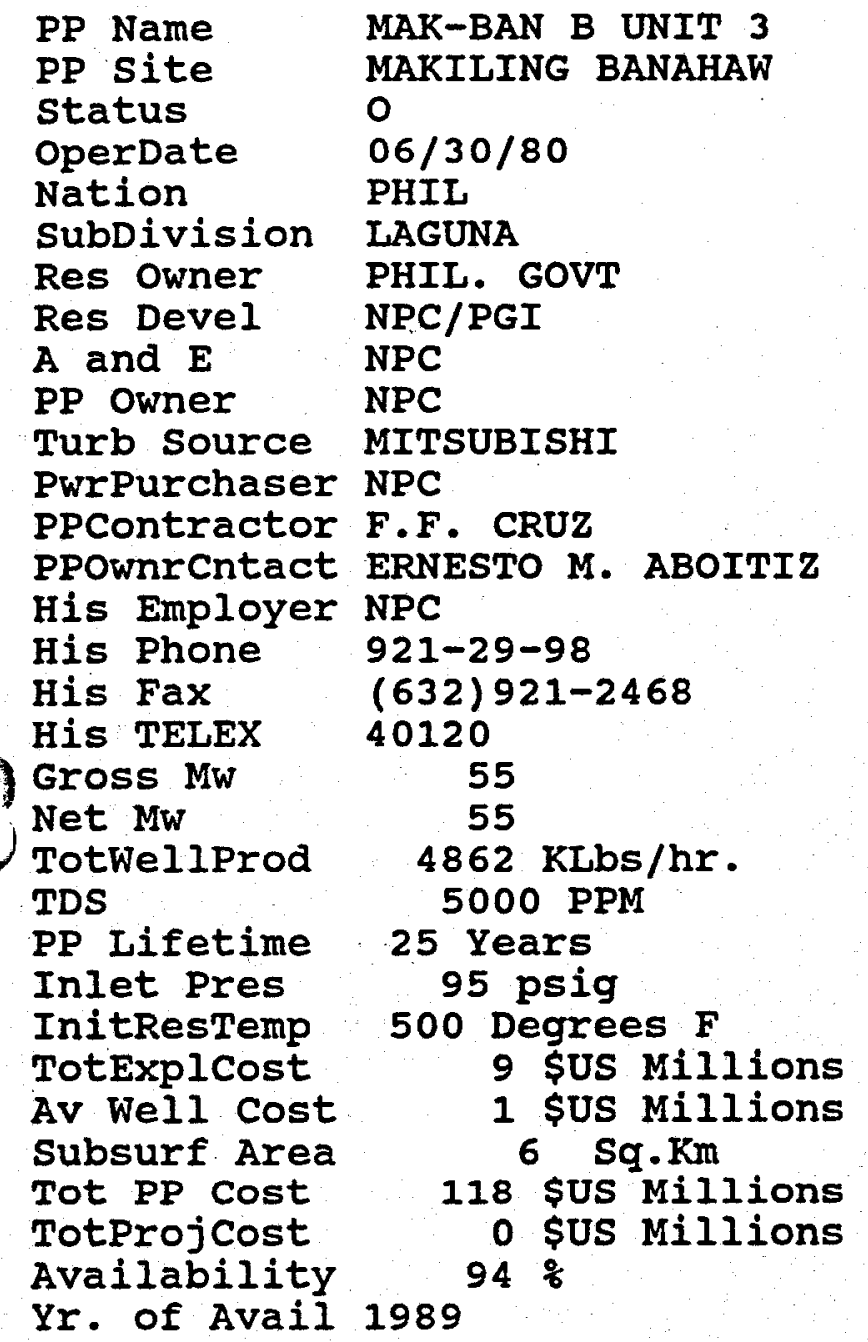

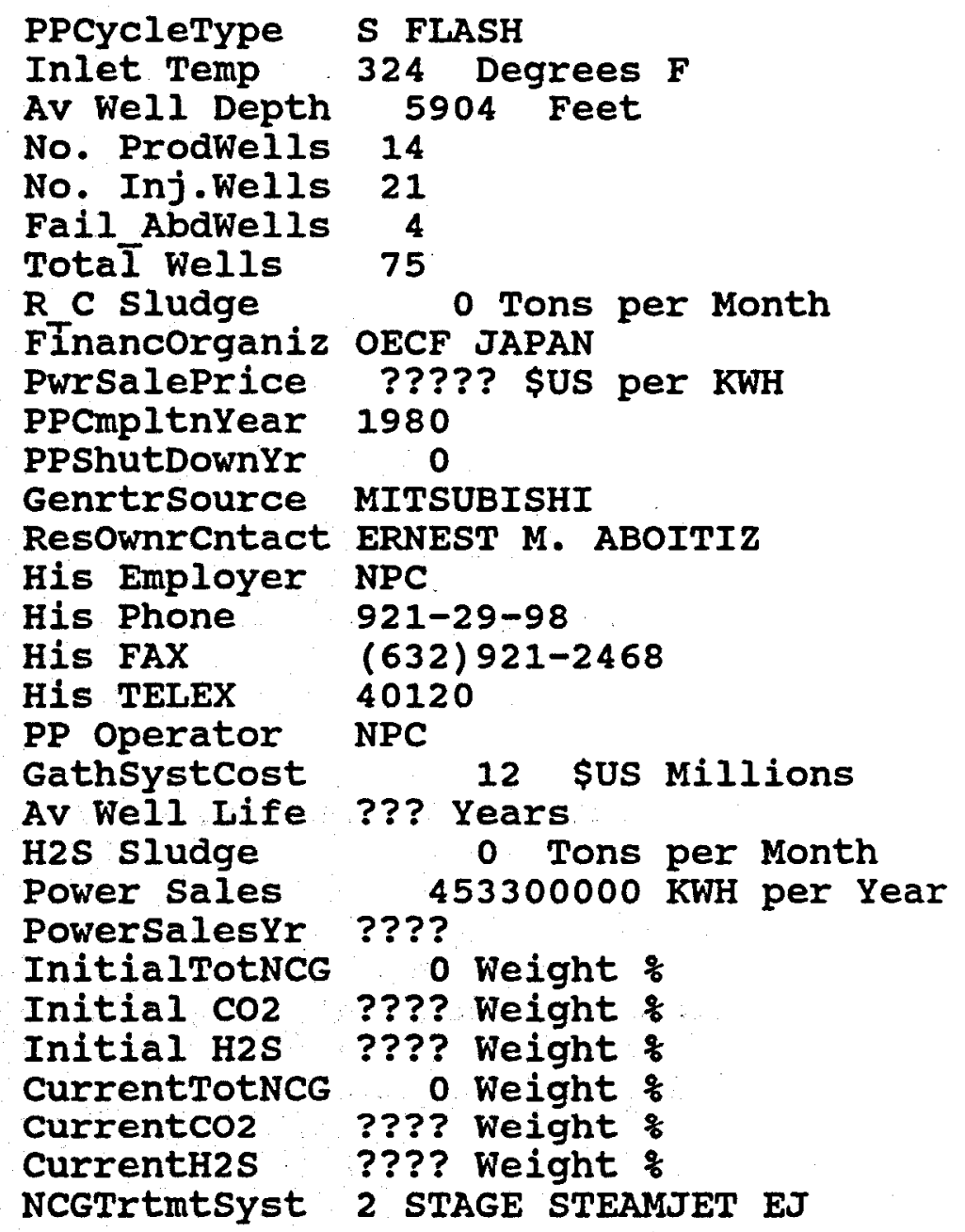




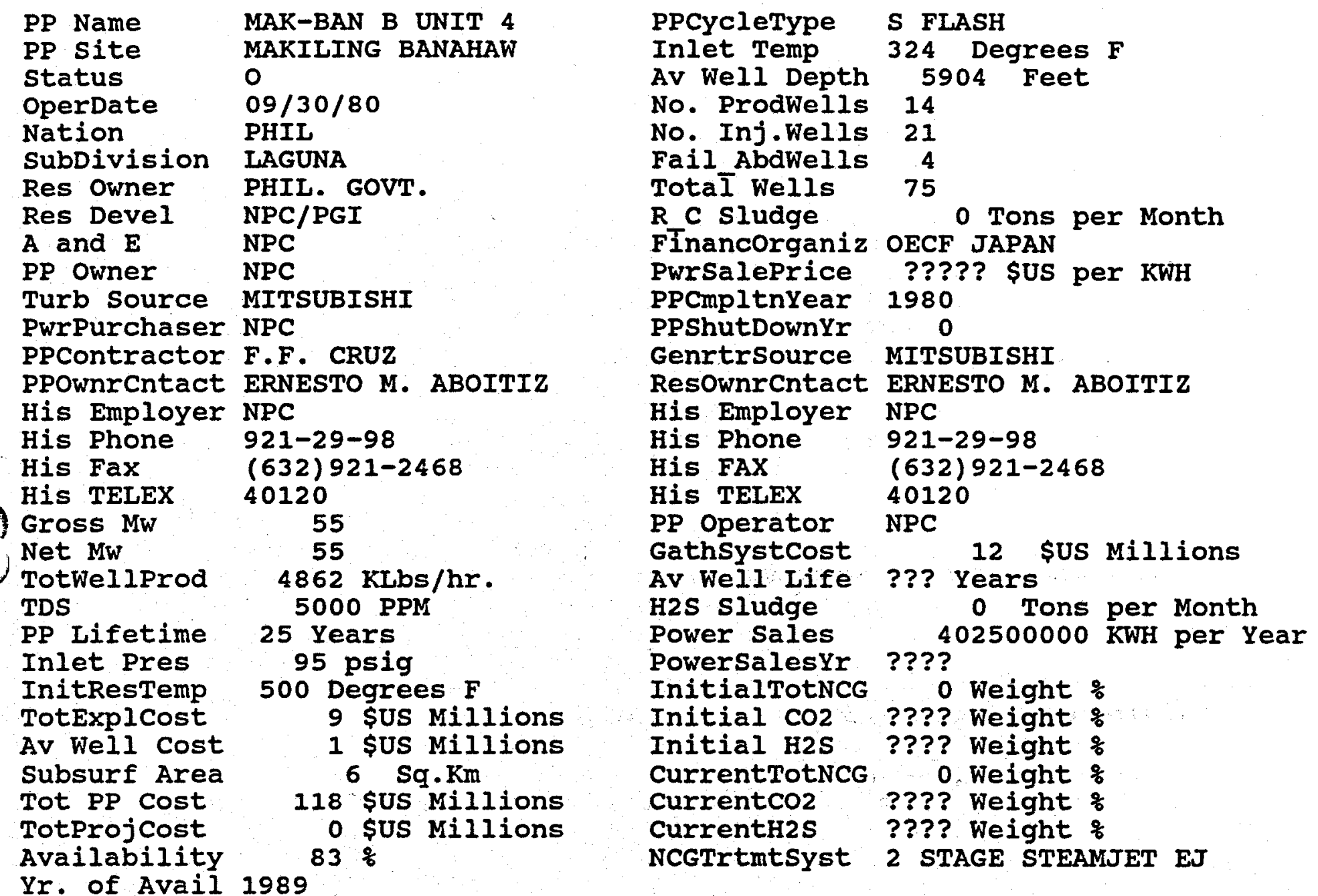




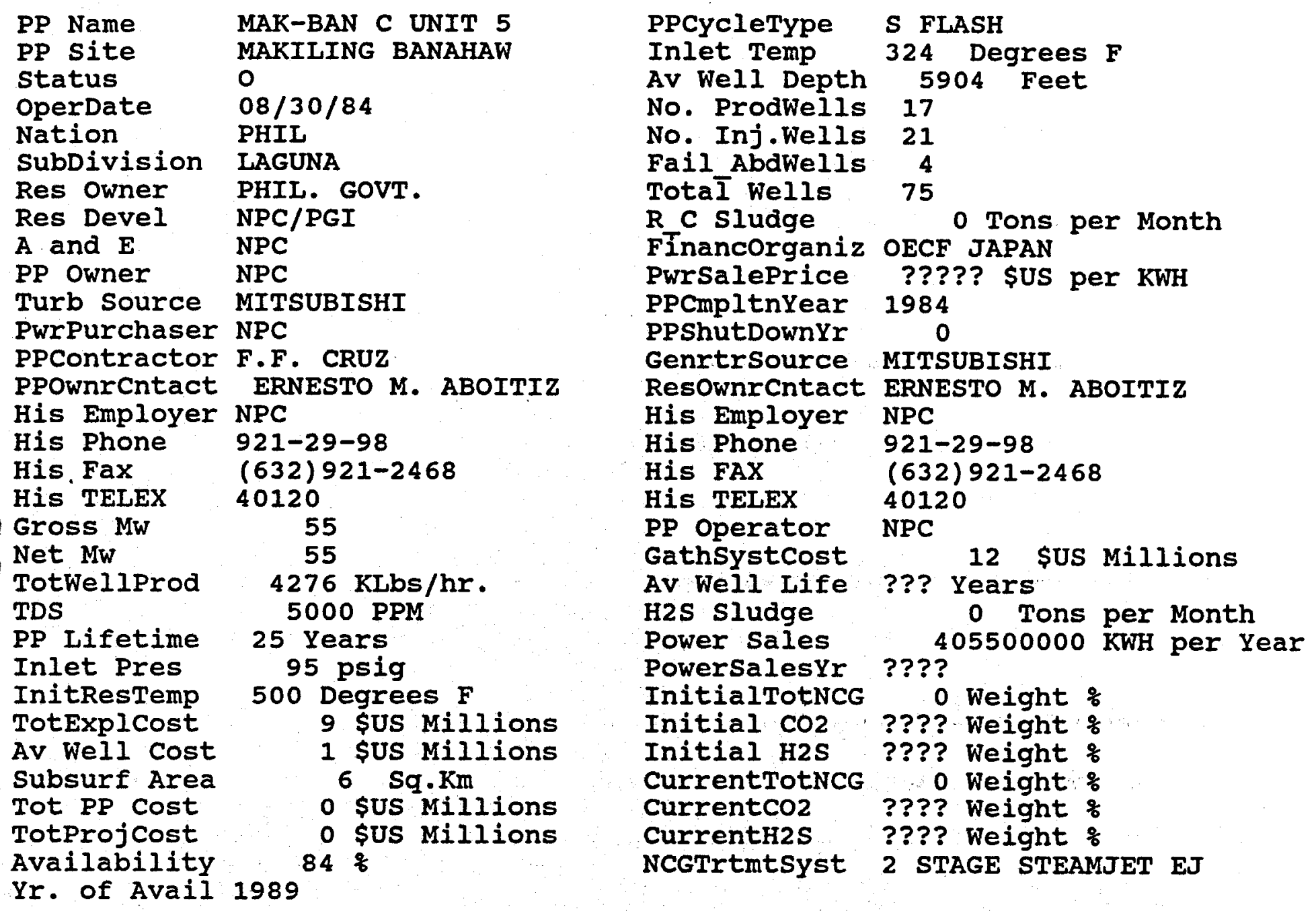




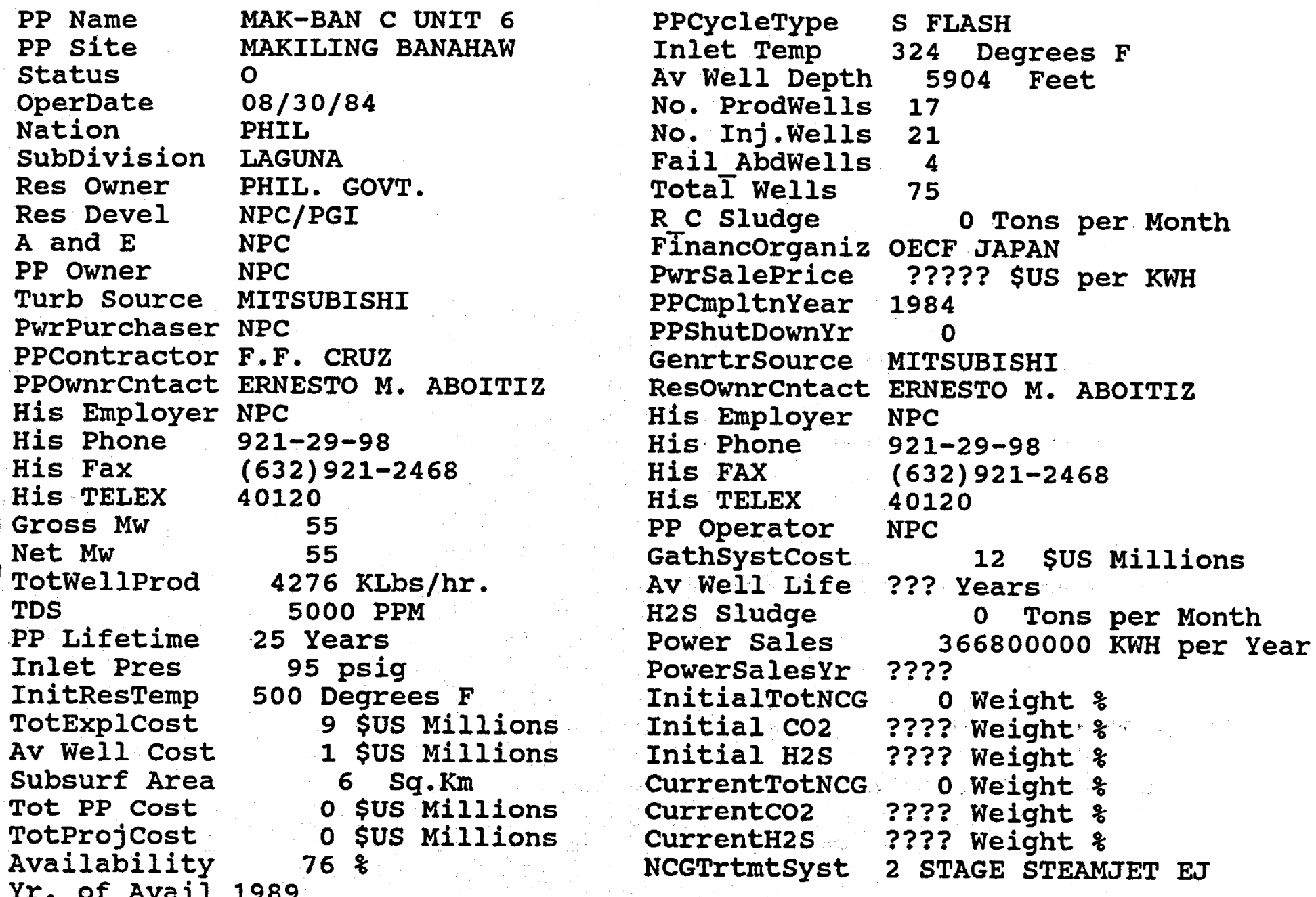




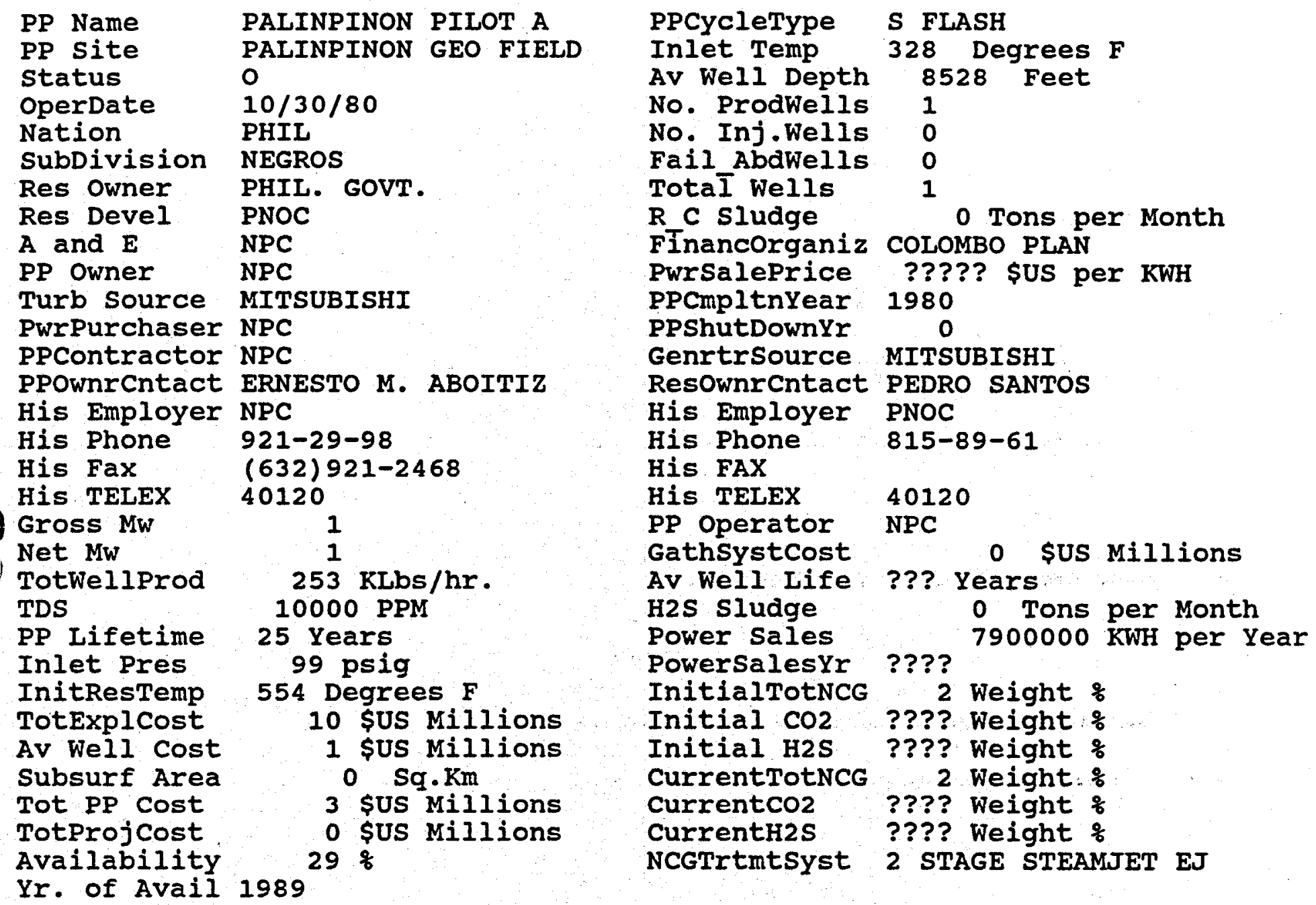




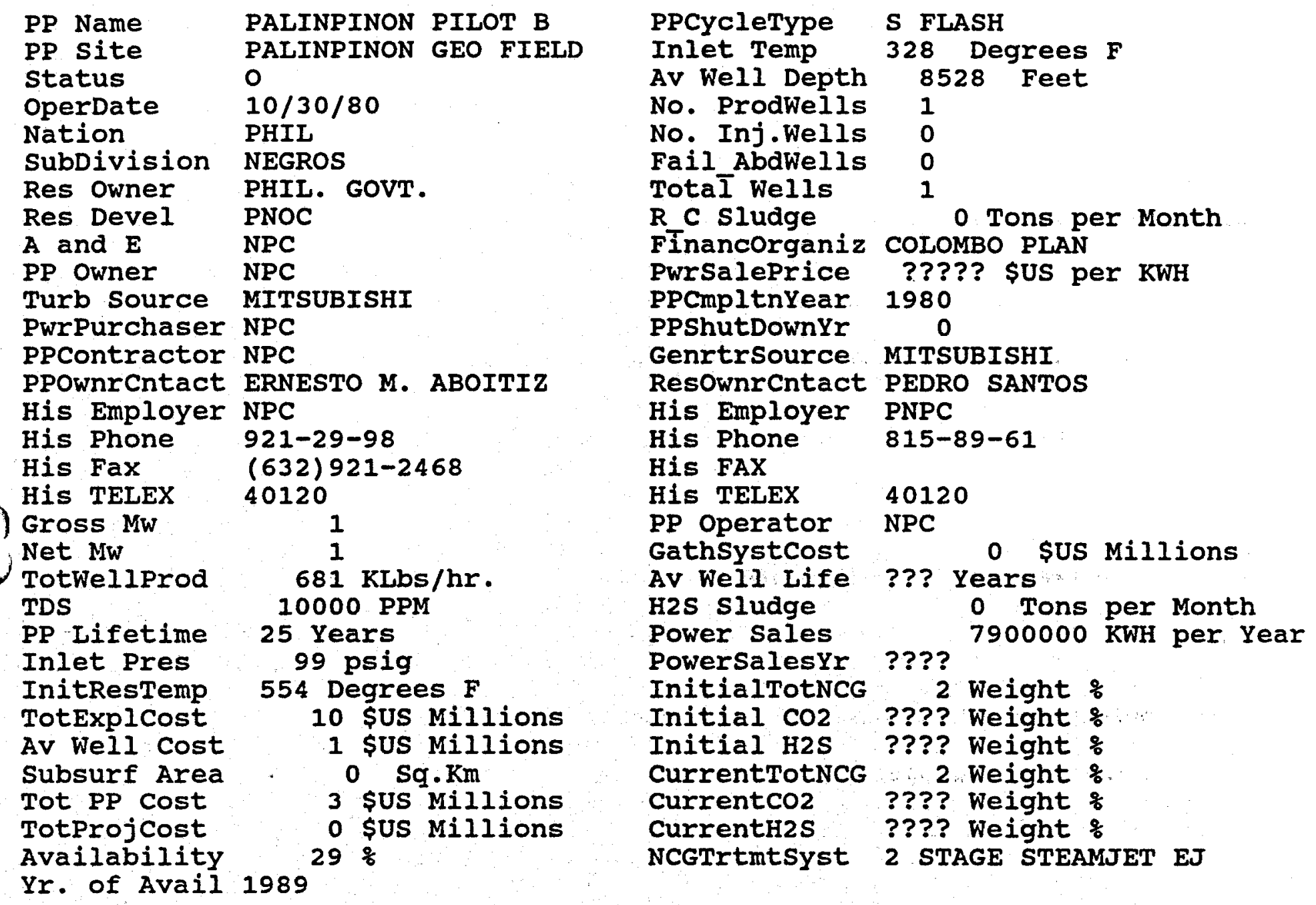




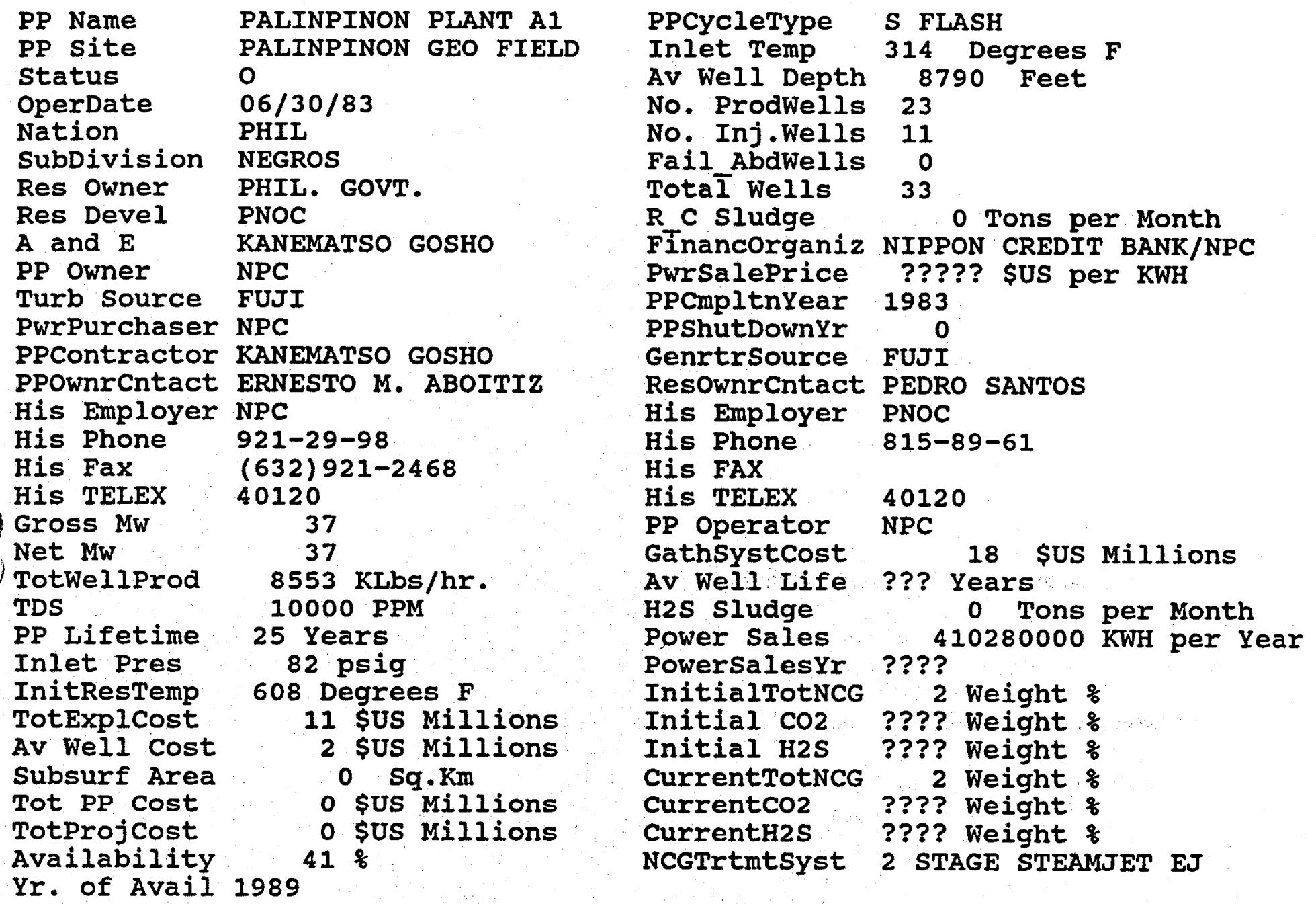




\begin{tabular}{|c|c|c|c|}
\hline PP Name & PALINPINON PLANT A2 & PPCycleType & S FLASH \\
\hline PP site & PALINPINON GEO FIELD & Inlet Temp & Degrees $\mathbf{F}$ \\
\hline $\begin{array}{l}\text { Status } \\
\text { OperDate }\end{array}$ & $\begin{array}{l}0 \\
07 / 30 / 83\end{array}$ & Av Well Depth & 8790 \\
\hline lation & PHIL & $\begin{array}{l}\text { No. Proawells } \\
\text { No. Inj.Wells }\end{array}$ & $\begin{array}{l}23 \\
11\end{array}$ \\
\hline ubDivision & NEGROS & Fail_AbaWells & 0 \\
\hline Res Owmer & PHIL. GOVT. & TotaI Wells & 33 \\
\hline $\begin{array}{l}\text { Res Devel } \\
\text { A and E } \\
\text { PP Owner }\end{array}$ & $\begin{array}{l}\text { PNOC } \\
\text { KANEMATSO GOSHO } \\
\text { NPC }\end{array}$ & $\begin{array}{l}\text { R C sludge } \\
\text { Fínancorganiz }\end{array}$ & $\begin{array}{l}0 \text { Tons per Nonth } \\
\text { NIPPON CREDIT BANK/NPC }\end{array}$ \\
\hline Turb source & FUJI & $\begin{array}{l}\text { Pwrsaleprice } \\
\text { PPCmpltnYear }\end{array}$ & $\begin{array}{l}\text { ????? \$US per KWH } \\
\text { 1983 }\end{array}$ \\
\hline PwrPurchaser & NPC & PPShutDownYr & 0 \\
\hline PPContractor & KANEMATSO & Genrtrsource & FUJI \\
\hline $\begin{array}{l}\text { PPOwnrcntact } \\
\text { His Employer }\end{array}$ & $\begin{array}{l}\text { ERNESTO M. ABOITIZ } \\
\text { NPC }\end{array}$ & Resownrcntact & PEDRO SANTOS \\
\hline $\begin{array}{l}\text { His Phone } \\
\text { His Fax }\end{array}$ & $\begin{array}{l}921-29-98 \\
(632) 921-2468\end{array}$ & $\begin{array}{l}\text { His Phone } \\
\text { His FAX }\end{array}$ & $815-89-61$ \\
\hline $\begin{array}{l}\text { His TELEX } \\
\text { Gross MW }\end{array}$ & $\begin{array}{r}40120 \\
37\end{array}$ & $\begin{array}{l}\text { His TELEX } \\
\text { PP operator }\end{array}$ & $\begin{array}{l}40120 \\
\text { NPC }\end{array}$ \\
\hline $\begin{array}{l}\text { Net } \mathrm{MW} \\
\text { Totwellprod }\end{array}$ & $37 \mathrm{KLbs} / \mathrm{hr}$. & $\begin{array}{l}\text { Gathsystcost } \\
\text { Av Well Life }\end{array}$ & $\begin{array}{c}18 \text { \$US Millions } \\
\text { ??? Years }\end{array}$ \\
\hline $\begin{array}{l}\text { TDS } \\
\text { PP Iifetime } \\
\text { Inlet Pres }\end{array}$ & $\begin{array}{l}0 \text { PPM } \\
0 \text { Years } \\
82 \text { psig }\end{array}$ & $\begin{array}{l}\text { H2s sludge } \\
\text { Power Sales } \\
\text { PowersalesYr }\end{array}$ & $\begin{array}{l}\text { 0 Tons per Month } \\
410280000 \text { KWH per Year } \\
\text { ???? }\end{array}$ \\
\hline $\begin{array}{l}\text { InitResTemp } \\
\text { TotExplCost } \\
\text { Av Well Cost }\end{array}$ & $\begin{array}{l}608 \text { Degrees } F \\
11 \text { \$US Millions } \\
2 \text { SUS Millions }\end{array}$ & $\begin{array}{l}\text { InitialTotNCG } \\
\text { Initial } \mathrm{CO} 2 \\
\text { Initial } \mathrm{H} 2 \mathrm{~S}\end{array}$ & $\begin{array}{l}2 \text { Weight } \% \\
\text { ???? Weight \% } \\
\text { ???? Weight \% }\end{array}$ \\
\hline $\begin{array}{l}\text { Subsurf Area } \\
\text { Tot PP Cost } \\
\text { TotProj Cost }\end{array}$ & $\begin{array}{l}0 \text { Sq. } \mathrm{Km} \\
0 \text { sUS Millions } \\
0 \text { sUS Millions }\end{array}$ & $\begin{array}{l}\text { CurrentTotNCG } \\
\text { CurrentCO2 } \\
\text { CurrentH2s }\end{array}$ & $\begin{array}{l}2 \text { Weight } \\
\text { ???? Weight } \\
\text { ???? Weight } \%\end{array}$ \\
\hline $\begin{array}{l}\text { Availability } \\
\text { Yr. of Avail }\end{array}$ & & NCGTrtmtSyst & EJ \\
\hline
\end{tabular}




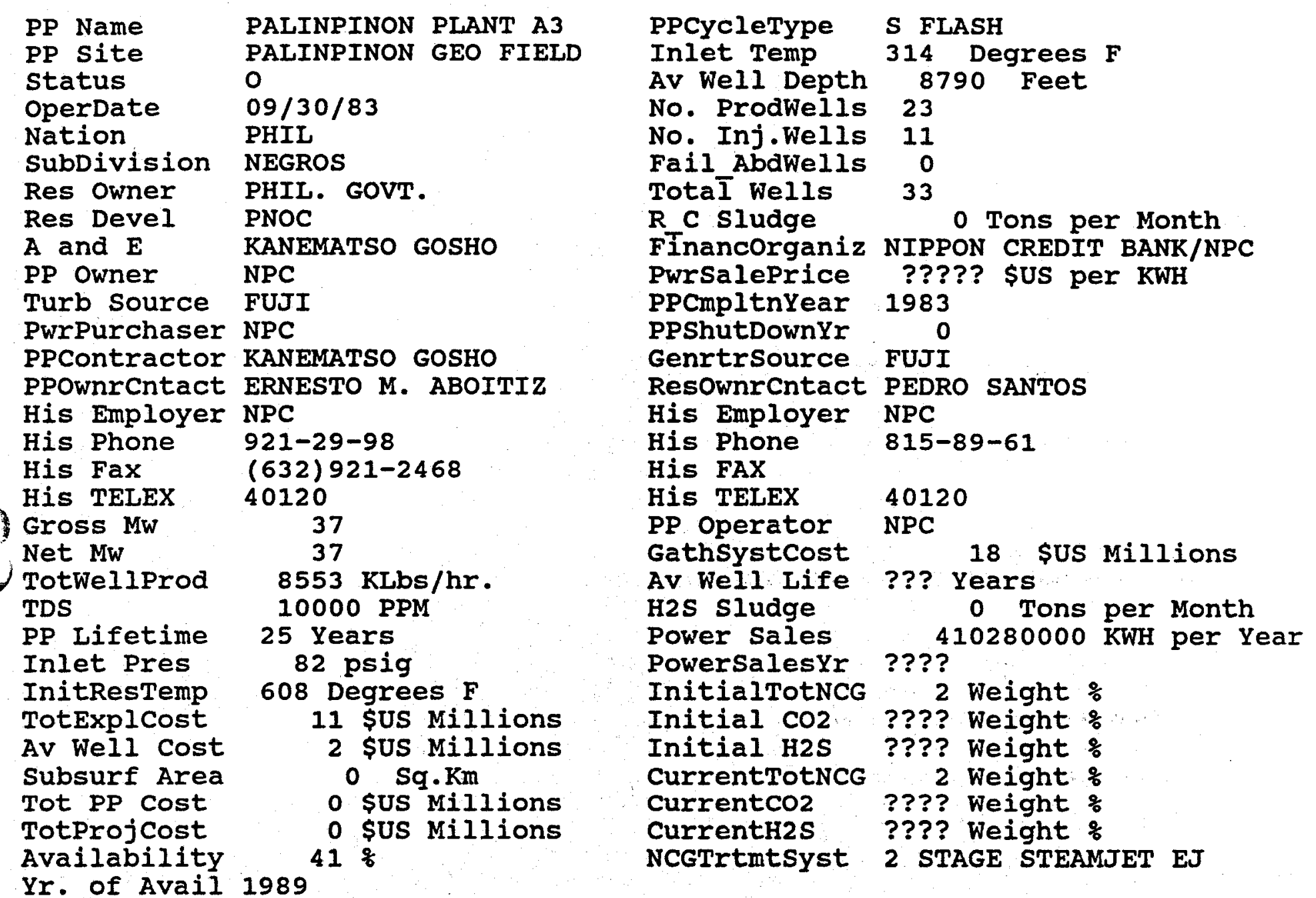




\begin{tabular}{|c|c|c|c|}
\hline PP Name & TIWI PLANT A UNIT 1 & PPCycleType & S FLASH \\
\hline PP site & TIWI GEO. FIELD & Inlet Temp & 324 Degrees $F$ \\
\hline Status & 0 & Av Well Depth & 4920 Feet \\
\hline $\begin{array}{l}\text { OperDate } \\
\text { Nation }\end{array}$ & $\begin{array}{l}01 / 30 / 79 \\
\text { PHIL }\end{array}$ & $\begin{array}{l}\text { No. ProdWells } \\
\text { No. Inj.Wells }\end{array}$ & $\begin{array}{r}19 \\
6\end{array}$ \\
\hline SubDivision & ALBAY & Fail_AbdWells & 20 \\
\hline Res Owner & PHIL. GOVT. & TotaI Wells & 69 \\
\hline $\begin{array}{l}\text { Res Devel } \\
A \text { and } E\end{array}$ & $\begin{array}{l}\text { NPC/PGI } \\
\text { MTTSUT }\end{array}$ & R C sluage & $\begin{array}{l}0 \text { Tons per Month } \\
\text { MTTSUT }\end{array}$ \\
\hline PP Owner & $\begin{array}{l}\text { MPSU1 } \\
\text { NPC }\end{array}$ & $\begin{array}{l}\text { Financorganiz } \\
\text { Pwrsaleprice }\end{array}$ & $\begin{array}{l}\text { MIISUI } \\
\text { ????? \$US per KWH }\end{array}$ \\
\hline Turb Source & TOSHIBA & PPCmpltnYear & 1979 \\
\hline PwrPurchaser & NPC & PPShutDownYr & 0 \\
\hline PPContractor & F.F.CRUZ & GenrtrSource & TOSHIBA \\
\hline PPOwnrcntact & ERNESTO M. ABOITIZ & ResownrCntact & ERNESTO M. ABOITIZ \\
\hline His Employer & NPC & His Employer & NPC \\
\hline His Phone & $921-29-98$ & His Phone & $921-29-98$ \\
\hline $\begin{array}{l}\text { His FaX } \\
\text { His TELEX }\end{array}$ & $\begin{array}{l}(632) 921-2468 \\
40120\end{array}$ & $\begin{array}{l}\text { His FAX } \\
\text { His TELEX }\end{array}$ & $\begin{array}{l}(632) 921-2468 \\
40120\end{array}$ \\
\hline Gross $\mathrm{MW}$ & 55 & PP operator & NPC \\
\hline $\begin{array}{l}\text { Net } M W \\
\text { Totwellprod }\end{array}$ & $\begin{array}{r}55 \\
2577\end{array}$ & Gathsystcost & ?2? Years 18 SUS Millions \\
\hline $\begin{array}{l}\text { TotwellProd } \\
\text { TDS }\end{array}$ & $\begin{array}{l}2677 \mathrm{KLbs} / \mathrm{hr} . \\
9000 \mathrm{PPM}\end{array}$ & $\begin{array}{l}\text { Av well Lite } \\
\text { H2S sludge }\end{array}$ & $\begin{array}{c}? \text { Years } \\
0 \text { Tons per Month }\end{array}$ \\
\hline $\begin{array}{l}\text { PP Lifetime } \\
\text { Inlet Pres }\end{array}$ & $\begin{array}{l}25 \text { Years } \\
90 \text { psig }\end{array}$ & $\begin{array}{l}\text { Power Sales } \\
\text { PowersalesYr }\end{array}$ & $\begin{array}{l}380900000 \mathrm{KWH} \text { per Year } \\
\text { ???? }\end{array}$ \\
\hline $\begin{array}{l}\text { InitResTemp } \\
\text { TotExplCost } \\
\text { AV Wel Cost }\end{array}$ & $\begin{array}{l}500 \text { Degrees } F \\
13 \text { SUS Millions }\end{array}$ & $\begin{array}{l}\text { InitialTotNCG } \\
\text { Initial } \mathrm{CO} 2\end{array}$ & $\begin{array}{l}3 \text { Weight \% } \\
\text { ???? Weight \% } \\
\text { j?j? }\end{array}$ \\
\hline $\begin{array}{l}\text { Av Well cost } \\
\text { Subsurf Area } \\
\text { Tot PP Cost } \\
\text { TotProjcost }\end{array}$ & $\begin{array}{l}2 \text { \$US Millions } \\
16 \text { Sq.Km } \\
97 \text { \$US Millions } \\
0 \text { \$US Millions }\end{array}$ & $\begin{array}{l}\text { Initial H2S } \\
\text { CurrentTotNCG } \\
\text { CurrentCO2 } \\
\text { CurrentH2S }\end{array}$ & $\begin{array}{r}\text { ???? Weight } \% \\
\text { 2 Weight } \% \\
\text { ???? Weight } \% \\
\text { ???? Weight } \%\end{array}$ \\
\hline ailability & $79 \%$ & GTrtmtsyst & 2 STAGE STEAMJET EJ \\
\hline
\end{tabular}




\begin{tabular}{|c|c|c|c|}
\hline & $\begin{array}{l}\text { TIWI PLANT A UNIT } 2 \\
\text { TIWI GEO FIELD } \\
\text { O } \\
\text { O5/30/79 } \\
\text { PHIL } \\
\text { ALBAY } \\
\text { PHIL. GOVT. } \\
\text { NPC/PGI } \\
\text { MITSUI } \\
\text { NPC } \\
\text { TOSHIBA } \\
\text { NPC } \\
\text { F.F. CRUZ } \\
\text { ERNESTO ABOITIZ } \\
\text { NPC } \\
921-29-98 \\
(632) 921-2468 \\
40120 \\
55 \\
55 \\
0 \text { KLbS/hr. } \\
9000 \text { PPM } \\
25 \text { Years } \\
90 \text { psig } \\
500 \text { Degrees F } \\
13 \text { SUS Millions } \\
2 \text { \$US Millions } \\
16 \text { Sq.Km } \\
97 \text { SUS Millions } \\
0 \text { \$US Millions } \\
69 \% \\
1989\end{array}$ & $\begin{array}{l}\text { PPCycleType } \\
\text { Inlet Temp } \\
\text { Av Well Depth } \\
\text { No. ProdWells } \\
\text { No. Inj. Wells } \\
\text { Fail AbdWells } \\
\text { Total Wells } \\
\text { RC Sludge } \\
\text { Financorganiz } \\
\text { PwrSalePrice } \\
\text { PPCmpltnYear } \\
\text { PPShutDownYr } \\
\text { GenrtrSource } \\
\text { ResOwnrCntact } \\
\text { His Employer } \\
\text { His Phone } \\
\text { His FAX } \\
\text { His TELEX } \\
\text { PP Operator } \\
\text { GathSystCost } \\
\text { Av Well Life } \\
\text { H2S Sludge } \\
\text { Power Sales } \\
\text { PowerSalesYr } \\
\text { InitialTotNCG } \\
\text { Initial CO2 } \\
\text { Initial H2S } \\
\text { CurrentTotNCG } \\
\text { CurrentCO2 } \\
\text { CurrentH2S } \\
\text { NCGTrtmtSyst }\end{array}$ & 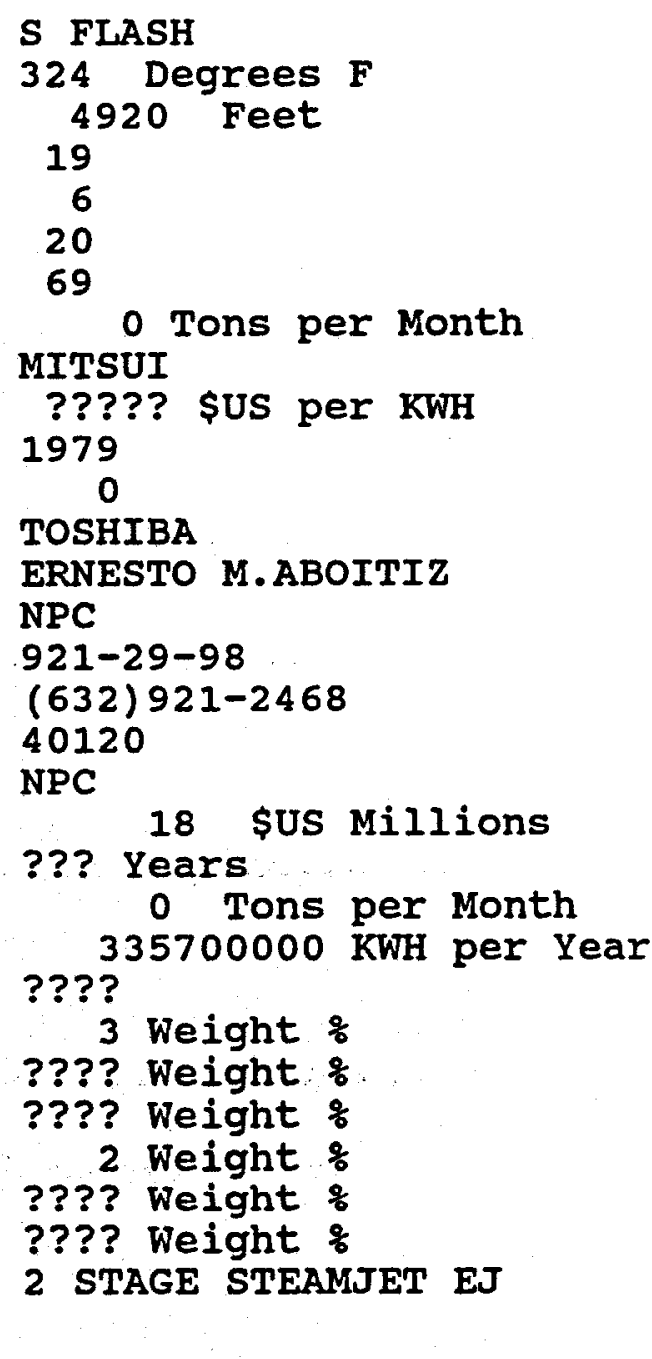 \\
\hline
\end{tabular}




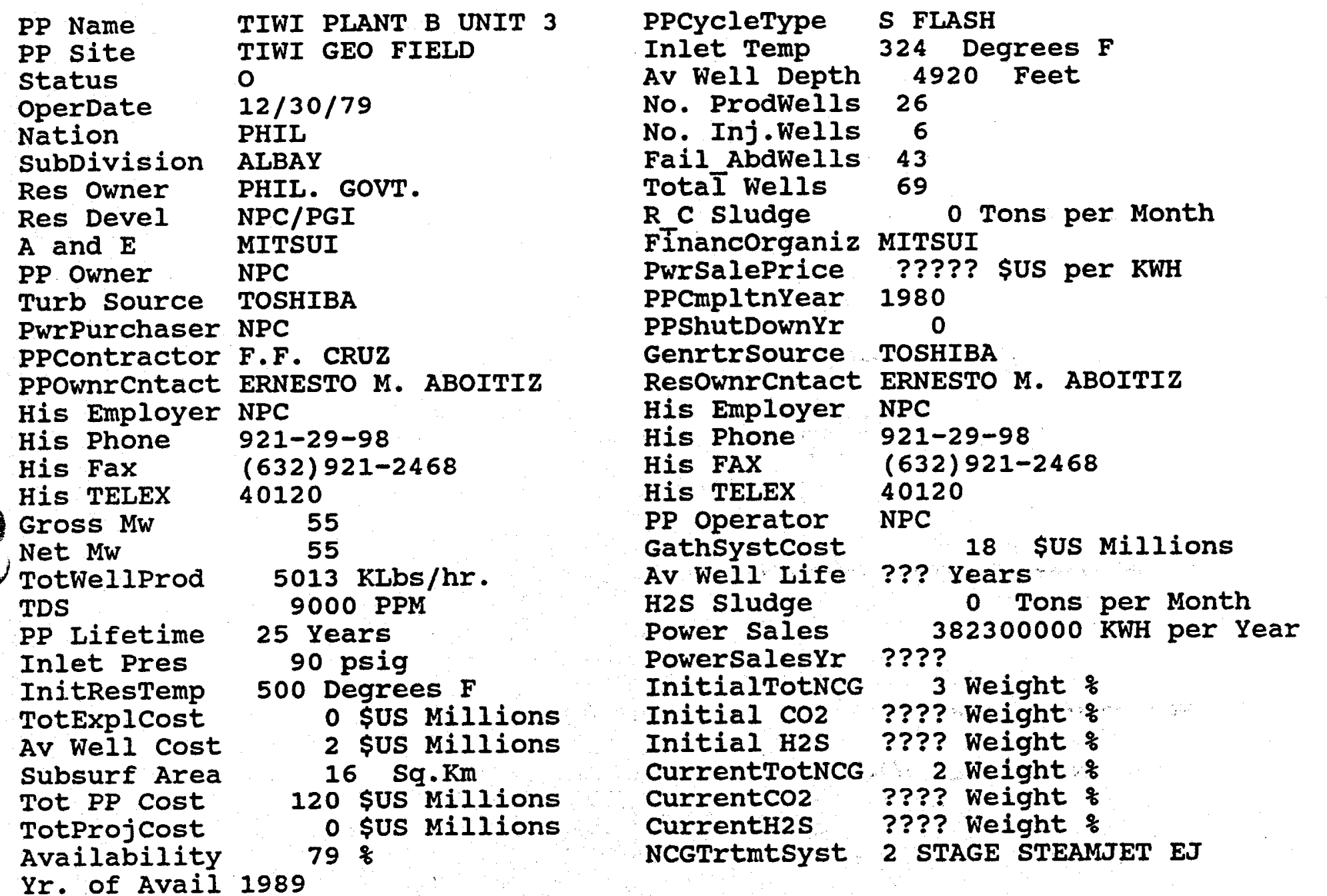




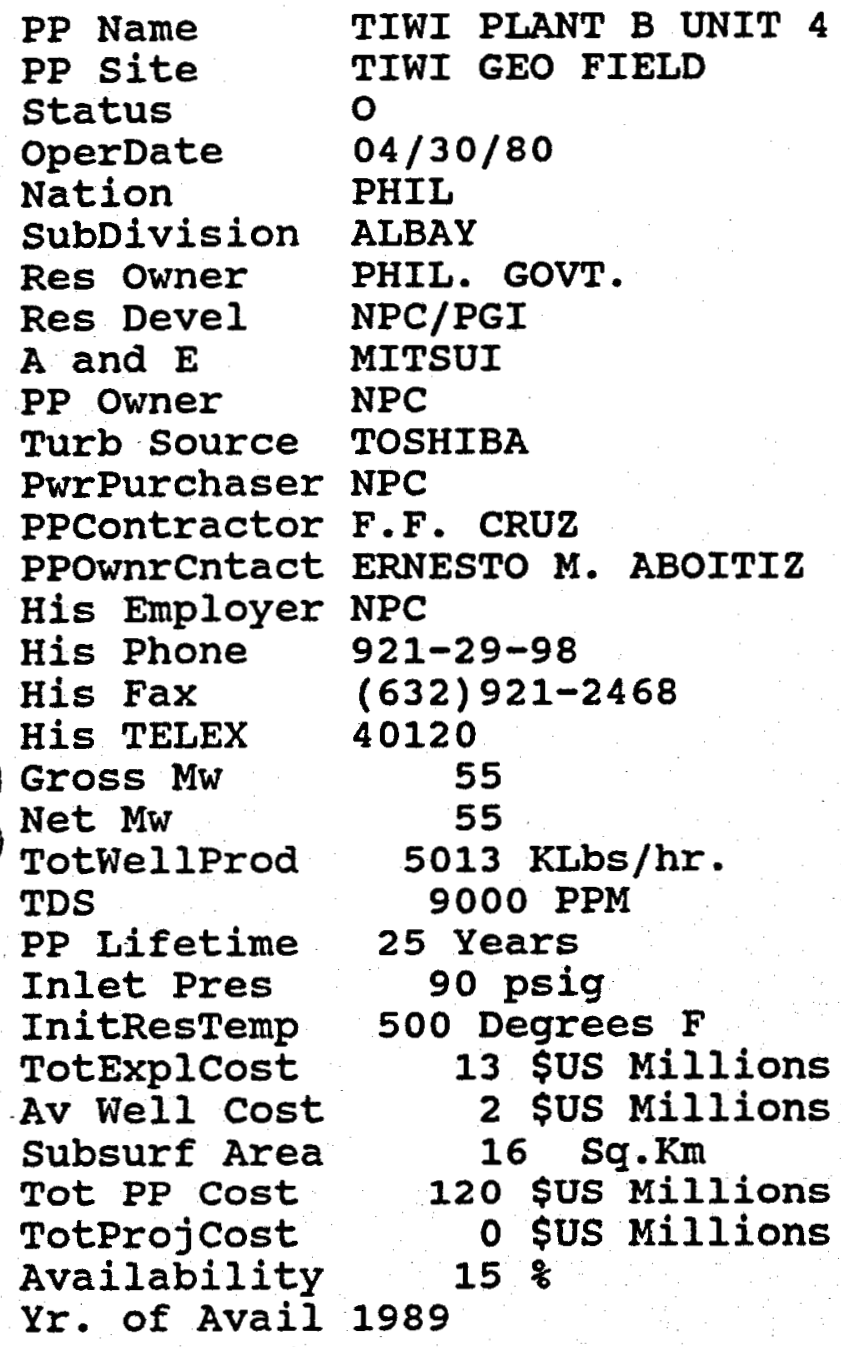

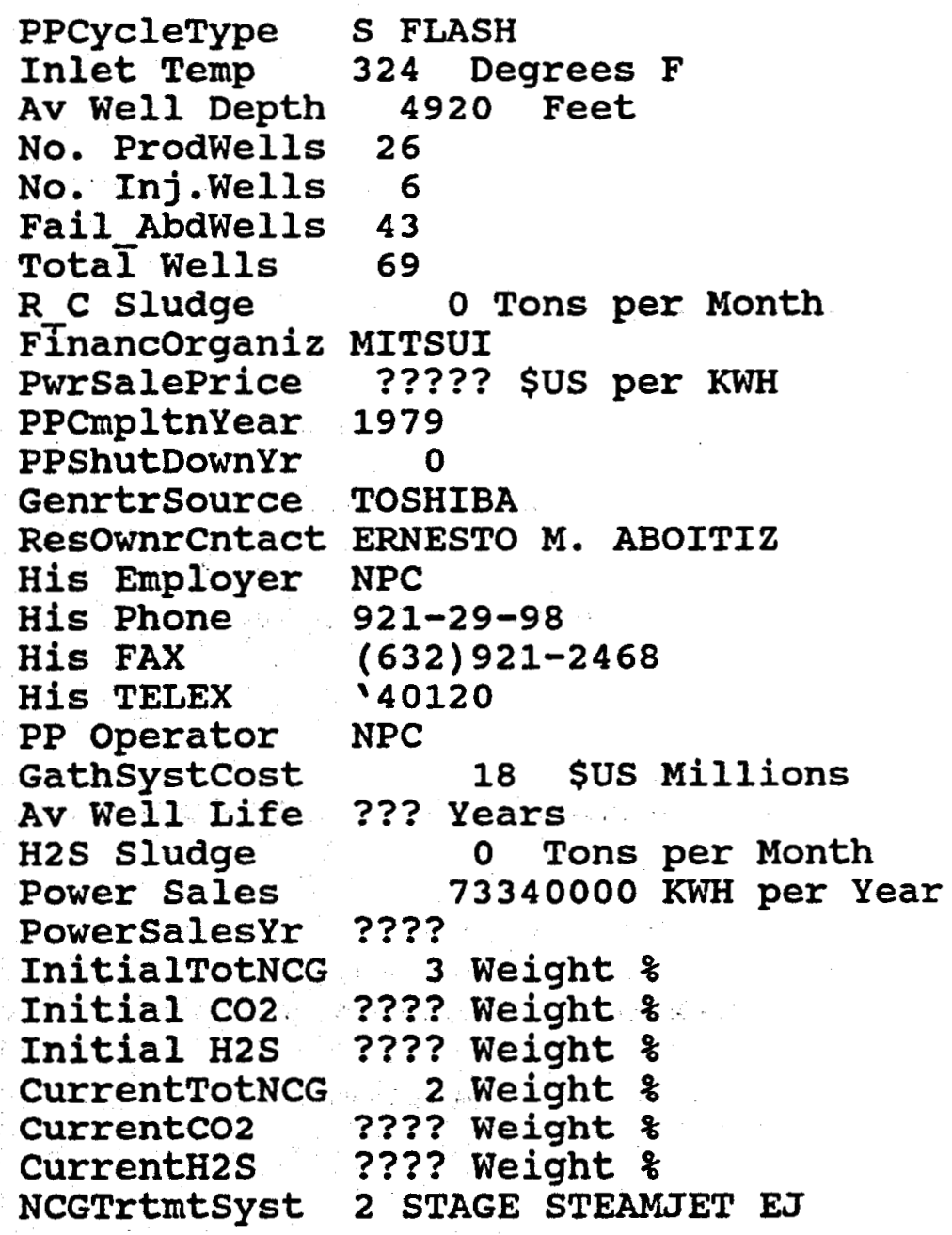




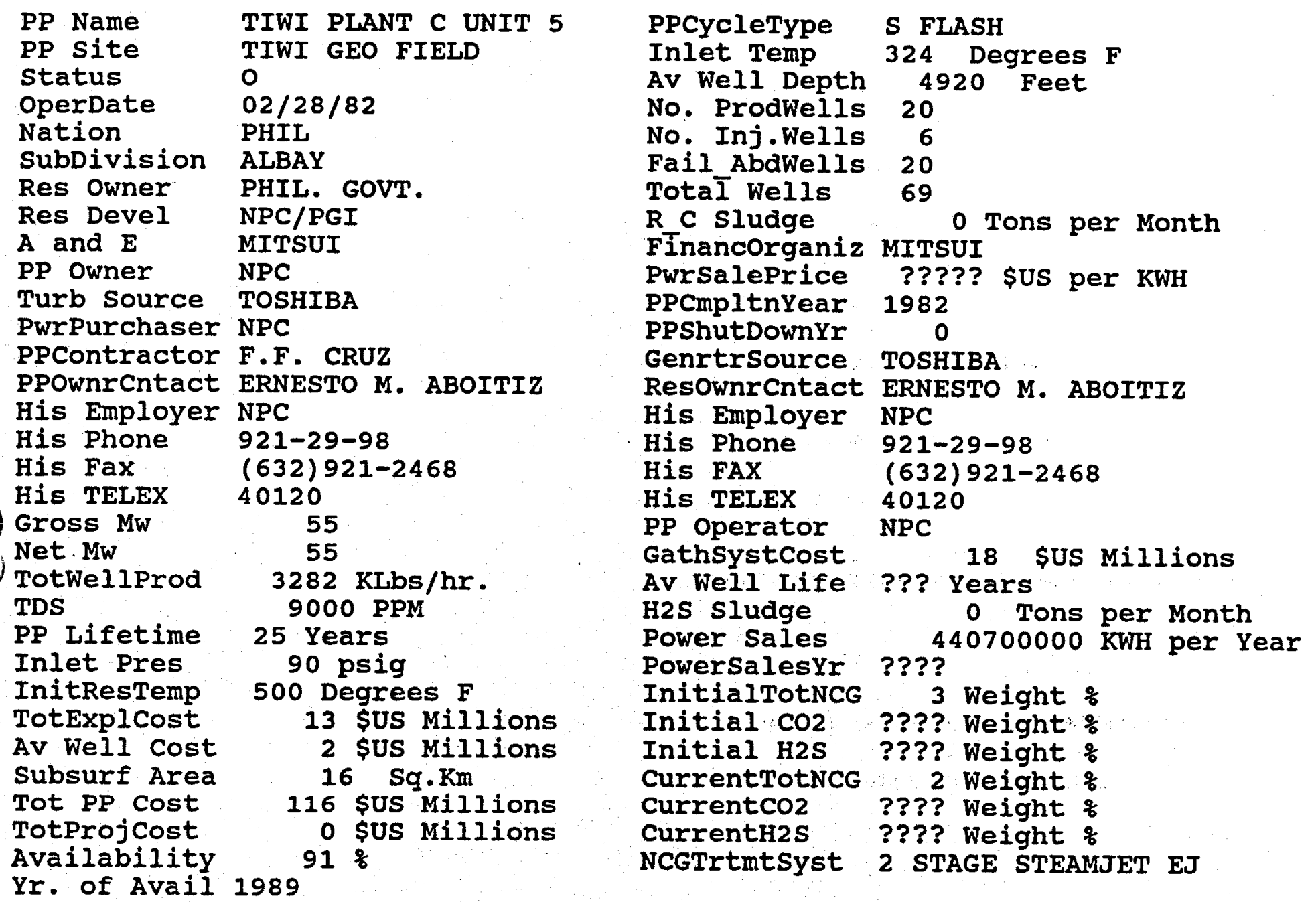




\begin{tabular}{|c|c|c|c|}
\hline PP Name & TIWI PLANT C UNIT 6 & PPCycleType & S FLASH \\
\hline PP site & TIWI GEO FIELD & Inlet Temp & 324 Degrees $F$ \\
\hline status & 0 & Av Well Depth & 4920 Feet \\
\hline $\begin{array}{l}\text { OperDace } \\
\text { Nation }\end{array}$ & PHIL & No. Inj.Wells & $\begin{array}{r}20 \\
6\end{array}$ \\
\hline ubDivision & ALBAY & Fail_AbdWells & 20 \\
\hline es owner & PHIL. GOVT & TotaI Wells & 69 \\
\hline $\begin{array}{l}\text { Res Devel } \\
A \text { and } E\end{array}$ & $\begin{array}{l}\text { NPC/PGI } \\
\text { MITSUI }\end{array}$ & $\begin{array}{l}\mathrm{R} \text { C Sludge } \\
\text { Fínancorganiz }\end{array}$ & $\begin{array}{l}0 \text { Tons per Month } \\
\text { MITSUI }\end{array}$ \\
\hline PP Owner & NPC & Pwrsaleprice & ????? \$US per KWH \\
\hline Turb source & TOSHIBA & PPCmpltnYear & 1982 \\
\hline PwrPurchaser & NPC & PPShutDownYr & 0 \\
\hline $\begin{array}{l}\text { PPContractor } \\
\text { PPOwnrcntact }\end{array}$ & $\begin{array}{l}\text { F.F. CRUZ } \\
\text { ERNESTO M. ABOITIZ }\end{array}$ & $\begin{array}{l}\text { Genrtrsource } \\
\text { ResownrCntact }\end{array}$ & $\begin{array}{l}\text { TOSHIBA } \\
\text { ERNESTO M. ABOITIZ }\end{array}$ \\
\hline $\begin{array}{l}\text { His Employer } \\
\text { His Phone } \\
\text { His Fax } \\
\text { His TELEX }\end{array}$ & $\begin{array}{l}\text { NPC } \\
921-29-98 \\
(632) 921-2468 \\
40120\end{array}$ & $\begin{array}{l}\text { His Employer } \\
\text { His Phone } \\
\text { His FAX } \\
\text { His TELEX }\end{array}$ & $\begin{array}{l}\text { NPC } \\
921-29-98 \\
(632) 921-2468 \\
40120\end{array}$ \\
\hline $\begin{array}{l}\text { Gross Mw } \\
\text { Net Mw }\end{array}$ & $\begin{array}{l}55 \\
55\end{array}$ & $\begin{array}{l}\text { PP Operator } \\
\text { Gathsystcost }\end{array}$ & US Millions \\
\hline $\begin{array}{l}\text { TotWellProd } \\
\text { TDS }\end{array}$ & $\begin{array}{l}3282 \mathrm{KLbs} / \mathrm{hr} \\
9000 \mathrm{PPM}\end{array}$ & $\begin{array}{l}\text { Av Well Life } \\
\text { H2S sludge }\end{array}$ & $\begin{array}{l}\text { ??? Years } \\
0 \text { Tons per Month }\end{array}$ \\
\hline $\begin{array}{l}\text { PP Lifetime } \\
\text { Inlet Pres }\end{array}$ & $\begin{array}{l}25 \text { Years } \\
90 \text { psig }\end{array}$ & $\begin{array}{l}\text { Power sales } \\
\text { PowersalesYr }\end{array}$ & $\begin{array}{l}391200000 \mathrm{KWH} \text { per Year } \\
\text { ???? }\end{array}$ \\
\hline $\begin{array}{l}\text { InitResTemp } \\
\text { TotExplCost } \\
\text { Av Well Cost }\end{array}$ & $\begin{array}{l}500 \text { Degrees F } \\
13 \text { SUS Militions } \\
2 \text { SUS Militons }\end{array}$ & $\begin{array}{l}\text { InitialTotNCG } \\
\text { Initial } \mathrm{CO} 2 \\
\text { Initial H2S }\end{array}$ & $\begin{array}{r}3 \text { Weight \% } \\
\text { ???? Weight \% } \\
\text { ???? Weight \% }\end{array}$ \\
\hline $\begin{array}{l}\text { Subsurf Area } \\
\text { Tot PP Cost } \\
\text { TotProjcost }\end{array}$ & $\begin{array}{l}16 \text { Sq.Km } \\
116 \text { SUS Millions } \\
0 \text { \$US Millions }\end{array}$ & $\begin{array}{l}\text { CurrentTotNCG } \\
\text { CurrentCO2 } \\
\text { CurrentH2S }\end{array}$ & $\begin{array}{l}2 \text { Weight } \% \\
\text { ???? Weight } \% \\
\text { ???? Weight } \%\end{array}$ \\
\hline $\begin{array}{l}\text { vailability } \\
\text { r. of Avail }\end{array}$ & $989^{81 \%}$ & NCGTrtmtSyst & 2 STAGE STEAM \\
\hline
\end{tabular}




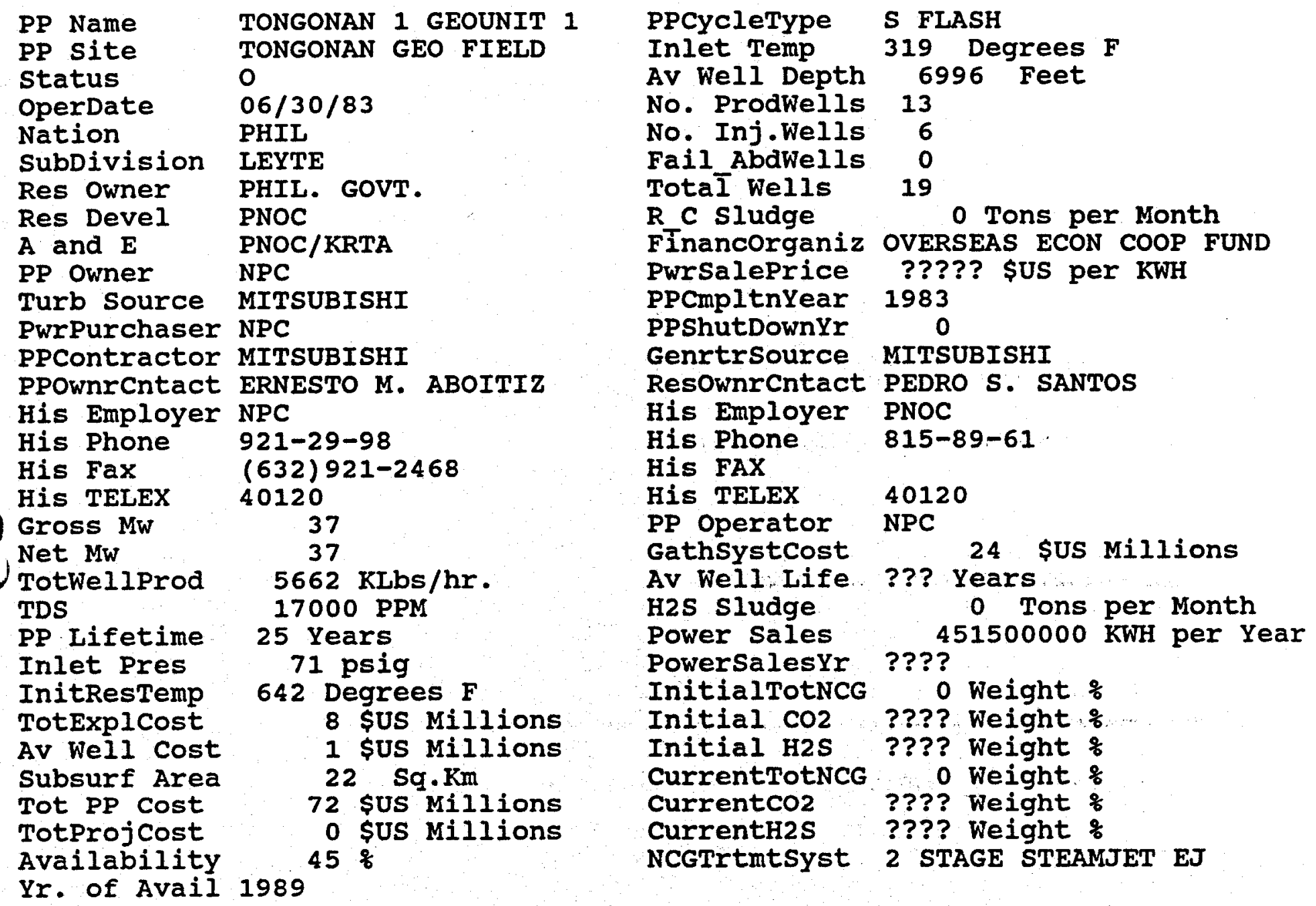




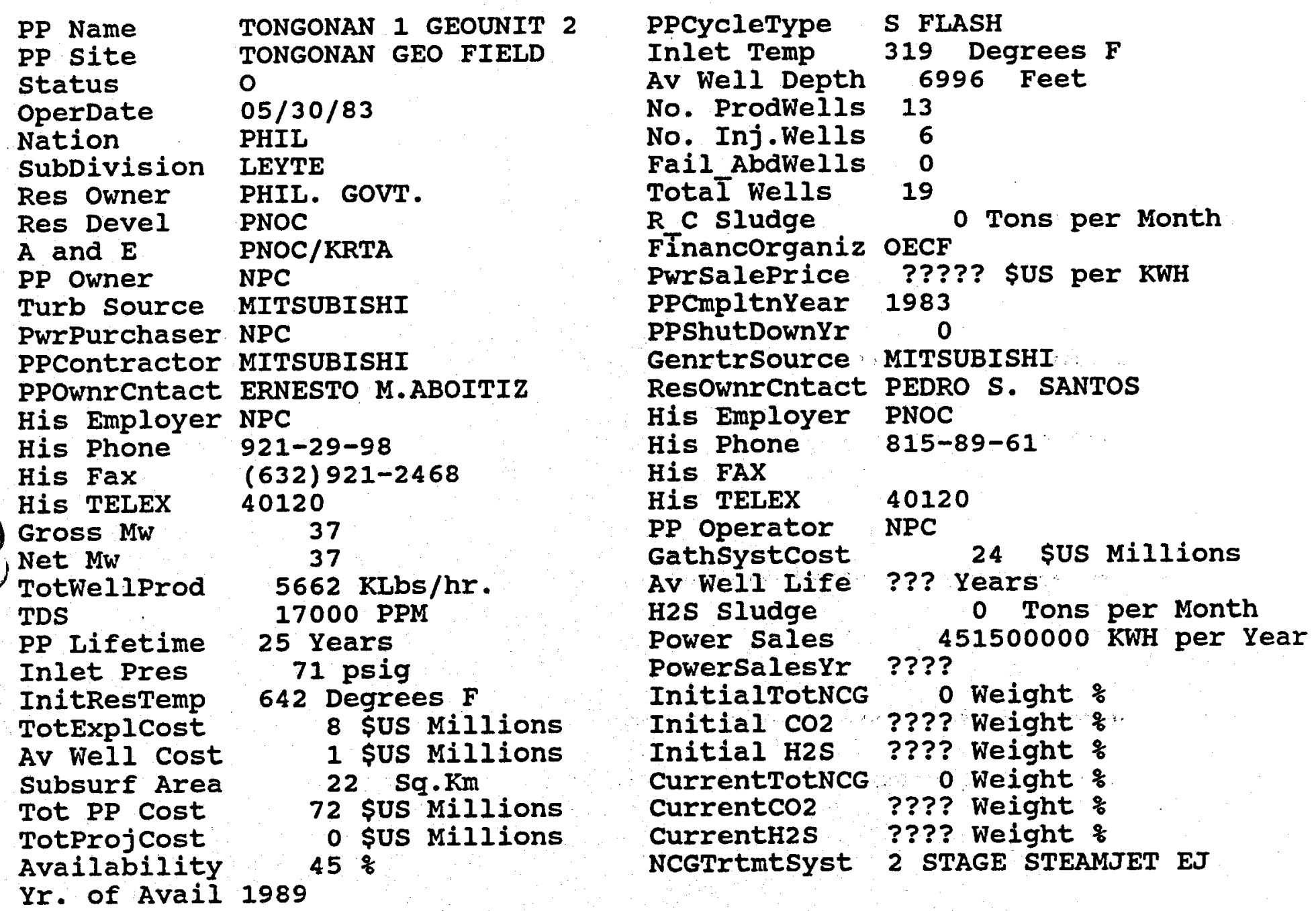




\begin{tabular}{|c|c|c|c|}
\hline $\begin{array}{l}\text { PP Name } \\
\text { PP Site }\end{array}$ & $\begin{array}{l}\text { TONGONAN } 1 \text { GEOUNIT } 3 \\
\text { TONGONAN GEO FIELD }\end{array}$ & $\begin{array}{l}\text { PPCycletype } \\
\text { Inlet Temp }\end{array}$ & $\begin{array}{l}\text { S FLASH } \\
319 \text { Deqrees F }\end{array}$ \\
\hline $\begin{array}{l}\text { PP site } \\
\text { status }\end{array}$ & $\begin{array}{l}\text { TONGONAN GEO FIELD } \\
\text { O }\end{array}$ & $\begin{array}{l}\text { Inlet Temp } \\
\text { Av Well Depth }\end{array}$ & $\begin{array}{c}319 \text { Degrees F } \\
6996 \text { Feet }\end{array}$ \\
\hline OperDate & $03 / 30 / 83$ & No. ProdWelis & 13 \\
\hline Nation & PHIL & No. Inj.Wells & 6 \\
\hline SubDivision & LEYTE & Fail AbdWells & 0 \\
\hline Res Owner & PHIL. GOVT. & TotaI Wells & 19 \\
\hline Res Devel & PNOC & R_C sludge & 0 Tons per Month \\
\hline$A$ and $E$ & PNOC/KRTA & Fínancorganiz & OECF \\
\hline PP Owner & NPC & Pwrsaleprice & ????? \$US per KWH \\
\hline Turb Source & MITSUBISHI & PPCmpltnYear & 1983 \\
\hline PwrPurchaser & NPC & PPShutDownYr & 0 \\
\hline PPContractor & MITSUBISHI & GenrtrSource & MITSUBISHI \\
\hline PPOwnrCntact & ERNESTO M. ABOITIZ & ResownrCntact & PEDRO S. SANTOS \\
\hline His Employer & NPC & His Employer & PNOC \\
\hline His Phone & $921-29-98$ & His Phone & $815-89-61$ \\
\hline His Fax & $(632) 921-2468$ & His FAX & \\
\hline HiS TELEX & 40120 & HiS TELEX & 40120 \\
\hline Gross Mw & 37 & PP operator & NPC \\
\hline Net Mw & 37 & Gathsystcost & sus Millions \\
\hline TotWellProd & $5662 \mathrm{KLbs} / \mathrm{hr}$. & Av Weill Life & ??? Years \\
\hline $\begin{array}{l}\text { TDS } \\
\text { PP Lifetime }\end{array}$ & $\begin{array}{l}17000 \mathrm{PF} \\
25 \text { Years }\end{array}$ & $\begin{array}{l}\text { H2s sludge } \\
\text { Power Sales }\end{array}$ & $\begin{array}{c}0 \text { Tons per Month } \\
451500000 \text { KWH per Year }\end{array}$ \\
\hline Inlet Pres & 71 psig & PowersalesYr & ???? \\
\hline InitResTemp & 642 Degrees F & InitialTotNCG & 0 Weight $\%$ \\
\hline $\begin{array}{l}\text { TotExplcost } \\
\text { Av Well Cost }\end{array}$ & 8 \$US Millions & Initial $\mathrm{CO} 2$ & ???? Weight \% \\
\hline Subsurf Area & $22 \mathrm{Sg} \cdot \mathrm{Km}$ & $\begin{array}{l}\text { Initial Has } \\
\text { CurrentTotNCG }\end{array}$ & $\begin{array}{r}\text { ??? Weight \% } \\
0 \text { Weight \% }\end{array}$ \\
\hline PP Cost & 72 sus Millions & Currentco2 & ???? Weight : \\
\hline & 0 \$US Milli & CurrentH2s & ???? Weight \\
\hline of Ava & & & \\
\hline
\end{tabular}


PORTUGAUL 


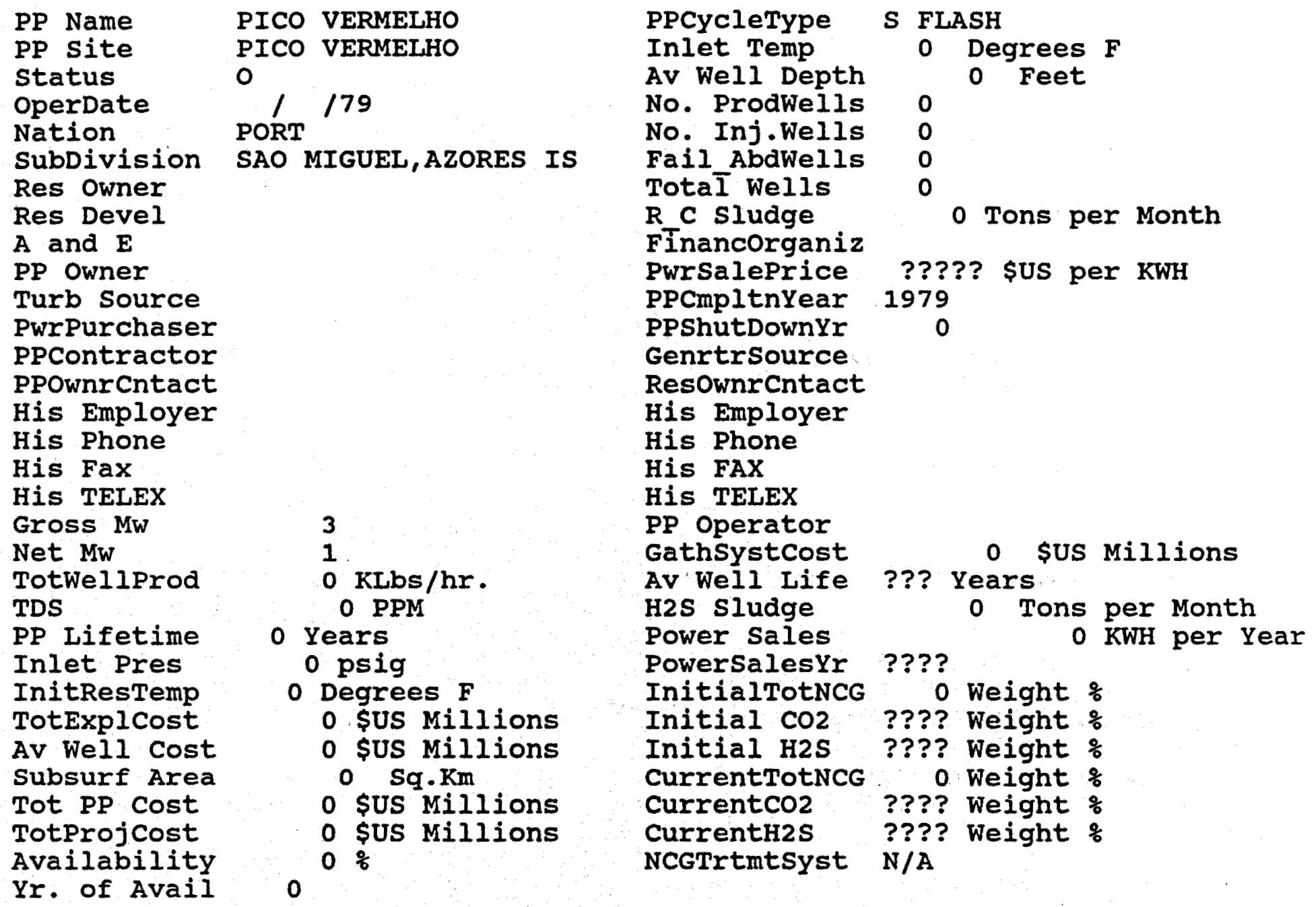


REPUBLIC OF CHINA 


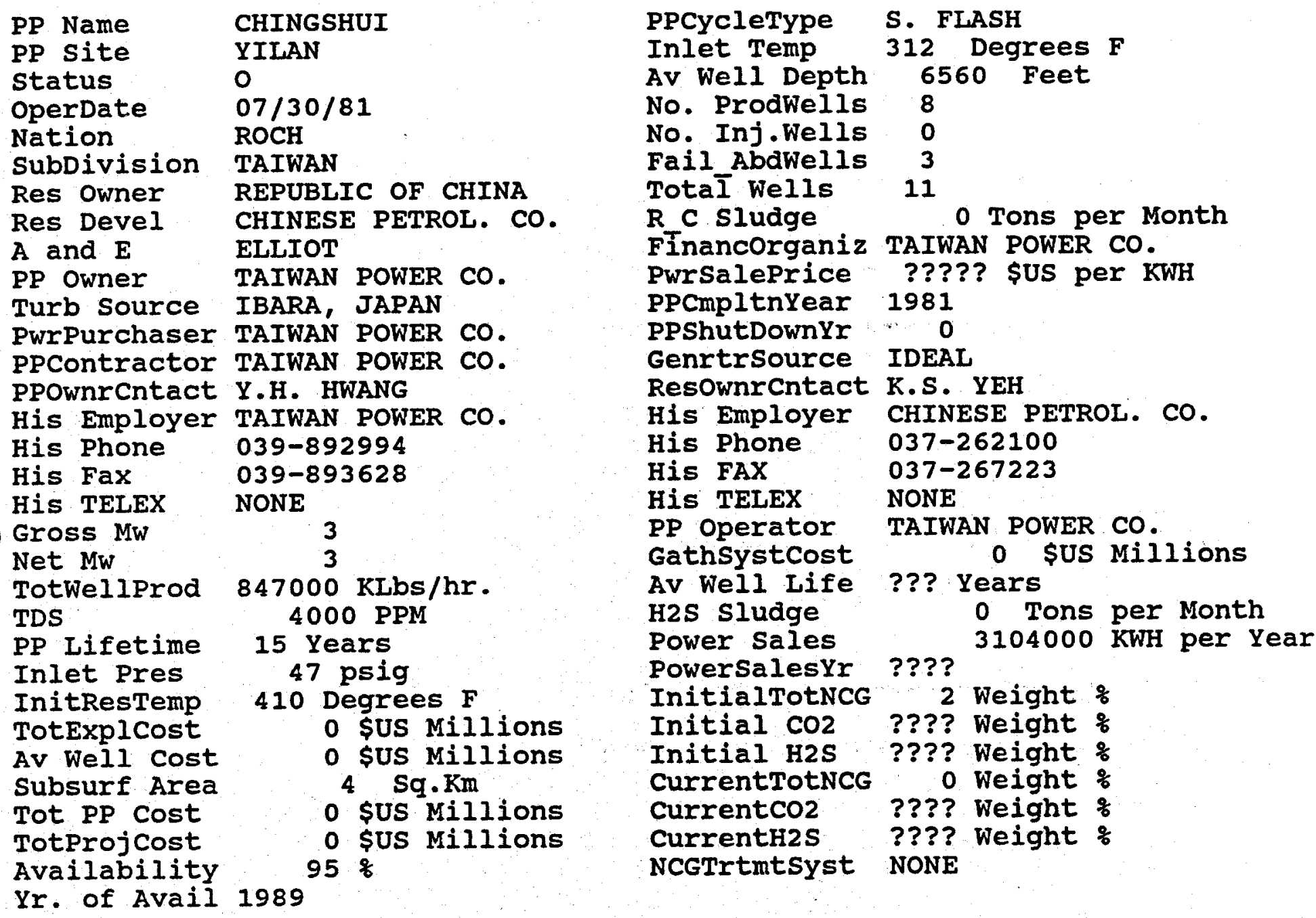




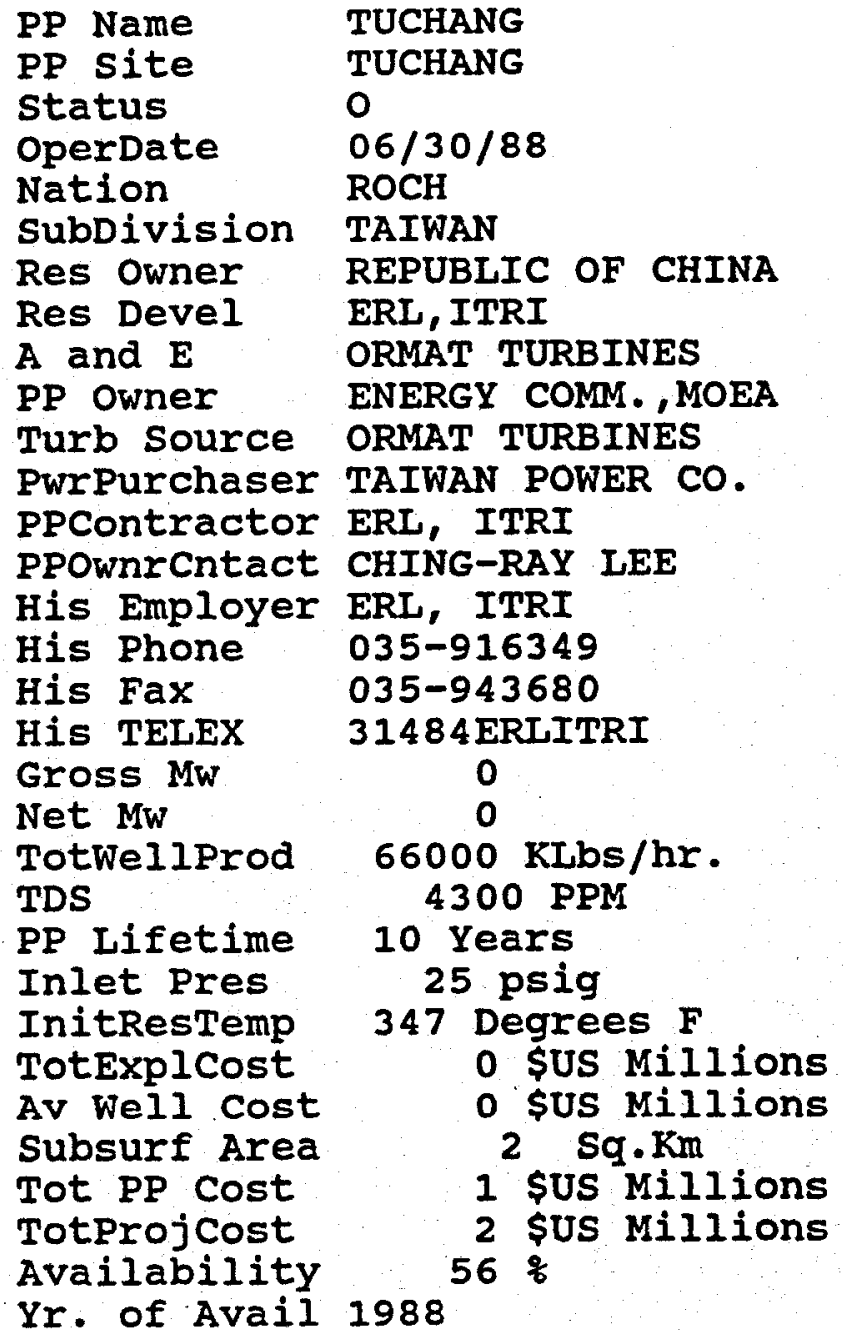

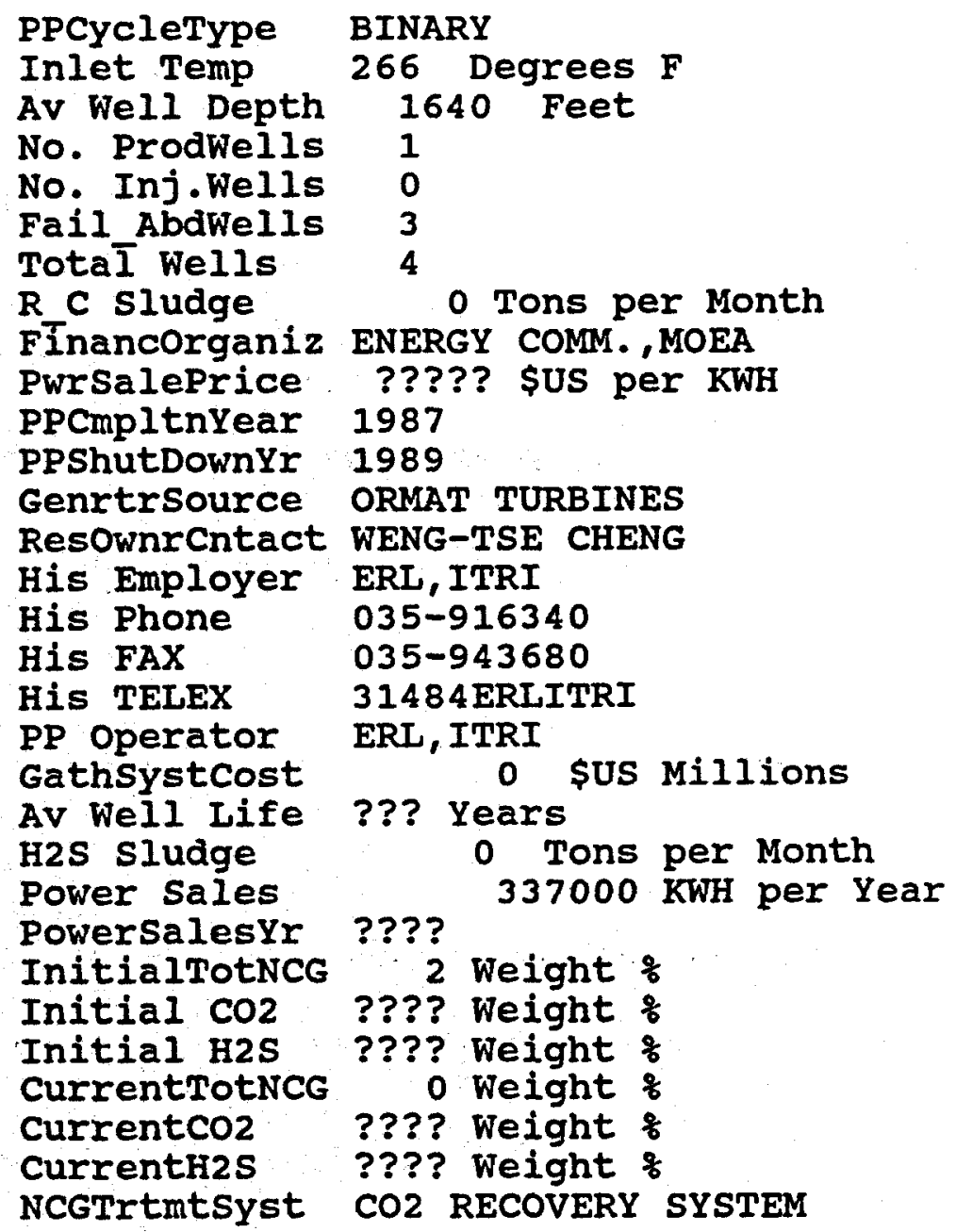


THAILAND 


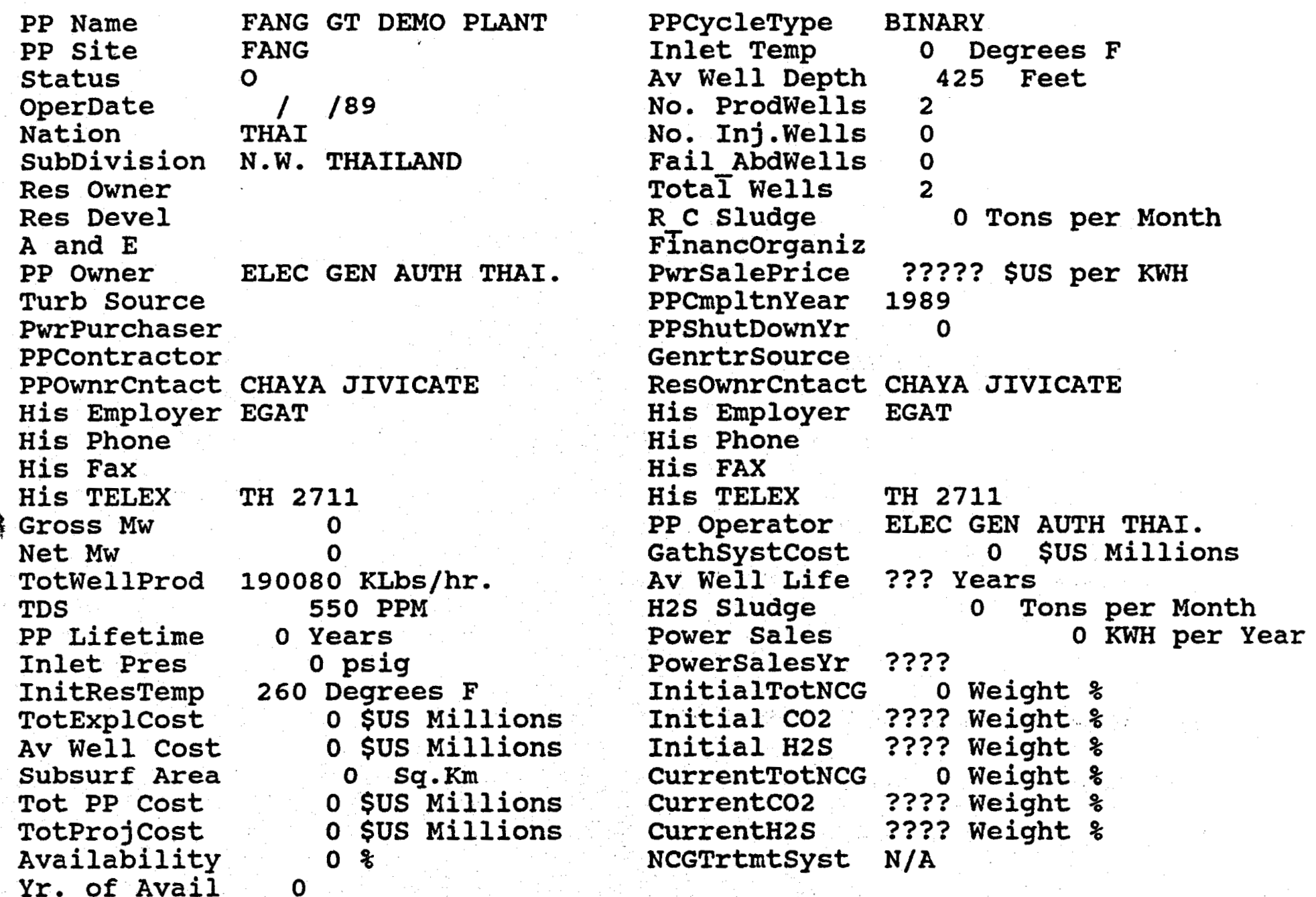




\section{TURKEY}




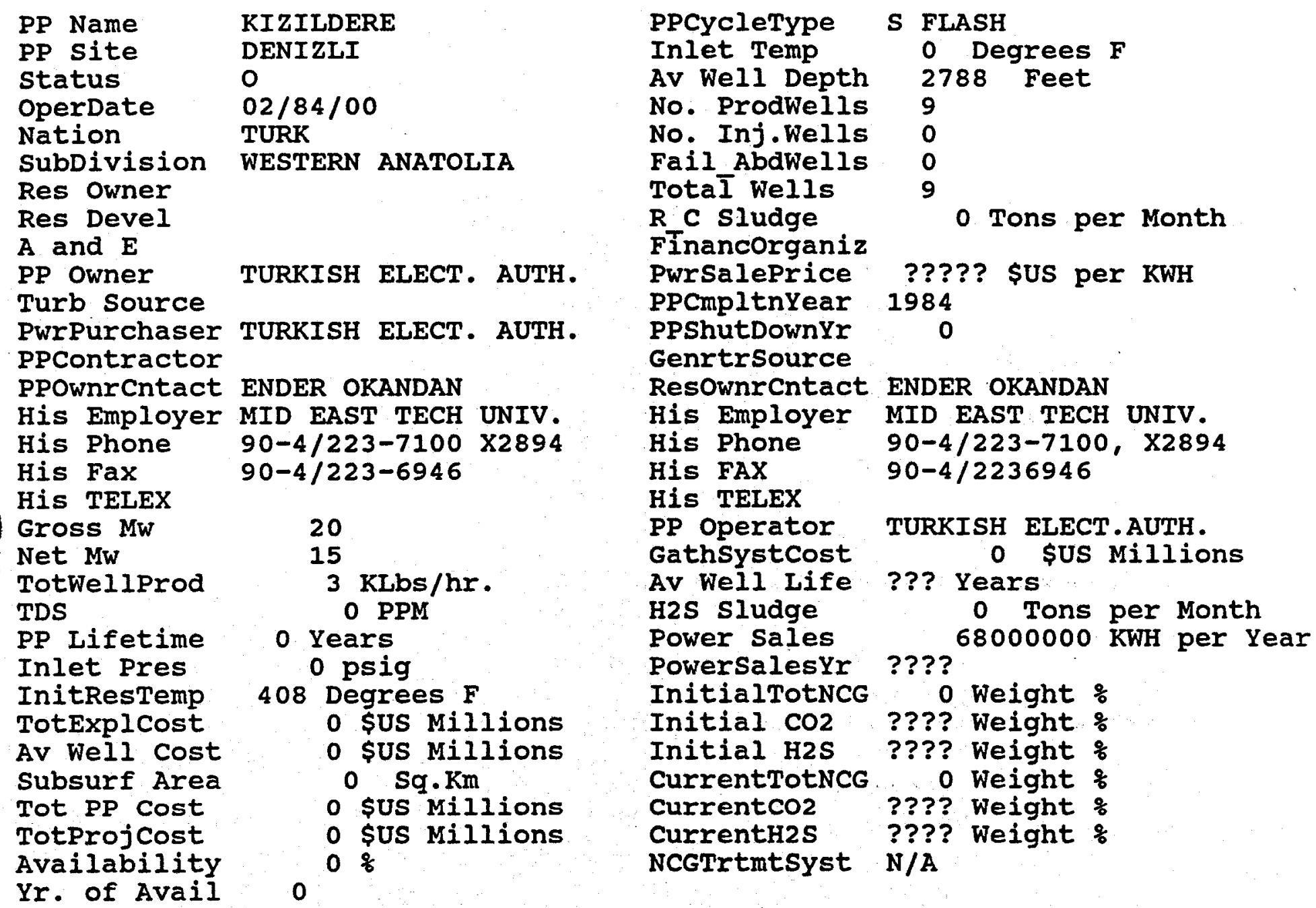


USSR 


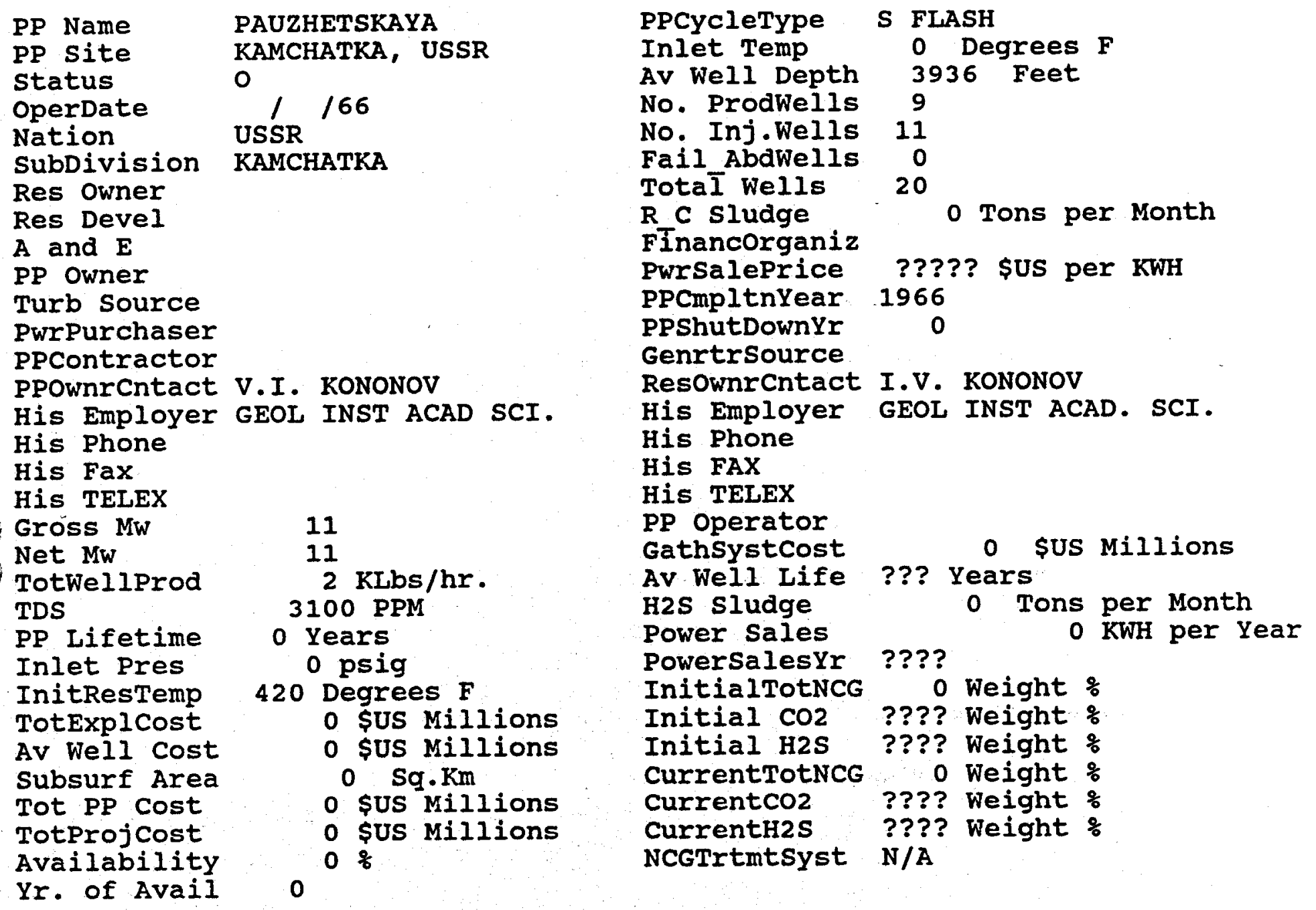


USA 


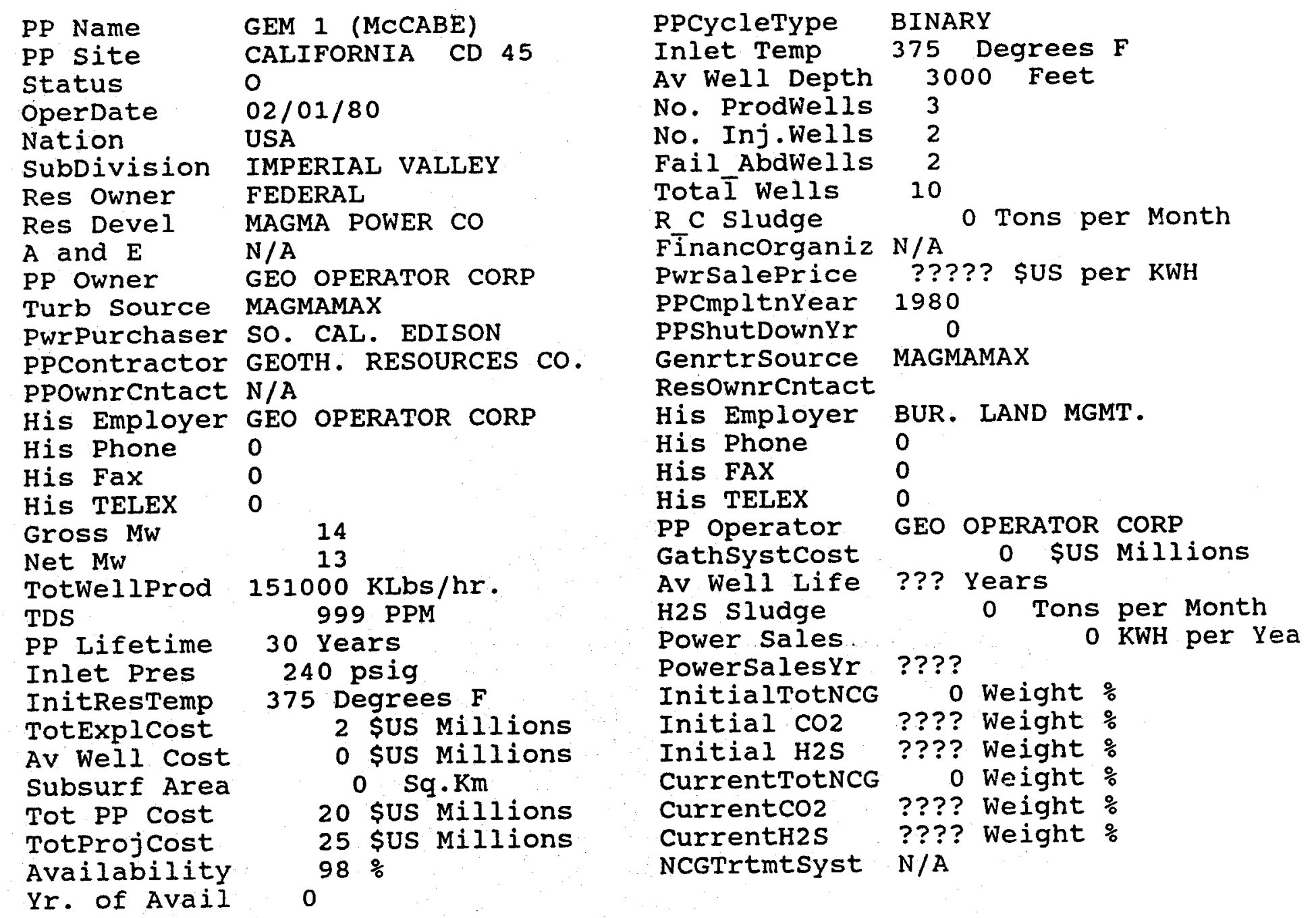




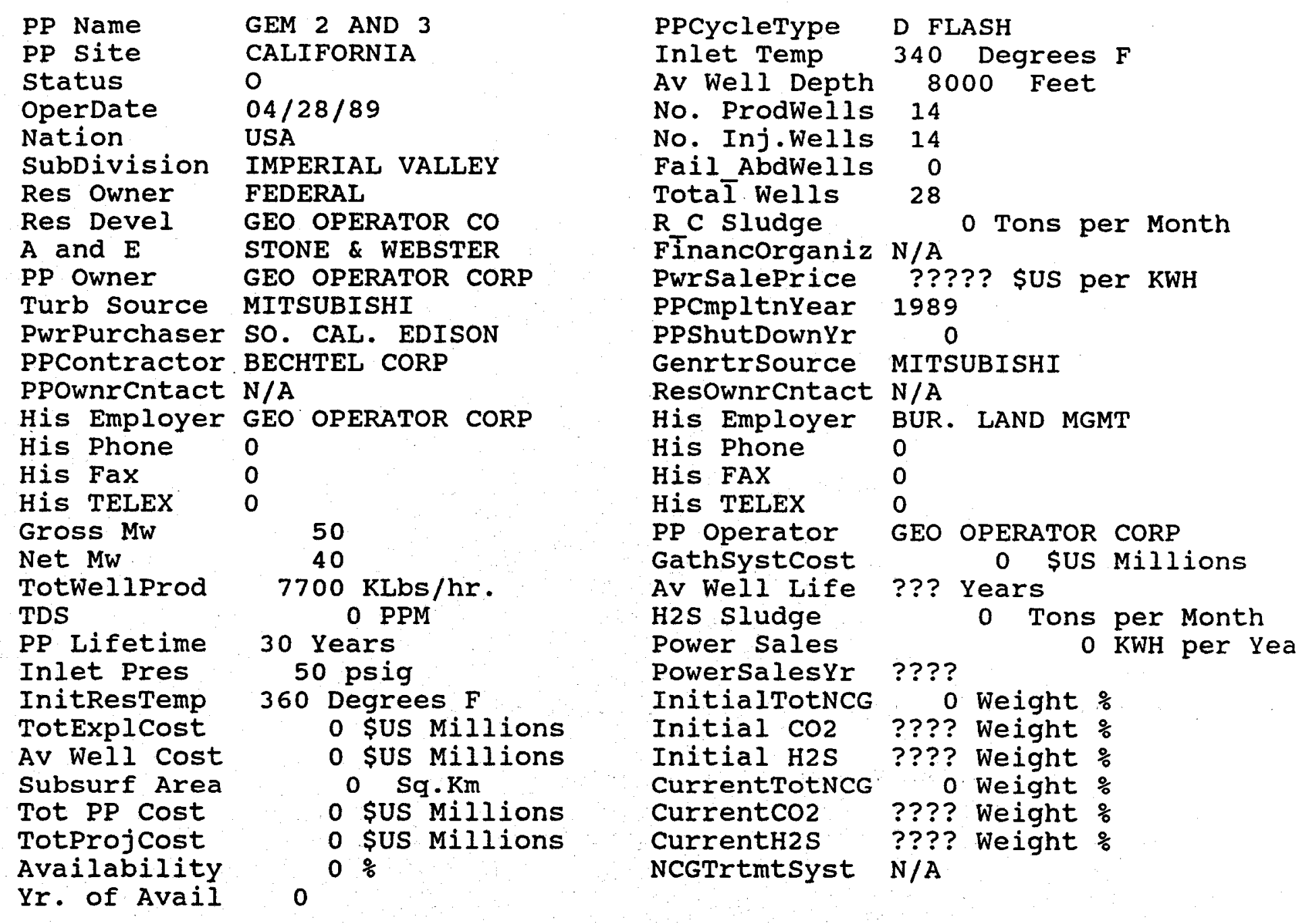




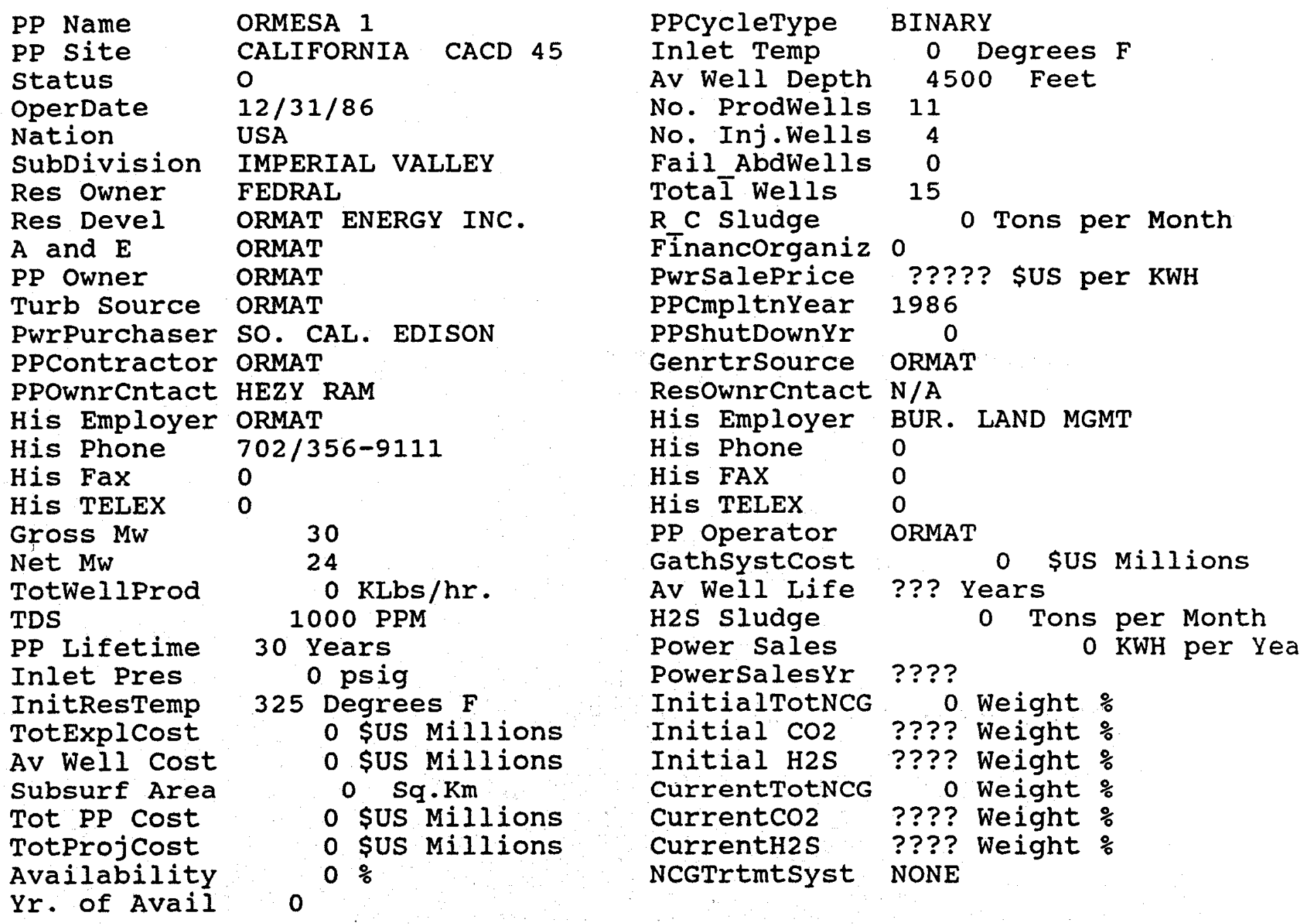




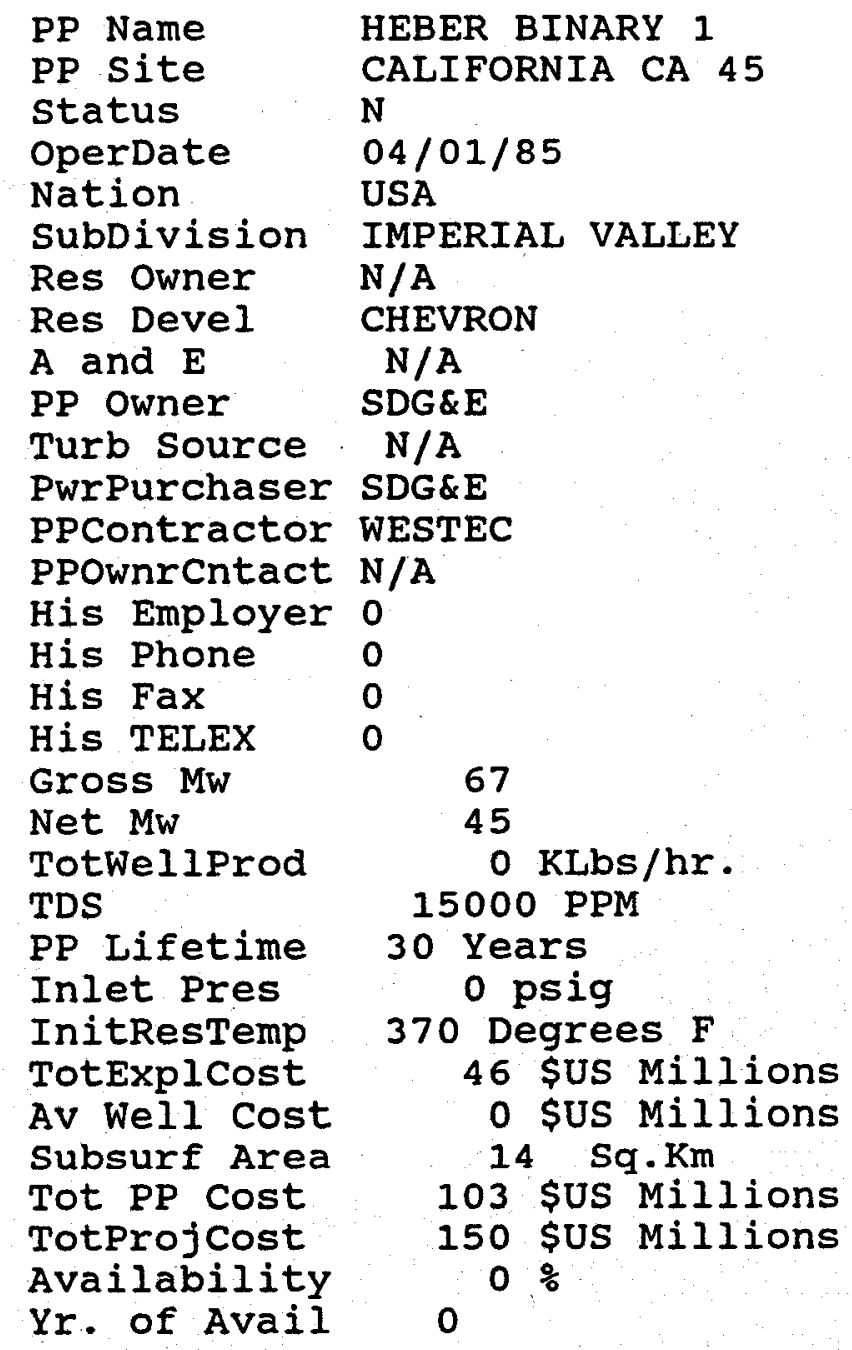

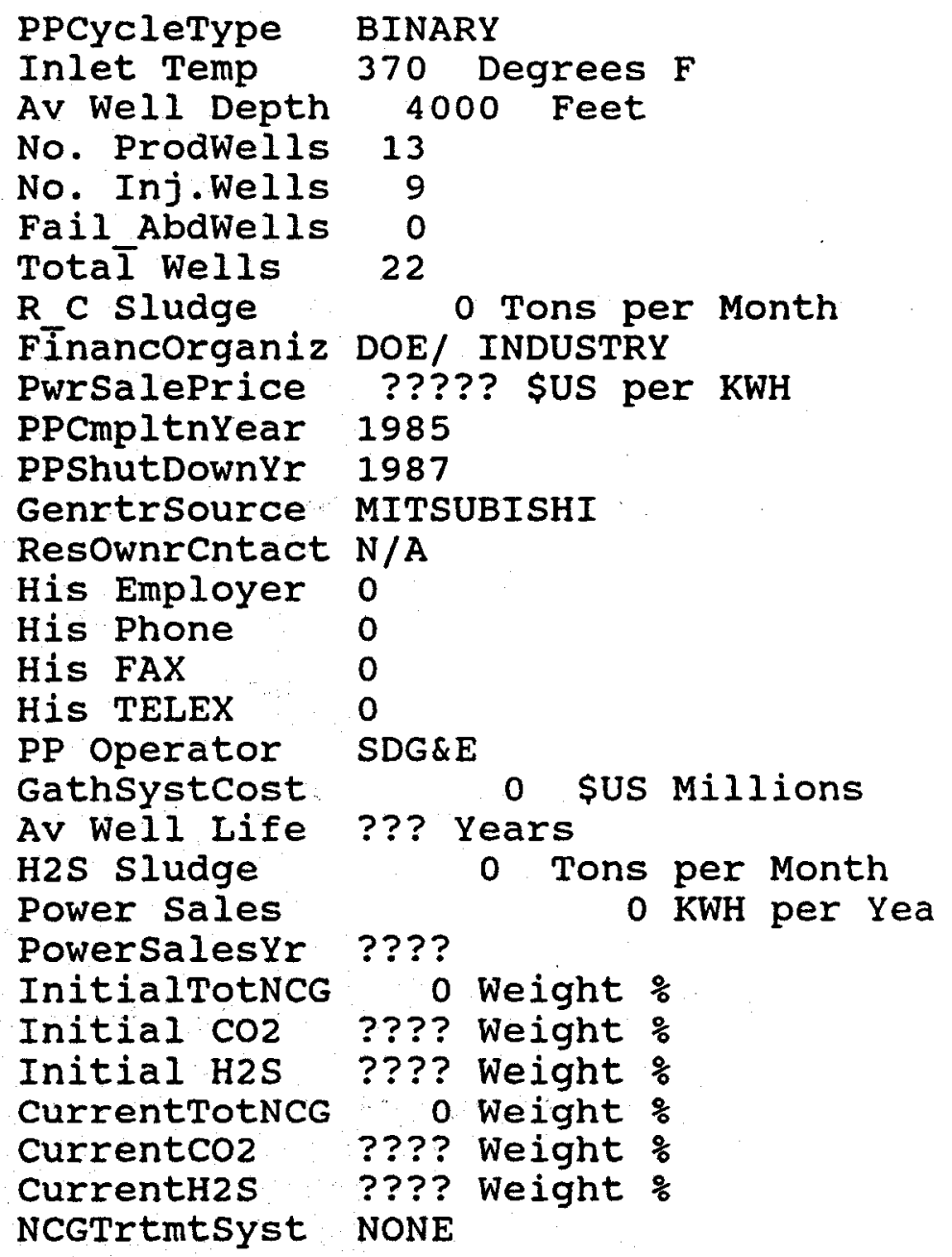




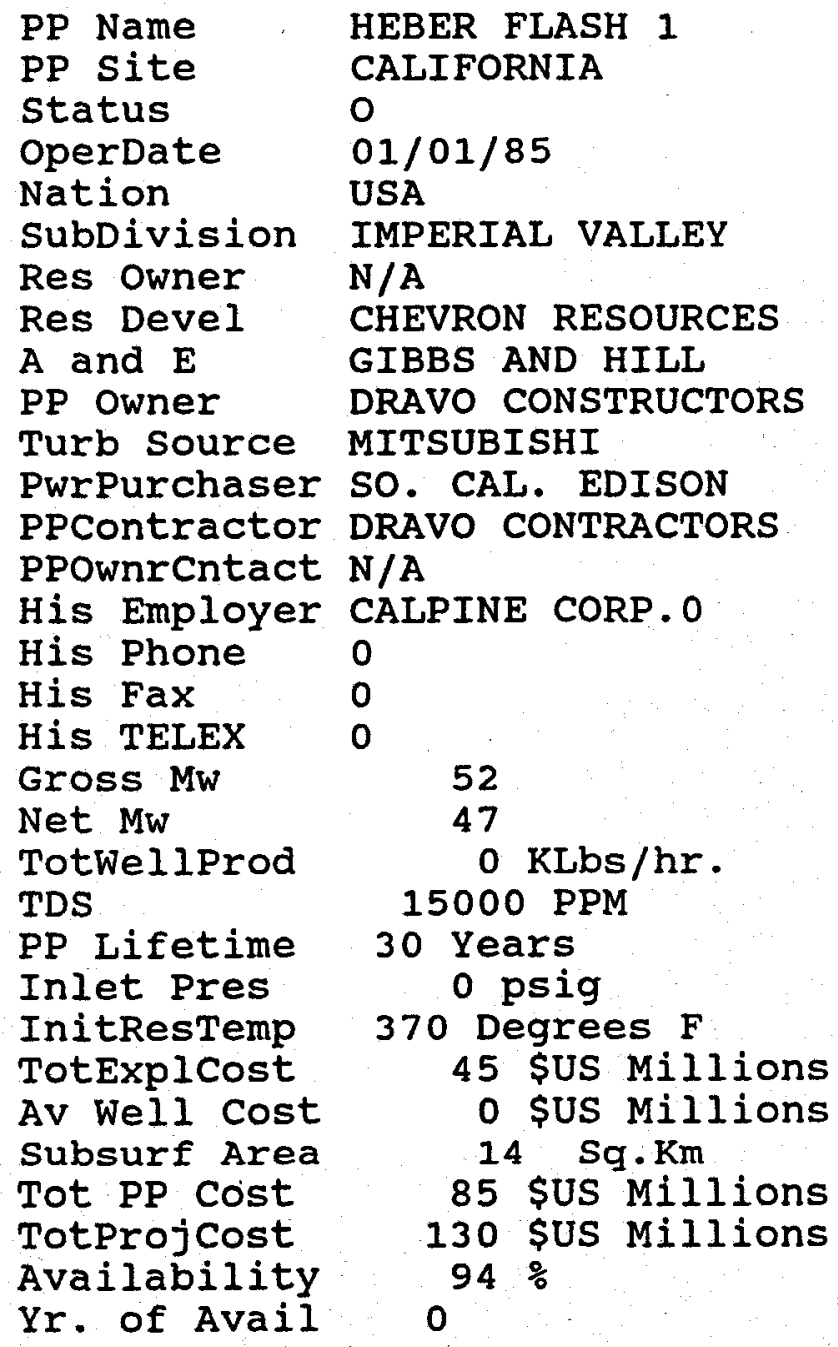

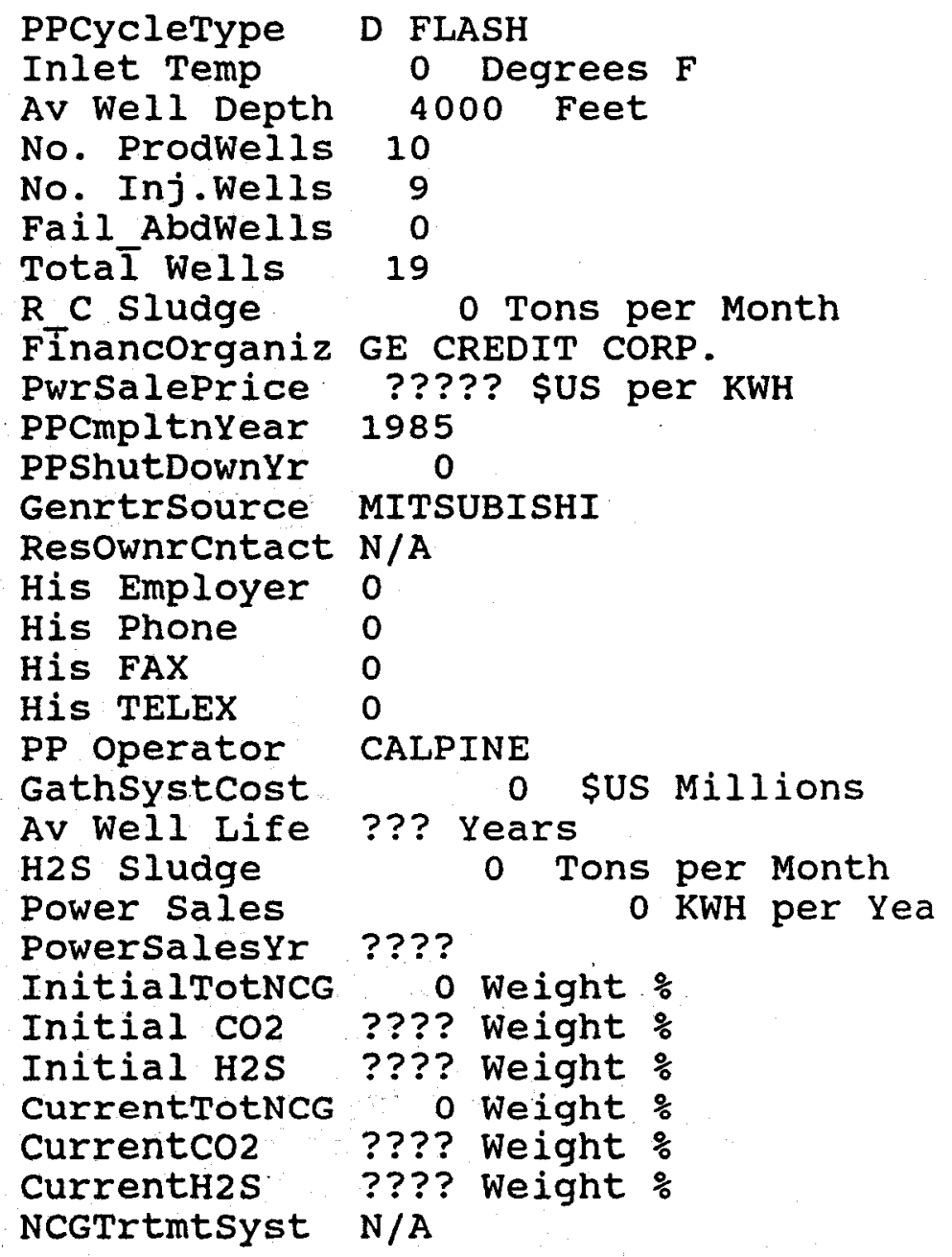




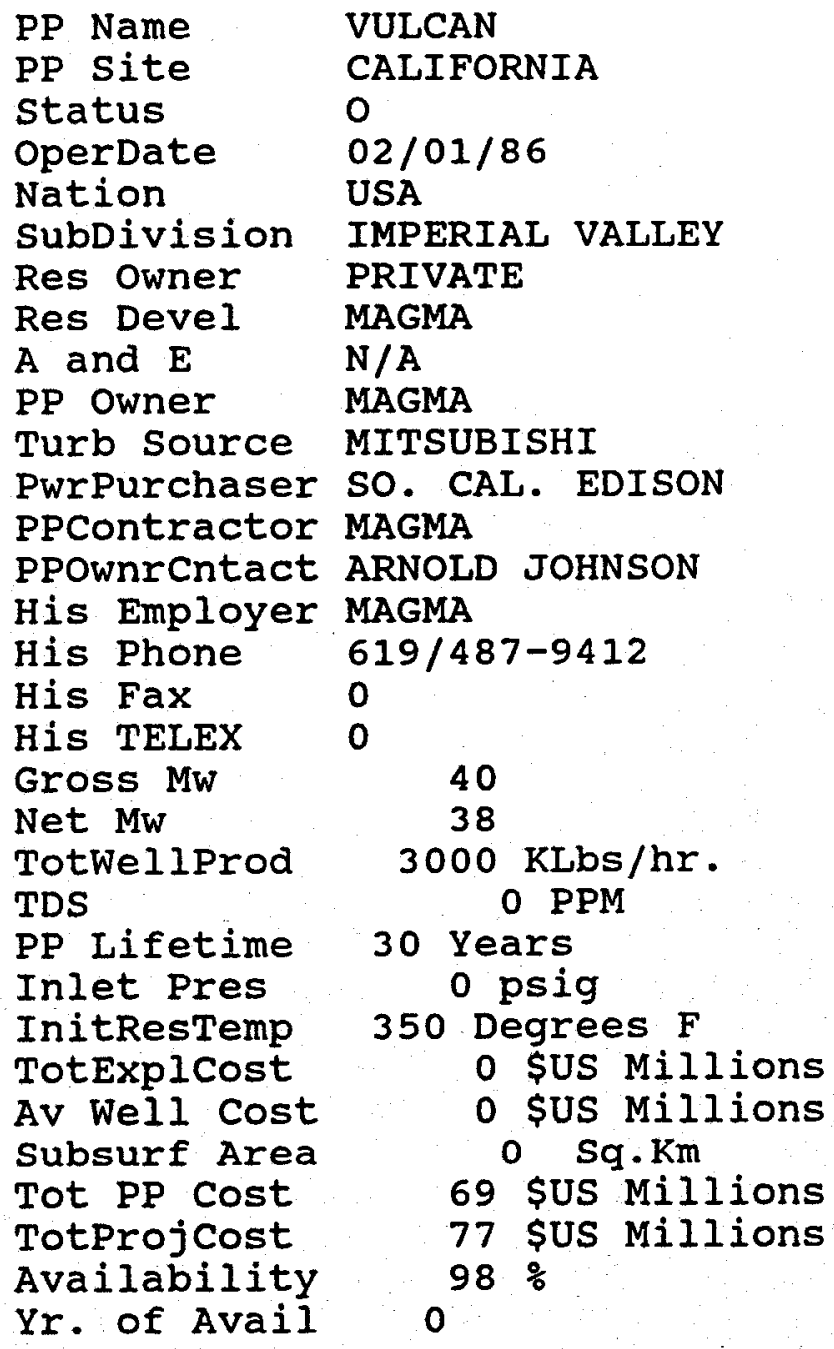

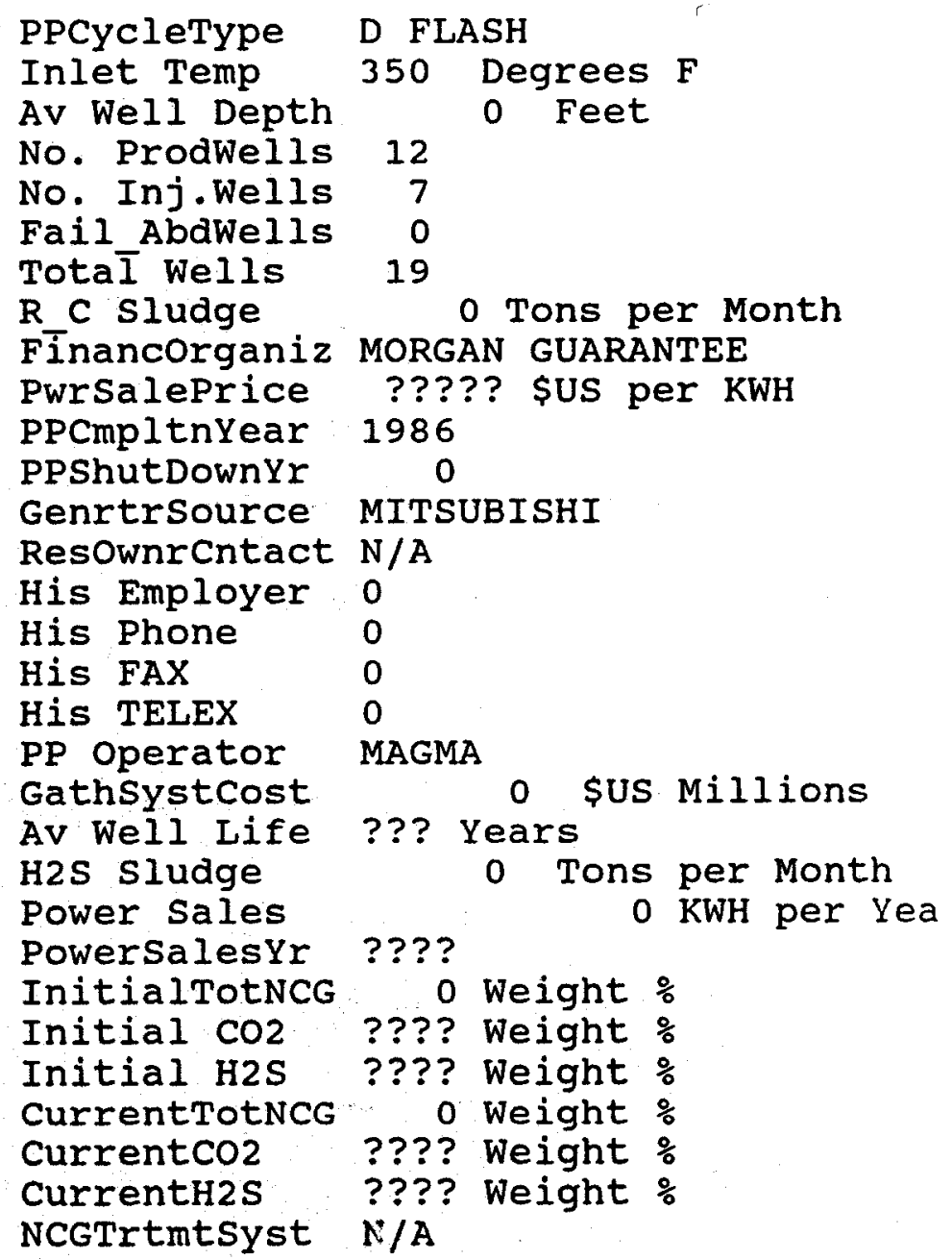




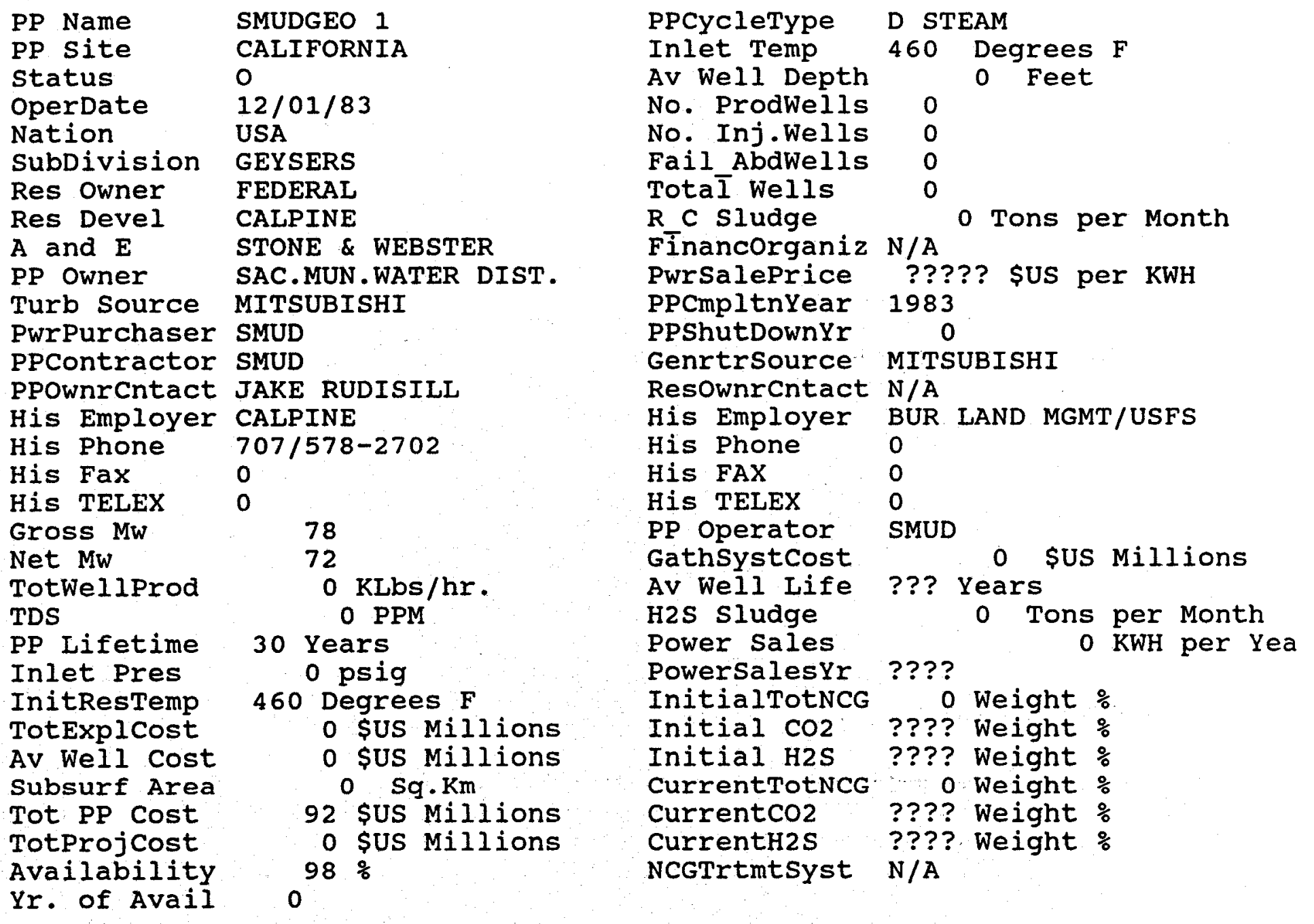




\begin{tabular}{|c|c|}
\hline PP Name & BLUNDELL UNIT 1 \\
\hline PP site & UTAH \\
\hline status & 0 \\
\hline OperDate & $07 / 01 / 84$ \\
\hline Nation & USA \\
\hline SubDivision & MILFORD AREA \\
\hline Res Owner & FEDERAL \\
\hline Res Devel & CALIFORNIA ENERGY \\
\hline$A$ and $E$ & GIBBS \& HILL \\
\hline PP Owner & PACIFICORPS \\
\hline Turb source & GENERAL ELECTRIC \\
\hline PwrPurchaser & UTAH POWER AND LIGHT \\
\hline PPcontractor & $N / A$ \\
\hline PPownrcntact & $N / A$ \\
\hline His Employer & N/A \\
\hline His Phone & 0 \\
\hline His Fax & 0 \\
\hline His TELEX & 0 \\
\hline Gross Mw & 23 \\
\hline Net MW & 20 \\
\hline $\begin{array}{l}\text { TotWel IProd } \\
\text { TDS }\end{array}$ & $\begin{array}{l}0 \mathrm{KLbs} / \mathrm{hr} \text {. } \\
7000 \mathrm{PPM}\end{array}$ \\
\hline $\begin{array}{l}\text { PP Lifetime } \\
\text { Inlet Pres }\end{array}$ & $\begin{array}{l}30 \text { Years } \\
0 \text { psig }\end{array}$ \\
\hline $\begin{array}{l}\text { InitResTemp } \\
\text { TotExplCost }\end{array}$ & $\begin{array}{l}383 \text { Degrees } F \\
12 \text { SUS Millions }\end{array}$ \\
\hline $\begin{array}{l}\text { Av Well cost } \\
\text { Subsurf Area } \\
\text { Tot PP Cost } \\
\text { TotProjcost } \\
\text { Availability }\end{array}$ & $\begin{array}{l}2 \text { Sq.Km } \\
23 \text { SUS Militions } \\
3.5 \text { SUS Millions } \\
89 \%\end{array}$ \\
\hline Yr. of Avail & 0 \\
\hline
\end{tabular}

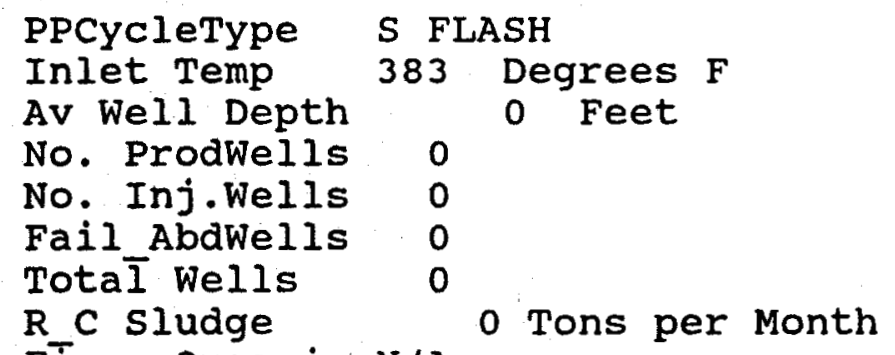

Financorganiz N/A

PwrsalePrice ????? \$US per KWH

PPCmpltnYear 1984

PPShutDownYr 0

GenrtrSource GENERAL ELECTRIC

ResownrCntact N/A

His Employer 0

His Phone 0

His FAX 0

His TELEX 0

PP operator UTAH POWER AND LIGHT

Gathsystcost 0 SUS Miliions

Av Well Life ??? Years

H2S Sludge 0 Tons per Month

Power Sales $0 \mathrm{KWH}$ per Yea

PowersalesYr ????

InitialTotNCG 0 weight. $\%$

Initial $\mathrm{CO} 2$ ???? Weight $\%$

Initial H2S ???? Weight $\%$

currentTotNCG 0 Weight $\%$

CurrentCO2 ???? Weight \%

CurrentH2s ???? Weight \%

NCGTrtmtsyst $\mathrm{N} / \mathrm{A}$ 


\begin{tabular}{|c|c|}
\hline PP Name & NAVY 1 UNIT \\
\hline PP site & CALIFORNIA \\
\hline ;tatus & 0 \\
\hline OperDate & $07 / 01 / 87$ \\
\hline Nation & USA \\
\hline SubDivision & COSO AREA \\
\hline Res Owner & US NAVY \\
\hline Res Devel & CALIFORNIA ENERGX CO \\
\hline$A$ and $E$ & CEC + MISSION ENERGY \\
\hline PP Owner & CEC+MISSION ENERGY \\
\hline Turb source & MITSUBISHI \\
\hline PwrPurchaser & SO. CAL. EDISON \\
\hline PPcontractor & CALIFORNIA ENERGY CO \\
\hline PPownrcntact & $N / A$ \\
\hline His Employer & 0 \\
\hline His Phone & 0 \\
\hline His Fax & 0 \\
\hline His TELEX & 0 \\
\hline Gross MW & 0 \\
\hline Net Mw & 30 \\
\hline $\begin{array}{l}\text { TotWel Prod } \\
\text { TDS }\end{array}$ & $\begin{array}{l}0 \mathrm{KLbs} / \mathrm{hr} . \\
6000 \mathrm{PPM}\end{array}$ \\
\hline PP Lifetime & 30 Years \\
\hline Inlet Pres & 0 psig \\
\hline $\begin{array}{l}\text { InitResTemp } \\
\text { TotExplCost }\end{array}$ & $\begin{array}{l}450 \text { Degrees } F \\
23 \text { SUS Millions }\end{array}$ \\
\hline Av Well cost & 0 SUS Millions \\
\hline Subsurf Area & $0 \mathrm{Sq} \cdot \mathrm{Km}$ \\
\hline Tot PP cost & 48 \$US Millions \\
\hline $\begin{array}{l}\text { TotProjcost } \\
\text { Availability }\end{array}$ & $\begin{array}{l}71 \text { \$US } \\
90 \%\end{array}$ \\
\hline Yr of Avail & 0 \\
\hline
\end{tabular}

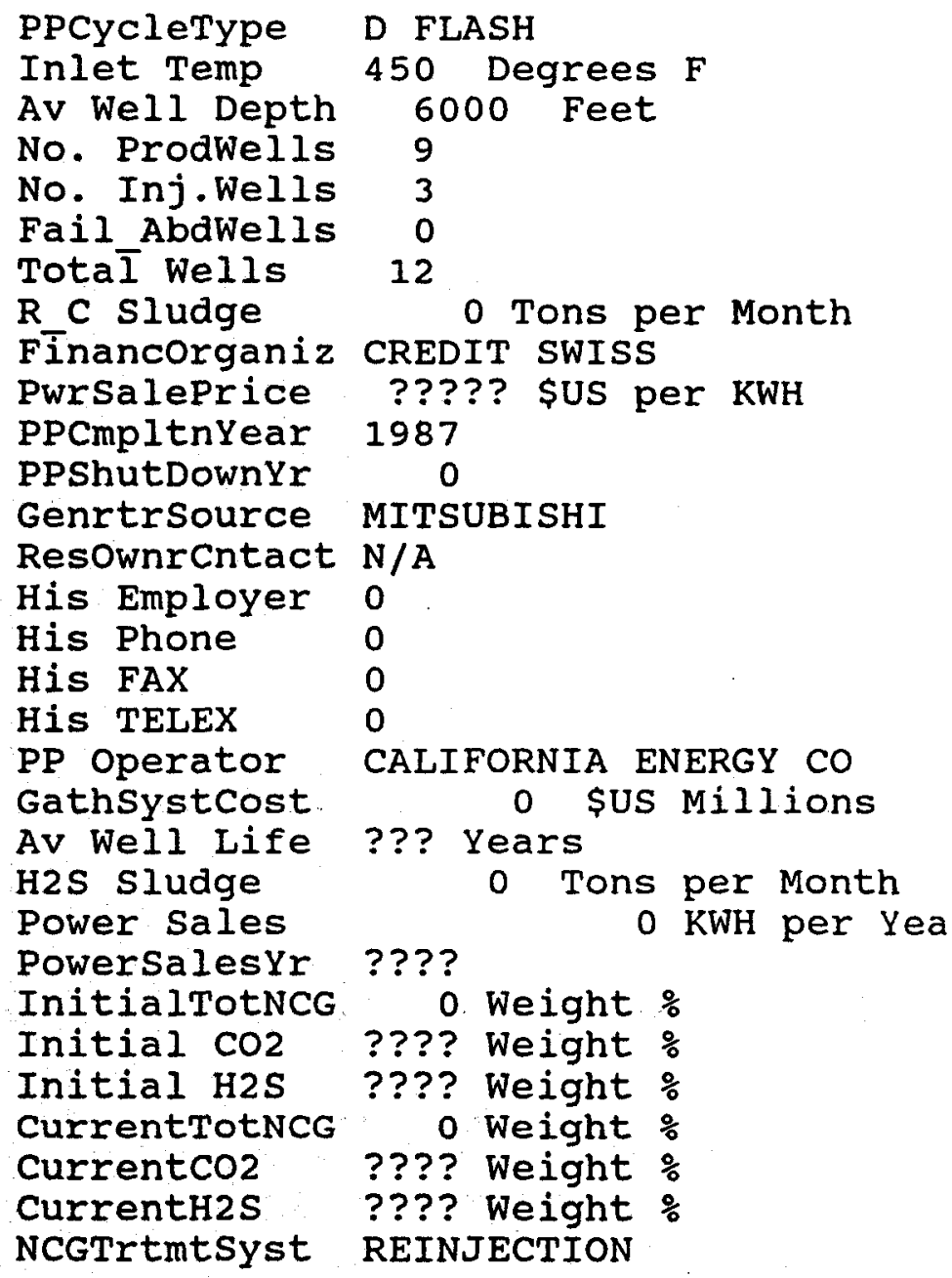




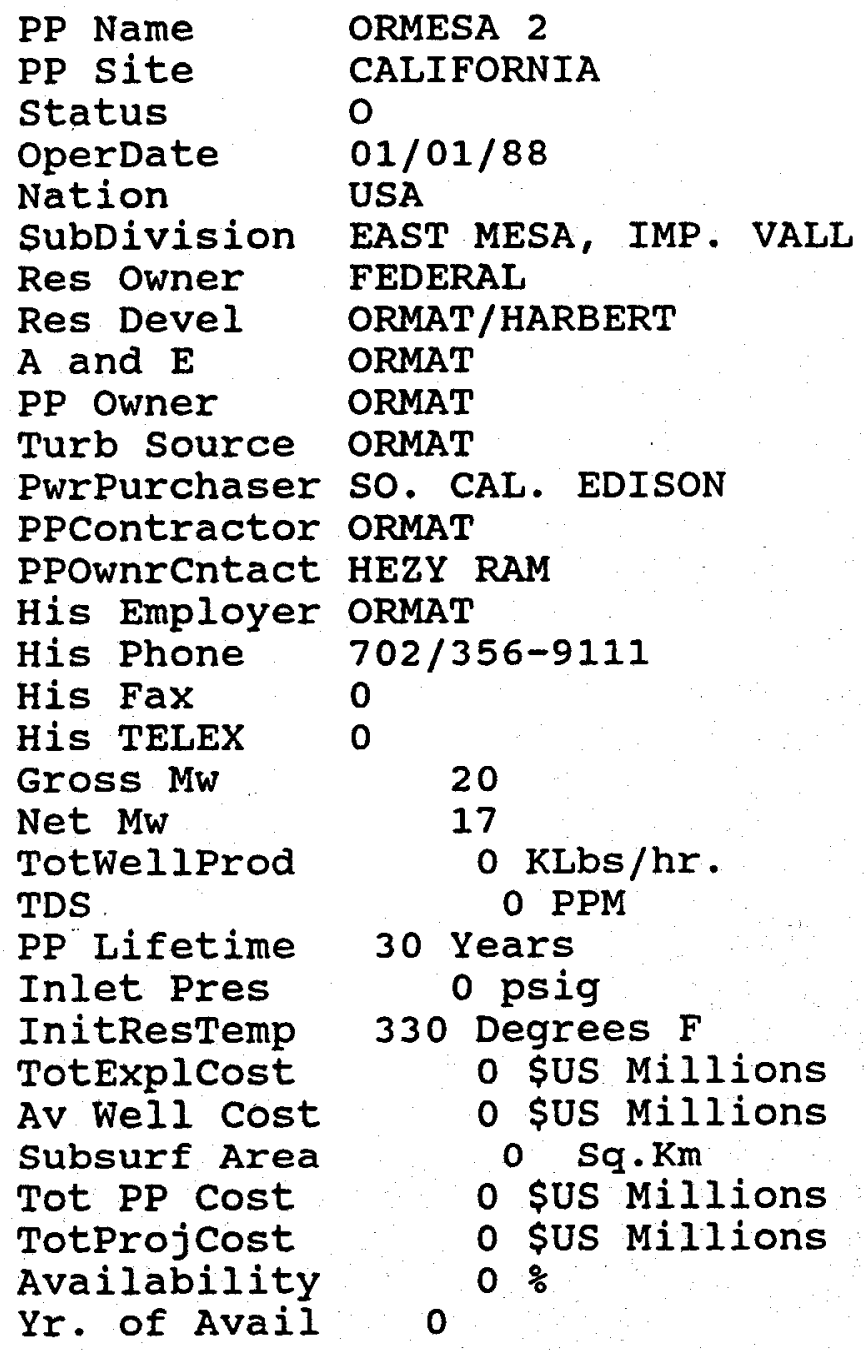

Net $\mathrm{Mw}$

$0 \mathrm{KLbs} / \mathrm{hr}$. 0 PPM

30 Years 0 psig 330 Degrees $F$ 0 \$US Millions 0 \$US Millions $0 \mathrm{Sq} \cdot \mathrm{Km}$

0 \$US Millions 0 SUS Militons $0 \%$ 


\begin{tabular}{|c|c|c|c|}
\hline PP Name & MAMMOTH PACIFIC 1 & PPCycleType & BINARY \\
\hline PP site & CALIFORNIA & Inlet Temp & Degrees $F$ \\
\hline status & & Av Well Depth & Feet \\
\hline perDate & $03 / 01 / 84$ & No. ProdWells & 4 \\
\hline lation & USA & No. Inj.Wells & 3 \\
\hline ubDivision & LONG VALLEY-MONO & Fail AbdWells & 0 \\
\hline Res Owner & MAGMA POWER & TotaI Wells & 7 \\
\hline $\begin{array}{l}\text { Res Devel } \\
\text { A and E } \\
\text { PP Owner }\end{array}$ & $\begin{array}{l}\text { PACIFIC ENERGY } \\
\text { BEN HOLT CO } \\
\text { PACIFIC ENERGY }\end{array}$ & $\begin{array}{l}\text { R C sludge } \\
\text { Financorganiz } \\
\text { PwrSaleprice }\end{array}$ & $\begin{array}{l}0 \text { Tons per Month } \\
\text { HOLT/PACIFIC LIGHTING } \\
\text { ????? \$US per KWH }\end{array}$ \\
\hline Turb source & BEN HOLT CO & PPCmpltnYear & 1984 \\
\hline PwrPurchaser & SO. CAL. EDISON & PPshutDownYr & 0 \\
\hline $\begin{array}{l}\text { PPContractor } \\
\text { PPownrcntact }\end{array}$ & $\begin{array}{l}\text { BEN HOLT CO } \\
\text { MICHAEL WALKER }\end{array}$ & $\begin{array}{l}\text { Genrtrsource } \\
\text { ResownrCntact }\end{array}$ & $\begin{array}{l}\text { THE BEN HOLT CO } \\
\text { N/A }\end{array}$ \\
\hline His Employer & PACIFIC ENERGY & His Employer & MAGMA POWER \\
\hline His Phone & $213 / 725-1139$ & His Phone & 0 \\
\hline His Fax & 0 & His FAX & 0 \\
\hline His TELEX & & His TELEX & 0 \\
\hline $\begin{array}{l}\text { Gross MW } \\
\text { Net Mw } \\
\text { TotwellProd }\end{array}$ & $\begin{array}{r}10 \\
7 \\
0 \mathrm{KLbs} / \mathrm{hr} .\end{array}$ & $\begin{array}{l}\text { PP Operator } \\
\text { Gathsystcost } \\
\text { Av Well Life }\end{array}$ & $\begin{array}{l}\text { PCIFIC ENERGY } \\
\text { o \$US Millions } \\
\text { ??? Years }\end{array}$ \\
\hline $\begin{array}{l}\text { TDS } \\
\text { PP Lifetime }\end{array}$ & $\begin{array}{l}0 \text { PPM } \\
30 \text { Years }\end{array}$ & $\begin{array}{l}\text { H2s sludge } \\
\text { Power Sales }\end{array}$ & $\begin{array}{l}0 \text { Tons per Month } \\
0 \text { KWH per Yea }\end{array}$ \\
\hline $\begin{array}{l}\text { Inlet Pres } \\
\text { InitResTemp }\end{array}$ & $\begin{array}{l}0 \text { psig } \\
330 \text { Degrees } F\end{array}$ & $\begin{array}{l}\text { PowersalesYr } \\
\text { InitialTotNCG }\end{array}$ & $\begin{array}{l}\text { ???? } \\
\text { o weight } \%\end{array}$ \\
\hline $\begin{array}{l}\text { TotExplCost } \\
\text { Av Well Cost }\end{array}$ & $\begin{array}{l}3 \text { \$US Millions } \\
0 \text { SUS Millions }\end{array}$ & $\begin{array}{ll}\text { Initial } & \mathrm{CO} 2 \\
\text { Initial } & \mathrm{H} 2 \mathrm{~S}\end{array}$ & $\begin{array}{l}\text { ???? Weight } \% \\
\text { ???? Weight \% }\end{array}$ \\
\hline Subsurf Area & $0 \mathrm{Sq} \cdot \mathrm{Km}$ & CurrentTotNCG & 0 Weight $\%$ \\
\hline $\begin{array}{l}\text { Tot PP Cost } \\
\text { Totprojcost } \\
\text { Availability }\end{array}$ & $\begin{array}{l}10 \text { \$US Millions } \\
13 \text { \$US Militions } \\
0 \%\end{array}$ & $\begin{array}{l}\text { CurrentCO2 } \\
\text { CurrentH2S } \\
\text { NCGTrtmtSyst }\end{array}$ & $\begin{array}{l}\text { ???? Weight \% } \\
\text { ???? Weight \% } \\
\text { N/A }\end{array}$ \\
\hline r. o & & & \\
\hline
\end{tabular}




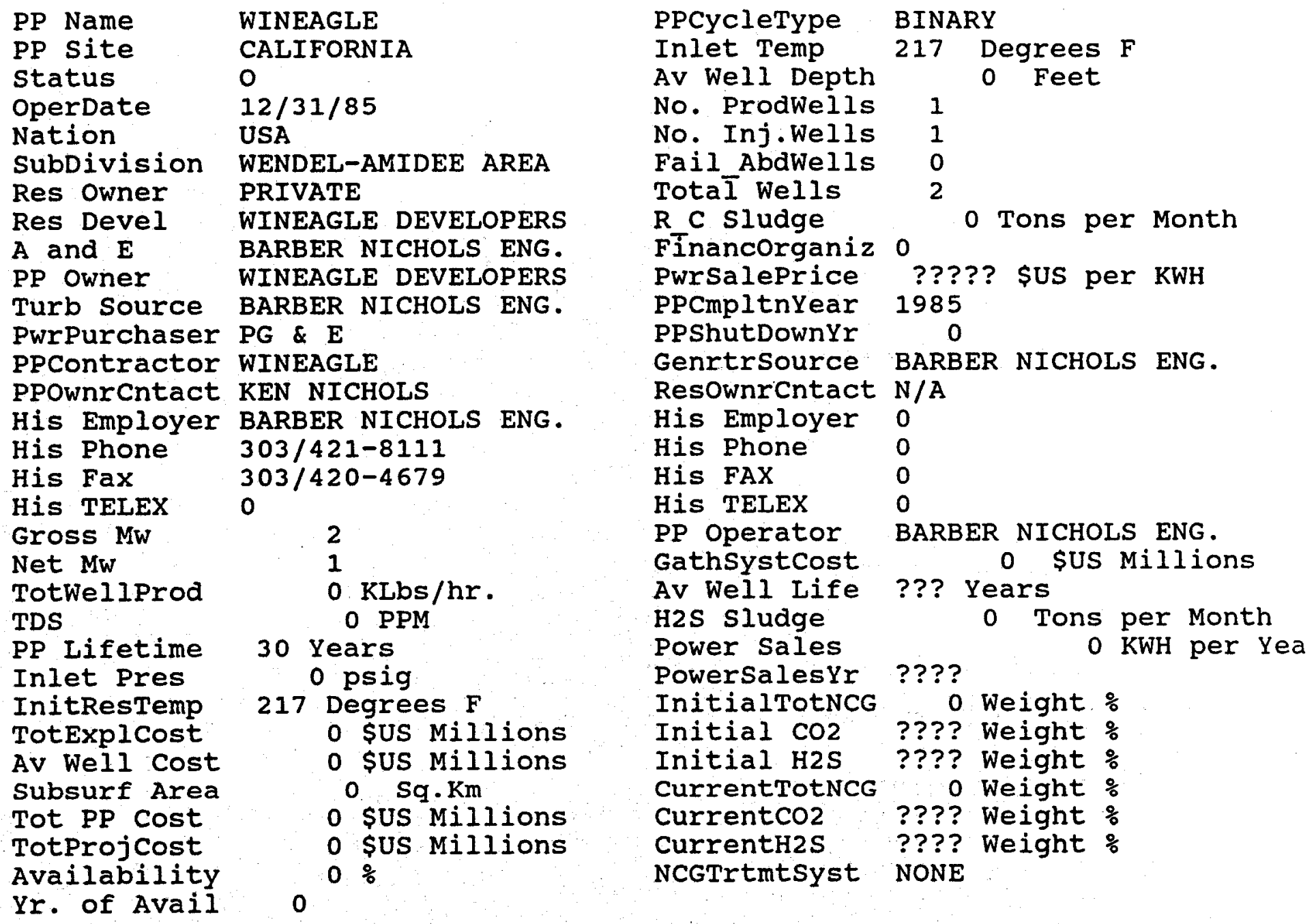




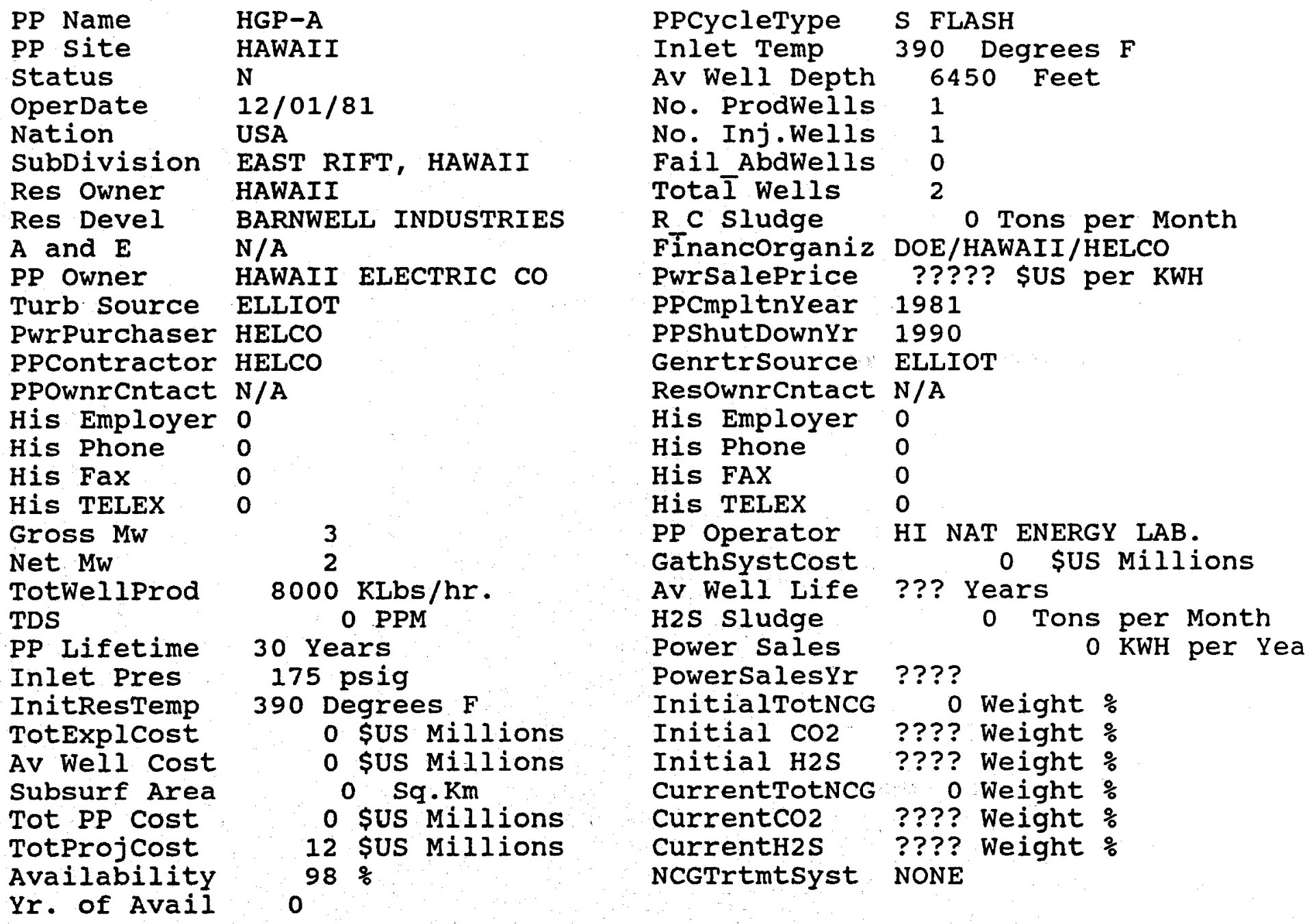




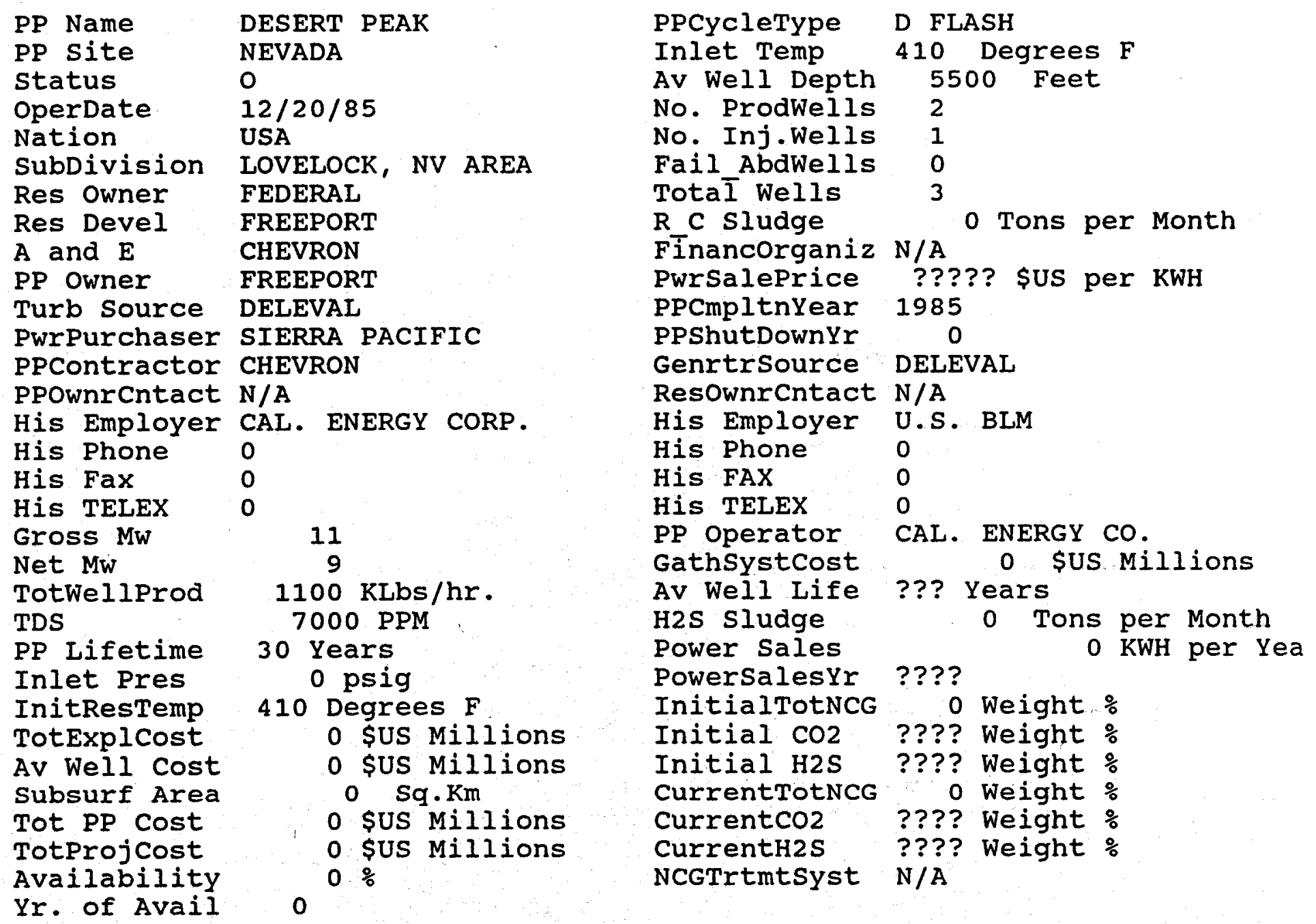

D FLASH
410 Degrees $F$ 5500 Feet

2

$$
1
$$

0 Tons per Month

0
0
0
CAL. ENERGY
0 \$US
??? Years
0 Tons
0
????
0 Weight
???? Weight \%
???? Weight \%
0 Weight \%
???? Weight \%
???? Weight \%
N/A




\begin{tabular}{|c|c|c|c|}
\hline $\begin{array}{l}\text { PP Name } \\
\text { PP site }\end{array}$ & $\begin{array}{l}\text { EMPIRE GEO. PROJECT } \\
\text { NEVADA }\end{array}$ & PPCycleType & $\begin{array}{l}\text { BINARY } \\
280 \text { Dearees } \mathrm{F}\end{array}$ \\
\hline $\begin{array}{l}\text { PP site } \\
\text { status }\end{array}$ & $\begin{array}{l}\text { NEVADA } \\
0\end{array}$ & $\begin{array}{l}\text { Inlet Temp } \\
\text { Av Well Depth }\end{array}$ & $\begin{array}{c}280 \text { Degrees } F \\
700 \text { Feet }\end{array}$ \\
\hline OperDate & $12 / 31 / 87$ & No. ProdWelis & 2 \\
\hline Nation & USA & No. Inj.Wells & 1 \\
\hline SubDivision & GERLACH, NV AREA & Fail AbdWells & 0 \\
\hline Res Owner & FEDERAL & TotaI Wells & -0 \\
\hline $\begin{array}{l}\text { Res Devel } \\
A \text { and } E\end{array}$ & $\begin{array}{l}\text { STEWART/ORMAT } \\
\text { ORMAT }\end{array}$ & $\begin{array}{l}\mathrm{R} C \text { sludge } \\
\text { Fínancorganiz }\end{array}$ & $\begin{array}{l}\text { o Tons per Month } \\
\text { ORMAT/CONSTELLATION }\end{array}$ \\
\hline PP Owner & ORMAT & Pwrsaleprice & ????? \$US per KWH \\
\hline Turb source & ORMAT & PPCmpltnYear & 1987 \\
\hline PwrPurchaser & SIERRA PACIFIC & PPShutDownYr & 0 \\
\hline PPContractor & ORMAT & Genrtrsource & ORMAT \\
\hline PPOwnrCntact & HEZY RAM & ResownrCntact & HEZY RAM \\
\hline His Employer & ORMAT & His Employer & ORMAT \\
\hline His Phone & $702 / 356-8111$ & His Phone & $702 / 356-8111$ \\
\hline His Fax & 0 & His FAX & 0 \\
\hline His TELEX & & His TELEX & 0 \\
\hline Gross $\mathrm{MW}$ & 0 & PP Operator & ORMAT \\
\hline Net $M w$ & 3 & Gathsystcost & \$US Millions \\
\hline $\begin{array}{l}\text { Totwellprod } \\
\text { TDS }\end{array}$ & $\begin{array}{l}0 \mathrm{KLbs} / \mathrm{hr} \text {. } \\
0 \mathrm{PPM}\end{array}$ & $\begin{array}{l}\text { Av Well Life } \\
\text { H2S sludge }\end{array}$ & $\begin{array}{l}\text { ??? Years } \\
\quad 0 \text { Tons per Month }\end{array}$ \\
\hline $\begin{array}{l}\text { PP Lifetime } \\
\text { Inlet Pres }\end{array}$ & $\begin{array}{l}30 \text { Years } \\
0 \text { psig }\end{array}$ & Power Sales & ???? 0 KWH per Yea \\
\hline InitResTemp & $\begin{array}{l}300 \text { Degrees } F \\
0 \text { \$US Millions }\end{array}$ & $\begin{array}{l}\text { InitialTotNCG } \\
\text { Initial CO2 } \\
\text { Initial } \mathrm{H} 2 \mathrm{~S}\end{array}$ & $\begin{array}{l}\text { 0 Weight \% } \\
\text { ???? Weight \% } \\
\text { ???? Weight \% }\end{array}$ \\
\hline $\begin{array}{l}\text { Av Well Cost } \\
\text { Subsurf Area }\end{array}$ & $\begin{array}{l}0 \mathrm{Sq} \cdot \mathrm{Km} \\
0\end{array}$ & currentTotNCG & ?! Weight $\%$ \\
\hline $\begin{array}{l}\text { Tot PP Cost } \\
\text { TotProjCost } \\
\text { Availability }\end{array}$ & $\begin{array}{l}0 \text { SUS Millions } \\
9 \text { \$US Militions } \\
0 \%\end{array}$ & $\begin{array}{l}\text { CurrentCO2 } \\
\text { CurrentH2S } \\
\text { NCGTrtmtsyst }\end{array}$ & $\begin{array}{l}\text { ???? Weight \% } \\
\text { ???? Weight \% } \\
\text { NONE }\end{array}$ \\
\hline r. of $\mathrm{Av}$ & 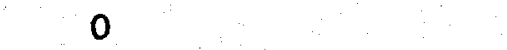 & & \\
\hline
\end{tabular}




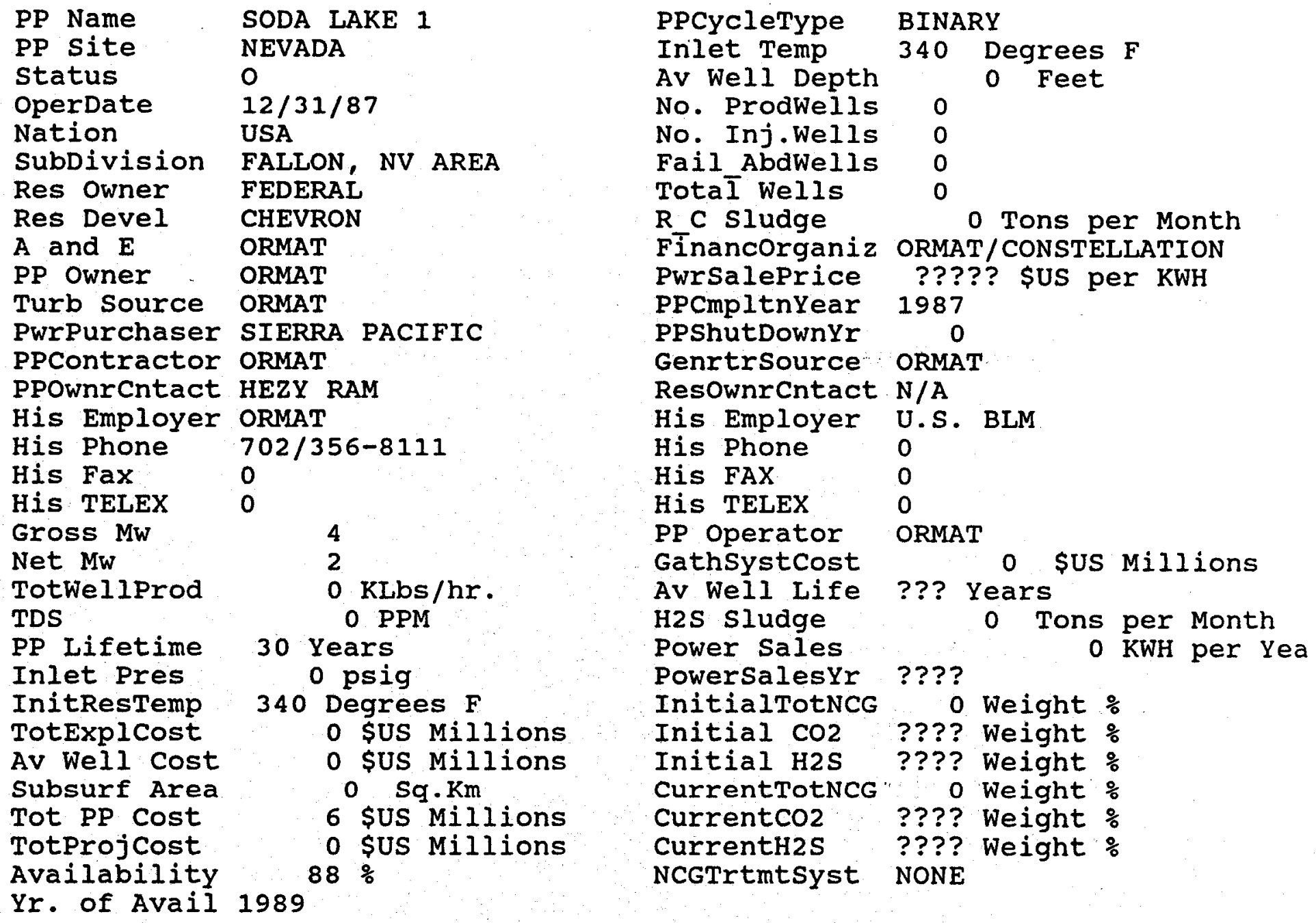

Av Well Depth

No. ProdWells

No. Inj.Wells

Fail AbdWells

TotaI Wells

$R$ C sludge Financorganiz

PwrSalePrice

PPCmpltnYear

PPShutDownYr

Genrtrsource ORMAT

ResownrCntact N/A

His Employer U.S. BLM

His Phone 0

His FAX 0

HiS TELEX 0

PP Operator ORMAT

Gathsystcost 0 sUS Millions

Av Well Life ??? Years

H2S sludge

Power Sales

PowersalesYr

InitialTotNCG

Initial $\mathrm{CO} 2$

Initial H2S

CurrentTotNCG

CurrentCO2

CurrentH2S

NCGTrtmtsyst

0 Tons per Month

$0 \mathrm{KWH}$ per Yea

\section{????}

0 Weight $\%$

???? Weight $\%$

???? Weight \%

0 Weight $\%$

???? Weight \%

???? Weight \%

NONE 


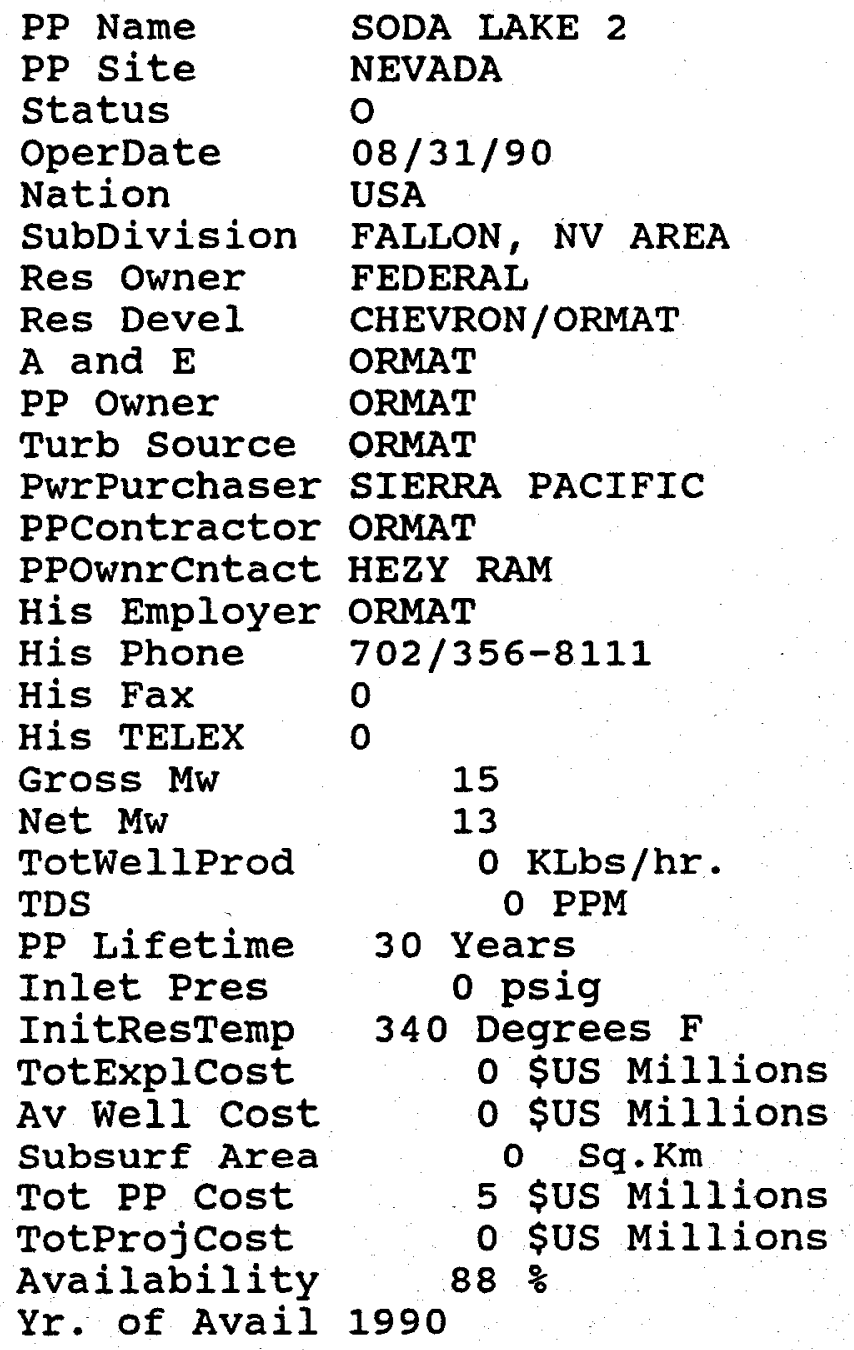

SODA LAKE 2

NEVADA

0

$08 / 31 / 90$

USA

FALLON, NV AREA

FEDERAL

CHEVRON / ORMAT

ORMAT

ORMAT

ORMAT

0

15
13
0 KLbs/hr.
0 PPM
30 Years
0 psig
340 Degrees F
0 \$US Millions
0 \$US Millions
0 Sq.Km
5 \$US Militions
0 \$US Millions
$88 \%$

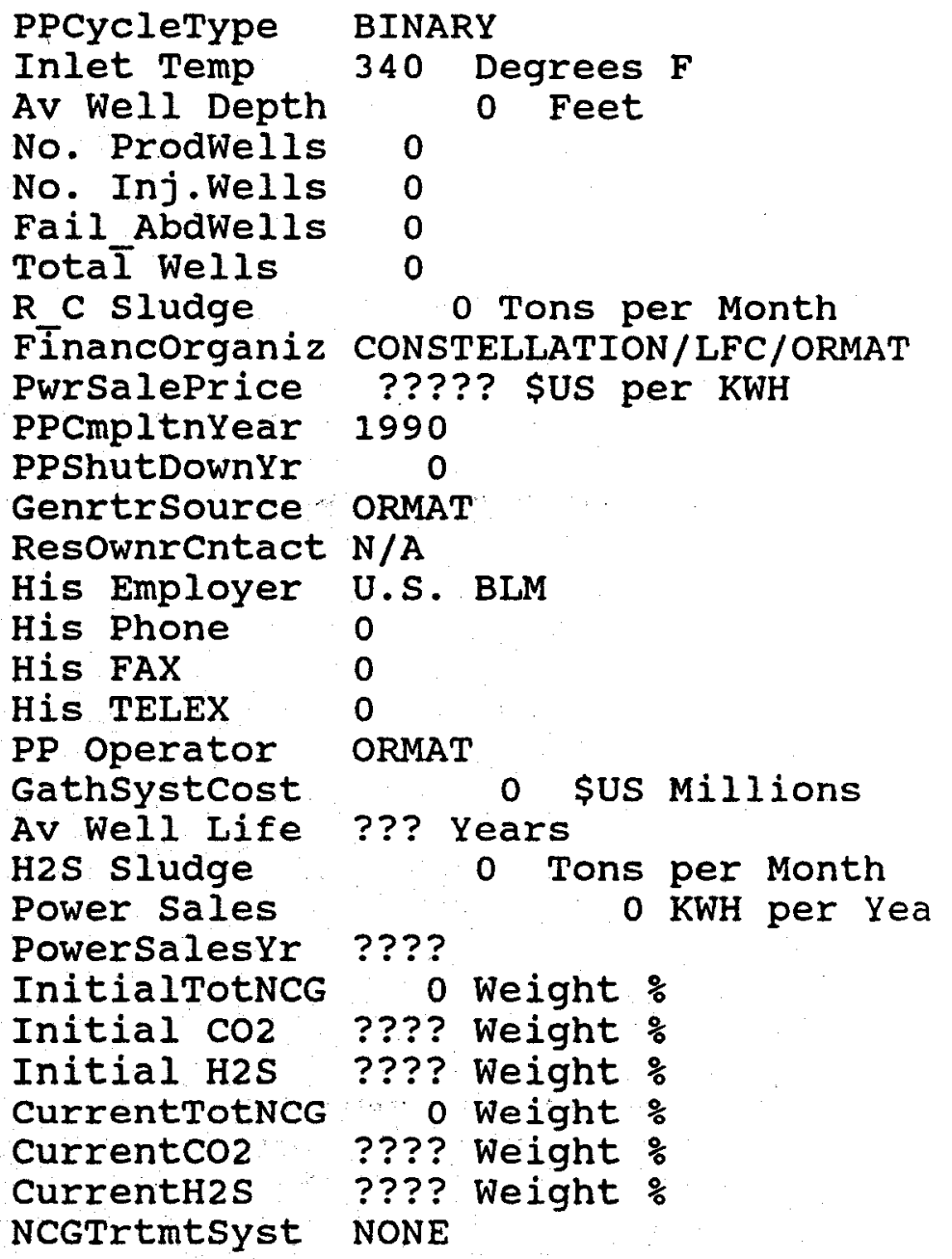




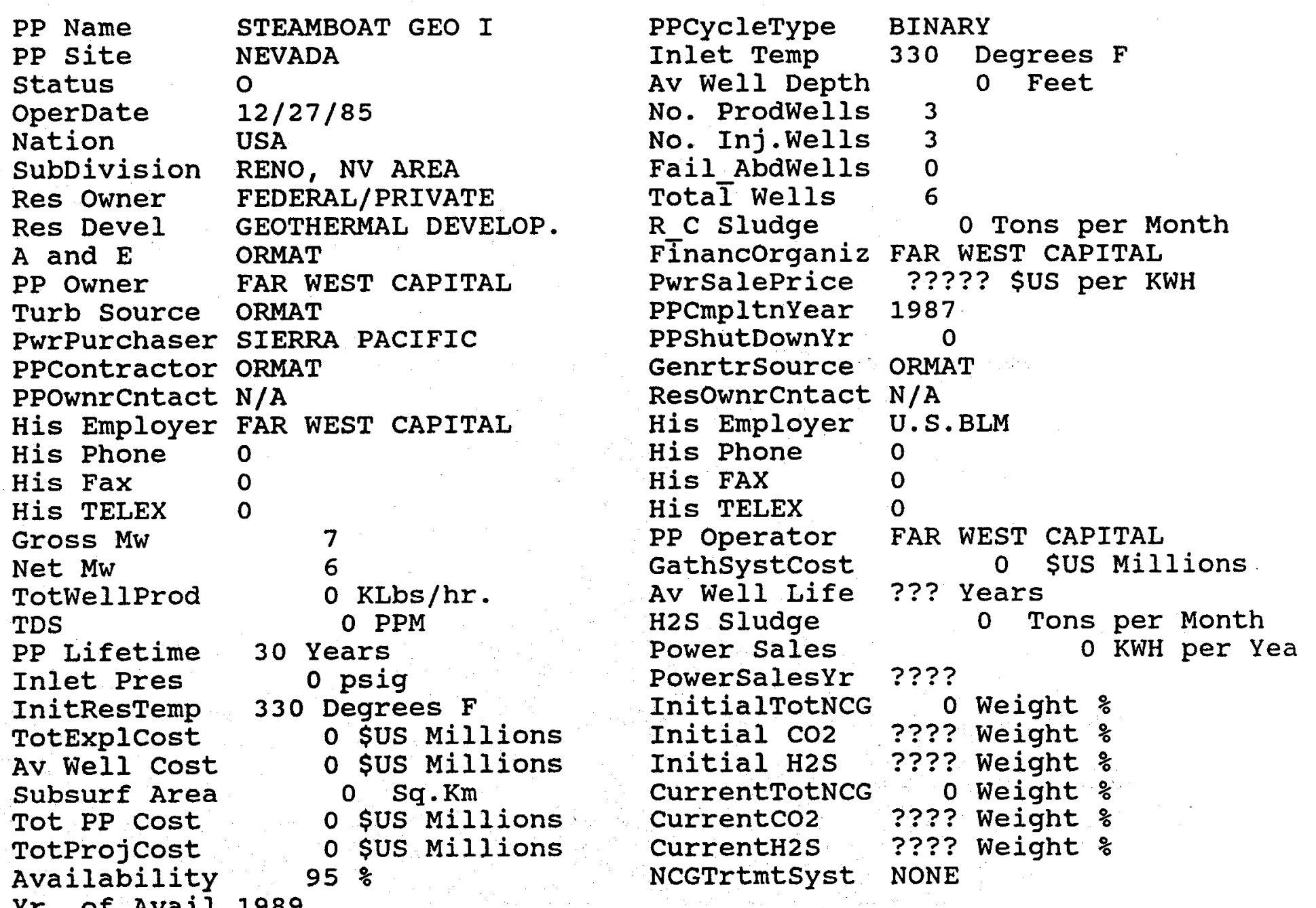




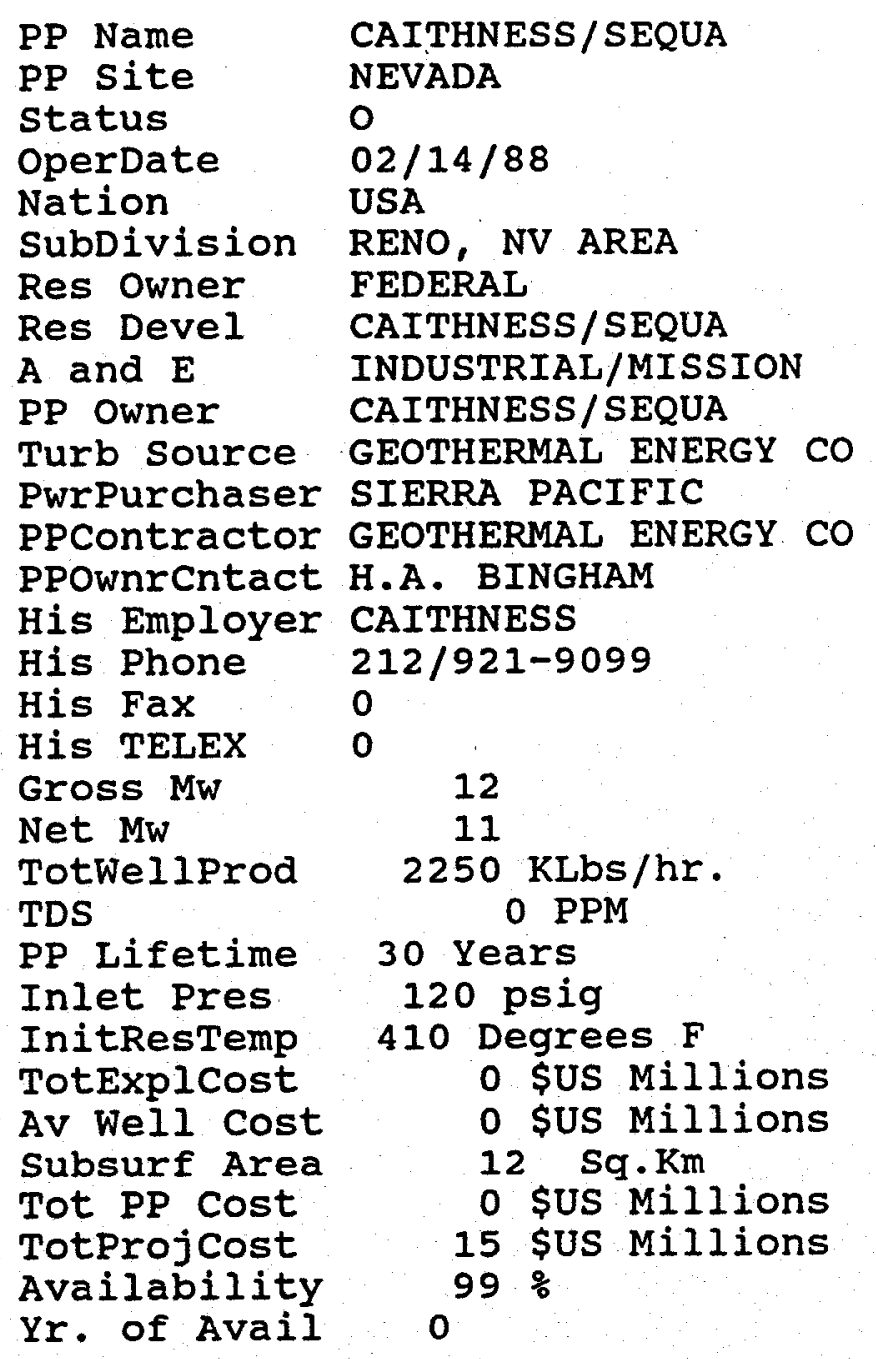

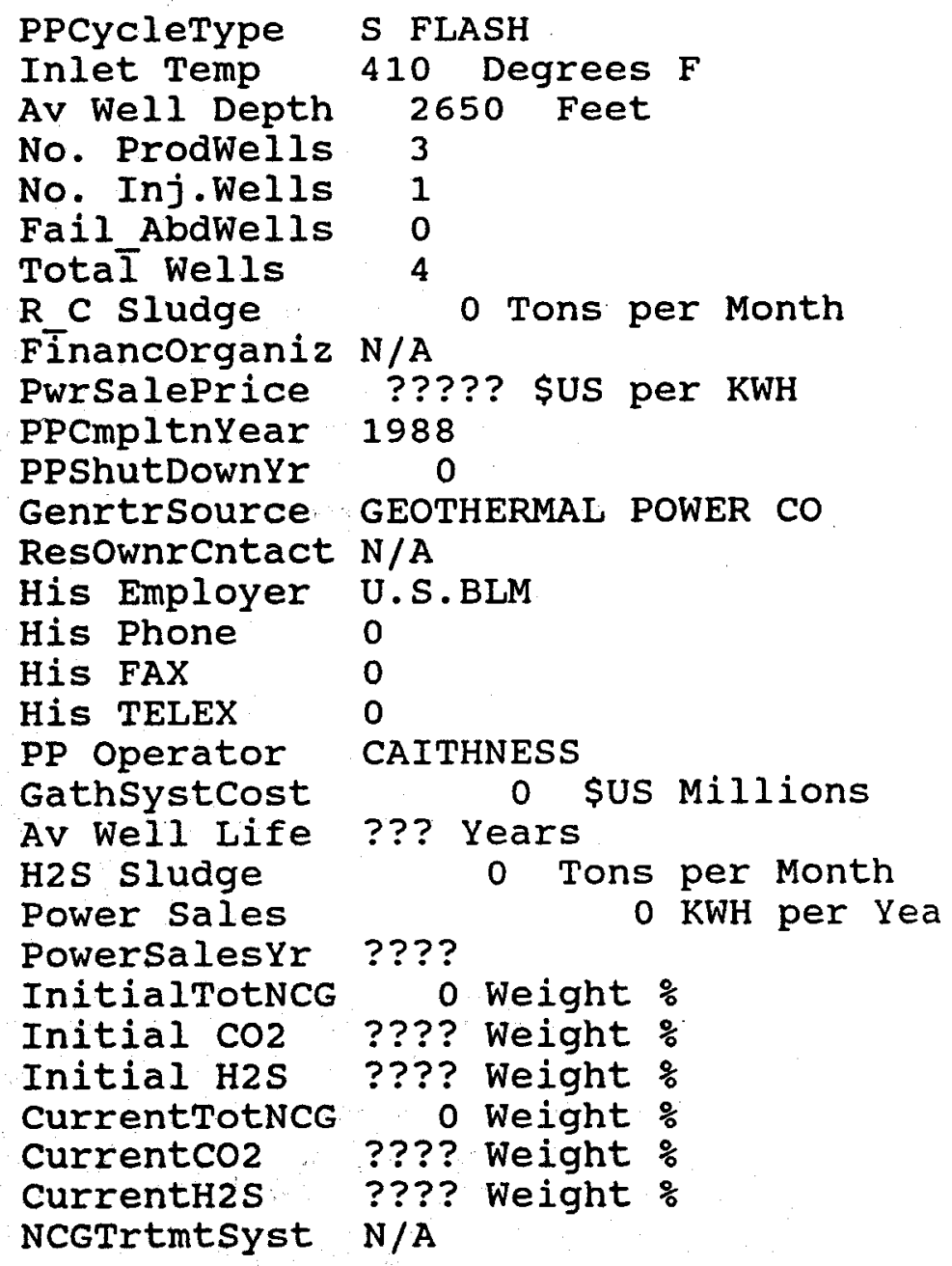




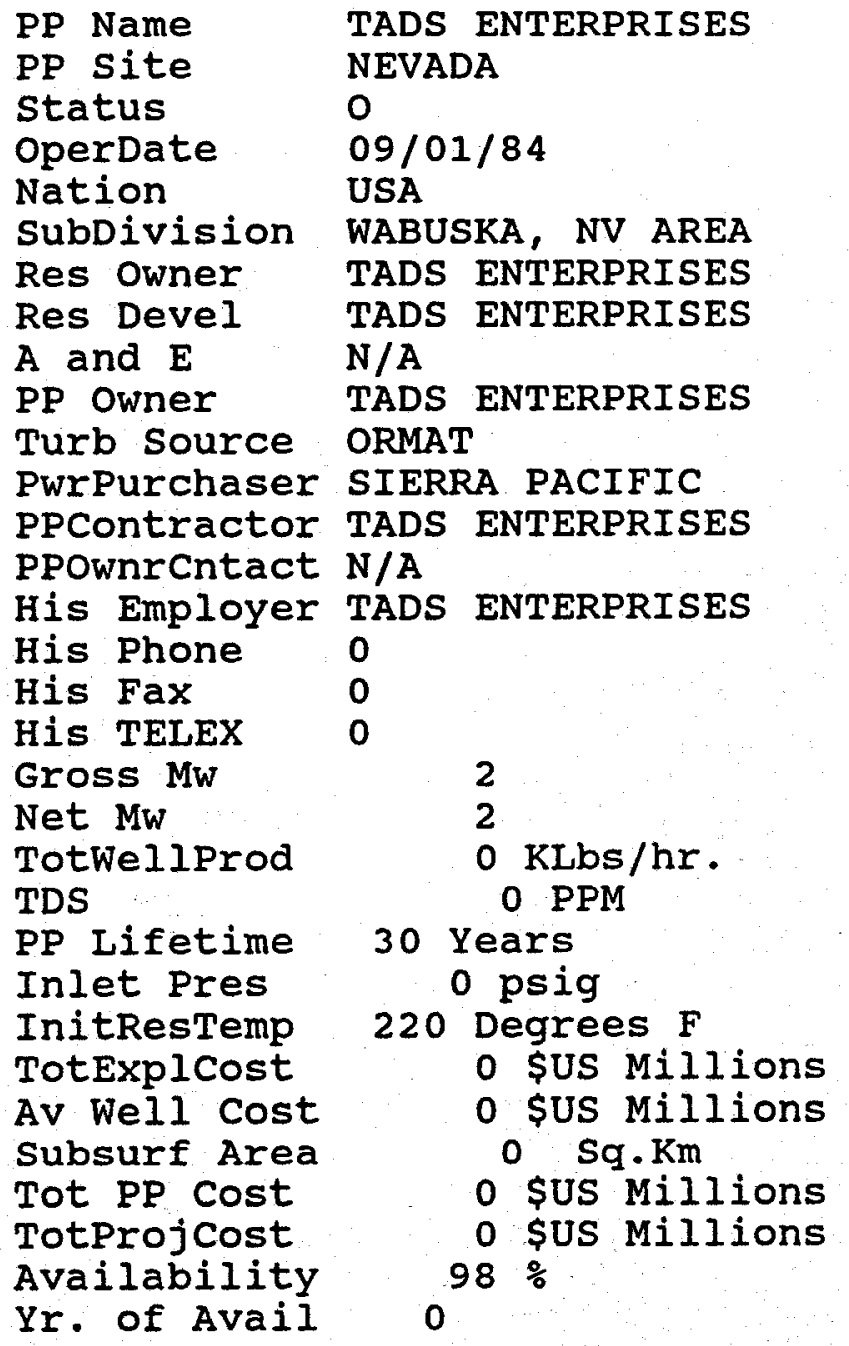

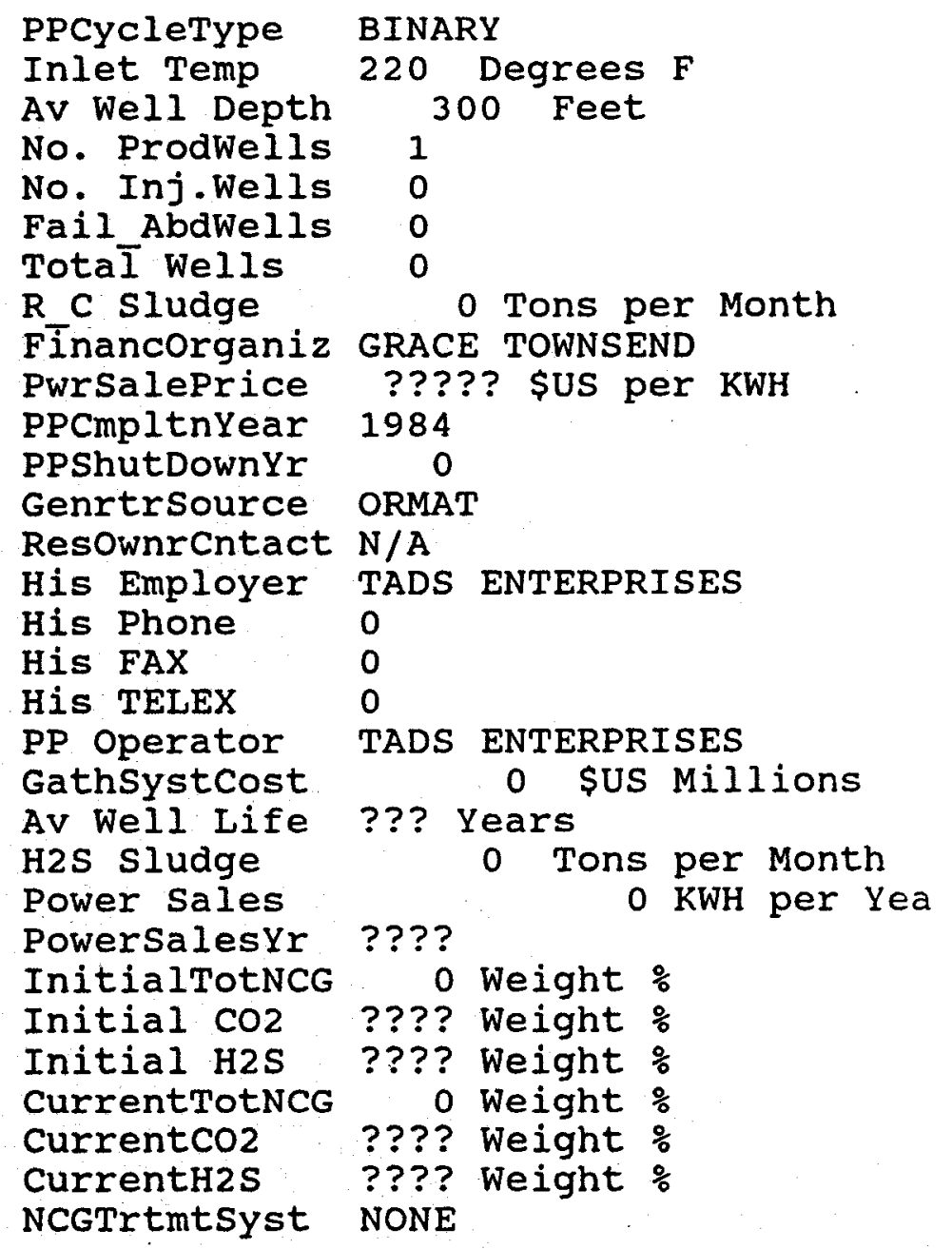




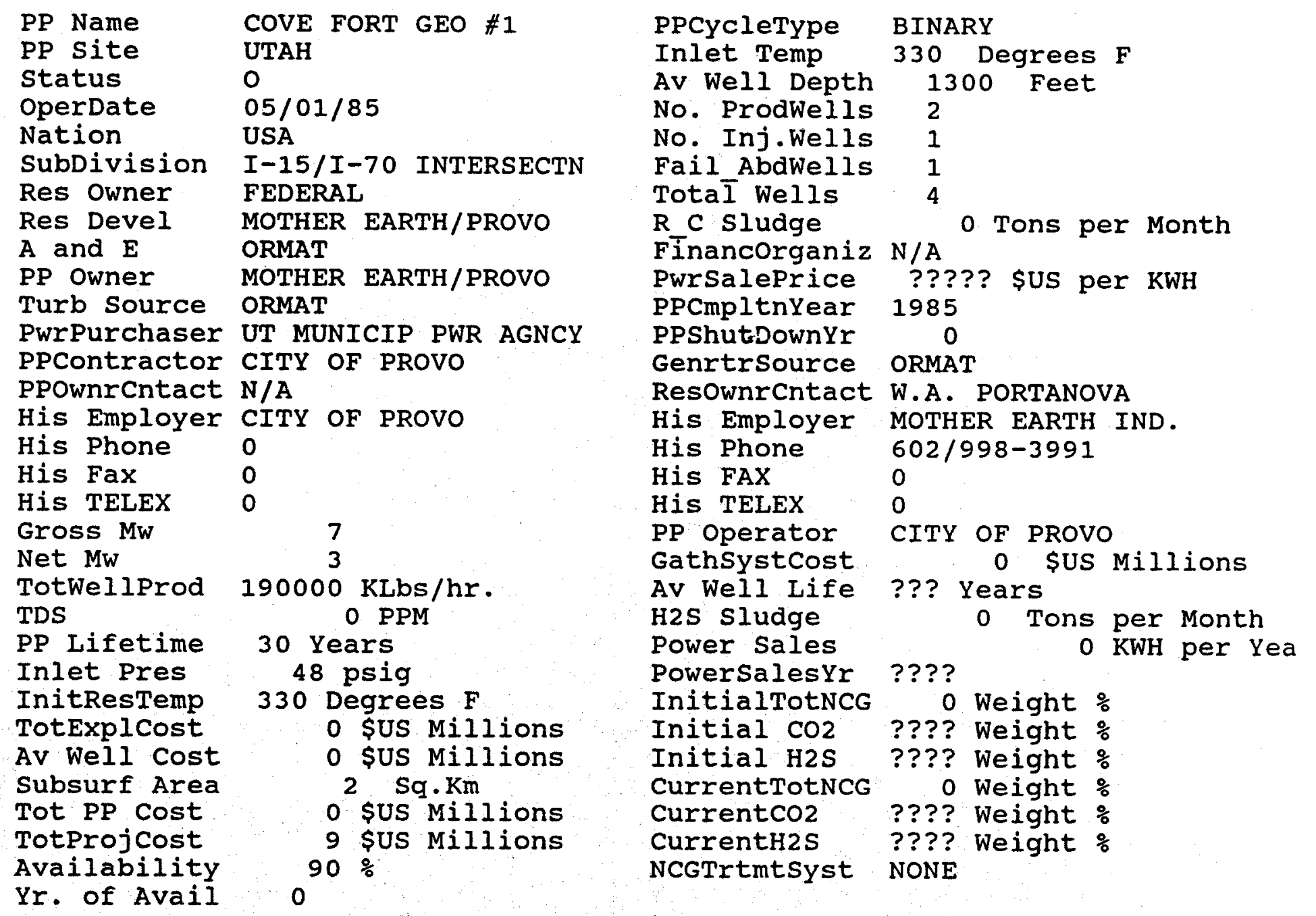




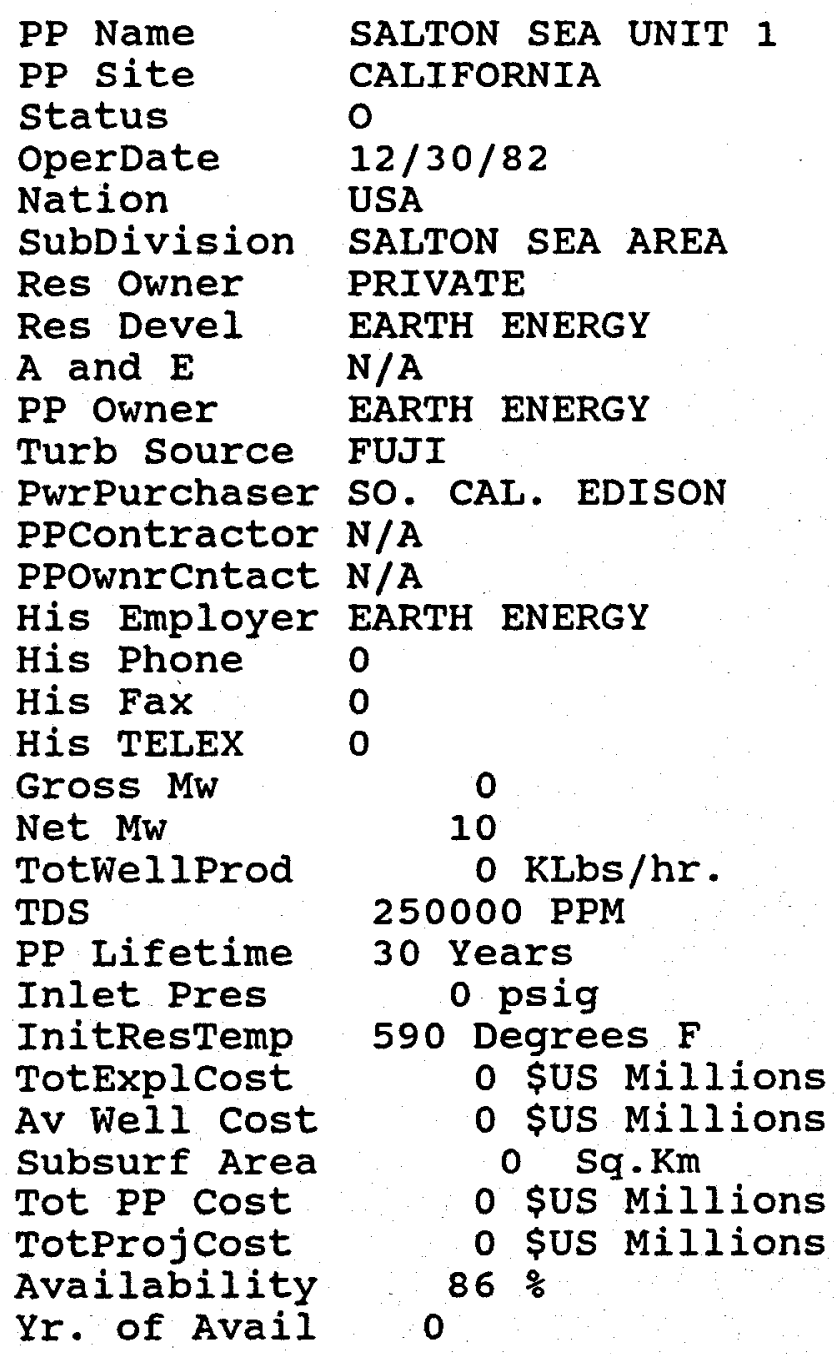

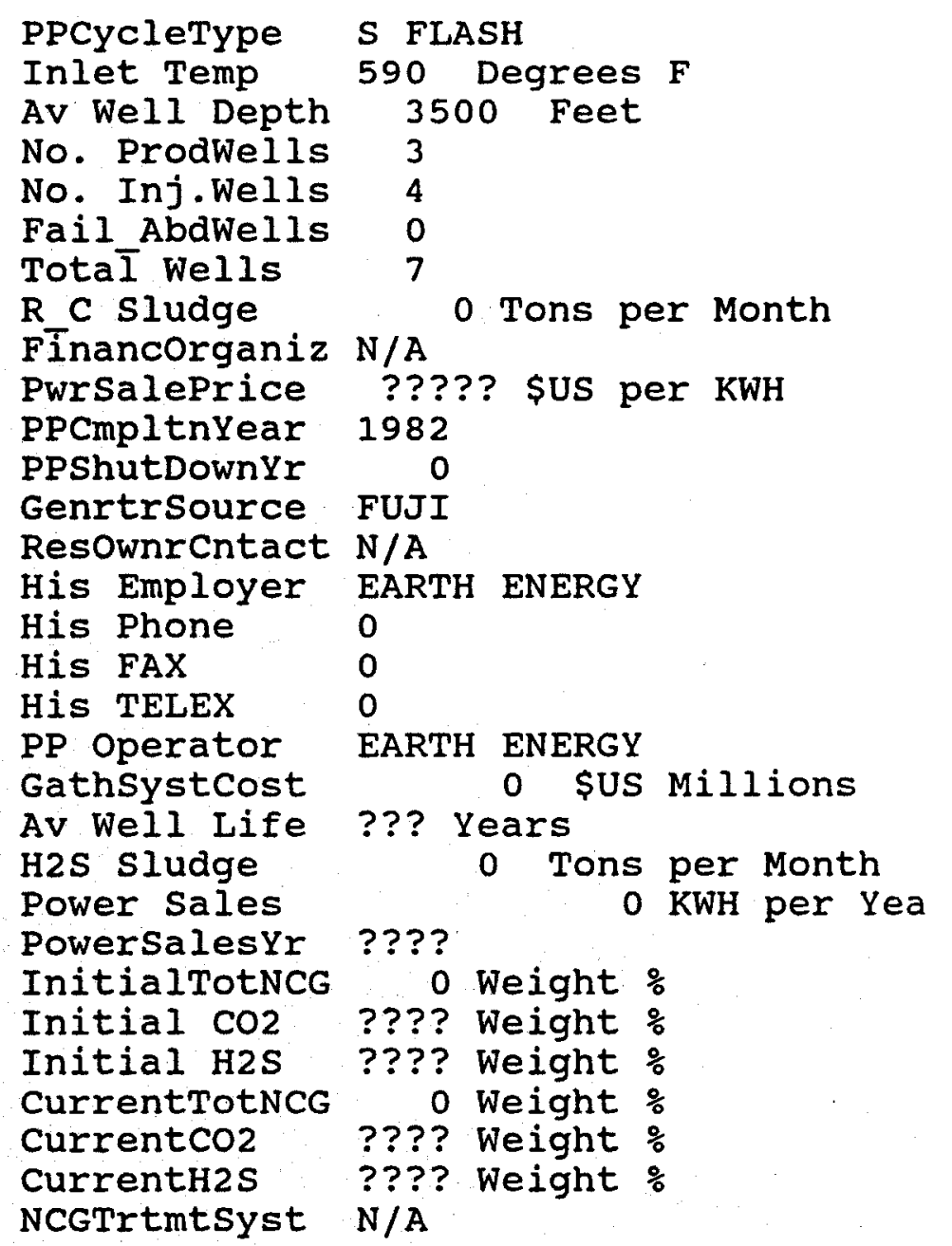




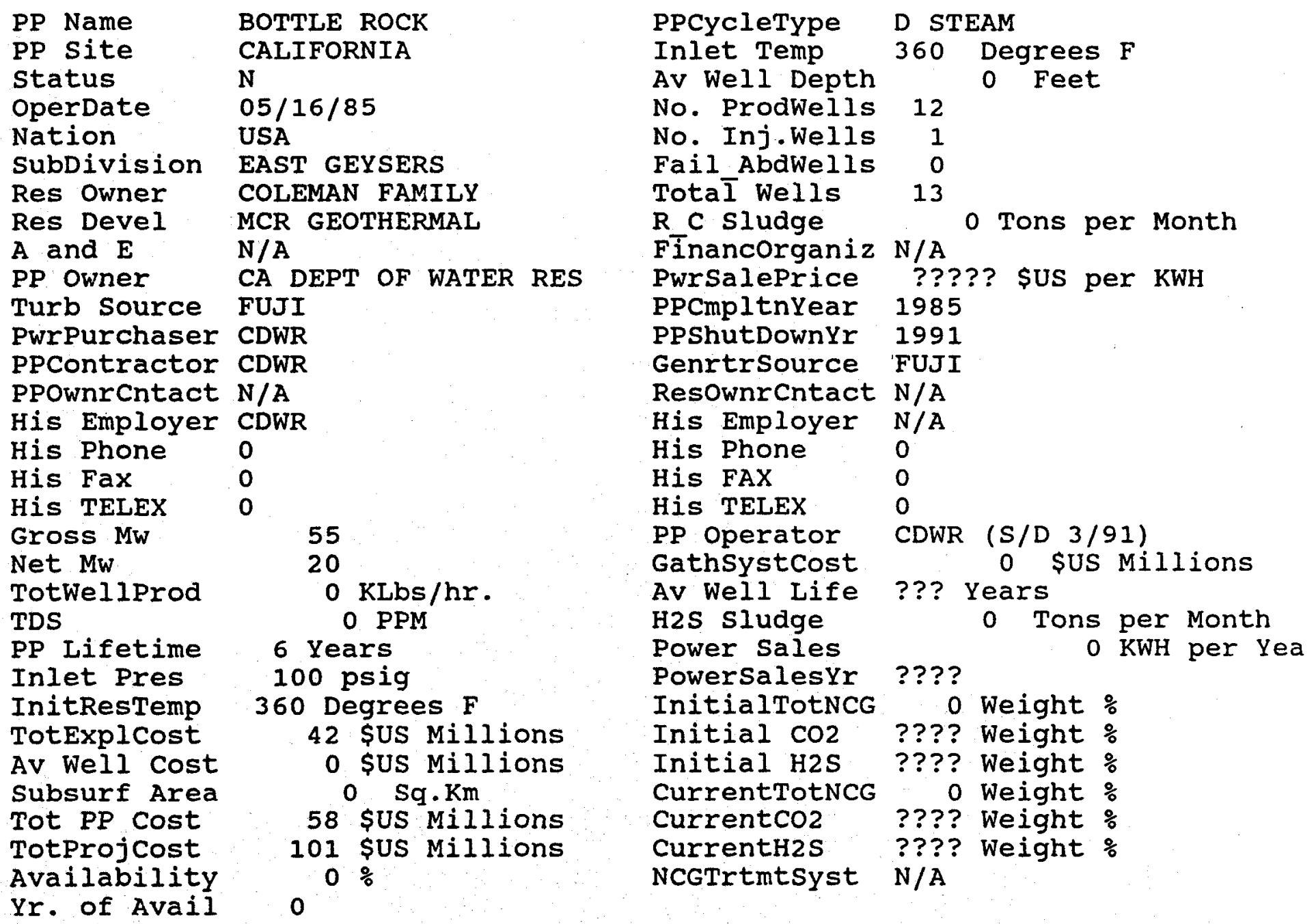




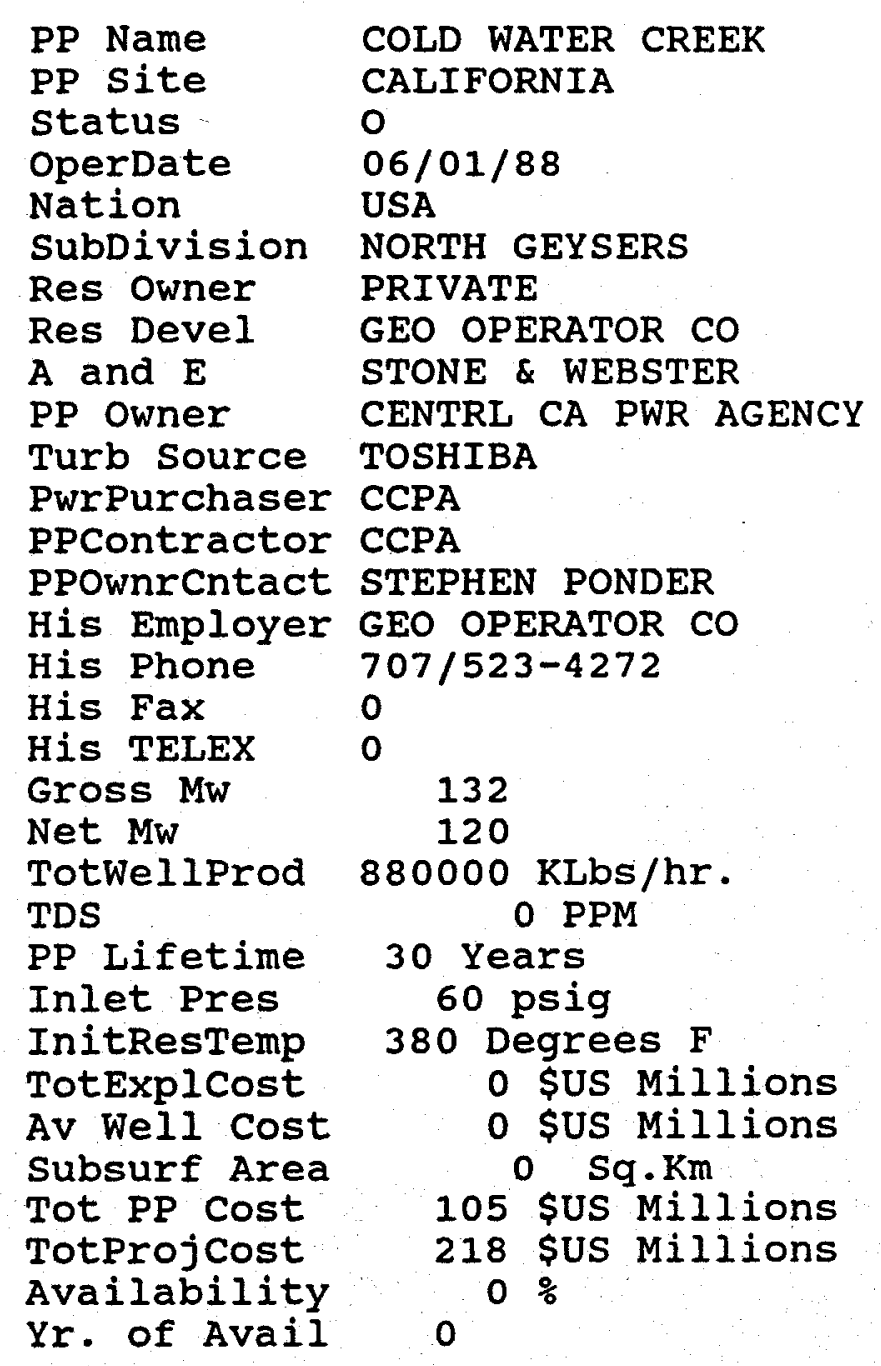

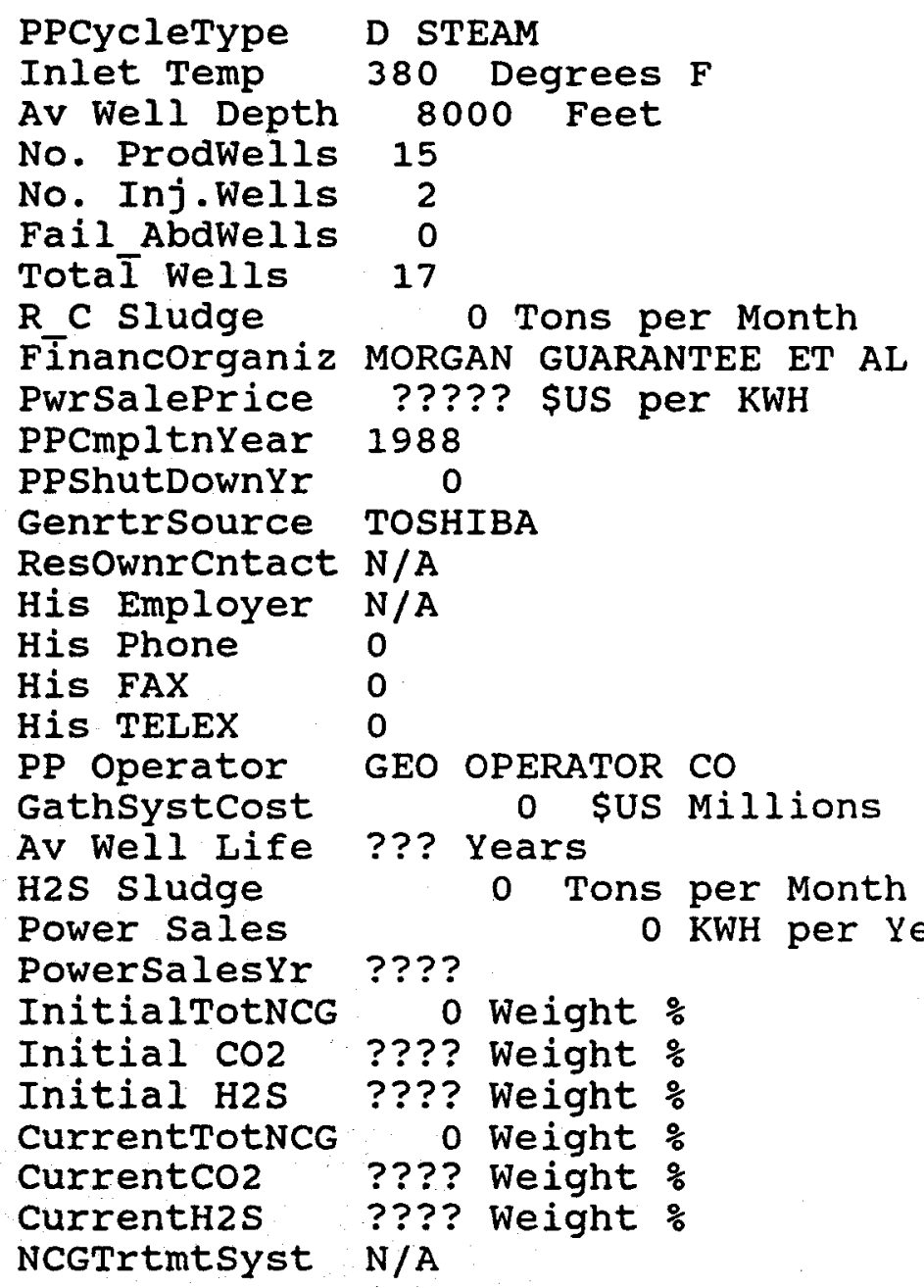




\begin{tabular}{|c|c|}
\hline PP Name & PG\&E \\
\hline PP site & CALIFORNIA \\
\hline status & $\mathbf{N}$ \\
\hline OperDate & $06 / 17 / 79$ \\
\hline Nation & USÁ \\
\hline SubDivision & WEST GEYSERS \\
\hline Res Owner & PG\&E 95\% \\
\hline Res Devel & GEO OPERATOR CO \\
\hline$A$ and $E$ & $\mathrm{~N} / \mathrm{A}$ \\
\hline PP Owner & PG\&E \\
\hline Turb Source & GENERAL ELECTRIC \\
\hline PwrPurchaser & PG\&E \\
\hline PPContractor & PG\&E \\
\hline PPownrcntact & GLEN HORTON \\
\hline His Employer & PG\&E \\
\hline His Phone & 0 \\
\hline His Fax & 0 \\
\hline His TELEX & 0 \\
\hline Gross Mw & 62 \\
\hline Net $M w$ & 57 \\
\hline $\begin{array}{l}\text { Totweliprod } \\
\text { TDS }\end{array}$ & $\begin{array}{l}0 \text { KLbs/hr. } \\
0 \text { PPM }\end{array}$ \\
\hline $\begin{array}{l}\text { PP Lifetime } \\
\text { Inlet Pres }\end{array}$ & $\begin{array}{l}11 \text { Years } \\
0 \text { psig }\end{array}$ \\
\hline $\begin{array}{l}\text { InitResTemp } \\
\text { TotExplCost }\end{array}$ & $\begin{array}{l}380 \text { Degrees } F \\
0 \text { \$US Militions }\end{array}$ \\
\hline Av Well Cost & 0 sus Millions \\
\hline Subsurf Area & $0 \quad \mathrm{Sq} \cdot \mathrm{Km}$ \\
\hline $\begin{array}{l}\text { Totprojcost } \\
\text { Availability }\end{array}$ & $\begin{array}{l}0 \text { \$US Millions } \\
0 \text { g }\end{array}$ \\
\hline Yr. of Avai & 0 \\
\hline
\end{tabular}

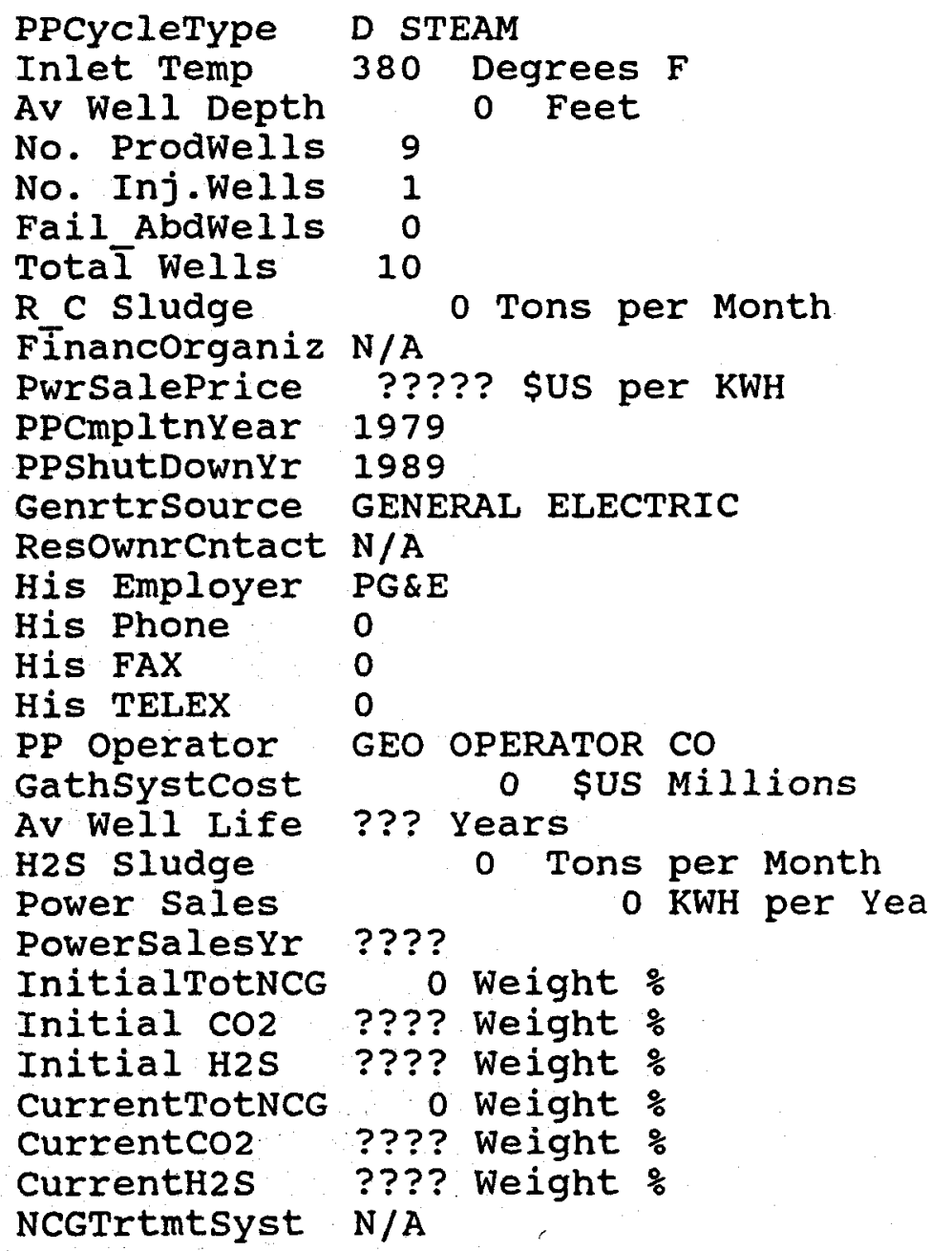




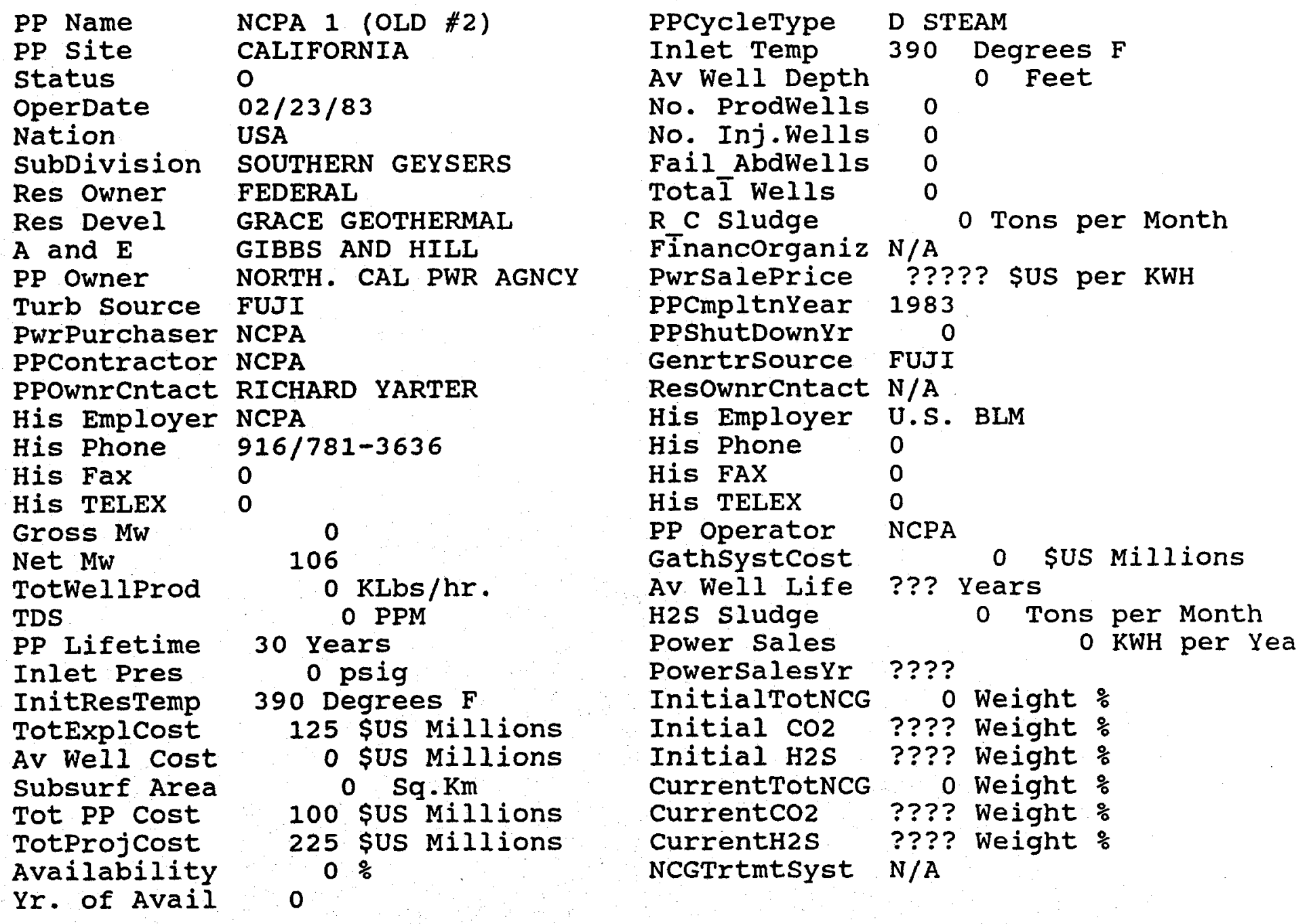




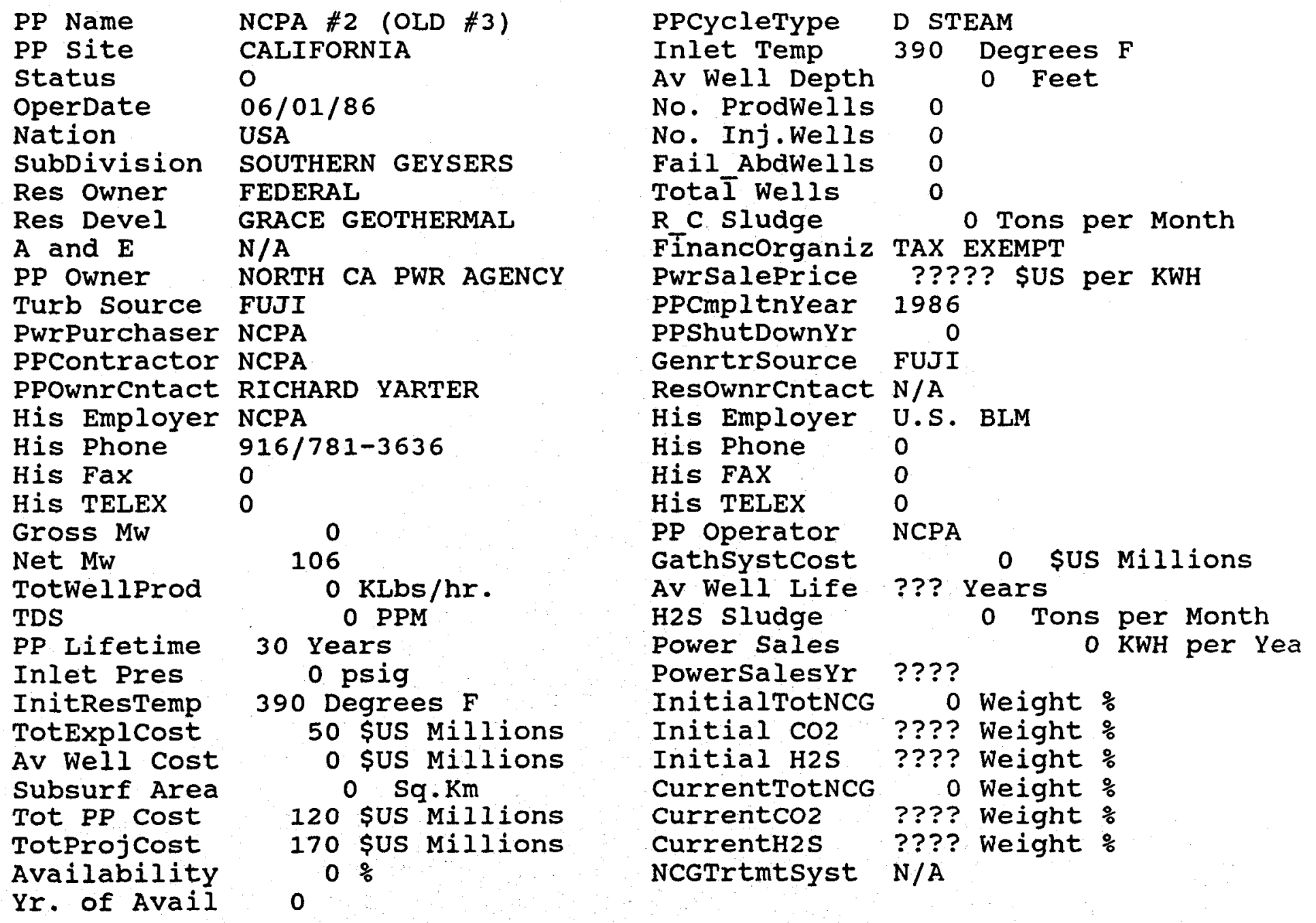

$0 \mathrm{KLbs} / \mathrm{hr}$. 0 PPM

30 Years 0 psig

390 Degrees $F$ 50 \$US Millions 0 SUS Millions $0 \mathrm{Sq} \cdot \mathrm{Km}$ 120 SUS Millions 170 SUS Militions $0 \%$ 


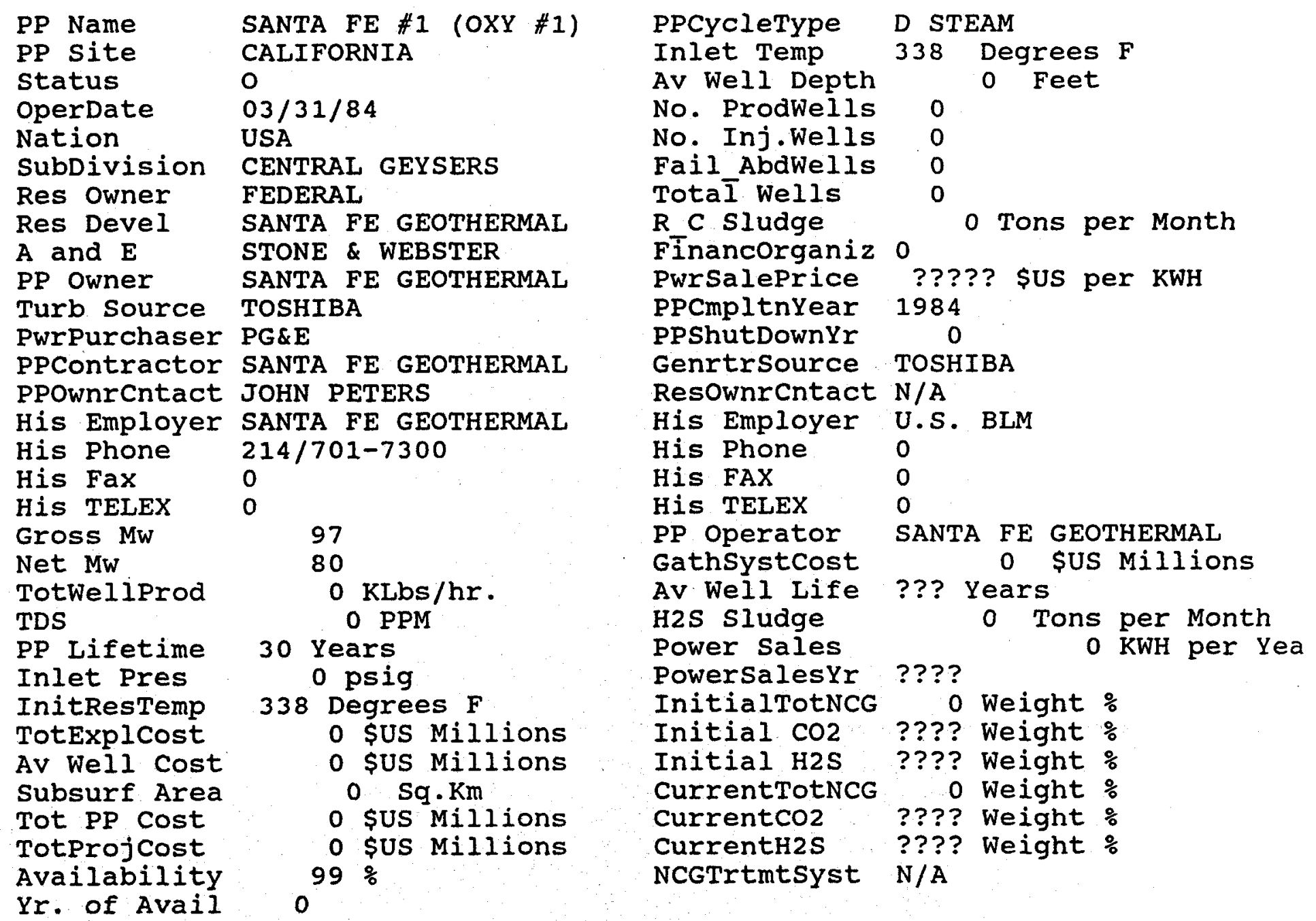




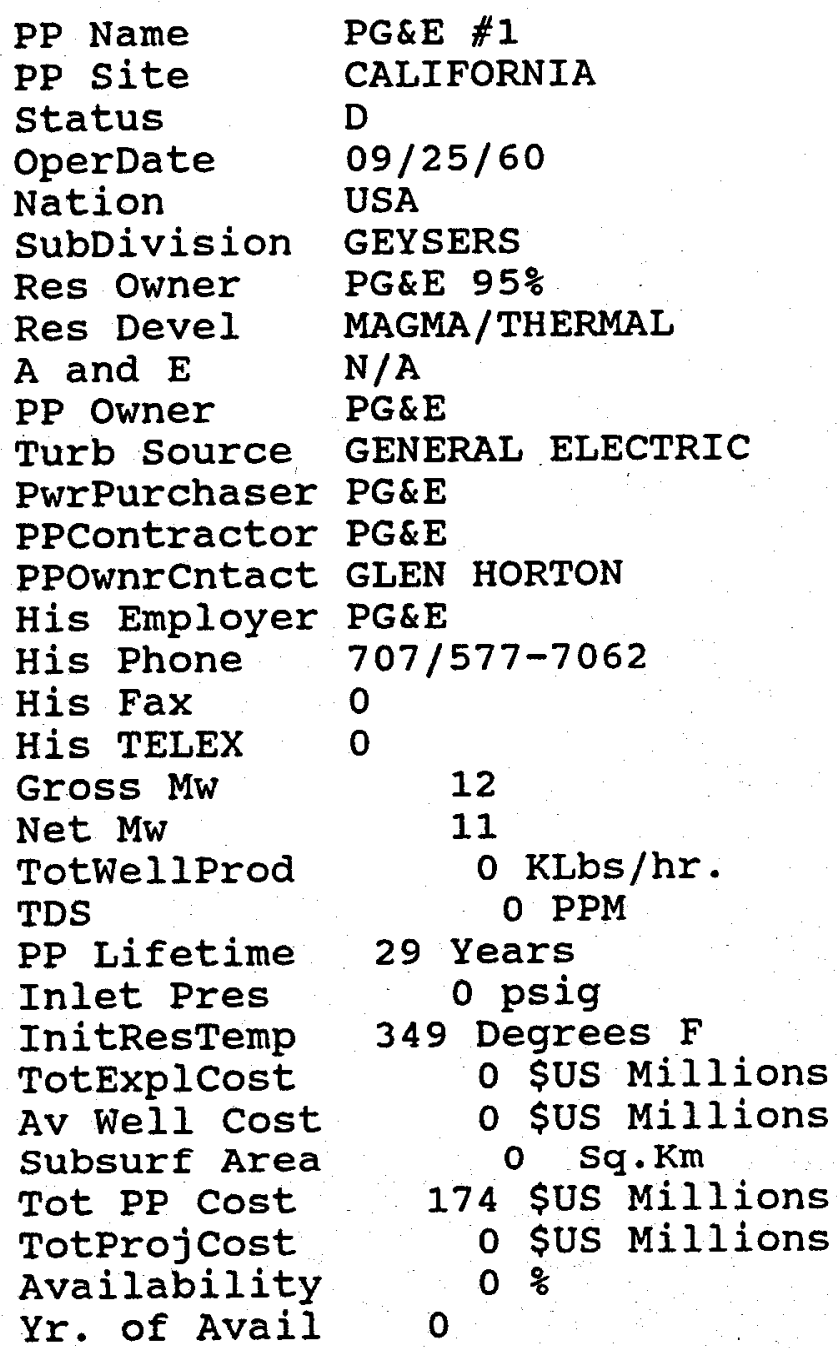

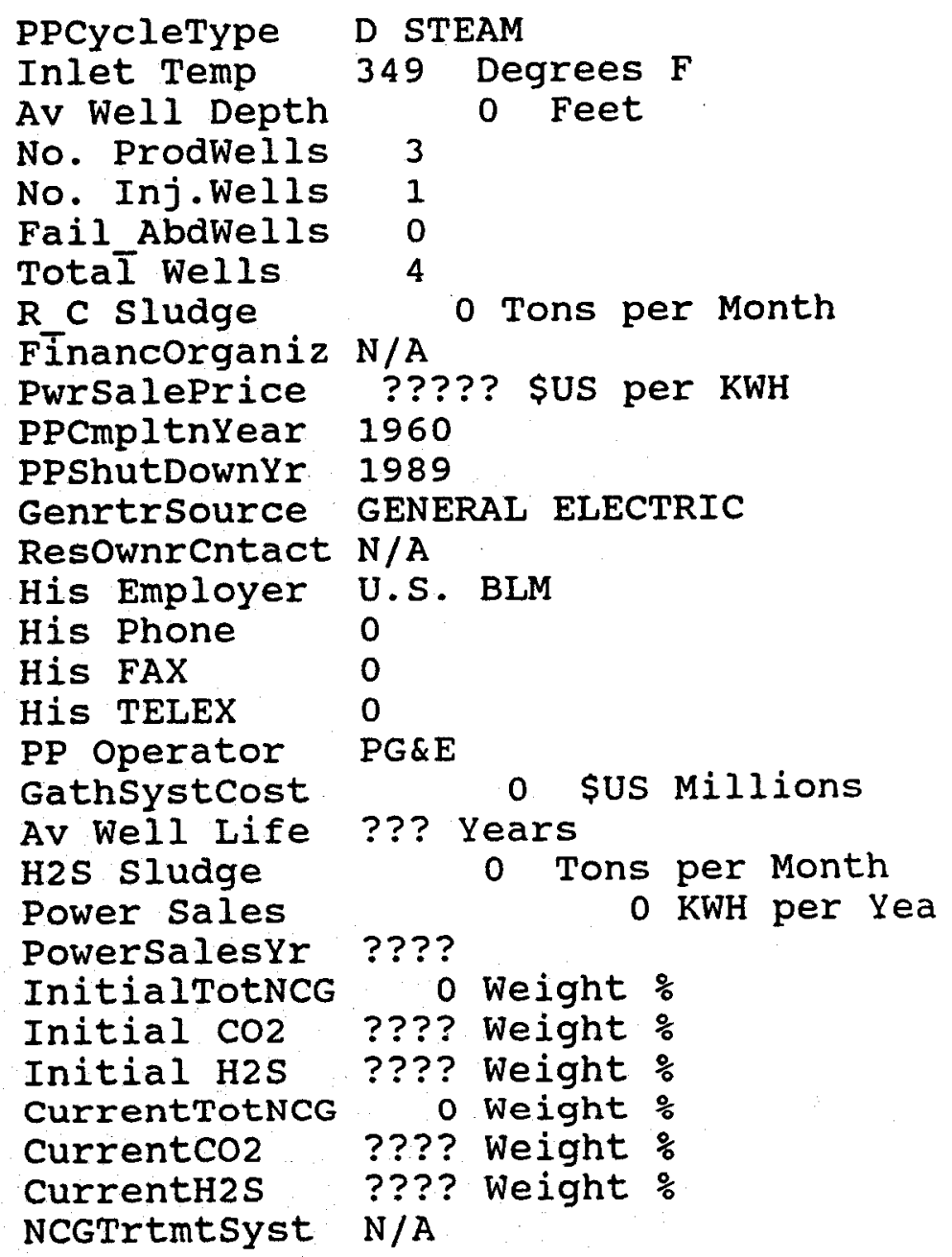




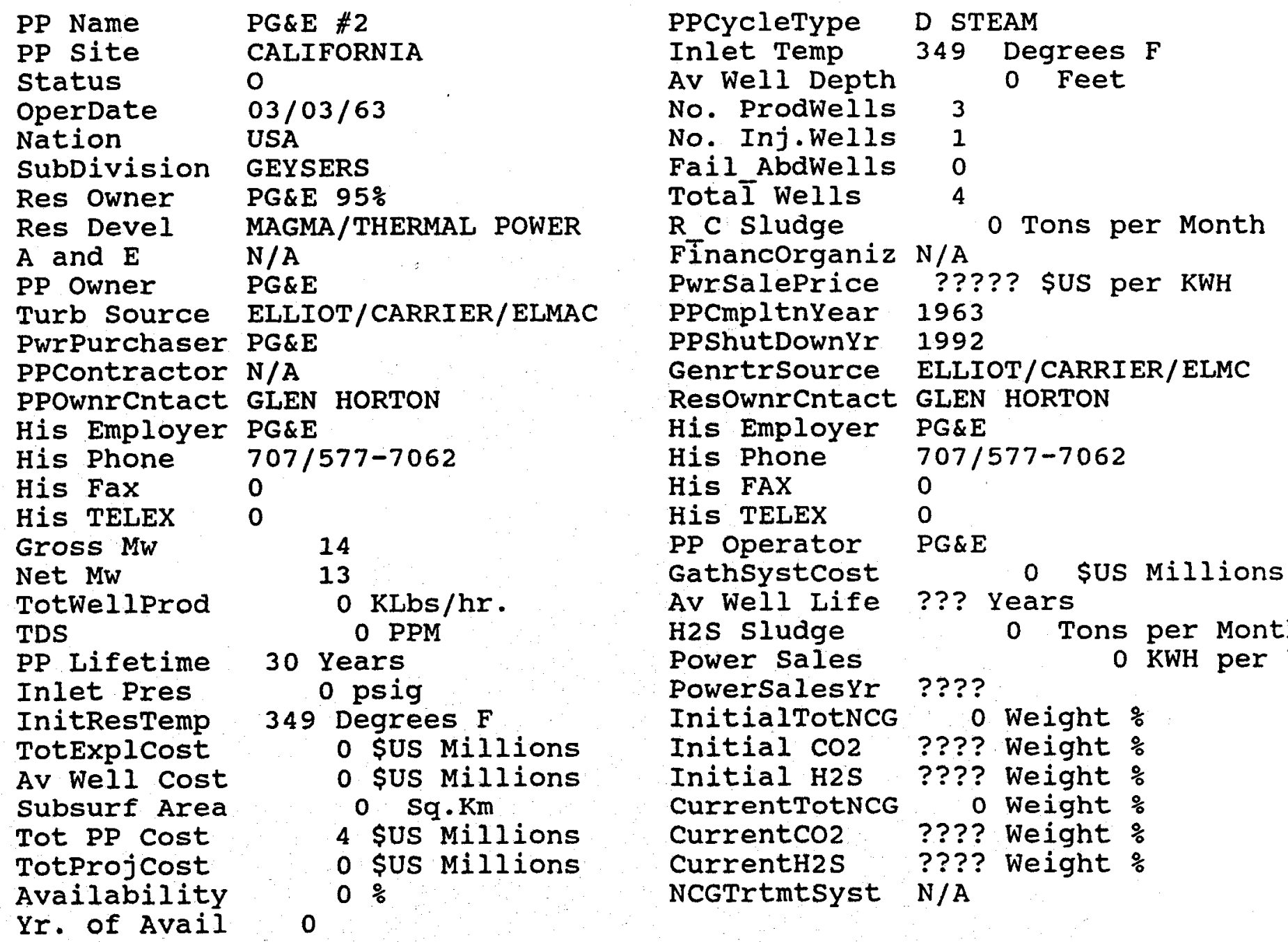

His TELEX 0

CALIFORNIA

$03 / 03 / 63$

USA

GEYSERS

PG\&E 95\%

MAGMA/THERMAL POWER

$N / A$

PG\&E$$
14
$$$$
13
$$$$
0 \mathrm{KLbs} / \mathrm{hr} \text {. }
$$$$
30 \text { Years }
$$$$
0 \text { psig }
$$$$
349 \text { Degrees } F
$$$$
0 \text { \$US Millions }
$$$$
0 \text { SUS Millions }
$$$$
0 \mathrm{Sq} \cdot \mathrm{Km}
$$$$
4 \text { SUS Millions }
$$$$
0 \text { \$US Millions }
$$$$
0 \%
$$

PPCycleType

Inlet Temp

Av Well Depth

No. ProdWells

No. Inj.Welis

Fail AbdWells

TotaI Wells

$R$ C sludge

Fīnancorganiz N/A

D STEAM

349 Degrees $\mathrm{F}$

3

1

0

4

0 Tons per Month

PwrSalePrice ????? \$US per KWH

PPCmpltnYear 1963

PPShutDownYr 1992

GenrtrSource ELLIOT/CARRIER/ELMC

ResOwnrCntact GLEN HORTON

His Employer PG\&E

His Phone 707/577-7062

His FAX 0

HiS TELEX 0

PP Operator PG\&E

Gathsystcost o sus Millions

Av Well Life ??? Years

H2S sludge

Power sales

PowersalesYr

InitialTotNCG

Initial $\mathrm{CO} 2$

Initial H2S ???? Weight \%

currentTotNCG o Weight $\%$

CurrentCO2 ???? Weight $\%$

CurrentH2S ???? Weight $\%$

NCGTrtmtsyst N/A 


\begin{tabular}{|c|c|c|c|}
\hline PP Name & PG\&E \#3 & PPCycleType & D STEAM \\
\hline PP site & CALIFORNIA & Inlet Temp & 342 Degrees $F$ \\
\hline status & 0 & Av Well Depth & 0 Feet \\
\hline perDate & $04 / 28 / 67$ & No. ProdWells & 0 \\
\hline ation & USA & No. Inj.Wells & 0 \\
\hline SubDivision & GEYSERS & Fail_AbaWells & 0 \\
\hline Res Owner & PG\&E & Total Wells & 0 \\
\hline $\begin{array}{l}\text { Res Devel } \\
A \text { and } E\end{array}$ & $\begin{array}{l}\text { UNOCAL/MAGMA/THERMAL } \\
\text { N/A }\end{array}$ & $\begin{array}{l}R \text { C sludge } \\
\text { Financorganiz }\end{array}$ & 0 Tons per Month \\
\hline PP Owner & PG\&E & PwrSalePrice & ????? \$US per KWH \\
\hline Turb Source & ELLIOT/CARRIER/ELMAC & PPCmpltnYear & 1967 \\
\hline PwrPurchaser & PG\&E & PPShutDownYr & 0 \\
\hline Pcontractor & PG\&E & GenrtrSource & ELLIOT/CARRIER/ELMC \\
\hline Pownrcntact & GLEN HORTON & Resownrcntact & GLEN HORTON \\
\hline iis Employer & $P G \& E$ & His Employer & PG\&E \\
\hline His Phone & $707 / 577-7062$ & His Phone & $707 / 577-7062$ \\
\hline His Fax & 0 & His FAX & 0 \\
\hline His TELEX & & His TELEX & 0 \\
\hline Gross MW & 28 & PP Operator & $P G \& E$ \\
\hline Net $M w$ & 27 & Gathsystcost & \$US Millions \\
\hline $\begin{array}{l}\text { Totwel1Prod } \\
\text { TDS }\end{array}$ & $\begin{array}{l}0 \text { KLbs/hr. } \\
0 \text { PPM }\end{array}$ & $\begin{array}{l}\text { Av Well Life } \\
\text { H2S sludge }\end{array}$ & $\begin{array}{l}\text { ??? Years } \\
0 \text { Tons per Month }\end{array}$ \\
\hline $\begin{array}{l}\text { PP Lifetime } \\
\text { Inlet Pres }\end{array}$ & $\begin{array}{l}30 \text { Years } \\
0 \text { psig }\end{array}$ & $\begin{array}{l}\text { Power sales } \\
\text { PowersalesYr }\end{array}$ & 0 KWH per Yea \\
\hline $\begin{array}{l}\text { InitResTemp } \\
\text { TotExplCost }\end{array}$ & $\begin{array}{l}342 \text { Degrees } F \\
\text { O \$US Millions }\end{array}$ & $\begin{array}{l}\text { InitialTotNCG } \\
\text { Initial } \mathrm{CO} 2\end{array}$ & $\begin{array}{r}0 \text { Weight } \% \\
\text { ???? Weight } \%\end{array}$ \\
\hline $\begin{array}{l}\text { Av Well Cost } \\
\text { subsurf Area }\end{array}$ & $\begin{array}{l}0 \text { SUS Millions } \\
0 \quad \mathrm{Sq} \cdot \mathrm{Km}\end{array}$ & $\begin{array}{l}\text { Initial H2S } \\
\text { currentTotNCG }\end{array}$ & $\begin{array}{r}\text { ???? Weight \% } \\
\text { o Weight \% }\end{array}$ \\
\hline $\begin{array}{l}\text { Tot PP cost } \\
\text { TotProjcost } \\
\text { Availability }\end{array}$ & $\begin{array}{l}4 \text { \$US Millions } \\
0 \text { \$US Militions } \\
0 \%\end{array}$ & $\begin{array}{l}\text { CurrentCO2 } \\
\text { CurrentH2S } \\
\text { NCGTrtmtsyst }\end{array}$ & $\begin{array}{l}\text { ???? Weight } \% \\
\text { ???? Weight } \\
\text { N/A }\end{array}$ \\
\hline inf $x=$ & 0 & & \\
\hline
\end{tabular}




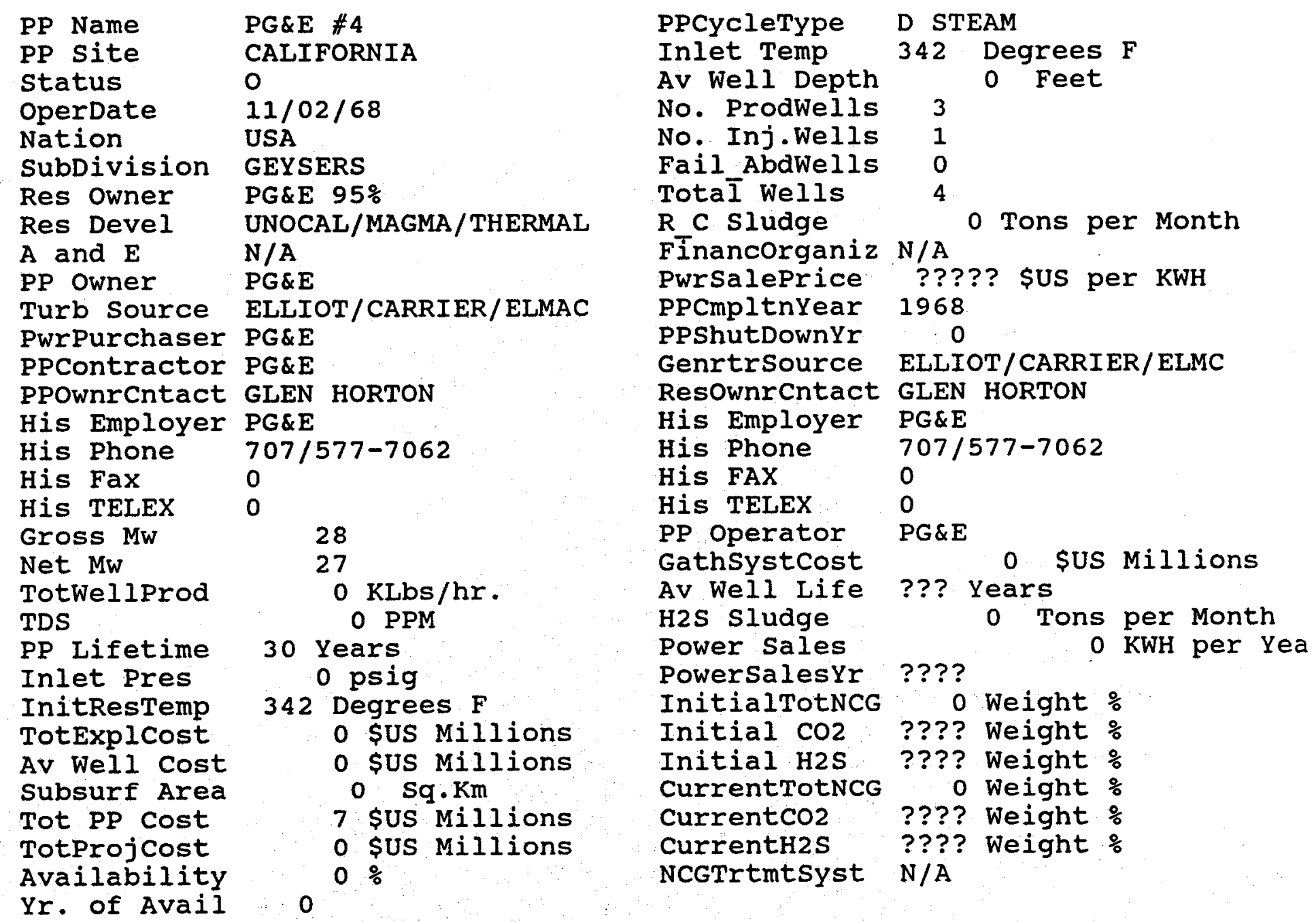




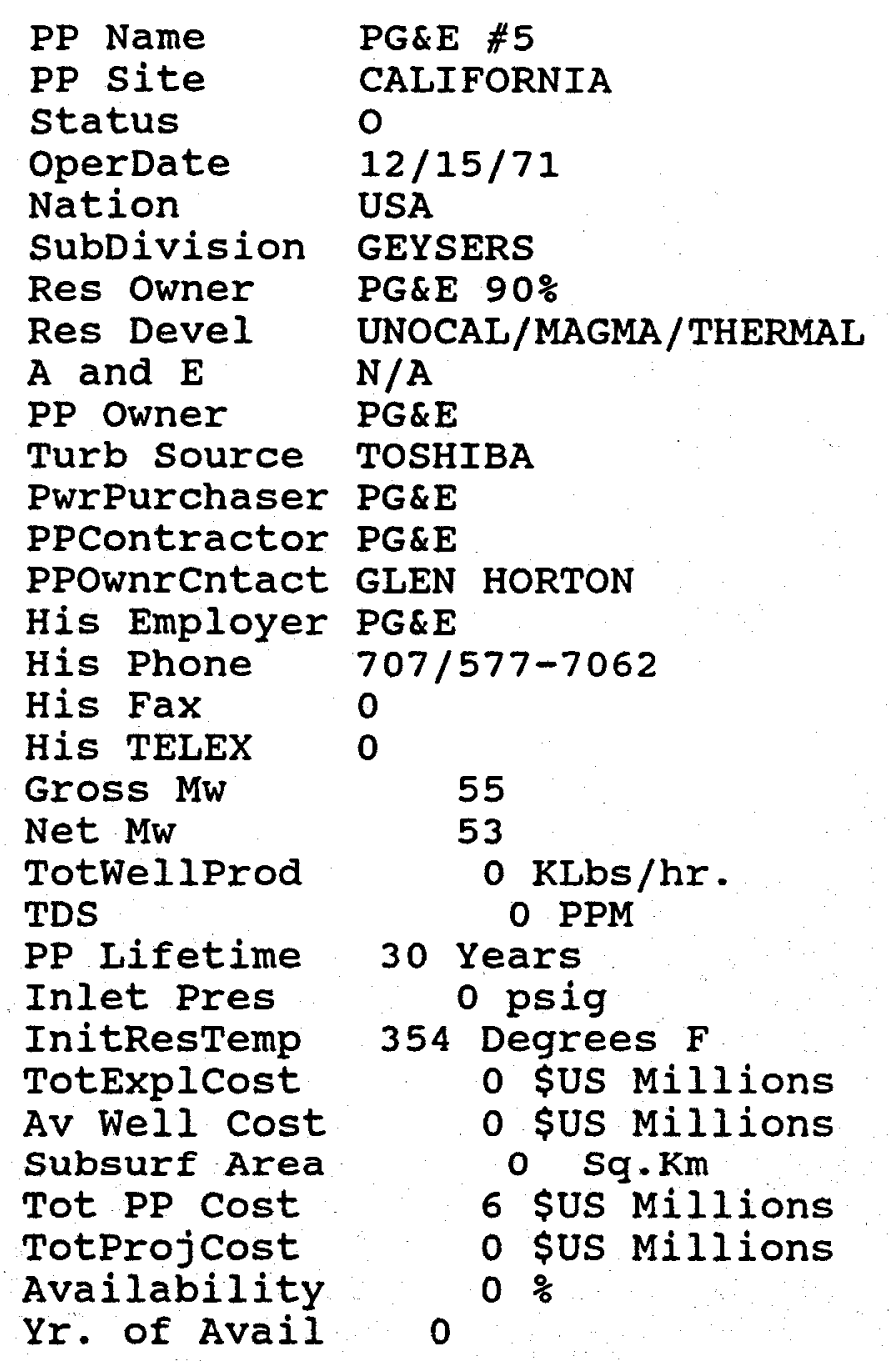

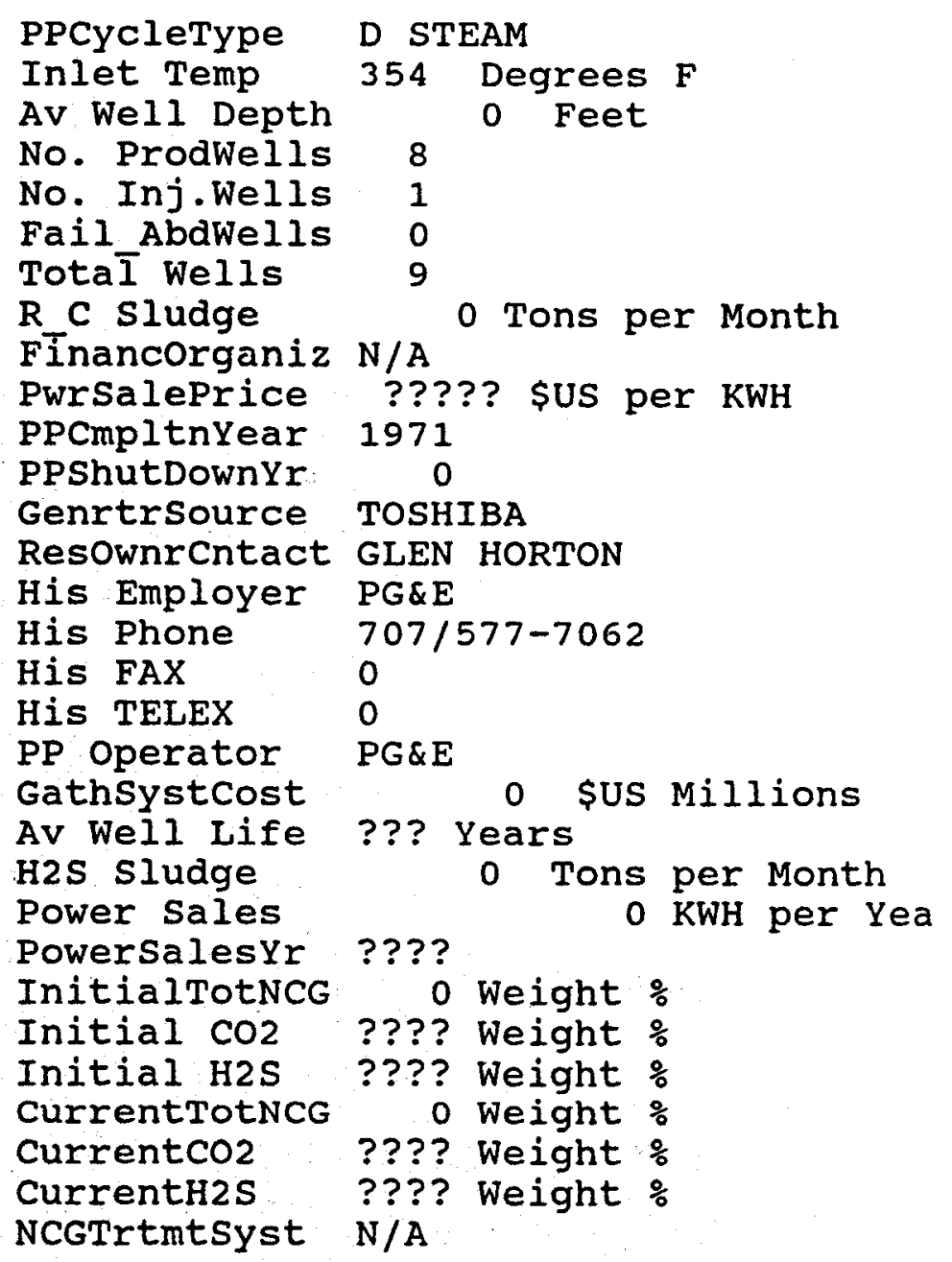




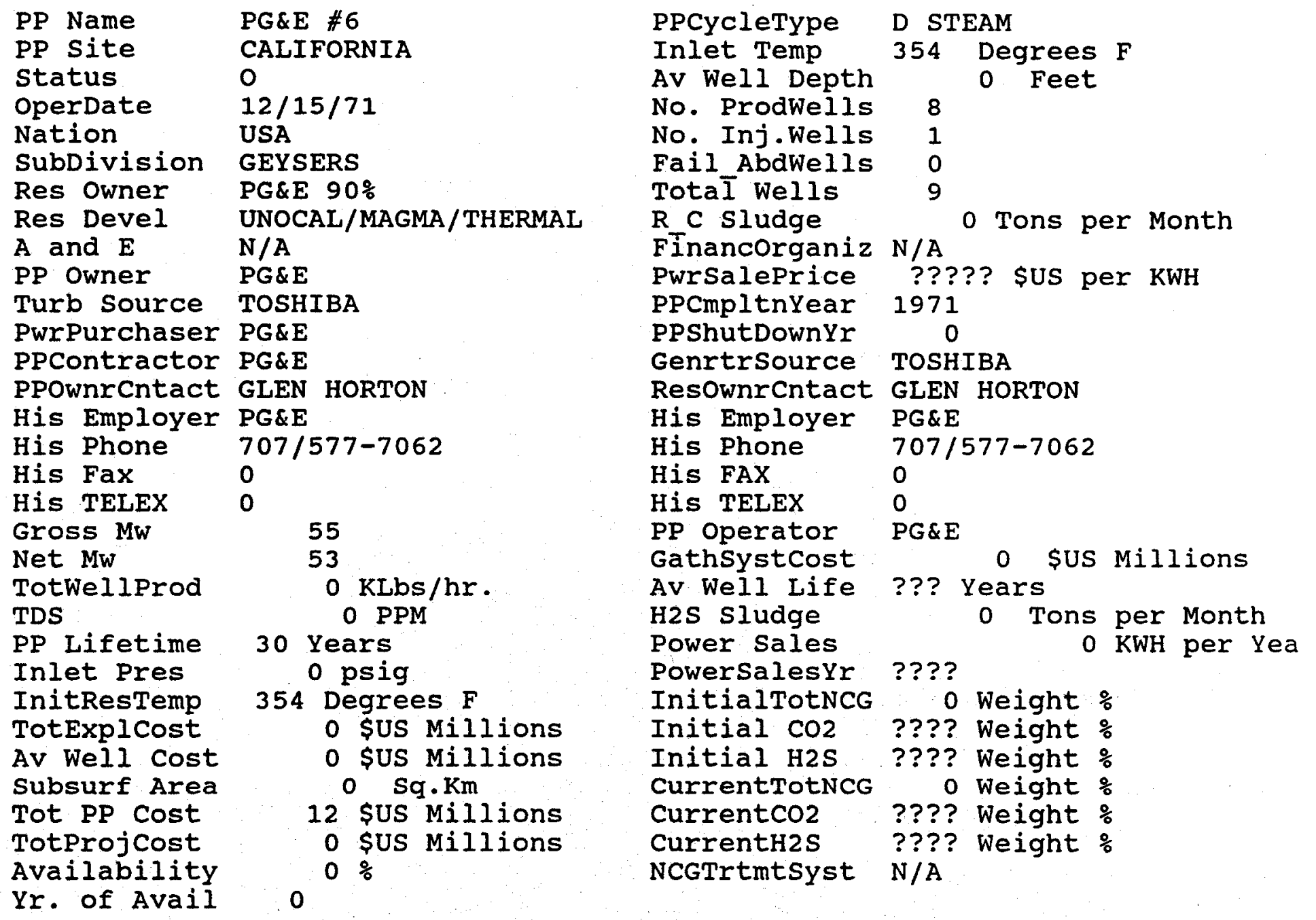

PwrPurchaser PG\&E

PPOwnrcntact GLEN HORTON

His Phone 707/577-7062

PG\&E \#6

CALIFORNIA

$12 / 15 / 71$

USA

GEYSERS

PG\&E 90\%

UNOCAL/MAGMA/THERMAL

N/A

PG\&E

5

53

$0 \mathrm{KLbs} / \mathrm{hr}$. 0 PPM

30 Years

0 psig

354 Degrees $F$

0 \$US Millions

0 \$US Millions

- $\mathrm{Sq} . \mathrm{Km}$

12 SUS Millions

0 SUS Millions

$0 \%$ 


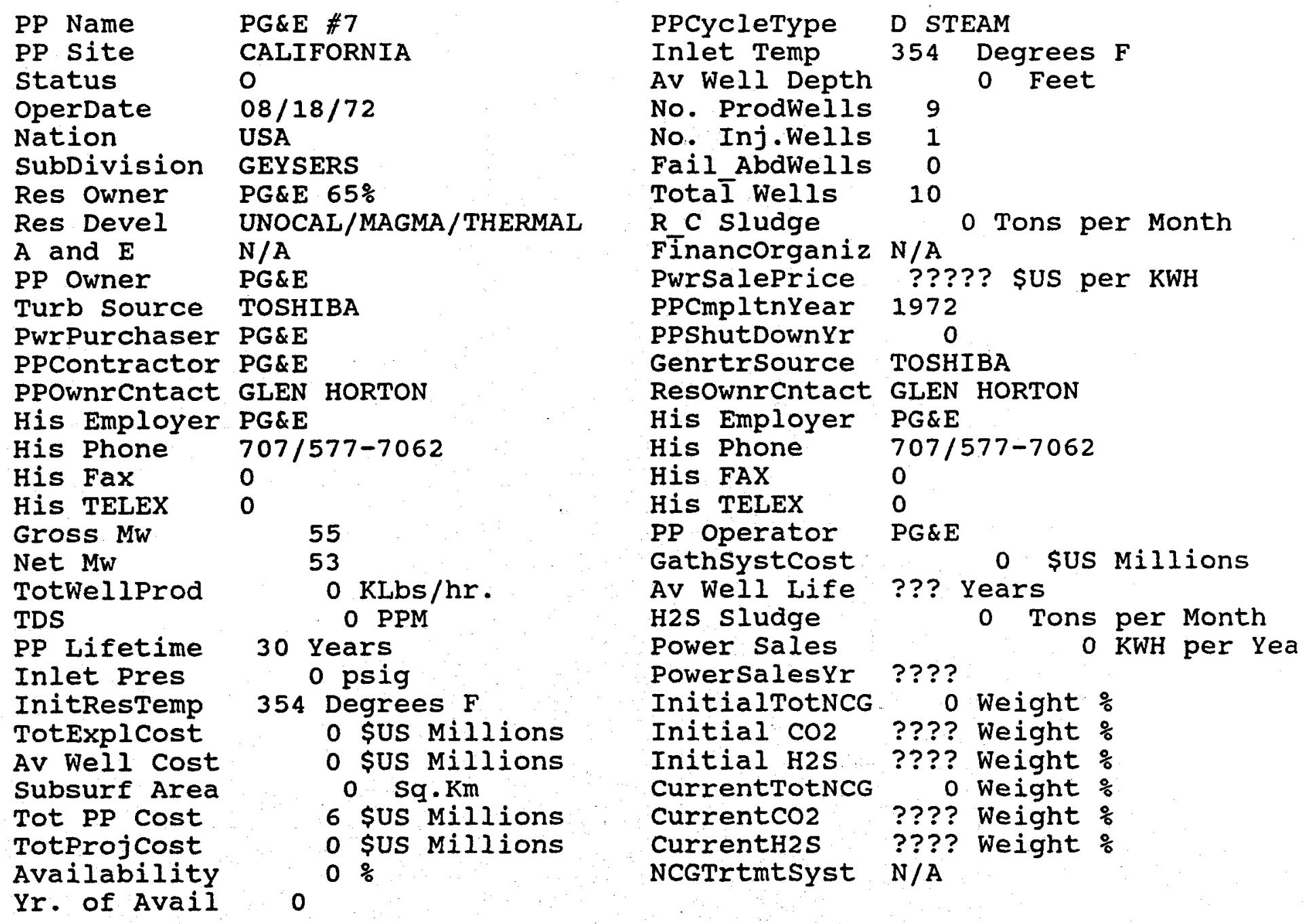




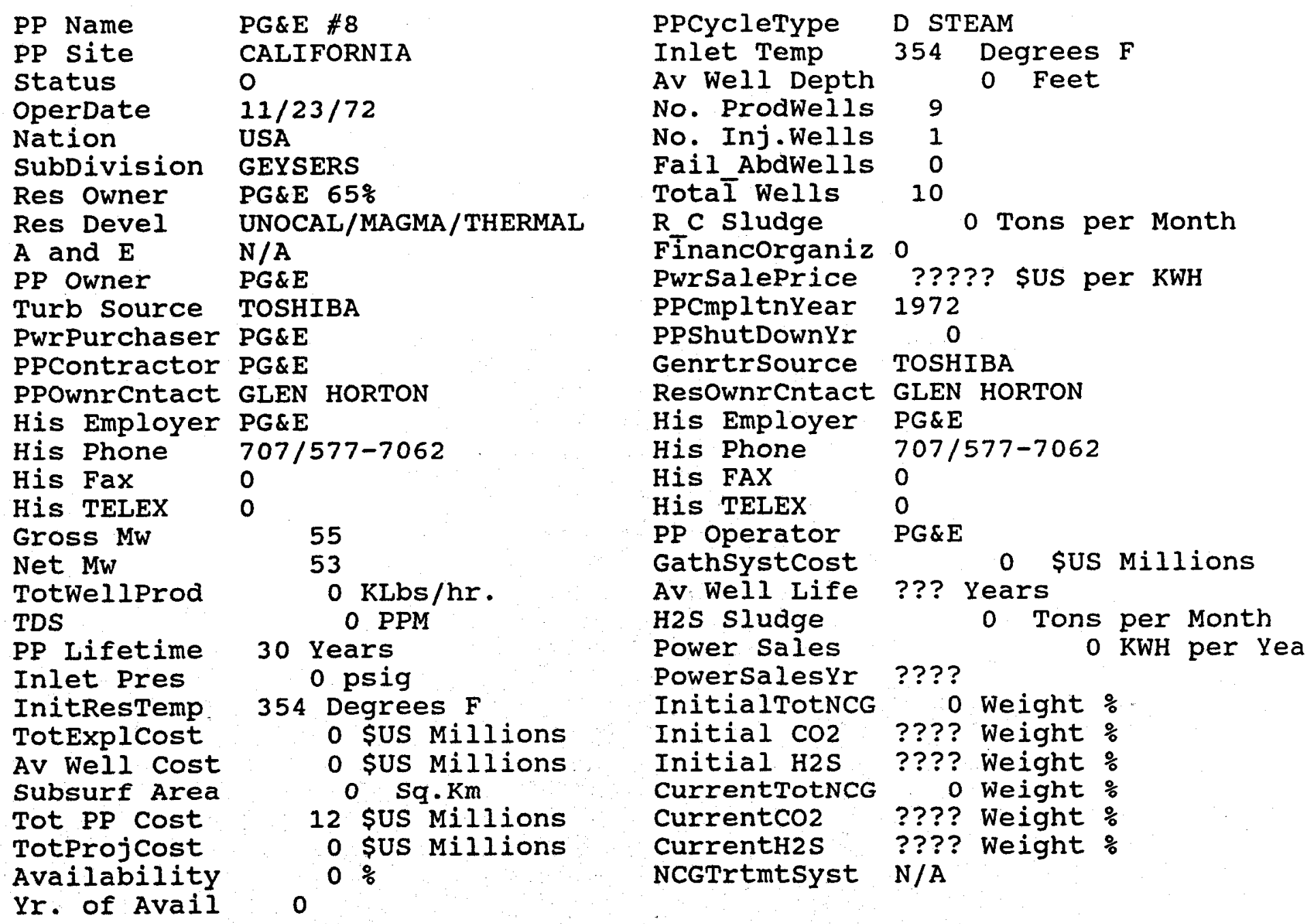




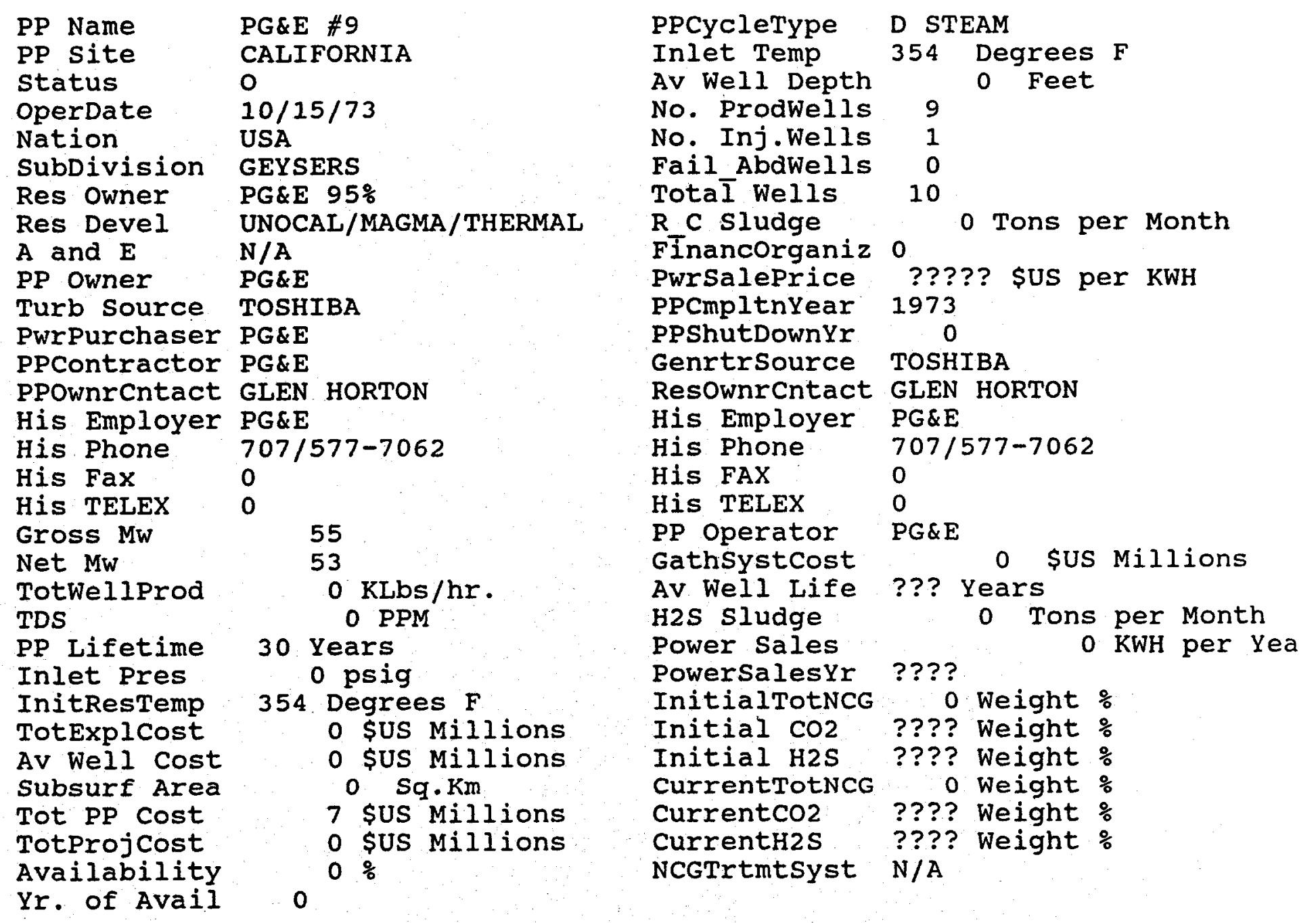




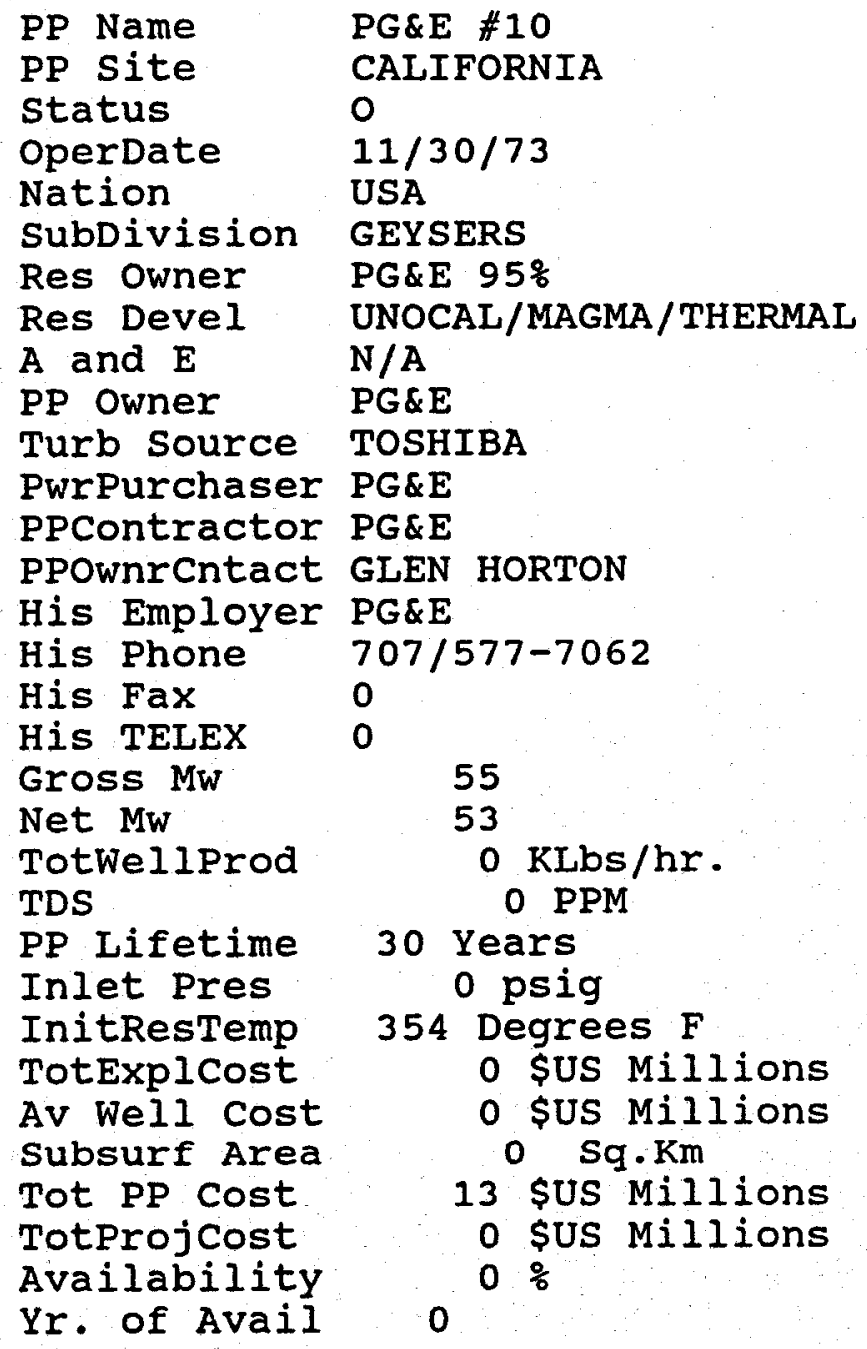

53 


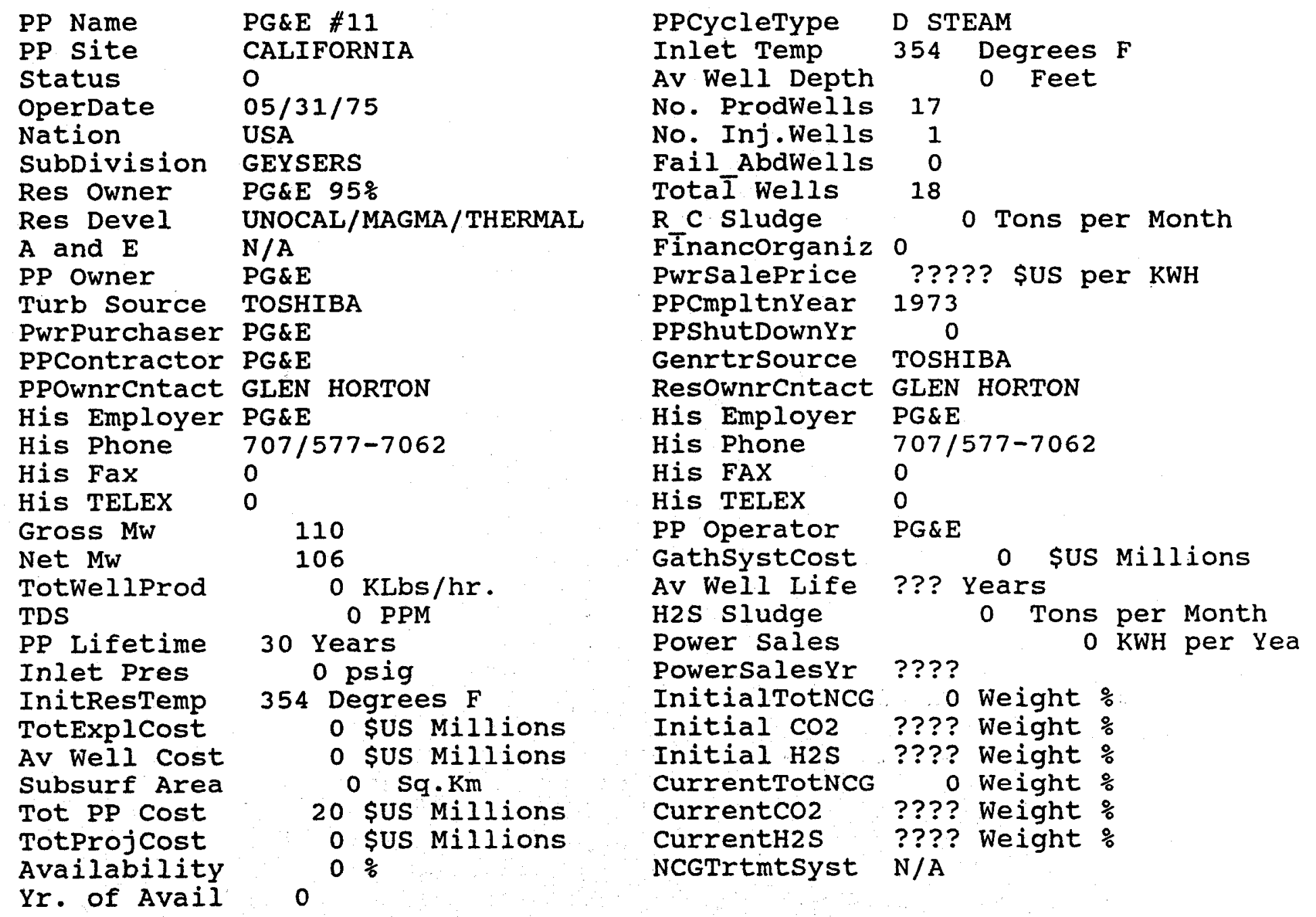




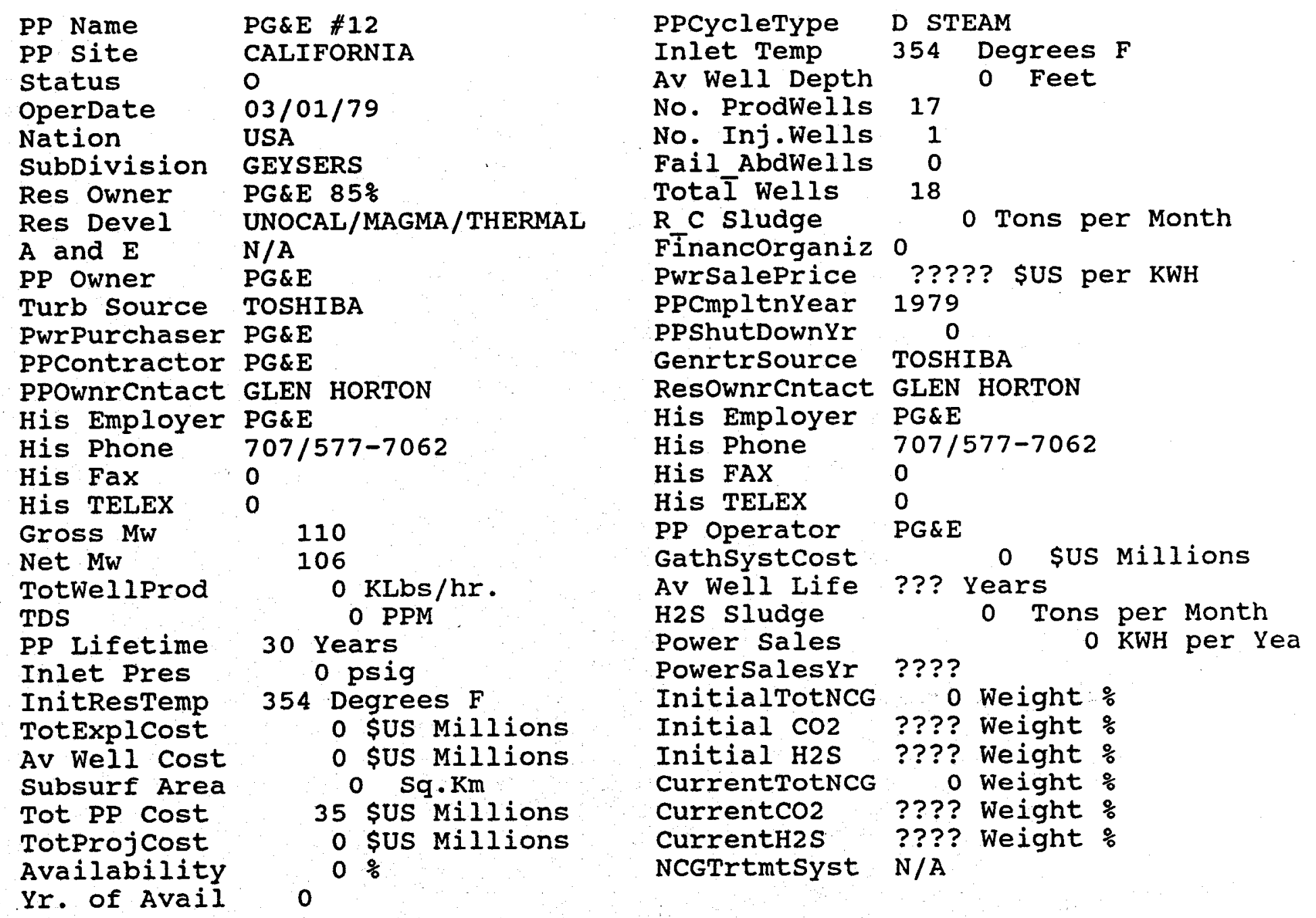




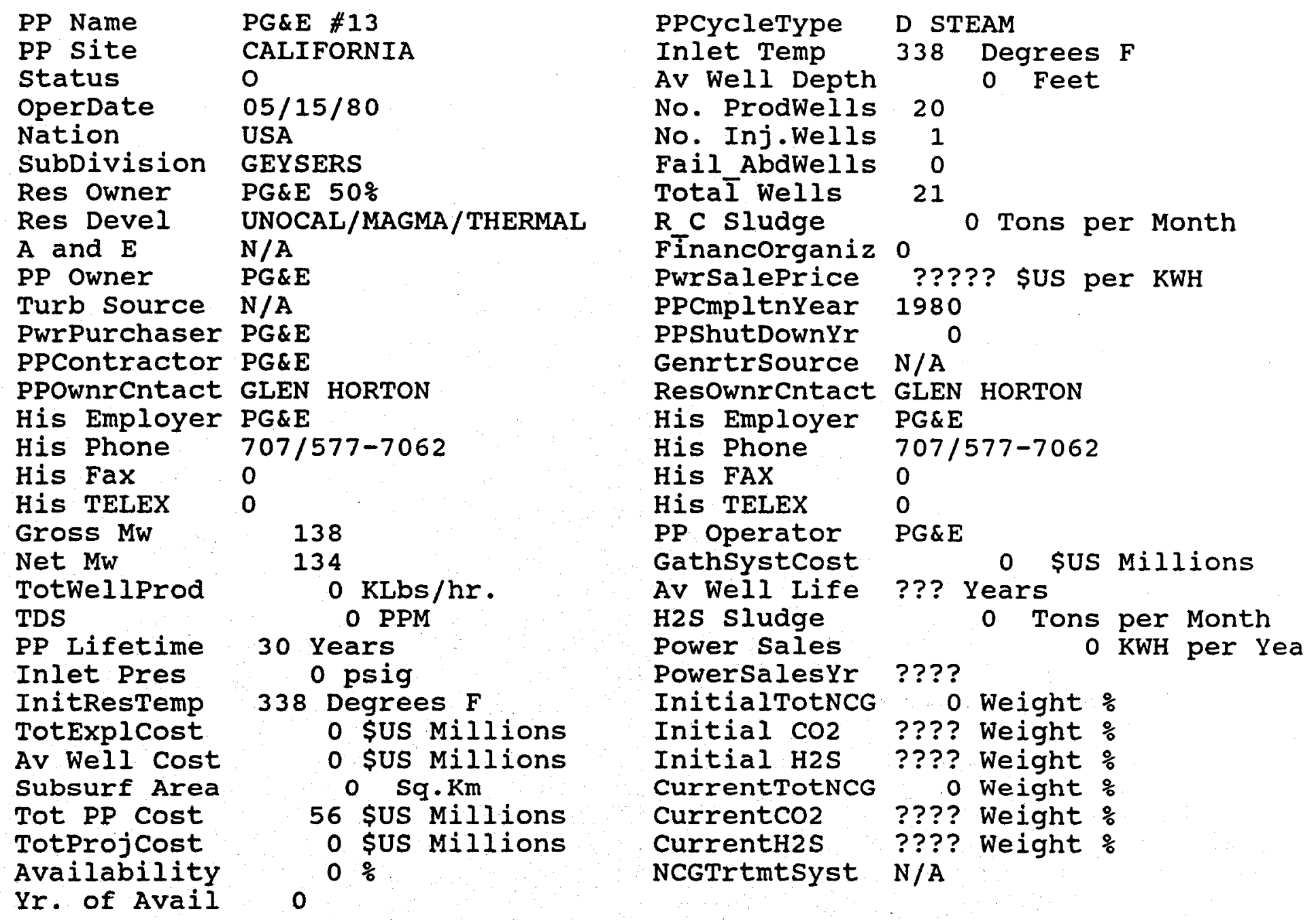




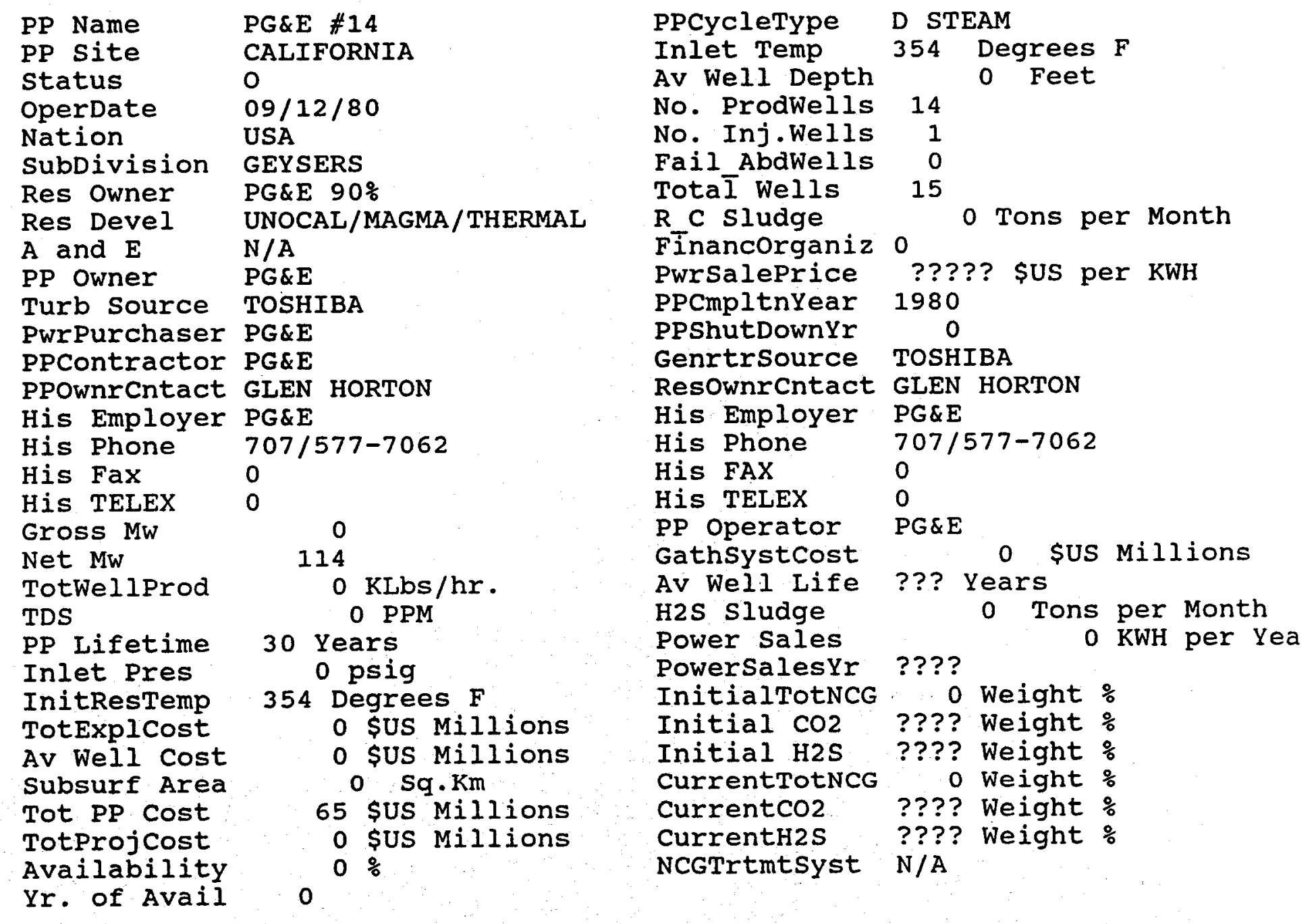




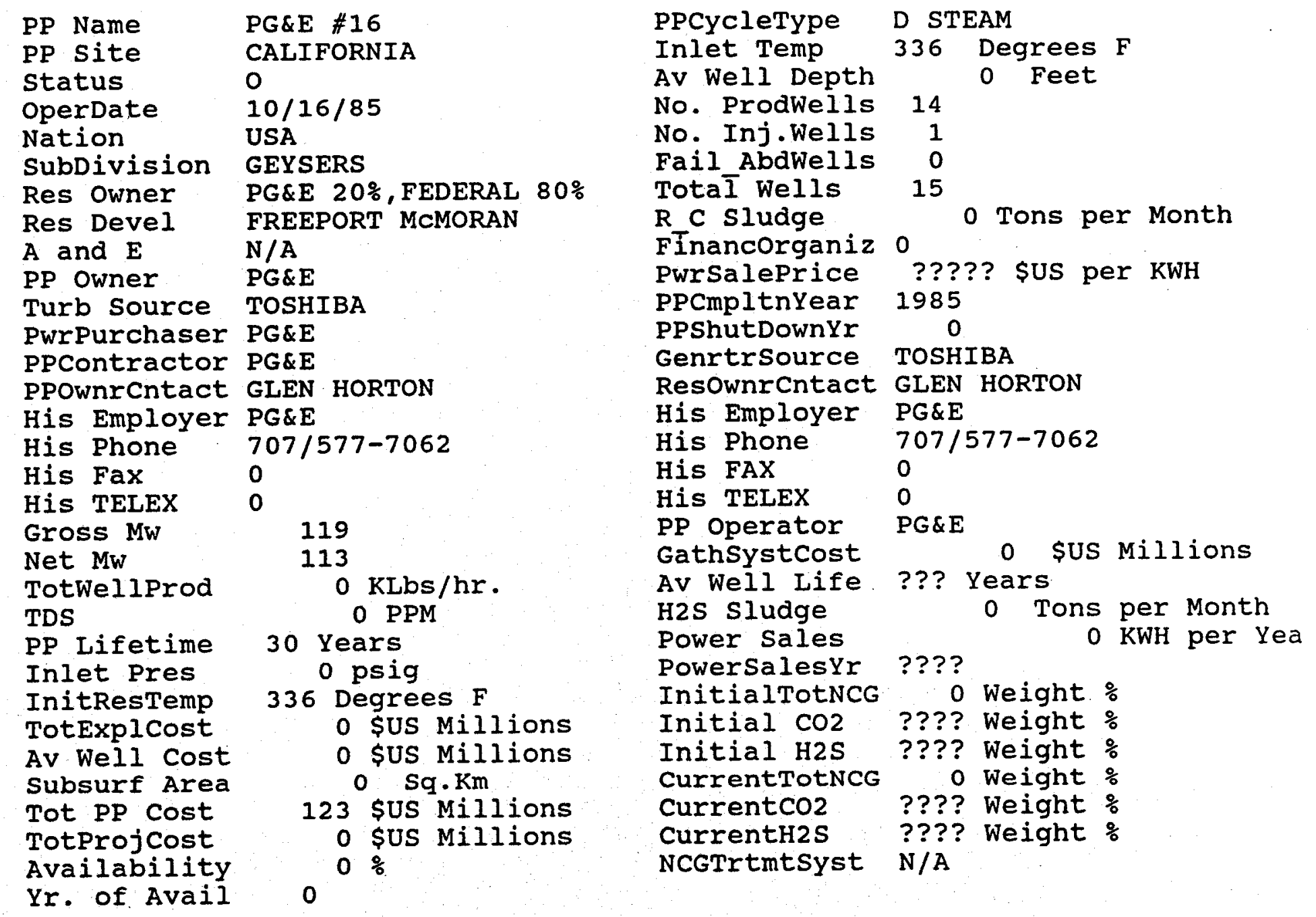




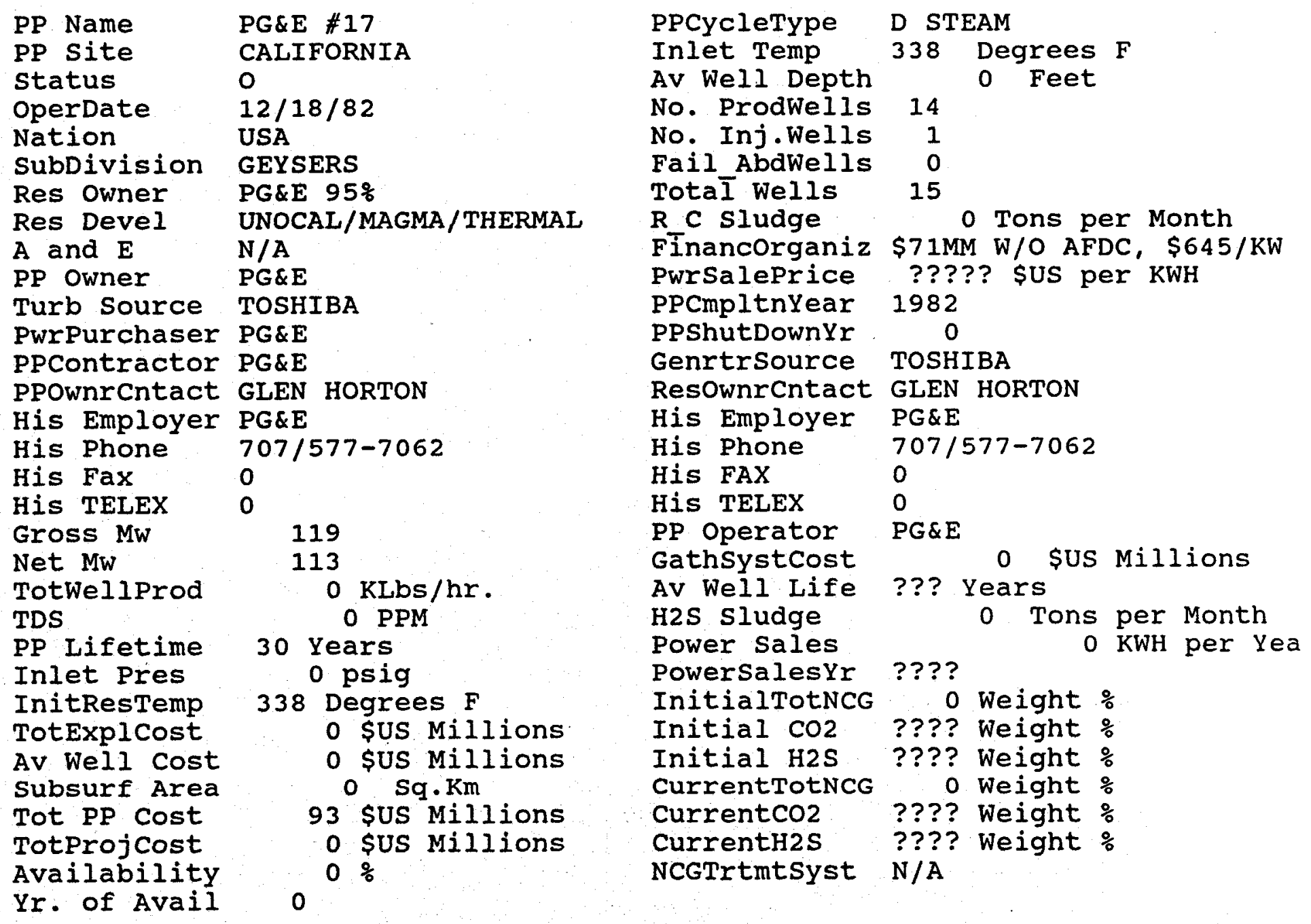




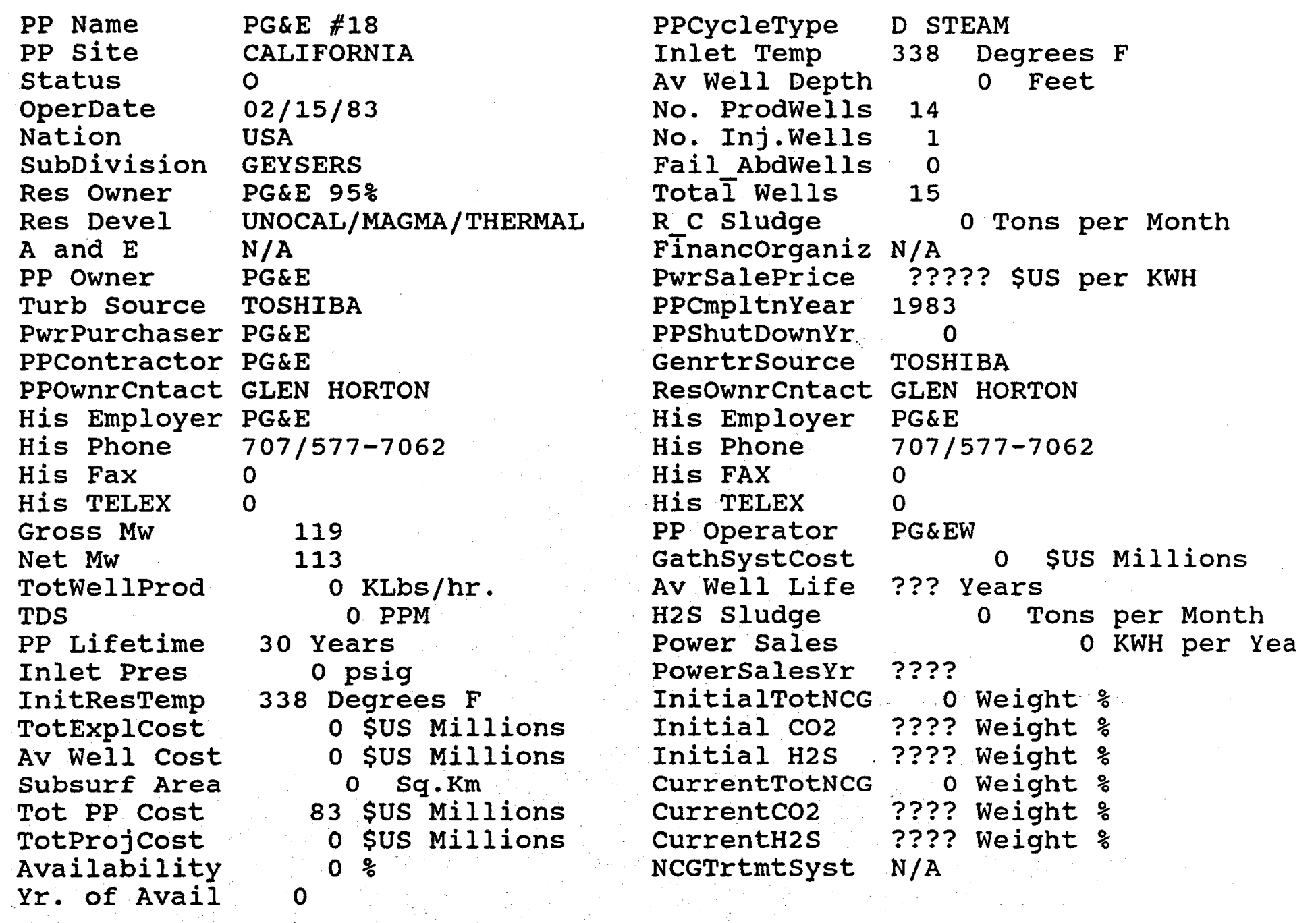




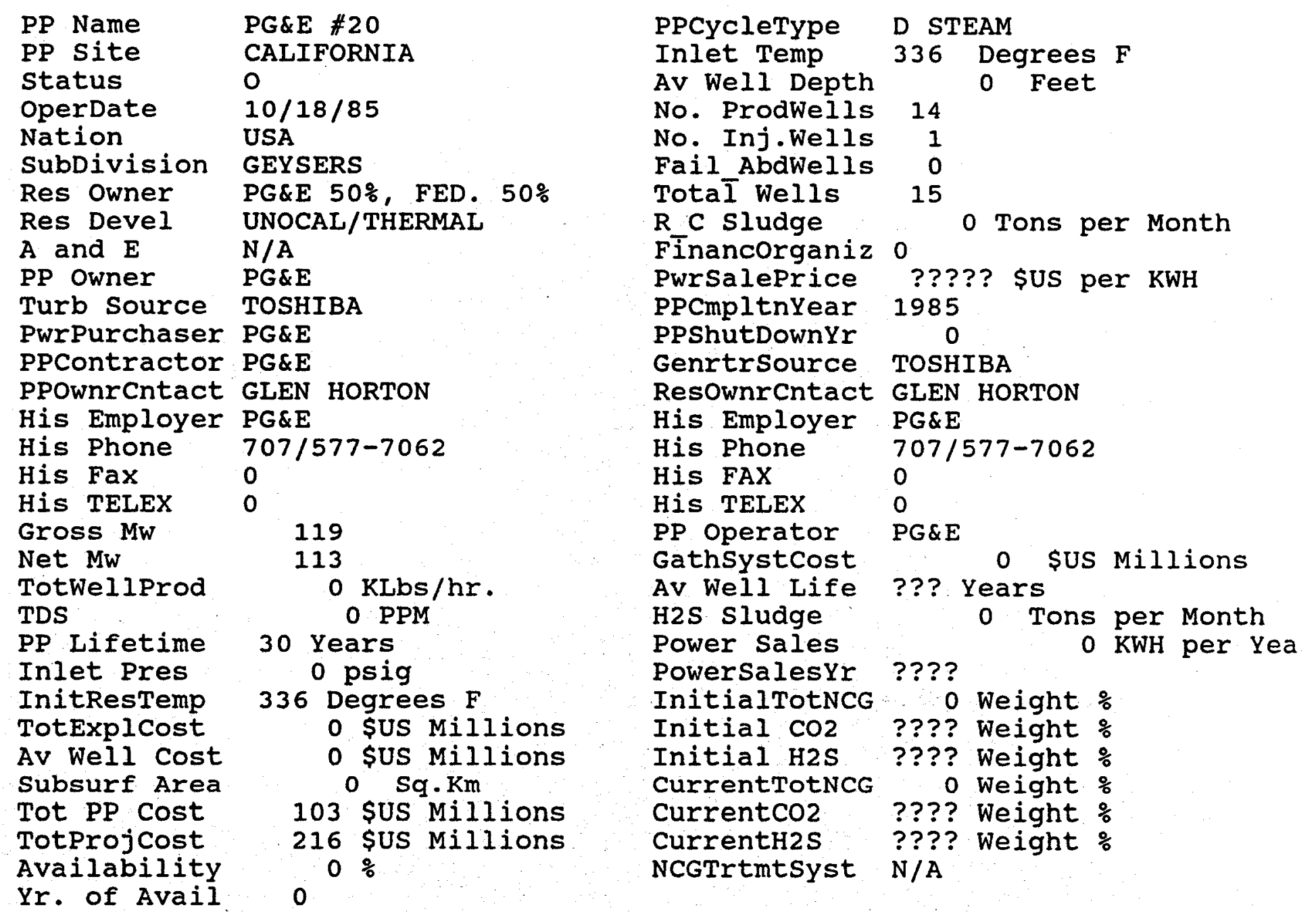




\begin{tabular}{|c|c|}
\hline PP Name & HONEY LAKE \\
\hline PP Site & CALIFORNIA \\
\hline status & 0 \\
\hline OperDate & $01 / 01 / 88$ \\
\hline lation & USA \\
\hline SubDivision & SUSANVILLE, CA \\
\hline es Owner & PRIVATE \\
\hline Res Devel & GEOPRODUCTS \\
\hline $\mathrm{A}$ and $\mathrm{E}$ & $\mathrm{N} / \mathrm{A}$ \\
\hline P Owner & GEOPRODUCTS \\
\hline rurb source & $N / A$ \\
\hline PwrPurchaser & PG\&E \\
\hline PPContractor & $N / A$ \\
\hline PPownrcntact & KEN BOREN \\
\hline His Employer & GEOPRODUCTS \\
\hline His Phone & $415 / 935-9627$ \\
\hline His Fax & 0 \\
\hline His TELEX & 0 \\
\hline Gross $\mathrm{MW}$ & 0 \\
\hline Net Mw & 30 \\
\hline $\begin{array}{l}\text { Totwell Prod } \\
\text { TDS }\end{array}$ & $\begin{array}{l}0 \mathrm{KLbs} / \mathrm{hr} \text {. } \\
2000 \mathrm{PPM}\end{array}$ \\
\hline $\begin{array}{l}\text { PP Lifetime } \\
\text { Inlet Pres }\end{array}$ & $\begin{array}{l}30 \text { Years } \\
0 \text { psiq }\end{array}$ \\
\hline $\begin{array}{l}\text { InitResTemp } \\
\text { TotExplCost }\end{array}$ & $\begin{array}{l}228 \text { Degrees } F \\
0 \text { SUS Militions }\end{array}$ \\
\hline Av Well cost & o \$US Millions \\
\hline Subsurf Area & $0 \mathrm{Sq} \cdot \mathrm{Km}$ \\
\hline $\begin{array}{l}\text { Tot PP cost } \\
\text { TotProjcost } \\
\text { Availability }\end{array}$ & $\begin{array}{l}0 \text { \$US Millions } \\
0 \text { \$US Millions } \\
0 \%\end{array}$ \\
\hline 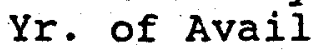 & 0 \\
\hline
\end{tabular}

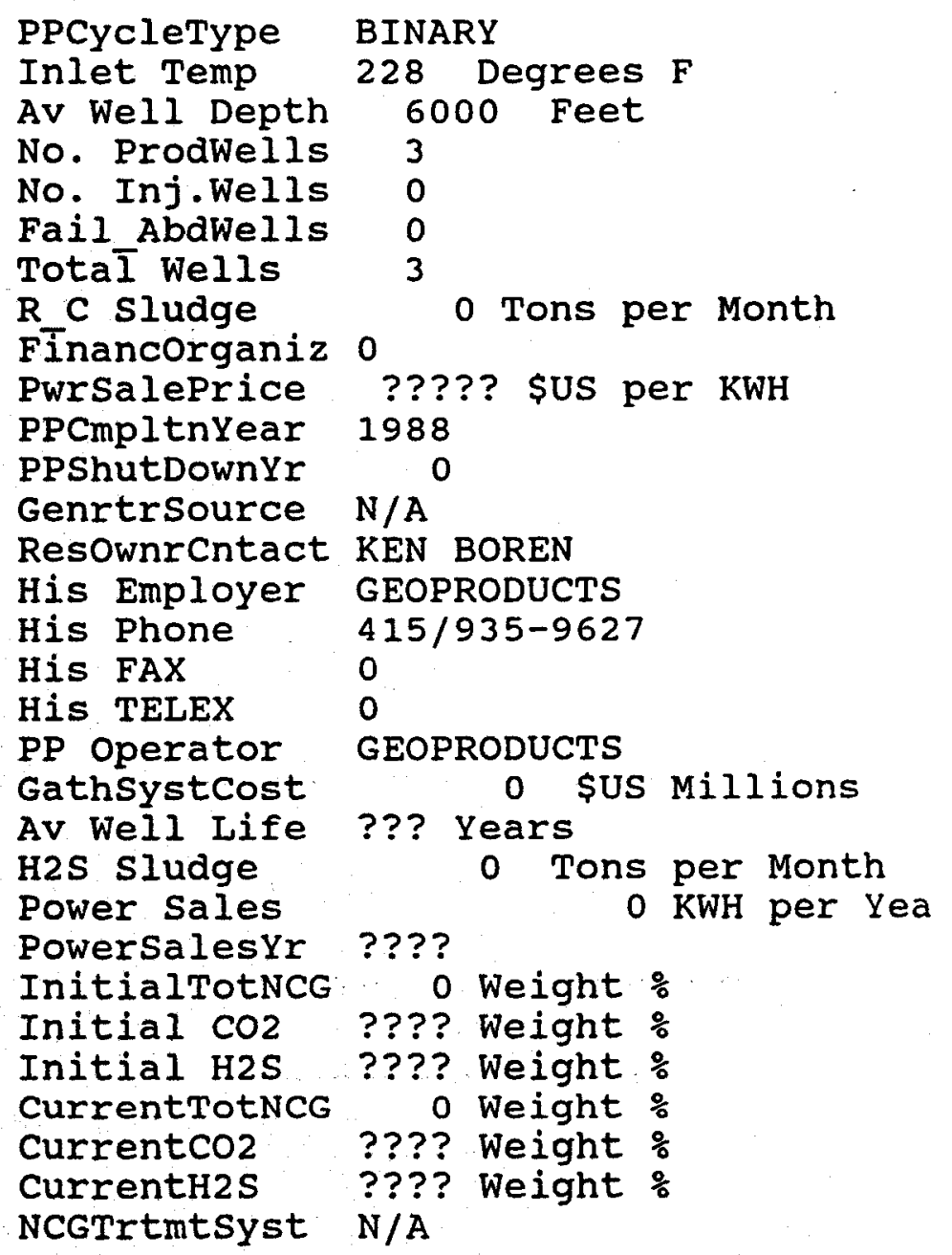




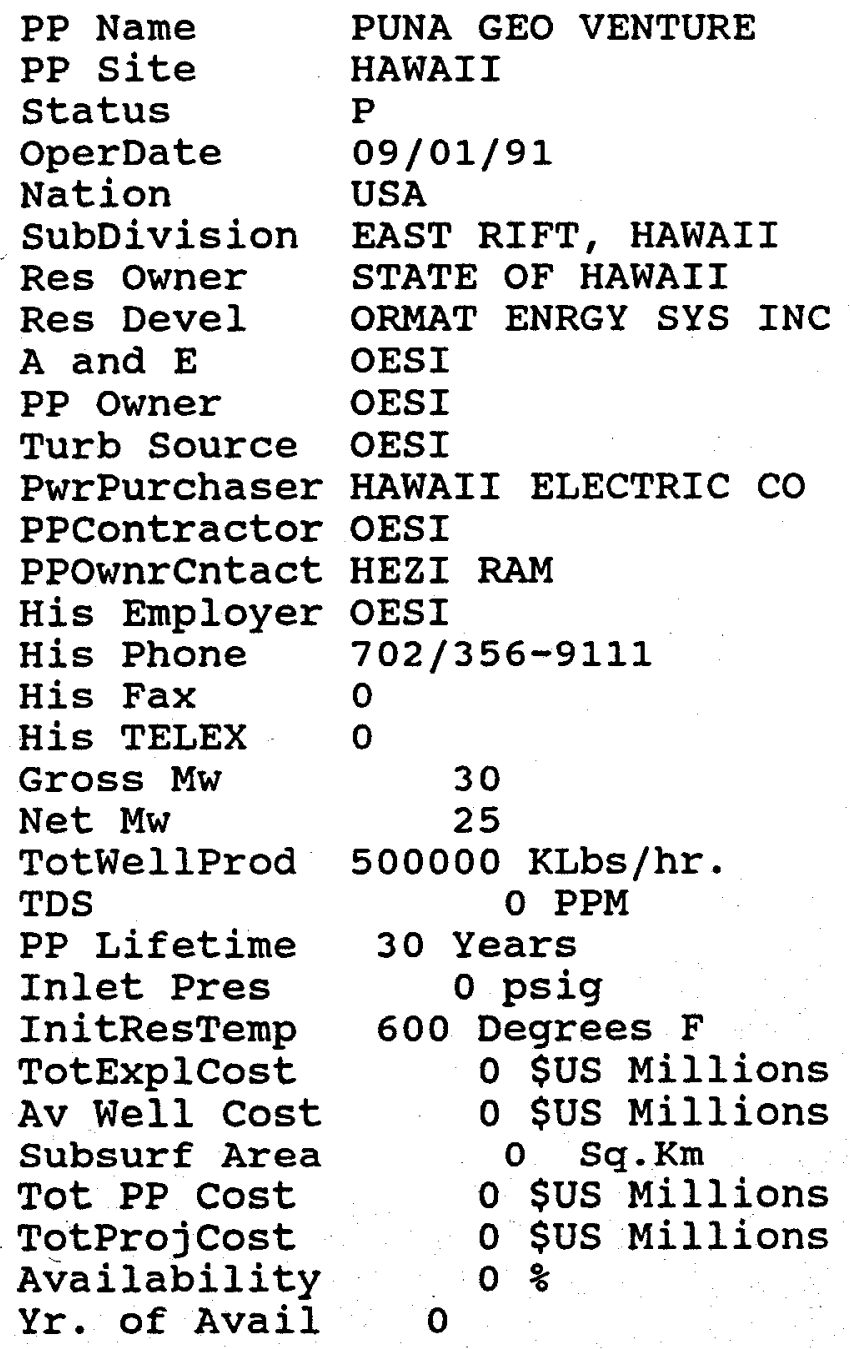

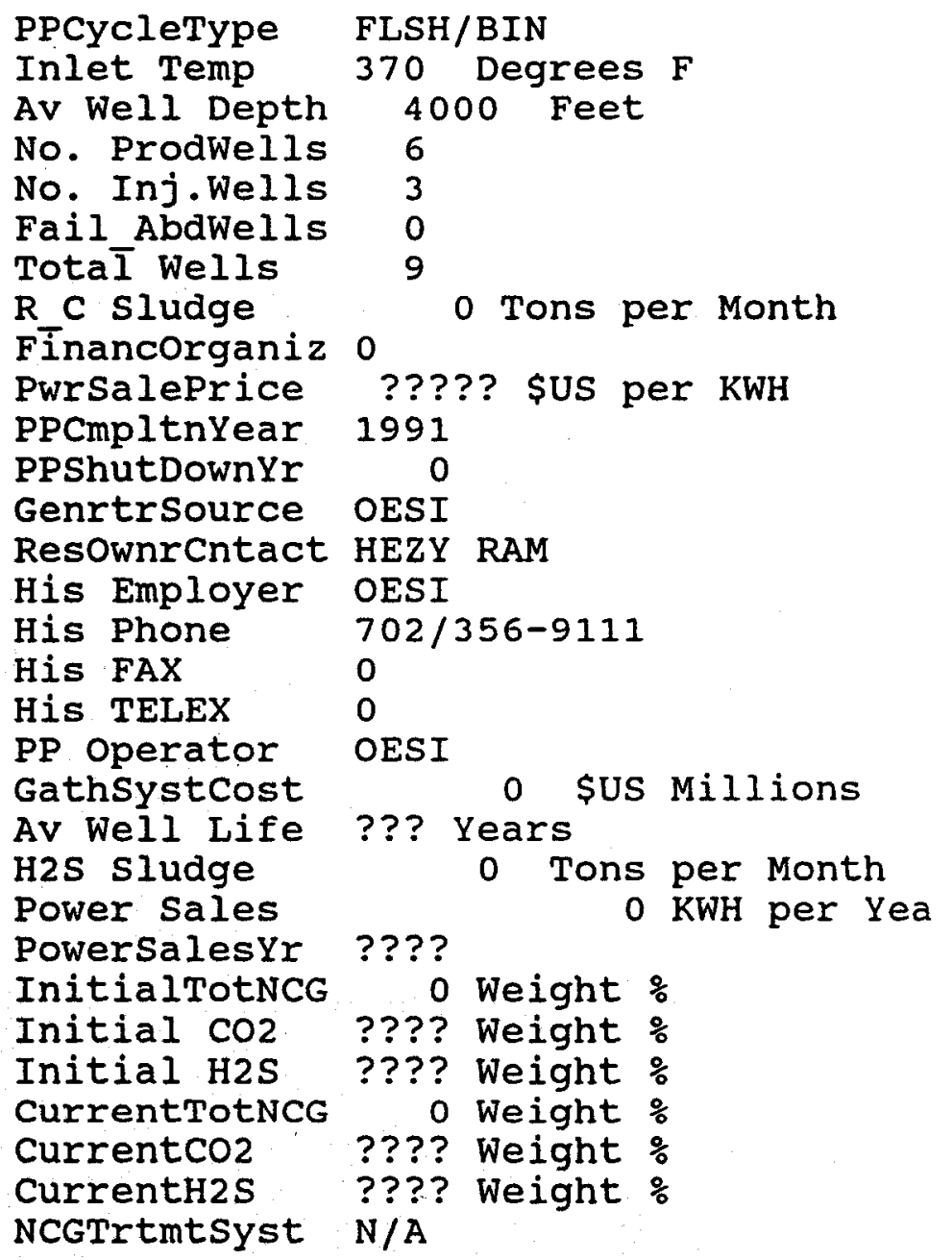




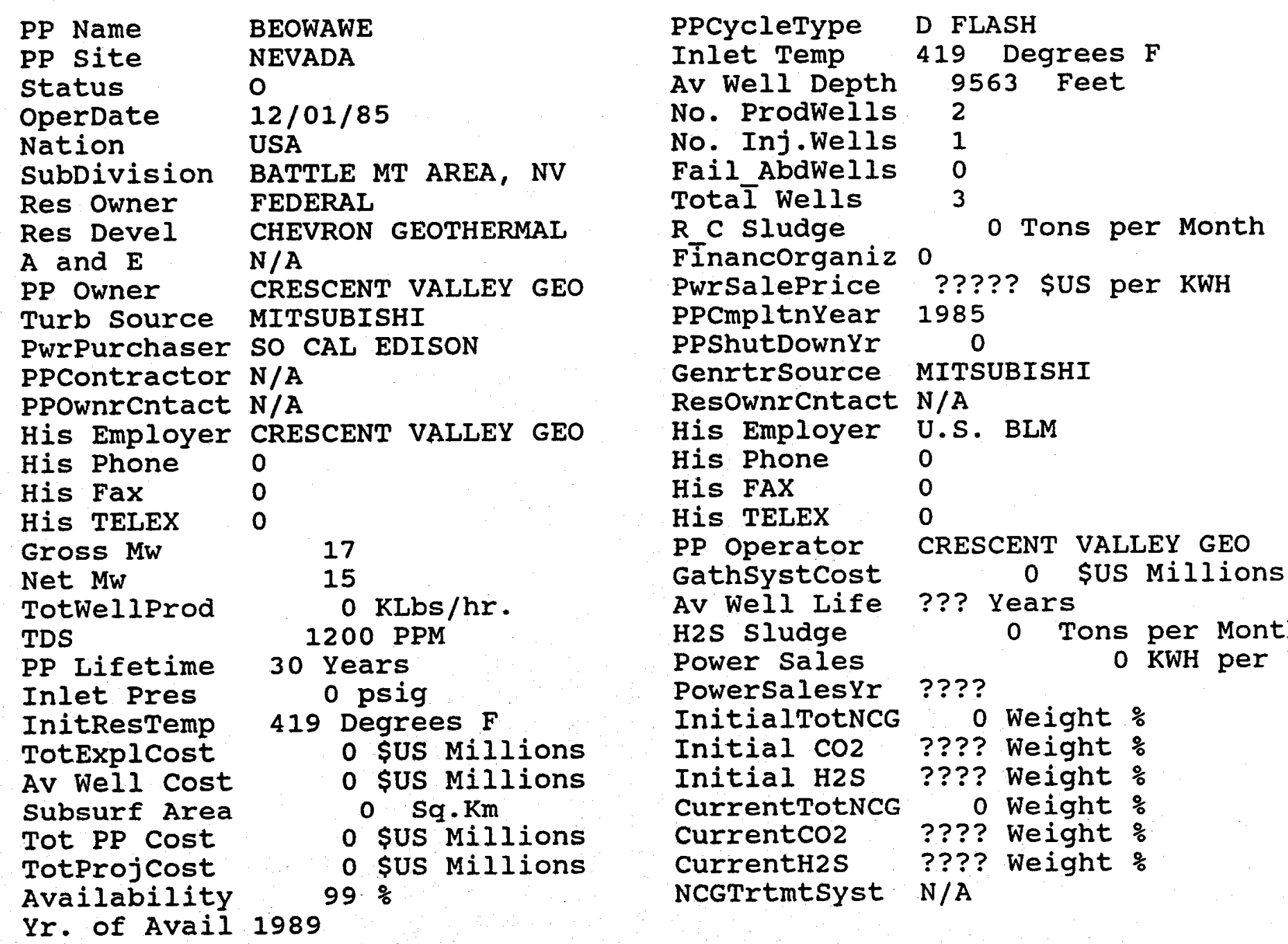

PPCycleType

Inlet Temp

Av Well Depth

No. ProdWells

No. Inj.Wells

Fail AbdWells

TotaI Wells

$R$ C sludge

Financorganiz 0

Pwrsaleprice

PPCmpltnYear

PPShutDownYr

Genrtrsource MITSUBISHI

Resownrcntact N/A

His Employer U.S. BLM

His Phone 0

His FAX 0

His TELEX 0

PP Operator CRESCENT VALLEY GEO

Gathsystcost 0 SUS Millions

Av Well Life ??? Years

H2s sludge

Power Sales

PowersalesYr ????

InitialTotNCG 0 weight $\%$

Initial $\mathrm{CO} 2$ ???? Weight $\%$

Initial H2S ???? Weight $\%$

CurrentTotNCG 0 Weight $\%$

CurrentCO2 ???? Weight $\%$

CurrentH2S ???? Weight \%

NCGTrtmtsyst $\mathrm{N} / \mathrm{A}$ 


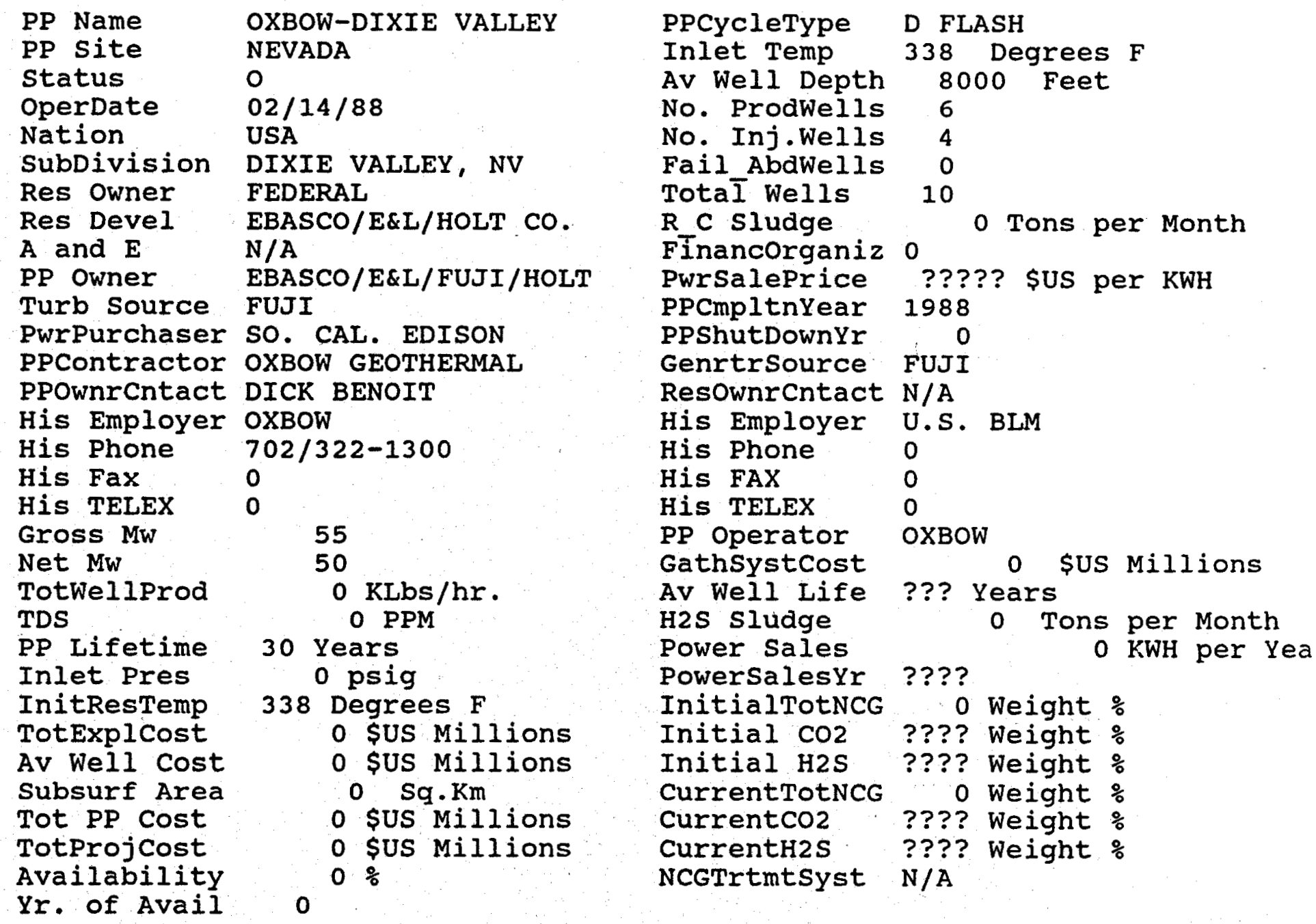




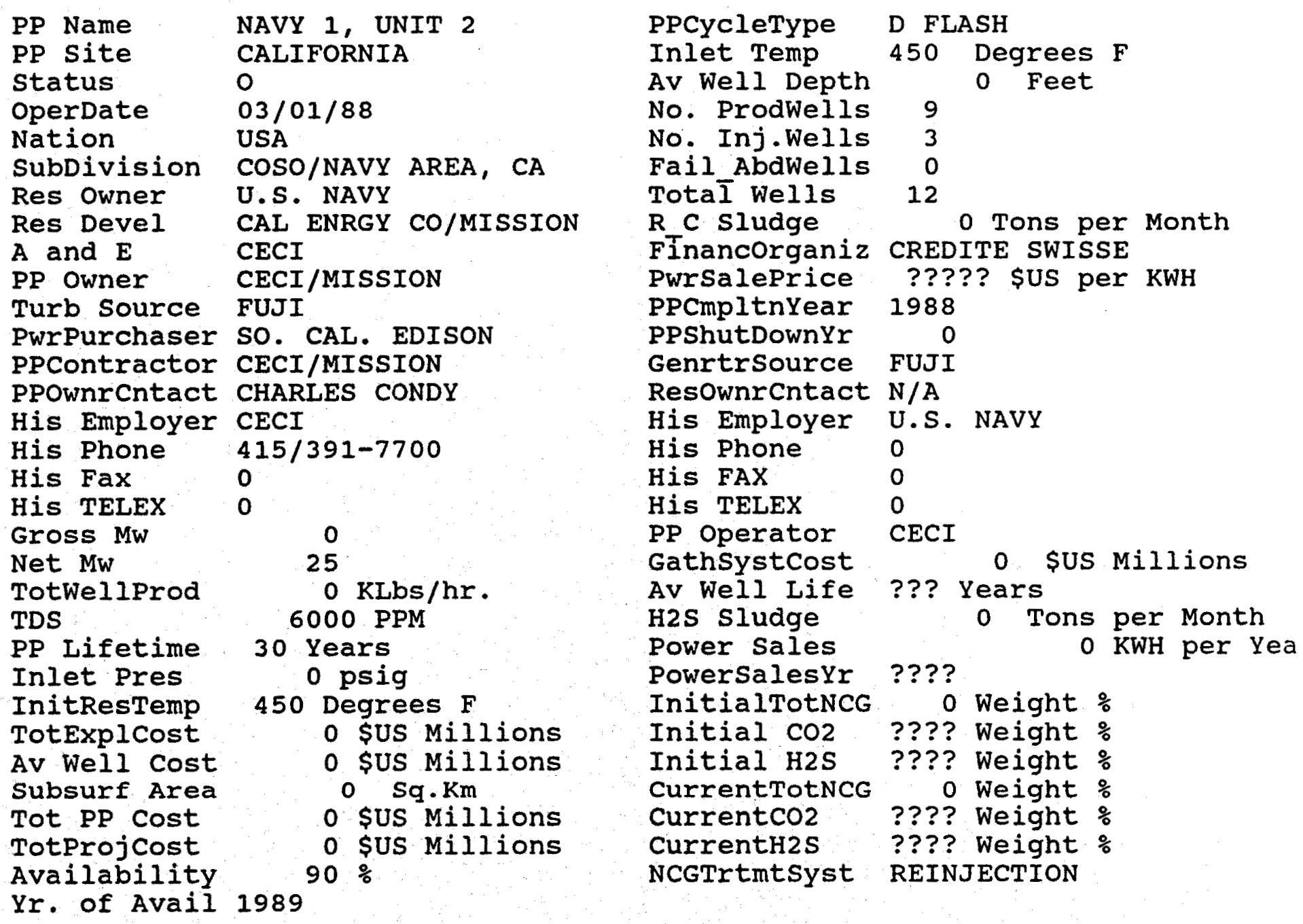




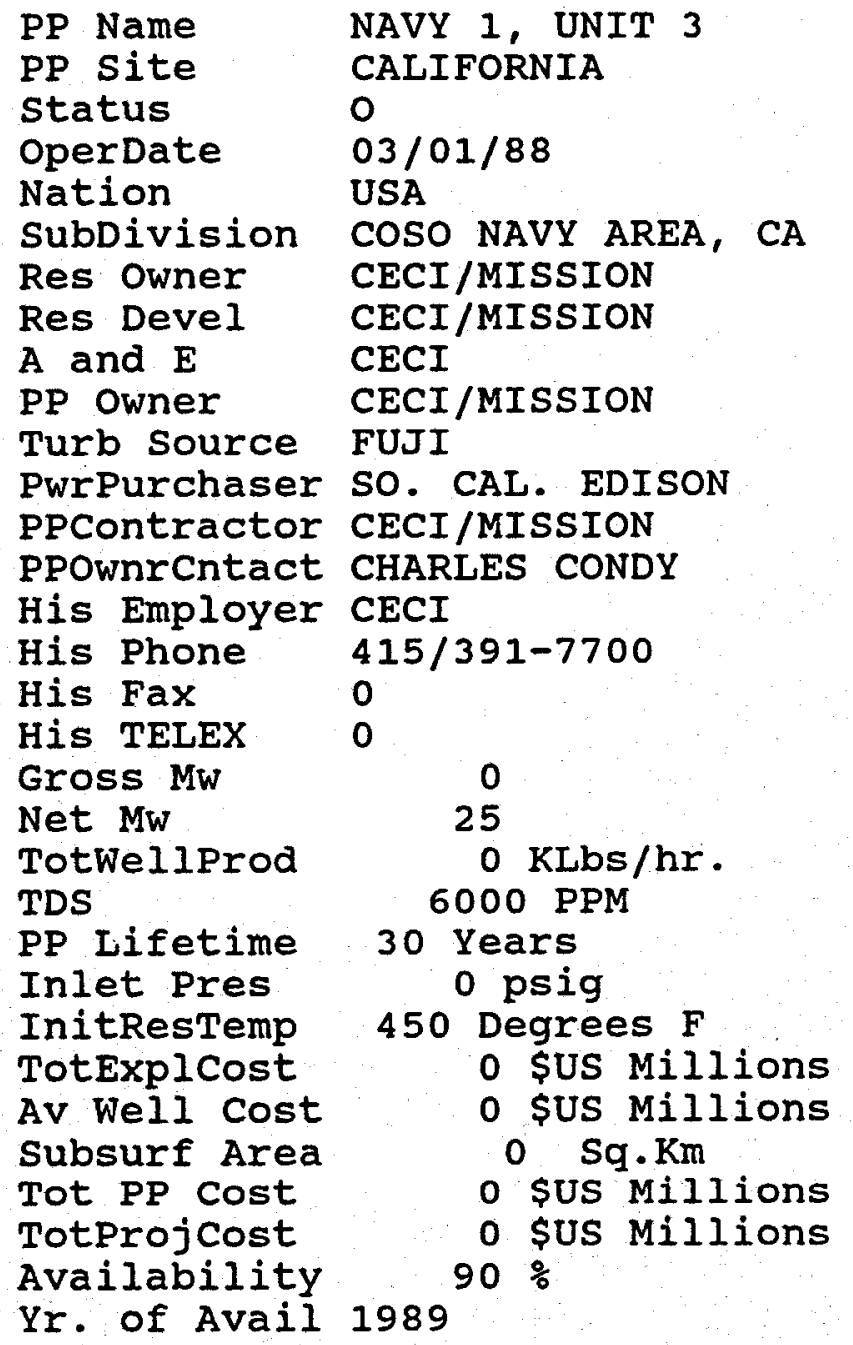

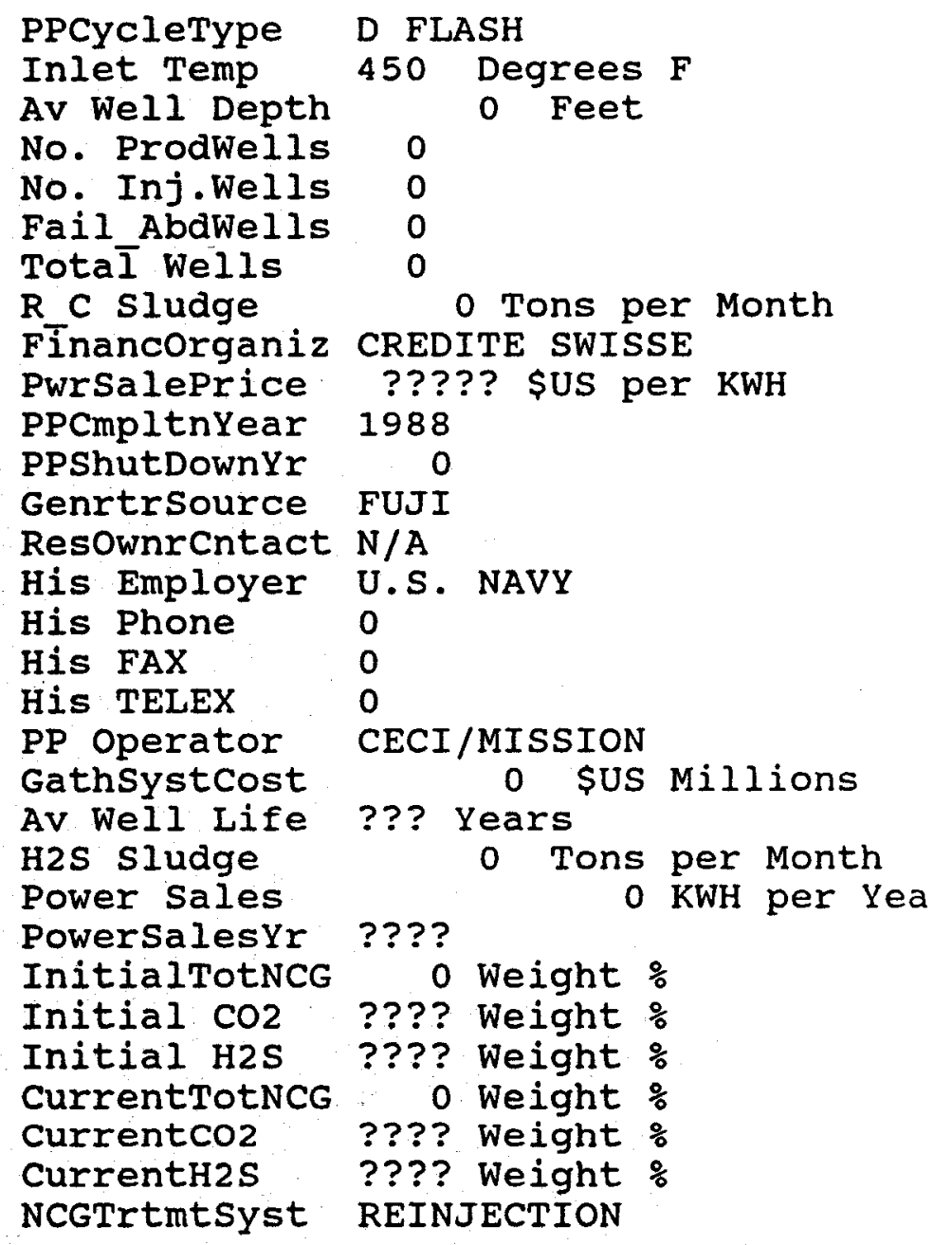




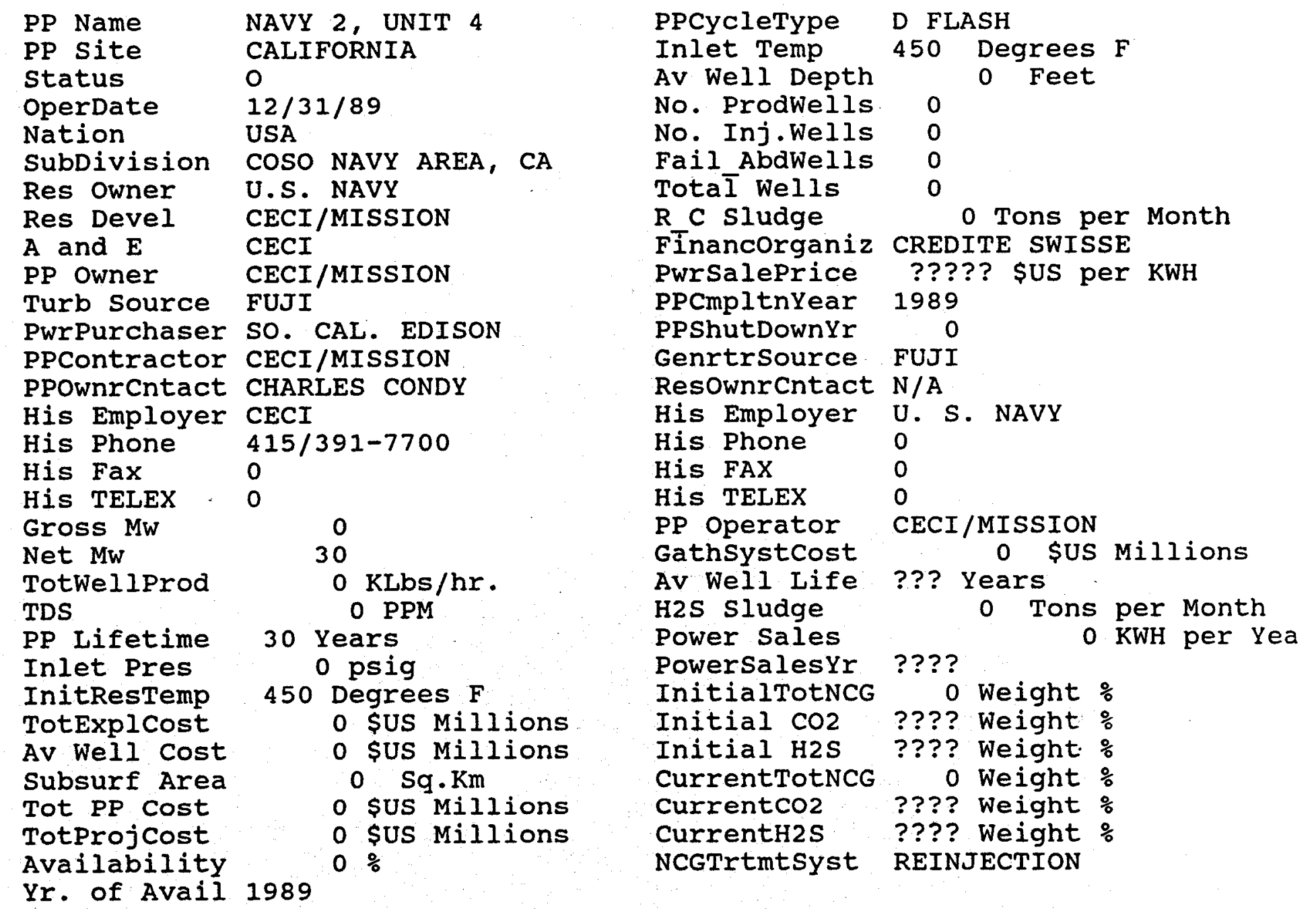




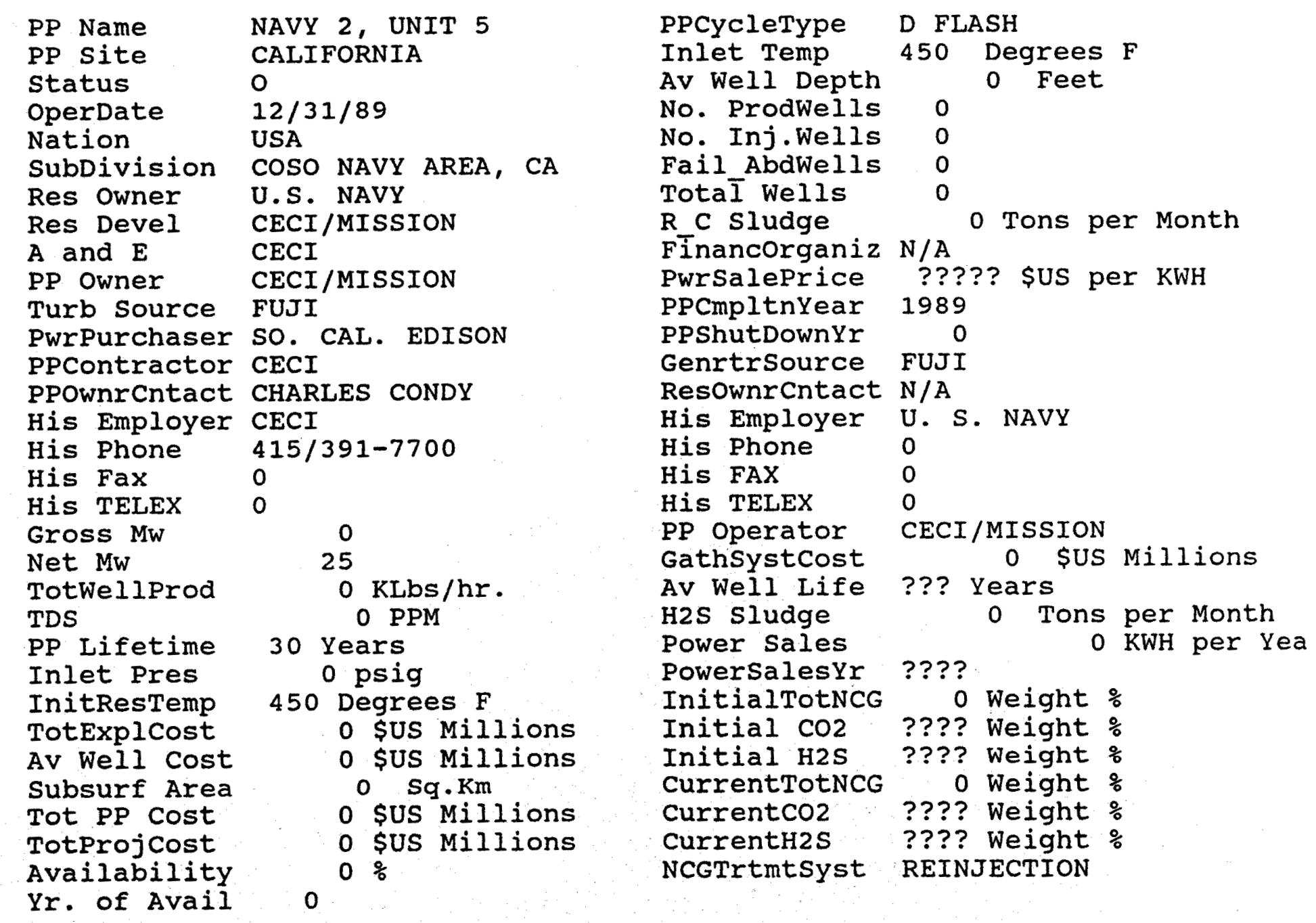




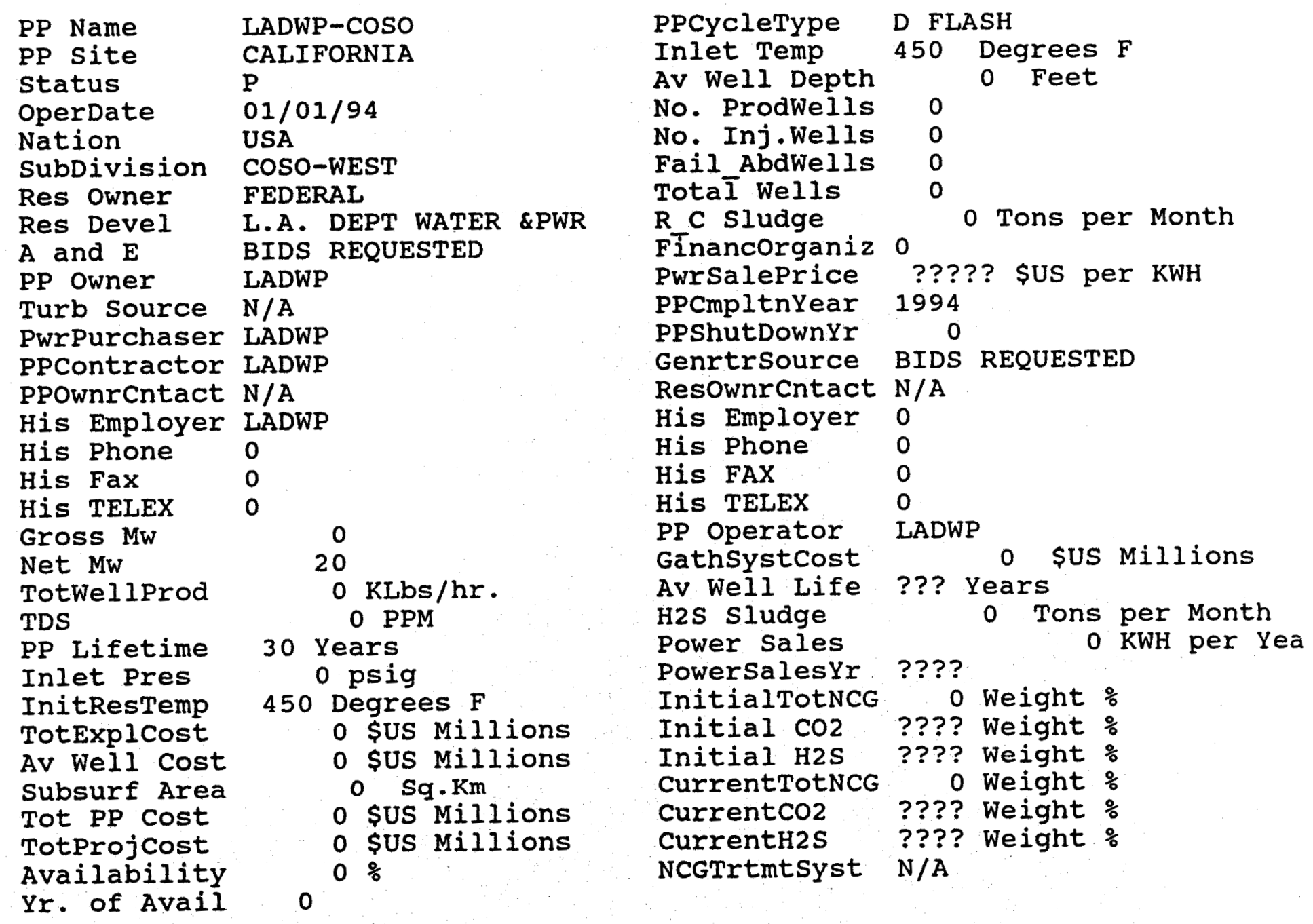




\begin{tabular}{|c|c|}
\hline PP Name & ORMESA $1 \mathrm{E}$ \\
\hline PP site & CALIFORNIA \\
\hline status & 0 \\
\hline OperDate & $12 / 31 / 88$ \\
\hline Nation & USA \\
\hline SubDivision & EAST MESA \\
\hline Res Owner & FEDERAL \\
\hline Res Devel & ORMAT \\
\hline$A$ and $E$ & ORMAT \\
\hline PP Owner & ORMAT \\
\hline Turb Source & ORMAT \\
\hline $\begin{array}{l}\text { PwrPurchaser } \\
\text { PPContractor }\end{array}$ & $\begin{array}{l}\text { SO. CAL. EDISON } \\
\text { ORMAT }\end{array}$ \\
\hline pPownrcntact & HEZY RAM \\
\hline His Employer & ORMAT \\
\hline His Phone & $702 / 356-9111$ \\
\hline His Fax & 0 \\
\hline His TELEX & 0 \\
\hline Gross $\mathrm{MW}$ & 10 \\
\hline Net MW & 8 \\
\hline $\begin{array}{l}\text { TotWel1Prod } \\
\text { TDS }\end{array}$ & $\begin{array}{c}280 \mathrm{KLbs} / \mathrm{hr} \text {. } \\
0 \mathrm{PPM}\end{array}$ \\
\hline $\begin{array}{l}\text { PP Lifetime } \\
\text { Inlet Pres }\end{array}$ & $\begin{array}{l}30 \text { Years } \\
0 \text { psig }\end{array}$ \\
\hline $\begin{array}{l}\text { InitResTemp } \\
\text { TotExplCost }\end{array}$ & 320 Degrees $F$ \\
\hline $\begin{array}{l}\text { Av Well cost } \\
\text { Subsurf Area } \\
\text { Tot PP Cost } \\
\text { TotProjcost } \\
\text { Availability }\end{array}$ & $\begin{array}{l}0 \text { sUS Millions } \\
0 \text { Sq. Km } \\
0 \text { \$US Millions } \\
0 \text { \$US Millions } \\
0 \%\end{array}$ \\
\hline ir. of Avail & \\
\hline
\end{tabular}

\begin{tabular}{|c|c|}
\hline PPCycletype & BINARY \\
\hline Inlet Temp & 293 Degrees $F$ \\
\hline Av Well Depth & 0 Feet \\
\hline No. ProdWelis & 4 \\
\hline No. Inj. Welis & 0 \\
\hline $\begin{array}{l}\text { Fail AbdWells } \\
\text { TotaI Wells }\end{array}$ & 0 \\
\hline $\begin{array}{l}\text { TotaI Wells } \\
\text { R C Sludge }\end{array}$ & 0 Tons per Month \\
\hline Financorganiz & $0 \quad 0$ Tons per Monen \\
\hline Pwrsaleprice & ????? \$US per KWH \\
\hline PPCmpltnYear & 1988 \\
\hline PPShutDownYr & 0 \\
\hline GenrtrSource & ORMAT \\
\hline Resownrcntact & $N / A$ \\
\hline His Employer & U.S. BLM \\
\hline His Phone & 0 \\
\hline His FAX & 0 \\
\hline His TELEX & 0 \\
\hline PP Operator & ORMAT \\
\hline Gathsystcost & \$US Millions \\
\hline Av Weil Life & ??? Years \\
\hline H2S sludge & 0 Tons per Month \\
\hline Power Sales & 0 KWH per Yea \\
\hline PowersalesYr & ???? \\
\hline InitialTotNCG & 0 Weight $\%$ \\
\hline Initial $\mathrm{CO} 2$ & ???? Weight $\%$ \\
\hline Initial H2S & ???? Weight $\%$ \\
\hline CurrentTotNCG & 0 Weight : \\
\hline CurrentCO2 & ???? Weight \% \\
\hline CurrentH2s & ???? Weight \% \\
\hline NCGTrtmtsyst & $N / A$ \\
\hline
\end{tabular}




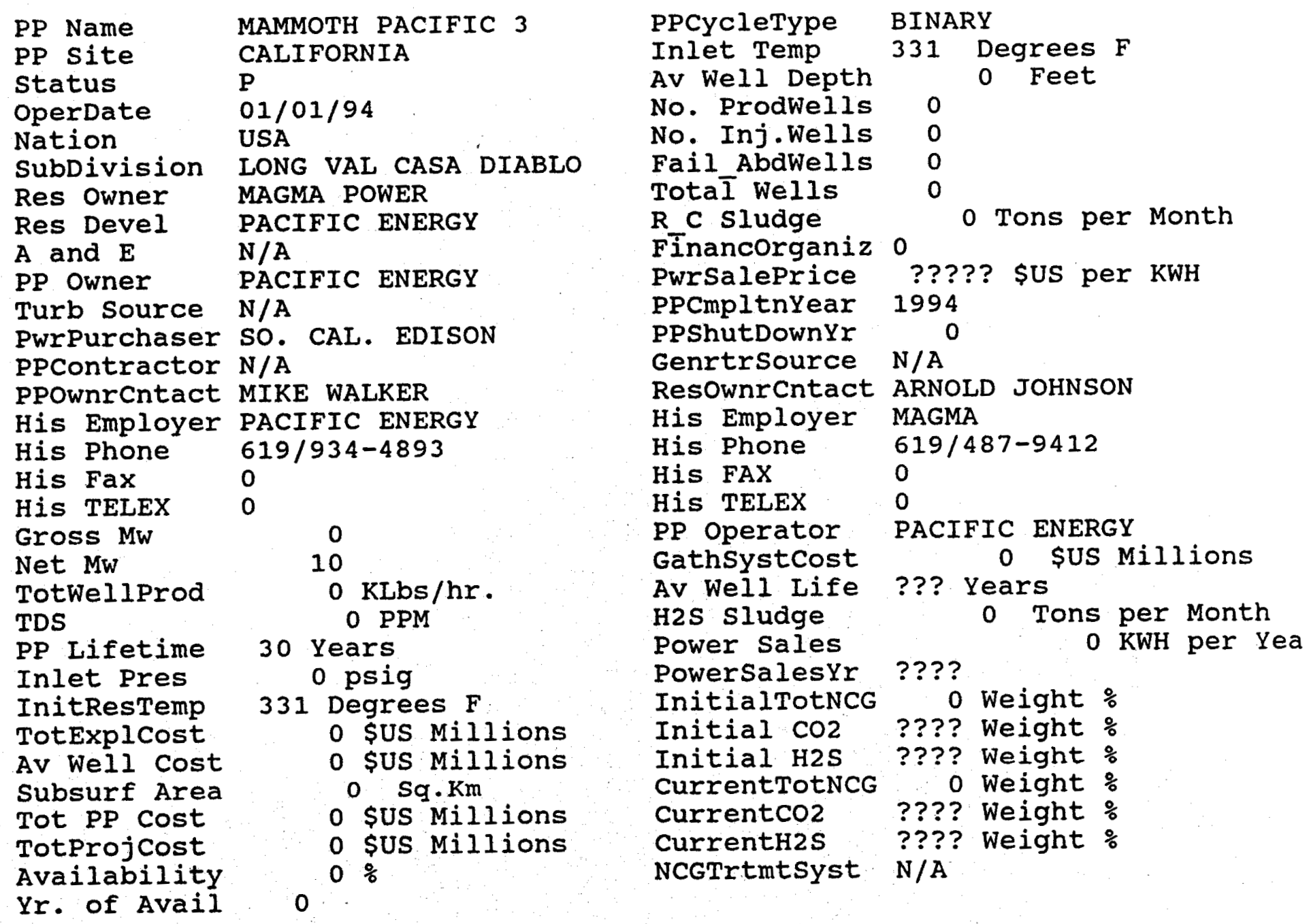




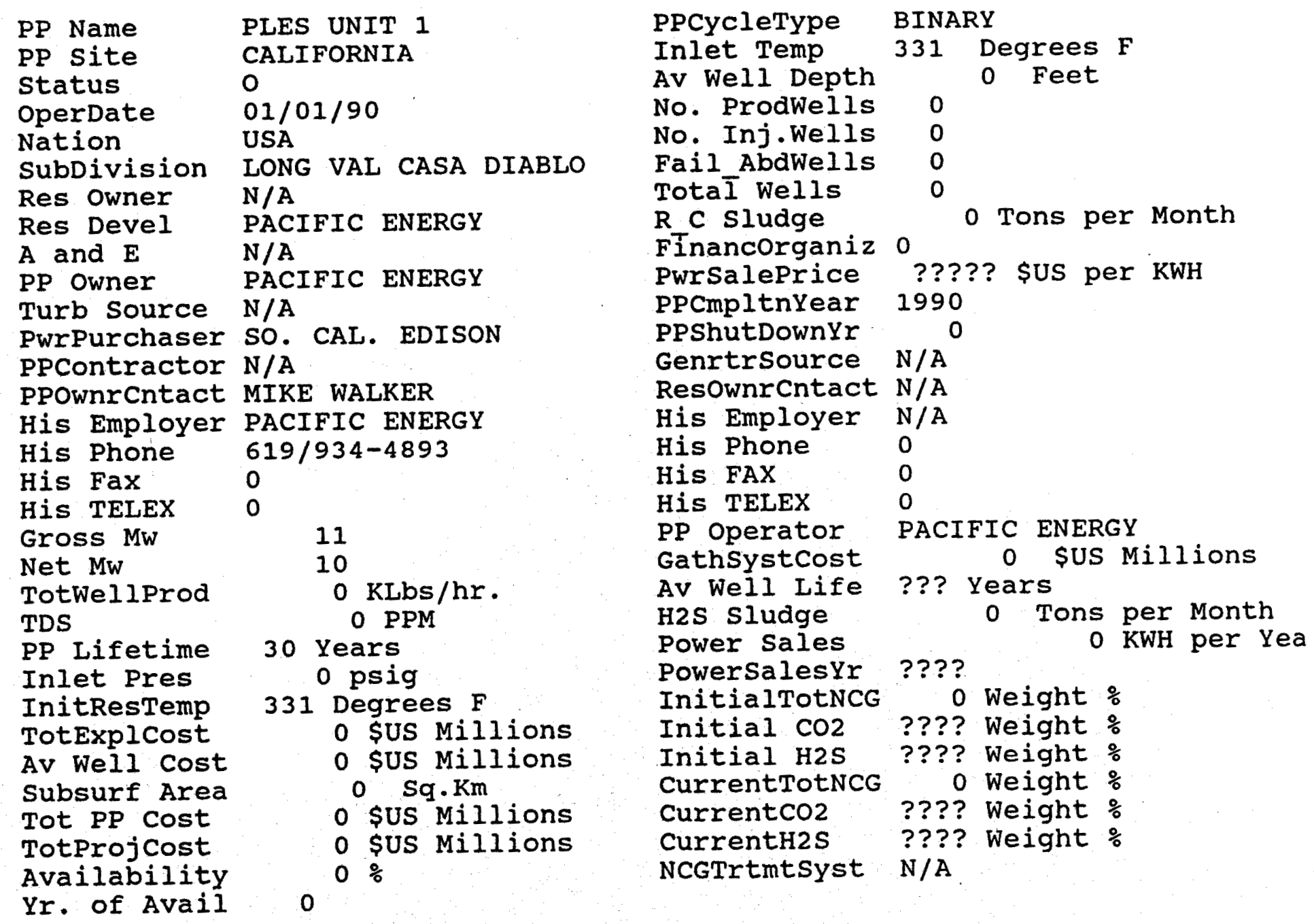




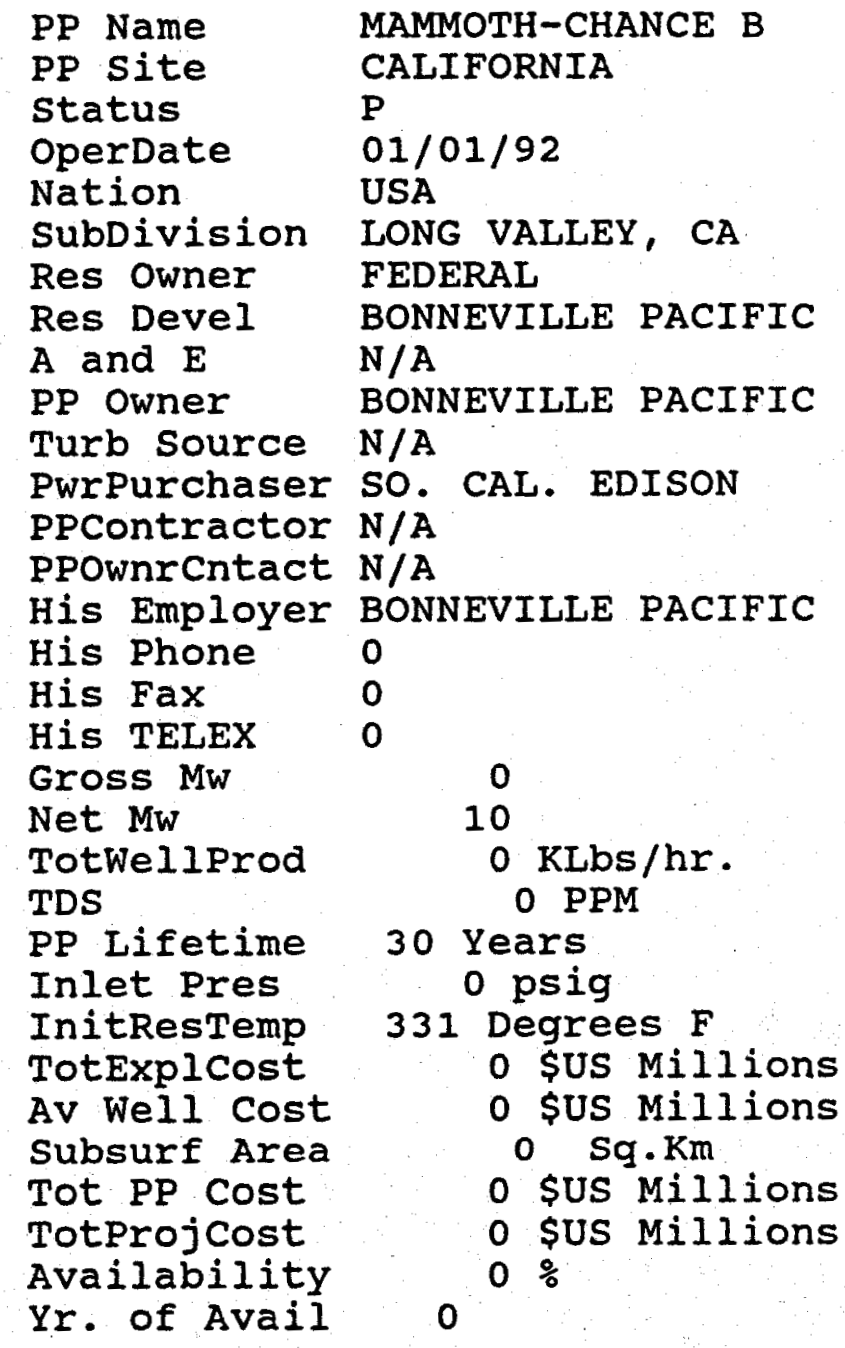

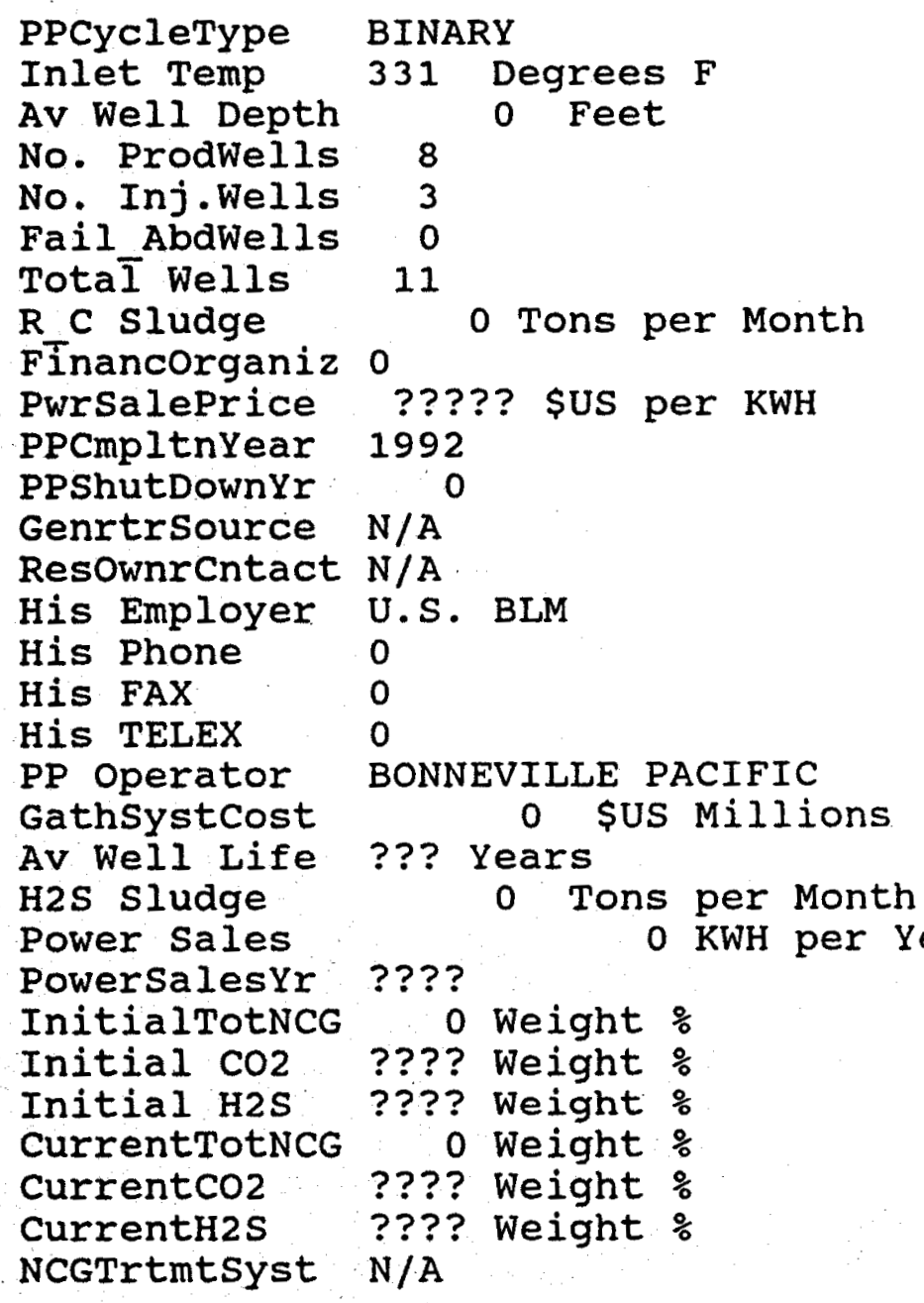




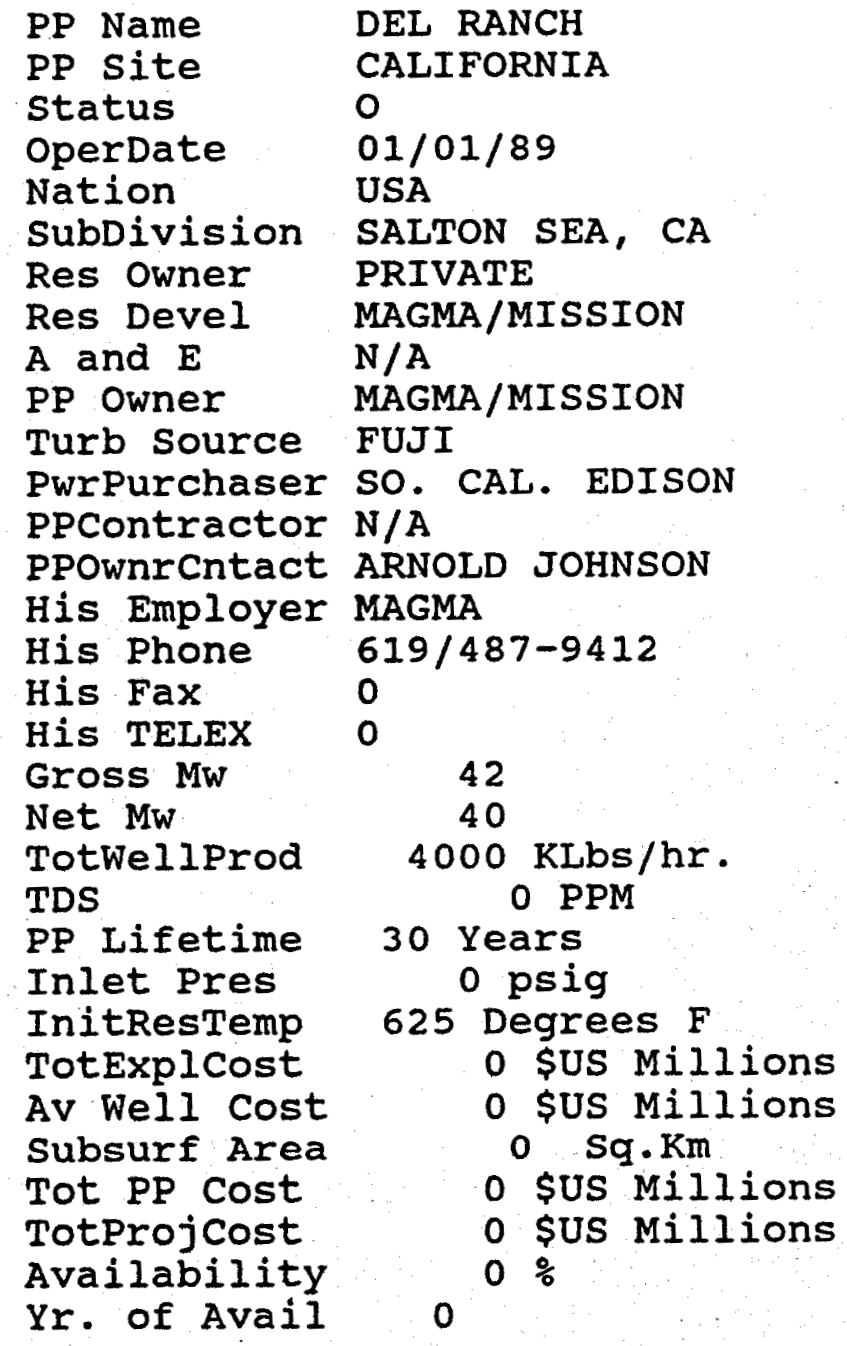

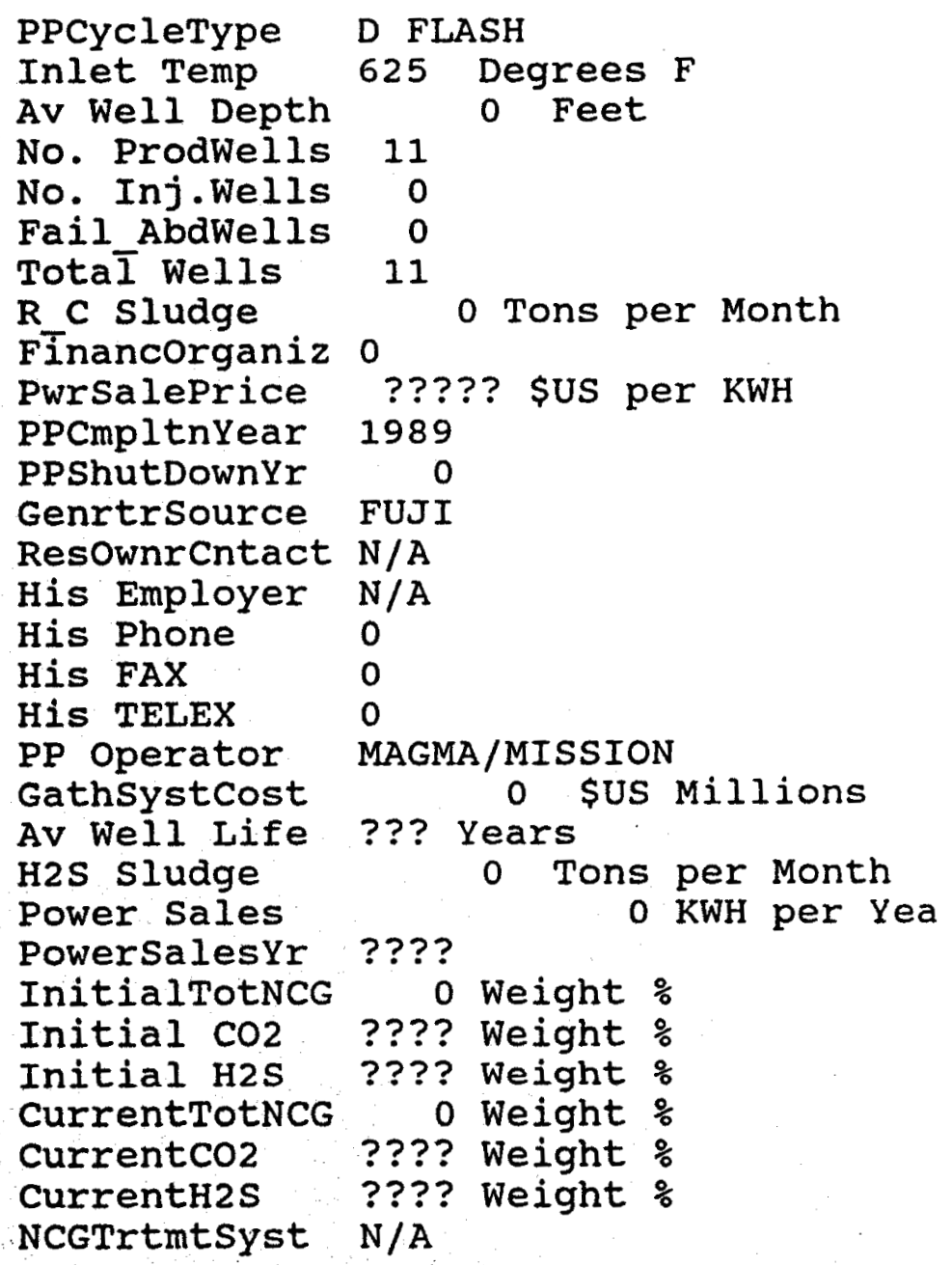




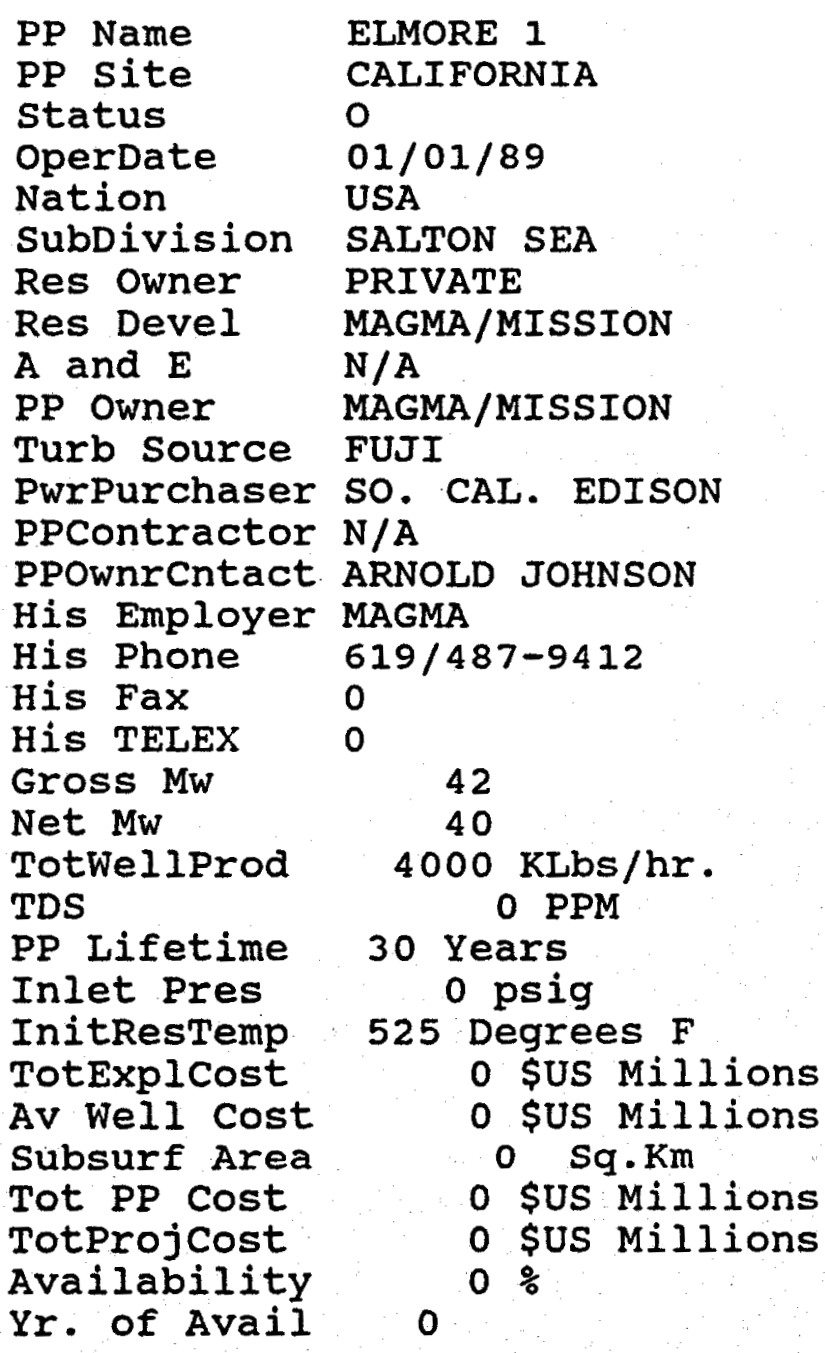

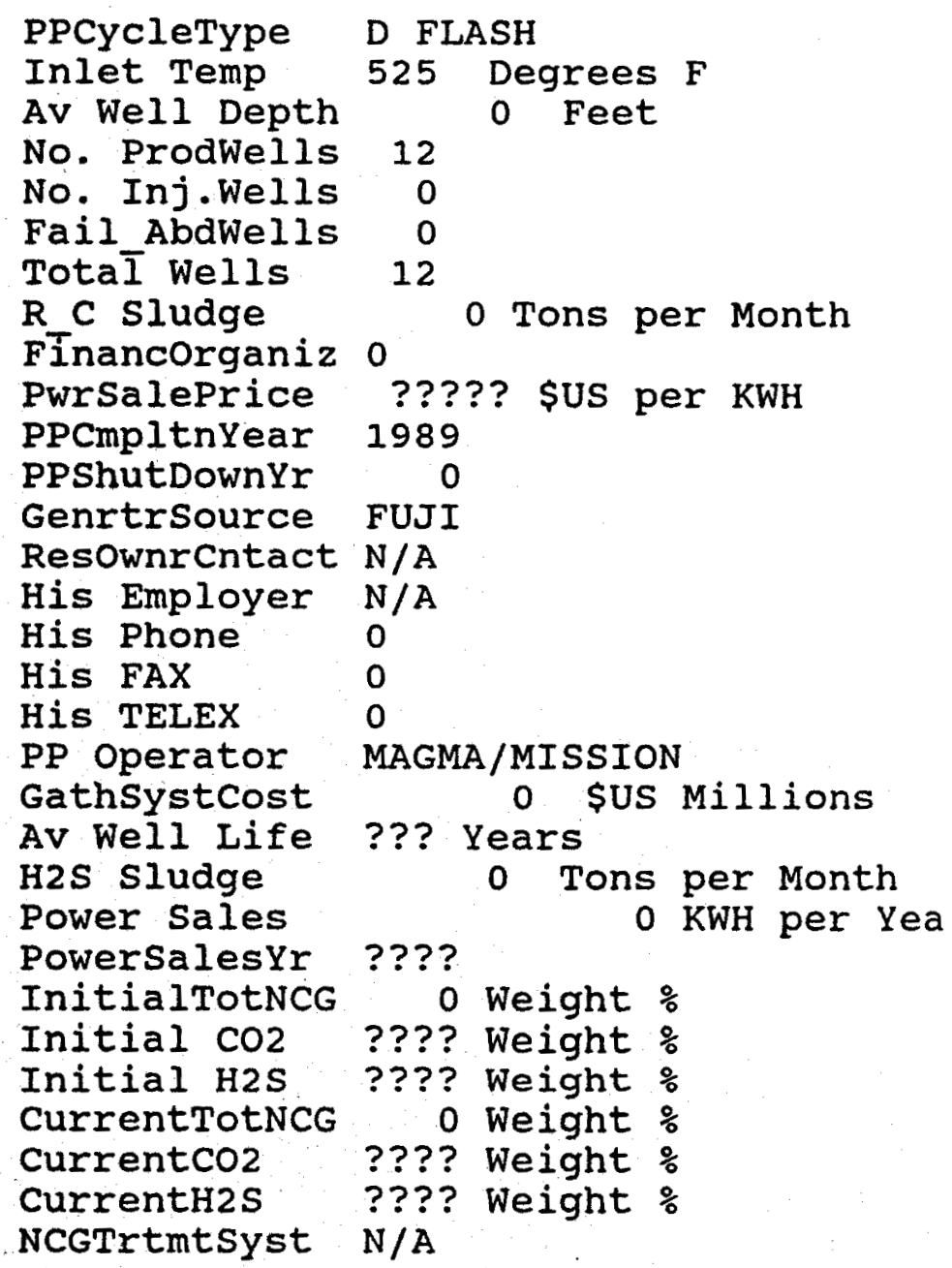




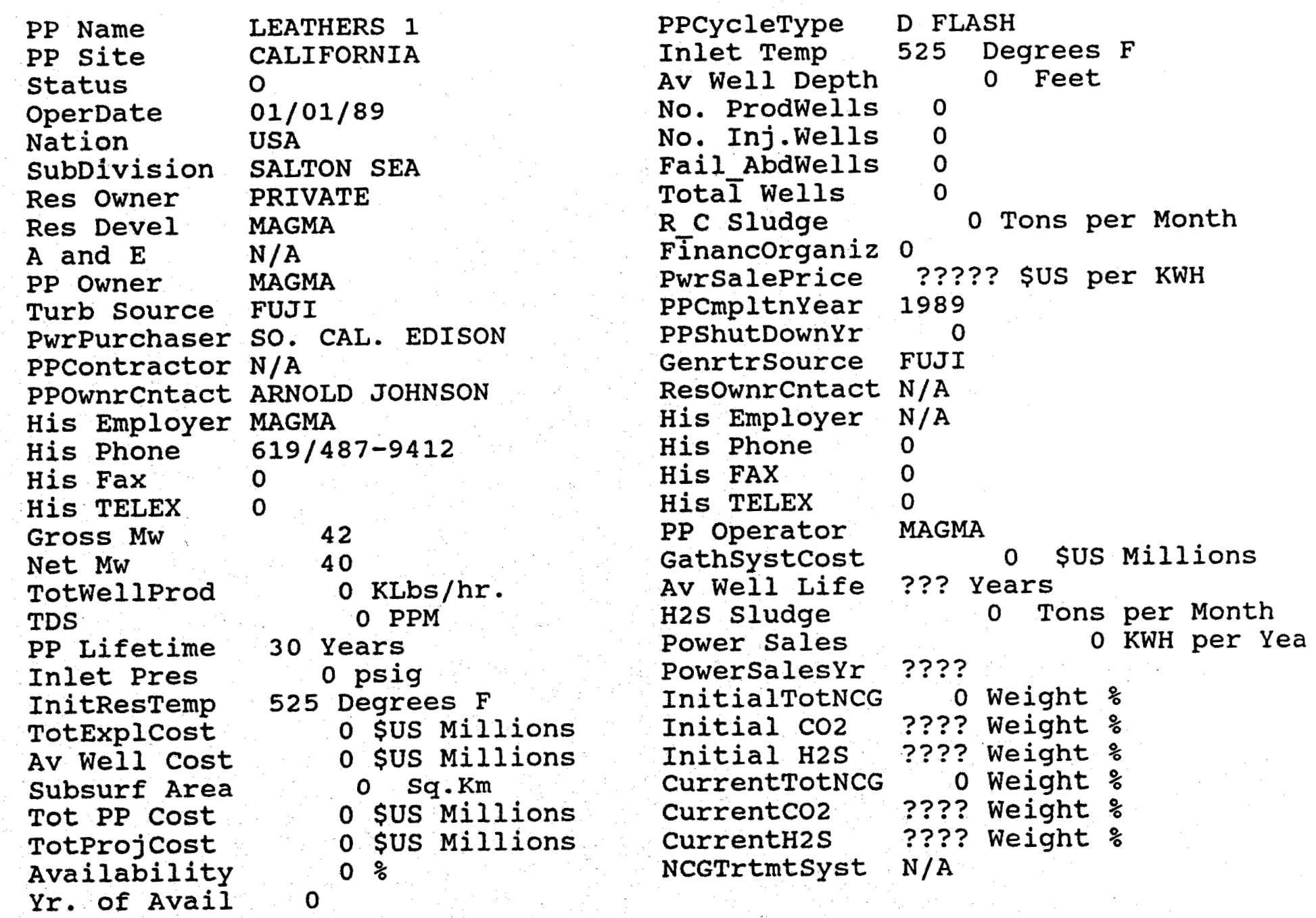




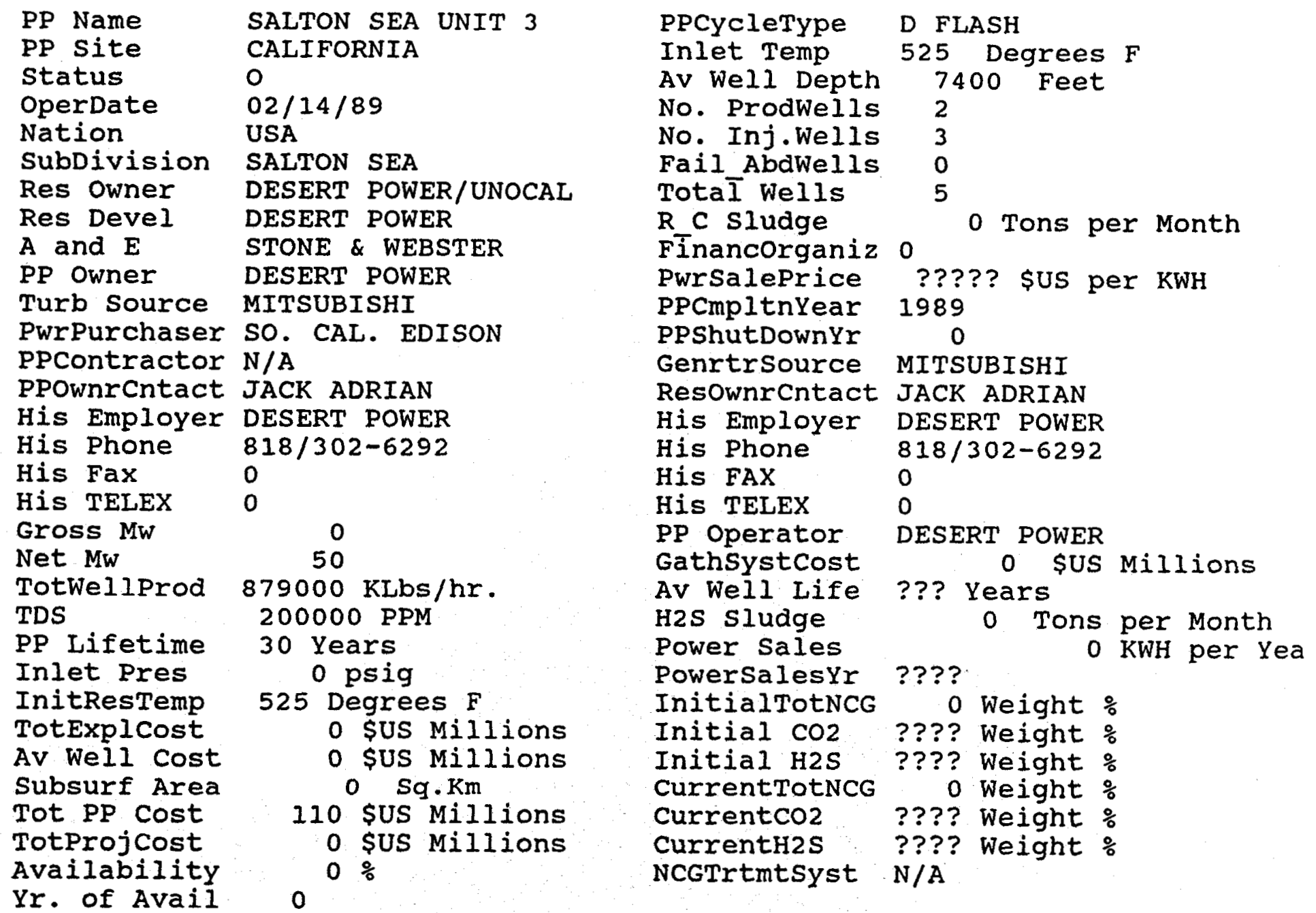




\begin{tabular}{|c|c|c|c|}
\hline $\begin{array}{l}\text { PP Name } \\
\text { PP Site }\end{array}$ & $\begin{array}{l}\text { WENDEL-AMEDEE UNIT } \\
\text { CALIFORNIA }\end{array}$ & $\begin{array}{l}\text { PPCycleType } \\
\text { Inlet Temp }\end{array}$ & $\begin{array}{l}\text { BINARY } \\
219 \text { Degrees } F\end{array}$ \\
\hline status & & Av Well Depth & 850 Feet \\
\hline OperDate & $01 / 01 / 88$ & No. Prodwells & 2 \\
\hline Nation & USA & No. Inj.Wells & 0 \\
\hline $\begin{array}{l}\text { SubDivision } \\
\text { Res Owner }\end{array}$ & WENDEL-AMEDEE, NE CAL & Fail AbdWells & 0 \\
\hline $\begin{array}{l}\text { Res Owner } \\
\text { Res Devel }\end{array}$ & $\begin{array}{l}\text { FEDERAL } \\
\text { TRANSPACIFIC GEOTH. }\end{array}$ & $\begin{array}{l}\text { Total Wells } \\
R_{-} C \text { sludge }\end{array}$ & 2 Tons per Month \\
\hline $\begin{array}{l}A \text { and } E \\
\text { PP Owner }\end{array}$ & $\begin{array}{l}\text { BARBER NICHOLS ENG. } \\
\text { TPG/US ENERGY }\end{array}$ & $\begin{array}{l}\text { Financorganiz } \\
\text { PwrsalePrice }\end{array}$ & $\begin{array}{l}\text { 0 } \\
\text { ????? sus per KWH }\end{array}$ \\
\hline Turb source & BARBER NICHOLS & PPCmpltnYear & $\begin{array}{l}\text { ???? SUS per KWH } \\
1988\end{array}$ \\
\hline $\begin{array}{l}\text { PwrPurchaser } \\
\text { PPContractor }\end{array}$ & LASSEN MINING UTLITY & PPShutDownYr & 0 \\
\hline $\begin{array}{l}\text { PPContractor } \\
\text { PPownrcntact }\end{array}$ & $\begin{array}{l}\text { N/A } \\
\text { AVIV GOLDSMITH }\end{array}$ & $\begin{array}{l}\text { GenrtrSource } \\
\text { ResownrCntact }\end{array}$ & $\begin{array}{l}\text { BARBER NICHOLS } \\
\text { N/A }\end{array}$ \\
\hline His Employer & U.S. ENERGY & His Employer & U.S. BLM \\
\hline His Phone & $702 / 323-2866$ & His Phone & 0 \\
\hline His Fax & 0 & His FAX & 0 \\
\hline $\begin{array}{l}\text { His TELEX } \\
\text { Gross MW }\end{array}$ & & His TELEX & 0 \\
\hline $\begin{array}{l}\text { Gross Mw } \\
\text { Net Mw } \\
\text { Totwellprod }\end{array}$ & $\begin{array}{r}3 \\
2 \\
850 \mathrm{kLbs} / \mathrm{hr} .\end{array}$ & $\begin{array}{l}\text { PP Operator } \\
\text { Gathsystcost } \\
\text { Av Well Life }\end{array}$ & $\begin{array}{l}\text { U.S. ENERGY } \\
0 \text { SUS Millions } \\
\text { ??? Years }\end{array}$ \\
\hline TDS & 0 PPM & H2S sludge & 0 Tons per Month \\
\hline $\begin{array}{l}\text { PP Lifetime } \\
\text { Inlet Pres }\end{array}$ & $\begin{array}{l}30 \text { Years } \\
0 \text { psig }\end{array}$ & $\begin{array}{l}\text { Power Sales } \\
\text { PowersalesIr }\end{array}$ & 0 KWH per Yea \\
\hline $\begin{array}{l}\text { InitRestemp } \\
\text { TotExplcost } \\
\text { Av Well cost }\end{array}$ & $\begin{array}{l}228 \text { Degrees } F \\
0 \text { sUS Militions } \\
0 \text { sUS Millions }\end{array}$ & $\begin{array}{l}\text { InitialTotNCG } \\
\text { Initial CO2 } \\
\text { Initial H2S }\end{array}$ & $\begin{array}{r}0 \text { Weight \% } \\
\text { ???? Weight \% } \\
\text { ???? Weight \% }\end{array}$ \\
\hline $\begin{array}{l}\text { Av well cost } \\
\text { Subsurf Area } \\
\text { Tot PP Cost } \\
\text { TotProjCost } \\
\text { Availability }\end{array}$ & $\begin{array}{l}0 \text { Sq.Km } \\
0 \text { \$US Militions } \\
9 \text { \$US Militions } \\
0 \text { \% }\end{array}$ & $\begin{array}{l}\text { CurrentTotNCG } \\
\text { CurrentCO2 } \\
\text { CurrentH2S } \\
\text { NCGTrtmtsyst }\end{array}$ & $\begin{array}{l}0 \text { Weight } \% \\
\text { ???? Weight } \% \\
\text { ???? Weight \% } \\
\text { N/A }\end{array}$ \\
\hline E. of $A$ & & & \\
\hline
\end{tabular}




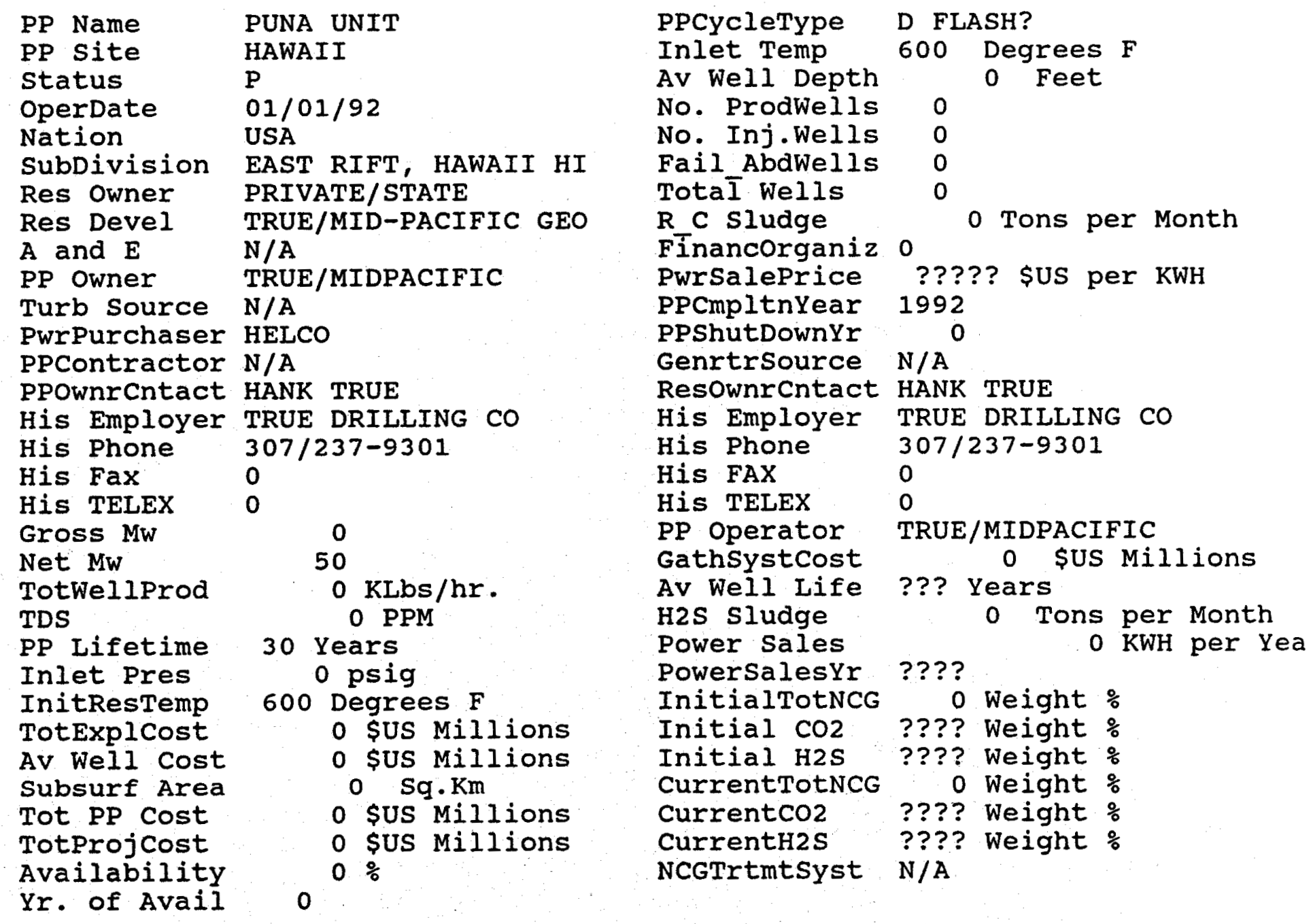




\begin{tabular}{|c|c|c|c|}
\hline PP Name & STILLWATER GEO. PROJ & PPCycleType & BINARY \\
\hline PP site & NEVADA & Inlet Temp & Degrees $F$ \\
\hline status & & Av Well Depth & Feet \\
\hline OperDate & $12 / 01 / 89$ & No. ProdWeils & 0 \\
\hline Nation & USA & No. Inj.Wells & 0 \\
\hline SubDivision & FALLON, NV AREA & Fail_AbdWells & 0 \\
\hline Res Owner & FEDERAL & TotaI Wells & 0 \\
\hline Res Devel & ORMAT ENERGY SYS INC & R_C sludge & per Month \\
\hline $\begin{array}{l}A \text { and } E \\
P P \text { Owner }\end{array}$ & $\begin{array}{l}\text { ORMAT } \\
\text { OESI }\end{array}$ & $\begin{array}{l}\text { Financorganiz } \\
\text { PwrSalePrice }\end{array}$ & $\begin{array}{l}\text { BNKRS TRST/NOVA SCOTIA B } \\
\text { ????? SUS per KWH }\end{array}$ \\
\hline Turb Source & OESI & PPCmpltnYear & $1 \ddot{98} \dot{9}$ \\
\hline PwrPurchaser & SIERRA PACIFIC PWR & PPShutDownYr & 0 \\
\hline PPContractor & OESI & GenrtrSource & OESI \\
\hline PPownrcntact & HEZY RAM & ResownrCntact & $N / A$ \\
\hline His Employer & OESI & His Employer & U.S. BLM \\
\hline His Phone & $702 / 356-9111$ & His Phone & 0 \\
\hline His Fax & 0 & His FAX & 0 \\
\hline His TELEX & 0 & His TELEX & 0 \\
\hline Gross Mw & 21 & PP operator & OESI \\
\hline Net $\mathrm{MW}$ & 13 & Gathsystcost & \$US Millions \\
\hline $\begin{array}{l}\text { TotWellProd } \\
\text { TDS }\end{array}$ & $\begin{array}{l}0 \text { KLbs/hr. } \\
0 \text { PPM }\end{array}$ & $\begin{array}{l}\text { Av Well Life } \\
\text { H2S sludge }\end{array}$ & $\begin{array}{l}\text { ??? Years } \\
0 \text { Tons per Month }\end{array}$ \\
\hline $\begin{array}{l}\text { PP Lifetime } \\
\text { Inlet Pres }\end{array}$ & $\begin{array}{l}30 \text { Years } \\
0 \text { psig }\end{array}$ & $\begin{array}{l}\text { Power sales } \\
\text { PowersalesYr }\end{array}$ & 0 KWH per Yea \\
\hline InitResTemp & 338 Degrees $F$ & InitialTotNCG & 0 Weight : \\
\hline TotExplCost & 0 sUS Millions & Initial $\mathrm{CO} 2$ & ???? Weight : \\
\hline $\begin{array}{l}\text { Av well cost } \\
\text { Subsurf Area }\end{array}$ & $\begin{array}{l}0 \text { \$US Millions } \\
0 \quad \mathrm{Sg} \cdot \mathrm{Km}\end{array}$ & $\begin{array}{l}\text { Initial H2S } \\
\text { currentTotNCG }\end{array}$ & $\begin{array}{r}\text { ???? Weight } \% \\
0 \text { Weight } \%\end{array}$ \\
\hline $\begin{array}{l}\text { Tot PP Cost } \\
\text { Totprojcost } \\
\text { Availability }\end{array}$ & $\begin{aligned} 0 & \text { SUS Millions } \\
36 & \text { \$US Millions } \\
0 & \frac{9}{6}\end{aligned}$ & $\begin{array}{l}\text { CurrentCO2 } \\
\text { CurrentH2S } \\
\text { NCGTrtmtSyst }\end{array}$ & $\begin{array}{l}\text { ???? Weight } \% \\
\text { ???? Weight } \% \\
\text { N/A }\end{array}$ \\
\hline c. of $A V$ & 0 & & \\
\hline
\end{tabular}




\begin{tabular}{|c|c|c|c|}
\hline $\begin{array}{l}\text { PP Name } \\
\text { PP site }\end{array}$ & $\begin{array}{l}\text { COVE FT TOPPING TRBN } \\
\text { UTAH }\end{array}$ & PPCycleType & D STEAM \\
\hline $\begin{array}{l}\text { PP site } \\
\text { Status }\end{array}$ & & Inlet Temp & 332 Degrees $F$ \\
\hline perDate & $\begin{array}{l}0 \\
01 / 01 / 88\end{array}$ & $\begin{array}{l}\text { Av Well Depth } \\
\text { No. Prodwel }\end{array}$ & 1100 \\
\hline ation & USA & $\begin{array}{l}\text { No. Proawells } \\
\text { No. Inj.Wells }\end{array}$ & $\begin{array}{l}2 \\
1\end{array}$ \\
\hline abDivision & BEAVER/FILMORE AREA & Fail AbaWells & 0 \\
\hline es Owner & FEDERAL/PRIVATE & TotaI Wells & 3 \\
\hline $\begin{array}{l}\text { Res Devel } \\
A \text { and } E\end{array}$ & $\begin{array}{l}\text { MOTHER EARTH INDUST. } \\
\text { BARBER NICHOLS }\end{array}$ & $\begin{array}{l}R \text { C sluage } \\
\text { Financorganiz }\end{array}$ & $\begin{array}{l}0 \text { Tons per Month } \\
\text { MOTHER EARTH INDUSTR }\end{array}$ \\
\hline $\begin{array}{l}\text { P Owner } \\
\text { urb Source }\end{array}$ & $\begin{array}{l}\text { MOTHER EARTH IND. } \\
\text { BARBER NICHOLS }\end{array}$ & $\begin{array}{l}\text { PwrSalePrice } \\
\text { PPCmpltnYear }\end{array}$ & $\begin{array}{l}\text { ????? \$US per KWH } \\
1988\end{array}$ \\
\hline rrPurchaser & PROVO POWER & PPShutDownYr & $\begin{array}{r}1700 \\
\quad 0\end{array}$ \\
\hline Contra & BARBER NI & urce & BARBER NI \\
\hline Ownrentact & KEN NICHOI & Resownr & W.A. PORI \\
\hline $\begin{array}{l}\text { is Employer } \\
\text { is Phone }\end{array}$ & $\begin{array}{l}\text { BARBER NI } \\
303 / 421-8\end{array}$ & His Employer & MOTHER EA \\
\hline $\begin{array}{l}\text { is Pnone } \\
\text { is Fax }\end{array}$ & $\begin{array}{l}30 \\
0\end{array}$ & $\begin{array}{l}\text { His Phor } \\
\text { His FAX }\end{array}$ & $\begin{array}{l}602 / \\
0\end{array}$ \\
\hline Iis TELEX & & His TELEX & 0 \\
\hline jross Mw & 1 & PP Operator & PROVO POWER \\
\hline $\begin{array}{l}\text { otWellProd } \\
\text { DS }\end{array}$ & $100000_{0}^{1} \mathrm{KLbs} / \mathrm{hr}$. & $\begin{array}{l}\text { Gathsystcost } \\
\text { Av Well Life } \\
\text { H2s sludae }\end{array}$ & ??? Years \\
\hline $\begin{array}{l}\text { P Lifetime } \\
\text { nlet Pres }\end{array}$ & $\begin{array}{l}30 \text { Years } \\
38 \text { psig }\end{array}$ & $\begin{array}{l}\text { Power Sales } \\
\text { PowersalesYr }\end{array}$ & $\begin{aligned} n s & \text { per Month } \\
0 & \text { KWH per Yea }\end{aligned}$ \\
\hline $\begin{array}{l}\text { InitResTemp } \\
\text { rotExplCost } \\
\text { Av Well Cost }\end{array}$ & $\begin{aligned} & 332 \text { Degrees } F \\
& 0 \text { SUS Millions } \\
& 0 \text { SUS Millions }\end{aligned}$ & $\begin{array}{l}\text { InitialTotNCG } \\
\text { Initial } \mathrm{CO} 2 \\
\text { Initial }\end{array}$ & $\begin{array}{r}0 \text { Weight } \% \\
\text { ???? Weight } \%\end{array}$ \\
\hline $\begin{array}{l}\text { ubsurf Area } \\
\text { ot PP Cost } \\
\text { otprojcost } \\
\text { vailability }\end{array}$ & $\begin{array}{l}1 \text { SUS Millions } \\
1 \text { SUS Millions } \\
0 \text { SUS } \\
90 \text { \% }\end{array}$ & $\begin{array}{l}\text { CurrentTotNCG } \\
\text { CurrentCO2 } \\
\text { CurrentH2S } \\
\text { NCGTrtmtsyst }\end{array}$ & $\begin{array}{l}? ? ? \text { Weight } \% \\
0 \text { Weight \% } \\
\text { ???? Weight \% } \\
\text { ???? Weight \% } \\
\text { STACK EMMISSION }\end{array}$ \\
\hline & & & \\
\hline
\end{tabular}




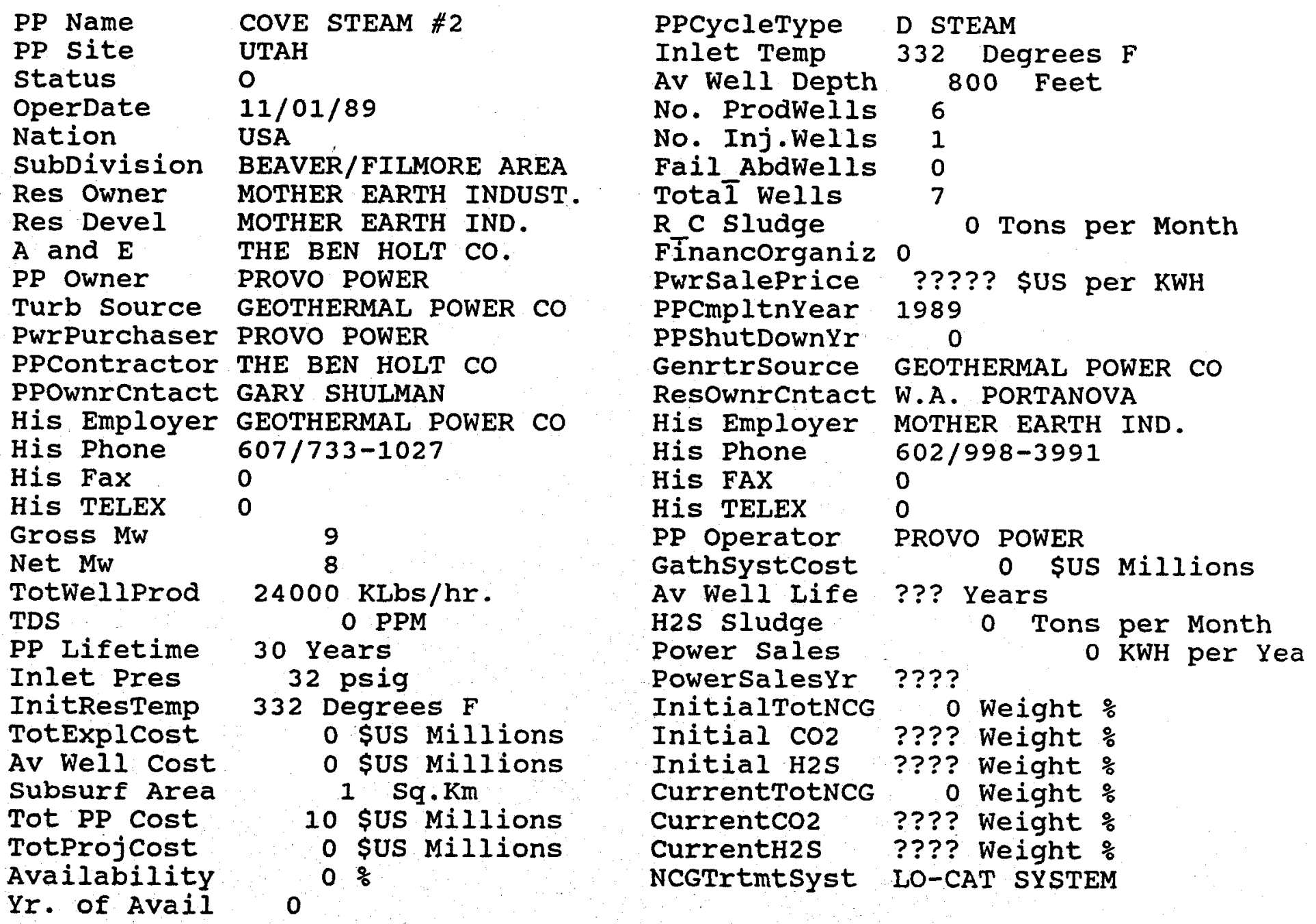




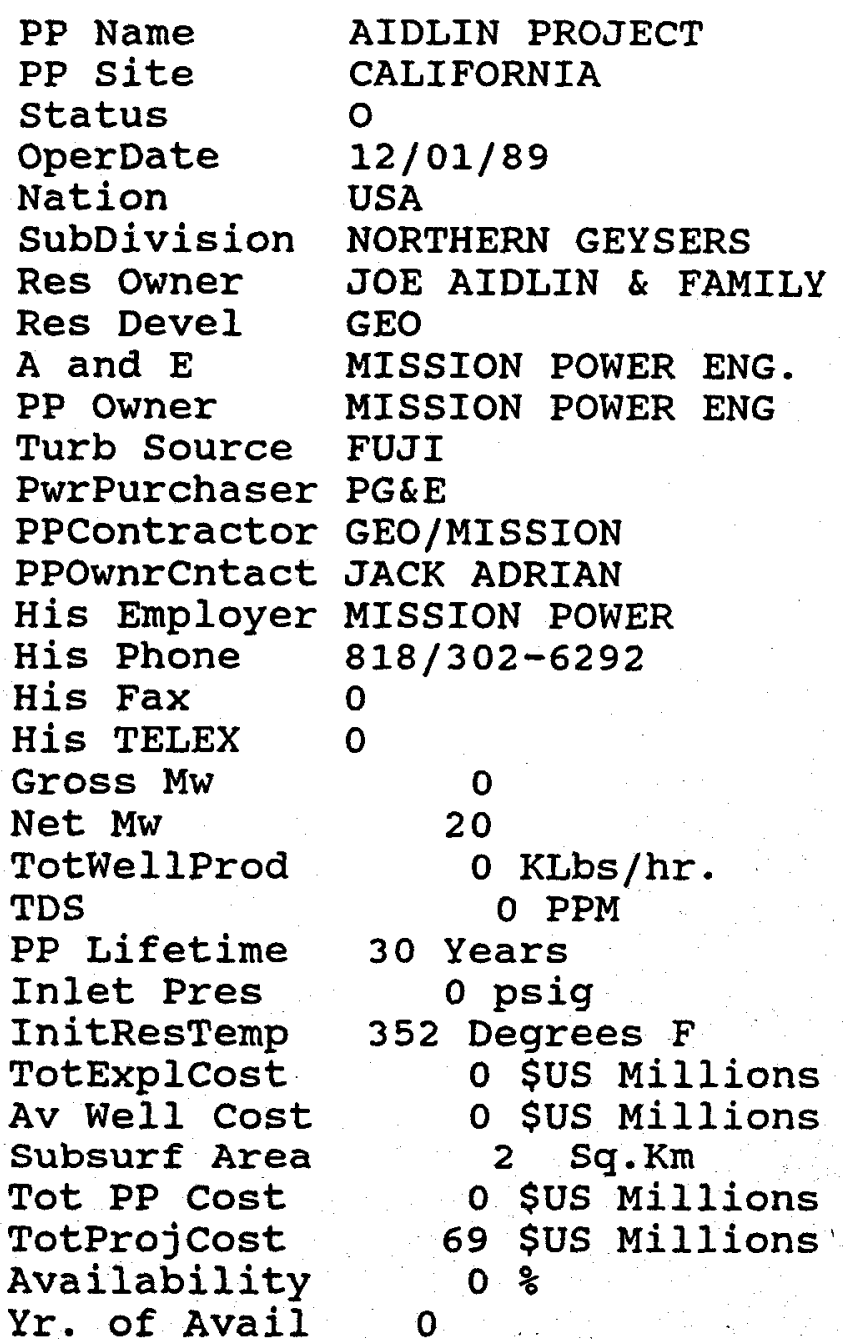

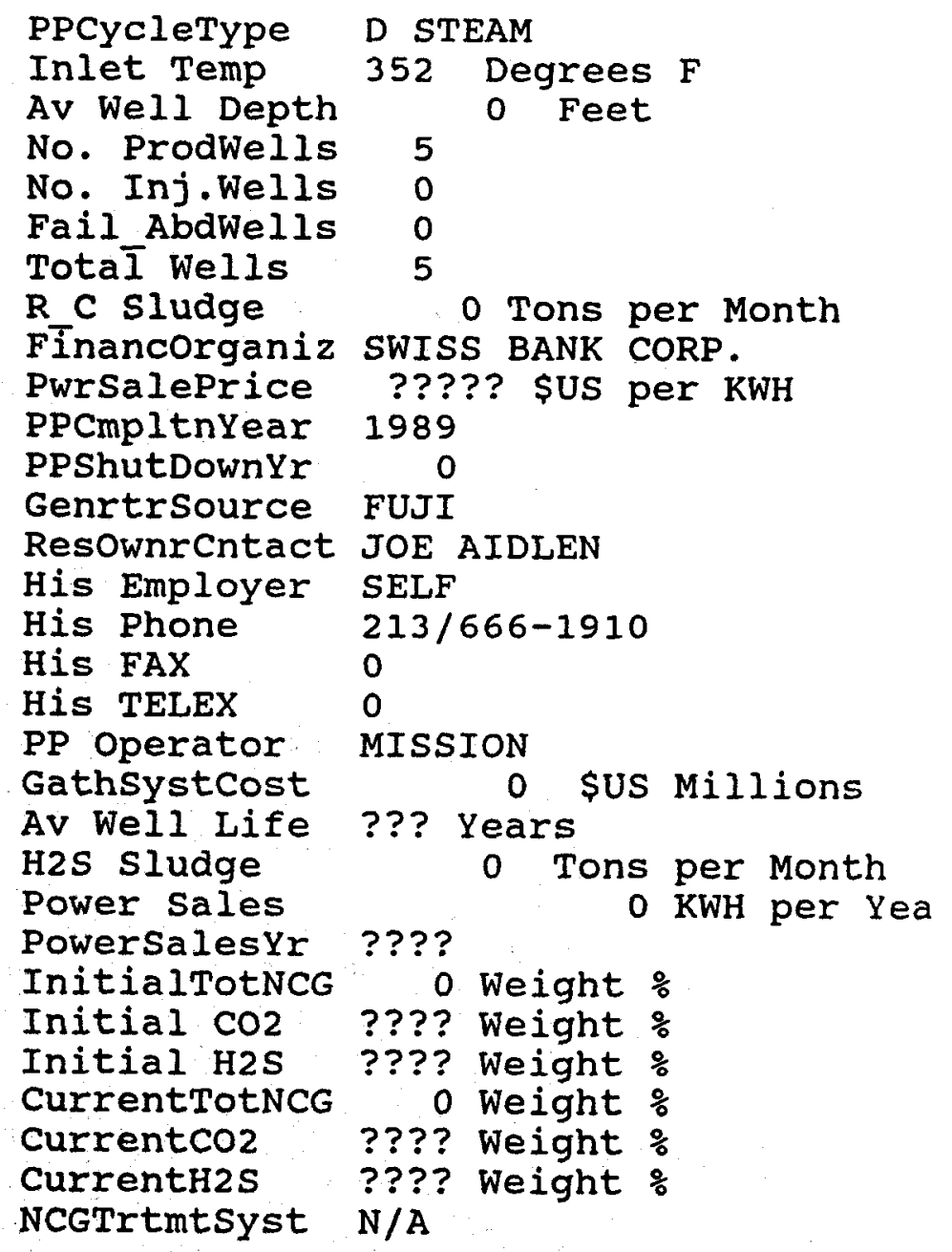




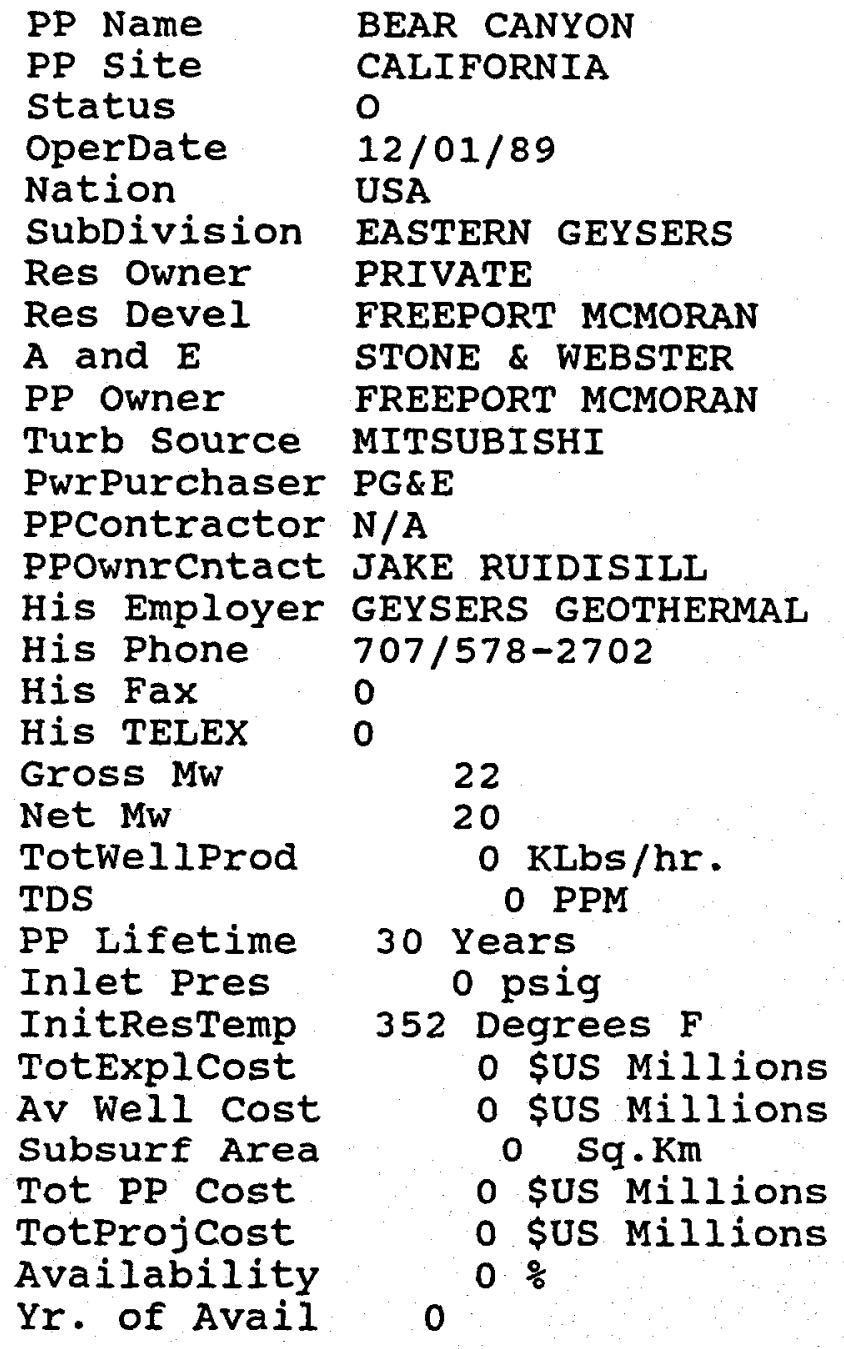

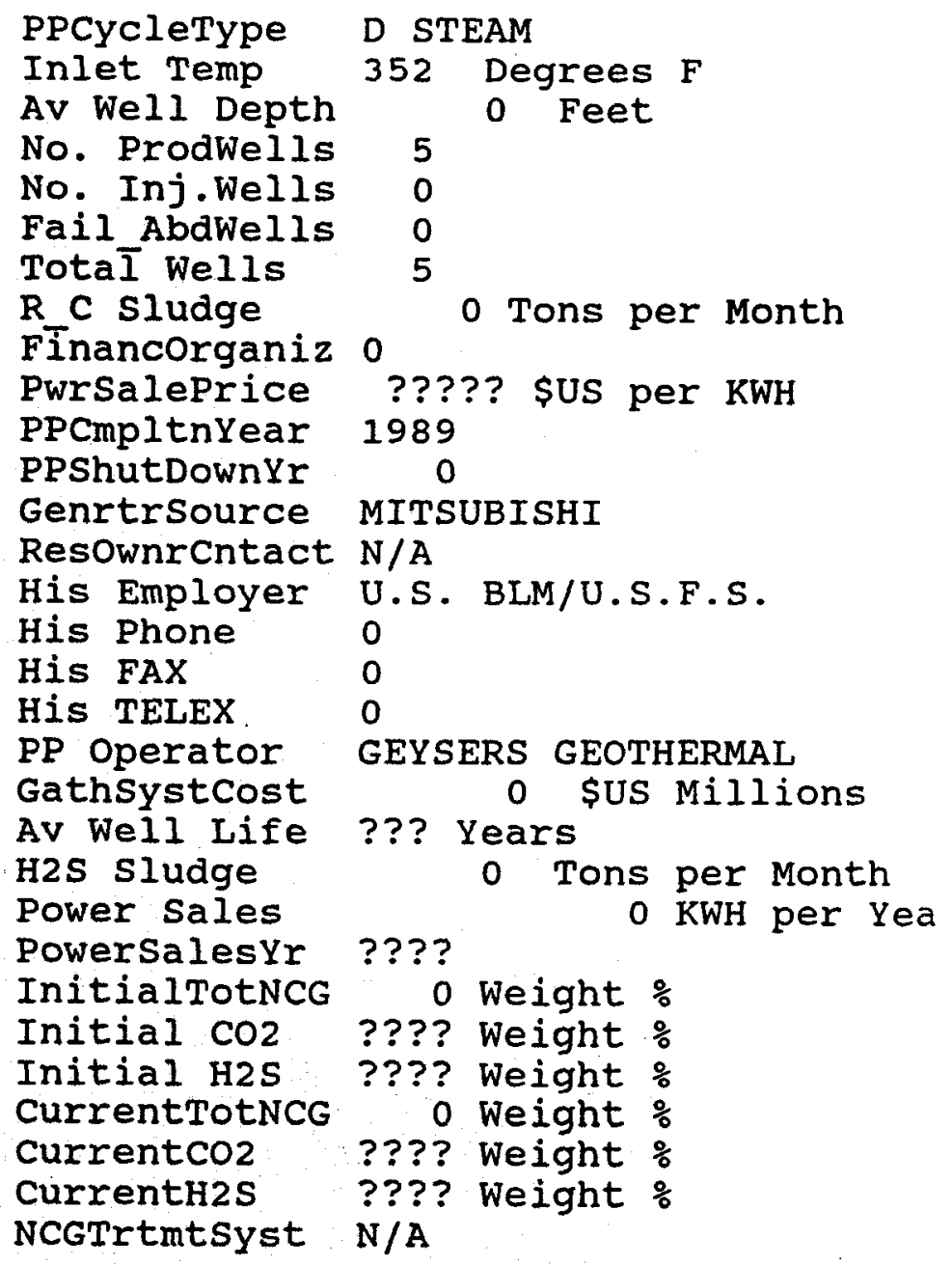




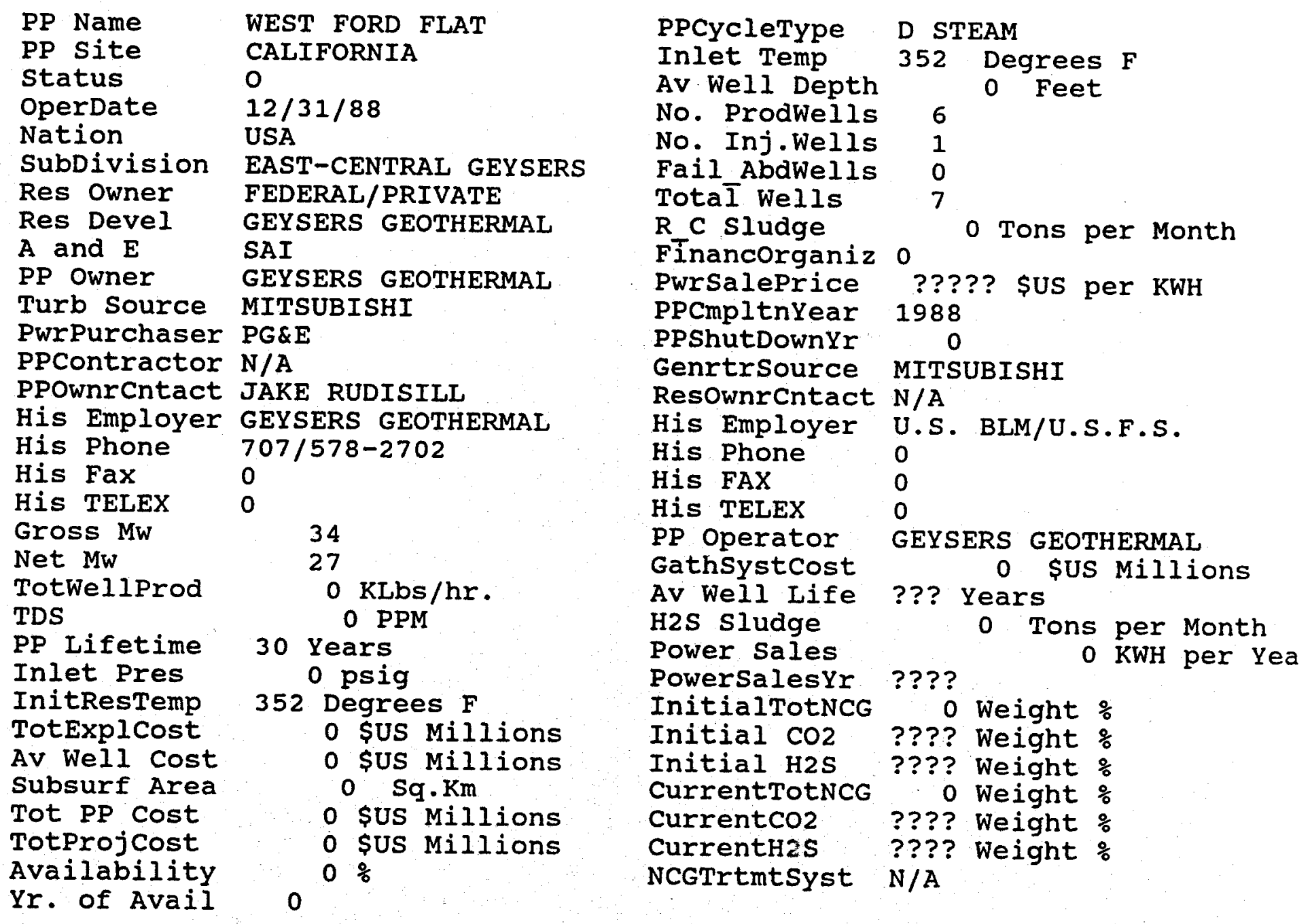




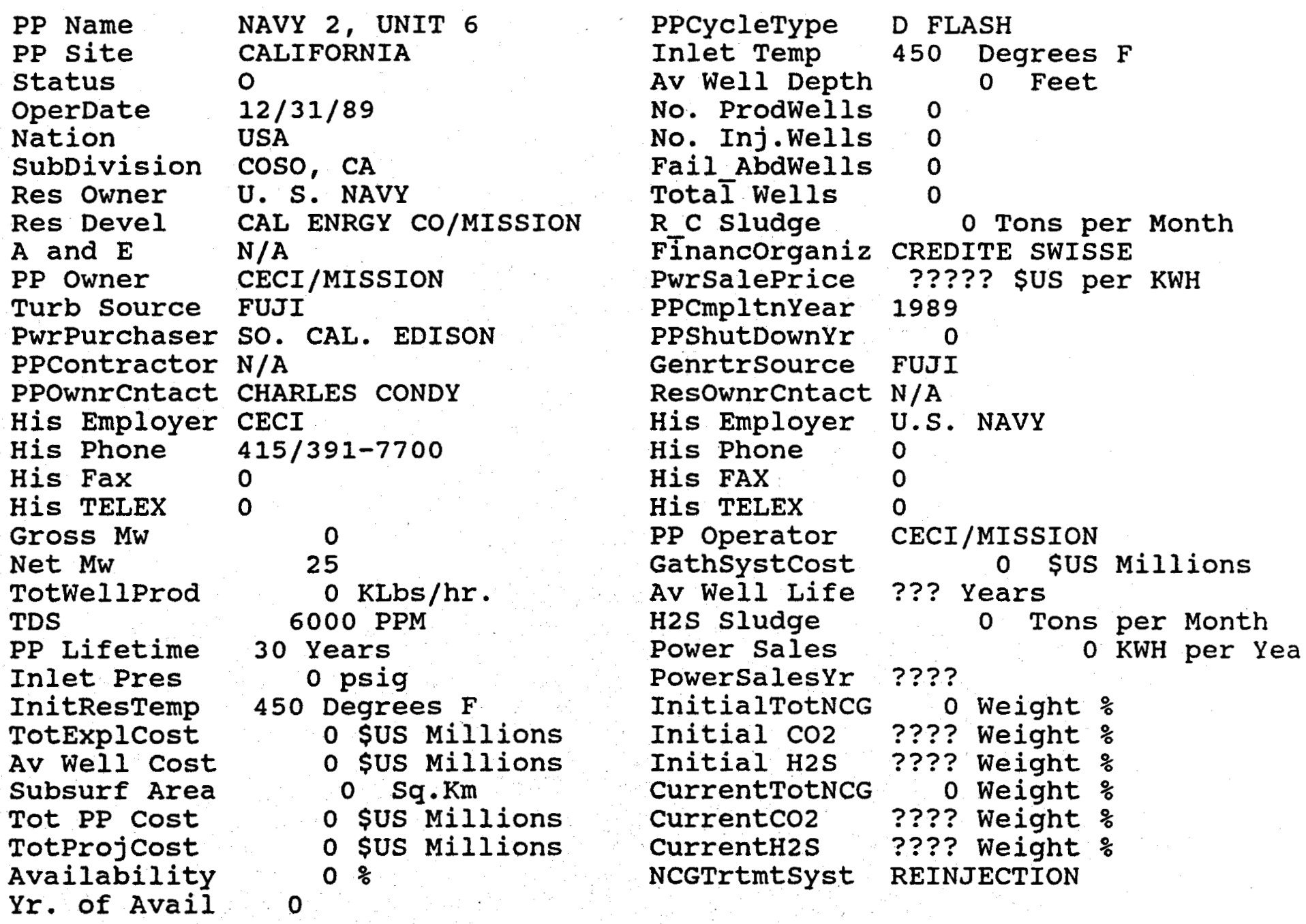




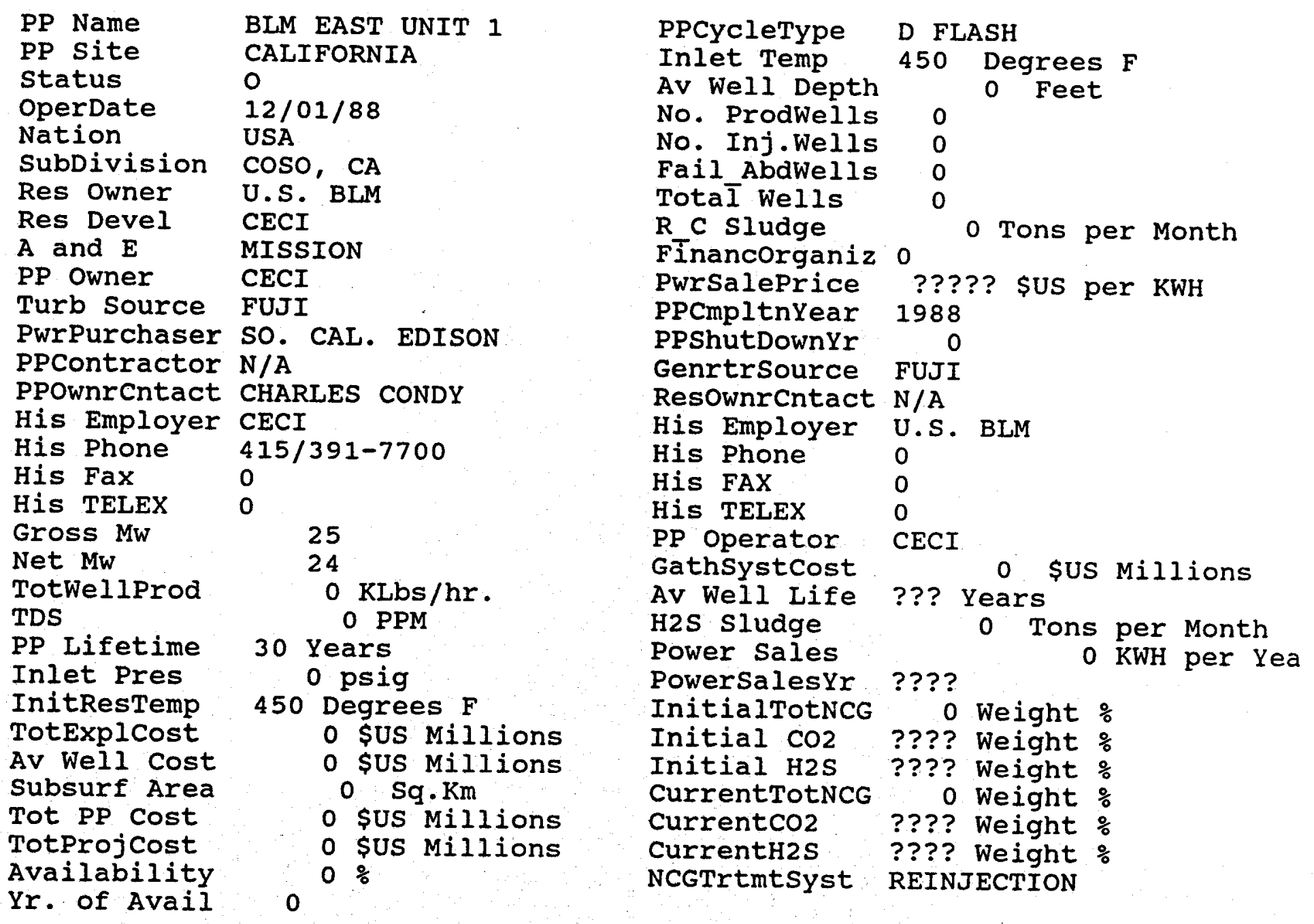




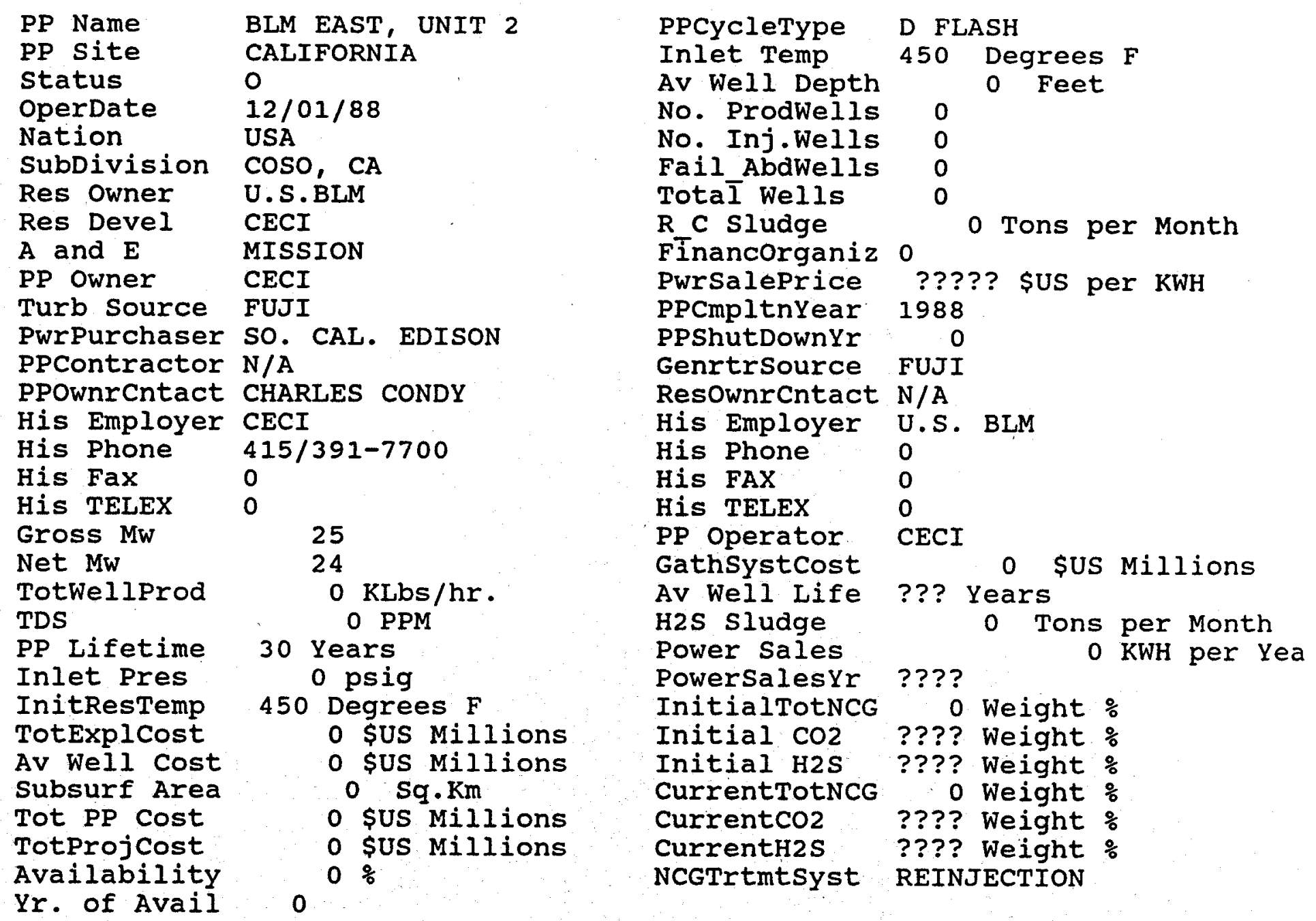




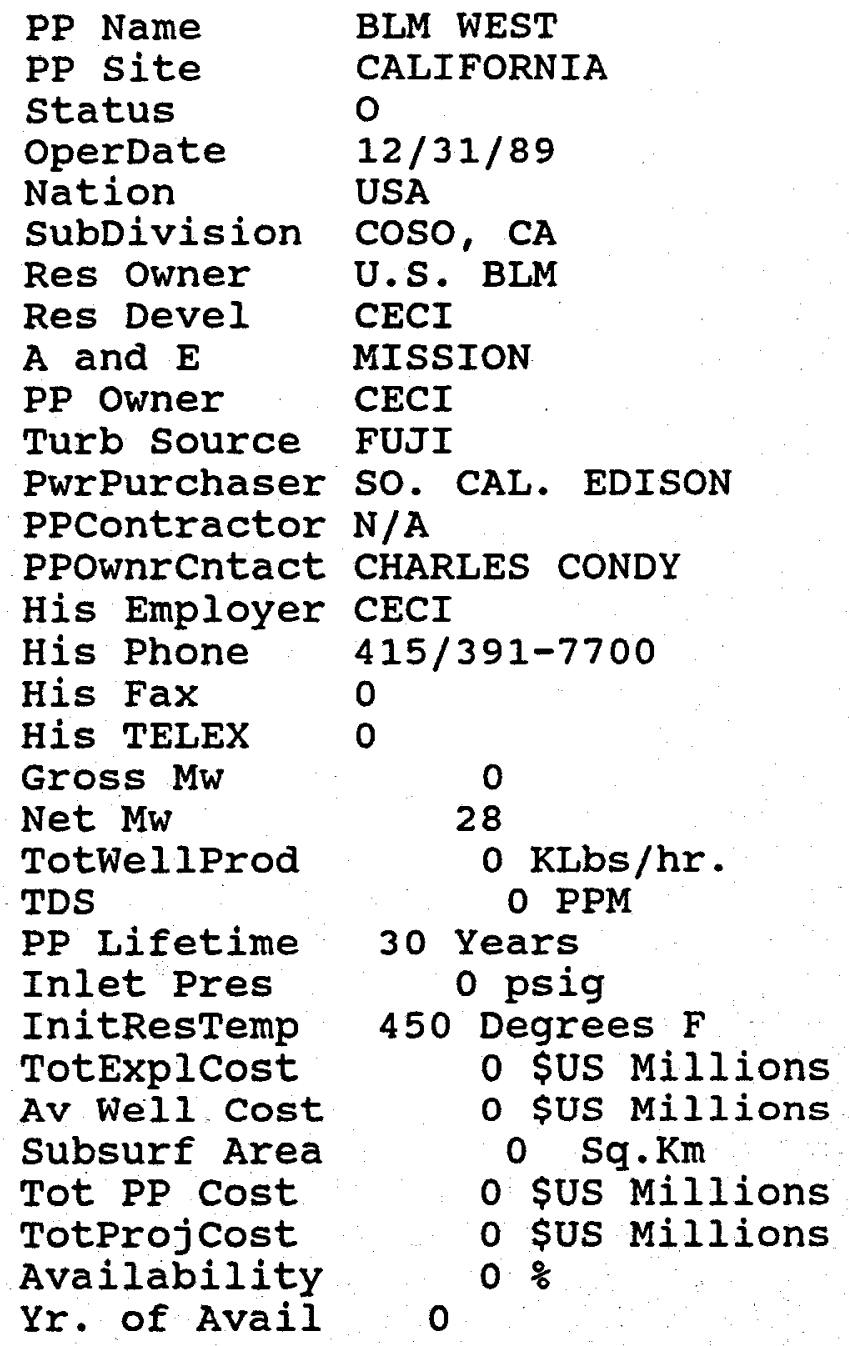

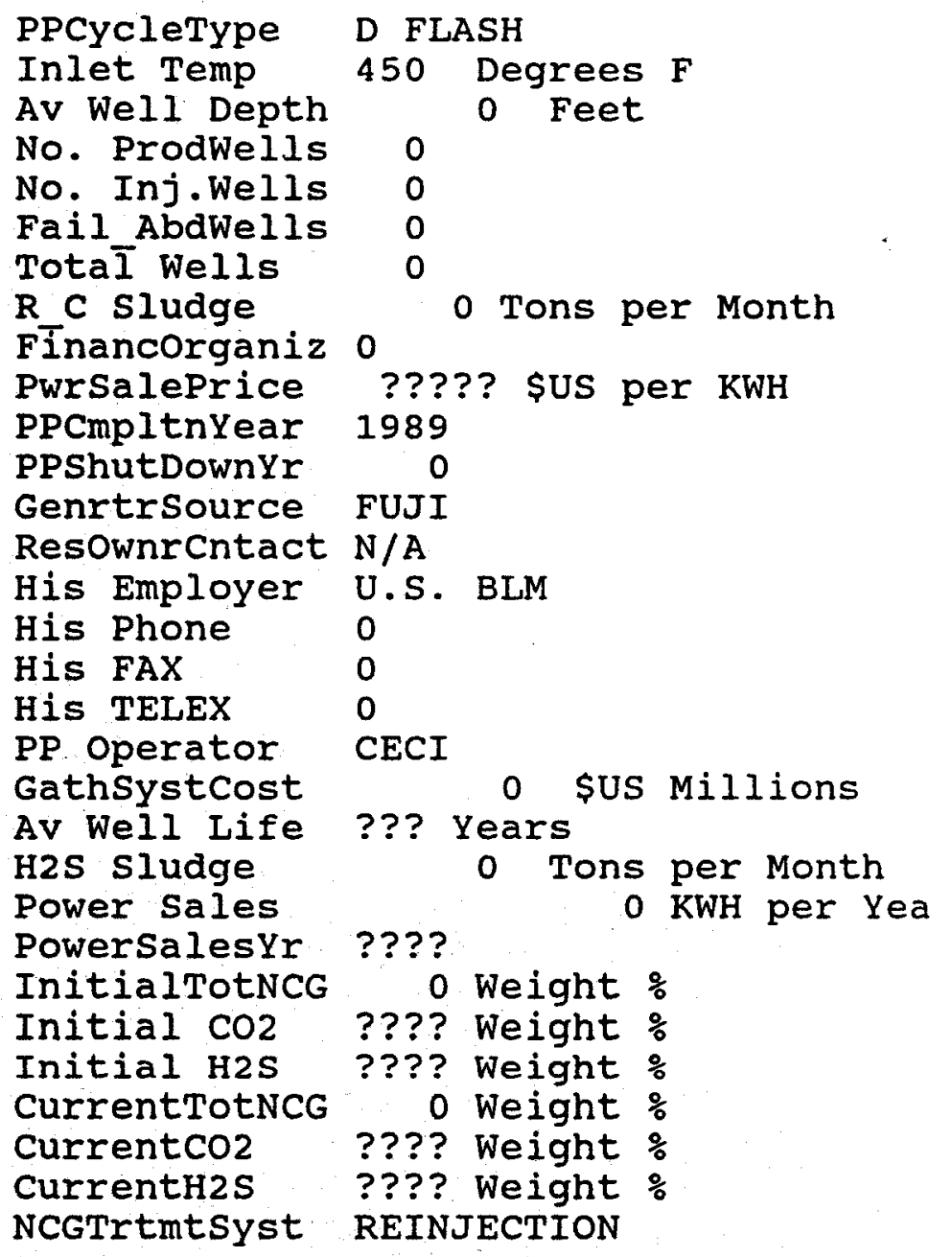




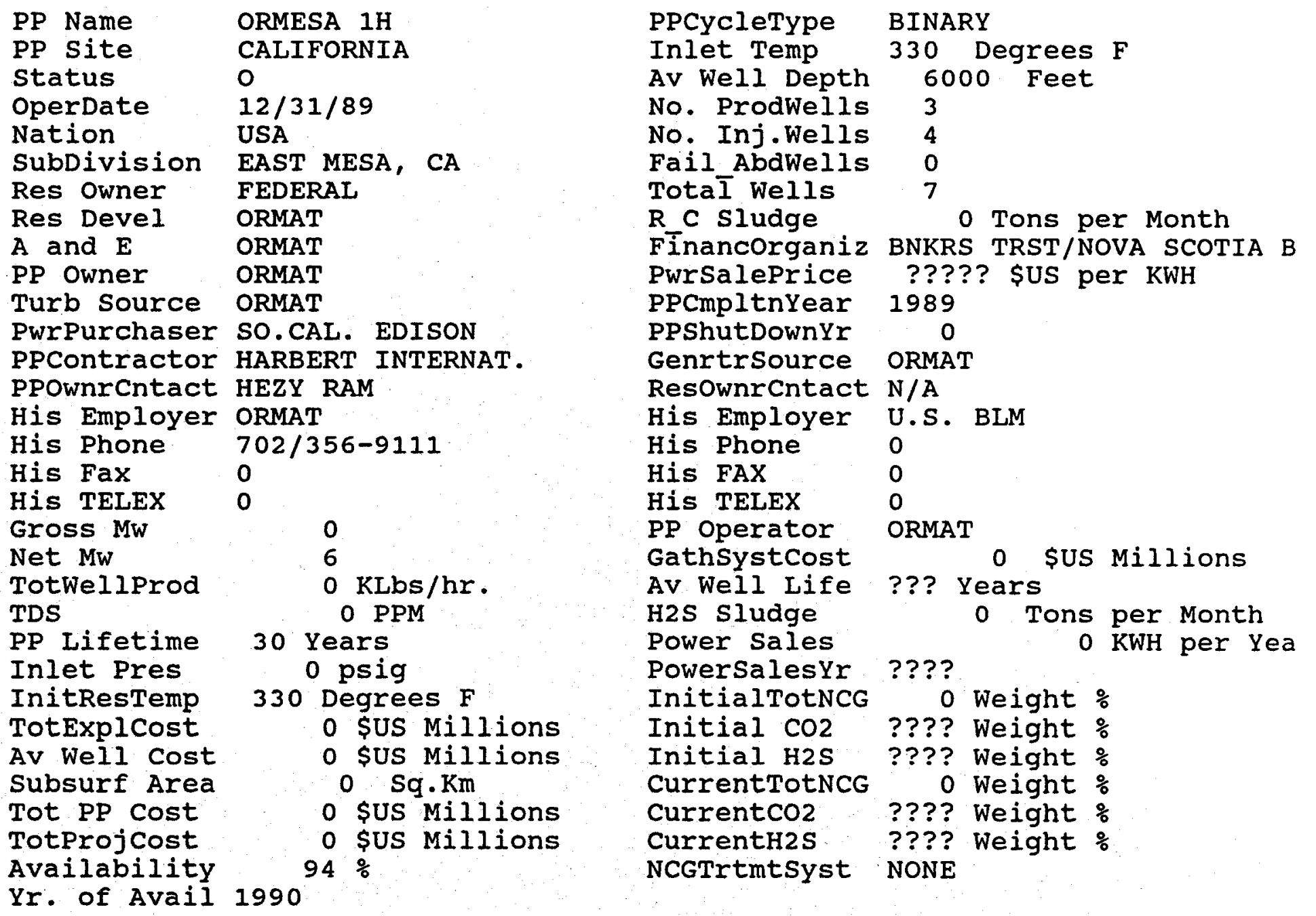




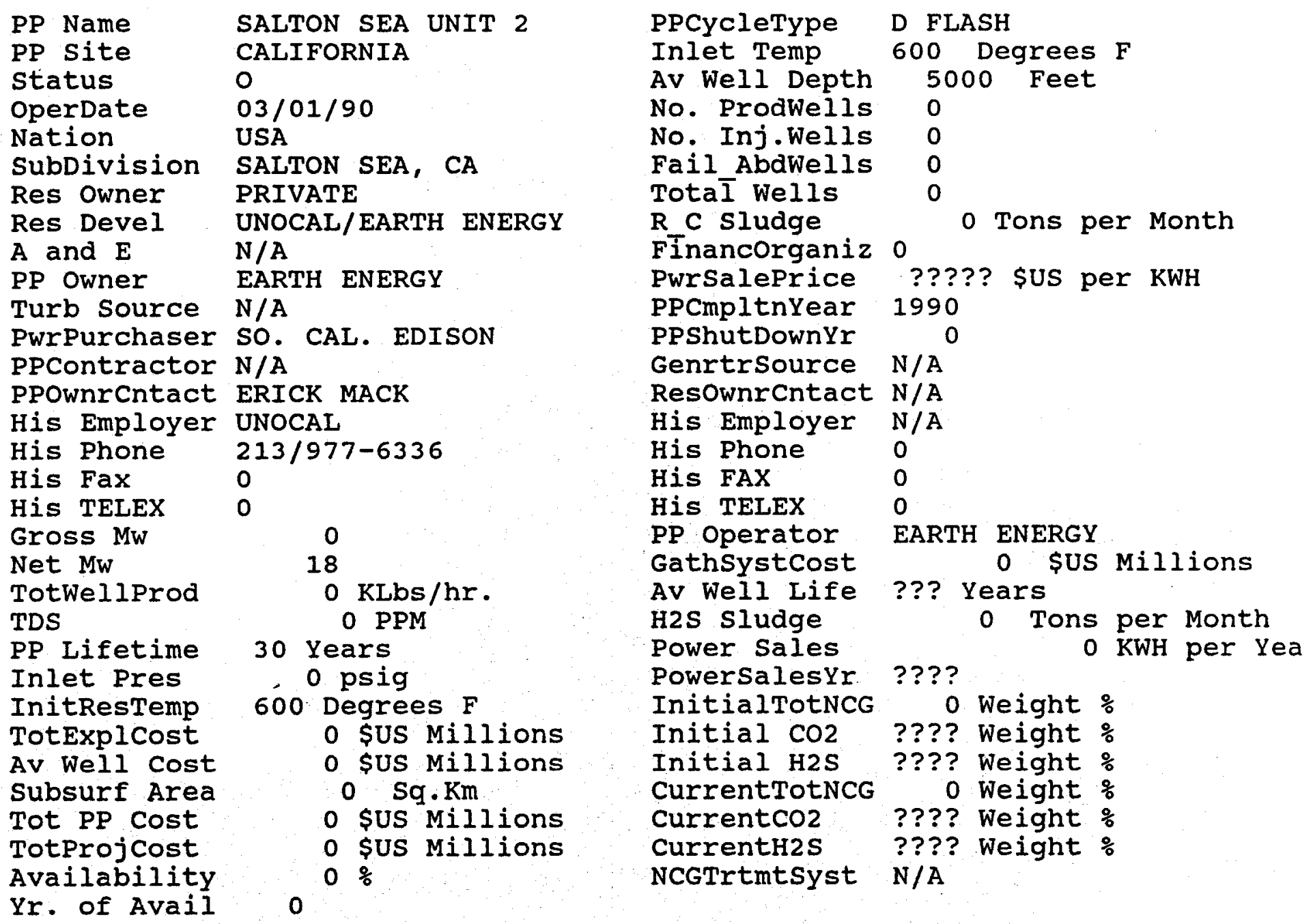




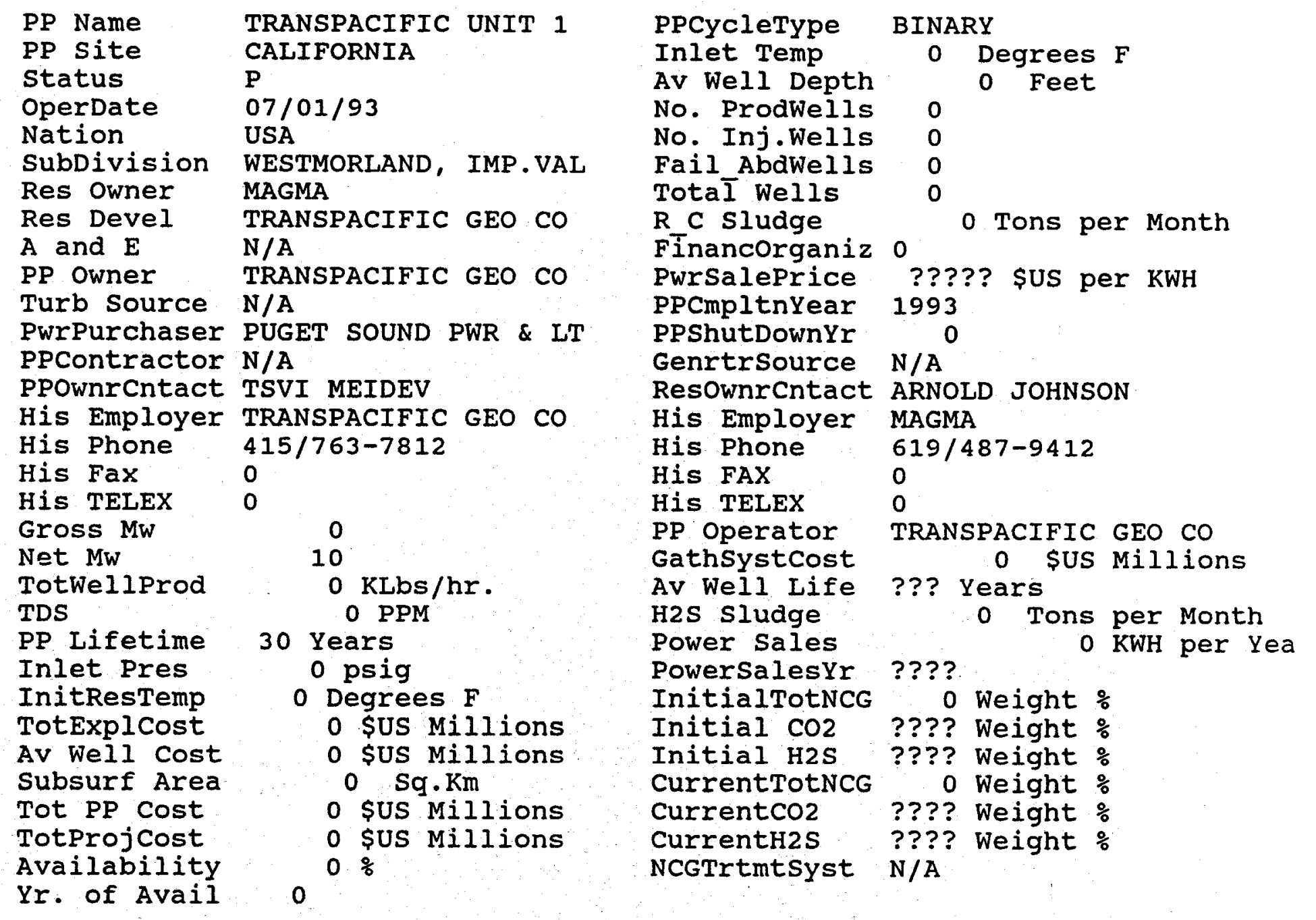




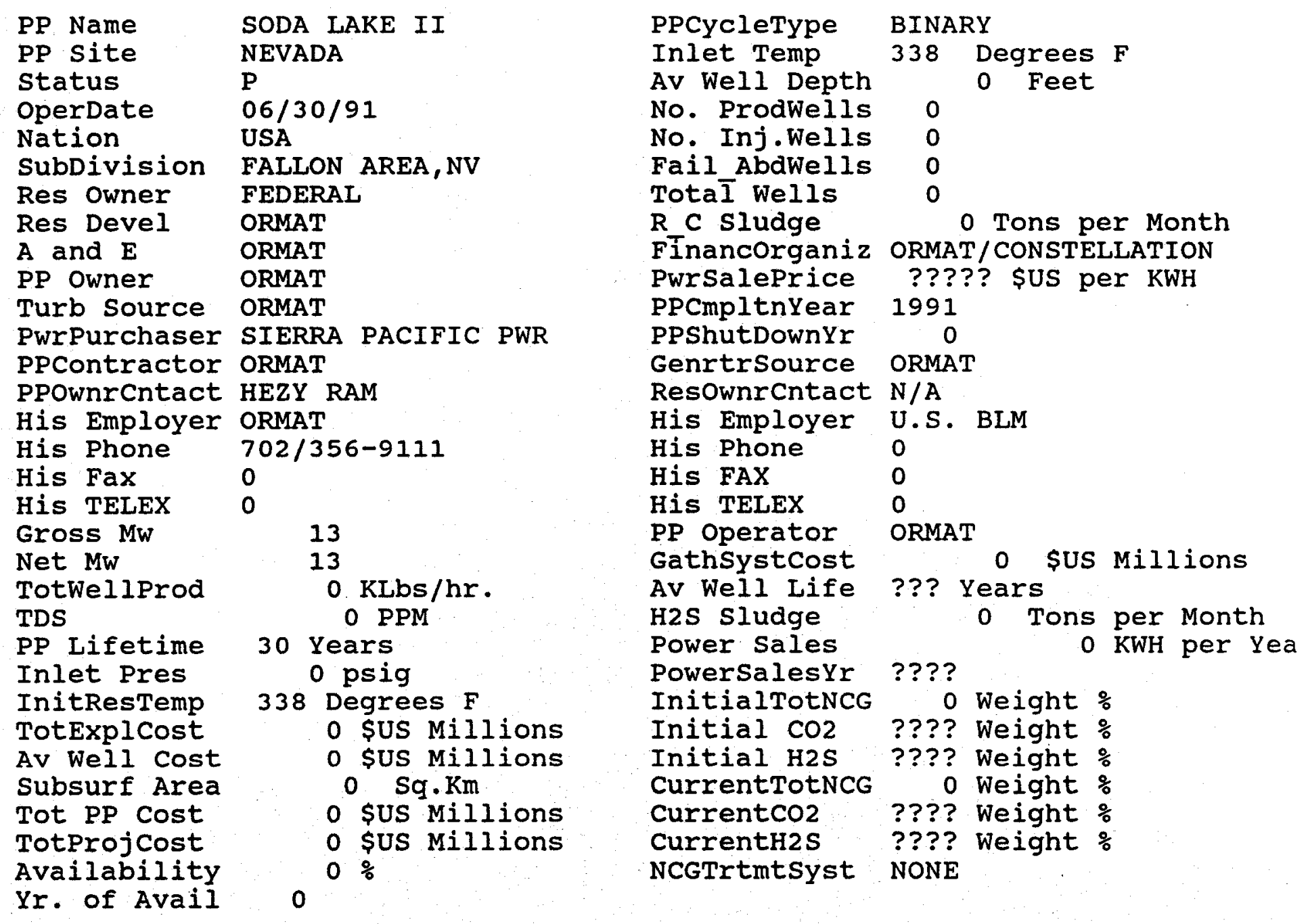




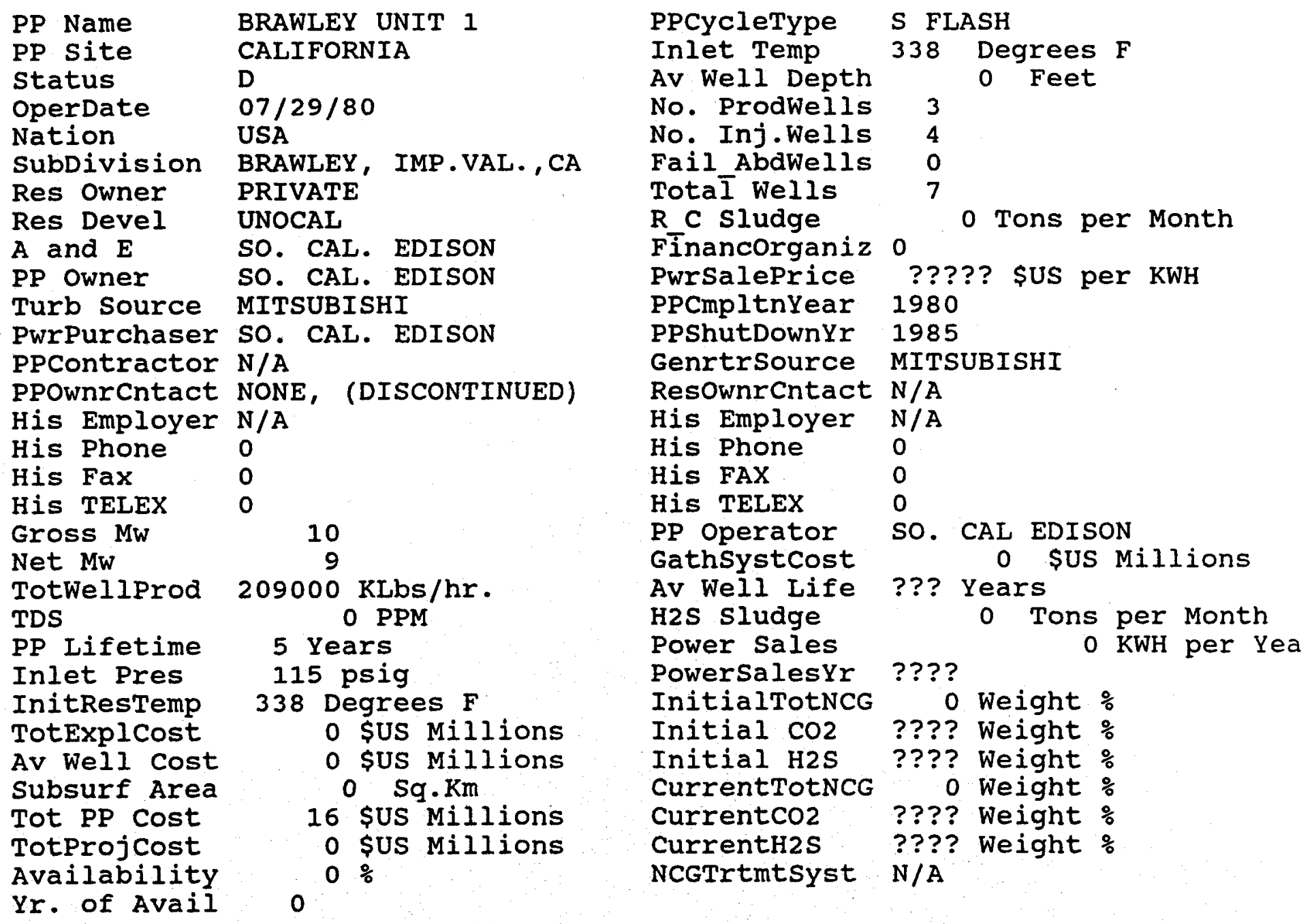




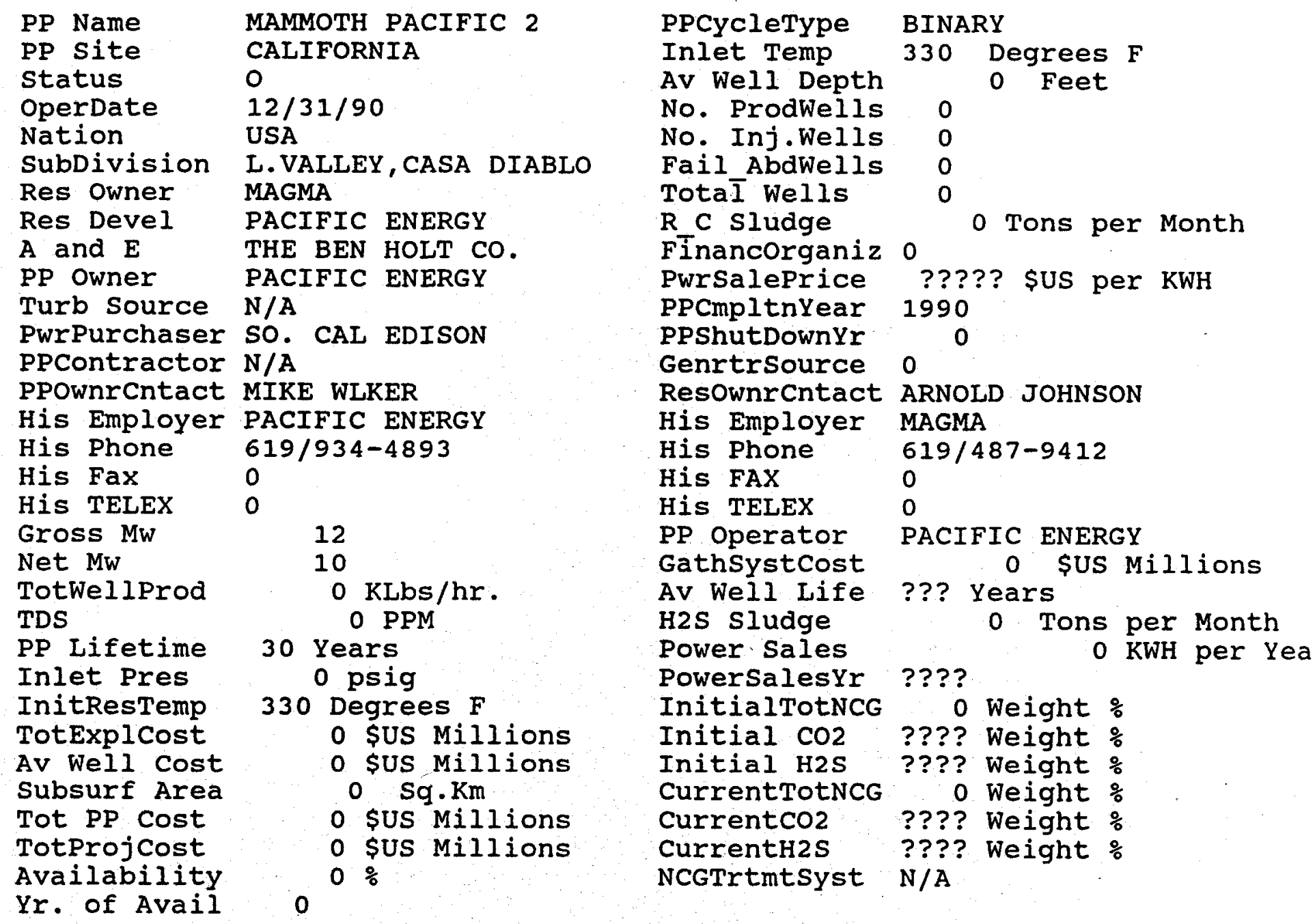


GEOTHERMALLY ACTIVE ENTITY DATABASE 
Algeria $\ldots \ldots \ldots \ldots \ldots \ldots \ldots \ldots \ldots \ldots \ldots \ldots \ldots \ldots \ldots \ldots$

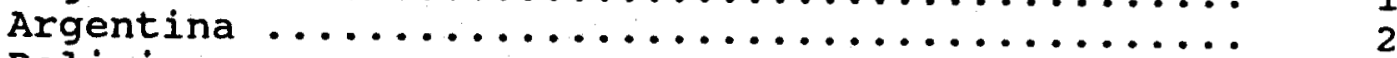

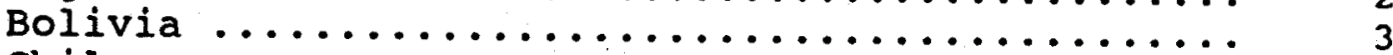

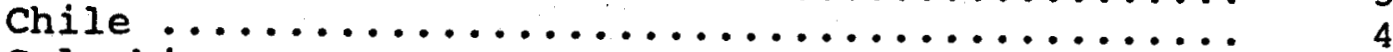

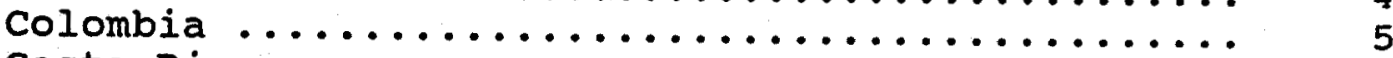

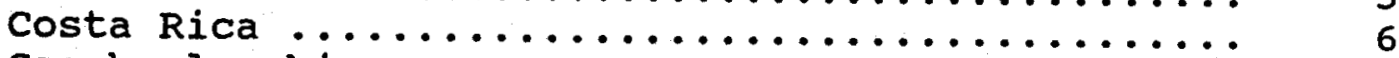

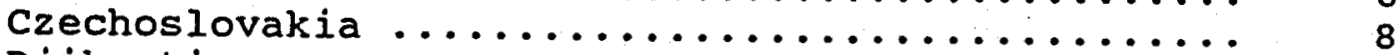

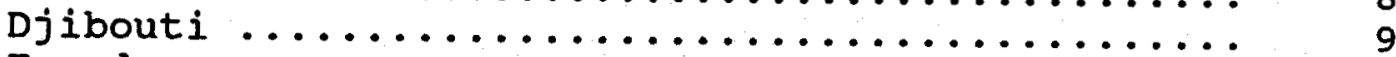

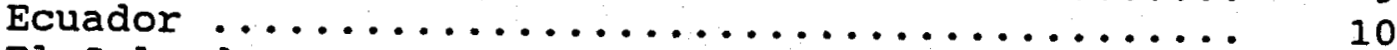

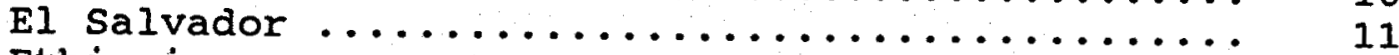

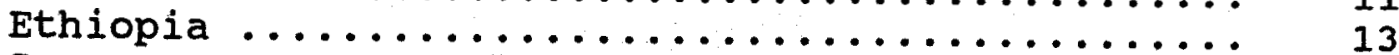

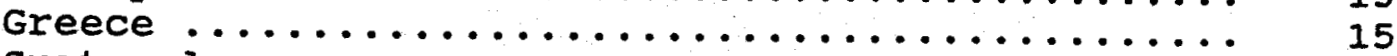

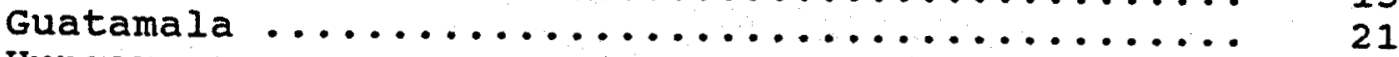

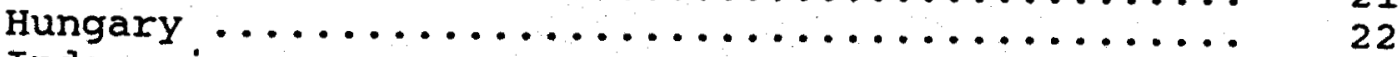

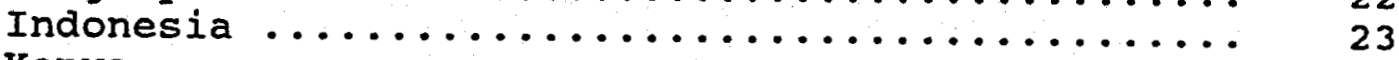

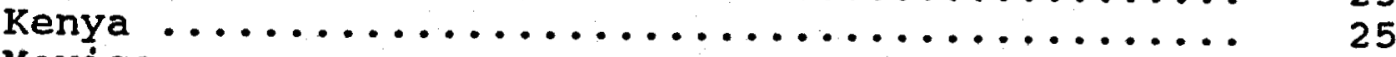

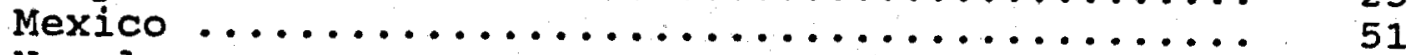

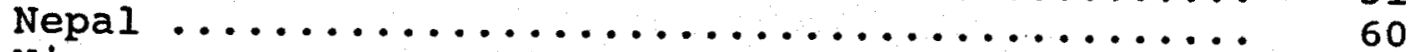

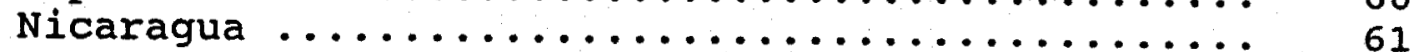

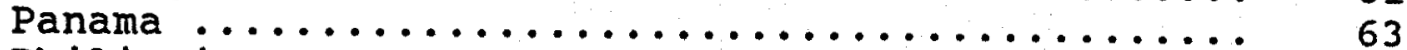

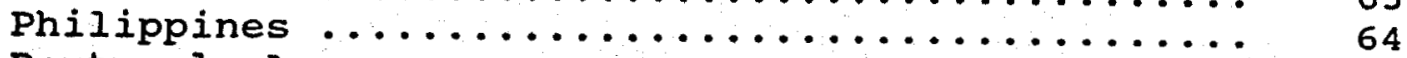

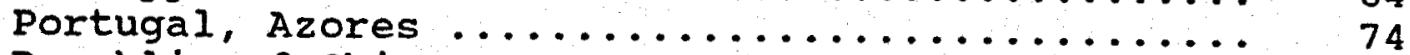

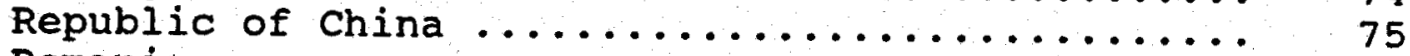

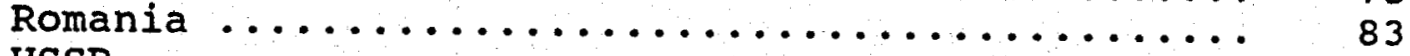

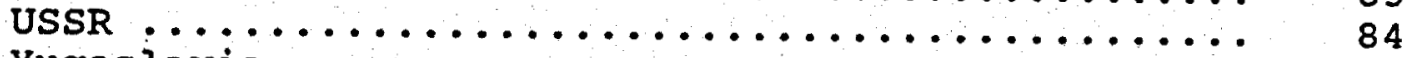

Yugoslavia $\ldots \ldots \ldots \ldots \ldots \ldots \ldots \ldots \ldots \ldots \ldots \ldots \ldots \ldots \ldots$

ngaentc.ont 
NGA LIST OF GEOTHERMALLY ACTIVE ENTITIES

PAGE

1

NATION ALGERIA

FIELD OF EXPERTISE

NAME OF ENTITY

DEPARTMENT NAME

FLOOR

SUITE OR BLDG. NO. STREET ADDRESS

COUNTY

CITY

POSTAL CODE

PERSON TO CONTACT

HIS/HER TITLE

PHONE NUMBER

FAX NUMBER

TELEX NUMBER

B.P. 82
DATE February 22, 1991
RENEWABLE ENERGY

CENTRE de DEV ENERGIES RENOUV

HAUT COMMISSARIAT a la RECHER

NONE

NONE

NONE

BOUZAREAH

CP 16.340

AMOR FEKRAOUI

NONE

NONE

NONE

NONE 
NGA LIST OF GEOTHERMALLY ACTIVE ENTITIES

PAGE 2

DATE February 22, 1991

NATION ARGENTINA

FIELD OF EXPERTISE

SCIENTIFIC STUDIES

NAME OF ENTITY

UNIVERSITY NAC DE SALTA

DEPARTMENT NAME

FLOOR

SUITE OR BLDG. NO. CONSYO DE INVESTIGACION

NONE

NONE

STREET ADDRESS

SALTA CAPITAL 4400

COUNTY

NONE

CITY

POSTAL CODE

PERSON TO CONTACT

SALTA

NONE

HIS/HER TITLE

PHONE NUMBER

SONIA AREDES

NONE

FAX NUMBER

NONE

TELEX NUMBER

548-725-0993

65121 UNSAT AR 
NGA LIST OF GEOTHERMALLY ACTIVE ENTITIES

PAGE 3

DATE February 22, 1991

NATION BOLIVIA

FIELD OF EXPERTISE NAME OF ENTITY DEPARTMENT NAME FLOOR

SUITE OR BLDG. NO. STREET ADDRESS COUNTY

CITY POSTAL CODE PERSON TO CONTACT HIS/HER TITLE PHONE NUMBER FAX NUMBER TELEX NUMBER
MANAGEMENT

EMPRESSA NACIONAL DE ELECTICI GEOTHERMAL NONE

NONE

CASILLA 565

NONE

COCHABAMBA

NONE

GONSALO RICO CALDRON

GENERAL MANAGER GEOTHERML

04214-6721

NONE

$3556251 \mathrm{EDE} \mathrm{BV}$ 
NGA LIST OF GEOTHERMALLY ACTIVE ENTITIES

PAGE 4

DATE February 22, 1991

NATION CHILE

FIELD OF EXPERTISE NAME OF ENTITY

DEPARTMENT NAME

FLOOR

SUITE OR BLDG. NO. STREET ADDRESS COUNTY

CITY

POSTAL CODE

PERSON TO CONTACT

HIS/HER TITLE

PHONE NUMBER

FAX NUMBER

TELEX NUMBER
RESOURCE PRODUCTION

CORP de FOMENTO de la PRODUCT

NONE

NONE

NONE

MONIDA 921

NONE

SANTIAGO

NONE

CLAUDIO CADIZ

NONE

$562-231654$

562-2235019

394-240 421 CORFO CL 
NGA LIST OF GEOTHERMALLY ACTIVE ENTITIES

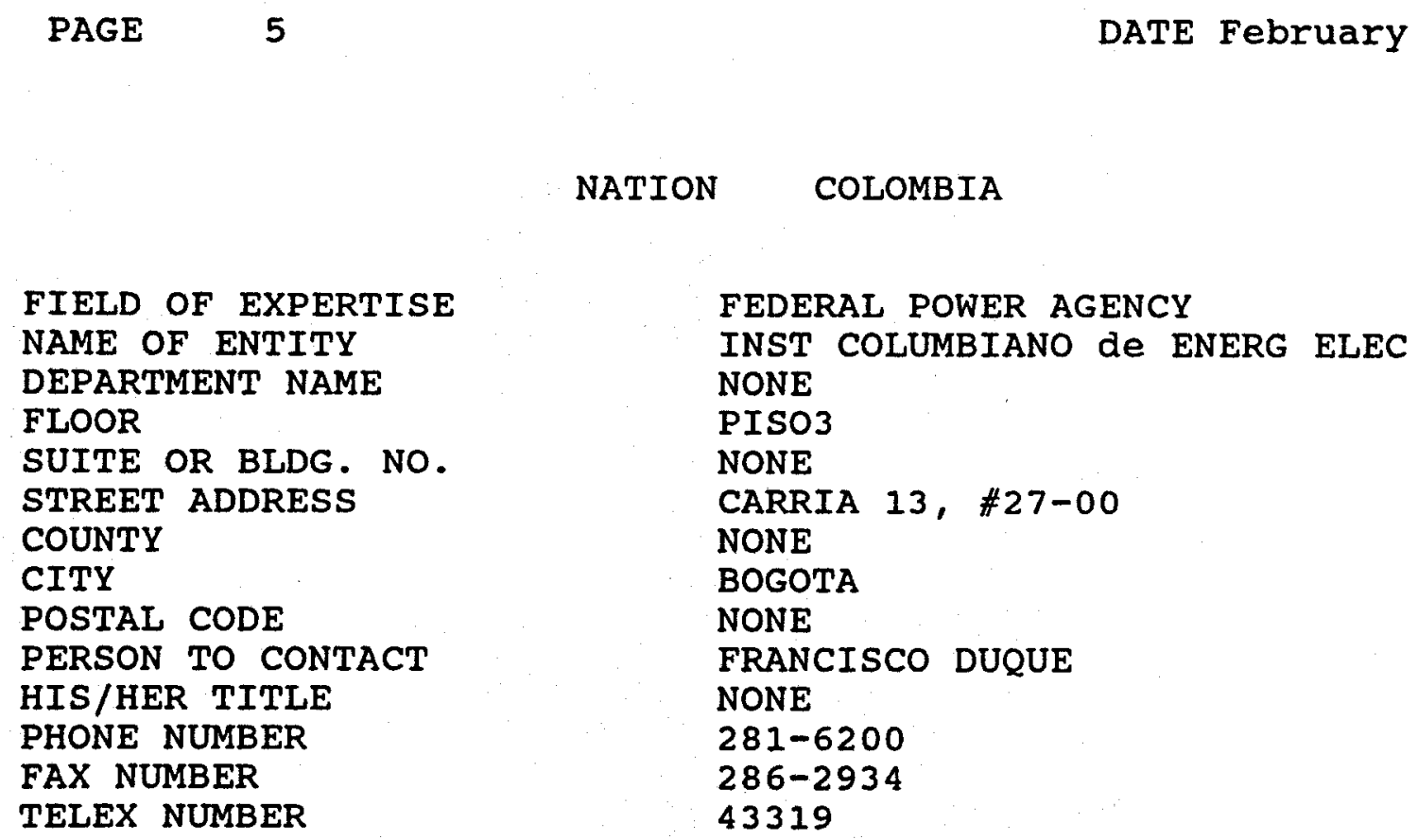

FIELD OF EXPERTISE NAME OF ENTITY DEPARTMENT NAME FLOOR

SUITE OR BLDG. NO. STREET ADDRESS COUNTY

CITY POSTAL CODE PERSON TO CONTACT HIS/HER TITLE PHONE NUMBER FAX NUMBER

TELEX NUMBER

FEDERAL POWER AGENCY INST COLUMBIANO de ENERG ELEC NONE

PISO3

NONE

CARRIA 13, \#27-00

NONE

BOGOTA

NONE

FRANCISCO DUQUE NONE

$281-6200$

286-2934

43319 
NGA LIST OF GEOTHERMALLY ACTIVE ENTITIES

PAGE 6

NATION COSTA RICA
FIELD OF EXPERTISE NAME OF ENTITY DEPARTMENT NAME FLOOR

SUITE OR BLDG. NO. STREET ADDRESS COUNTY

CITY POSTAL CODE PERSON TO CONTACT HIS/HER TITLE PHONE NUMBER FAX NUMBER TELEX NUMBER
DATE February 22, 1991

\author{
MANAGEMENT \\ INST. COSTARRICENSE DE ELECTR \\ NONE \\ NONE \\ NONE \\ APARTADO 10032-1000 \\ NONE \\ SAN JOSE \\ NONE \\ TEOFILO de la TORRE \\ DEPUTY DIRECTOR \\ 303-207720 \\ 506-32-2824 \\ 303-2600 ICEGER CR
}


NGA LIST OF GEOTHERMALLY ACTIVE ENTITIES

PAGE 7

DATE February 22, 1991

NATION COSTA RICA

FIELD OF EXPERTISE

GEOTHERMAL OPERATIONS

NAME OF ENTITY

DEPARTMENT NAME

FLOOR

SUITE OR BLDG. NO.

STREET ADDRESS

COUNTY

CITY

POSTAL CODE

PERSON TO CONTACT

HIS/HER TITLE

PHONE NUMBER

INST. COSTARRICENSE DE ELECTR

NONE

NONE

NONE

P.O. BOX 471

NONE

SAN JOSE

NONE

ALFREDO MAINIERI

MANAGER, GEOTH OPERATIONS

FAX NUMBER

506-20-7533

506-303-2140

TELEX NUMBER

NONE 
NGA LIST OF GEOTHERMALLY ACTIVE ENTITIES

PAGE 8

NATION CZECHOSLOVAKIA
FIELD OF EXPERTISE NAME OF ENTITY DEPARTMENT NAME FLOOR

SUITE OR BLDG. NO. STREET ADDRESS COUNTY

CITY

POSTAL CODE

PERSON TO CONTACT

HIS/HER TITLE

PHONE NUMBER

FAX NUMBER

TELEX NUMBER
DATE February 22, 1991

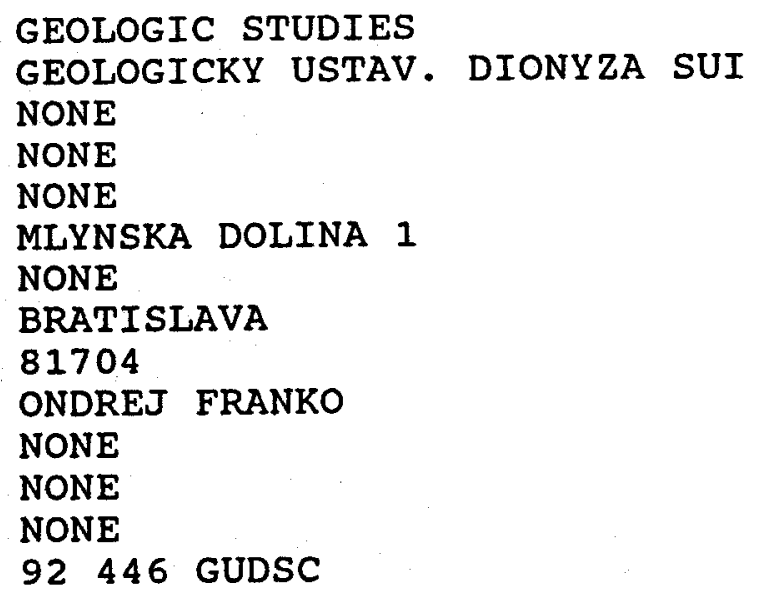


NGA LIST OF GEOTHERMALLY ACTIVE ENTITIES

PAGE 9

DATE February 22, 1991

NATION DJIBOUTI

FIELD OF EXPERTISE

PUBLIC UTILITY

NAME OF ENTITY

DEPARTMENT NAME

FLOOR

SUITE OR BLDG. NO.

STREET ADDRESS

COUNTY

CITY

POSTAL CODE

PERSON TO CONTACT

ELECTRIC DE DJIBOUTI

NONE

NONE

NONE

BOULVARD de la REPUBLIQUE

NONE

DJIBOUTI

NONE

DJAMA A. GUELLEH

HIS/HER TITLE

PHONE NUMBER

ING. DIPLOME, DIRECTOR

FAX NUMBER

$35 \quad 28 \quad 51-352852$

TELEX NUMBER

NONE

5842 DJIBELEC 
NGA LIST OF GEOTHERMALLY ACTIVE ENTITIES

PAGE $\quad 10$

DATE February 22, 1991

NATION ECUADOR

FIELD OF EXPERTISE NAME OF ENTITY DEPARTMENT NAME FLOOR

SUITE OR BLDG. NO. STREET ADDRESS COUNTY

\section{CITY} POSTAL CODE PERSON TO CONTACT HIS/HER TITLE PHONE NUMBER

FAX NUMBER

TELEX NUMBER
FEDERAL POWER AGENCY

INST. ECUATORIANO ELECTRIC GEOTHERMAL

NONE

NONE

AVENIDA 10 de AGOSTO Y ROCA

NONE

QUITO

P.O. BOX 565 A

EDUARDO ALMEIDA

HEAD OF GEOTHERMAL PROJTS

593-246-4643

593-256-9997

3932437 DEIC 
NGA LIST OF GEOTHERMÁLLY ACTIVE ENTITIES

PAGE 11

DATE February 22, 1991

NATION EL SALVADOR

FIELD OF EXPERTISE

GEOTHERMAL CONSULTATION

NAME OF ENTITY

DEPARTMENT NAME NONE

NONE

FLOOR

SUITE OR BLDG. NO. STREET ADDRESS

COUNTY

CITY

POSTAL CODE

PERSON TO CONTACT

NONE

NONE

P.O. BOX 01478

NONE

SAN SALVADOR

NONE

HIS/HER TITLE

GUSTAVO CUELLAR

PHONE NUMBER

PRIVATE CONSULTANT

FAX NUMBER

TELEX NUMBER

503-23-5183

503-23-1654

NONE 
NGA LIST OF GEOTHERMALLY ACTIVE ENTITIES

PAGE 12

DATE February 22, 1991

NATION EL SALVADOR

FIELD OF EXPERTISE

NAME OF ENTITY

DEPARTMENT NAME

FLOOR

SUITE OR BLDG. NO. STREET ADDRESS

COUNTY

CITY

POSTAL CODE

PERSON TO CONTACT

HIS/HER TITLE

PHONE NUMBER

FAX NUMBER

TELEX NUMBER
GEOTHERMAL EXPLORATION

CENTRO INVESTIG. GEOTERMICAS EXPLORATION DEPT.

NONE

NONE

NONE

NONE

NUEVA SAN SALVADOR

NONE

ALEJANDOR CAMPOS ROMERO

HEAD-GEOTH EXPLOR. DEPT

NONE

503-28-1911

20303 CEL-SAL 
NGA LIST OF GEOTHERMALLY ACTIVE ENTITIES

PAGE 13

DATE February 22, 1991

NATION ETHIOPIA

FIELD OF EXPERTISE

NAME OF ENTITY

DEPARTMENT NAME

FLOOR

SUITE OR BLDG. NO. STREET ADDRESS

COUNTY

CITY

POSTAL CODE

PERSON TO CONTACT

HIS/HER TITLE

PHONE NUMBER

FAX NUMBER

TELEX NUMBER
GEOTHERMAL EXPLORATION

ETHIOPIAN INST.OF GEOL.SURVEY

GEOTHERMAL EXPLORATION DEPT.

NONE

NONE

P.O. BOX 2616

NONE

ADDIS ABABA

NONE

TESHOME ABERA

NONE

NONE

NONE

21448 MME ET 
NGA LIST OF GEOTHERMALLY ACTIVE ENTITIES

PAGE $\quad 14$

DATE February 22, 1991

NATION ETHIOPIA

FIELD OF EXPERTISE NAME OF ENTITY

DEPARTMENT NAME

FLOOR

SUITE OR BLDG. NO. STREET ADDRESS COUNTY

CITY

POSTAL CODE

PERSON TO CONTACT

HIS/HER TITLE

PHONE NUMBER

FAX NUMBER

TELEX NUMBER
GEOTHERMAL EXPLORATION

ETHIOPIAN INST.OF GEOL. SURVEY GEOTHERMAL EXPLORATION DEPT.

NONE

NONE

P.O. BOX 2616

NONE

ADDIS ABABA

NONE

TEKLU HUADGU

DR.

NONE

NONE

21448 MME ET 
NGA LIST OF GEOTHERMALLLY ACTIVE ENTITIES

PAGE 15 DATE February 22, 1991

NATION GREECE

FIELD OF EXPERTISE NAME OF ENTITY DEPARTMENT NAME FLOOR

SUITE OR BLDG. NO. STREET ADDRESS COUNTY

CITY

POSTAL CODE PERSON TO CONTACT HIS/HER TITLE PHONE NUMBER FAX NUMBER TELEX NUMBER
GEOTHERMAL EXPLORATION INST. OF GEOL AND MIN EXPLOR. IGME , DEPY NONE NONE 70, MESSOGHION STREET NONE ATHENS 10680 K. KOUKOUZAS MANAGER NONE LISTED NONELISTED NONE LISTED 
NGA LIST OF GEOTHERMALLY ACTIVE ENTITIES

PAGE 16

DATE February 22, 1991

NATION GREECE

FIELD OF EXPERTISE

NAME OF ENTITY

DEPARTMENT NAME

FLOOR

SUITE OR BLDG. NO.

STREET ADDRESS

COUNTY

CITY

POSTAL CODE

PERSON TO CONTACT

HIS/HER TITLE

PHONE NUMBER

FAX NUMBER

TELEX NUMBER
POWER PIANT CONSTRUCTION

PUBLIC POWER CORPORATION

DIR ALTERN ENERGY SOURCES

NONE

NONE

10, NAVARINOU STREET

NONE

ATHENS

10680

MRS. R. TASSIOU

MANAGER PPC, DEME

NONE LISTED

NONE LISTED

NONE LISTED 
NGA IIST OF GEOTHERMALLY ACTIVE ENTITIES

PAGE 17

NATION GREECE
DATE February 22, 1991

\author{
GEOTHERMAL DEVELOPMENT \\ PUBLIC POWER CORPORATION \\ DIR OF ALTERN ENERGY SOURCES \\ NONE \\ NONE \\ 10, NAVARINOU STREET \\ NONE \\ ATHENS \\ 10680 \\ MRS. R. TASSIOU \\ MANAGER \\ NONE LISTED \\ NONE LISTED \\ NONE LISTED
}


NGA LIST OF GEOTHERMALLY ACTIVE ENTITIES

PAGE 18

DATE February 22, 1991

NATION GREECE

FIELD OF EXPERTISE NAME OF ENTITY

DEPARTMENT NAME

FLOOR

SUITE OR BLDG. NO. STREET ADDRESS COUNTY

CITY

POSTAL CODE

PERSON TO CONTACT

HIS/HER TITLE

PHONE NUMBER

FAX NUMBER

TELEX NUMBER
GRANTING OF GT LEASES, CONCES

MINISTRY OF IND, ENERGY, TECHN

DIRECTION OF PUBLIC MINES

NONE

NONE

80, MIHALACOPOULOU STREET

NONE

ATHENS

10192

NONE LISTED

NONE LISTED

NONE LISTED

NONE LISTED

NONE LISTED 
NGA LIST OF GEOTHERMALLY ACTIVE ENTITIES

PAGE $\quad 19$

DATE February 22, 1991

NATION GREECE

FIELD OF EXPERTISE NAME OF ENTITY

DEPARTMENT NAME

FLOOR

SUITE OR BLDG. NO. STREET ADDRESS COUNTY

CITY

POSTAL CODE

PERSON TO CONTACT

HIS/HER TITLE

PHONE NUMBER

FAX NUMBER

TELEX NUMBER
ENVIRONMENTAL MATTERS

MINISTRY OF PUBLIC WORKS

NONE LISTED

NONE

NONE

PATTISON 147

NONE

ATHENS

GREECE

NONE LISTED

NONE LISTED

NONE LISTED

NONE LISTED

NONE LISTED 
NGA LIST OF GEOTHERMALLY ACTIVE ENTITIES

PAGE 20

DATE February 22, 1991

NATION GREECE

FIELD OF EXPERTISE NAME OF ENTITY

DEPARTMENT NAME

FLOOR

SUITE OR BLDG. NO. STREET ADDRESS COUNTY

CITY POSTAL CODE PERSON TO CONTACT HIS/HER TITLE PHONE NUMBER FAX NUMBER TELEX NUMBER
EXPLORATION

INST. OF GEOL. \& MINERAL EXPL DIV. OF ENERGY RES. GEOTH. DI NONE

NONE

70 MESSOGHION ST.

NONE

ATHENS

NONE

E. CHIOTIS

NONE

775-2211

NONE

$30 \quad 77 \quad 98 \quad 412$ 
NGA LIST OF GEOTHERMALLY ACTIVE ENTITIES

PAGE 21

DATE February 22, 1991

NATION GUATEMALA

FIELD OF EXPERTISE NAME OF ENTITY DEPARTMENT NAME FLOOR SUITE OR BLDG. NO. STREET ADDRESS COUNTY

CITY POSTAL CODE PERSON TO CONTACT HIS/HER TITLE PHONE NUMBER FAX NUMBER TELEX NUMBER

\author{
MANAGEMENT \\ NATIONAL INSTIT. OF ELECTRICI \\ GEOTHERMAL DEVELOPMENT UNIT \\ NONE \\ NONE \\ GA AVENIDA 2-73, ED. C.HORJAL \\ NONE \\ GUATEMALA CITY \\ ZONA 4 \\ ANDRES CAICEDO \\ COORDINATOR GEOTH ENERGY \\ NONE \\ $502-2-345785$ \\ 305-5234 GU
}


NGA LIST OF GEOTHERMALLY ACTIVE ENTITIES

PAGE 22

DATE February 22, 1991

NATION HUNGARY

FIELD OF EXPERTISE

NAME OF ENTITY

DEPARTMENT NAME

FLOOR

SUITE OR BLDG. NO.

STREET ADDRESS

COUNTY

CITY

POSTAL CODE

PERSON TO CONTACT

HIS/HER TITLE

PHONE NUMBER

FAX NUMBER

TELEX NUMBER
ENERGY SCIENCE

INSTITUTE OF ENERGETICS

GEOTHERMAL

NONE

NONE

Bem RKp 33-34

NONE

BUDAPEST

H-1027

PETER OTTLIK

GEOTHERMAL ADVISOR

NONE

NONE

$86 \quad 1223476 \mathrm{H}$ 
NGA LIST OF GEOTHERMALLY ACTIVE ENTITIES

PAGE 23

DATE February 22, 1991

NATION INDONESIA

FIELD OF EXPERTISE NAME OF ENTITY DEPARTMENT NAME FLOOR SUITE OR BLDG. NO. STREET ADDRESS COUNTY

CITY POSTAL CODE PERSON TO CONTACT HIS/HER TITLE PHONE NUMBER FAX NUMBER TELEX NUMBER
PUBLIC UTILITY

PERTAMINA

DINAS GEOTHERMAL NONE

NONE

J1 KRAMAT RAYA NO. 59

NONE

JAKARTA

NONE

AMIR FAUZI NONE

$021-310-3690$

021-35-9177

44302 PTM JKTIA 
NGA LIST OF GEOTHERMALLY ACTIVE ENTITIES

PAGE $\quad 24$

DATE February 22, 1991

NATION INDONESIA

FIELD OF EXPERTISE

NAME OF ENTITY

DEPARTMENT NAME

FLOOR

SUITE OR BLDG. NO.

STREET ADDRESS

COUNTY

CITY

POSTAL CODE

PERSON TO CONTACT

HIS/HER TITLE

PHONE NUMBER

FAX NUMBER

TELEX NUMBER
FEDERAL POWER AGENCY

STATE ELECTRICITY CORP. TRUNOJOYO BLOCK MI/135

NONE

NONE

KEYBAYORAN BARU

NONE

JAKARTA

10450

VINCENT RADJA

NONE

NONE

NONE

NONE 
NGA LIST OF GEOTHERMALLY ACTIVE ENTITIES

PAGE $\quad 25$

DATE February 22, 1991

NATION KENYA

FIELD OF EXPERTISE NAME OF ENTITY DEPARTMENT NAME FLOOR SUITE OR BLDG. NO. STREET ADDRESS COUNTY

CITY POSTAL CODE PERSON TO CONTACT HIS/HER TITLE PHONE NUMBER FAX NUMBER TELEX NUMBER
GEOTHERMAL EXPLOR. \& DEVELOPM

KENYA POWER COMPANY LTD

NONE

NONE

NONE

P.O. BOX 30099

NONE

NAIROBI

NONE

E.D.WASUNNA CHIEF PROJ.DEVEL. MANAGER 254-2-21251 254-2-337351 22253 ELECTRIC 
NGA LIST OF GEOTHERMALLY ACTIVE ENTITIES

PAGE 26

DATE February 22, 1991

NATION KENYA

FIELD OF EXPERTISE NAME OF ENTITY

DEPARTMENT NAME

FLOOR

SUITE OR BLDG. NO. STREET ADDRESS

COUNTY

CITY

POSTAL CODE

PERSON TO CONTACT

HIS/HER TITLE

PHONE NUMBER

FAX NUMBER

TELEX NUMBER
POWER PLANT CONSTRUCTION

KENYA POWER CO LTD

NONE

NONE

NONE

P.O.BOX 30099

NONE

NAIROBI

NONE

E.D.WASUNNA

CHIEF PROJ. DEVEL MANAGER

254-2-21251

254-2-337351

22253 ELECTRIC 
NGA LIST OF GEOTHERMALLY ACTIVE ENTITIES

PAGE 27

DATE February 22, 1991

NATION KENYA

FIELD OF EXPERTISE

NAME OF ENTITY

DEPARTMENT NAME

FLOOR

SUITE OR BLDG. NO. STREET ADDRESS

COUNTY

CITY

POSTAL CODE

PERSON TO CONTACT

HIS/HER TITLE

PHONE NUMBER

FAX NUMBER

TELEX NUMBER
ELECTRICITY PURCHASES \& SALES

KENYA POWER \& LIGHTING CO

NONE

NONE

NONE

P.O. BOX 30099

NONE

NAIROBI

NONE

MR. JOSEPH OUMA

COMMERCIAL MANAGER

254-2-21251

254-2-337351

22253-ELECTRIC 
NGA LIST OF GEOTHERMALLY ACTIVE ENTITIES

PAGE 28

DATE February 22, 1991

NATION KENYA

FIELD OF EXPERTISE

NAME OF ENTITY

DEPARTMENT NAME

FLOOR

SUITE OR BLDG. NO. STREET ADDRESS

COUNTY

CITY

POSTAL CODE

PERSON TO CONTACT

HIS/HER TITLE

PHONE NUMBER

FAX NUMBER

TELEX NUMBER

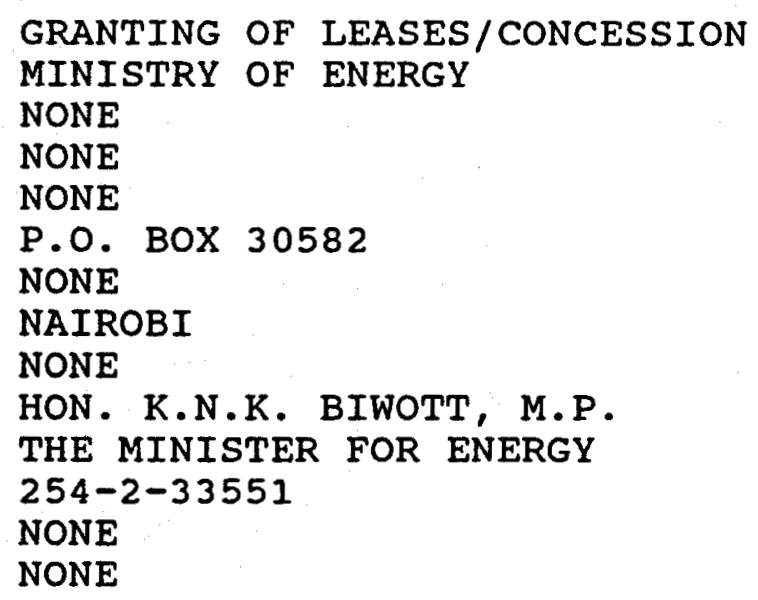


NGA LIST OF GEOTHERMALIY ACTIVE ENTITIES

PAGE 29

DATE February 22, 1991

NATION KENYA

FIELD OF EXPERTISE NAME OF ENTITY

DEPARTMENT NAME FLOOR

SUITE OR BLDG. NO. STREET ADDRESS COUNTY

CITY

POSTAL CODE

PERSON TO CONTACT

HIS/HER TITLE

PHONE NUMBER

FAX NUMBER

TELEX NUMBER
ENVIRONMENTAL MATTERS

KENYA POWER CO LTD

NONE

NONE

NONE

P.O. BOX 30099

NONE

NAIROBI

NONE

MR E.D. WASUNNA

CHIEF PROJ. DEVEL MANAGER

254-2-21251

254-2-337351

22253-ELECTRIC 
NGA LIST OF GEOTHERMALLY ACTIVE ENTITIES

PAGE 30

DATE February 22, 1991

NATION KENYA

FIELD OF EXPERTISE NAME OF ENTITY DEPARTMENT NAME FLOOR

SUITE OR BLDG. NO. STREET ADDRESS

COUNTY

CITY

POSTAL CODE

PERSON TO CONTACT

HIS/HER TITLE

PHONE NUMBER

FAX NUMBER

TELEX NUMBER
INTERNATIONAL BANKING

INT.BANK FOR RECON \& DEVEL.

NONE

NONE

NONE

P.O. BOX

30577

NAIROBI

NONE

NONE

NONE

254-2-338858

NONE

NONE 
NGA LIST OF GEOTHERMALLY ACTIVE ENTITIES

PAGE 31

DATE February 22, 1991

NATION KENYA

FIELD OF EXPERTISE NAME OF ENTITY

DEPARTMENT NAME

FLOOR

SUITE OR BLDG. NO. STREET ADDRESS

COUNTY

CITY

POSTAL CODE

PERSON TO CONTACT

HIS/HER TITLE

PHONE NUMBER

FAX NUMBER

TELEX NUMBER
INTERNATIONAL BANKING

COMMONWEALTH DEVELOPMENT CORP

NONE

NONE

NONE

P.O. BOX 43233

NONE

NAIROBI

NONE

MR C.J. STEPHENSON

REGIONAL CONTROLLER

254-2-340183-5

NONE

NONE 
NGA LIST OF GEOTHERMALLY ACTIVE ENTITIES

PAGE $\quad 32$

DATE February 22, 1991

NATION KENYA

FIELD OF EXPERTISE

CONSULTANTS

NAME OF ENTITY

DEPARTMENT NAME

FLOOR

SUITE OR BLDG. NO.

STREET ADDRESS

COUNTY

CITY

POSTAL CODE

PERSON TO CONTACT

HIS/HER TITLE

PHONE NUMBER

FAX NUMBER

TELEX NUMBER

GENZL (K) ITD

NONE

NONE

NONE

P.O. BOX 43183

NONE

NAIROBI

NONE

NONE

NONE

NONE

NONE

NONE 
NGA LIST OF GEOTHERMALLY ACTIVE ENTITIES

PAGE 33

DATE February 22, 1991

NATION KENYA

FIELD OF EXPERTISE NAME OF ENTITY

DEPARTMENT NAME FLOOR

SUITE OR BLDG. NO. STREET ADDRESS COUNTY

CITY

POSTAL CODE

PERSON TO CONTACT

HIS/HER TITLE

PHONE NUMBER

FAX NUMBER

TELEX NUMBER
CONSULTANTS

MERTZ, MCLELLAN CNSLTNG ENG.

4M ENTERPRICES LTD

NONE

NONE

P.O. BOX 48340

NONE

NAIROBI

NONE

MR CHRIS MALAVU

NONE

254-2-333795

NONE

NONE 
NGA LIST OF GEOTHERMALLY ACTIVE ENTITIES

PAGE

NATION KENYA

FIELD OF EXPERTISE NAME OF ENTITY DEPARTMENT NAME FLOOR SUITE OR BLDG. NO. STREET ADDRESS COUNTY

CITY POSTAL CODE PERSON TO CONTACT HIS/HER TITLE PHONE NUMBER FAX NUMBER TELEX NUMBER
CONSULTANTS

VIRKIR/ORKINT CONSULTING GROU NONE

NONE

NONE

P.O. BOX 45000

NONE

NAIROBI

NONE

DR GESTUR GISLASSON

NONE

254-2-567053

NONE

NONE 
NGA LIST OF GEOTHERMALLY ACTIVE ENTITIES

PAGE $\quad 35$

DATE February 22, 1991

NATION KENYA

FIELD OF EXPERTISE

CONSULTANTS

NAME OF ENTITY

DEPARTMENT NAME

FLOOR

SUITE OR BLDG. NO. STREET ADDRESS

COUNTY

CITY

POSTAL CODE

PERSON TO CONTACT

HIS/HER TITLE

PHONE NUMBER

FAX NUMBER

GEOCONSULT LTD

NONE

NONE

NONE

P.O. BOX 58454

NONE

NAIROBI

NONE

PROFESSOR ISAAC NYAMBOK

NONE

254-2-560549

TELEX NUMBER

NONE

NONE 
NGA LIST OF GEOTHERMALLY ACTIVE ENTITIES

PAGE $\quad 38$

DATE February 22, 1991

NATION KENYA

FIELD OF EXPERTISE NAME OF ENTITY

DEPARTMENT NAME

FLOOR

SUITE OR BLDG. NO. STREET ADDRESS COUNTY

CITY

POSTAL CODE

PERSON TO CONTACT

HIS/HER TITLE

PHONE NUMBER

FAX NUMBER

TELEX NUMBER
EXPLORATIONISTS

GEOTERMICA ITALIANA srI

NONE

NONE

NONE

P.O. BOX 44637

NONE

NAIROBI

NONE

DR DANIELE CIOPPI

NONE

254-2-339715 EXT 372

NONE

NONE 
NGA LIST OF GEOTHERMALLY ACTIVE ENTITIES

PAGE 39

DATE February 22, 1991

NATION KENYA

FIELD OF EXPERTISE NAME OF ENTITY

DEPARTMENT NAME

FLOOR

SUITE OR BLDG. NO. STREET ADDRESS

COUNTY

CITY

POSTAL CODE

PERSON TO CONTACT

HIS/HER TITLE

PHONE NUMBER

FAX NUMBER

TELEX NUMBER
EXPLORATIONISTS

BRITISH GEOLOGICAL SURVEY

C/O MINISTRY OF ENERGY

NONE

NONE

UTALII HOUSE, P.O. BOX 30582

NONE

NAIROBI

NONE

NONE

NONE

$254-2-340800$

NONE

NONE 
NGA LIST OF GEOTHERMÄLLY ACTIVE ENTITIES

PAGE $\quad 40$ DATE February 22, 1991

NATION KENYA

FIELD OF EXPERTISE NAME OF ENTITY DEPARTMENT NAME FLOOR

SUITE OR BLDG. NO. STREET ADDRESS COUNTY

CITY POSTAL CODE PERSON TO CONTACT HIS/HER TITLE PHONE NUMBER FAX NUMBER TELEX NUMBER
DRILLING COMPANIES FORAKI DRILLING COMPANY C/O KENYA POWER CO LTD NONE NONE P.O. BOX 30099 NONE NAIROBI NONE MR PIERRE ORGONOUSKY NONE $254-2-21251$ 254-2-337351 22253-ELECTRIC 
NGA LIST OF GEOTHERMALLY ACTIVE ENTITIES

PAGE 41

DATE February 22, 1991

NATION KENYA

FIELD OF EXPERTISE

NAME OF ENTITY

DEPARTMENT NAME

FLOOR

SUITE OR BLDG. NO. STREET ADDRESS

COUNTY

CITY

POSTAL CODE

PERSON TO CONTACT

HIS/HER TITLE

PHONE NUMBER

FAX NUMBER

TELEX NUMBER
DRILLING COMPANIES

BOWDEN DRILLING INTERNATIONAL

NONE

NONE

NONE

P.O. BOX 62216

NONE

NAIROBI

NONE

NONE

NONE

254-2-725088

NONE

NONE 
NGA LIST OF GEOTHERMALLY ACTIVE ENTITIES

PAGE 42

DATE February 22, 1991

NATION KENYA

FIELD OF EXPERTISE

NAME OF ENTITY

DEPARTMENT NAME

FLOOR

SUITE OR BLDG. NO. STREET ADDRESS COUNTY

CITY

POSTAL CODE

PERSON TO CONTACT

HIS/HER TITLE

PHONE NUMBER

FAX NUMBER

TELEX NUMBER
PLANT OR FIELD OPERATORS

KENYA OILFIELD SERVICES LTD

NONE

NONE

NONE

P.O.BOX 14662

NONE

NAIROBI

NONE

NONE

NONE

254-2-334307

NONE

NONE 
NGA LIST OF GEOTHERMALLY ACTIVE ENTITIES

PAGE 43

DATE February 22, 1991

NATION KENYA

FIELD OF EXPERTISE

PLANT OR FIELD OPERATORS

NAME OF ENTITY

DEPARTMENT NAME

HALLIBURTON LTD

FLOOR

SUITE OR BLDG. NO.

STREET ADDRESS

COUNTY

CITY

POSTAL CODE

PERSON TO CONTACT

HIS/HER TITLE

PHONE NUMBER

FAX NUMBER

KENYA BRANCH, MUTHAIGA ROAD

NONE

NONE

P.O BOX 62216

NONE

NAIROBI

NONE

NONE

NONE

254-2-65437

TELEX NUMBER

NONE

NONE 
NGA LIST OF GEOTHERMALLY ACTIVE ENTITIES

PAGE 44

DATE February 22, 1991

NATION KENYA

FIELD OF EXPERTISE

NAME OF ENTITY

DEPARTMENT NAME

FLOOR

SUITE OR BLDG. NO.

STREET ADDRESS

COUNTY

CITY

POSTAL CODE

PERSON TO CONTACT

HIS/HER TITLE

PHONE NUMBER

FAX NUMBER

TELEX NUMBER
PLANT OR FIELD CONSTRUCTION

UNIVERSAL F \& B CONTRACTORS

NONE

NONE

NONE

P.O. BOX 45695

NONE

NAIROBI

NONE

MR P.S. SAIMBI

NONE

254-2-822084

NONE

NONE

) 
NGA LIST OF GEOTHERMALLY ACTIVE ENTITIES

PAGE $\quad 45$

DATE February 22, 1991

NATION KENYA

FIELD OF EXPERTISE

NAME OF ENTITY

DEPARTMENT NAME

FLOOR

SUITE OR BLDG. NO.

STREET ADDRESS

COUNTY

CITY

POSTAL CODE

PERSON TO CONTACT

HIS/HER TITLE

PHONE NUMBER

FAX NUMBER

TELEX NUMBER
PLANT OR FIELD CONSTRUCTION

INDUSTRIAL PLANT (E.A.) LTD

NONE

NONE

NONE

P.O. BOX 44717

NONE

NAIROBI

NONE

MR H.S. SAGOO

NONE

254-2-542055

NONE

NONE 
NGA LIST OF GEOTHERMALLY ACTIVE ENTITIES

PAGE $\quad 46$

NATION KENYA
FIELD OF EXPERTISE NAME OF ENTITY DEPARTMENT NAME FLOOR SUITE OR BLDG. NO. STREET ADDRESS COUNTY

CITY

POSTAL CODE PERSON TO CONTACT HIS/HER TITLE PHONE NUMBER FAX NUMBER TELEX NUMBER
DATE February 22, 1991

PLANT OR FIELD CONSTRUCTION

H. YOUNG \& CO. (E.A.) LTD

NONE

NONE

NONE

P.O. BOX 30118

NONE

NAIROBI

NONE

MR JOSEPH SCHWARTZAN

NONE

254-2-555653

NONE

NONE 
NGA IIST OF GEOTHERMALLY ACTIVE ENTITIES

PAGE $\quad 47$

DATE February 22, 1991

NATION KENYA

FIELD OF EXPERTISE

NAME OF ENTITY

DEPARTMENT NAME

FLOOR

SUITE OR BLDG. NO. STREET ADDRESS

COUNTY

CITY

POSTAL CODE

PERSON TO CONTACT

HIS/HER TITLE

PHONE NUMBER

FAX NUMBER

TELEX NUMBER
PLANT OR FIELD CONSTRUCTION

RICHFIELD ENGINEERING LTD

NONE

NONE

NONE

P.O. BOX 67321

NONE

NAIROBI

NONE

MR BRAUMBLATT

NONE

254-2-540917

NONE

NONE 
NGA LIST OF GEOTHERMALLY ACTIVE ENTITIES

PAGE $\quad 48$

DATE February 22, 1991

NATION KENYA

FIELD OF EXPERTISE

PLANT OR FIELD CONSTRUCTION

NAME OF ENTITY

DEPARTMENT NAME

AFRICAN STEEL PIPES CO. LTD

NONE

FLOOR

SUITE OR BLDG. NO.

NONE

NONE

STREET ADDRESS

COUNTY

CITY

POSTAL CODE

PERSON TO CONTACT

HIS/HER TITLE

PHONE NUMBER

FAX NUMBER

P.O. BOX 66038

NONE

NAIROBI

NONE

MR C.I. GEY

NONE

TELEX NUMBER

254-2-822920 TO 924

254-2-822925

22078 PIPECO KE 


\section{NGA LIST OF GEOTHERMALLY ACTIVE ENTITIES}

PAGE 49

NATION KENYA
FIELD OF EXPERTISE NAME OF ENTITY DEPARTMENT NAME FLOOR SUITE OR BLDG. NO. STREET ADDRESS COUNTY

CITY POSTAL CODE PERSON TO CONTACT HIS/HER TITLE PHONE NUMBER FAX NUMBER

TELEX NUMBER
DATE February 22, 1991

\author{
DRILLING COMPANIES \\ THE KENYA POWER CO. LTD \\ NONE \\ NONE \\ NONE \\ P.O. BOX 30099 \\ NONE \\ NAIROBI \\ NONE \\ MR E.D. WASUNNA \\ NONE \\ 254-2-21251 \\ 254-2-337351 \\ 22253-ELECTRIC
}


NGA LIST OF GEOTHERMALLY ACTIVE ENTITIES

PAGE 50

DATE February 22, 1991

NATION KENYA

FIELD OF EXPERTISE

NAME OF ENTITY

DEPARTMENT NAME

FLOOR

SUITE OR BLDG. NO. STREET ADDRESS

COUNTY

CITY

POSTAL CODE

PERSON TO CONTACT

HIS/HER TITLE

PHONE NUMBER

FAX NUMBER

TELEX NUMBER
PUBLIC UTILITY

KENYA POWER \& LIGHTING LTD.

GEOTHERMAL PROJECT

NONE

NONE

P.O. BOX 785

NONE

NAIVASHA

NONE

GEOFFREY G. MUCHEMI

NONE

NONE

$\begin{array}{lll}254 & 2 & 337351\end{array}$

$22253 \mathrm{KY}$ 
NGA LIST OF GEOTHERMALLY ACTIVE ENTITIES

PAGE 51

DATE February 22, 1991

NATION MEXICO

FIELD OF EXPERTISE

NAME OF ENTITY

DEPARTMENT NAME

FLOOR

SUITE OR BLDG. NO.

STREET ADDRESS

COUNTY

CITY

POSTAL CODE

PERSON TO CONTACT

HIS/HER TITLE

PHONE NUMBER

FAX NUMBER

TELEX NUMBER
POWER PLANT CONSTRUCTION

ANSALDO COMPONENTI di MEXICO

NONE

1ST

NONE

MARIANO ESCOBEDO 388

DISTRICT FEDERAL

MEXICO CITY

11590

GIAN PIETRO GIOLITO

MANAGER (ING.)

(5) 255-1242, 3162

NONE LISTED

1763170-ANELME 
NGA LIST OF GEOTHERMALLY ACTIVE ENTITIES

PAGE $\quad 52$

DATE February 22, 1991

NATION MEXICO

\author{
FIELD OF EXPERTISE \\ NAME OF ENTITY \\ DEPARTMENT NAME \\ FLOOR \\ SUITE OR BLDG. NO. \\ STREET ADDRESS \\ COUNTY \\ CITY \\ POSTAL CODE \\ PERSON TO CONTACT \\ HIS/HER TITLE \\ PHONE NUMBER \\ FAX NUMBER \\ TELEX NUMBER
}

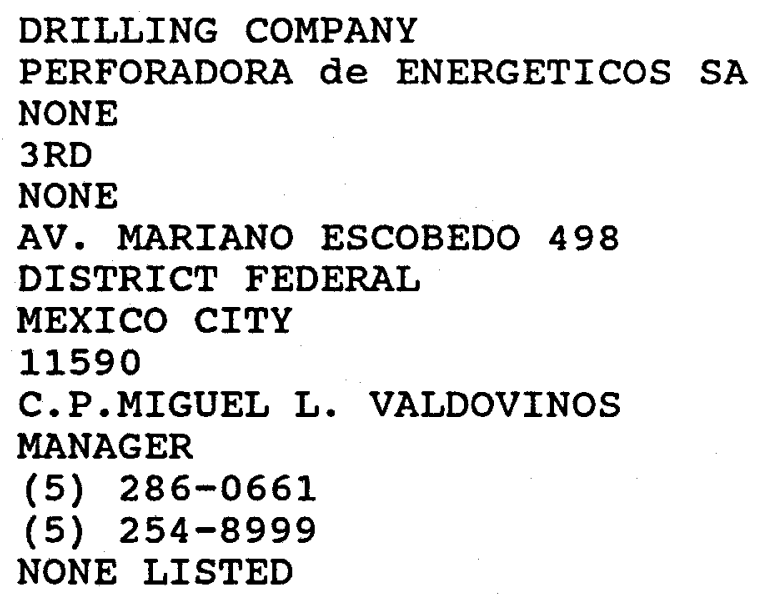


NGA LIST OF GEOTHERMALLY ACTIVE ENTITIES

PAGE $\quad 53$

DATE February 22, 1991

NATION MEXICO

FIELD OF EXPERTISE

NAME OF ENTITY

DEPARTMENT NAME

FLOOR

SUITE OR BLDG. NO. STREET ADDRESS

COUNTY

CITY

POSTAL CODE

PERSON TO CONTACT

HIS/HER TITLE

PHONE NUMBER

FAX NUMBER

TELEX NUMBER
ELECTRICITY SALES TO PUBLIC COMISION FEDERAL ELECTRICIDAD SUBDIRECCION DE DISTRIBUCION NONE

NONE

NONE

MICHOACAN

MORELIA

APDO. POSTAL 31-C

MIGUEL RAMIREZ GUTIERREZ

ING.

NONE LISTED

NONE LISTED

NONE LISTED 
NGA LIST OF GEOTHERMALLY ACTIVE ENTITIES

PAGE 54

DATE February 22, 1991

NATION MEXICO

FIELD OF EXPERTISE

NAME OF ENTITY

DEPARTMENT NAME

FLOOR

SUITE OR BLDG. NO.

STREET ADDRESS

COUNTY

CITY

POSTAL CODE

PERSON TO CONTACT

HIS/HER TITLE

PHONE NUMBER

FAX NUMBER

TELEX NUMBER
POWER PLANT CONSTRUCTION

MAKROTEK SA de CV

NONE

9TH

NONE

AV. INSURGENTES SUR 667

DISTRICT FEDERAL

MEXICO CITY

03810

ROBERTO SANDOVAL FASCIO

MANAGER (ING.)

(5) $536-1383,2258$

NONE LISTED

$1764152-$ GALVME 
NGA LIST OF GEOTHERMALLY ACTIVE ENTITIES

PAGE $\quad 55$

DATE February 22, 1991

NATION MEXICO

FIELD OF EXPERTISE NAME OF ENTITY DEPARTMENT NAME FLOOR

SUITE OR BLDG. NO. STREET ADDRESS COUNTY

CITY

POSTAL CODE

PERSON TO CONTACT

HIS/HER TITLE

PHONE NUMBER

FAX NUMBER

TELEX NUMBER
DRILLING COMPANY

PERF. Y CONSTRUCT. TERMAL， SA NONE

NONE

NONE

RIO ELBA NO. 10-401

DISTRICT FEDERAL

MEXICO CITY

06500

JOSE GIJON GUTIERREZ

MANAGER (ING.)

(5) 286-0661

(5) $553-7150$

NONE LISTED 
NGA LIST OF GEOTHERMALLY ACTIVE ENTITIES

PAGE $\quad 56$

DATE February 22, 1991

NATION MEXICO

FIELD OF EXPERTISE NAME OF ENTITY DEPARTMENT NAME FLOOR SUITE OR BLDG. NO. STREET ADDRESS

COUNTY

CITY

POSTAL CODE

PERSON TO CONTACT

HIS/HER TITLE

PHONE NUMBER

FAX NUMBER

TELEX NUMBER
REGULATIONS, ENVIRON, LICENSE SEC DE DESARROLO URBÁNO Y ECO NONE LISTED

NONE

NONE

NONE

NONE

NONE

NONE

NONE

NONE

NONE

NONE

NONE 
NGA LIST OF GEOTHERMALLY ACTIVE ENTITIES

PAGE 57

DATE February 22, 1991

NATION MEXICO

FIELD OF EXPERTISE

DRILLING COMPANY

NAME OF ENTITY

DEPARTMENT NAME

CONST. Y PERFORADORA LATINA $S$

FLOOR

SUITE OR BLDG. NO. NONE

1ST

NONE

STREET ADDRESS

COUNTY

CITY

POSTAL CODE

PERSON TO CONTACT

HIS/HER TITLE

PHONE NUMBER

FAX NUMBER

TELEX NUMBER

SIERRA MOJADA 626, LOMAS de C DISTRICT FEDERAL

MEXICO CITY

11050

ERNESTO REGUERA ROA

MANAGER

(5) 284-3377

(5) $540-1786$

1761121-EPNME 
NGA LIST OF GEOTHERMALLY ACTIVE ENTITIES

PAGE $\quad 58$

DATE February 22, 1991

NATION MEXICO

FIELD OF EXPERTISE NAME OF ENTITY

DEPARTMENT NAME

FLOOR

SUITE OR BLDG. NO. STREET ADDRESS COUNTY

CITY

POSTAL CODE

PERSON TO CONTACT

HIS/HER TITLE

PHONE NUMBER

FAX NUMBER

TELEX NUMBER
GEOTHERMAL EXPLOR, DEVEL, CONST

COMISION FEDERAL ELECTRICIDAD SUBDIRECTION DE CONSTRUCTION

NONE

NONE

NONE

MICHOACAN

MORELIA

APDO.POSTAL 31-C

MIGUEL RAMIREZ GUTIERREZ

ING.

NONE LISTED

NONE LISTED

NONE LISTED 
NGA LIST OF GEOTHERMALLY ACTIVE ENTITIES

PAGE $\quad 59$

DATE February 22, 1991

NATION MEXICO

FIELD OF EXPERTISE

NAME OF ENTITY

DEPARTMENT NAME

FLOOR

SUITE OR BLDG. NO. STREET ADDRESS

COUNTY

CITY

POSTAL CODE

PERSON TO CONTACT

HIS/HER TITLE

PHONE NUMBER

FAX NUMBER

TELEX NUMBER

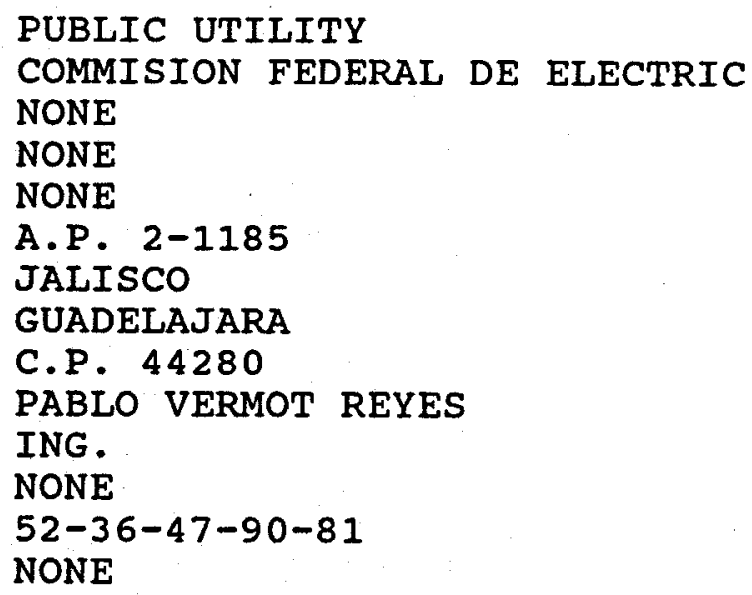


NGA LIST OF GEOTHERMALLY ACTIVE ENTITIES

PAGE 60

DATE February 22, 1991

NATION NEPAL

FIELD OF EXPERTISE NAME OF ENTITY DEPARTMENT NAME FLOOR SUITE OR BLDG. NO. STREET ADDRESS COUNTY

\section{CITY}

POSTAL CODE PERSON TO CONTACT HIS/HER TITLE PHONE NUMBER FAX NUMBER TELEX NUMBER
FEDERAL AGENCY

DEPARTMENT OF MINES \& GEOLOGY NONE

NONE

NONE

LAINCHAUR

NONE

KATMANDU

NONE

BHARAT M. JNAWALI

NONE

NONE

NONE

2320 MINES NP 
NGA LIST OF GEOTHERMALLY ACTIVE ENTITIES

PAGE 61

DATE February 22, 1991

NATION NICARAGUA

\author{
FIELD OF EXPERTISE \\ NAME OF ENTITY \\ DEPARTMENT NAME \\ FLOOR \\ SUITE OR BLDG. NO. \\ STREET ADDRESS \\ COUNTY \\ CITY \\ POSTAL CODE \\ PERSON TO CONTACT \\ HIS/HER TITLE \\ PHONE NUMBER \\ FAX NUMBER \\ TELEX NUMBER
}

MANAGEMENT

INSTIT. NICARAGUENSE DE ENERG GEOTHERMAL

NONE

NONE

APARTADO POSTAL 55

NONE

MANAGUA

NONE

ERNESTO MARTINEZ TIFFER

DIRECTOR GENERAL GEO. RES

505-2-27846

505-2-27893

1364 INEPERF 
NGA LIST OF GEOTHERMALLY ACTIVE ENTITIES

PAGE 62

DATE February 22, 1991

NATION NICARAGUA

FIELD OF EXPERTISE
NAME OF ENTITY
DEPARTMENT NAME
FLOOR
SUITE OR BLDG. NO.
STREET ADDRESS
COUNTY
CITY
POSTAL CODE
PERSON TO CONTACT
HIS/HER TITLE
PHONE NUMBER
FAX NUMBER
TELEX NUMBER

MANAGEMENT

INST. NICARAGUENSE DE ENERGIA GEOTHERMAL

NONE

NONE

APARTADO POSTAL 55

NONE

MANAGUA

NONE

ROGERACIA LACAYO

DIRECTOR GEOTHERMAL

505-2-27846

505-2-27893

1364 INEPERF 
NGA LIST OF GEOTHERMALLY ACTIVE ENTITIES

PAGE 63

DATE February 22, 1991

NATION PANAMA

FIELD OF EXPERTISE NAME OF ENTITY DEPARTMENT NAME FLOOR SUITE OR BLDG. NO. STREET ADDRESS COUNTY

CITY

POSTAL CODE PERSON TO CONTACT HIS/HER TITLE PHONE NUMBER FAX NUMBER TELEX NUMBER
MANAGEMENT

INST. RECUR. HYDR. Y ELECTRIF IRHE

NONE

NONE

EDIFICIO POLI, AVENIDA CUBA NONE

PANAMA CITY

NONE

CARLOS ALGONDONA

DIRECTOR GEOTHERMAL NONE

NONE

3282158 IRHE PG 
PAGE $\quad 64$

DATE February 22, 1991

NATION PHILIPPINES

FIELD OF EXPERTISE

NAME OF ENTITY

DEPARTMENT NAME

FLOOR

SUITE OR BLDG. NO.

STREET ADDRESS

COUNTY

CITY

POSTAL CODE

PERSON TO CONTACT

HIS/HER TITLE

PHONE NUMBER

FAX NUMBER

TELEX NUMBER
GEOTH. EXPLORATION AND DEVELO

PHILIPPINE NATIONAL OIL COMPA

ENERGY DEVELOPMENT CORPORATIO

NONE

NONE

MERRIT RD, FORT BONIFACIO

METRO MANILA

MANILA

MAKATI

PEDRO S. SANTOS

VICE PRESIDENT

815-89-61

NONE LISTED

NONE LISTED 
NGA LIST OF GEOTHERMALLY ACTIVE ENTITIES

PAGE $\quad 65$

DATE February 22, 1991

NATION PHILIPPINES

FIELD OF EXPERTISE NAME OF ENTITY

DEPARTMENT NAME

FLOOR

SUITE OR BLDG. NO. STREET ADDRESS COUNTY

CITY

POSTAL CODE

PERSON TO CONTACT

HIS/HER TITLE

PHONE NUMBER

FAX NUMBER

TELEX NUMBER
POWER PURCHASES AND SALES

NATIONAL POWER CORPORATION

NONE LISTED

NONE

NONE

CORNER AGHAM AVE - QUEZON AVE

NONE LISTED

QUEZON CITY

DILIMAN

ERNESTO M. ABOITIZ

PRESIDENT

921-29-98

632921-2468

40120 
NGA LIST OF GEOTHERMALLY ACTIVE ENTITIES

PAGE $\quad 66$

DATE February 22, 1991

NATION PHILIPPINES

FIELD OF EXPERTISE NAME OF ENTITY

DEPARTMENT NAME FLOOR

SUITE OR BLDG. NO. STREET ADDRESS

COUNTY

CITY

POSTAL CODE

PERSON TO CONTACT

HIS/HER TITLE

PHONE NUMBER

FAX NUMBER

TELEX NUMBER
ENVIRONMENTAL MATTERS

DEPT OF ENVIRON AND NAT RESOU NONE LISTED

NONE

NONE

VISAYAS AVE

NONE LISTED

QUEZON CITY

NONE LISTED

FULGENCIO FACTORAN

SECRETARY

96-65-76

NONE LISTED

NONE LISTED 
NGA LIST OF GEOTHERMALLY ACTIVE ENTITIES

PAGE 67

DATE February 22, 1991

NATION PHILIPPINES

FIELD OF EXPERTISE

NAME OF ENTITY

DEPARTMENT NAME

FLOOR

SUITE OR BLDG. NO. STREET ADDRESS

COUNTY

CITY

POSTAL CODE

PERSON TO CONTACT

HIS/HER TITLE

PHONE NUMBER

FAX NUMBER

TELEX NUMBER
POWER PLANT CONSTRUCTION

F.F.CRUZ AND CO. INC

NONE LISTED

NONE

NONE

800 EPIFANO de LOS SANTOS AVE

NONE

QUEZON CITY

NONE

FELIPE F. CRUZ

PRESIDENT

NONE LISTED

NONE LISTED

NONE LISTED 
NGA LIST OF GEOTHERMALLY ACTIVE ENTITIES

PAGE 68

DATE February 22, 1991

NATION PHILIPPINES

FIELD OF EXPERTISE

NAME OF ENTITY

DEPARTMENT NAME

FLOOR

SUITE OR BLDG. NO.

STREET ADDRESS

COUNTY

CITY

POSTAL CODE

PERSON TO CONTACT

HIS/HER TITLE

PHONE NUMBER

FAX NUMBER

TELEX NUMBER
GEOTH. EXPLORATION AND DEVEL. PHILIPPINE GEOTHERMAL INC.

NONE

$15 \mathrm{TH}$

NONE

METROBANK PLAZA, GIL PUYAT AVE

METRO MANILA

MANILA

MAKATI

PAUL ATKINSON

MANAGER, RESERVOIR ENG.

817-88-76 TO 80

NONE LISTED

NONE LISTED 
NGA LIST OF GEOTHERMALLY ACTIVE ENTITIES

PAGE $\quad 69$

DATE February 22, 1991

NATION PHILIPPINES

FIELD OF EXPERTISE

NAME OF ENTITY

DEPARTMENT NAME

FLOOR

SUITE OR BLDG. NO. STREET ADDRESS

COUNTY

CITY

POSTAL CODE

PERSON TO CONTACT

HIS/HER TITLE

PHONE NUMBER

FAX NUMBER

TELEX NUMBER
GRNTING OF GEOTHERMAL LEASES

OFFICE OF ENERGY AFFAIRS

NONE LISTED

NONE

NONE

MERRIT RD., FORT BONIFACIO

METRO MANILA

MANILA

MAKATI

WENCESLAO Q. DE LA PAZ

DIRECTOR

85-10-21 TO 31

NONE LISTED

NONE LISTED 
NGA LIST OF GEOTHERMALLY ACTIVE ENTITIES

PAGE $\quad 70$

DATE February 22, 1991

NATION PHILIPPINES

FIELD OF EXPERTISE NAME OF ENTITY DEPARTMENT NAME FLOOR SUITE OR BLDG. NO. STREET ADDRESS COUNTY

CITY POSTAL CODE PERSON TO CONTACT HIS/HER TITLE PHONE NUMBER FAX NUMBER TELEX NUMBER
NATIONAL POWER UTILITY NATIONAL POWER CORPORATION GEOTHERMAL

NONE

NONE

QUEZON \& AGHAM RD, BOX 10183 NONE

QUEZON CITY

NONE

ROGELIO DATUIN

SPECIAL ASSISTNT TO CHRMN $632-921-3451$ 632-921-2468 NONE 
NGA LIST OF GEOTHERMALLY ACTIVE ENTITIES

PAGE 71

DATE February 22, 1991

NATION PHILIPPINES

FIELD OF EXPERTISE NAME OF ENTITY DEPARTMENT NAME FLOOR

SUITE OR BLDG. NO. STREET ADDRESS

COUNTY

CITY

POSTAL CODE

PERSON TO CONTACT

HIS/HER TITLE

PHONE NUMBER

FAX NUMBER

TELEX NUMBER

\author{
GEOTHERMAL ENGINEERING \\ NATIONAL POWER CORPORATION \\ GEOTHERMAL \\ NONE \\ NONE \\ BOX 10183, \\ NONE \\ QUEZON CITY \\ DILIMAN \\ FELICITO M. GAZO \\ GENERAL RESEARCH ENGINEER \\ $632-921-3451$ \\ 632-921-2468 \\ NONE
}


NGA LIST OF GEOTHERMALLY ACTIVE ENTITIES

PAGE 72

NATION PHILIPPINES

FIELD OF EXPERTISE NAME OF ENTITY DEPARTMENT NAME FLOOR

SUITE OR BLDG. NO. STREET ADDRESS COUNTY

CITY POSTAL CODE PERSON TO CONTACT HIS/HER TITLE PHONE NUMBER FAX NUMBER TELEX NUMBER
DATE February 22, 1991

\author{
EXPLORATION \\ PNOC ENERGY COMPANIES \\ GEOTHERMAL \\ NONE \\ NONE \\ BOX 1031, MCPO \\ METRO MANILA \\ MAKATI \\ NONE \\ CARLI M. RICIO \\ GEOSCIENTIFIC MANAGER \\ NONE \\ 63-2-815-2747 \\ NONE
}


NGA LIST OF GEOTHERMALLYY ACTIVE ENTITIES

PAGE $\quad 75$

DATE February 22, 1991

NATION REPUBLIC OF CHINA

FIELD OF EXPERTISE

NAME OF ENTITY

DEPARTMENT NAME

FLOOR

SUITE OR BLDG. NO. STREET ADDRESS

COUNTY

CITY

POSTAL CODE

PERSON TO CONTACT

HIS/HER TITLE

PHONE NUMBER

FAX NUMBER

TELEX NUMBER
PLANT OR FIELD OPERATORS

CHINESE PETROLEUM CORPORATION

PRODUCTION DEPARTMENT

NONE

NONE

90 CHUNG CHENG ROAD

TAIMAN

MIAOLI

NONE LISTED

K.S.YEH

DEPARTMENT MANAGER

037-262100

886-037-267223

NONE LISTED 
NGA LIST OF GEOTHERMALLY ACTIVE ENTITIES

PAGE $\quad 76$

DATE February 22, 1991

NATION REPUBLIC OF CHINA

FIELD OF EXPERTISE NAME OF ENTITY

DEPARTMENT NAME

FLOOR

SUITE OR BLDG. NO. STREET ADDRESS COUNTY

CITY

POSTAL CODE

PERSON TO CONTACT

HIS/HER TITLE

PHONE NUMBER

FAX NUMBER

TELEX NUMBER
LEASE OR CONCESSION GRANTOR

MINISTRY OF ECONOMIC AFFAIRS

DEPARTMENT OF MINES

NONE

NONE

15 FU CHOU STREET

TAIWAN

TAIPEI

NONE LISTED

NONE LISTED

NONE LISTED

NONE LISTED

NONE LISTED

NONE LISTED 
NGA LIST OF GEOTHERMALLY ACTIVE ENTITIES

PAGE $\quad 77$

DATE February 22, 1991

NATION REPUBLIC OF CHINA

FIELD OF EXPERTISE

NAME OF ENTITY

DEPARTMENT NAME

FLOOR

SUITE OR BLDG. NO. STREET ADDRESS COUNTY

CITY

POSTAL CODE

PERSON TO CONTACT

HIS/HER TITLE

PHONE NUMBER

FAX NUMBER

TELEX NUMBER
EXPLORATION AND DEVELOPMENT

ENERGY COMMISSION

NONE LISTED

FL. 13

$\mathrm{N} / \mathrm{A}$

2 FU-HSING NORTH ROAD

TAIWAN

TAIPEI

NONE LISTED

K.H.LO

DEPARTMENT MANAGER

NONE LISTED

886-02-7769417

NONE LISTED 
NGA LIST OF GEOTHERMALLY ACTIVE ENTITIES

PAGE $\quad 78$

DATE February 22, 1991

NATION REPUBLIC OF CHINA

FIELD OF EXPERTISE

NAME OF ENTITY

DEPARTMENT NAME

FLOOR

SUITE OR BLDG. NO.

STREET ADDRESS

COUNTY

CITY

POSTAL CODE

PERSON TO CONTACT

HIS/HER TITLE

PHONE NUMBER

FAX NUMBER

TELEX NUMBER
PROJECT MANAGEMENT, CONSULTING

ENER RES LAB, IND TECH RES INS

RESOURCES DEVELOPMENT DIVISIO

NONE

NO 64

195 CHUNG HSING ROAD

TAIWAN

CHUTUNG, HSINCHU

SECTION 4

WENG-TSE CHENG

DEPARTMENT MANAGER

035-916340

886-035-943680

NONE LISTED 
NGA LIST OF GEOTHERMALLY ACTIVE ENTITIES

PAGE $\quad 79$

DATE February 22, 1991

NATION REPUBLIC OF CHINA

\begin{abstract}
FIELD OF EXPERTISE
NAME OF ENTITY

DEPARTMENT NAME

FLOOR

SUITE OR BLDG. NO. STREET ADDRESS

COUNTY

CITY

POSTAL CODE

PERSON TO CONTACT

HIS/HER TITLE

PHONE NUMBER

FAX NUMBER

TELEX NUMBER
\end{abstract}

\author{
ENGINEERING \\ TIANJIN UNIVERSITY \\ CIVIL ENGINEERING \\ NONE \\ NONE \\ NONE \\ NONE \\ TIANJIN \\ 10072 \\ JING-YU CHANG \\ NONE \\ NONE \\ NONE \\ NONE
}


NGA LIST OF GEOTHERMALLY ACTIVE ENTITIES

PAGE $\quad 80$

DATE February 22, 1991

NATION REPUBLIC OF CHINA

FIELD OF EXPERTISE NAME OF ENTITY DEPARTMENT NAME FLOOR

SUITE OR BLDG. NO. STREET ADDRESS

COUNTY

CITY

POSTAL CODE

PERSON TO CONTACT

HIS/HER TITLE

PHONE NUMBER

FAX NUMBER

TELEX NUMBER
GEOTHERMAL STUDIES

ACADEMICA SINICA INSTITUTE OF GEOLOGY

NONE

NONE

NONE

NONE

BEIJING

100044

JI-YANG WANG

PROFESSOR

861-401-661-1472

8611-401-4031

NONE 
NGA LIST OF GEOTHERMALLY ACTIVE ENTITIES

PAGE

81

DATE February 22, 1991

NATION REPUBLIC OF CHINA

\author{
FIELD OF EXPERTISE \\ NAME OF ENTITY \\ DEPARTMENT NAME \\ FLOOR \\ SUITE OR BLDG. NO. \\ STREET ADDRESS \\ COUNTY \\ CITY \\ POSTAL CODE \\ PERSON TO CONTACT \\ HIS/HER TITLE \\ PHONE NUMBER \\ FAX NUMBER \\ TELEX NUMBER
}

\author{
GEOLOGIC STUDIES \\ PEKING UNIVERSITY \\ GEOLOGY DEPARTMENT \\ NONE \\ NONE \\ NONE \\ NONE \\ BEJING \\ 100871 \\ SHEN MINZI \\ PROFESSOR \\ NONE \\ NONE
}

71211215 BEUN CN 
NGA LIST OF GEOTHERMALLY ACTIVE ENTITIES

PAGE $\quad 82$

DATE February 22, 1991

NATION REPUBLIC OF CHINA

FIELD OF EXPERTISE

NAME OF ENTITY

DEPARTMENT NAME

FLOOR

SUITE OR BLDG. NO. STREET ADDRESS

COUNTY

CITY

POSTAL CODE

PERSON TO CONTACT

HIS/HER TITLE

PHONE NUMBER

FAX NUMBER

TELEX NUMBER
POWER PURCHASES AND SALES

TAIWAN POWER COMPANY

THERMAL POWER RESOURCES DIVIS

NONE

NONE

242 ROOSEVELT ROAD

TAIWAN

TAIPEI

SECTION 3

SHENG-HSIUNG YU

DIVISION CHIEF

NONE LISTED

NONE LISTED

NONE LISTED 
NGA LIST OF GEOTHERMALLY ACTIVE ENTITIES

PAGE 83

DATE February 22, 1991

NATION ROMANIA

FIELD OF EXPERTISE NAME OF ENTITY DEPARTMENT NAME FLOOR SUITE OR BLDG. NO. STREET ADDRESS COUNTY

CITY

POSTAL CODE PERSON TO CONTACT HIS/HER TITLE PHONE NUMBER

FAX NUMBER

TELEX NUMBER
NOT KNOWN

PARCUL PETOFI NOLL NONE

NONE

NONE

NONE

NONE

ORADEA

3700

ROMONA PLAVITA

NONE

NONE

NONE

11223 MIMURE R 


\section{NGA LIST OF GEOTHERMALLY ACTIVE ENTITIES}

PAGE $\quad 84$

DATE February 22, 1991

NATION USSR

FIELD OF EXPERTISE
NAME OF ENTITY
DEPARTMENT NAME
FLOOR
SUITE OR BLDG. NO.
STREET ADDRESS
COUNTY
CITY
POSTAL CODE
PERSON TO CONTACT
HIS/HER TITLE
PHONE NUMBER
FAX NUMBER
TELEX NUMBER

FIELD OF EXPERTISE

NAME OF ENTITY

DEPARTMENT NAME

FLOOR

SUITE OR BLDG. NO.

STREET ADDRESS

COUNTY

CITY

POSTAL CODE

PERSON TO CONTACT

HIS/HER TITLE

FAX NUMBER

TELEX NUMBER

\author{
GEOPHYSICS \\ INSTITUTE OF GEOPHYSICS \\ LABORATORY OF GEOTHERMICS \\ NONE \\ NONE \\ NONE \\ NONE \\ PAYZHESKY PX7 \\ 10917 \\ VLADIMIR KONONOV \\ PROFESSOR \\ NONE \\ NONE \\ 871411848 GIN SU
}


NGA LIST OF GEOTHERMALLY ACTIVE ENTITIES

PAGE 85

DATE February 22, 1991

NATION USSR

FIELD OF EXPERTISE

NAME OF ENTITY

DEPARTMENT NAME

FLOOR

SUITE OR BLDG. NO. STREET ADDRESS

COUNTY

CITY

POSTAL CODE

PERSON TO CONTACT

HIS/HER TITLE

PHONE NUMBER

FAX NUMBER

TELEX NUMBER
GENERAL LOW TEMP. GEOTHERMICS

INSTIT. OF GEOTHERMAL PROBLEM

NONE

NONE

NONE

C/O GEO-HEAT CENTER, OIT

NONE

KLAMATH FALLS, OR

97601 (USA)

SARD SARDAROV

DR.

503-882-6371 (USA)

503-882-4136 (USA)

NONE 
NGA LIST OF GEOTHERMALLY ACTIVE ENTITIES

PAGE $\quad 86$

DATE February 22, 1991

NATION USSR

FIELD OF EXPERTISE

NAME OF ENTITY

DEPARTMENT NAME

FLOOR

SUITE OR BLDG. NO.

STREET ADDRESS

COUNTY

CITY

POSTAL CODE

PERSON TO CONTACT

HIS/HER TITLE

PHONE NUMBER

FAX NUMBER

TELEX NUMBER
ACADEMICS

ACADEMY OF SCIENCE OF GEORGIA

NONE

NONE

NONE

NONE

GEORGIA

TIBILISI

38008

GURAM BUACHIDZE

PROFESSOR

NONE

NONE

212912 PTBSU 
NGA LIST OF GEOTHERMALLY ACTIVE ENTITIES

PAGE

87

DATE February 22, 1991

NATION YUGOSLAVIA

FIELD OF EXPERTISE

NAME OF ENTITY

DEPARTMENT NAME

FLOOR

SUITE OR BLDG. NO.

STREET ADDRESS

COUNTY

CITY

POSTAL CODE

PERSON TO CONTACT

HIS/HER TITLE

PHONE NUMBER

FAX NUMBER

TELEX NUMBER
GEOTHERMICS

UNIVERSITY CYRIL AND METHODIC

MASTNEKI FAHULTET

NONE

NONE

KARPOS II bb

MACEDONIA

SKOPJE

91000

KONSTANTINE DIMITROV

PROFESSOR

091-259645

503-882-4136 (USA)

51884 MaFakyw 
NGA LIST OF GEOTHERMALLY ACTIVE ENTITIES

PAGE 88

DATE February 22, 1991

NATION YUGOSLAVIA

FIELD OF EXPERTISE NAME OF ENTITY DEPARTMENT NAME FLOOR SUITE OR BLDG. NO. STREET ADDRESS COUNTY

CITY POSTAL CODE PERSON TO CONTACT HIS/HER TITLE PHONE NUMBER FAX NUMBER TELEX NUMBER

\author{
GEOLOGY \\ GEOLOSKI ZAVOD \\ NONE \\ NONE \\ NONE \\ GAZI BABABB \\ MACEDONIA \\ SKOPJE \\ 91000 \\ MIRJANA GORGIEVA \\ NONE \\ NONE \\ NONE \\ NONE
}


MAPS OF GEOTHERMAL

INSTALLATIONS 


\title{
GEOTHERMAL MANAGEMENT CO., Inc.
}

\author{
NGA DATABASE MAP FILES
}

\section{Comments and Explanations}

The maps in the following section of this document were produced by hand scanning redrafted versions of original maps submitted by data base respondants from each nation. The illustrations were redrafted in a uniform style by the National Geothermal Association (NGA) and returned to Geothermal Management Company, Inc. (GMC) for scanning into machine readable files that are available to the geothermal community.

scanning - Each map was first copy- reduced in size until its minimum dimension was slightly less than 4.0 inches. The reduced image was then positioned in a "Scan:Align" device (made by American Business Concepts, Dallas, Texas) to facilitate use of a single pass with a hand scanner.

A 4.5 inch wide NISCAN/GS hand scanner was then placed in the appropriate Scan:Align template and each image was scanned into "Scanxpress" software (made by NISCA, Dallas, Texas). The files thus generated were saved in the *.PCX and * TIF formats that are importable by most graphics and word processing programs. File sizes range from 50,335 to 331,930 bytes with an average TIF size of 260,000 bytes and an average PCX size of 55,000 bytes.

The TIF and PCX files comprising the map portion of the NGA database have been copied onto diskettes for distribution to industry users. The contents of the diskettes may be copied onto the user's hard disk and then imported useing a graphics or word processing program by following the appropriate instructions in the manual. The maps can then be printed by the user at any scale allowed by his software and printer.

The importation of a graphic map file using Microsoft word is described as an example.

1. Assume that the the map files are located in the D: ISCANXIITALY.PCX (or ITALY TIF) Path and File.

2. In Microsoft Word (MSWORD), choose Library Link Graphics. 
3. Type in the Path and File (See 1. above)

4. Type <Return>

5. Choose Printer, print and wait until print file is loaded, a period of several minutes, before obtaining the final map of Italy from the printer.

Note that the printer must have enough memory to accomodate 350,000 byte graphic files. Laser printers work especially well and can produce maps at virtually any scale within 4-5 minutes per map.

ngadbmap. inf 


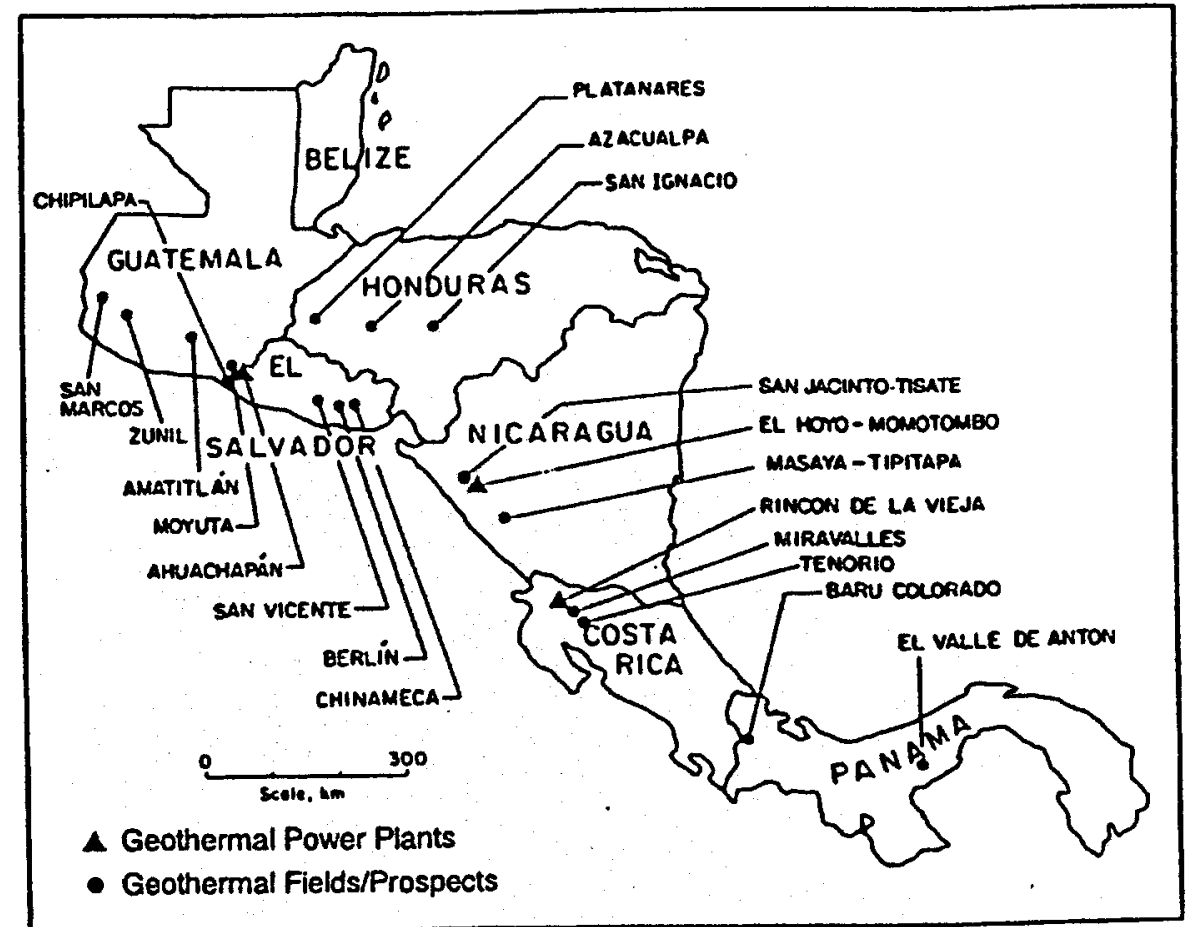

CENTRAL AMERICA 


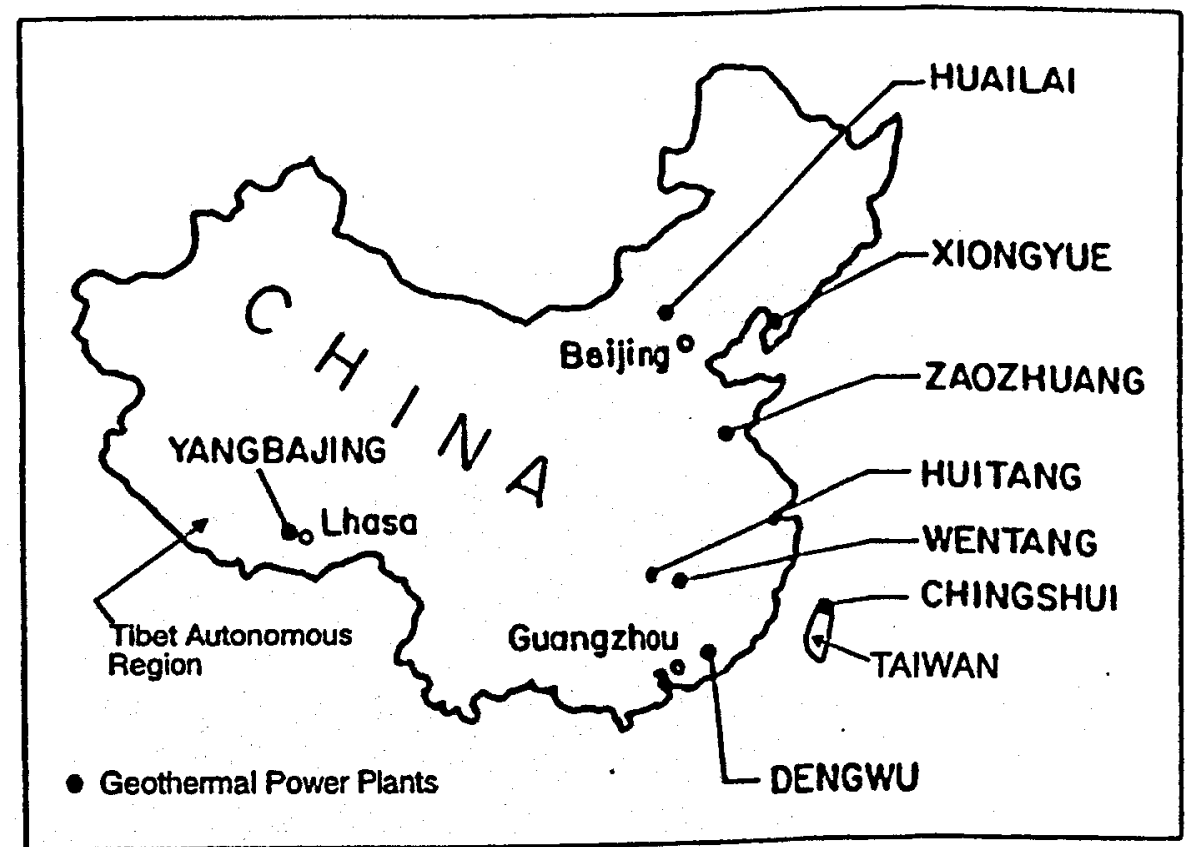

CHINA AND TAIWAN 


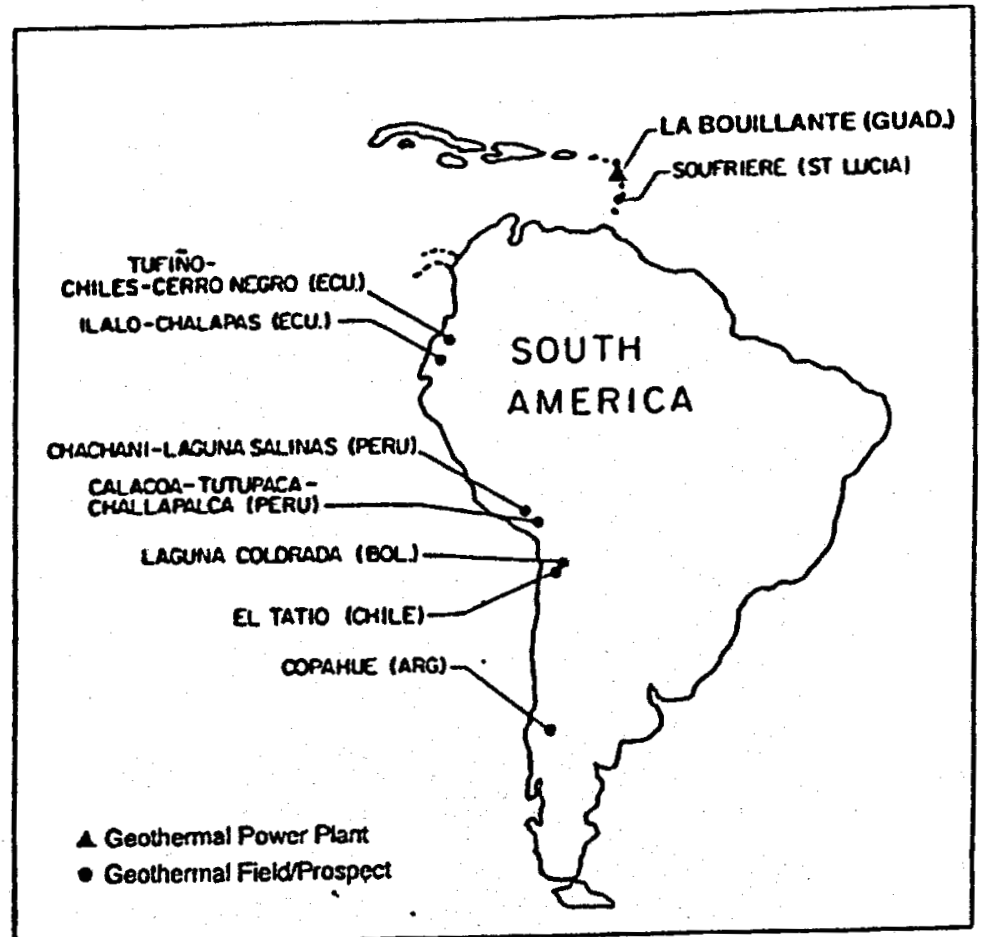

FRANCE (Guadeloup)

\section{D:ISCANXIFRANCE.TIF}




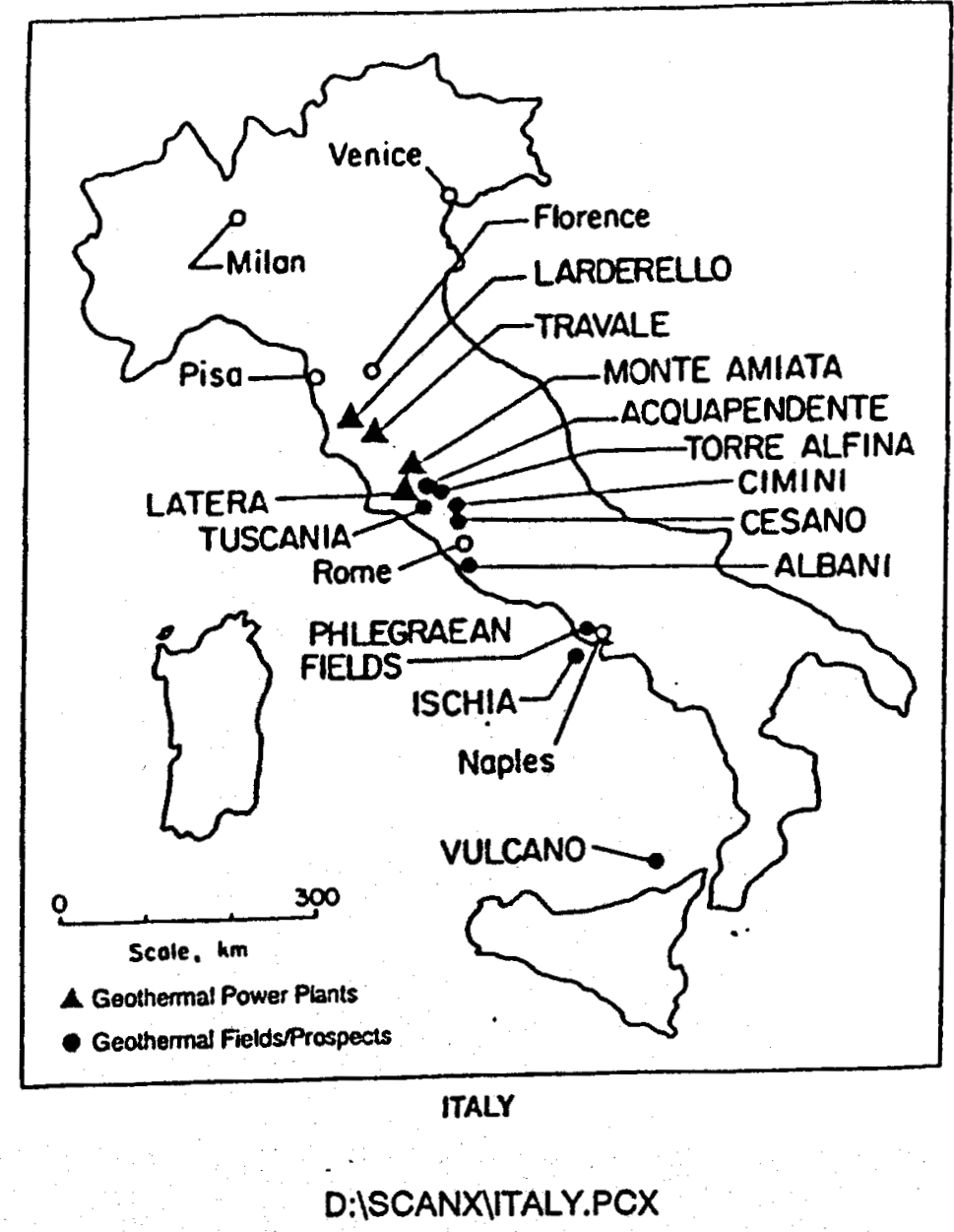




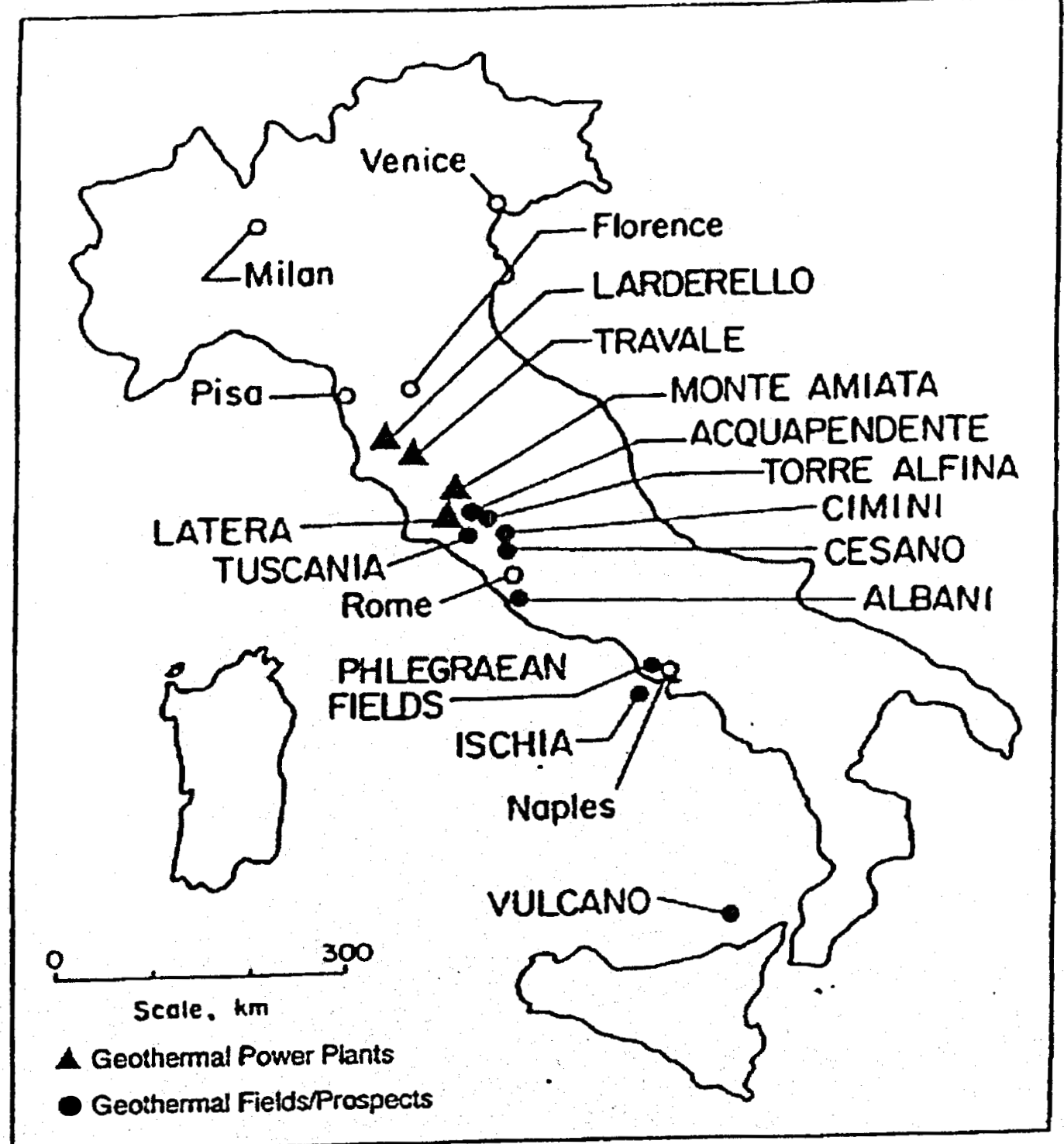

ITALY 


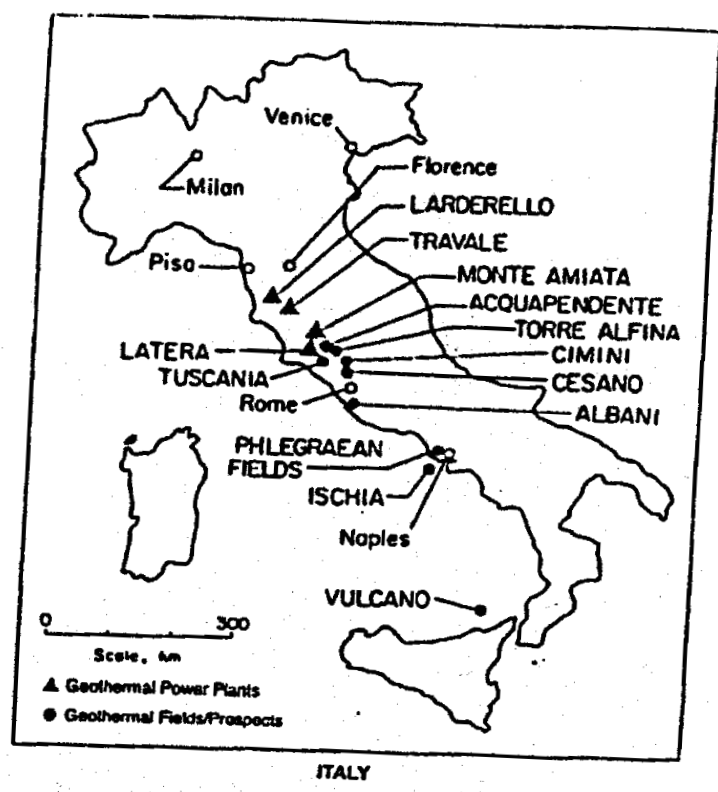




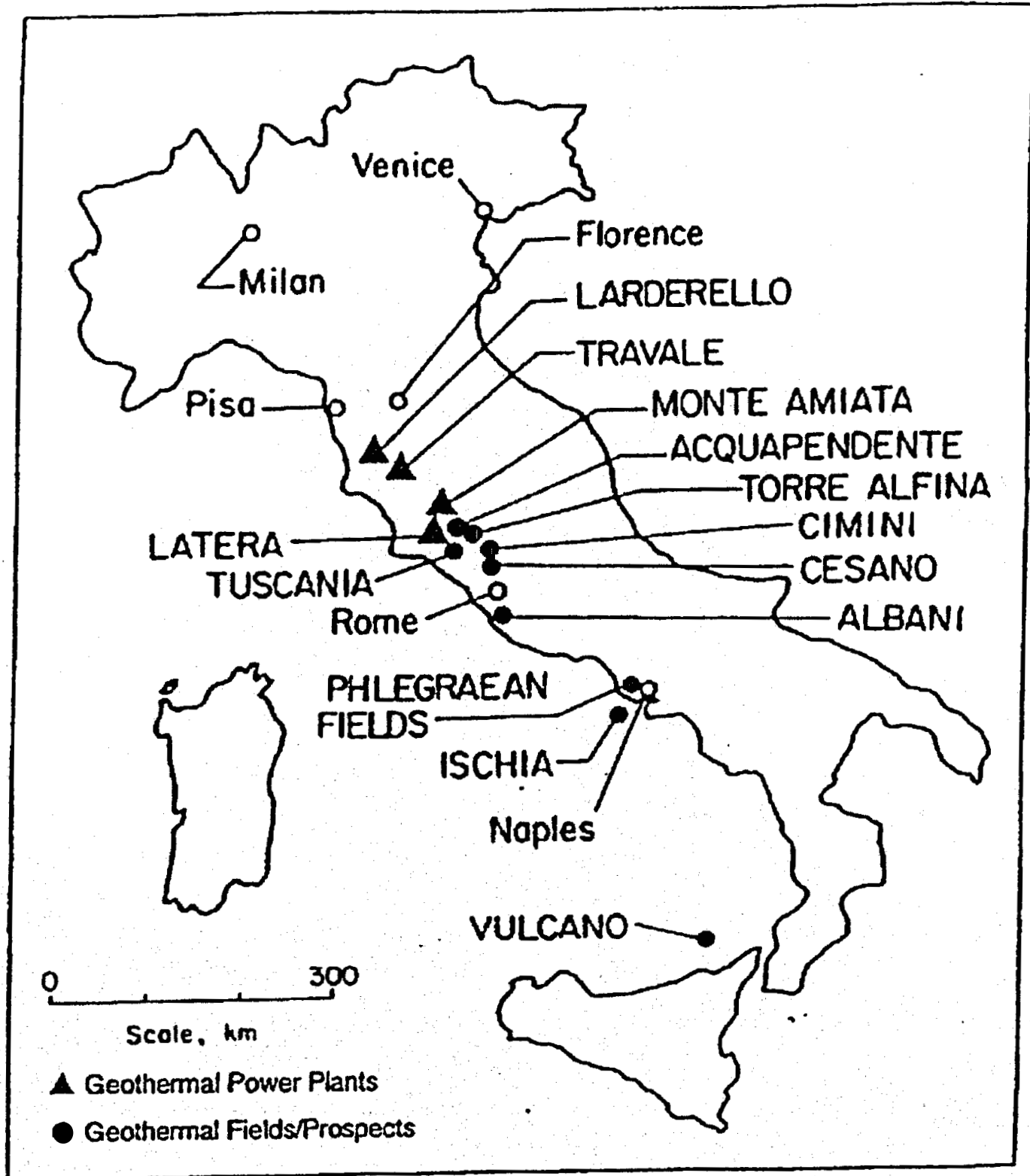

ITALY 


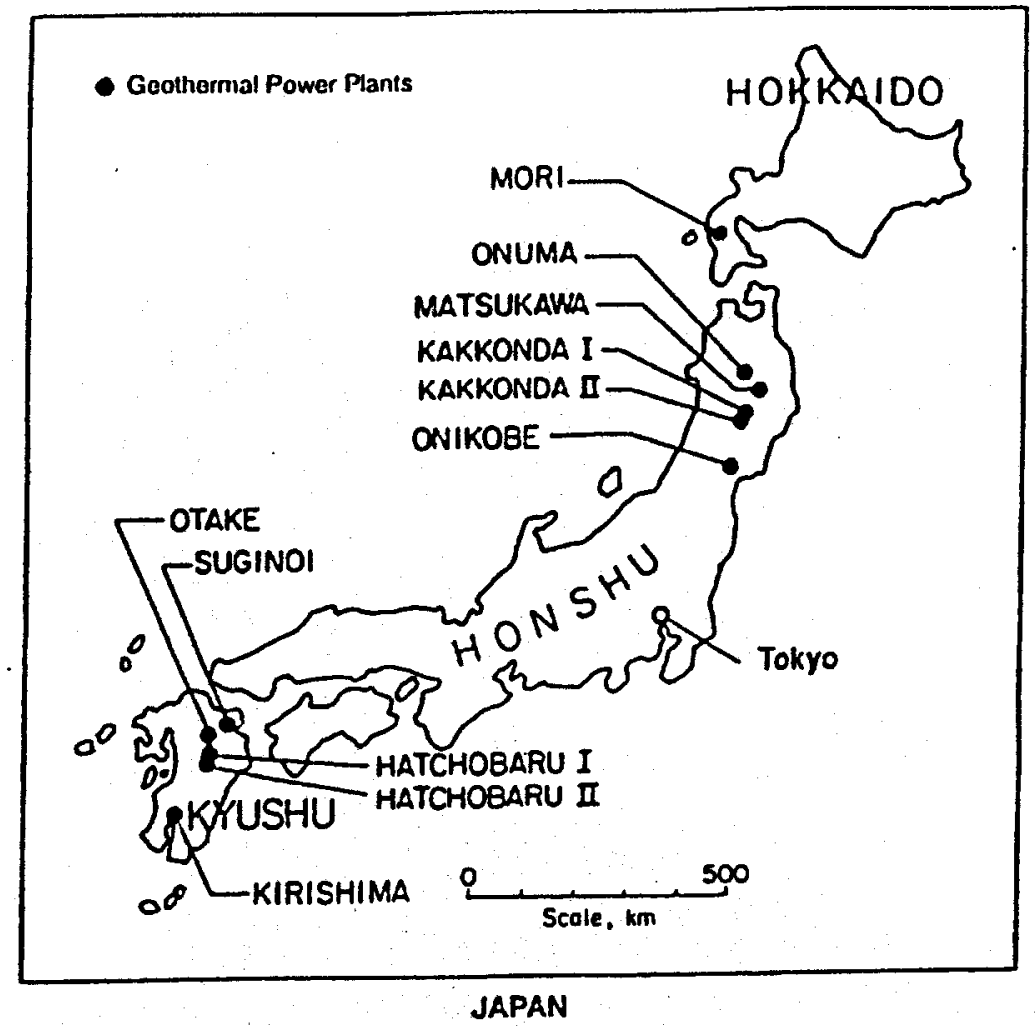

D:ISCANXIJAPAN.TIF 


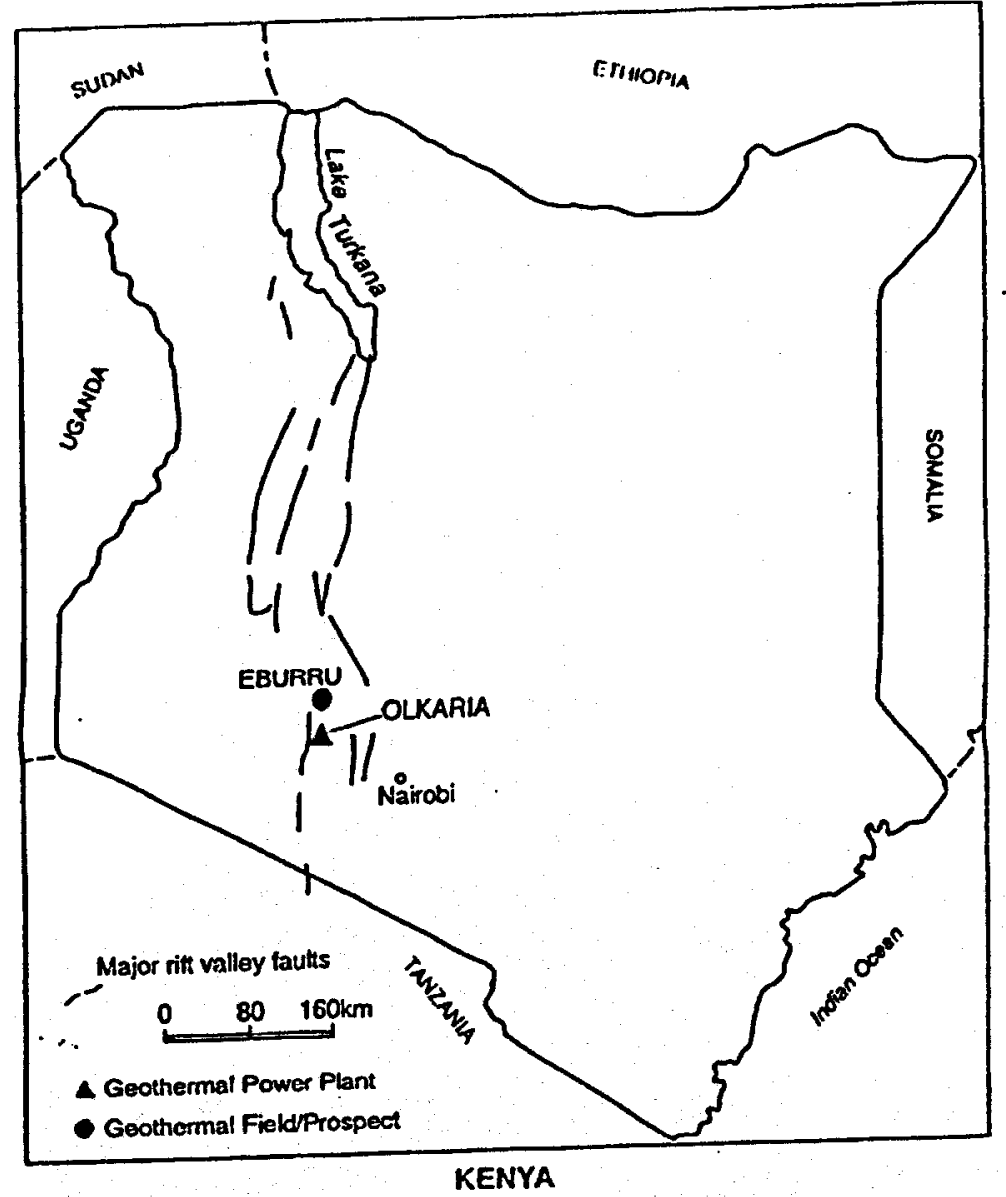

D:ISCANXIKENYA.TIF 


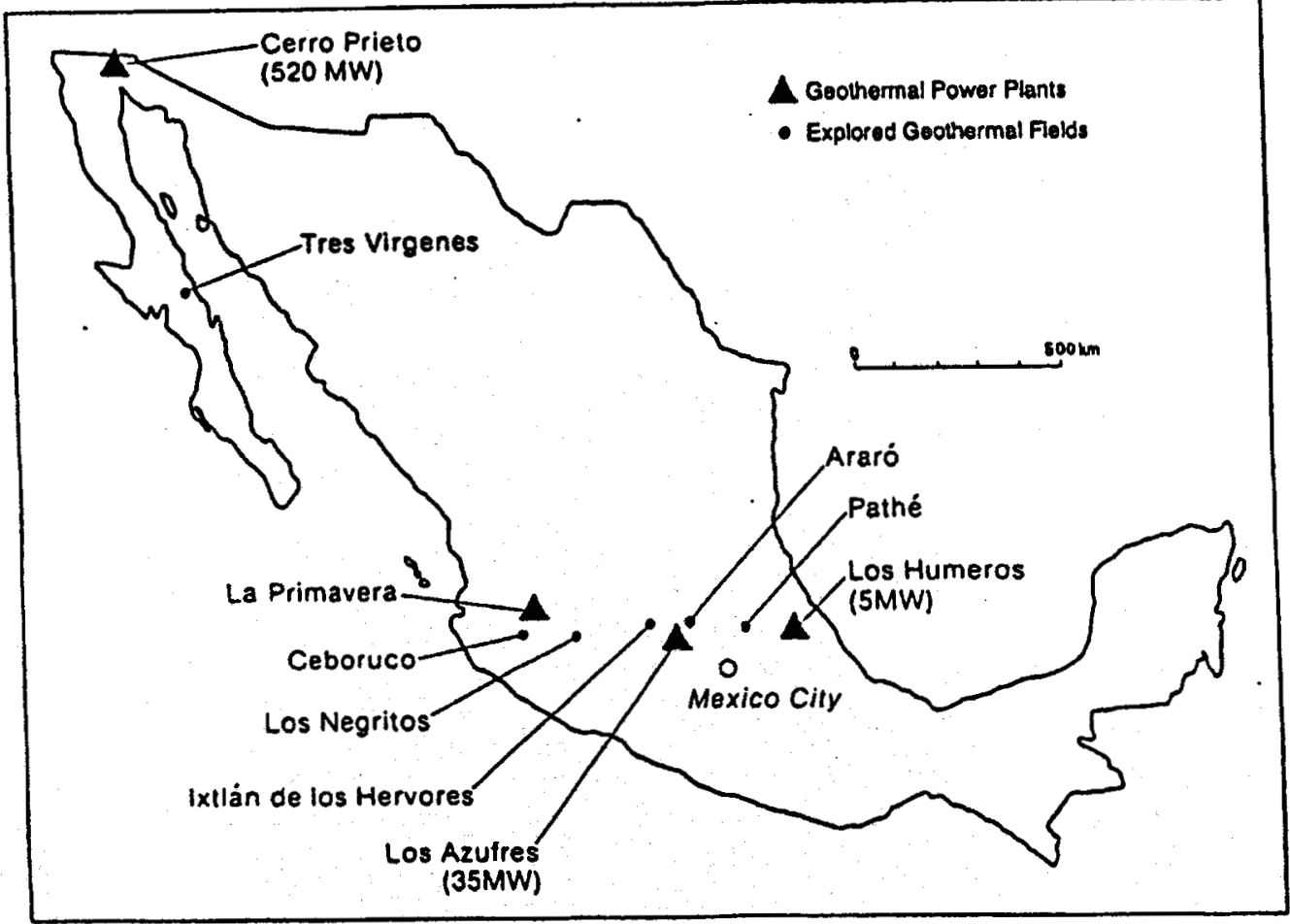

MEXICO 


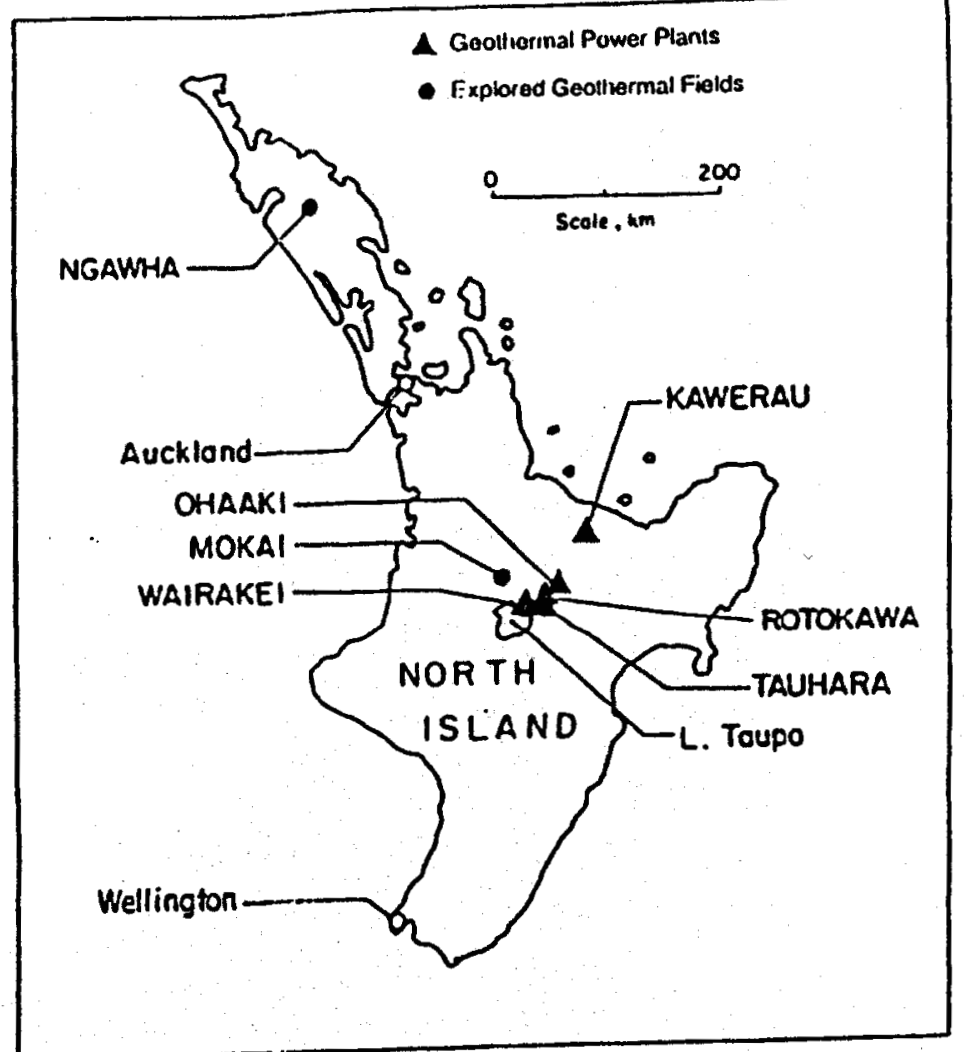

NEW ZEALANO (North Island)

D:ISCANXINEWZEAL.TIF 


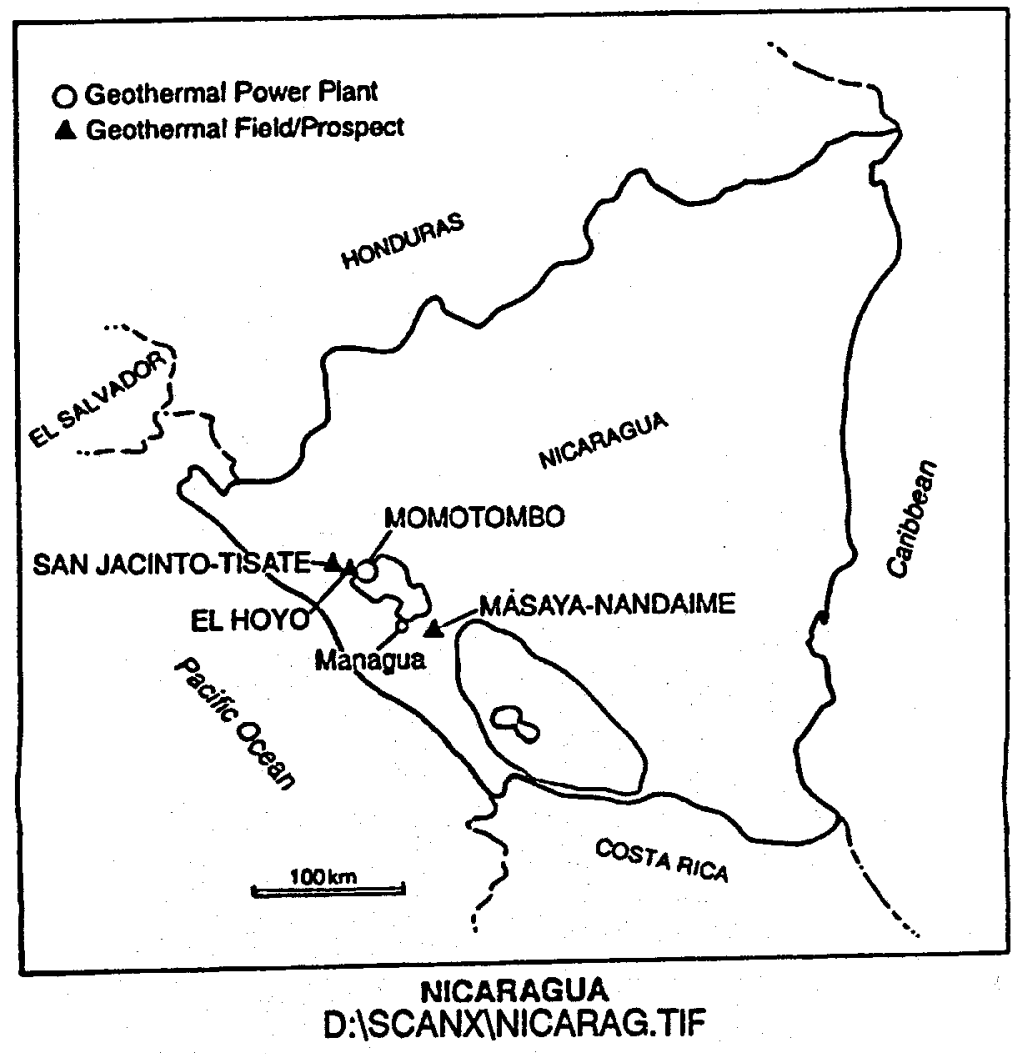




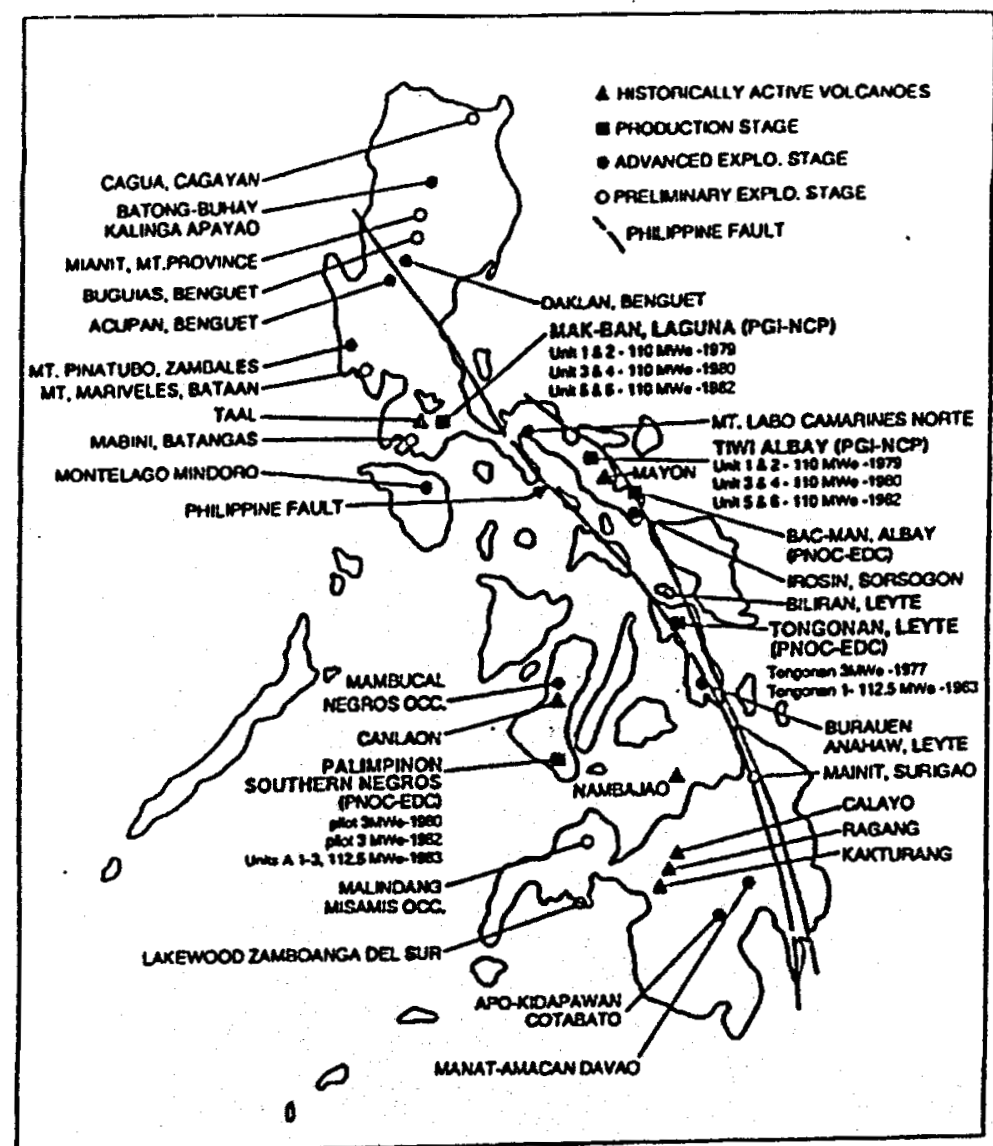

PHILIPPINES

D:ISCANXIPHILIPP.TIF 


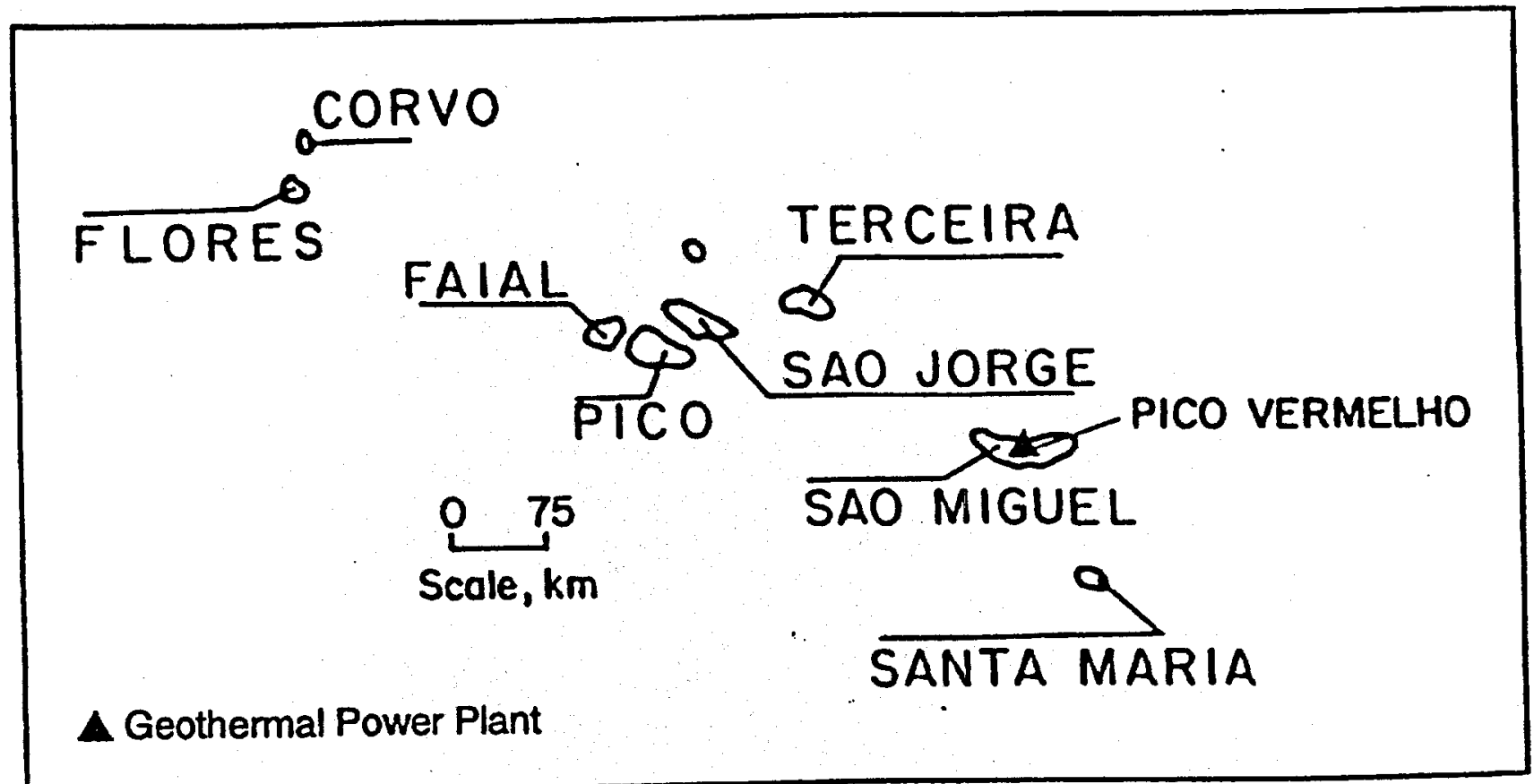

PORTUGAL (Azores Islands) 


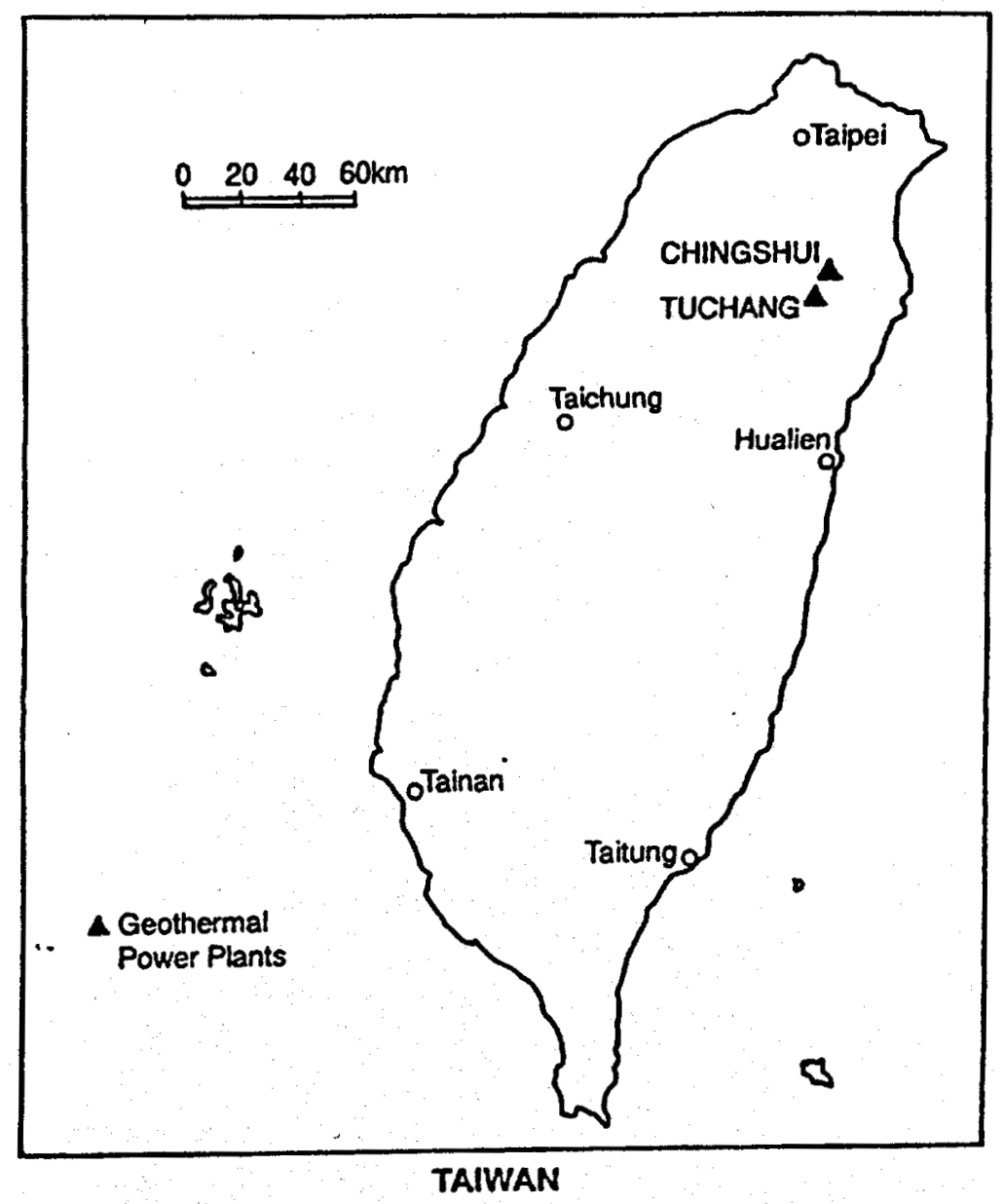

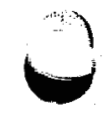




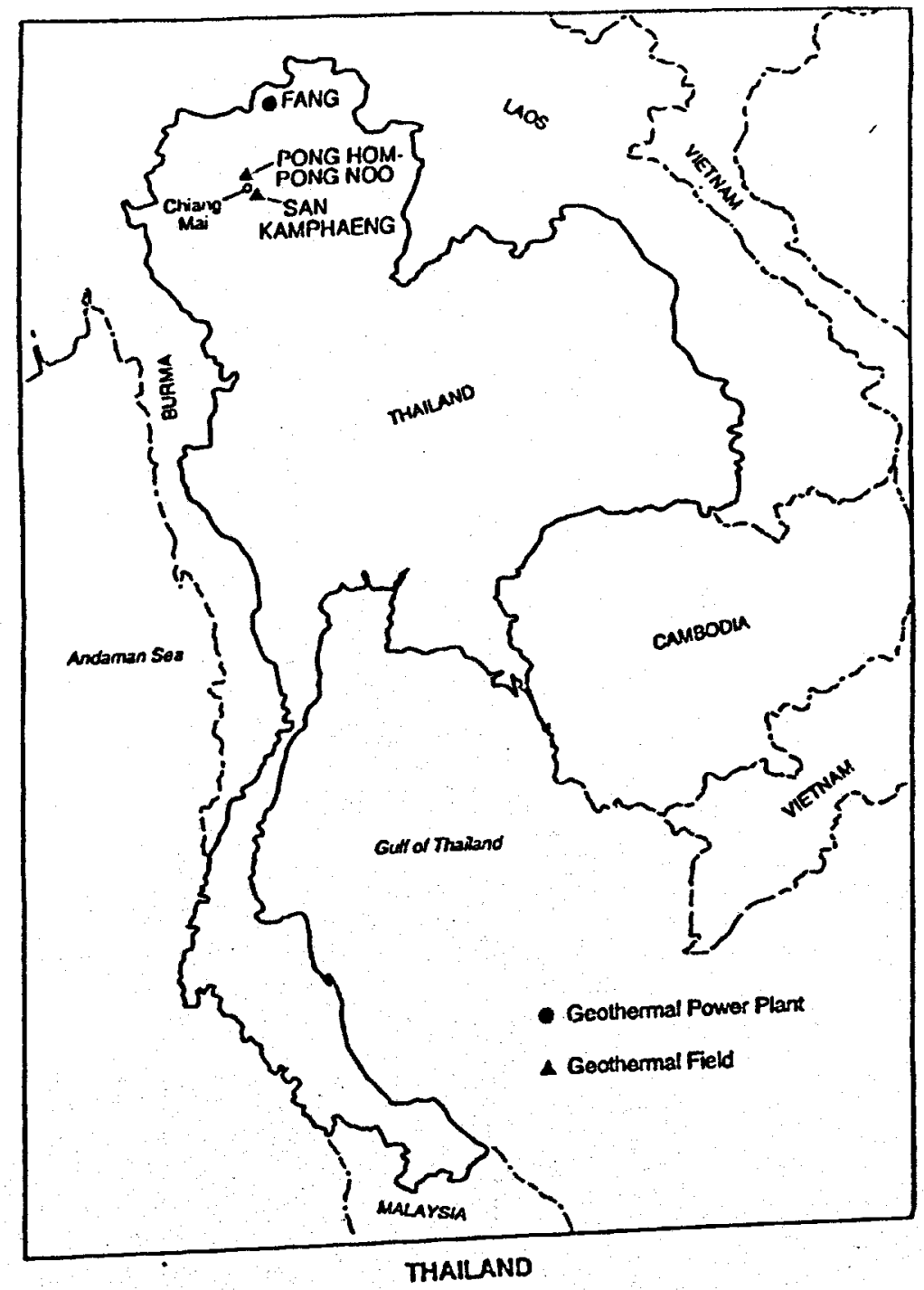




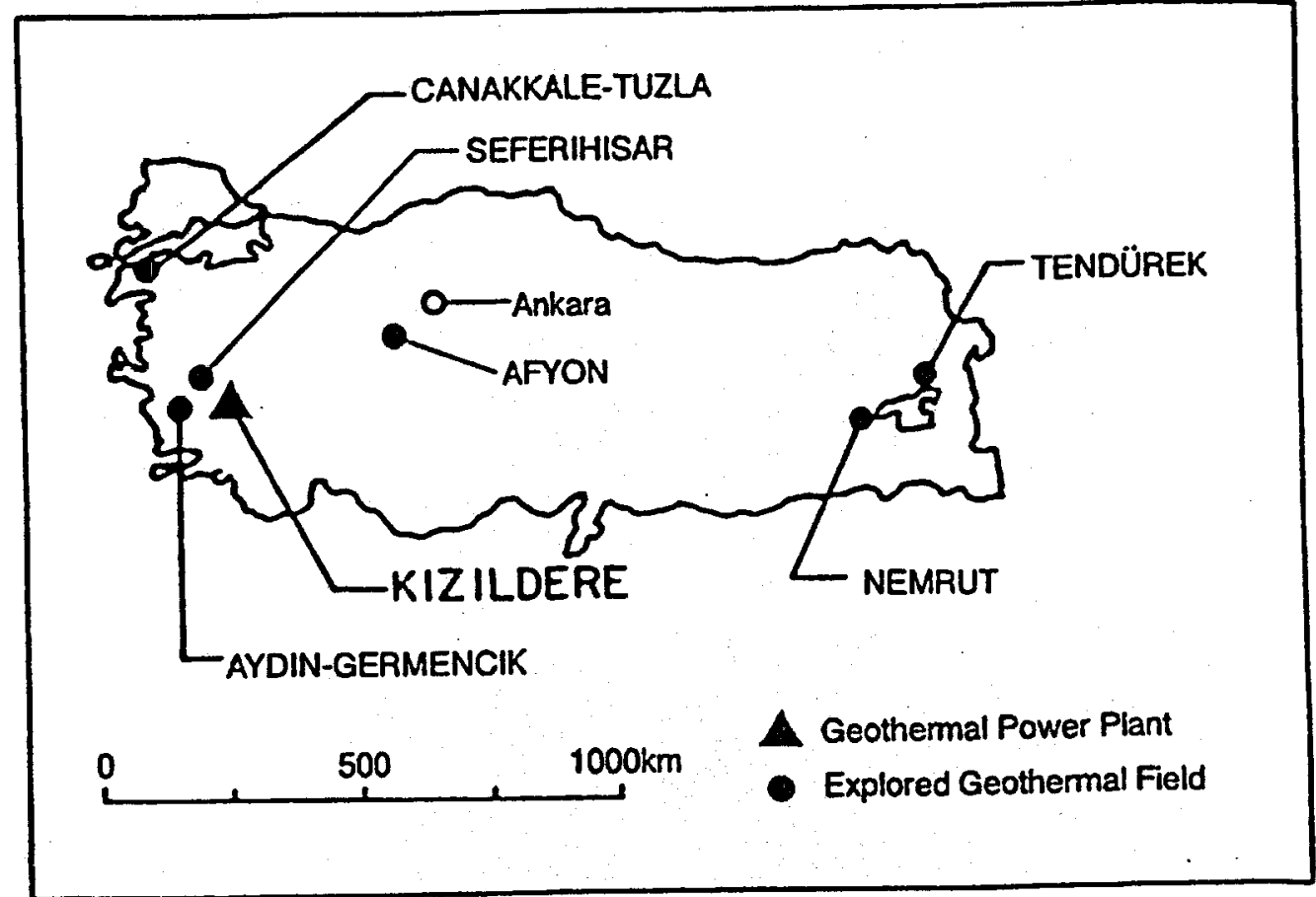

TURKEY 


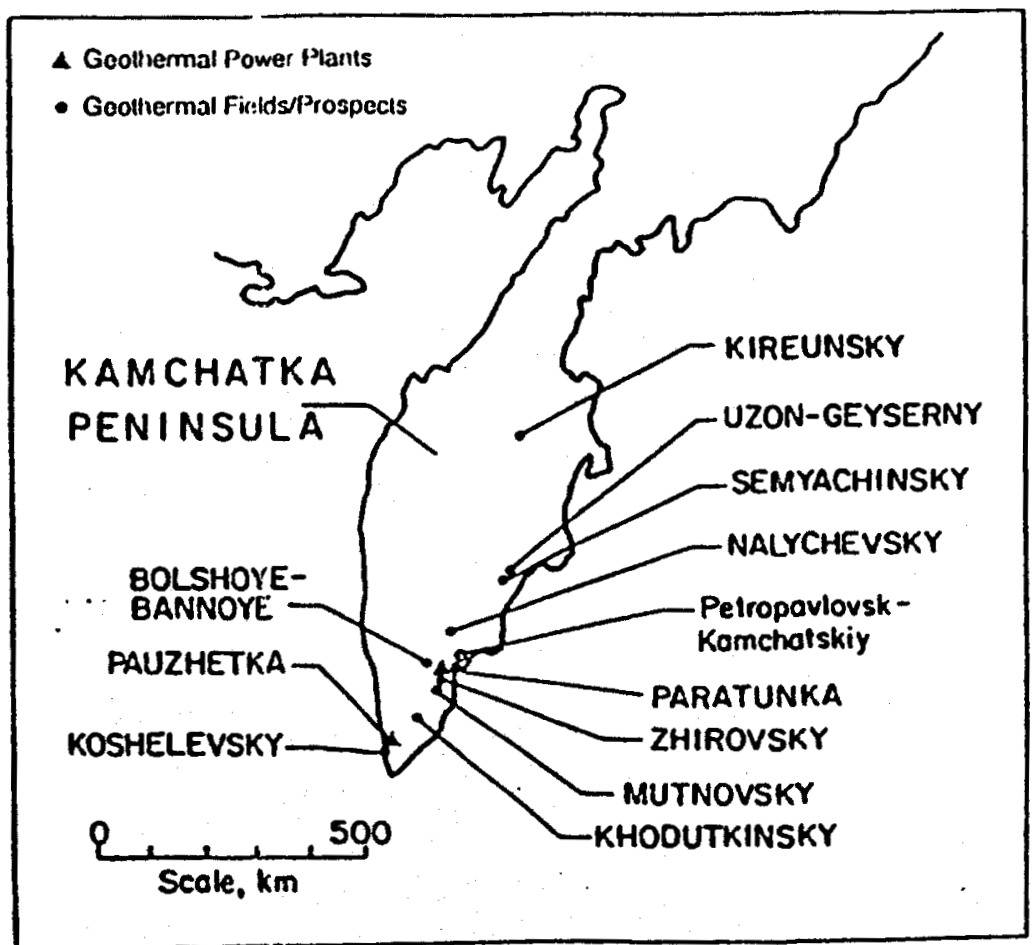

USSR (Kamchatka) 


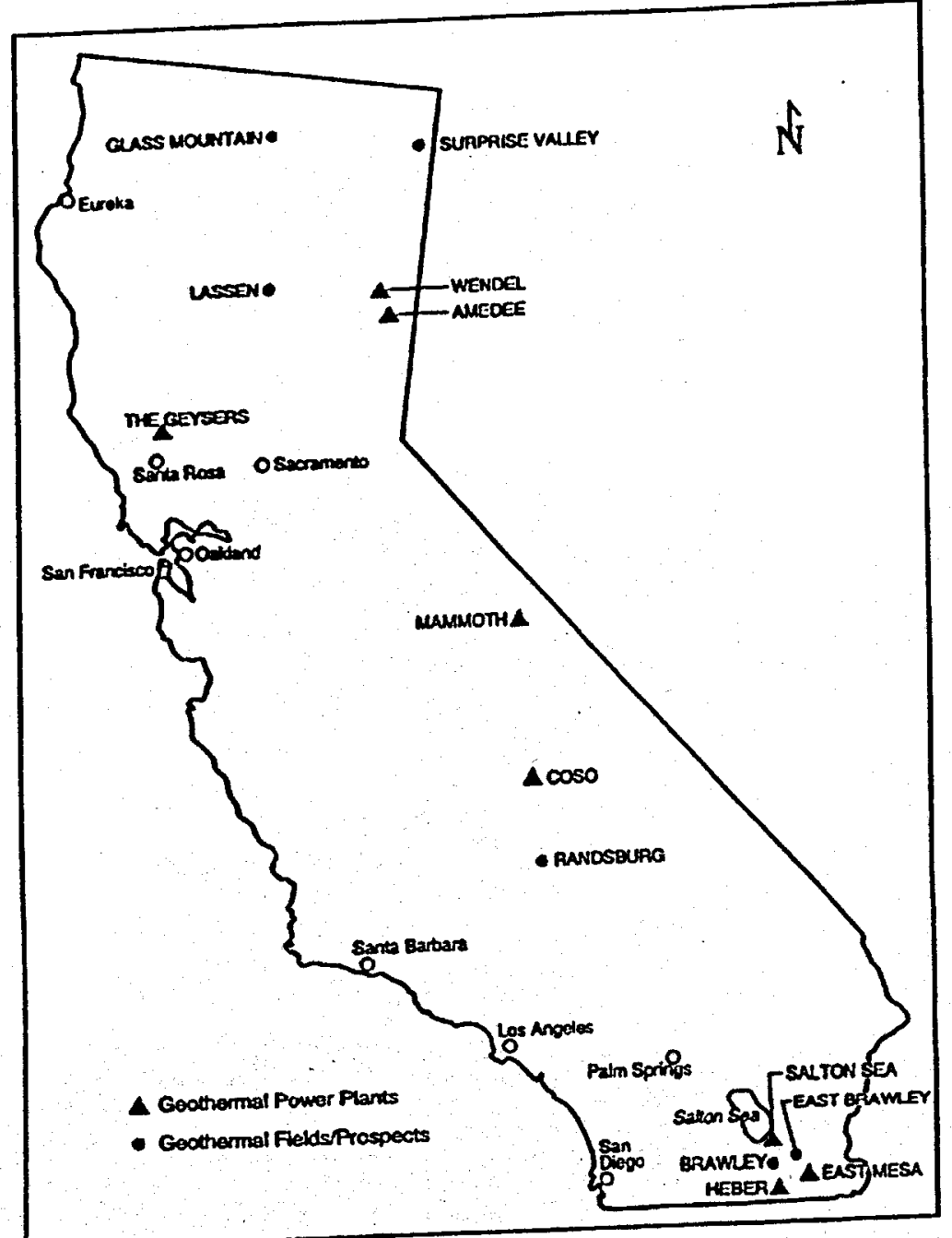

CALUFORNIA, USA 


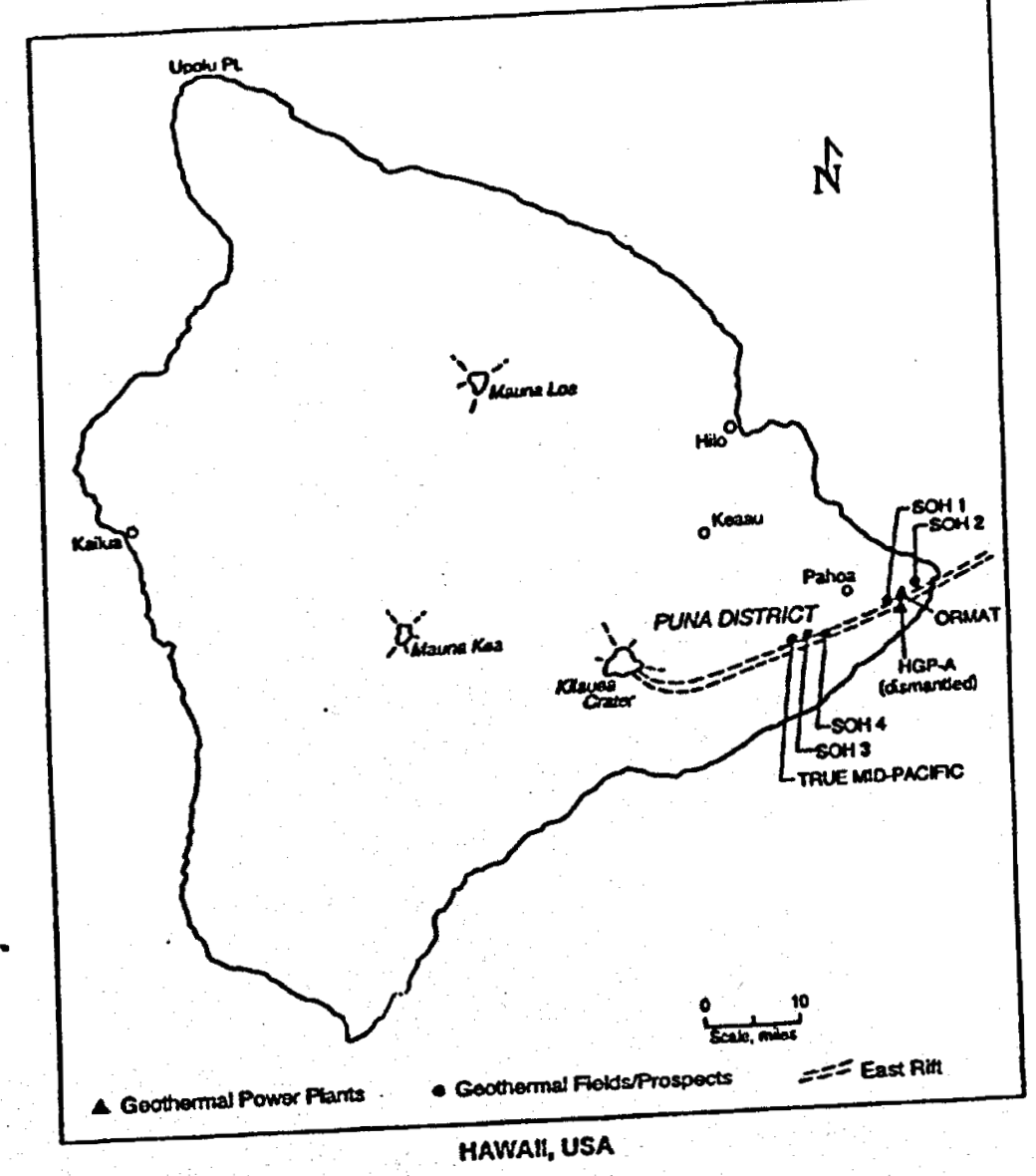

D:ISCANXIHAWAII.TIF 


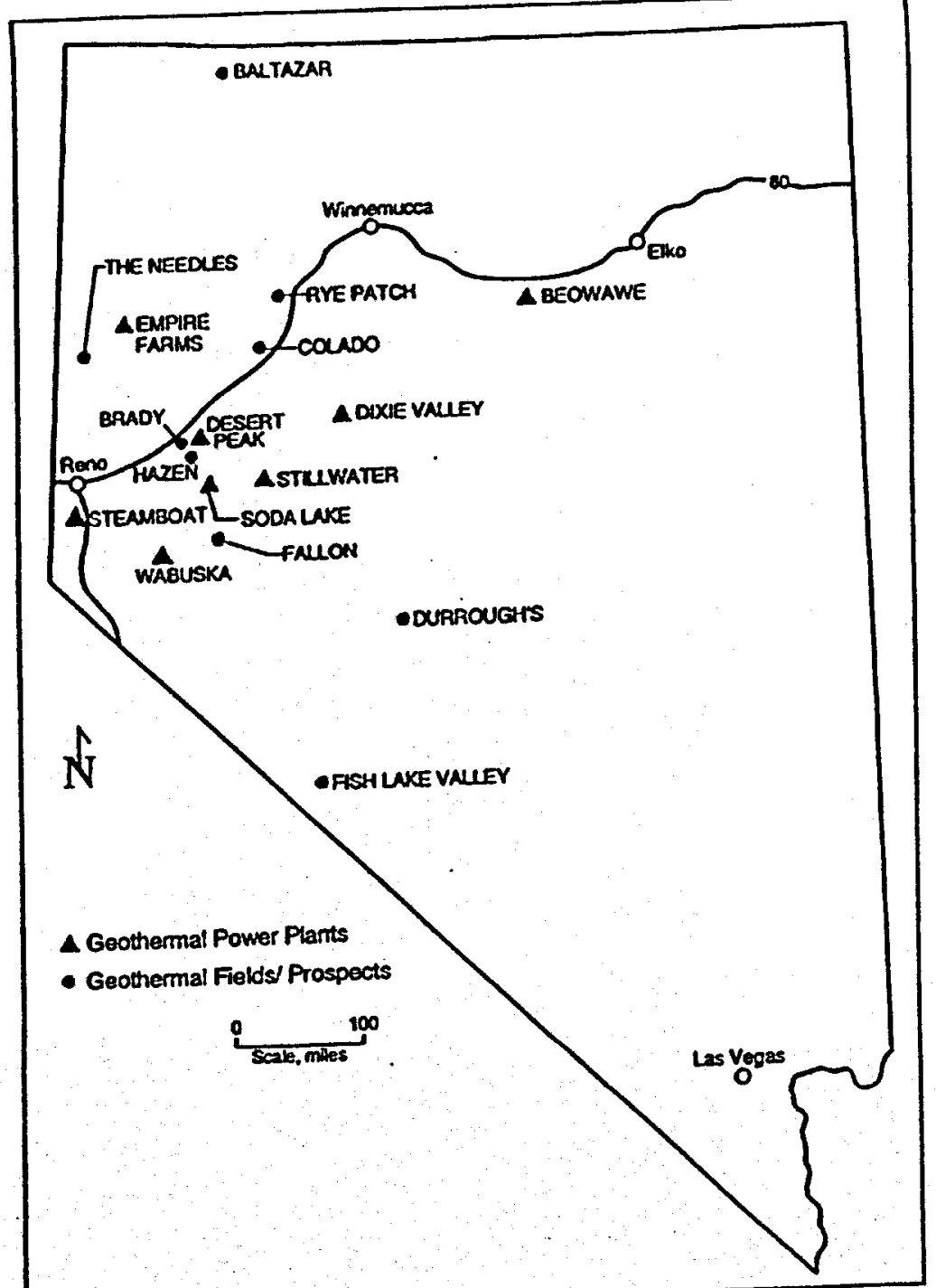

NEVADA, USA

D:ISCANXINEVADA.PCX 


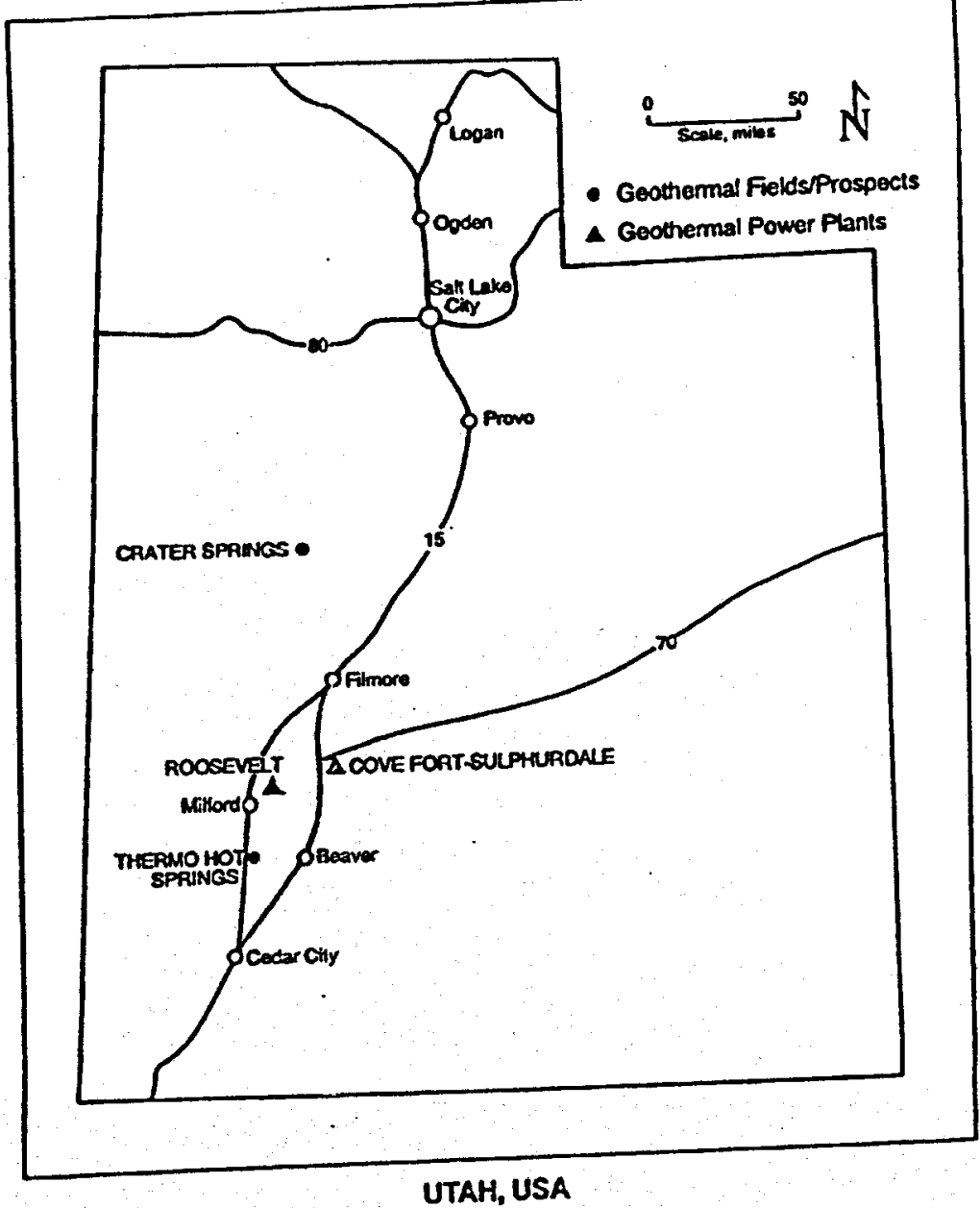

D:ISCANXIUTAH.TIF 
DATABASE QUESTIONS 


\section{ANSWER SHEET \\ For Use with \\ THE NGA DATA BASE QUESTIONNAIRE}

Instructions:

1. Please use a separate answer sheet for EACH GEOTHERMAL POWER PLANT IN YOUR NATION. Several answer sheets have been supplied for your convenience.

2. Please supply the responses to questions $64 \mathrm{a}, 64 \mathrm{~b}$, and $64 \mathrm{C}$ on separate, dedicated sheet(s) of paper.

1.

2.

3. $-(p, u, o, n, d)$

4. (Mo/ Yr)

5 .

6.

7.

8.

9.

10.

11.

12 .

13.

14.

15.

16.

17 .
18.

19. (Gross MWe)

20 . (Net MWe)

21. ___ $(\mathrm{Klbs} / \mathrm{hr}$ or $\mathrm{Kg} / \mathrm{sec})$

22 - $(\mathrm{Mg} / \mathrm{L}$ or PPM)

23. (years)

24. (psig or $\mathrm{Kg} / \mathrm{Cm}^{2}$ )

25. (Degrees F)

26. $=$ (

27. -_ (\$US Millions)

28. - $\quad\left(\mathrm{km}^{2}\right)$

29. -_ (\$US Millions)

30. -_ (\$US Militons)

31. (\%)

32.

33.

34. (Degrees F) 


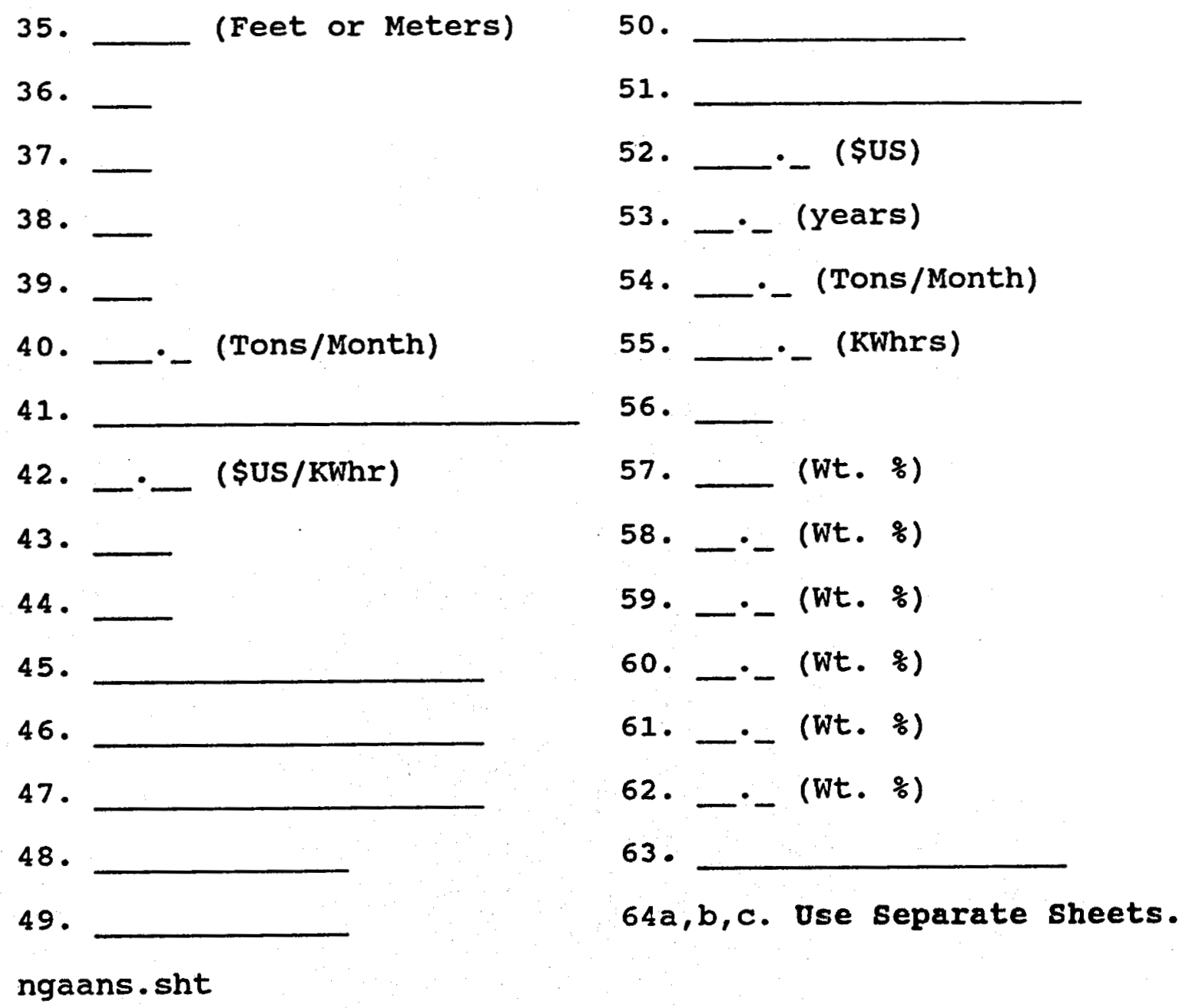




\section{NATIONAL GEOTHERMAL ASSOCIATION}

P.O. Box 1350

Davis, California 95617-1350 USA

(916) $758-2360$ Telex: 882410

INTENT TO RESPOND

NAME

ORGANIZATION

TELEPHONE

FAX

TELEX

1. WE WILI COMPLETE THE NGA QUESTIONNAIRE

Please mark (x) AND SUBMIT SOME TEXT AND OUTLINE MAPS

your intent. FOR THE NGA DATA BASE.

2. WE WILL NOT BE ABLE TO SUBMIT INFORMTION FOR THE NGA DATA BASE.

3. THE NGA LETTER - REQUEST HAS BEEN GIVEN TO THE FOLLOWING ORGANIZATION FOR RESPONSE. Contact Person

organization

Phone FAX TWX

signed Date

PLEASE FAX THIS TO: 303-674-1971 (Evergreen, Colorado, USA) or

MAIL TO: GERALD W. HUTTRER GEOTHERMAI MANAGEMENT COMPANY, INC. P. O. BOX 2980 27972 MEADOW DRIVE, SUITE 340 EVERGREEN, COLORADO 80439-2980 USA

ngaintto.res 


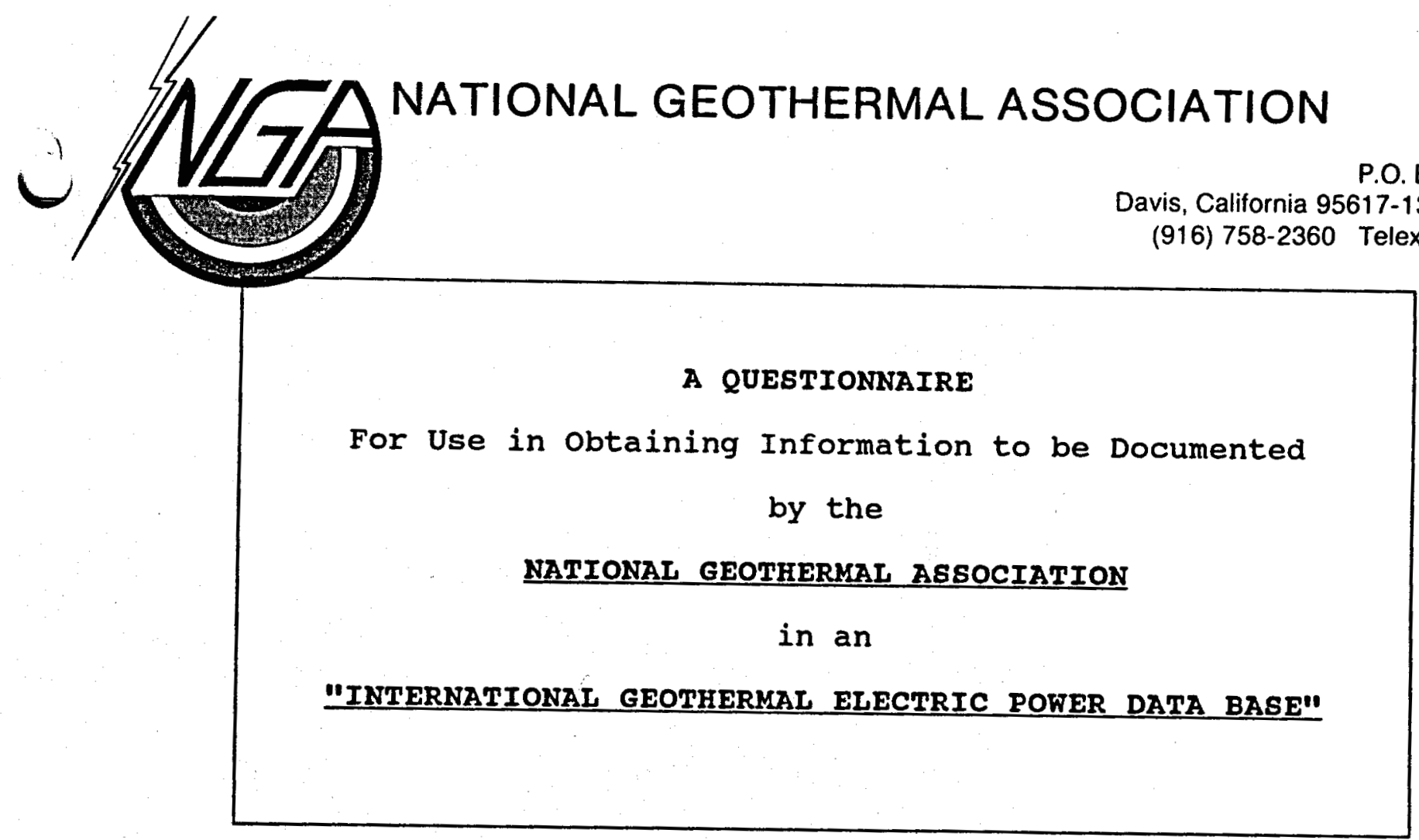

\section{Instructions:}

a. It is likely that a large geothermal field will support several generating units. Please answer all questions for EACH separate unit on copies of this questionnaire.

b. Please answer all questions; use $\mathrm{N} / \mathrm{A}$ for questions that are not applicable.

c. Please provide explanatory text whenever it will amplify or clarify your responses to the NGA questions.

d. Please send AN OUTLINE MAP of your nation showing the sites of the generating units operating, planned, or out of service.

\section{QUESTIONS}

1. Plant Name - The name designating the unique unit organized to produce electricity. If two independent units are located at the same site, they should be listed as unit number 1 and unit number 2. Text, up to 20 characters.

2. Site - Name of the site or geothermal field where the unit is located. Text, up to 20 characters. 
3. status - (Planned, Under Construction, Operating, Nonoperating, Decommissioned). The plant status is limited to these 5 categories. Text, 1 character $(P, U, O, N$, or $D)$.

4. Date of Firm Operation - Date that the power purchaser accepts power from the unit on a firm basis. (Mo/Yr).

5. Nation - The nation within which the unit is located. Text. Should be abbreviated to 4 characters.

6. Political subdivision - The region or state or island or other identifiable district in which the unit is located. Text. Should be abbreviated to 4 characters.

7. Resource Owner - The beneficial owner of the geothermal resource. Text, up to 20 characters.

8. Resource Lessee or Field Developer - The entity that has leased or has a concession for or who is developing the resource. If it is the resource owner, then reenter the owners name. Text, up to 20 characters.

9. Architect/Engineer - The name of the firm that was responsible for design of the power plant unit beginning at the exit of the gathering system and ending at the entrance to the disposal system. Text, up to 20 characters.

10. Power Plant owner - The beneficial owner of the power plant. Text, up to 20 characters.

11. Turbine source - The manufacturer of the turbine or the supplier, if significant modifications were made to it. Text, up to 20 characters.

12. Power Purchaser - The utility or industrial firm that purchases the electricity or the steam or the hot water produced by the unit. Text, up to 20 characters.

13. Plant contractor - The name of the firm or other entity acting as the general contractor for the power plant construction. (This entity is responsible for integrating all construction activities and delivering to the owner an operating power plant. Text, up to 20 characters. 
14. Power plant contact Person - The name of the person to be contacted in order to obtain information regarding the power plant. Text, up to 20 characters.

15. Entity Employing the Power Plant contact Person - The name of the firm or other entity for whom the power plant contact person works. Text, up to 20 characters.

16. Telephone - The phone number of the power plant contact person. Numerical, up to 13 numbers.

17. Facsimile (FAX) - The FAX number of the power plant contact person. Numerical, up to 13 numbers.

18. TELEX - The TELEX identification letters of the power plant contact person. Text, up to 14 characters.

19. Design Capacity (GROSS MWe) - The GROSS power capacity that the power plant was designed to deliver. Numerical, up to 6 numbers including a decimal point and 2 decimal places.

20. Design Capacity (NET MWe) - The NET power capacity that the power plant was designed to deliver. Numerical, up to 6 numbers including a decimal point and 2 decimal places.

21. Total Well Production (Lbs/hr or $\mathrm{kg} / \mathrm{sec}$ ) - The total production, in thousands of pounds per hour, or in kilograms per second, from all wells as measured at the outlet of the gathering system. Numerical, up to 6 numbers including a decimal point and 2 decimal places.

22. Total Dissolved solids (PPM or $\mathrm{Mg} / \mathrm{L}$ ) - The total dissolved solids content of the fluids produced at the outlet of the gathering system as of the date of firm operation. Numerical, up to 6 numbers including a decimal point and 2 decimal places.

23. Design Lifetime (Years) - The economic life span over which the power plant was designed to function. Numerical, up to 3 integers.

24. Inlet pressure (PSI or $\mathrm{kg} / \mathrm{cm}^{2}$ ) - The initial design inlet pressure as measured at the outlet of the gathering system. This is important for flash or dry steam systems but less important for binary systems. Numerical, up to 5 integers. 
25. Initial Reservoir Temperature (Degrees F) - The initial temperature of the reservoir at depth as measured in degrees Fahrenheit. Numerical, up to 3 integers.

26. Exploration costs (\$) - A reasonable estimate of the exploration costs associated with the availability of the geothermal resources fueling this power plant in millions of Us dollars. Numerical, up to 6 numbers including a decimal point and 1 decimal place.

27. Average Nell cost (\$OS Millions) - The average cost of completed production, injection, and standby wells, including casings, wellhead equipment, but not including pumps in millions of us dollars. Numerical, up to 6 numbers including a decimal point and 1 decimal place.

28. Subsurface Area of the Geothermal Field $\left(\mathrm{Km}^{2}\right)$ - The approximate subsurface area occupied by the geothermal field if directional wells are included. Numerical, up to 6 numbers including a decimal point and 1 decimal place.

29. Total cost of the Power plant (\$US Millions) - cost of the power plant from the output of the gathering system, including the "switch yard", but not including the transmission lines in millions of US dollars. Numerical, up to 6 numbers including a decimal point and 1 decimal place.

30. Total Project cost (\$os Millions) - Total cost (equity and debt) of the project, not including the transmission lines, in millions of US dollars. Numerical, up to 6 numbers including a decimal point and 1 decimal place.

31. Availability ( $\%$ - The number of hours that the power plant was available to produce power during the last calendar year period DIVIDED by 8760 hours TIMES 100 . Numerical, up to 4 numbers including a decimal point and 1 decimal place.

32. Availability Year - The year for which the Availability $\%$ was calculated. Numerical, 4 integers.

33. Type of Power Cycle Used - The power plant generation system type. Respond only as follows: Binary, $S$ Flash, $D$ Flash, or D Steam. Text, up to 8 characters. 
34. Inlet Temperature (Degrees F) - The resource temperature as measured at the outlet of the gathering system in degrees Fahrenheit. Numerical, up to 5 numbers including a decimal point and 1 decimal place.

35. Average Well Depth (Feet or Meters) - The average depth of all production, injection and standby wells used for power production. Numerical, up to 5 numbers including a decimal point and 1 decimal place.

36. Number of Production Fells - The number of producing wells needed to fully supply resource to the power plant. This does not include standby wells. Numerical, up to 3 integers.

37. Injection Fells - The number of injection wells needed to dispose of fluids resulting from full power plant operation. This does not include standby wells. Numerical, up to 3 integers.

38. Failed or Abandoned Fells - In reference to this power plant: includes non-producable exploration wells as well as production, injection, and standby wells unsuccessfully drilled or abandoned. Numerical, up to 3 integers.

39. Total Number of Wells - This includes all wells on the site that are functioning or are capable of functioning properly as production, injection, or standby wells for this power plant. Numerical, up to 3 integers.

40. Reactor/Clarifier sludge Produces (Tons/Month) - If a Reactor/Clarifier is in use, please enter the tons per month of sludge that are produced. Numerical, up to 5 numbers including a decimal point and 1 decimal place.

41. Financing organization - The name of the financing organization that took the lead in providing funds for the power plant. The lead lending organization/syndicator. Text, up to 25 characters.

42. Electricity sales Price per KWhr. - The average price, preferably converted to \$US/KWhr, received for electricity generated at the geothermal power plant during 1989. Numerical, up to 4 numbers including a decimal point and 1 decimal place. 
43. Year Power Plant was Completed - Numerical, 4 integers. 44. Year Power Plant stopped Operating - Numerical, 4 integers.

45. Generator source - The manufacturer of the generator or the supplier, if the generator was significantly modified. Text, up to 20 characters.

46. Resource Owner/Lessee contact Person - The name of the person to contact in order to obtain information regarding the resource on behalf of the owner or lessee. Text, up to 20 characters.

47. Entity Employing the Resource contact Person - The name of the firm or other entity for which the resource contact person works. Text, up to 20 characters.

48. Telephone - The phone number of the person to contact regarding the resource on behalf of the owner or lessee. Numerical, up to 13 integers.

49. Facsimile (FAX) - The FAX number of the resource contact person. Numerical, up to 13 integers.

50. TELEX - The TELEX identification letters of the resource contact person. Text, up to 14 characters.

51. Power Plant operator - The name of the power plant operator in case that it is not the same as the power plant owner. Text, up to 20 characters.

52. Cost of the Gathering systems (\$Us) - The cost of the fluid gathering and disposal systems for both production and injection in millions of Us dollars. This includes the cost of pumps (lineshaft or downhole) if used. Numerical, up to 6 numbers including a decimal point and 1 decimal place.

53. Average Well uife (years) - The average number of years that wells function properly with normal maintenance. Numerical, up to 4 numbers including a decimal point and 1 decimal place.

54. $\mathrm{H}_{2} \mathrm{~s}$ scrubber sludge produced (Tons/Month) - If an $\mathrm{H}_{2} \mathrm{~S}$ scrubber is used, then the sludge produced should be entered 
in tons per month. Numerical, up to. 5 numbers including a decimal point and 1 decimal place.

55. Electricity sales per Year(KWhr) - The local electricity sales from the power plant for a designated year (1989) in KWhrs. Numerical, up to 6 numbers including a decimal point and one decimal place.

56. Year of Electricity sales - The year for which the sale information requested in question number 55 was provided, (if not for 1989). Numerical, 4 integers.

57. Initial Total Non-Condensible Gas production (Weight \%) - The weight percent of the total amount of non-condensible gases extracted from the produced resource upstream of the power production units when the power plant was first operated, i.e. $\mathrm{H}_{2} \mathrm{~S}, \mathrm{CO}_{2}$, etc. Numerical, up to 4 integers.

58. $\mathrm{CO}_{2}$ Produced initially (weight $\%$ ) - The total amount of carbon dioxide produced initially in weight percent. Numerical, up to 4 numbers incuding a decimal point and 1 decimal place.

59. $\mathrm{H}_{2} \mathrm{~s}$ Produced Initially (Weight $\%$ ) - The total amount of hydrogen sulfide produced initially in weight percent. Numerical, up to 4 numbers including a decimal point and 1 decimal place.

60. Current Total Non-Condensible Gas Production (Weight $\%$ ) - The weight percent of the total amount of non-condensible gases extracted from the produced resource upstream of the power production units in 1989. Numerical, up to 4 numbers including a decimal point and 1 decimal place.

61. $\mathrm{CO}_{2}$ currently Being produced (weight \%) - The amount of carbon dioxide produced in 1989 in weight percent. Numerical, up to 4 numbers including a decimal point and 1 decimal place.

62. $\mathrm{H}_{2} \mathrm{~s}$ currently Being produced (weight $\%$ ) - The amount of hydrogen sulfide produced in 1989 in weight percent. Numerical, up to 4 numbers including a decimal point and 1 decimal place.

63. Non-Condensible Gas Treatment system Used - The name of the system used to remove non-condensible gases from the 
produced resource or from power plant emissions. Text, up to 20 characters.

In order to facilitate contacts within the international geothermal community, the NGA wishes to obtain the names addresses and phone/FAX/TELEX numbers for the major geothermally-involved entities in each nation. Please supply the information requested in questions $64 \mathrm{a}, 64 \mathrm{~b}$, and $64 \mathrm{c}$ in as much detailas possible. Please use separate sheets of paper for this purpose.

64a. Please supply the names, addresses and numbers for the Federal, District and/or local agencies responsible for :

1. Geothermal Exploration and Development

2. Power Plant Construction

3. Electricity Purchases and sales

4. Granting of Geothermal Concessions or Leases

5. Environmental Matters

The names of current department managers are also requested.

$64 \mathrm{~b}$. Please supply the names, addresses, and numbers for the geothermally active major lending agencies in your nation including, but not limited to:

1. Federal Agencies

2. International Banks or their local branches

3. Regional Funding Sources

The names of current department managers are also requested.

64c. Please supply the names, addresses, and numbers for the major companies currently engaged in geothermally-related activities on a commercial basis in your nation and/or in countries outside your nation. This list might include:

1. Consultants (Local, Regional, and/or International)

2. Trading Companies

3. Explorationists

4. Drilling Companies

5. Plant or Field Operators

6. Plant or Field Construction

The names of the current managers of these companies are also requested.

THE NGA THANKS YOU FOR YOUR TIME AND EFFORT IN PROVIDING THIS INFORMATION. HOPEFULLY, THE DATA BASE WILL SOME DAY BE USEFUL TO YOU IN YOUR GEOTHERMAL ACTIVITIES, SAVING YOU AND YOUR PERSONNEL TIME AND EFFORT.

ngaques1.doc 
MAILING LISTS 


\section{A national geothermal association}

P.O. Box 1350

Davis, California 95617-1350 USA

(916) $758-2360$ Telex: 882410

MAILING LIST FOR NGA/DOE GEOTHERMAL POWER PLANT DATA BASE DEVELOPMENT

$12 / 13 / 89$ - G.W. HUTTRER

1.

Prof. Ji-Yang Wang

Chairman, Geothermal Research Council

Institute of Geology

Academia Sinica

P.O. Box 634

Beijing 100011

THE PEOPLES REPUBLIC OF CHINA

2 .

Dr. Alfredo Mainieri

Instituto Costarricense

de Electricidad

Apartado 10

032-1000 San Jose

COSTA RICA

3.

Dr. Gustavo Cuellar

CEL (GEOCEL)

P.O. Box 01-478

San Salvador

EL SALVADOR

4.

Dr. Andre Girard

Bureau de Recherches Geologiques et Minieres

Institut Mixte de Recherches Geothermiques

B.P. 6009

45060 Orleans Cedex 2

FRANCE

5.

Dr. Michael Fytikas

Institute of Geology and

Mineral Exploration

70 Messogheion St.

Athens 11527

GREECE 
6.

Angel Andres Caicedo Anchissi

Unidad de Desarrollo Geotermico Guatemala sur

San Jose Villa Nueva

GUATEMALA

7.

Dr. Gudmundur Palmason

Geothermal Division

orkustofnun

Grensasvegur 9

108 Reykjavik

ICELAND

8.

B.R. Moon

Central Electricity Authority

Sewa Bhawan

New Delhi 110066

INDIA

9.

Ing. Vincent Radja

Perusahaan Listrik Negara (PLN)

Trunojoyo MI/135

Kebayoran Baru

Jakarta, 10450

INDONESIA

10.

Ing. Guido Civis Paimerini

Deputy General Director

ENEL/DPT

Via Andrea Pisano 120

56122 Pisa

ITALY

11.

Mooto Higo

Japan Geothermal Energy Association

Yuurakucho Denki Building

1-7-1 Yurakucho, Chiyoda-ku

Tokyo 100

JAPAN 
12 .

Mr. Sebastian Bwire-ojiambo

Kenya Power and Lighting Co. Ltd.

Geothermal Project

Electricity House, Harambee Ave.

P.O. Box 30099

Nairobi

KENYA

13.

Ing. Miguel Ramirez Gutierrez

Gerente de Estudios Geotermicos

Comision Federal de Electricidad

APDO 31-C

Morelia, Michocan

MEXICO

14.

Prof. Derek Freeston

Geothermal Institute

University of Auckland

Private Bag

Auckland

NEW ZEALAND

15.

Ernesto Martinez Tiffer

Instituto Nicaraguense de Energia

P.O. Box 55

Managua

NICARAGUA

16.

Dr.Roger Datuin, Vice President

National Power Corporation

Quezon Ave. Corner Agham st.

Diliman

Quezon City

PHILIPPINES

17.

Dr. Antonio M. Rodrigues da silva

Laboratorio de Geosciencias

e Technologia

Rua de Sao Miguel, 41

9500 Ponta Delgada

Acores

PORTUGAL 
18.

Mr. Alaxandru Butac, Manager

Ministry of Geology

Str. Mendeleev nr. 36

Bucharest

ROMANIA

19.

Weng-Tse Cheng

Energy and Mining

Research/Service organization

Industrial Technology Research Inst.

1 Tun-Hwa South Road

Tapei 105

Taiwan

REPUBLIC OF CHINA

20.

Prof. Ender Okandan

Midale East Technical University

Petroleum Engineering Dept.

Ionu Bulvari

Ankara

TURKEY

21.

Prof. Vladimir Kononov

Chairman of the scientific Council

on Geothermal Investigations

USSR Academy of Sciences

Staromonetny Per. 35, Room 414

Moscow 109017

USSR

22.

Dr. James Rannels

U.S. Department of Energy

1000 Independence Avenue SW

CE-342

Washington, DC 20589

USA 
23.

Mr. David Anderson

Executive Director

National Geothermal Association

P.O. Box 1350

Davis California 95617-1350

USA

24.

Gerald W. Huttrer

Geothermal Management Company, Inc.

P.O. Box 2980

27972 Meadow Dr. Suite 340

Evergreen, Colorado 80439

USA

ngamail.lst 